Supporting information

\title{
Multiscale Quantum Refinement Approaches for Metalloproteins
}

\author{
Zeyin Yan, Xin Li, Lung Wa Chung* \\ Shenzhen Grubbs Institute, Department of Chemistry and Guangdong Provincial Key \\ Laboratory of Catalysis, Southern University of Science and Technology, Shenzhen \\ 518055, China
}

1. Protein structure preparation. S2

2. Crystallography \& NMR System (CNS) program preparation... ... S3

3. Molecular mechanics force field parameters . S3

4. ONIOM layers and optimized regions definition ....................................................S4

5. Energy computations of multicentered (MC) ONIOM .................................. S6

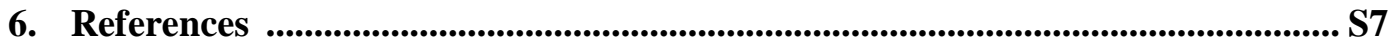

7. Quantum refined results of Rusticyanin (RC) .................................................... S9

8. Quantum refined results of MYST histone acetyltransferase ........................... S16

9. Quantum refined results of Amyloid- $\beta$....................................................................S41

10. Cartesian coordinates of quantum refinement results ........................................... S46 


\section{Protein structure preparation}

In our quantum refinement applications, three protein structures taken from protein data bank (PDB) were used as the initial structures for the quantum refinement calculations. Before the quantum refinement calculations, protonated state of amino acid residues was checked. WHATCHECK ${ }^{1,2}$ was also used to check the quality of protein structures. Moreover, the rotamer of Asn, Gln, His residues and ligands were checked and determined on the basis of their local structures and hydrogen bond network. Furthermore, $\mathrm{pKa}$ values of amino acid residues were calculated by PROPKA 3.0 program at the $\mathrm{pH}$ of crystallization and hydrogen atoms were added by PDB2PQR 2.0.0 program. ${ }^{3,4}$.

\section{A. Rusticyanin (RC)}

Protonation state: HID39, HID57, HIE85, HID128, CYM138, HIE143; the other titratable residues remain the standard protonation state.

The flipped residues: His57, Gln139

\section{B. Histone acetyltransferase}

Protonation state: HID513, CYM540, CYM543, HID556, CYM563, HID579, HID586, HIE603, HIP627, HID743, HID755; ASP520 (anionic amide form); the other titratable residues remain the standard protonation state.

The flipped residues: His579, His603, His627, Gln717, Gln731, Asn734

\section{Amyloid- $\beta(20-34)$ with L-isoAsp23}

Protonation state: PHE20 (with $-\mathrm{NH}_{3}{ }^{+}$terminus), LEU34 (with $-\mathrm{CO}_{2}{ }^{-}$terminus); the other titratable residues remain the standard protonation state. 


\section{Crystallography \& NMR System (CNS) program preparation}

In our quantum refinement calculations, CNS program calculated the crystallographic penalty function and its corresponding derivatives $\left(\mathrm{E}_{\mathrm{xray}} \& \nabla \mathrm{E}_{\mathrm{xray}}\right)$ based on the given structure and X-ray structure factors. Therefore, the minimization step is set to zero in the CNS refinement input file. The topology and parameters of the ligands (such as the mutated residue ALY from lysine) for our CNS refinement calculations were downloaded from HIC-UP database 5 . Whereas, AceDRG and the CNS tool (cns_import_cif) were used to generate topology and parameter files for the unknown ligands (such as FCV in histone acetyltransferase).

\section{Molecular mechanics force field parameters}

Since $\mathrm{ONIOM}^{6}$ method implemented in Gaussian 16 package was used, ${ }^{7}$ amber force field parameters implemented were used for our MM calculations except for metal ions and unknown ligands.

For the metal ions, the published Lennard-Jones non-bonded model parameters for copper $^{8}$, zinc ${ }^{9}$ and magnesium ${ }^{10}$ ions were used.

RESP (Restrained Electrostatic Potential) ${ }^{11}$ charges calculated at HF/6-31G(d) level ${ }^{12}$ and the similar AMBER atom types were applied for the mutated residue ALY and FCV ligand in the case of the histone acetyltransferase system.

For the magnesium binding site in the histone acetyltransferase, its amide should become deprotonated, due to a short distance between $\mathrm{N}_{\mathrm{ASP} 520}$ and magnesium ion (3.2 A, Figure S1). The revised force field parameter (RESP atomic charges) for this binding site are given in Table S1, based on standard RESP re-fitting calculations (at HF/6-31(d) level) on a dipeptide model of ILE519 and ASP520.

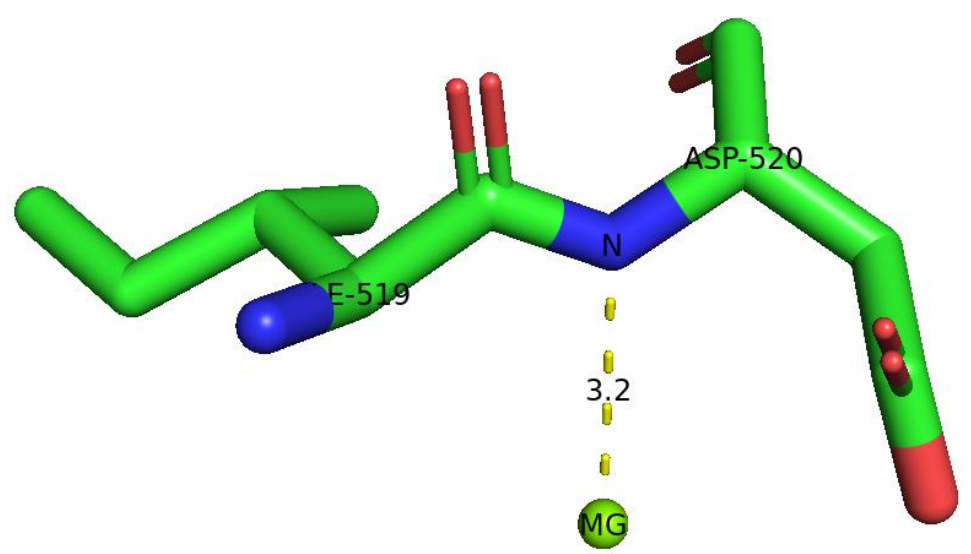

Figure S1. Local structure for the Mg binding site in the histone acetyltransferase 
Table S1. RESP charges calculated at HF/6-31G(d) level and atom type in the histone acetyltransferase

\begin{tabular}{|c|c|c|c|c|c|c|c|c|c|c|}
\hline \multicolumn{11}{|l|}{ ALY604: } \\
\hline Atom type & $\mathrm{N}$ & $\mathrm{H}$ & $\mathrm{CT}$ & $\mathrm{CT}$ & $\mathrm{CT}$ & $\mathrm{CT}$ & $\mathrm{CT}$ & $\mathrm{N}$ & $\mathrm{C}$ & $\mathrm{O}$ \\
\hline charge & -0.6081 & 0.3518 & -0.0206 & -0.5133 & -0.0226 & -0.1307 & -0.3000 & -0.5793 & 0.8771 & -0.6258 \\
\hline Atom type & $\mathrm{CT}$ & $\mathrm{HC}$ & $\mathrm{HC}$ & $\mathrm{HC}$ & $\mathrm{H}$ & H1 & H1 & $\mathrm{HC}$ & $\mathrm{HC}$ & $\mathrm{HC}$ \\
\hline charge & -0.5526 & 0.1531 & 0.1531 & 0.1531 & 0.3012 & 0.1854 & 0.1854 & 0.1013 & 0.1013 & 0.0858 \\
\hline Atom type & $\mathrm{HC}$ & $\mathrm{HC}$ & $\mathrm{HC}$ & $\mathrm{H} 1$ & $\mathrm{C}$ & $\mathrm{O}$ & & & & \\
\hline charge & 0.0858 & 0.1942 & 0.1942 & 0.1364 & 0.6616 & -0.5675 & & & & \\
\hline \multicolumn{11}{|l|}{ FCV801: } \\
\hline Atom type & $\mathrm{CA}$ & HA & $\mathrm{CA}$ & HA & $\mathrm{CA}$ & HA & $\mathrm{CA}$ & $\mathrm{H} 4$ & $\mathrm{NC}$ & $\mathrm{CA}$ \\
\hline charge & -0.5492 & 0.1812 & 0.1657 & 0.1179 & -0.4574 & 0.1792 & 0.3857 & 0.0690 & -0.6868 & 0.7469 \\
\hline Atom type & $\mathrm{CA}$ & $\mathrm{CA}$ & $\mathrm{CA}$ & $\mathrm{CA}$ & HA & $\mathrm{F}$ & HA & $\mathrm{CA}$ & HA & $\mathrm{CA}$ \\
\hline charge & -0.0215 & -0.2847 & 0.3872 & -0.2887 & 0.1919 & -0.2275 & 0.1708 & -0.2444 & 0.1746 & -0.0696 \\
\hline Atom type & $\mathrm{C}$ & $\mathrm{O}$ & $\mathrm{N}$ & $\mathrm{H}$ & NT & $\mathrm{H}$ & SO & $\mathrm{O}$ & $\mathrm{O}$ & $\mathrm{CA}$ \\
\hline charge & 0.7728 & -0.5062 & -0.5160 & 0.3593 & -0.5628 & 0.4651 & 0.9182 & -0.5210 & -0.5210 & -0.0973 \\
\hline Atom type & $\mathrm{CA}$ & HA & $\mathrm{CA}$ & HA & $\mathrm{CA}$ & HA & $\mathrm{CA}$ & HA & $\mathrm{CA}$ & $\mathrm{F}$ \\
\hline charge & -0.0200 & 0.1535 & -0.2395 & 0.1789 & -0.0465 & 0.1653 & -0.3351 & 0.2012 & 0.4473 & -0.2365 \\
\hline \multicolumn{11}{|l|}{ ILE519: } \\
\hline Atom type & $\mathrm{N}$ & $\mathrm{CT}$ & $\mathrm{C}$ & $\mathrm{O}$ & $\mathrm{CT}$ & $\mathrm{CT}$ & $\mathrm{CT}$ & $\mathrm{CT}$ & H1 & $\mathrm{HC}$ \\
\hline charge & -0.5562 & -0.4434 & 1.1689 & -0.9351 & 0.3264 & 0.0677 & -0.1574 & -0.2506 & 0.2117 & 0.0255 \\
\hline Atom type & $\mathrm{HC}$ & $\mathrm{HC}$ & $\mathrm{HC}$ & $\mathrm{HC}$ & $\mathrm{HC}$ & $\mathrm{H}$ & $\mathrm{HC}$ & $\mathrm{HC}$ & $\mathrm{HC}$ & \\
\hline charge & 0.0255 & -0.0476 & -0.0201 & -0.0201 & 0.0323 & 0.3057 & 0.0323 & 0.0255 & 0.0323 & \\
\hline \multicolumn{11}{|l|}{ ASP520: } \\
\hline Atom type & $\mathrm{N}$ & $\mathrm{CT}$ & $\mathrm{C}$ & $\mathrm{O}$ & $\mathrm{CT}$ & $\mathrm{C}$ & $\mathrm{O} 2$ & $\mathrm{O} 2$ & $\mathrm{H} 1$ & $\mathrm{HC}$ \\
\hline charge & -1.0512 & 0.4485 & 0.5530 & -0.6284 & -0.3790 & 0.9318 & -0.8712 & -0.8712 & -0.0426 & 0.0434 \\
\hline Atom type & $\mathrm{HC}$ & & & & & & & & & \\
\hline charge & 0.0434 & & & & & & & & & \\
\hline
\end{tabular}

\section{Definition of ONIOM layers and optimized regions}

\section{A. Rusticyanin (RC)}

ONIOM layers were defined with the copper ion as the center atom:

High layer: all residues within a radius $\sim 4.0 \AA$ to the $\mathrm{Cu}$ center (117 atoms, including 17 link atoms):

PRO52, HIE85, SER86, CYM138, ILE140, HIE143, MET148, CU156

Medium layer for the ONIOM3 cases: all residues within a radius $\sim 8.0 \AA$ to the $\mathrm{Cu}$ center, (449 atoms, including 15 link atoms and the high-layer atoms):

ALA43, ALA44, VAL45, LEU46, PRO50, PHE51, SER53, PHE54, GLU55, THR75, PHE76, ILE77, ASN78, THR79, ASN80, LYS81, GLY82, PHE83, GLY84, PHE87, ASP88, GLY110, PHE111, SER112, TYR136, VAL137, GLN139, ILE140, PRO141, GLY142, HIE143, ALA144, ALA145, THR146, GLY147, MET148, PHE149, 
HOH212, HOH221, HOH238, PHE51, PRO52, SER53, GLY84, HIE85, SER86, PHE87, VAL137, CYM138, GLN139, ILE140, HIE143, MET148, CU156

Optimized regions:

R1a: all atoms in the high layer (117 atoms):

PHE51, PRO52, SER53, GLY84, HIE85, SER86, PHE87, VAL137, CYM138, GLN139, ILE140, HIE143, MET148 and CU156

$\mathrm{R} 2 \mathrm{a}$ : all residues within a radius of $\sim 8.0 \AA$ to the $\mathrm{Cu}$ center (396 atoms):

ALA44, VAL45, PHE51, PRO52, SER53, PHE54, PHE76, ASN78, ASN80, PHE83, GLY84, HIE85, SER86, PHE87, PHE111, SER112, VAL137, CYM138, GLN139, ILE140, PRO141, GLY142, HIE143, ALA144, THR146, MET148, CU156, $\mathrm{HOH} 212, \mathrm{HOH} 221, \mathrm{HOH} 238$

R3a: all atoms in the medium layer for the ONIOM3 cases (449 atoms):

ALA43, ALA44, VAL45, LEU46, PRO50, PHE51, PRO52, SER53, PHE54, GLU55, THR75, PHE76, ILE77, ASN78, THR79, ASN80, LYS81, GLY82, PHE83, GLY84, HIE85, SER86, PHE87, ASP88, GLY110, PHE111, SER112, TYR136, VAL137, CYM138, GLN139, ILE140, PRO141, GLY142, HIE143, ALA144, ALA145, THR146, GLY147, MET148, PHE149, CU156, HOH212, HOH221, HOH238

\section{B. Histone acetyltransferase}

ONIOM layers were defined with the zinc ion and FCV ligand as the center atoms:

High layer: FCV ligand and all residues within a $\sim .0 \AA$ to the $\mathrm{Zn}$ center (112 atoms, including 8 link atoms):

CYM540, CYM543, HID556 and CYM560, FCV801, ZN802

Medium layer: all residues within a radius $\sim 3.0 \AA$ to the FCV center and all residues within a radius $\sim 5.0 \AA$ to the $\mathrm{Zn}$ center (592 atoms, including 24 link atoms and the high-layer atoms):

GLU541, TYR542, LEU544, LYS545, TYR546, GLN561, TRP562, ARG563, LEU599, PHE600, LEU601, ASP602, CYS646, ILE647, MET648, ILE649, LEU650, TYR653，GLN654，ARG655，ARG656，GLY657，TYR658，GLY659，ARG660, PHE661, LEU662, ILE663, ALA664, LEU683, SER684, ASP685, LEU686, GLY687, LYS688, LEU689, SER690, TYR691, ARG692, SER693, TYR694, TRP695, HOH904, HOH938, HOH950, HOH951, HOH981, HOH983, HOH1008, HOH1022, LEU539, CYM540, GLU541, TYR542, CYM543, LEU544, PHE555, HID556, LEU557, GLN559, CYM560, GLN561, FCV801, ZN802

Optimized regions:

R1b: all atoms in the high layer (112 atoms): 
LEU539, CYM540, GLU541, TYR542, CYM543, LEU544, PHE555, HID556, LEU557, GLN559, CYM560, GLN561, FCV801, ZN802

R2b: all residues within a radius of $\sim 3.0 \AA$ to the FCV center and all residues within a radius to $\sim 5.0 \AA$ around the $\mathrm{Zn}$ center (528 atoms):

LEU539, CYM540, GLU541, TYR542, CYM543, LEU544, LYS545, PHE555, HID556, LEU557, GLN559, CYM560, GLN561, TRP562, PHE600, LEU601, ILE647, MET648, ILE649, GLN654, ARG655, ARG656, GLY657, TYR658, GLY659, ARG660, ILE663, SER684, LEU686, GLY687, SER690, SER693, TYR694, FCV801, ZN802, HOH904, HOH938, HOH950, HOH951, HOH981, HOH983, HOH1008, HOH1022

R3b: all atoms in the medium layer for ONIOM3 cases (592 atoms).

LEU539, CYM540, GLU541, TYR542, CYM543, LEU544, LYS545, TYR546, PHE555, HID556, LEU557, GLN559, CYM560, GLN561, TRP562, ARG563, LEU599, PHE600, LEU601, ASP602, CYS646, ILE647, MET648, ILE649, LEU650, TYR653, GLN654, ARG655, ARG656, GLY657, TYR658, GLY659, ARG660, PHE661, LEU662, ILE663, ALA664, LEU683, SER684, ASP685, LEU686, GLY687, LYS688, LEU689, SER690, TYR691, ARG692, SER693, TYR694, TRP695, FCV801, ZN802, HOH904, HOH938, HOH950, HOH951, HOH981, HOH983, HOH1008, HOH1022

\section{Energy expression for multicentered (MC) ONIOM schemes}

\section{ONIOM2:}

$\mathbf{E}(1 \mathrm{C}-$ ONIOM2 $)=\mathrm{E}_{\mathrm{Low}}($ Real $)+\mathrm{E}_{\mathrm{High}}\left(\operatorname{Model}_{1}+\operatorname{Model}_{2}\right)-\mathrm{E}_{\mathrm{Low}}\left(\mathrm{Model}_{1}+\mathrm{Model}_{2}\right)$

$$
\begin{aligned}
\mathbf{E}(2 \mathrm{C}-\text { ONIOM2 })= & \mathrm{E}_{\mathrm{Low}}(\text { Real })+\mathrm{E}_{\mathrm{High}}\left(\operatorname{Model}_{1}\right)-\mathrm{E}_{\mathrm{Low}}\left(\mathrm{Model}_{1}\right) \\
& +\mathrm{E}_{\mathrm{High}}\left(\operatorname{Model}_{2}\right)-\mathrm{E}_{\mathrm{Low}}\left(\operatorname{Model}_{2}\right)
\end{aligned}
$$

\section{ONIOM3:}

$$
\begin{aligned}
\mathbf{E}(\mathbf{1 C}-\text { ONIOM3 })= & \mathrm{E}_{\mathrm{Low}}(\text { Real })+\mathrm{E}_{\text {Medium }}(\text { intermediate })-\mathrm{E}_{\text {Low }}(\text { intermediate }) \\
& +\mathrm{E}_{\mathrm{High}}\left(\operatorname{Model}_{1}+\operatorname{Model}_{2}\right)-\mathrm{E}_{\mathrm{Medium}}\left(\operatorname{Model}_{1}+\mathrm{Model}_{2}\right)
\end{aligned}
$$

$\mathbf{E}(\mathbf{2 C}-\mathbf{O N I O M 3})=\mathrm{E}_{\text {Low }}($ Real $)+\mathrm{E}_{\text {Medium }}($ intermediate $)-\mathrm{E}_{\text {Low }}($ intermediate $)$

$$
\begin{aligned}
& +\mathrm{E}_{\mathrm{High}}\left(\operatorname{Model}_{1}\right)-\mathrm{E}_{\text {Medium }}\left(\operatorname{Model}_{1}\right) \\
& +\mathrm{E}_{\mathrm{High}}\left(\operatorname{Model}_{2}\right)-\mathrm{E}_{\text {Medium }}\left(\operatorname{Model}_{2}\right)
\end{aligned}
$$




\section{References:}

(1) Vriend, G. WHAT IF: A Molecular Modeling and Drug Design Program. $J$. Mol. Graphics 1990, 8, 52-56.

(2) Hooft, R. W.; Vriend, G.; Sander, C.; Abola, E. E. Errors in protein structures. Nature 1996, 381, 272.

(3) Olsson, M. H.; Sondergaard, C. R.; Rostkowski, M.; Jensen, J. H. PROPKA3: Consistent Treatment of Internal and Surface Residues in Empirical pKa Predictions. J. Chem. Theory Comput. 2011, 7, 525-537.

(4) Dolinsky, T. J.; Nielsen, J. E.; McCammon, J. A.; Baker, N. A. PDB2PQR: an automated pipeline for the setup of Poisson-Boltzmann electrostatics calculations. Nucleic Acids Res. 2004, 32, W665-W667.

(5) Kleywegt, G. J. Crystallographic refinement of ligand complexes. Acta Crystallogr. Sect. D., Biol. Crystallogr. 2007, 63, 94-100.

(6) Dapprich, S.; Komaromi, I.; Byun, K. S.; Morokuma, K.; Frisch, M. J. A new ONIOM implementation in Gaussian98. Part I. The calculation of energies, gradients, vibrational frequencies and electric field derivatives. J. Mol. Struct.: THEOCHEM 1999, 461, 1-21.

(7) Frisch, M. J.; Trucks, G. W.; Schlegel, H. B.; Scuseria, G. E.; Robb, M. A.; Cheeseman, J. R.; Scalmani, G.; Barone, V.; Petersson, G. A.; Nakatsuji, H.; Li, X.; Caricato, M.; Marenich, A. V.; Bloino, J.; Janesko, B. G.; Gomperts, R.; Mennucci, B.; Hratchian, H. P.; Ortiz, J. V.; Izmaylov, A. F.; Sonnenberg, J. L.; Williams; Ding, F.; Lipparini, F.; Egidi, F.; Goings, J.; Peng, B.; Petrone, A.; Henderson, T.; Ranasinghe, D.; Zakrzewski, V. G.; Gao, J.; Rega, N.; Zheng, G.; Liang, W.; Hada, M.; Ehara, M.; Toyota, K.; Fukuda, R.; Hasegawa, J.; Ishida, M.; Nakajima, T.; Honda, Y.; Kitao, O.; Nakai, H.; Vreven, T.; Throssell, K.; Montgomery Jr., J. A.; Peralta, J. E.; Ogliaro, F.; Bearpark, M. J.; Heyd, J. J.; Brothers, E. N.; Kudin, K. N.; Staroverov, V. N.; Keith, T. A.; Kobayashi, R.; Normand, J.; Raghavachari, K.; Rendell, A. P.; Burant, J. C.; Iyengar, S. S.; Tomasi, J.; Cossi, M.; Millam, J. M.; Klene, M.; Adamo, C.; Cammi, R.; Ochterski, J. W.; Martin, R. L.; Morokuma, K.; Farkas, O.; Foresman, J. B.; Fox, D. J. Gaussian 16 Rev. A.03, Gaussian Inc.: Wallingford, CT, 2016.

(8) Comba, P.; Remenyi, R. A new molecular mechanics force field for the oxidized form of blue copper proteins. J. Comput. Chem. 2002, 23, 697-705.

(9) Hoops, S. C.; Anderson, K. W.; Merz, K. M. Force field design for metalloproteins. J. Am. Chem. Soc. 1991, 113, 8262-8270.

(10) Aqvist, J. Ion Water Interaction Potentials Derived from Free-Energy Perturbation Simulations. J. Phys. Chem. 1990, 94, 8021-8024.

(11) Bayly, C. I.; Cieplak, P.; Cornell, W. D.; Kollman, P. A. A Well-Behaved Electrostatic Potential Based Method Using Charge Restraints for Deriving Atomic Charges - the Resp Model. J. Phys. Chem. 1993, 97, 10269-10280.

(12) Roothaan, C. C. J. New Developments in Molecular Orbital Theory. Rev. Mod. Phys. 1951, 23, 69-89.

(13) Weigend, F.; Ahlrichs, R. Balanced basis sets of split valence, triple zeta valence and quadruple zeta valence quality for $\mathrm{H}$ to $\mathrm{Rn}$ : Design and assessment of accuracy. Phys. Chem. Chem. Phys. 2005, 7, 3297-3305.

(14) Weigend, F. Accurate Coulomb-fitting basis sets for H to Rn. Phys. Chem. Chem. Phys. 2006, 8, 1057-1065. 
(15) Neese, F.; Wennmohs, F.; Hansen, A.; Becker, U. Efficient, approximate and parallel Hartree-Fock and hybrid DFT calculations. A 'chain-of-spheres' algorithm for the Hartree-Fock exchange. Chem. Phys. 2009, 356, 98-109.

(16) Neese, F. Software update: the ORCA program system, version 4.0. Wiley Interdiscip. Rev.: Comput. Mol. Sci. 2018, 8, No. e1327.

(17) Li, W.; Li, S. H.; Jiang, Y. S. Generalized energy-based fragmentation approach for computing the ground-state energies and properties of large molecules. J. Phys. Chem. A 2007, 111, 2193-2199.

(18) Yang, Z.; Hua, S. G.; Hua, W. J.; Li, S. H. Low-Lying Structures and Stabilities of Large Water Clusters: Investigation Based on the Combination of the AMOEBA Potential and Generalized Energy-Based Fragmentation Approach. $J$. Phys. Chem. A 2010, 114, 9253-9261.

(19) Hua, W. J.; Fang, T.; Li, W.; Yu, J. G.; Li, S. H. Geometry Optimizations and Vibrational Spectra of Large Molecules from a Generalized Energy-Based Fragmentation Approach. J. Phys. Chem. A 2008, 112, 10864-10872.

(20) Li, S. H.; Li, W.; Ma, J. Generalized Energy-Based Fragmentation Approach and Its Applications to Macromolecules and Molecular Aggregates. Acc. Chem. Res. 2014, 47, 2712-2720.

(21) Li, W.; Chen, C. H.; Zhao, D. B.; Li, S. H. LSQC: Low Scaling Quantum Chemistry Program. Int. J. Quantum Chem. 2015, 115, 641-646. 


\section{Quantum refined results of Rusticyanin (RC)}

Table S2. Our quantum refined key structural results for the copper binding site using different DFT functionals (B3LYP, B3LYP-D3, or M06L) and basis set (6-31G(d) or $\mathrm{SDD}$ for copper) in the ONIOM(QM/MM) scheme with the R1a-optimized region and $\omega_{\alpha}$ value of 1.0. M6 R2a results are used as reference for RMSD and MAD computaions.

\begin{tabular}{ccccc}
\hline & M1 & M2 & M3 & M4 \\
\hline $\mathrm{Cu}-\mathrm{N}_{\mathrm{H} 85}$ & 2.14 & 2.14 & 2.14 & 2.16 \\
$\mathrm{Cu}-\mathrm{N}_{\mathrm{H} 143}$ & 1.96 & 1.96 & 1.96 & 1.99 \\
$\mathrm{Cu}-\mathrm{S}_{\mathrm{C} 138}$ & 2.26 & 2.26 & 2.26 & 2.27 \\
$\mathrm{Cu}-\mathrm{S}_{\mathrm{M} 148}$ & 2.87 & 2.88 & 2.86 & 2.88 \\
\hline $\mathrm{RMSD}$ (bonds) & 0.02 & 0.02 & 0.02 & 0.00 \\
$\mathrm{MAD}$ (bonds) & 0.02 & 0.02 & 0.02 & 0.00 \\
\hline $\mathrm{N}_{\mathrm{H} 85}-\mathrm{Cu}-\mathrm{N}_{\mathrm{H} 143}$ & 102.1 & 102.5 & 101.8 & 102.4 \\
$\mathrm{~N}_{\mathrm{H} 85-\mathrm{Cu}-\mathrm{S}_{\mathrm{C} 138}}$ & 127.5 & 127.1 & 127.4 & 127.0 \\
$\mathrm{~N}_{\mathrm{H} 85}-\mathrm{Cu}-\mathrm{S}_{\mathrm{M} 148}$ & 88.0 & 88.0 & 88.2 & 88.0 \\
$\mathrm{~S}_{\mathrm{M} 148}-\mathrm{Cu}-\mathrm{S}_{\mathrm{C} 138}$ & 105.8 & 105.8 & 105.8 & 105.7 \\
$\mathrm{~N}_{\mathrm{H} 143}-\mathrm{Cu}-\mathrm{S}_{\mathrm{C} 138}$ & 120.9 & 121.0 & 121.1 & 121.3 \\
\hline $\mathrm{RMSD}($ angles) & 0.5 & 0.4 & 0.5 & 0.3 \\
$\mathrm{MAD}$ (angles) & 0.5 & 0.3 & 0.5 & 0.2 \\
\hline $\mathrm{R} / \mathrm{R}_{\text {free }}$ & $0.162 / 0.221$ & $0.162 / 0.22$ & $0.163 / 0.221$ & $0.162 / 0.221$ \\
\hline
\end{tabular}

Table S3. Our key structural results for the copper binding site from quantum refinement $\left(\omega_{\alpha}\right.$ value of 1.0$)$ in the $\operatorname{ONIOM}(\mathrm{QM} / \mathrm{MM})$ scheme with the R1aoptimized region in protein and optimization of $\mathrm{R} 1 \mathrm{a}$-optimized region in gas phase using B3LYP/6-31G(d) and B3LYP-D3/6-31G(d) (in parenthesis).

\begin{tabular}{ccc}
\hline & Protein & Gas \\
\hline $\mathrm{Cu}-\mathrm{N}_{\mathrm{H} 85}$ & $2.14(2.14)$ & $1.99(2.00)$ \\
$\mathrm{Cu}-\mathrm{N}_{\mathrm{H} 143}$ & $1.96(1.96)$ & $1.98(1.95)$ \\
$\mathrm{Cu}-\mathrm{S}_{\mathrm{C} 138}$ & $2.26(2.26)$ & $2.23(2.22)$ \\
$\mathrm{Cu}-\mathrm{S}_{\mathrm{M} 148}$ & $2.87(2.88)$ & $2.46(2.41)$ \\
\hline $\mathrm{N}_{\mathrm{H} 85}-\mathrm{Cu}-\mathrm{N}_{\mathrm{H} 143}$ & $102.1(102.5)$ & $96.3(98.0)$ \\
$\mathrm{N}_{\mathrm{H} 85}-\mathrm{Cu}-\mathrm{S}_{\mathrm{C} 138}$ & $127.5(127.1)$ & $139.8(135.0)$ \\
$\mathrm{N}_{\mathrm{H} 85-\mathrm{Cu}-\mathrm{S}_{\mathrm{M} 148}}$ & $88.0(88.0)$ & $88.6(88.9)$ \\
$\mathrm{S}_{\mathrm{M} 148}-\mathrm{Cu}-\mathrm{S}_{\mathrm{C} 138}$ & $105.8(105.8)$ & $109.2(106.6)$ \\
$\mathrm{N}_{\mathrm{H} 143}-\mathrm{Cu}-\mathrm{S}_{\mathrm{C} 138}$ & $120.9(121.0)$ & $94.5(93.4)$ \\
\hline
\end{tabular}


Table S4. RSZD scores of residues around the $\mathrm{Cu}(\mathrm{II})$ binding site in the rusticyanin protein from quantum refinement approaches using B3LYP/6-31G(d) (M1) and B3LYP-D3/6-31G(d) (M2) with $\omega_{\alpha}$ value of 1.0. RSZD scores of the FCV binding site were also given.

\begin{tabular}{cccc}
\hline & X-ray & M1 & M2 \\
\hline PRO52 & 1.3 & 1.3 & 1.4 \\
HIE85 & 1.4 & 0.2 & 0.3 \\
SER86 & 2.3 & 0.5 & 0.6 \\
CYM138 & 1.0 & 0.8 & 0.8 \\
ILE140 & 0.3 & 0.3 & 0.3 \\
HIE143 & 1.5 & 0.4 & 0.4 \\
MET148 & 0.8 & 0.9 & 0.8 \\
Cu(II) & 2.7 & 3.1 & 3.0 \\
\hline Sum & 11.3 & 7.5 & 7.6 \\
\hline
\end{tabular}

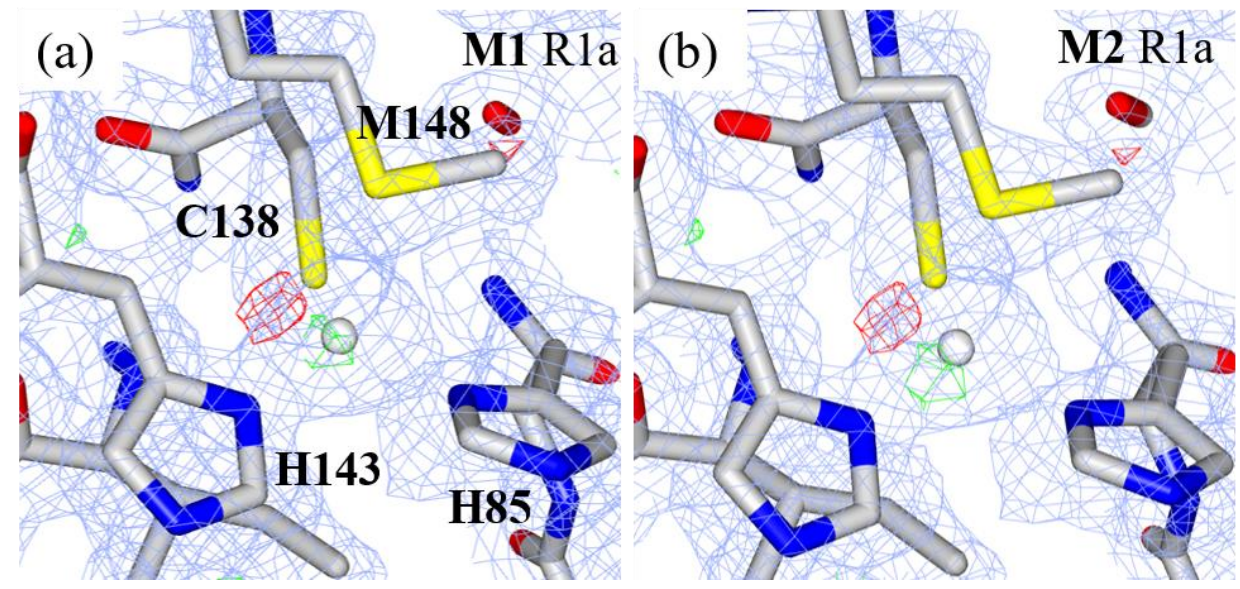

Figure S2. Structures for the $\mathrm{Cu}(\mathrm{II})$ binding site in the rusticyanin protein from various quantum refinement schemes with $\omega_{\alpha}$ value of 1.0 using (a) M1 (with the R1a-optimized region), (b) M2 (with the R1a-optimized region), including the electron density map (2mFo-DFc maps, contoured at $1.0 \sigma$ (blue), mFo-DFc maps, contoured at $+3.0 \sigma$ (green), and $\mathrm{mFo}-\mathrm{DFc}$ maps, contoured at $-3.0 \sigma$ (red)). 
Table S5. Our quantum refined key structural results for the copper binding site using mechanical embedding (ONIOM-ME) and electrostatic embedding (ONIOM-EE; in parenthesis) ONIOM (M1) schemes with the R1a- and R2a-optimized regions and $\omega_{\alpha}$ value of 1.0. M6 R2a results are used as reference for RMSD and MAD computaions.

\begin{tabular}{ccc}
\hline & M1 R1a & M1 R2a \\
\hline $\mathrm{Cu}-\mathrm{N}_{\mathrm{H} 85}$ & $2.14(2.14)$ & $2.14(2.14)$ \\
$\mathrm{Cu}-\mathrm{N}_{\mathrm{H} 143}$ & $1.96(1.98)$ & $1.96(1.98)$ \\
$\mathrm{Cu}-\mathrm{S}_{\mathrm{C} 138}$ & $2.26(2.26)$ & $2.26(2.25)$ \\
$\mathrm{Cu}-\mathrm{S}_{\mathrm{M} 148}$ & $2.87(2.88)$ & $2.87(2.88)$ \\
\hline $\mathrm{RMSD}$ (bonds) & $0.02(0.02)$ & $0.01(0.01)$ \\
$\mathrm{MAD}$ (bonds) & $0.02(0.02)$ & $0.01(0.01)$ \\
\hline $\mathrm{N}_{\mathrm{H} 85}-\mathrm{Cu}-\mathrm{N}_{\mathrm{H} 143}$ & $102.1(102.0)$ & $102.6(102.4)$ \\
$\mathrm{N}_{\mathrm{H} 85}-\mathrm{Cu}-\mathrm{S}_{\mathrm{C} 138}$ & $127.5(127.4)$ & $126.8(126.9)$ \\
$\mathrm{N}_{\mathrm{H} 85}-\mathrm{Cu}-\mathrm{S}_{\mathrm{M} 148}$ & $88.0(88.0)$ & $88.2(88.2)$ \\
$\mathrm{S}_{\mathrm{M} 148}-\mathrm{Cu}-\mathrm{S}_{\mathrm{C} 138}$ & $105.8(105.9)$ & $105.6(105.7)$ \\
$\mathrm{N}_{\mathrm{H} 143}-\mathrm{Cu}-\mathrm{S}_{\mathrm{C} 138}$ & $120.9(121.0)$ & $121.4(121.4)$ \\
\hline $\mathrm{RMSD}($ angles $)$ & $0.5(0.5)$ & $0.2(0.2)$ \\
$\mathrm{MAD}$ (angles) & $0.5(0.5)$ & $0.1(0.1)$ \\
\hline \multirow{2}{*}{$\mathrm{R} / \mathrm{R}_{\text {free }}$} & $0.162 / 0.221$ & $0.162 / 0.216$ \\
& $(0.162 / 0.221)$ & $(0.162 / 0.216)$ \\
\hline
\end{tabular}

Table S6. Our quantum refined key structural results for the copper binding site using different multiscale schemes with different optimized regions (R1a, R2a or R3a). Different values of the weighting factor (1.0 and 0.606 (in parenthesis)) are used. The key structural results from the X-ray crystal structure were also given. M6 R2a results are used as reference for RMSD and MAD computaions.

\begin{tabular}{|c|c|c|c|c|c|c|c|}
\hline & M1 R1a & M1 R2a & M5 R1a & M5 R3a & M6 R1a & M6 R2a & X-ray \\
\hline $\mathrm{Cu}-\mathrm{N}_{\mathrm{H} 85}$ & $2.14(2.11)$ & $2.14(2.11)$ & $2.17(2.14)$ & $2.16(2.14)$ & $2.17(2.14)$ & $2.16(2.14)$ & 2.04 \\
\hline $\mathrm{Cu}-\mathrm{N}_{\mathrm{H} 143}$ & $1.96(1.97)$ & $1.98(1.98)$ & $1.98(1.99)$ & $2.00(1.99)$ & $2.00(1.98)$ & $1.99(1.98)$ & 1.89 \\
\hline $\mathrm{Cu}-\mathrm{S}_{\mathrm{C} 138}$ & $2.26(2.25)$ & $2.26(2.25)$ & $2.26(2.25)$ & $2.25(2.25)$ & $2.26(2.25)$ & $2.26(2.25)$ & 2.26 \\
\hline $\mathrm{Cu}-\mathrm{S}_{\mathrm{M} 148}$ & $2.87(2.87)$ & $2.88(2.88)$ & $2.87(2.88)$ & $2.88(2.88)$ & $2.87(2.87)$ & $2.88(2.88)$ & 2.88 \\
\hline RMSD (bonds) & $0.02(0.02)$ & $0.01(0.01)$ & $0.01(0.00)$ & $0.00(0.00)$ & $0.01(0.00)$ & $0.00(0.00)$ & $0.08(0.07)$ \\
\hline MAD (bonds) & $0.02(0.01)$ & $0.01(0.01)$ & $0.01(0.00)$ & $0.00(0.00)$ & $0.01(0.00)$ & $0.00(0.00)$ & $0.06(0.05)$ \\
\hline $\mathrm{N}_{\mathrm{H} 85}-\mathrm{Cu}-\mathrm{N}_{\mathrm{H} 143}$ & $102.1(102.1)$ & $102.6(102.8)$ & $101.8(102.1)$ & $102.0(102.3)$ & $102.1(102.1)$ & $102.5(102.3)$ & 105.4 \\
\hline $\mathrm{N}_{\mathrm{H} 85}-\mathrm{Cu}-\mathrm{S}_{\mathrm{C} 138}$ & $127.5(127.5)$ & $126.8(126.6)$ & $127.4(127.1)$ & $127.2(126.9)$ & $127.2(127.0)$ & $126.7(126.9)$ & 127.9 \\
\hline $\mathrm{N}_{\mathrm{H} 85}-\mathrm{Cu}-\mathrm{S}_{\mathrm{M} 148}$ & $88.0(87.6)$ & $88.2(88.2)$ & $88.0(87.8)$ & $88.2(88.0)$ & $87.9(87.9)$ & $88.5(88.2)$ & 85.3 \\
\hline $\mathrm{S}_{\mathrm{M} 148}-\mathrm{Cu}-\mathrm{S}_{\mathrm{C} 138}$ & $105.8(105.7)$ & $105.6(105.5)$ & $105.8(105.7)$ & $105.4(105.5)$ & $105.7(105.8)$ & 105.6(105.6) & 105.5 \\
\hline $\mathrm{N}_{\mathrm{H} 143}-\mathrm{Cu}-\mathrm{S}_{\mathrm{C} 138}$ & $120.9(121.2)$ & $121.4(121.5)$ & 121.3(121.6) & $121.8(121.9)$ & $121.3(121.5)$ & 121.5(121.6) & 119.1 \\
\hline RMSD (angles) & $0.5(0.4)$ & $0.2(0.3)$ & $0.5(0.2)$ & $0.3(0.2)$ & $0.4(0.2)$ & $0.0(0.0)$ & $2.3(2.3)$ \\
\hline MAD (angles) & $0.5(0.4)$ & $0.1(0.2)$ & $0.4(0.2)$ & $0.3(0.1)$ & $0.4(0.2)$ & $0.0(0.0)$ & $2.0(1.9)$ \\
\hline $\mathrm{R}$ & $0.162(0.163)$ & $0.162(0.162)$ & $0.163(0.163)$ & $0.162(0.162)$ & $0.162(0.163)$ & $0.161(0.162)$ & 0.163 \\
\hline $\mathrm{R}_{\text {free }}$ & $0.221(0.221)$ & $0.216(0.216)$ & $0.221(0.221)$ & $0.215(0.215)$ & $0.220(0.221)$ & $0.216(0.217)$ & 0.223 \\
\hline
\end{tabular}


Table S7. Strain energy $(\Delta \mathrm{E}, \mathrm{kcal} / \mathrm{mol})$ at $\mathrm{B} 3 \mathrm{LYP} / 6-31 \mathrm{G}(\mathrm{d})$ level for the R1aoptimized active-site region in the copper protein determined by various quantum refinement schemes. Different values of the weighting factor (1.0 and 0.606 (values given in parenthesis)) are used

\begin{tabular}{cc}
\hline & $\Delta \mathrm{E}$ \\
\hline X-ray & 258.0 \\
M1 R1a & $88.74(82.05)$ \\
M1 R2a & $89.85(80.44)$ \\
M5 R1a & $93.15(82.88)$ \\
M5 R3a & $91.57(82.31)$ \\
M6 R1a & $92.85(82.64)$ \\
M6 R2a & $89.92(82.53)$ \\
\hline
\end{tabular}


Table S8. Atomic displacements of copper binding site using different multiscale schemes with different optimized regions (R1a, R2a or R3a). Different values of the weighting factor 1.0 and 0.606 (values given in parenthesis) are used

\begin{tabular}{|c|c|c|c|c|c|c|}
\hline & M1 R1a & M1 R2a & M5 R1a & M5 R3a & M6 R1a & M6 R2a \\
\hline \multicolumn{7}{|l|}{ HIE85 } \\
\hline $\mathrm{N}$ & $0.07(0.05)$ & $0.10(0.09)$ & $0.07(0.03)$ & $0.11(0.09)$ & $0.06(0.03)$ & $0.09(0.08)$ \\
\hline $\mathrm{CA}$ & $0.05(0.04)$ & $0.07(0.08)$ & $0.04(0.02)$ & $0.07(0.06)$ & $0.03(0.03)$ & $0.06(0.06)$ \\
\hline $\mathrm{C}$ & $0.05(0.07)$ & $0.09(0.11)$ & $0.05(0.07)$ & $0.07(0.09)$ & $0.05(0.08)$ & $0.09(0.10)$ \\
\hline $\mathrm{O}$ & $0.07(0.06)$ & $0.09(0.09)$ & $0.08(0.08)$ & $0.09(0.09)$ & $0.08(0.09)$ & $0.10(0.10)$ \\
\hline $\mathrm{CB}$ & $0.10(0.09)$ & $0.11(0.09)$ & $0.13(0.11)$ & $0.12(0.11)$ & $0.13(0.12)$ & $0.12(0.11)$ \\
\hline $\mathrm{CG}$ & $0.11(0.08)$ & $0.12(0.10)$ & $0.13(0.11)$ & $0.13(0.11)$ & $0.13(0.11)$ & $0.14(0.11)$ \\
\hline ND1 & $0.08(0.07)$ & $0.08(0.07)$ & $0.11(0.09)$ & $0.10(0.08)$ & $0.11(0.10)$ & $0.10(0.09)$ \\
\hline $\mathrm{CD} 2$ & $0.09(0.09)$ & $0.09(0.07)$ & $0.12(0.10)$ & $0.11(0.09)$ & $0.12(0.11)$ & $0.10(0.10)$ \\
\hline CE1 & $0.06(0.06)$ & $0.09(0.09)$ & $0.05(0.05)$ & $0.08(0.08)$ & $0.05(0.05)$ & $0.07(0.06)$ \\
\hline NE2 & $0.05(0.06)$ & $0.07(0.08)$ & $0.05(0.06)$ & $0.07(0.08)$ & $0.05(0.05)$ & $0.07(0.06)$ \\
\hline \multicolumn{7}{|c|}{ CYM138 } \\
\hline $\mathrm{N}$ & $0.13(0.15)$ & $0.15(0.14)$ & $0.13(0.13)$ & $0.13(0.13)$ & $0.13(0.14)$ & $0.14(0.14)$ \\
\hline $\mathrm{CA}$ & $0.04(0.04)$ & $0.03(0.04)$ & $0.04(0.03)$ & $0.05(0.03)$ & $0.04(0.03)$ & $0.03(0.03)$ \\
\hline $\mathrm{C}$ & $0.07(0.06)$ & $0.09(0.08)$ & $0.09(0.07)$ & $0.10(0.07)$ & $0.10(0.06)$ & $0.09(0.06)$ \\
\hline $\mathrm{O}$ & $0.02(0.02)$ & $0.02(0.02)$ & $0.02(0.01)$ & $0.01(0.01)$ & $0.02(0.01)$ & $0.01(0.01)$ \\
\hline $\mathrm{CB}$ & $0.06(0.07)$ & $0.05(0.06)$ & $0.05(0.05)$ & $0.04(0.04)$ & $0.04(0.05)$ & $0.03(0.04)$ \\
\hline SG & $0.08(0.08)$ & 0.08(0.07) & $0.09(0.10)$ & $0.08(0.09)$ & $0.09(0.10)$ & $0.08(0.08)$ \\
\hline \multicolumn{7}{|l|}{ HIE143 } \\
\hline $\mathrm{N}$ & $0.00(0.00)$ & $0.02(0.02)$ & $0.00(0.00)$ & $0.03(0.04)$ & $0.00(0.00)$ & $0.04(0.04)$ \\
\hline $\mathrm{CA}$ & $0.04(0.05)$ & $0.06(0.05)$ & $0.06(0.06)$ & $0.07(0.08)$ & $0.06(0.06)$ & $0.08(0.07)$ \\
\hline $\mathrm{C}$ & $0.04(0.05)$ & $0.07(0.08)$ & $0.04(0.05)$ & $0.06(0.06)$ & $0.05(0.05)$ & $0.06(0.05)$ \\
\hline $\mathrm{O}$ & $0.05(0.06)$ & $0.10(0.10)$ & $0.06(0.06)$ & $0.10(0.10)$ & $0.06(0.06)$ & $0.10(0.09)$ \\
\hline $\mathrm{CB}$ & $0.08(0.08)$ & $0.11(0.11)$ & $0.11(0.11)$ & $0.13(0.11)$ & $0.13(0.10)$ & $0.12(0.10)$ \\
\hline CG & $0.09(0.11)$ & $0.12(0.12)$ & $0.12(0.13)$ & $0.13(0.13)$ & $0.13(0.12)$ & $0.12(0.12)$ \\
\hline ND1 & $0.14(0.15)$ & $0.15(0.13)$ & $0.17(0.17)$ & $0.17(0.15)$ & $0.19(0.16)$ & $0.16(0.14)$ \\
\hline $\mathrm{CD} 2$ & $0.11(0.12)$ & $0.13(0.11)$ & $0.15(0.15)$ & $0.15(0.13)$ & $0.17(0.14)$ & $0.14(0.12)$ \\
\hline CE1 & $0.00(0.00)$ & $0.03(0.03)$ & $0.00(0.00)$ & $0.05(0.04)$ & $0.00(0.00)$ & $0.04(0.04)$ \\
\hline NE2 & $0.00(0.00)$ & $0.06(0.05)$ & $0.00(0.00)$ & $0.05(0.04)$ & $0.00(0.00)$ & $0.05(0.04)$ \\
\hline \multicolumn{7}{|c|}{ MET148 } \\
\hline $\mathrm{N}$ & $0.00(0.00)$ & $0.03(0.03)$ & $0.00(0.00)$ & $0.03(0.04)$ & $0.00(0.00)$ & $0.03(0.04)$ \\
\hline CA & $0.04(0.05)$ & $0.04(0.04)$ & $0.04(0.05)$ & $0.03(0.02)$ & $0.04(0.05)$ & $0.03(0.02)$ \\
\hline $\mathrm{C}$ & $0.08(0.09)$ & $0.07(0.07)$ & $0.11(0.13)$ & $0.06(0.06)$ & $0.12(0.12)$ & $0.09(0.06)$ \\
\hline $\mathrm{O}$ & $0.06(0.08)$ & $0.09(0.09)$ & $0.07(0.07)$ & $0.09(0.09)$ & $0.08(0.07)$ & $0.09(0.09)$ \\
\hline $\mathrm{CB}$ & $0.03(0.03)$ & $0.04(0.03)$ & $0.04(0.03)$ & $0.04(0.04)$ & $0.04(0.03)$ & $0.04(0.04)$ \\
\hline CG & $0.06(0.08)$ & $0.08(0.08)$ & $0.07(0.07)$ & $0.08(0.07)$ & $0.08(0.07)$ & $0.07(0.07)$ \\
\hline SD & $0.00(0.00)$ & $0.03(0.03)$ & $0.00(0.00)$ & $0.05(0.04)$ & $0.00(0.00)$ & $0.05(0.05)$ \\
\hline $\mathrm{CE}$ & $0.00(0.00)$ & $0.05(0.04)$ & $0.00(0.00)$ & $0.04(0.04)$ & $0.00(0.00)$ & $0.06(0.06)$ \\
\hline $\mathrm{Cu}$ & $0.02(0.01)$ & $0.02(0.01)$ & $0.02(0.02)$ & 0.02(0.02) & 0.02(0.02) & $0.02(0.02)$ \\
\hline
\end{tabular}




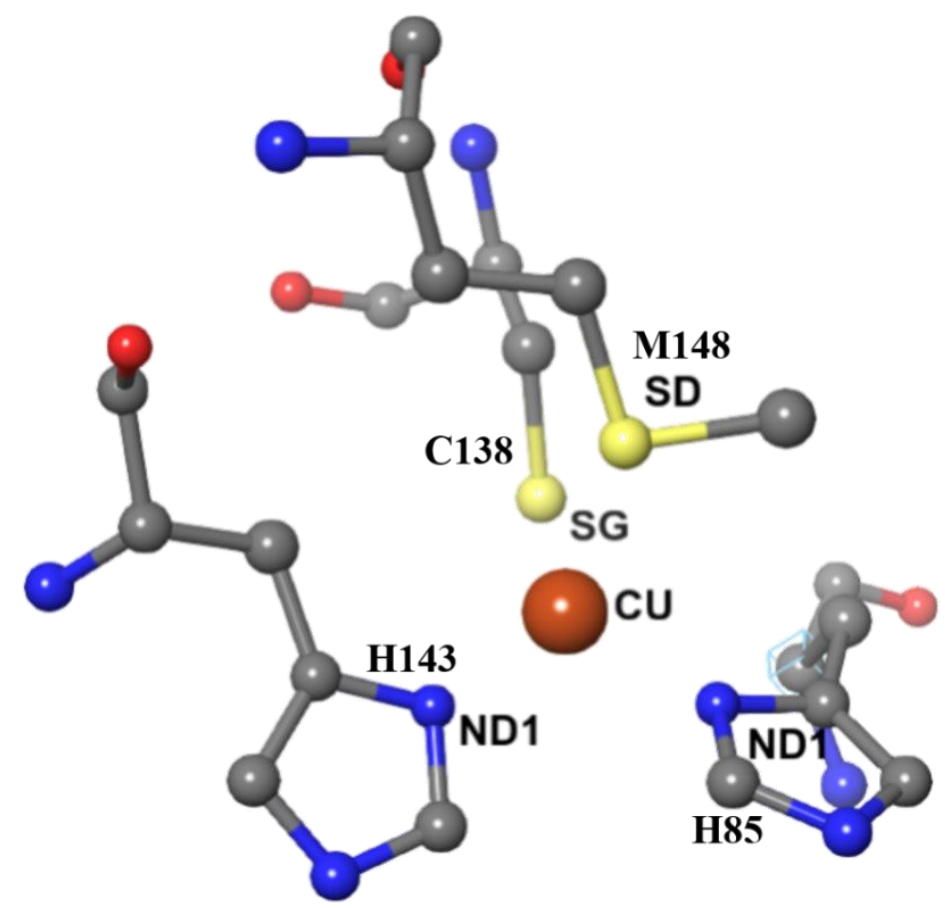

Figure S3. Local structure for the $\mathrm{Cu}$ binding site in X-ray crystal strcuture of the rusticyanin $(\mathrm{RC})$.
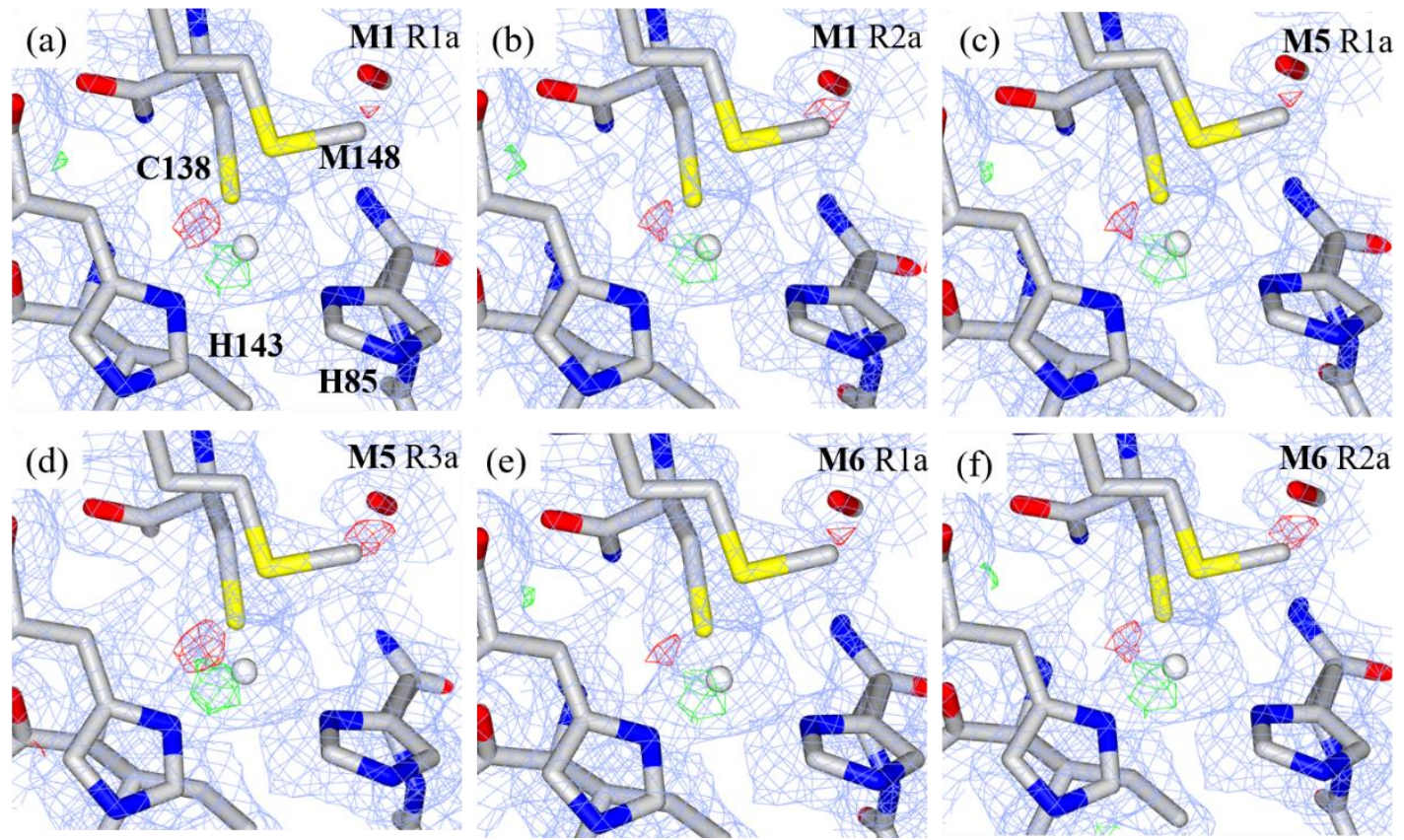

Figure S4. Strucutures for the $\mathrm{Cu}$ binding site in the rusticyanin (RC) protein from various quantum refinement schemes with $\omega_{\alpha}$ value of 0.606 using (a) M1 (with the R1a-optimized region), (b) M1 (with the R2a-optimized region), (c) M5 (with the R1a-optimized region), (d) M5 (with the R3a-optimized region), (e) M6 (with the R1a-optimized region) and (f) M6 (with the R2a-optimized region), including the electron density map (2mFo-DFc maps, contoured at $1.0 \sigma$ (blue), mFo-DFc maps, contoured at $+3.0 \sigma$ (green), and mFo-DFc maps, contoured at $-3.0 \sigma$ (red)). 


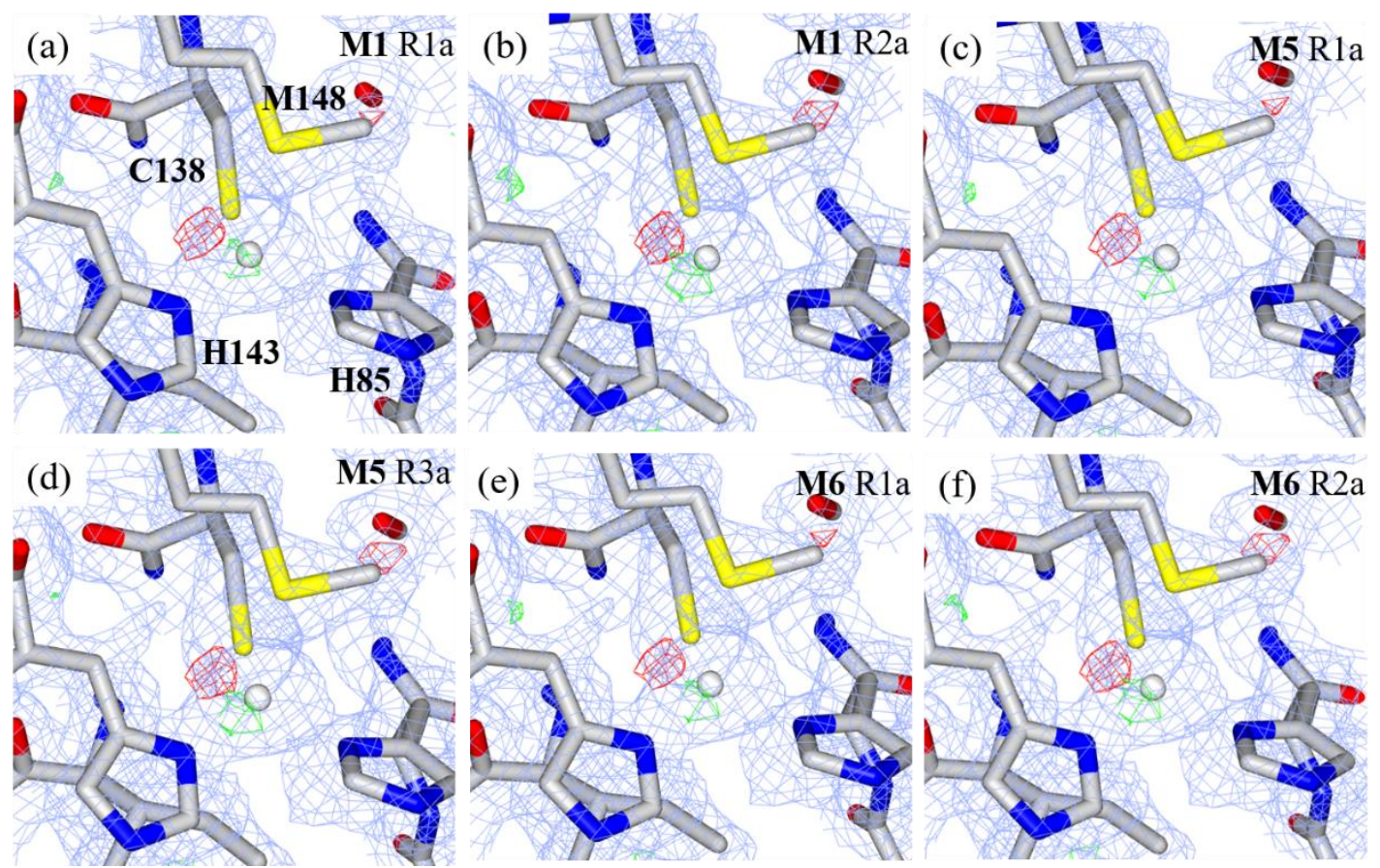

Figure S5. Strucutures for the $\mathrm{Cu}$ binding site in the rusticyanin $(\mathrm{RC})$ protein from various quantum refinement schemes with $\omega_{\alpha}$ value of 1.0 using (a) M1 (with the R1a-optimized region), (b) M1 (with the R2a-optimized region), (c) M5 (with the R1a-optimized region), (d) M5 (with the R3a-optimized region), (e) M6 (with the R1a-optimized region) and (f) M6 (with the R2a-optimized region), including the electron density map (2mFo-DFc maps, contoured at $1.0 \sigma$ (blue), mFo-DFc maps, contoured at $+3.0 \sigma$ (green), and mFo-DFc maps, contoured at $-3.0 \sigma($ red)). 


\section{Quantum refined results of histone acetyltransferase}

Table S9. Our key structural results for the zinc binding site from quantum refinement $\left(\omega_{\alpha}\right.$ value of 1.0) in the ONIOM(QM/MM) scheme with the R1boptimized region in protein and optimization of $\mathrm{R} 1 \mathrm{~b}$-optimized region in gas phase using B3LYP/6-31G(d) and B3LYP-D3/6-31G(d) (in parenthesis).

\begin{tabular}{ccc}
\hline & Protein & Gas \\
\hline $\mathrm{Zn}-\mathrm{S}_{\mathrm{C} 540}$ & $2.39(2.41)$ & $2.33(2.33)$ \\
$\mathrm{Zn}-\mathrm{S}_{\mathrm{C} 543}$ & $2.34(2.34)$ & $2.17(2.16)$ \\
$\mathrm{Zn}-\mathrm{N}_{\mathrm{H} 556}$ & $2.09(2.07)$ & $2.07(2.05)$ \\
$\mathrm{Zn}-\mathrm{S}_{\mathrm{C} 560}$ & $2.35(2.32)$ & $2.19(2.19)$ \\
\hline $\mathrm{N}_{\mathrm{H} 556}-\mathrm{Zn}-\mathrm{S}_{\mathrm{C} 540}$ & $103.3(97.7)$ & $100.8(100.1)$ \\
$\mathrm{N}_{\mathrm{H} 556}-\mathrm{Zn}-\mathrm{S}_{\mathrm{C} 543}$ & $98.2(97.6)$ & $97.2(97.7)$ \\
$\mathrm{N}_{\mathrm{H} 556}-\mathrm{Zn}-\mathrm{S}_{\mathrm{C} 560}$ & $109.3(108.6)$ & $104.7(104.6)$ \\
$\mathrm{S}_{\mathrm{C} 540}-\mathrm{Zn}-\mathrm{S}_{\mathrm{C} 543}$ & $113.7(108.7)$ & $110.9(111.1)$ \\
$\mathrm{S}_{\mathrm{C} 540}-\mathrm{Zn}-\mathrm{S}_{\mathrm{C} 560}$ & $111.0(117.7)$ & $110.7(110.6)$ \\
$\mathrm{S}_{\mathrm{C} 543}-\mathrm{Zn}-\mathrm{S}_{\mathrm{C} 560}$ & $119.3(121.6)$ & $127.6(127.7)$ \\
\hline
\end{tabular}

Table S10. RSZD scores of residues around the $\mathrm{Zn}$ (II) binding site in the histone acetyltransferase from quantum refinement approaches using B3LYP/6-31G(d) (M1) and B3LYP-D3/6-31G(d) (M2) with $\omega_{\alpha}$ value of 1.0. RSZD scores of the FCV binding site were also given.

\begin{tabular}{cccc}
\hline & X-ray & M1 & M2 \\
\hline CYM540 & 0.6 & 2.1 & 2.1 \\
CYM543 & 1.5 & 1.7 & 1.8 \\
HID556 & 3.0 & 0.6 & 0.5 \\
CYM560 & 1.7 & 1.6 & 1.6 \\
Zn & 2.7 & 3.6 & 3.6 \\
Sum $^{\mathrm{a}}$ & 9.5 & 9.6 & 9.6 \\
(Zn(II) center) & & 1.2 & 1.3 \\
FCV & 1.7 &
\end{tabular}

a: The sum for $\mathrm{Zn}(\mathrm{II})$ center is the sum of CYM540, CYM543, HID556, CYM560 and $\mathrm{Zn}$ (II). 


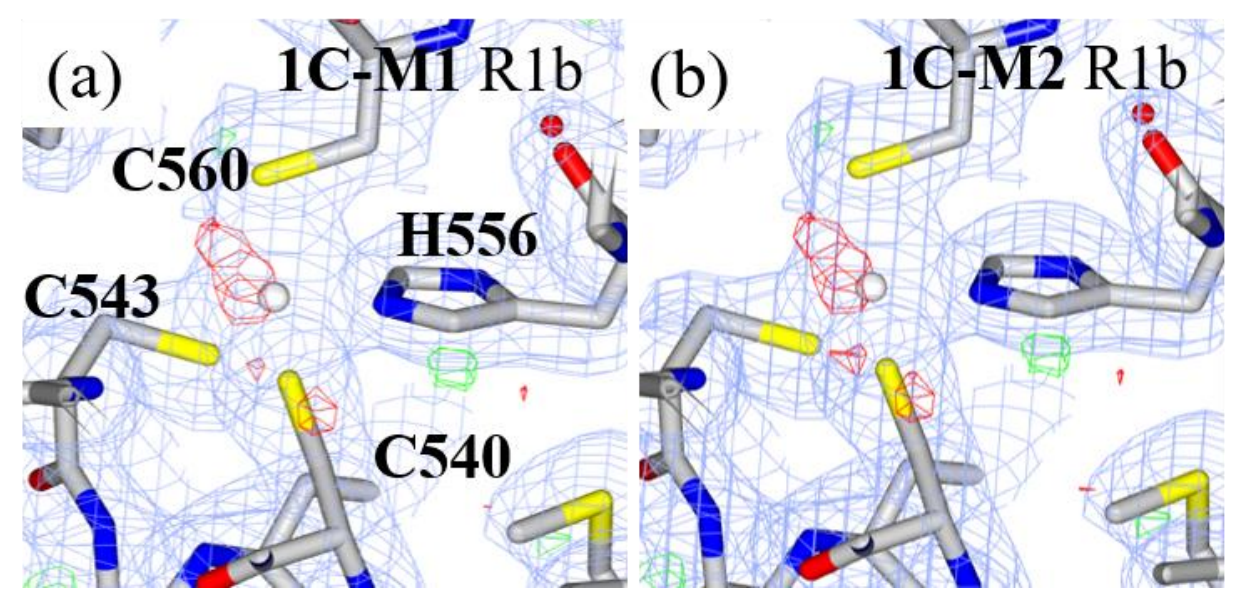

Figure S6. Structures for the Zinc active-site in the MYST histone acetyltransferase KAT6A/B protein from various quantum refinement schemes with $\omega_{\alpha}$ value of 1.0 using (a) 1C-M1 (with the R1b-optimized region), (b) 1C-M2 (with the R1boptimized region), including the electron density map ( $2 \mathrm{mFo}-\mathrm{DFc}$ maps, contoured at $1.0 \sigma$ (blue), mFo-DFc maps, contoured at $+3.0 \sigma$ (green), and mFo-DFc maps, contoured at $-3.0 \sigma(\mathrm{red}))$.

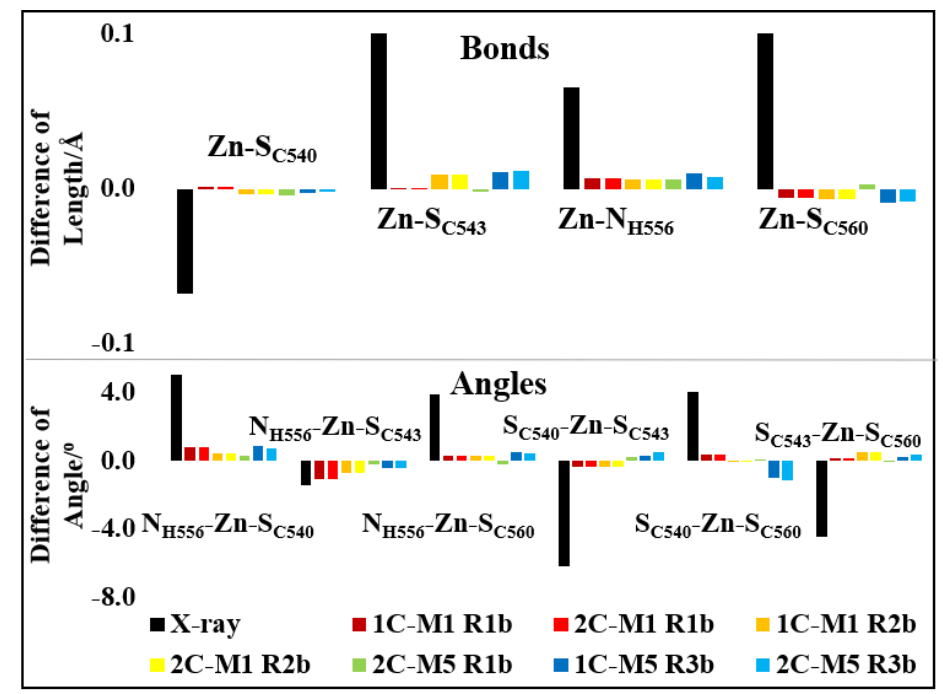

Figure S7. Difference in the key bond lengths and bond angles involving the zinc metal-ligand in our quantum refinement with $\omega_{\alpha}$ value of 1.667 of the histone acetyltransferase using different methods compared to that obtained from the most reliable 1C-M5 scheme with R1b-optimized region (detailed data are given in Tables S12 and S14). 

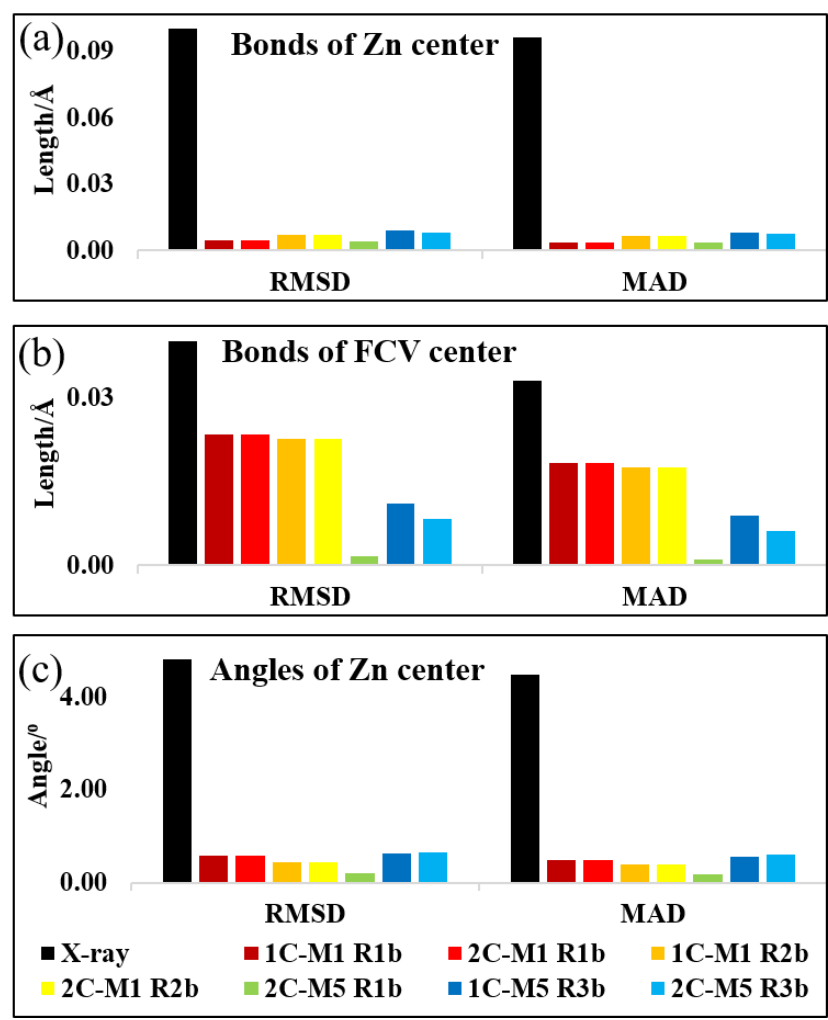

Figure S8. RMSD and MAD analysis of the refined (a) bond lengths for the $\mathrm{Zn}$ (II) center; (b) bond lengths for the FCV center; (c) bond angles around the $\mathrm{Zn}$ (II) center of the histone acetyltransferase obtained using different methods with $\omega_{\alpha}$ value of 1.667, which are compared to that obtained from the most reliable 1C-M5 scheme with R1b-optimized region (detailed data are given in Tables S12 and S14).

The key results for the conventional two- and three-layer ONIOM-based quantum refinements (M1 and M5) are compared and summarized in Figures S7-S8 and Tables S12-S17. In comparison with our quantum refinement calculations with $\omega_{\alpha}$ value of 1.667 (suggested by CNS) and 1.0 (Tables S16-S17), their maximum difference of the metal-ligand bond lengths on the $\mathrm{Zn}$ (II) site between various quantum refinement schemes is less than $0.03 \AA$ and that of the related angle is less than $0.7^{\circ}$. The computed RSZD scores for these $\omega_{\alpha}$ values are also similar with a deviation of less than 0.2 (Table $\mathrm{S} 11$ ). In addition, the $\mathrm{R}$ and $\mathrm{R}_{\text {free }}$ factors for these $\omega_{\alpha}$ values are computed to be identical. These results reveal that our quantum refinement 
calculations give similar local geometries for the two different $\omega_{\alpha}$ cases. Hereafter, the refined results for $\omega_{\alpha}$ value of 1.667 are mainly discussed.

Table S11. RSZD scores of residues around the $\mathrm{Zn}$ (II) binding site in the histone acetyltransferase from various quantum refinement approaches with $\omega_{\alpha}$ value of 1.667 and 1.0 (in parenthesis). RSZD scores of the FCV binding site were also given.

\begin{tabular}{ccccccccc}
\hline & & M1 R1b & M1 R2b & M5 R1b & M5 R3b & M7 R1b & M8 R1b $^{\text {a }}$ & X-ray \\
\hline \multirow{2}{*}{ CYM540 } & 1C & $2.0(2.1)$ & $2.0(2.1)$ & $1.9(2.1)$ & $2.0(2.1)$ & - & - & \\
& 2C & $2.0(2.1)$ & $2.2(2.2)$ & $1.9(2.1)$ & $1.9(2.1)$ & $(2.3)$ & $(2.3)$ & 0.6 \\
CYM543 & 1C & $1.4(1.7)$ & $1.3(1.6)$ & $1.3(1.6)$ & $1.3(1.5)$ & - & - & \\
& 2C & $1.4(1.7)$ & $1.3(1.6)$ & $1.3(1.6)$ & $1.2(1.6)$ & $(2.0)$ & $(1.9)$ & 1.5 \\
HID556 & 1C & $0.8(0.6)$ & $0.8(0.8)$ & $0.5(0.8)$ & $0.9(1.1)$ & - & - & \\
& 2C & $0.8(0.5)$ & $2.0(1.2)$ & $0.5(0.6)$ & $0.8(0.8)$ & $(0.6)$ & $(0.7)$ & 3.0 \\
CYM560 & 1C & $1.9(1.6)$ & $1.9(1.7)$ & $1.7(1.6)$ & $1.8(1.7)$ & - & - & \\
& 2C & $1.9(1.6)$ & $1.7(1.7)$ & $1.6(1.5)$ & $1.8(1.7)$ & $(1.4)$ & $(1.5)$ & 1.7 \\
Zn & 1C & $3.7(3.6)$ & $3.9(3.8)$ & $3.7(3.4)$ & $3.7(3.4)$ & - & - & \\
Sum & 2C & $3.8(3.5)$ & $3.9(3.7)$ & $3.7(3.5)$ & $3.8(3.3)$ & $(3.5)$ & $(3.3)$ & 2.7 \\
(Zn(II) center) & 1C & $9.8(9.6)$ & $9.9(10.0)$ & $9.1(9.5)$ & $9.7(9.8)$ & - & - & \\
FCV & 2C & $9.9(9.4)$ & $11.1(10.4)$ & $9.0(9.3)$ & $9.5(9.5)$ & $(9.8)$ & $(9.7)$ & 9.5 \\
& 1C & $1.1(1.2)$ & $1.0(1.0)$ & $0.9(1.0)$ & $0.8(1.0)$ & - & - & \\
\hline & 2C & $1.1(1.2)$ & $1.0(1.0)$ & $0.9(1.1)$ & $0.7(1.1)$ & $(1.2)$ & $(1.1)$ & 1.7 \\
\hline
\end{tabular}

a: values computed by the 2C-M7 and 2C-M8 schemes with the CCSD-in-DFT method as the QM method. b: The sum for Zn(II) center is the sum of CYM540, CYM543, HID556, CYM560 and $\mathrm{Zn}(\mathrm{II})$.

Compared to the X-ray crystal structure, the refined $\mathrm{Zn}-\mathrm{S}_{\mathrm{C} 540}$ bond obtained by different multiscale schemes (M1 and M5) becomes longer by $0.07 \AA$, while the Zn$\mathrm{S}_{\mathrm{C} 543}, \mathrm{Zn}-\mathrm{N}_{\mathrm{H} 556}$ and $\mathrm{Zn}-\mathrm{S}_{\mathrm{C} 560}$ bonds become shorter by $0.11-0.12,0.05-0.06$ and $0.08-$ $0.09 \AA$, respectively (Figure S7). In addition, our different quantum refinement calculations generally provide similar active-site bonding based on their RMSD, MAD and RSZD scores. Compared to the best computational combination (1C-M5 R1b), the RMSD and MAD values for the bonds involving the zinc ion refined by different approaches deviate by 0.01 and $0.01 \AA$ only (Figure S8), respectively. Similarly, the RMSD and MAD values for the bond angles refined by different 
approaches deviate by approximately $0.1-0.7^{\circ}$ and $0.1-0.6^{\circ}$, respectively. Likewise, the RSZD scores of the zinc and FCV centers refined by different approaches deviate by less than 1.3 and 0.2 , respectively (Table S11). The electron density maps are almost identical relative to those by 1C-M5 R1b (Figures S10 and S14). These computational results show again that different quantum refinement schemes generally lead to the similar active-site geometry.

Furthermore, compared to the X-ray crystal structure, the strain energy of the R1boptimized region (which was calculated as the QM energy difference between the fully-optimized R1b-optimized geometry in vacuum and the quantum refined R1boptimized geometry extracted from the protein at B3LYP/6-31G(d) level) is considerably decreased by more than $17.0-24.2 \mathrm{kcal} / \mathrm{mol}$ and $61.9-66.3 \mathrm{kcal} / \mathrm{mol}$ for the FCV and zinc centers, respectively, after these quantum refinement calculations (M1 and M5; Figure S9 and Table S23).
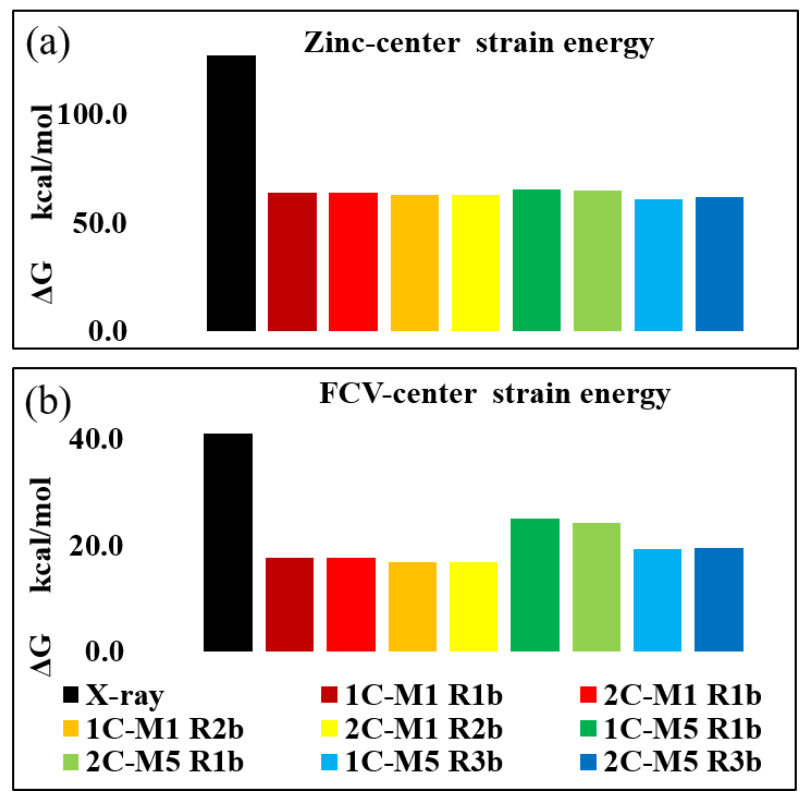

Figure S9. Strain energy $(\Delta \mathrm{E}, \mathrm{kcal} / \mathrm{mol})$ at $\mathrm{B} 3 \mathrm{LYP} / 6-31 \mathrm{G}(\mathrm{d})$ level for the R1boptimized region for (a) the $\mathrm{Zn}$ (II) center and (b) the FCV center in the histone acetyltransferase determined by various quantum refinement schemes. 
However, the computed RZSD scores of $\mathrm{Zn}(\mathrm{II})$ center for the different quantum refined calculations (M1 and M5) become higher than that obtained from the X-ray crystal structure by $0.4-1.7$. As shown in Figures S10 and S14, a less positive (green contours) difference density around the zinc ion was observed after these quantum refinement calculations. Moreover, the key atomic displacement for these refined results (Figure S12a and Tables S21-S22) with respect to the X-ray crystal structure show that the zinc metal moves only 0.07-0.08 $\AA$, whereas the Zn-ligated N$\varepsilon_{\text {HID556 }}$ atom was found to have the largest displacement $(0.18-0.22 \AA)$ than the other $\mathrm{Zn}$ ligated $\mathrm{S}_{\mathrm{CYM} 540}, \mathrm{~S}_{\mathrm{CYM} 543}$ and $\mathrm{S}_{\mathrm{CYM} 560}$ atoms $(0.12-0.17 \AA)$ and the $\mathrm{Zn}(\mathrm{II})$ metal (0.07$0.08 \AA$ ). On the basis of the RSZD scores results (Table S11), that for the sum of the zinc center modestly raises from 9.5 (crystal structure) to 9.1-9.8 (for the small R1boptimized region with M1 and M5 approaches) and 9.7-9.9 (for the larger R2b- and R3b-optimized regions with M1 and M5 approaches). These M1- and M5-refined results reveal that the RSZD score for the $\mathrm{Zn}$ (II) binding site which is positioned on the surface of the protein obviously becomes larger with the larger optimization region. Therefore, the flexible $\mathrm{Zn}(\mathrm{II})$ binding on this protein surface (Figure $\mathrm{S} 12 \mathrm{~b}$ ) should be attributed to the more negative (red contours) difference density around the zinc center, and the flexible optimization region on the protein surface should be carefully selected in quantum refinement calculations. 

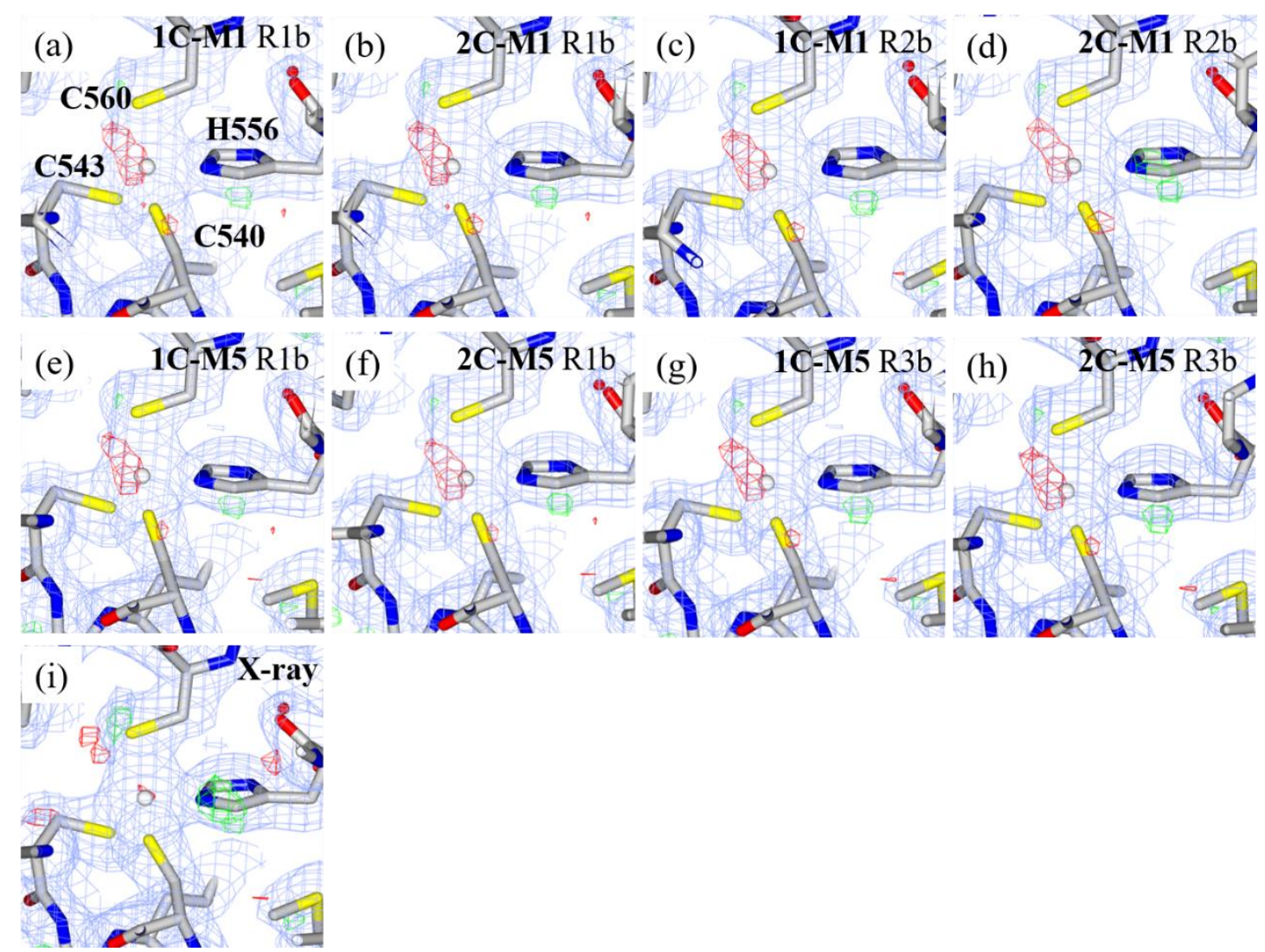

Figure S10. Structures for the Zinc active-site in the MYST histone acetyltransferase KAT6A/B protein from various quantum refinement schemes with $\omega_{\alpha}$ value of 1.667 using (a) 1C-M1 (with the R1b-optimized region), (b) 2C-M1 (with the R1boptimized region), (c) 1C-M1 (with the R2b-optimized region), (d) 2C-M1 (with the R2b-optimized region), (e) 1C-M5 (with the R1b-optimized region), (f) 2C-M5 (with the R1b-optimized region), (g) 1C-M5 (with the R3b-optimized region), (h) 2C-M5 (with the R3b-optimized region) and (i) the X-ray crystal structure, including the electron density map $\left(2 \mathrm{mF}_{\mathrm{o}}-\mathrm{DF}_{\mathrm{c}}\right.$ maps, contoured at $1.0 \sigma$ (blue), $\mathrm{mF}_{\mathrm{o}}-\mathrm{DF}_{\mathrm{c}}$ maps, contoured at $+3.0 \sigma$ (green), and $\mathrm{mF}_{\mathrm{o}}-\mathrm{DF}_{\mathrm{c}}$ maps, contoured at $-3.0 \sigma($ red)). 

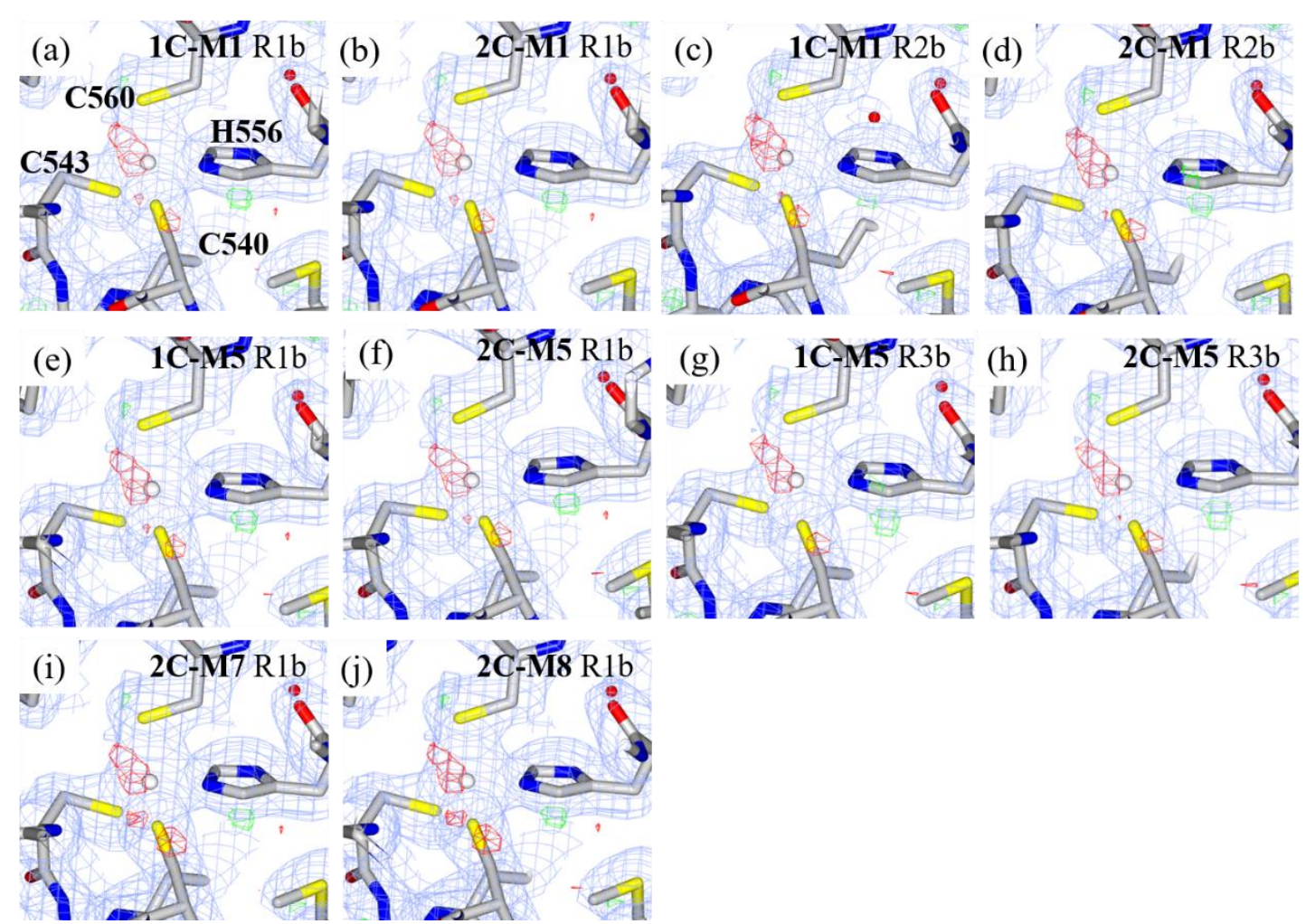

Figure S11. Structures for the $\mathrm{Zn}$ binding site in the histone acetyltransferase protein from various quantum refinement schemes with $\omega_{\alpha}$ value of 1.0 using (a) 1C-M1 (with the R1b-optimized region), (b) 2C-M1 (with the R1b-optimized region), (c) 1CM1 (with the R2b-optimized region), (d) 2C-M1 (with the R2b-optimized region), (e) 1C-M5 (with the R1b-optimized region), (f) 2C-M5 (with the R1b-optimized region), (g) 1C-M5 (with the R3b-optimized region), (h) 2C-M5 (with the R3b-optimized region), (i) 2C-M7 (with the R1b-optimized region) and (j) 2C-M8 (with the R1boptimized region), including the electron density map $\left(2 \mathrm{mF}_{\mathrm{o}}-\mathrm{DF}_{\mathrm{c}}\right.$ maps, contoured at $1.0 \sigma$ (blue), $\mathrm{mF}_{\mathrm{o}}-\mathrm{DF}_{\mathrm{c}}$ maps, contoured at $+3.0 \sigma$ (green), and $\mathrm{mF}_{\mathrm{o}}-\mathrm{DF}_{\mathrm{c}}$ maps, contoured at $-3.0 \sigma(\mathrm{red}))$. 


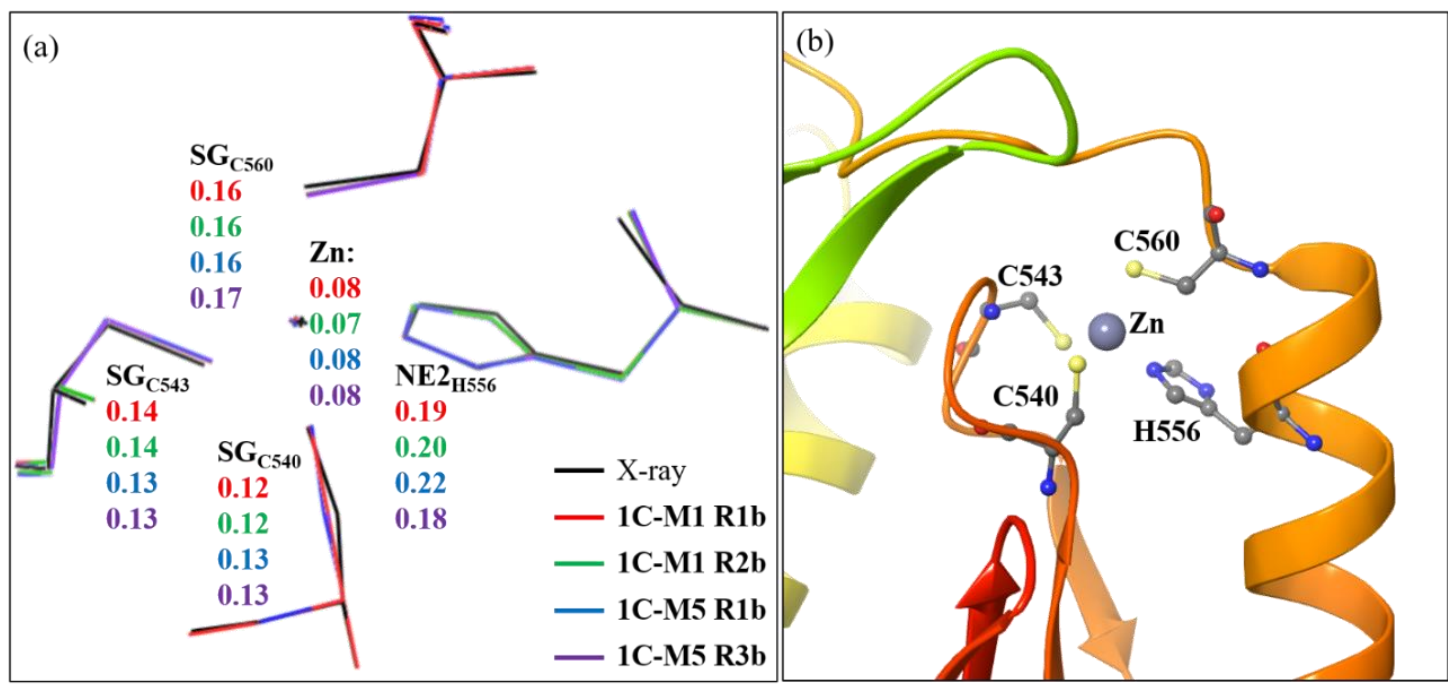

Figure S12. (a) Superposition of the Zinc binding site in the histone acetyltransferase protein from X-ray structure (black) and quantum refinements with $\omega_{\alpha}$ value of 1.667 using 1C-M1 (with the R1b-optimized region, red), 1C-M1 (with the R2b-optimized region, green), 1C-M5 (with the R1b-optimized region, blue), 1C-M5 (with the R3boptimized region, purple). The atomic displacements $(\AA)$ of the key atoms in our quantum refinement calculations relative to the $\mathrm{X}$-ray crystal structure are given. (b) The flexible Zinc binding site in X-ray structure of the histone acetyltransferase protein.

Table S12. Our quantum refined key structural results with $\omega_{\alpha}$ value of 1.667 for the zinc binding site using different multiscale schemes with different optimized regions (R1b, R2b or R3b) for histone acetyltransferase. The two-center (2C) ONIOM approximation was also used (values given in parenthesis). The key structural results from the X-ray crystal structure were also given. 1C-M5 R1b results are used as reference for RMSD and MAD computaions.

\begin{tabular}{|c|c|c|c|c|c|}
\hline & M1 R1b & M1 R2b & M5 R1b & M5 R3b & X-ray \\
\hline $\mathrm{Zn}-\mathrm{S}_{\mathrm{C} 540}$ & $2.33(2.33)$ & $2.33(2.33)$ & $2.33(2.33)$ & $2.33(2.33)$ & 2.26 \\
\hline $\mathrm{Zn}-\mathrm{S}_{\mathrm{C} 543}$ & $2.17(2.17)$ & $2.17(2.17)$ & $2.16(2.16)$ & $2.17(2.17)$ & 2.28 \\
\hline $\mathrm{Zn}-\mathrm{N}_{\mathrm{H} 556}$ & $2.07(2.07)$ & $2.08(2.09)$ & $2.08(2.08)$ & $2.08(2.07)$ & 2.13 \\
\hline $\mathrm{Zn}-\mathrm{S}_{\mathrm{C} 560}$ & $2.19(2.19)$ & $2.19(2.19)$ & $2.20(2.20)$ & $2.19(2.19)$ & 2.28 \\
\hline RMSD (bonds) & $0.00(0.00)$ & $0.01(0.01)$ & $0.00(0.00)$ & $0.01(0.01)$ & 0.10 \\
\hline MAD (bonds) & $0.00(0.00)$ & $0.01(0.01)$ & $0.00(0.00)$ & $0.01(0.01)$ & 0.10 \\
\hline $\mathrm{N}_{\mathrm{H} 556}-\mathrm{Zn}-\mathrm{S}_{\mathrm{C} 540}$ & $100.8(100.5)$ & $100.8(101.9)$ & $101.2(100.8)$ & $101.8(101.1)$ & 107.8 \\
\hline $\mathrm{N}_{\mathrm{H} 556}-\mathrm{Zn}-\mathrm{S}_{\mathrm{C} 543}$ & $97.2(97.5)$ & 97.3(96.8) & 97.6(97.9) & 97.6(98.0) & 96.7 \\
\hline $\mathrm{N}_{\mathrm{H} 556}-\mathrm{Zn}-\mathrm{S}_{\mathrm{C} 560}$ & $104.7(104.7)$ & $104.7(104.7)$ & $104.7(104.5)$ & $105.1(105.1)$ & 108.1 \\
\hline $\mathrm{S}_{\mathrm{C} 540}-\mathrm{Zn}-\mathrm{S}_{\mathrm{C} 543}$ & $110.9(110.9)$ & $111.0(110.8)$ & $111.2(111.3)$ & $111.5(111.8)$ & 105.2 \\
\hline $\mathrm{S}_{\mathrm{C} 540}-\mathrm{Zn}-\mathrm{S}_{\mathrm{C} 560}$ & $110.7(110.7)$ & $110.1(110.3)$ & $110.1(110.1)$ & 109.2(108.9) & 114.0 \\
\hline $\mathrm{S}_{\mathrm{C} 543}-\mathrm{Zn}-\mathrm{S}_{\mathrm{C} 560}$ & $127.6(127.7)$ & $128.1(127.8)$ & $127.4(127.5)$ & $127.5(127.6)$ & 123.0 \\
\hline RMSD (angles) & $0.6(0.6)$ & $0.4(0.4)$ & $0.0(0.2)$ & $0.6(0.7)$ & 4.8 \\
\hline MAD (angles) & $0.5(0.5)$ & $0.4(0.4)$ & $0.0(0.2)$ & $0.5(0.6)$ & 4.5 \\
\hline $\mathrm{R} /$ & $0.218(0.218 /$ & $0.218(0.217 /$ & $0.218(0.218 /$ & $0.217(0.217 /$ & $0.210 /$ \\
\hline$R_{\text {free }}$ & $0.244(0.244)$ & $0.244(0.243)$ & $0.244(0.244)$ & $0.243(0.243)$ & 0.241 \\
\hline
\end{tabular}


Table S13. Our quantum refined key structural results with $\omega_{\alpha}$ value of 1.0 for the zinc binding site using different multiscale schemes with different optimized regions (R1b, R2b or R3b) for histone acetyltransferase. The two-center (2C) ONIOM approximation was also used (values given in parenthesis). The key structural results from the X-ray crystal structure were also given. 1C-M5 R1b results are used as reference for RMSD and MAD computaions.

\begin{tabular}{|c|c|c|c|c|c|}
\hline & M1 R1b & M1 R2b & M5 R1b & M5 R3b & X-ray \\
\hline $\mathrm{Zn}-\mathrm{S}_{\mathrm{C} 540}$ & $2.33\left(2.33,2.35^{\mathrm{a}}\right)$ & $2.33(2.33)$ & $2.33\left(2.33,2.35^{\mathrm{a}}\right)$ & $2.33(2.33)$ & 2.26 \\
\hline $\mathrm{Zn}-\mathrm{S}_{\mathrm{C} 543}$ & $2.17(2.17,2.19)$ & $2.17(2.17)$ & $2.16(2.16,2.19)$ & $2.17(2.17)$ & 2.28 \\
\hline $\mathrm{Zn}-\mathrm{N}_{\mathrm{H} 556}$ & $2.07(2.07,2.08)$ & $2.08(2.09)$ & $2.08(2.08,2.09)$ & $2.08(2.07)$ & 2.13 \\
\hline $\mathrm{Zn}-\mathrm{S}_{\mathrm{C} 560}$ & $2.19(2.19,2.22)$ & $2.19(2.19)$ & $2.20(2.20,2.22)$ & $2.19(2.19)$ & 2.28 \\
\hline RMSD (bonds) & $0.01(0.01,0.02)$ & $0.01(0.00)$ & $0.00(0.00,0.02)$ & $0.01(0.01)$ & 0.09 \\
\hline MAD (bonds) & $0.00(0.01,0.01)$ & $0.00(0.00)$ & $0.00(0.00,0.02)$ & $0.00(0.01)$ & 0.08 \\
\hline $\mathrm{N}_{\mathrm{H} 556}-\mathrm{Zn}-\mathrm{S}_{\mathrm{C} 540}$ & $100.8(100.5,100.7)$ & $100.8(101.9)$ & $101.2(100.8,101.1)$ & $101.8(101.1)$ & 107.8 \\
\hline $\mathrm{N}_{\mathrm{H} 556}-\mathrm{Zn}-\mathrm{S}_{\mathrm{C} 543}$ & $97.2(97.5,97.0)$ & $97.3(96.8)$ & $97.6(97.9,97.5)$ & $97.6(98.0)$ & 96.7 \\
\hline $\mathrm{N}_{\mathrm{H} 556}-\mathrm{Zn}-\mathrm{S}_{\mathrm{C} 560}$ & $104.7(104.7,104.9)$ & $104.7(104.7)$ & $104.7(104.5,104.8)$ & $105.1(105.1)$ & 108.1 \\
\hline $\mathrm{S}_{\mathrm{C} 540}-\mathrm{Zn}-\mathrm{S}_{\mathrm{C} 543}$ & $110.9(110.9,110.9)$ & $111.0(110.8)$ & $111.2(111.3,111.3)$ & $111.5(111.8)$ & 105.2 \\
\hline $\mathrm{S}_{\mathrm{C} 540}-\mathrm{Zn}-\mathrm{S}_{\mathrm{C} 560}$ & $110.7(110.7,110.7)$ & $110.1(110.3)$ & $110.1(110.1,110.2)$ & 109.2(108.9) & 114.0 \\
\hline $\mathrm{S}_{\mathrm{C} 543}-\mathrm{Zn}-\mathrm{S}_{\mathrm{C} 560}$ & $127.6(127.7,127.7)$ & $128.1(127.8)$ & $127.4(127.5,127.4)$ & $127.5(127.6)$ & 123.0 \\
\hline RMSD (angles) & $0.4(0.4,0.5)$ & $0.4(0.5)$ & $0.0(0.3,0.1)$ & $0.5(0.6)$ & 4.6 \\
\hline MAD (angles) & $0.3(0.3,0.4)$ & $0.3(0.4)$ & $0.0(0.2,0.1)$ & $0.4(0.5)$ & 4.2 \\
\hline $\begin{array}{c}\mathrm{R} / \\
\mathrm{R}_{\text {free }}\end{array}$ & $\begin{array}{c}0.218(0.218,0.218) / \\
0.244(0.244,0.244)\end{array}$ & $\begin{array}{l}0.218(0.217) / \\
0.244(0.243)\end{array}$ & $\begin{array}{c}0.218(0.218,0.218) / \\
0.244(0.244,0.244)\end{array}$ & $\begin{array}{c}0.217(0.217) / \\
0.243(0.243)\end{array}$ & $\begin{array}{l}0.214 / \\
0.241\end{array}$ \\
\hline
\end{tabular}
a: values computed by the 2C-M7 and 2C-M8 method with the CCSD-in-DFT method as the QM method. 
Table S14. Our quantum refined key structural results with $\omega_{\alpha}$ value of 1.667 for the FCV ligand using different multiscale schemes with different optimized regions (R1b, $\mathrm{R} 2 \mathrm{~b}$ or $\mathrm{R} 3 \mathrm{~b}$ ). The two-center (2C) ONIOM approximation (values given in parenthesis) was also used. 1C-M5 R1b results are used as reference for RMSD and MAD computaions.

\begin{tabular}{cccccc}
\hline & M1 R1b & M1 R2b & M5 R1b & M5 R3b & X-ray \\
\hline C2-C7 & $1.40(1.40)$ & $1.40(1.40)$ & $1.42(1.42)$ & $1.41(1.41)$ & 1.39 \\
C2-C3 & $1.41(1.42)$ & $1.41(1.40)$ & $1.43(1.44)$ & $1.42(1.43)$ & 1.39 \\
C6-C7 & $1.40(1.40)$ & $1.39(1.39)$ & $1.39(1.39)$ & $1.39(1.39)$ & 1.39 \\
C7-F1 & $1.37(1.37)$ & $1.37(1.37)$ & $1.38(1.38)$ & $1.38(1.38)$ & 1.36 \\
C6-C5 & $1.39(1.39)$ & $1.39(1.39)$ & $1.41(1.41)$ & $1.40(1.40)$ & 1.38 \\
C5-C4 & $1.40(1.40)$ & $1.40(1.39)$ & $1.41(1.41)$ & $1.40(1.40)$ & 1.39 \\
C4-C3 & $1.41(1.41)$ & $1.40(1.40)$ & $1.39(1.40)$ & $1.40(1.40)$ & 1.39 \\
C2-S1 & $1.86(1.86)$ & $1.85(1.87)$ & $1.81(1.81)$ & $1.83(1.82)$ & 1.82 \\
S1-O3 & $1.46(1.46)$ & $1.47(1.47)$ & $1.48(1.48)$ & $1.48(1.48)$ & 1.47 \\
S1-O2 & $1.46(1.47)$ & $1.47(1.47)$ & $1.49(1.49)$ & $1.48(1.48)$ & 1.46 \\
S1-N1 & $1.66(1.66)$ & $1.66(1.67)$ & $1.67(1.66)$ & $1.67(1.66)$ & 1.70 \\
N1-N2 & $1.37(1.37)$ & $1.38(1.40)$ & $1.38(1.38)$ & $1.39(1.38)$ & 1.40 \\
N2-C1 & $1.39(1.39)$ & $1.39(1.39)$ & $1.40(1.40)$ & $1.40(1.40)$ & 1.45 \\
C1-O1 & $1.23(1.23)$ & $1.23(1.23)$ & $1.27(1.27)$ & $1.26(1.27)$ & 1.19 \\
C1-C8 & $1.48(1.48)$ & $1.48(1.49)$ & $1.44(1.44)$ & $1.46(1.45)$ & 1.52 \\
C8-C9 & $1.41(1.41)$ & $1.41(1.41)$ & $1.44(1.44)$ & $1.43(1.43)$ & 1.38 \\
C9-C10 & $1.39(1.39)$ & $1.39(1.39)$ & $1.38(1.38)$ & $1.38(1.38)$ & 1.38 \\
C10-F2 & $1.36(1.36)$ & $1.35(1.35)$ & $1.37(1.37)$ & $1.36(1.36)$ & 1.36 \\
C10-C11 & $1.40(1.40)$ & $1.40(1.39)$ & $1.40(1.40)$ & $1.40(1.40)$ & 1.39 \\
C11-C12 & $1.41(1.41)$ & $1.41(1.40)$ & $1.43(1.43)$ & $1.42(1.42)$ & 1.39 \\
C12-C18 & $1.39(1.39)$ & $1.39(1.39)$ & $1.39(1.39)$ & $1.39(1.39)$ & 1.38 \\
C18-C8 & $1.39(1.39)$ & $1.39(1.38)$ & $1.40(1.40)$ & $1.39(1.40)$ & 1.38 \\
C12-C13 & $1.48(1.48)$ & $1.47(1.46)$ & $1.47(1.47)$ & $1.46(1.47)$ & 1.38 \\
C13-C14 & $1.38(1.38)$ & $1.38(1.38)$ & $1.39(1.39)$ & $1.39(1.39)$ & 1.38 \\
C14-C15 & $1.39(1.39)$ & $1.39(1.38)$ & $1.39(1.38)$ & $1.38(1.39)$ & 1.38 \\
C15-C16 & $1.39(1.40)$ & $1.39(1.39)$ & $1.40(1.40)$ & $1.40(1.40)$ & 1.39 \\
C16-C17 & $1.39(1.39)$ & $1.39(1.39)$ & $1.39(1.39)$ & $1.39(1.39)$ & 1.39 \\
C17-N3 & $1.35(1.35)$ & $1.35(1.35)$ & $1.36(1.36)$ & $1.35(1.36)$ & 1.32 \\
C13-N3 & $1.34(1.34)$ & $1.34(1.34)$ & $1.36(1.35)$ & $1.35(1.35)$ & 1.32 \\
RMSD & $0.02(0.02)$ & $0.02(0.02)$ & $0.00(0.00)$ & $0.01(0.01)$ & 0.04 \\
MAD & $0.02(0.02)$ & $0.02(0.02)$ & $0.00(0.00)$ & $0.01(0.01)$ & 0.03 \\
\hline & & & & & \\
& & & & \\
C.
\end{tabular}


Table S15. Our quantum refined key structural results with $\omega_{\alpha}$ value of 1.0 for the FCV ligand using different multiscale schemes with different optimized regions (R1b, $\mathrm{R} 2 \mathrm{~b}$ or $\mathrm{R} 3 \mathrm{~b}$ ). The two-center (2C) ONIOM approximation (values given in parenthesis) was also used. 1C-M5 R1b results are used as reference for RMSD and MAD computaions.

\begin{tabular}{|c|c|c|c|c|c|}
\hline & M1 R1b & M1 R2b & M5 R1b & M5 R3b & X-ray \\
\hline $\mathrm{C} 2-\mathrm{C} 7$ & $1.40\left(1.40,1.40^{\mathrm{a}}\right)$ & $1.40(1.40)$ & $1.42\left(1.42,1.42^{\mathrm{a}}\right)$ & $1.41(1.41)$ & 1.39 \\
\hline $\mathrm{C} 2-\mathrm{C} 3$ & $1.41(1.42,1.41)$ & $1.41(1.40)$ & $1.43(1.44,1.44)$ & $1.42(1.43)$ & 1.39 \\
\hline C6-C7 & $1.40(1.40,1.40)$ & $1.39(1.39)$ & $1.39(1.39,1.39)$ & $1.39(1.39)$ & 1.39 \\
\hline $\mathrm{C} 7-\mathrm{F} 1$ & $1.37(1.37,1.37)$ & $1.37(1.37)$ & $1.38(1.38,1.38)$ & $1.38(1.38)$ & 1.36 \\
\hline C6-C5 & $1.39(1.39,1.39)$ & $1.39(1.39)$ & $1.41(1.41,1.41)$ & $1.40(1.40)$ & 1.38 \\
\hline $\mathrm{C} 5-\mathrm{C} 4$ & $1.40(1.40,1.40)$ & $1.40(1.39)$ & $1.41(1.41,1.41)$ & $1.40(1.40)$ & 1.39 \\
\hline $\mathrm{C} 4-\mathrm{C} 3$ & $1.41(1.41,1.41)$ & $1.40(1.40)$ & $1.39(1.40,1.40)$ & $1.40(1.40)$ & 1.39 \\
\hline $\mathrm{C} 2-\mathrm{S} 1$ & $1.86(1.86,1.86)$ & $1.85(1.87)$ & $1.81(1.81,1.81)$ & $1.83(1.82)$ & 1.82 \\
\hline $\mathrm{S} 1-\mathrm{O} 3$ & $1.46(1.46,1.46)$ & $1.47(1.47)$ & $1.48(1.48,1.48)$ & $1.48(1.48)$ & 1.47 \\
\hline $\mathrm{S} 1-\mathrm{O} 2$ & $1.46(1.47,1.47)$ & $1.47(1.47)$ & $1.49(1.49,1.49)$ & $1.48(1.48)$ & 1.46 \\
\hline S1-N1 & $1.66(1.66,1.66)$ & $1.66(1.67)$ & $1.67(1.66,1.66)$ & $1.67(1.66)$ & 1.70 \\
\hline N1-N2 & $1.37(1.37,1.37)$ & $1.38(1.40)$ & $1.38(1.38,1.38)$ & $1.39(1.38)$ & 1.40 \\
\hline $\mathrm{N} 2-\mathrm{C} 1$ & $1.39(1.39,1.39)$ & $1.39(1.39)$ & $1.40(1.40,1.40)$ & $1.40(1.40)$ & 1.45 \\
\hline $\mathrm{C} 1-\mathrm{O} 1$ & $1.23(1.23,1.23)$ & $1.23(1.23)$ & $1.27(1.27,1.27)$ & $1.26(1.27)$ & 1.19 \\
\hline $\mathrm{C} 1-\mathrm{C} 8$ & $1.48(1.48,1.48)$ & $1.48(1.49)$ & $1.44(1.44,1.44)$ & $1.46(1.45)$ & 1.52 \\
\hline $\mathrm{C} 8-\mathrm{C} 9$ & $1.41(1.41,1.41)$ & $1.41(1.41)$ & $1.44(1.44,1.44)$ & $1.43(1.43)$ & 1.38 \\
\hline C9-C10 & $1.39(1.39,1.39)$ & $1.39(1.39)$ & $1.38(1.38,1.38)$ & $1.38(1.38)$ & 1.38 \\
\hline C10-F2 & $1.36(1.36,1.36)$ & $1.35(1.35)$ & $1.37(1.37,1.37)$ & $1.36(1.36)$ & 1.36 \\
\hline $\mathrm{C} 10-\mathrm{C} 11$ & $1.40(1.40,1.40)$ & $1.40(1.39)$ & $1.40(1.40,1.40)$ & $1.40(1.40)$ & 1.39 \\
\hline $\mathrm{C} 11-\mathrm{C} 12$ & $1.41(1.41,1.41)$ & $1.41(1.40)$ & $1.43(1.43,1.43)$ & $1.42(1.42)$ & 1.39 \\
\hline $\mathrm{C} 12-\mathrm{C} 18$ & $1.39(1.39,1.39)$ & $1.39(1.39)$ & $1.39(1.39,1.39)$ & $1.39(1.39)$ & 1.38 \\
\hline C18-C8 & $1.39(1.39,1.39)$ & $1.39(1.38)$ & $1.40(1.40,1.40)$ & $1.39(1.40)$ & 1.38 \\
\hline C12-C13 & $1.48(1.48,1.48)$ & $1.47(1.46)$ & $1.47(1.47,1.47)$ & $1.46(1.47)$ & 1.38 \\
\hline $\mathrm{C} 13-\mathrm{C} 14$ & $1.38(1.38,1.38)$ & $1.38(1.38)$ & $1.39(1.39,1.39)$ & $1.39(1.39)$ & 1.38 \\
\hline $\mathrm{C} 14-\mathrm{C} 15$ & $1.39(1.39,1.39)$ & $1.39(1.38)$ & $1.39(1.38,1.38)$ & $1.38(1.39)$ & 1.38 \\
\hline $\mathrm{C} 15-\mathrm{C} 16$ & $1.39(1.40,1.40)$ & $1.39(1.39)$ & $1.40(1.40,1.40)$ & $1.40(1.40)$ & 1.39 \\
\hline $\mathrm{C} 16-\mathrm{C} 17$ & $1.39(1.39,1.39)$ & $1.39(1.39)$ & $1.39(1.39,1.39)$ & $1.39(1.39)$ & 1.39 \\
\hline C17-N3 & $1.35(1.35,1.35)$ & $1.35(1.35)$ & $1.36(1.36,1.36)$ & $1.35(1.36)$ & 1.32 \\
\hline C13-N3 & $1.34(1.34,1.34)$ & $1.34(1.34)$ & $1.36(1.35,1.35)$ & $1.35(1.35)$ & 1.32 \\
\hline RMSD & $0.02(0.02,0.02)$ & $0.02(0.02)$ & $0.00(0.00,0.00)$ & $0.01(0.00)$ & 0.04 \\
\hline MAD & $0.01(0.01,0.01)$ & $0.01(0.02)$ & $0.00(0.00,0.00)$ & $0.01(0.00)$ & 0.03 \\
\hline
\end{tabular}

a: values computed by the 2C-M7 and 2C-M8 method with the CCSD-in-DFT method as the QM method. 
Table S16. Our quantum refined key structural results for the zinc binding site using different multiscale schemes with different optimized regions (R1b, R2b or R3b) for histone acetyltransferase. Different values of the weighting factor (1.0 and 1.667 (in parenthesis)) are used. The key structural results from the X-ray crystal structure were also given. 1C-M5 R1b results are used as reference for RMSD and MAD computaions.

\begin{tabular}{|c|c|c|c|c|c|}
\hline & M1 R1b & M1 R2b & M5 R1b & M5 R3b & X-ray \\
\hline $\mathrm{Zn}-\mathrm{S}_{\mathrm{C} 540}$ & $2.33(2.33)$ & $2.33(2.32)$ & $2.33(2.33)$ & $2.33(2.32)$ & 2.26 \\
\hline $\mathrm{Zn}-\mathrm{S}_{\mathrm{C} 543}$ & $2.17(2.14)$ & $2.17(2.15)$ & $2.16(2.14)$ & $2.17(2.15)$ & 2.28 \\
\hline $\mathrm{Zn}-\mathrm{N}_{\mathrm{H} 556}$ & $2.07(2.07)$ & $2.08(2.07)$ & $2.08(2.06)$ & $2.08(2.07)$ & 2.13 \\
\hline $\mathrm{Zn}-\mathrm{S}_{\mathrm{C} 560}$ & $2.19(2.17)$ & $2.19(2.17)$ & $2.20(2.17)$ & $2.19(2.16)$ & 2.28 \\
\hline RMSD (bonds) & $0.01(0.00)$ & $0.01(0.01)$ & $0.00(0.00)$ & $0.01(0.01)$ & $0.09(0.10)$ \\
\hline MAD (bonds) & $0.00(0.00)$ & $0.00(0.01)$ & $0.00(0.00)$ & $0.00(0.01)$ & $0.08(0.10)$ \\
\hline $\mathrm{N}_{\mathrm{H} 556}-\mathrm{Zn}-\mathrm{S}_{\mathrm{C} 540}$ & $100.8(101.8)$ & $100.8(101.5)$ & $101.2(101.0)$ & $101.8(101.9)$ & 107.8 \\
\hline $\mathrm{N}_{\mathrm{H} 556}-\mathrm{Zn}-\mathrm{S}_{\mathrm{C} 543}$ & $97.2(97.1)$ & $97.3(97.4)$ & $97.6(98.1)$ & $97.6(97.7)$ & 96.7 \\
\hline $\mathrm{N}_{\mathrm{H} 556}-\mathrm{Zn}-\mathrm{S}_{\mathrm{C} 560}$ & $104.7(104.5)$ & $104.7(104.5)$ & $104.7(104.2)$ & 105.1(104.7) & 108.1 \\
\hline $\mathrm{S}_{\mathrm{C} 540}-\mathrm{Zn}-\mathrm{S}_{\mathrm{C} 543}$ & $110.9(111.1)$ & 111.0(111.0) & 111.2(111.4) & $111.5(111.7)$ & 105.2 \\
\hline $\mathrm{S}_{\mathrm{C} 540}-\mathrm{Zn}-\mathrm{S}_{\mathrm{C} 560}$ & $110.7(110.3)$ & $110.1(109.9)$ & 110.1(109.9) & 109.2(109.0) & 114.0 \\
\hline $\mathrm{S}_{\mathrm{C} 543}-\mathrm{Zn}-\mathrm{S}_{\mathrm{C} 560}$ & $127.6(127.6)$ & $128.1(128.0)$ & $127.4(127.5)$ & $127.5(127.7)$ & 123.0 \\
\hline RMSD (angles) & $0.4(0.6)$ & $0.4(0.4)$ & $0.0(0.0)$ & $0.5(0.6)$ & $4.6(4.8)$ \\
\hline MAD (angles) & $0.3(0.5)$ & $0.3(0.4)$ & $0.0(0.0)$ & $0.4(0.5)$ & $4.2(4.5)$ \\
\hline $\mathrm{R} /$ & $0.218(0.218 /$ & $0.218(0.217 /$ & $0.218(0.218 /$ & $0.217(0.217 /$ & $0.214 /$ \\
\hline$R_{\text {free }}$ & $0.244(0.244)$ & $0.244(0.243)$ & $0.244(0.244)$ & $0.243(0.243)$ & 0.241 \\
\hline
\end{tabular}


Table S17. Our quantum refined key structural results for the FCV ligand using different multiscale schemes with different optimized regions (R1b, R2b or R3b) for histone acetyltransferase. Different values of the weighting factor (1.0 and 1.667 (in parenthesis)) are used. The key structural results from the X-ray crystal structure were also given. 1C-M5 R1b results are used as reference for RMSD and MAD computaions.

\begin{tabular}{cccccc}
\hline & M1 R1b & M1 R2b & M5 R1b & M5 R3b & X-ray \\
\hline C2-C7 & $1.40(1.40)$ & $1.40(1.40)$ & $1.42(1.42)$ & $1.41(1.41)$ & 1.39 \\
C2-C3 & $1.41(1.41)$ & $1.41(1.41)$ & $1.43(1.45)$ & $1.42(1.42)$ & 1.39 \\
C6-C7 & $1.40(1.40)$ & $1.39(1.40)$ & $1.39(1.40)$ & $1.39(1.40)$ & 1.39 \\
C7-F1 & $1.37(1.38)$ & $1.37(1.38)$ & $1.38(1.39)$ & $1.38(1.39)$ & 1.36 \\
C6-C5 & $1.39(1.38)$ & $1.39(1.39)$ & $1.41(1.41)$ & $1.40(1.40)$ & 1.38 \\
C5-C4 & $1.40(1.39)$ & $1.40(1.39)$ & $1.41(1.41)$ & $1.40(1.40)$ & 1.39 \\
C4-C3 & $1.41(1.40)$ & $1.40(1.40)$ & $1.39(1.40)$ & $1.40(1.40)$ & 1.39 \\
C2-S1 & $1.86(1.88)$ & $1.85(1.88)$ & $1.81(1.82)$ & $1.83(1.85)$ & 1.82 \\
S1-O3 & $1.46(1.47)$ & $1.47(1.48)$ & $1.48(1.48)$ & $1.48(1.48)$ & 1.47 \\
S1-O2 & $1.46(1.47)$ & $1.47(1.47)$ & $1.49(1.50)$ & $1.48(1.49)$ & 1.46 \\
S1-N1 & $1.66(1.64)$ & $1.66(1.64)$ & $1.67(1.64)$ & $1.67(1.64)$ & 1.70 \\
N1-N2 & $1.37(1.40)$ & $1.38(1.40)$ & $1.38(1.37)$ & $1.39(1.39)$ & 1.40 \\
N2-C1 & $1.39(1.39)$ & $1.39(1.40)$ & $1.40(1.40)$ & $1.40(1.40)$ & 1.45 \\
C1-O1 & $1.23(1.23)$ & $1.23(1.23)$ & $1.27(1.27)$ & $1.26(1.26)$ & 1.19 \\
C1-C8 & $1.48(1.48)$ & $1.48(1.48)$ & $1.44(1.43)$ & $1.46(1.45)$ & 1.52 \\
C8-C9 & $1.41(1.41)$ & $1.41(1.42)$ & $1.44(1.45)$ & $1.43(1.44)$ & 1.38 \\
C9-C10 & $1.39(1.39)$ & $1.39(1.39)$ & $1.38(1.38)$ & $1.38(1.38)$ & 1.38 \\
C10-F2 & $1.36(1.36)$ & $1.35(1.36)$ & $1.37(1.37)$ & $1.36(1.37)$ & 1.36 \\
C10-C11 & $1.40(1.39)$ & $1.40(1.40)$ & $1.40(1.41)$ & $1.40(1.40)$ & 1.39 \\
C11-C12 & $1.41(1.41)$ & $1.41(1.41)$ & $1.43(1.44)$ & $1.42(1.42)$ & 1.39 \\
C12-C18 & $1.39(1.38)$ & $1.39(1.39)$ & $1.39(1.39)$ & $1.39(1.38)$ & 1.38 \\
C18-C8 & $1.39(1.39)$ & $1.39(1.38)$ & $1.40(1.40)$ & $1.39(1.39)$ & 1.38 \\
C12-C13 & $1.48(1.46)$ & $1.47(1.46)$ & $1.47(1.48)$ & $1.46(1.46)$ & 1.38 \\
C13-C14 & $1.38(1.37)$ & $1.38(1.38)$ & $1.39(1.39)$ & $1.39(1.38)$ & 1.38 \\
C14-C15 & $1.39(1.38)$ & $1.39(1.38)$ & $1.39(1.39)$ & $1.38(1.38)$ & 1.38 \\
C15-C16 & $1.39(1.39)$ & $1.39(1.39)$ & $1.40(1.40)$ & $1.40(1.40)$ & 1.39 \\
C16-C17 & $1.39(1.39)$ & $1.39(1.39)$ & $1.39(1.39)$ & $1.39(1.39)$ & 1.39 \\
C17-N3 & $1.35(1.35)$ & $1.35(1.35)$ & $1.36(1.37)$ & $1.35(1.36)$ & 1.32 \\
C13-N3 & $1.34(1.34)$ & $1.34(1.34)$ & $1.36(1.35)$ & $1.35(1.35)$ & 1.32 \\
RMSD & $0.02(0.02)$ & $0.02(0.02)$ & $0.00(0.00)$ & $0.01(0.01)$ & $0.04(0.04)$ \\
MAD & $0.01(0.02)$ & $0.01(0.02)$ & $0.00(0.00)$ & $0.01(0.01)$ & $0.03(0.03)$ \\
\hline & & & & & \\
& & & & \\
\hline
\end{tabular}




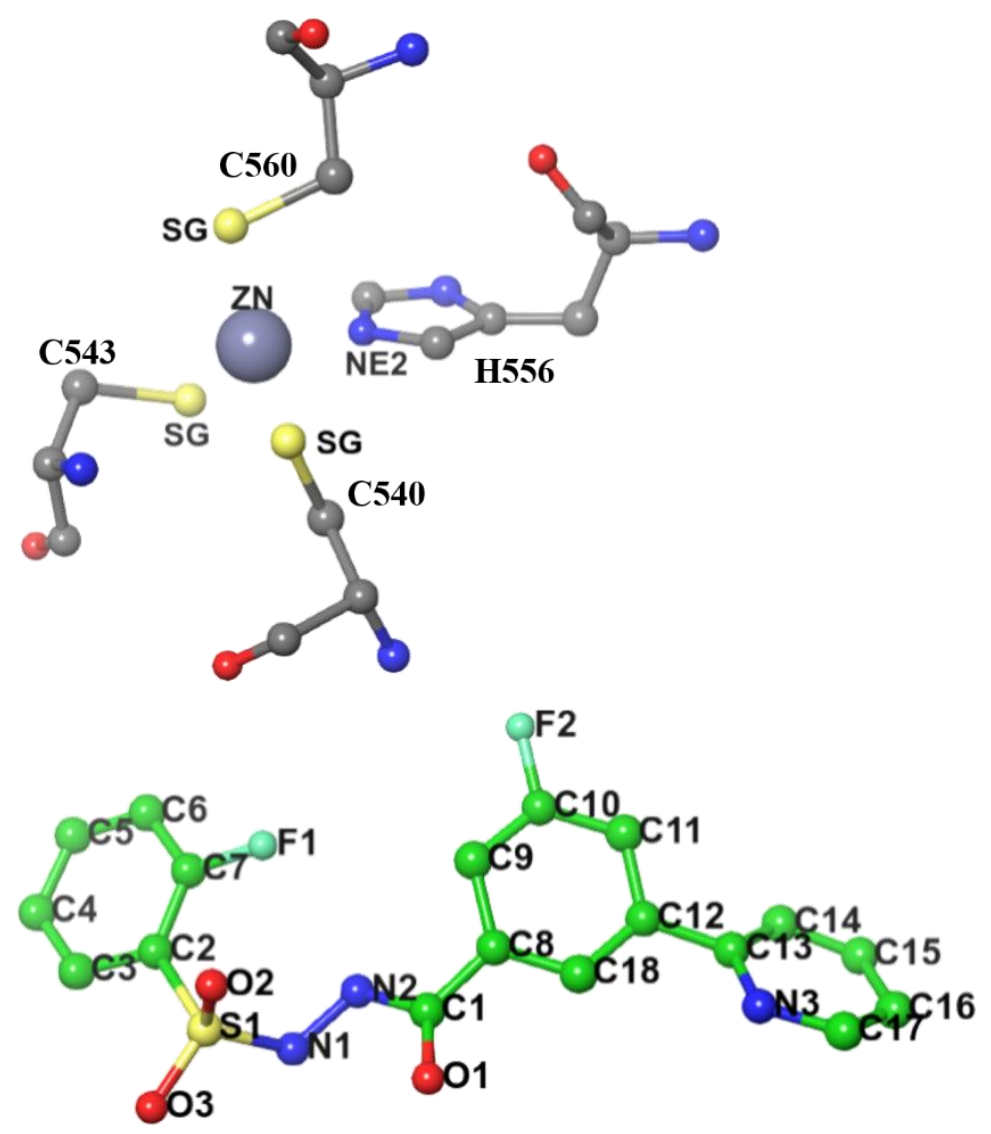

Figure S13. Local structure for the $\mathrm{Zn}$ and FCV binding site in X-ray crystal structure of the histone acetyltransferase.

Table S18. RSZD scores of residues around the $\mathrm{Zn}$ binding site in the histone acetyltransferase from various quantum refinement calculations with $\omega_{\alpha}$ value of 1.667. The two-center (2C) ONIOM approximation was also used (values given in parenthesis).

\begin{tabular}{cccccc}
\hline & M1 R1b & M1 R2b & M5 R1b & M5 R3b & X-ray \\
\hline CYM540 & $2.0(2.0)$ & $2.0(2.2)$ & $1.9(1.9)$ & $2.0(1.9)$ & 0.6 \\
CYM543 & $1.4(1.4)$ & $1.3(1.3)$ & $1.3(1.3)$ & $1.3(1.2)$ & 1.5 \\
HID556 & $0.8(0.8)$ & $0.8(2.0)$ & $0.5(0.5)$ & $0.9(0.8)$ & 3.0 \\
CYM560 & $1.9(1.9)$ & $1.9(1.7)$ & $1.7(1.6)$ & $1.8(1.8)$ & 1.7 \\
Zn & $3.7(3.8)$ & $3.9(3.9)$ & $3.7(3.7)$ & $3.7(3.8)$ & 2.7 \\
Sum (Zn center) & $9.8(9.9)$ & $9.9(11.1)$ & $9.1(9.0)$ & $9.7(9.5)$ & 9.5 \\
FCV & $1.1(1.1)$ & $1.0(1.0)$ & $0.9(0.9)$ & $0.8(0.7)$ & 1.7 \\
\hline
\end{tabular}


Table S19. RSZD scores of residues around the $\mathrm{Zn}$ binding site in the histone acetyltransferase from various quantum refinement calculations with $\omega_{\alpha}$ value of 1.0. The two-center (2C) ONIOM approximation was also used (values given in parenthesis).

\begin{tabular}{cccccc}
\hline & M1 R1b & M1 R2b & M5 R1b & M5 R3b & X-ray \\
\hline CYM540 & $2.1\left(2.1,2.3^{\mathrm{a}}\right)$ & $2.1(2.2)$ & $2.1\left(2.1,2.3^{\mathrm{a}}\right)$ & $2.1(2.1)$ & 0.6 \\
CYM543 & $1.7(1.7,2.0)$ & $1.6(1.6)$ & $1.6(1.6,1.9)$ & $1.5(1.6)$ & 1.5 \\
HID556 & $0.6(0.5,0.6)$ & $0.8(1.2)$ & $0.8(0.6,0.7)$ & $1.1(0.8)$ & 3.0 \\
CYM560 & $1.6(1.6,1.4)$ & $1.7(1.7)$ & $1.6(1.5,1.5)$ & $1.7(1.7)$ & 1.7 \\
Zn & $3.6(3.5,3.5)$ & $3.8(3.7)$ & $3.4(3.5,3.3)$ & $3.4(3.3)$ & 2.7 \\
Sum (Zn center) & $9.6(9.4,9.8)$ & $10.0(10.4)$ & $9.5(9.3,9.7)$ & $9.8(9.5)$ & 9.5 \\
FCV & $1.2(1.2,1.2)$ & $1.0(1.0)$ & $1.0(1.1,1.1)$ & $1.0(1.1)$ & 1.7 \\
\hline
\end{tabular}

a: values computed by the 2C-M7 and 2C-M8 method with the CCSD-in-DFT method as the QM method.

Table S20. RSZD scores of residues around the $\mathrm{Zn}$ binding site in the histone acetyltransferase from various quantum refinement calculations using 1C-ONIOM methods with $\omega_{\alpha}$ value of 1.0 and 1.667 (values given in parenthesis).

\begin{tabular}{cccccc}
\hline & M1 R1b & M1 R2b & M5 R1b & M5 R3b & X-ray \\
\hline CYM540 & $2.0(2.1)$ & $2.0(2.1)$ & $1.9(2.1)$ & $2.0(2.1)$ & 0.6 \\
CYM543 & $1.4(1.7)$ & $1.3(1.6)$ & $1.3(1.6)$ & $1.3(1.5)$ & 1.5 \\
HID556 & $0.8(0.6)$ & $0.8(0.8)$ & $0.5(0.8)$ & $0.9(1.1)$ & 3.0 \\
CYM560 & $1.9(1.6)$ & $1.9(1.7)$ & $1.7(1.6)$ & $1.8(1.7)$ & 1.7 \\
Zn & $3.7(3.6)$ & $3.9(3.8)$ & $3.7(3.4)$ & $3.7(3.4)$ & 2.7 \\
Sum (Zn center) & $9.8(9.6)$ & $9.9(10.0)$ & $9.1(9.5)$ & $9.7(9.8)$ & 9.5 \\
FCV & $1.1(1.2)$ & $1.0(1.0)$ & $0.9(1.0)$ & $0.8(1.0)$ & 1.7 \\
\hline
\end{tabular}


Table S21. Atomic displacements of zinc center through our quantum refinements using different multiscale schemes with different optimized regions (R1b, R2b or $\mathrm{R} 3 \mathrm{~b})$ for histone acetyltransferase with $\omega_{\alpha}$ value of 1.667 . The two-center (2C) ONIOM approximation was also used (values given in parenthesis).

\begin{tabular}{|c|c|c|c|c|}
\hline & M1 R1b & M1 R2b & M5 R1b & M5 R3b \\
\hline \multicolumn{5}{|c|}{ CYM540 } \\
\hline $\mathrm{N}$ & $0.08(0.08)$ & $0.07(0.04)$ & $0.05(0.05)$ & $0.06(0.06)$ \\
\hline $\mathrm{CA}$ & $0.05(0.05)$ & $0.04(0.01)$ & $0.04(0.04)$ & $0.03(0.03)$ \\
\hline $\mathrm{CB}$ & $0.13(0.13)$ & $0.13(0.06)$ & $0.21(0.21)$ & $0.15(0.19)$ \\
\hline SG & $0.12(0.12)$ & $0.12(0.11)$ & $0.13(0.13)$ & $0.13(0.13)$ \\
\hline $\mathrm{C}$ & $0.05(0.05)$ & $0.04(0.01)$ & $0.04(0.04)$ & $0.03(0.03)$ \\
\hline $\mathrm{O}$ & $0.07(0.07)$ & $0.06(0.04)$ & $0.07(0.07)$ & $0.07(0.08)$ \\
\hline \multicolumn{5}{|c|}{ CYM543 } \\
\hline $\mathrm{N}$ & $0.18(0.18)$ & $0.17(0.10)$ & $0.16(0.16)$ & $0.17(0.18)$ \\
\hline $\mathrm{CA}$ & $0.14(0.14)$ & $0.14(0.11)$ & $0.12(0.12)$ & $0.14(0.14)$ \\
\hline $\mathrm{CB}$ & $0.06(0.06)$ & $0.07(0.04)$ & $0.07(0.06)$ & $0.06(0.07)$ \\
\hline SG & $0.14(0.14)$ & $0.14(0.12)$ & $0.13(0.13)$ & $0.13(0.13)$ \\
\hline $\mathrm{C}$ & $0.07(0.07)$ & $0.08(0.04)$ & $0.08(0.08)$ & $0.09(0.09)$ \\
\hline $\mathrm{O}$ & $0.08(0.08)$ & $0.09(0.04)$ & $0.12(0.11)$ & $0.11(0.11)$ \\
\hline \multicolumn{5}{|c|}{ HID556 } \\
\hline $\mathrm{N}$ & $0.11(0.11)$ & $0.10(0.06)$ & $0.10(0.10)$ & $0.11(0.12)$ \\
\hline $\mathrm{CA}$ & $0.12(0.12)$ & $0.11(0.05)$ & $0.13(0.13)$ & $0.12(0.13)$ \\
\hline $\mathrm{CB}$ & $0.12(0.12)$ & $0.12(0.03)$ & $0.16(0.15)$ & $0.13(0.15)$ \\
\hline $\mathrm{CG}$ & $0.10(0.10)$ & $0.11(0.03)$ & $0.14(0.14)$ & $0.11(0.13)$ \\
\hline $\mathrm{CD} 2$ & $0.13(0.13)$ & $0.14(0.05)$ & $0.17(0.16)$ & $0.14(0.14)$ \\
\hline ND1 & $0.16(0.16)$ & $0.16(0.07)$ & $0.18(0.18)$ & $0.17(0.17)$ \\
\hline CE1 & $0.20(0.20)$ & $0.21(0.09)$ & $0.23(0.22)$ & $0.20(0.20)$ \\
\hline NE2 & $0.19(0.19)$ & $0.20(0.08)$ & $0.22(0.21)$ & $0.18(0.18)$ \\
\hline $\mathrm{C}$ & $0.08(0.08)$ & $0.08(0.05)$ & $0.06(0.06)$ & $0.07(0.07)$ \\
\hline $\mathrm{O}$ & $0.26(0.26)$ & $0.25(0.14)$ & $0.29(0.29)$ & $0.26(0.27)$ \\
\hline \multicolumn{5}{|c|}{ CYM560 } \\
\hline $\mathrm{N}$ & $0.05(0.05)$ & $0.04(0.03)$ & $0.05(0.05)$ & $0.06(0.09)$ \\
\hline $\mathrm{CA}$ & $0.05(0.05)$ & $0.06(0.03)$ & $0.06(0.05)$ & $0.08(0.10)$ \\
\hline $\mathrm{CB}$ & $0.12(0.12)$ & $0.12(0.04)$ & $0.13(0.13)$ & $0.10(0.12)$ \\
\hline SG & $0.16(0.16)$ & $0.16(0.14)$ & $0.16(0.16)$ & $0.17(0.17)$ \\
\hline $\mathrm{C}$ & $0.02(0.02)$ & $0.03(0.03)$ & $0.04(0.03)$ & $0.03(0.05)$ \\
\hline $\mathrm{O}$ & $0.07(0.07)$ & $0.08(0.03)$ & $0.15(0.14)$ & $0.12(0.14)$ \\
\hline \multicolumn{5}{|c|}{ ZN802 } \\
\hline $\mathbf{Z N}$ & $0.08(0.08)$ & $0.07(0.07)$ & $0.08(0.08)$ & $0.08(0.08)$ \\
\hline
\end{tabular}


Table S22. Atomic displacements of zinc center through our quantum refinements using different multiscale schemes with different optimized regions (R1b, R2b or $\mathrm{R} 3 \mathrm{~b}$ ) for histone acetyltransferase with $\omega_{\alpha}$ value of 1.0. The two-center (2C) ONIOM approximation was also used (values given in parenthesis).

\begin{tabular}{|c|c|c|c|c|}
\hline & M1 R1b & M1 R2b & M5 R1b & M5 R3b \\
\hline \multicolumn{5}{|c|}{ CYM540 } \\
\hline $\mathrm{N}$ & $0.06(0.06)$ & $0.06(0.07)$ & $0.04(0.04)$ & $0.05(0.05)$ \\
\hline $\mathrm{CA}$ & $0.03(0.03)$ & $0.03(0.04)$ & $0.02(0.02)$ & $0.02(0.02)$ \\
\hline $\mathrm{CB}$ & $0.18(0.18)$ & $0.17(0.09)$ & $0.18(0.17)$ & $0.13(0.17)$ \\
\hline SG & $0.13(0.13)$ & $0.13(0.12)$ & $0.13(0.13)$ & $0.13(0.14)$ \\
\hline $\mathrm{C}$ & $0.04(0.04)$ & $0.03(0.03)$ & $0.03(0.03)$ & $0.02(0.02)$ \\
\hline $\mathrm{O}$ & $0.07(0.07)$ & $0.07(0.06)$ & $0.07(0.07)$ & $0.07(0.08)$ \\
\hline \multicolumn{5}{|c|}{ CYM543 } \\
\hline $\mathrm{N}$ & $0.15(0.15)$ & $0.15(0.14)$ & $0.13(0.13)$ & $0.14(0.16)$ \\
\hline $\mathrm{CA}$ & $0.12(0.13)$ & $0.14(0.12)$ & $0.11(0.12)$ & $0.12(0.13)$ \\
\hline $\mathrm{CB}$ & $0.08(0.08)$ & $0.06(0.06)$ & $0.05(0.06)$ & $0.04(0.05)$ \\
\hline SG & $0.13(0.13)$ & $0.13(0.13)$ & $0.12(0.13)$ & $0.12(0.12)$ \\
\hline $\mathrm{C}$ & $0.05(0.05)$ & $0.07(0.06)$ & $0.06(0.06)$ & $0.07(0.08)$ \\
\hline $\mathrm{O}$ & $0.05(0.05)$ & $0.08(0.07)$ & $0.09(0.09)$ & $0.09(0.10)$ \\
\hline \multicolumn{5}{|c|}{ HID556 } \\
\hline $\mathrm{N}$ & $0.12(0.12)$ & $0.12(0.11)$ & $0.10(0.10)$ & $0.11(0.12)$ \\
\hline $\mathrm{CA}$ & $0.14(0.14)$ & $0.13(0.10)$ & $0.11(0.12)$ & $0.11(0.13)$ \\
\hline $\mathrm{CB}$ & $0.15(0.16)$ & $0.14(0.09)$ & $0.14(0.14)$ & $0.12(0.15)$ \\
\hline $\mathrm{CG}$ & $0.13(0.13)$ & $0.12(0.08)$ & $0.13(0.13)$ & $0.11(0.13)$ \\
\hline $\mathrm{CD} 2$ & $0.14(0.15)$ & $0.14(0.10)$ & $0.13(0.15)$ & $0.11(0.14)$ \\
\hline ND1 & $0.15(0.16)$ & $0.14(0.13)$ & $0.16(0.18)$ & $0.16(0.17)$ \\
\hline CE1 & $0.22(0.24)$ & $0.22(0.18)$ & $0.20(0.22)$ & $0.18(0.21)$ \\
\hline NE2 & $0.21(0.22)$ & $0.21(0.17)$ & $0.20(0.22)$ & $0.17(0.20)$ \\
\hline $\mathrm{C}$ & $0.08(0.09)$ & $0.08(0.08)$ & $0.06(0.07)$ & $0.07(0.08)$ \\
\hline $\mathrm{O}$ & $0.25(0.26)$ & $0.24(0.21)$ & $0.25(0.26)$ & $0.23(0.25)$ \\
\hline \multicolumn{5}{|c|}{ CYM560 } \\
\hline $\mathrm{N}$ & $0.06(0.06)$ & $0.08(0.04)$ & $0.06(0.06)$ & $0.08(0.12)$ \\
\hline $\mathrm{CA}$ & $0.06(0.06)$ & $0.07(0.04)$ & $0.05(0.04)$ & $0.07(0.09)$ \\
\hline $\mathrm{CB}$ & $0.11(0.12)$ & $0.12(0.10)$ & $0.11(0.12)$ & $0.09(0.10)$ \\
\hline SG & $0.13(0.13)$ & $0.14(0.13)$ & $0.13(0.13)$ & $0.14(0.14)$ \\
\hline $\mathrm{C}$ & $0.03(0.03)$ & $0.04(0.04)$ & $0.02(0.02)$ & $0.02(0.02)$ \\
\hline $\mathrm{O}$ & $0.07(0.07)$ & $0.09(0.06)$ & $0.06(0.05)$ & $0.06(0.08)$ \\
\hline \multicolumn{5}{|l|}{ ZN802 } \\
\hline $\mathbf{Z N}$ & $0.07(0.07)$ & $0.07(0.07)$ & $0.07(0.08)$ & $0.07(0.07)$ \\
\hline
\end{tabular}


Table S23. Strain energy $(\Delta \mathrm{E}, \mathrm{kcal} / \mathrm{mol})$ at $\mathrm{B} 3 \mathrm{LYP} / 6-31 \mathrm{G}(\mathrm{d})$ level for the R1boptimized region of the $\mathrm{FCV}$ and $\mathrm{Zn}$ centers in the histone acetyltransferase determined by various quantum refinement schemes with $\omega_{\alpha}$ value of 1.667 . The twocenter (2C) ONIOM approximation (values given in parenthesis) was also used.

\begin{tabular}{ccc}
\hline & \multicolumn{2}{c}{$\Delta \mathrm{E}$} \\
& FCV & Zn \\
\hline X-ray & 41.1 & 127.2 \\
M1 R1b & $17.6(17.6)$ & $64.1(64.1)$ \\
M1 R2b & $16.9(16.9)$ & $63.1(63.1)$ \\
M5 R1b & $25.0(24.1)$ & $65.3(64.8)$ \\
M5 R3b & $19.3(19.5)$ & $60.9(62.0)$ \\
\hline
\end{tabular}

Table S24. Strain energy $(\Delta \mathrm{E}, \mathrm{kcal} / \mathrm{mol})$ at $\mathrm{B} 3 \mathrm{LYP} / 6-31 \mathrm{G}(\mathrm{d})$ level for the R1boptimized region of the FCV and $\mathrm{Zn}$ centers in the histone acetyltransferase determined by various quantum refinement schemes with $\omega_{\alpha}$ value of 1.0. The twocenter (2C) ONIOM approximation (values given in parenthesis) was also used.

$\Delta \mathrm{E}$

\begin{tabular}{ccc} 
& FCV & Zn \\
\hline X-ray & 41.1 & 127.2 \\
M1 R1b & $13.9(14.1)$ & $55.9(56.6)$ \\
M1 R2b & $12.5(13.1)$ & $55.8(56.2)$ \\
M5 R1b & $16.3(17.4)$ & $55.4(56.1)$ \\
M5 R3b & $14.3(15.0)$ & $53.7(54.9)$ \\
\hline
\end{tabular}

Table S25. Strain energy $(\Delta \mathrm{E}, \mathrm{kcal} / \mathrm{mol})$ at B3LYP/6-31G(d) level for the R1boptimized region of the $\mathrm{FCV}$ and $\mathrm{Zn}$ centers in the histone acetyltransferase determined by various quantum refinement schemes. Different values of the weighting factor (1.0 and 1.667 (values given in parenthesis)) are used.

\begin{tabular}{ccc}
\hline & \multicolumn{2}{c}{$\Delta \mathrm{E}$} \\
& FCV & Zn \\
\hline X-ray & 41.1 & 127.2 \\
M1 R1b & $13.9(17.6)$ & $55.9(64.1)$ \\
M1 R2b & $12.5(16.9)$ & $55.8(63.1)$ \\
M5 R1b & $16.3(25.0)$ & $55.4(65.3)$ \\
M5 R3b & $14.3(19.3)$ & $53.7(60.9)$ \\
\hline
\end{tabular}




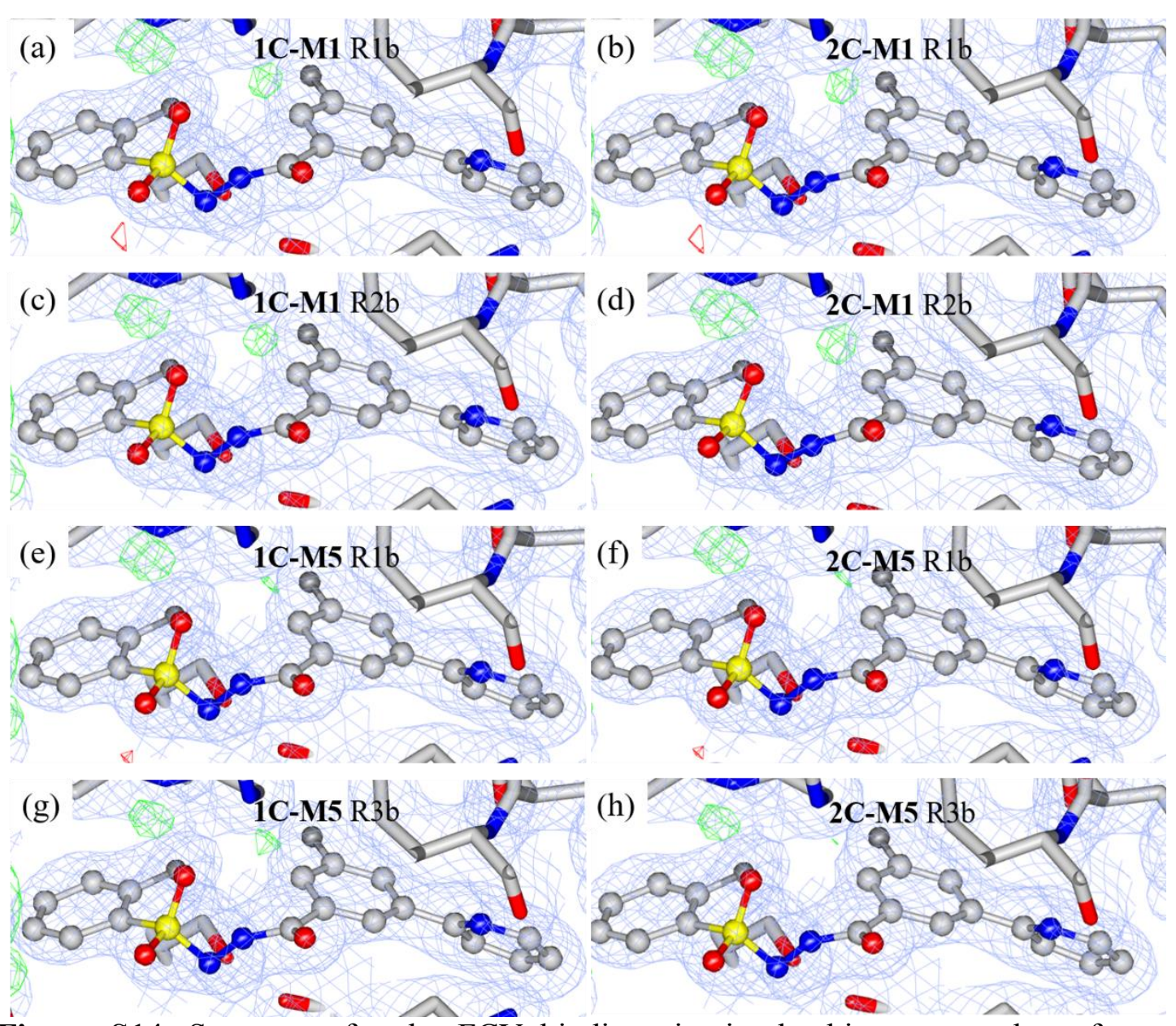

Figure S14. Strucutres for the FCV binding site in the histone acetyltransferase protein from various quantum refinement schemes with $\omega_{\alpha}$ value of 1.667 using (a) 1C-M1 (with the R1b-optimized region), (b) 2C-M1 (with the R1b-optimized region), (c) 1C-M1 (with the R2b-optimized region), (d) 2C-M1 (with the R2b-optimized region), (e) 1C-M5 (with the R1b-optimized region), (f) 2C-M5 (with the R1boptimized region), (g) 1C-M5 (with the R3b-optimized region), (h) 2C-M5 (with the R3b-optimized region), including the electron density map $\left(2 \mathrm{mF}_{\mathrm{o}}-\mathrm{DF}_{\mathrm{c}}\right.$ maps, contoured at $1.0 \sigma$ (blue), $\mathrm{mF}_{\mathrm{o}}-\mathrm{DF}_{\mathrm{c}}$ maps, contoured at $+3.0 \sigma$ (green), and $\mathrm{mF}_{\mathrm{o}}-\mathrm{DF}_{\mathrm{c}}$ maps, contoured at $-3.0 \sigma$ (red)). 


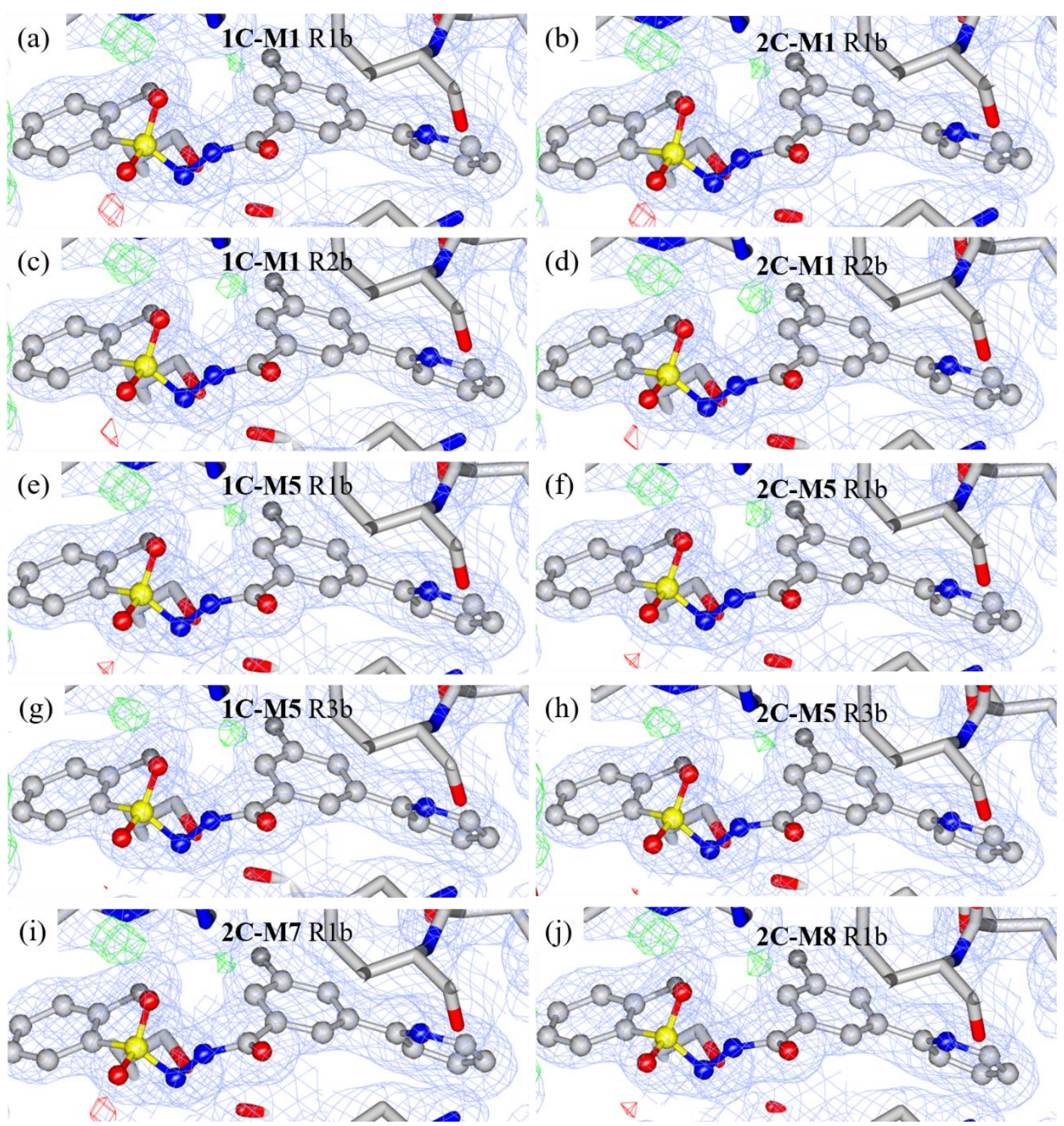

Figure S15. Strucutres for the FCV binding site in the histone acetyltransferase protein from various quantum refinement schemes with $\omega_{\alpha}$ value of 1.0 using (a) 1CM1 (with the R1b-optimized region), (b) 2C-M1 (with the R1b-optimized region), (c) 1C-M1 (with the R2b-optimized region), (d) 2C-M1 (with the R2-boptimized region), (e) 1C-M5 (with the R1b-optimized region), (f) 2C-M5 (with the R1b-optimized region), (g) 1C-M5 (with the R3b-optimized region), (h) 2C-M5 (with the R3boptimized region), (i) 2C-M7 (with the R1b-optimized region) and (j) 2C-M8 (with the R1b-optimized region), including the electron density map $\left(2 \mathrm{mF}_{\mathrm{o}}-\mathrm{DF}_{\mathrm{c}}\right.$ maps, contoured at $1.0 \sigma$ (blue), $\mathrm{mF}_{\mathrm{o}}-\mathrm{DF}_{\mathrm{c}}$ maps, contoured at $+3.0 \sigma$ (green), and $\mathrm{mF}_{\mathrm{o}}-\mathrm{DF}_{\mathrm{c}}$ maps, contoured at $-3.0 \sigma($ red $))$. 
Table S26. Our quantum refined key structural results with different $\omega_{\alpha}$ values for the zinc binding site using 1C-M1 R1b. The key structural results from the X-ray crystal structure were also given.

\begin{tabular}{cccccccc}
\hline & $\mathbf{1 . 0}$ & $\mathbf{1 . 6 6 7}$ & $\mathbf{3 . 0}$ & $\mathbf{5 . 0}$ & $\mathbf{1 0 . 0}$ & $\mathbf{2 0 . 0}$ & X-ray \\
\hline $\mathrm{Zn}_{\mathrm{S}}$ & 2.33 & 2.33 & 2.33 & 2.32 & 2.31 & 2.29 & 2.26 \\
$\mathrm{Zn}-\mathrm{S}_{\mathrm{C} 543}$ & 2.17 & 2.14 & 2.11 & 2.10 & 2.07 & 2.07 & 2.28 \\
$\mathrm{Zn}-\mathrm{N}_{\mathrm{H} 556}$ & 2.07 & 2.07 & 2.06 & 2.06 & 2.10 & 2.14 & 2.13 \\
$\mathrm{Zn}_{\mathrm{S}} \mathrm{S}_{\mathrm{C} 560}$ & 2.19 & 2.17 & 2.14 & 2.13 & 2.12 & 2.11 & 2.28 \\
$\mathrm{~N}_{\mathrm{H} 556}-\mathrm{Zn}-\mathrm{S}_{\mathrm{C} 540}$ & 100.8 & 101.8 & 101.2 & 101.8 & 101.2 & 100.7 & 107.8 \\
$\mathrm{~N}_{\mathrm{H} 556}-\mathrm{Zn}-\mathrm{S}_{\mathrm{C} 543}$ & 97.2 & 97.1 & 97.3 & 97.3 & 96.8 & 97.9 & 96.7 \\
$\mathrm{~N}_{\mathrm{H} 556}-\mathrm{Zn}-\mathrm{S}_{\mathrm{C} 560}$ & 104.7 & 104.5 & 104.4 & 104.8 & 105.0 & 105.7 & 108.1 \\
$\mathrm{~S}_{\mathrm{C} 540}-\mathrm{Zn}-\mathrm{S}_{\mathrm{C} 543}$ & 110.9 & 111.1 & 111.3 & 111.4 & 111.8 & 111.2 & 105.2 \\
$\mathrm{~S}_{\mathrm{C} 540}-\mathrm{Zn}-\mathrm{S}_{\mathrm{C} 560}$ & 110.7 & 110.3 & 109.7 & 109.6 & 109.3 & 109.3 & 114.0 \\
$\mathrm{~S}_{\mathrm{C} 543}-\mathrm{Zn}-\mathrm{S}_{\mathrm{C} 560}$ & 127.6 & 127.6 & 128.2 & 127.6 & 128.1 & 127.6 & 123.0 \\
\hline
\end{tabular}

Table S27. RSZD scores of residues around the $\mathrm{Zn}$ (II) binding site in the histone acetyltransferase from various quantum refinement approaches using 1C-M1 R1b with different $\omega_{\alpha}$ values. RSZD scores of the FCV binding site were also given. Results for X-ray were taken from the experimental structure without our further refinement.

\begin{tabular}{cccccccc}
\hline & $\mathbf{1 . 0}$ & $\mathbf{1 . 6 6 7}$ & $\mathbf{3 . 0}$ & $\mathbf{5 . 0}$ & $\mathbf{1 0 . 0}$ & $\mathbf{2 0 . 0}$ & X-ray \\
\hline CYM540 & 2.1 & 2.0 & 1.8 & 1.7 & 1.6 & 1.2 & 0.6 \\
CYM543 & 1.7 & 1.4 & 1.1 & 1.2 & 1.8 & 2.1 & 1.5 \\
HID556 & 0.6 & 0.8 & 0.4 & 0.5 & 0.7 & 0.7 & 3.0 \\
CYM560 & 1.6 & 1.9 & 2.3 & 2.2 & 2.4 & 2.2 & 1.7 \\
Zn(II) & 3.6 & 3.7 & 4.3 & 3.9 & 4.3 & 3.8 & 2.7 \\
Sum $^{\text {a }}$ & 9.6 & 9.8 & 9.9 & 9.5 & 10.8 & 10.0 & 9.5 \\
(Zn center) $_{\text {FCV }}$ & 1.2 & 1.1 & 0.6 & 0.5 & 0.1 & 0.1 & 1.7 \\
\hline
\end{tabular}

a: The sum for Zn center is the sum of CYM540, CYM543, HID556, CYM560 and $\mathrm{Zn}$. 


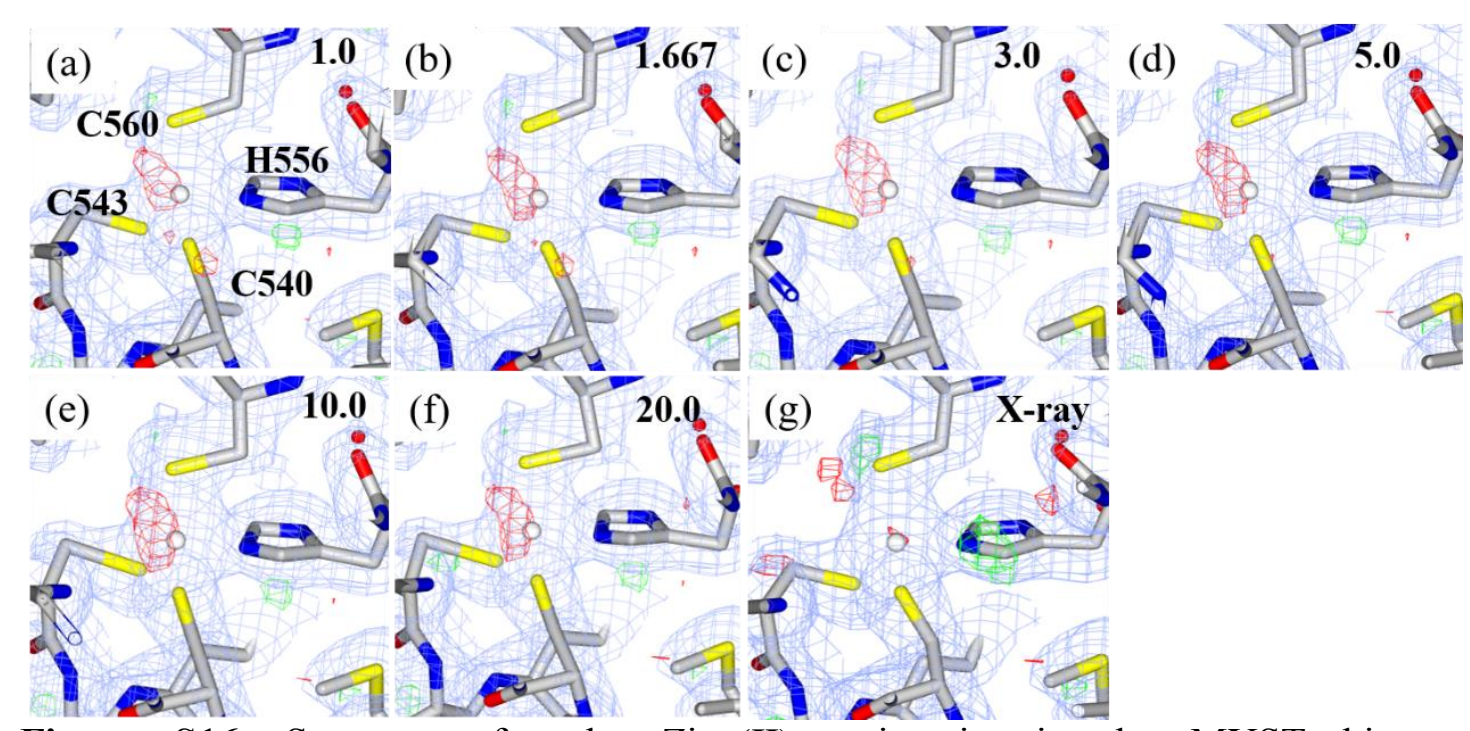

Figure S16. Structures for the Zinc(II) active-site in the MYST histone acetyltransferase KAT6A/B protein from various quantum refinement schemes with different $\omega_{\alpha}$ values using 1C-M1 (with the R1b-optimized region), (a) 1.0, (b) 1.667, (c) 3.0 , (d) 5.0 , (e) 10.0 , (f) 20.0 and (g) the X-ray crystal structure, including the electron density map $\left(2 \mathrm{mF}_{\mathrm{o}}-\mathrm{DF}_{\mathrm{c}}\right.$ maps, contoured at $1.0 \sigma$ (blue), $\mathrm{mF}_{\mathrm{o}}-\mathrm{DF}_{\mathrm{c}}$ maps, contoured at $+3.0 \sigma$ (green), and $\mathrm{mF}_{\mathrm{o}}-\mathrm{DF}_{\mathrm{c}}$ maps, contoured at $-3.0 \sigma$ (red)). Results for $\mathrm{X}$-ray were taken from the experimental structure without our further refinement.

Table S28. Our quantum refined key structural results with $\omega_{\alpha}$ value of 1.667 for the zinc binding site using different multiscale schemes with different optimized regions (R1b, R2b or R3b) for histone acetyltransferase including individual B factors for all atoms. The two-center (2C) ONIOM approximation was also used (values given in parenthesis). The key structural results from the X-ray crystal structure were also given. 1C-M5 R1b results are used as reference for RMSD and MAD computaions.

\begin{tabular}{|c|c|c|c|c|c|c|c|}
\hline & M1 R1b & M1 R2b & M5 R1b & M5 R3b & M7 R1b & M8 R1b & X-ray \\
\hline $\mathrm{Zn}-\mathrm{S}_{\mathrm{C} 540}$ & $2.35(2.36)$ & $2.36(2.36)$ & $2.35(2.36)$ & $2.36(2.37)$ & $(2.36)$ & $(2.37)$ & 2.26 \\
\hline $\mathrm{Zn}-\mathrm{S}_{\mathrm{C} 543}$ & $2.15(2.14)$ & $2.14(2.14)$ & $2.15(2.14)$ & $2.15(2.15)$ & $(2.16)$ & $(2.17)$ & 2.28 \\
\hline $\mathrm{Zn}-\mathrm{N}_{\mathrm{H} 556}$ & $2.06(2.07)$ & $2.07(2.07)$ & $2.09(2.08)$ & $2.09(2.09)$ & $(2.08)$ & (2.09) & 2.13 \\
\hline $\mathrm{Zn}-\mathrm{S}_{\mathrm{C} 560}$ & $2.18(2.18)$ & $2.17(2.17)$ & $2.18(2.18)$ & $2.18(2.18)$ & $(2.19)$ & $(2.21)$ & 2.28 \\
\hline RMSD (bonds) & $0.01(0.01)$ & $0.01(0.01)$ & $0.00(0.01)$ & $0.01(0.01)$ & $(0.01)$ & $(0.02)$ & 0.10 \\
\hline MAD (bonds) & $0.01(0.01)$ & $0.01(0.01)$ & $0.00(0.01)$ & $0.00(0.01)$ & $(0.01)$ & $(0.02)$ & 0.09 \\
\hline $\mathrm{N}_{\mathrm{H} 556}-\mathrm{Zn}-\mathrm{S}_{\mathrm{C} 540}$ & $100.4(100.6)$ & $100.1(100.1)$ & $101.4(100.5)$ & $100.9(100.9)$ & $(100.7)$ & $(100.7)$ & 107.8 \\
\hline $\mathrm{N}_{\mathrm{H} 556}-\mathrm{Zn}-\mathrm{S}_{\mathrm{C} 543}$ & $97.6(96.6)$ & $96.5(96.6)$ & $97.2(97.2)$ & $97.0(96.9)$ & $(96.4)$ & $(96.8)$ & 96.7 \\
\hline $\mathrm{N}_{\mathrm{H} 556}-\mathrm{Zn}-\mathrm{S}_{\mathrm{C} 560}$ & $105.2(106.0)$ & $105.8(105.9)$ & $105.3(105.9)$ & $106.3(106.3)$ & (106.1) & (106.2) & 108.1 \\
\hline $\mathrm{S}_{\mathrm{C} 540}-\mathrm{Zn}-\mathrm{S}_{\mathrm{C} 543}$ & $110.8(110.0)$ & $110.3(110.3)$ & $110.9(110.4)$ & $110.4(110.4)$ & $(110.0)$ & $(110.3)$ & 105.2 \\
\hline $\mathrm{S}_{\mathrm{C} 540}-\mathrm{Zn}-\mathrm{S}_{\mathrm{C} 560}$ & 109.9(109.6) & $108.7(108.7)$ & $109.5(109.1)$ & $108.4(108.4)$ & (109.7) & (109.2) & 114.0 \\
\hline $\mathrm{S}_{\mathrm{C} 543}-\mathrm{Zn}-\mathrm{S}_{\mathrm{C} 560}$ & $128.1(129.2)$ & $130.1(130.1)$ & $128.1(129.1)$ & $129.4(129.3)$ & (129.1) & $(129.0)$ & 123.0 \\
\hline RMSD (angles) & $0.5(0.8)$ & 1.1(1.1) & $0.0(0.6)$ & $0.8(0.8)$ & $(0.8)$ & $(0.7)$ & 4.6 \\
\hline MAD (angles) & $0.3(0.7)$ & $1.0(1.0)$ & $0.0(0.6)$ & $0.8(0.7)$ & $(0.7)$ & $(0.6)$ & 4.2 \\
\hline $\mathrm{R} /$ & $0.221(0.221) /$ & $0.220(0.220) /$ & $0.221(0.221) /$ & $0.220(0.220) /$ & $(0.221) /$ & $(0.221) /$ & $0.214 /$ \\
\hline$R_{\text {free }}$ & $0.235(0.235)$ & $0.236(0.236)$ & $0.235(0.235)$ & $0.235(0.235)$ & $(0.235)$ & $(0.235)$ & 0.241 \\
\hline
\end{tabular}


Table S29. Our quantum refined key structural results with $\omega_{\alpha}$ value of 1.667 for the FCV ligand using different multiscale schemes with different optimized regions (R1b, $\mathrm{R} 2 \mathrm{~b}$ or R3b) including individual $\mathrm{B}$ factors for all atoms. The two-center (2C) ONIOM approximation (values given in parenthesis) was also used. 1C-M5 R1b results are used as reference for RMSD and MAD computaions.

\begin{tabular}{|c|c|c|c|c|c|c|c|}
\hline & M1 R1b & M1 R2b & M5 R1b & M5 R3b & M7 R1b & M8 R1b & X-ray \\
\hline $\mathrm{C} 2-\mathrm{C} 7$ & $1.40(1.40)$ & $1.40(1.40)$ & $1.42(1.42)$ & $1.41(1.41)$ & $(1.40)$ & $(1.42)$ & 1.39 \\
\hline $\mathrm{C} 2-\mathrm{C} 3$ & $1.42(1.41)$ & $1.41(1.41)$ & $1.43(1.43)$ & $1.42(1.42)$ & $(1.41)$ & (1.43) & 1.39 \\
\hline C6-C7 & $1.41(1.40)$ & $1.40(1.40)$ & $1.39(1.39)$ & $1.39(1.39)$ & (1.40) & (1.39) & 1.39 \\
\hline C7-F1 & $1.39(1.39)$ & $1.38(1.38)$ & $1.39(1.39)$ & $1.39(1.39)$ & (1.39) & (1.40) & 1.36 \\
\hline C6-C5 & $1.39(1.38)$ & $1.39(1.39)$ & $1.40(1.40)$ & $1.39(1.39)$ & (1.38) & (1.40) & 1.38 \\
\hline $\mathrm{C} 5-\mathrm{C} 4$ & $1.40(1.39)$ & $1.40(1.40)$ & $1.40(1.40)$ & $1.40(1.40)$ & (1.39) & (1.40) & 1.39 \\
\hline C4-C3 & $1.41(1.41)$ & $1.41(1.41)$ & $1.40(1.40)$ & $1.40(1.41)$ & $(1.41)$ & (1.40) & 1.39 \\
\hline $\mathrm{C} 2-\mathrm{S} 1$ & $1.88(1.88)$ & $1.86(1.87)$ & $1.85(1.83)$ & $1.84(1.84)$ & $(1.88)$ & (1.83) & 1.82 \\
\hline $\mathrm{S} 1-\mathrm{O} 3$ & $1.47(1.47)$ & $1.47(1.47)$ & $1.49(1.49)$ & $1.48(1.48)$ & (1.47) & (1.49) & 1.47 \\
\hline $\mathrm{S} 1-\mathrm{O} 2$ & $1.48(1.48)$ & $1.48(1.48)$ & $1.50(1.50)$ & $1.50(1.50)$ & $(1.48)$ & (1.50) & 1.46 \\
\hline S1-N1 & $1.64(1.65)$ & $1.65(1.65)$ & $1.65(1.65)$ & $1.65(1.65)$ & $(1.65)$ & (1.65) & 1.70 \\
\hline $\mathrm{N} 1-\mathrm{N} 2$ & $1.38(1.40)$ & $1.38(1.38)$ & $1.41(1.40)$ & $1.40(1.40)$ & $(1.40)$ & $(1.40)$ & 1.40 \\
\hline $\mathrm{N} 2-\mathrm{C} 1$ & $1.40(1.40)$ & $1.40(1.40)$ & $1.40(1.41)$ & $1.41(1.41)$ & (1.40) & (1.41) & 1.45 \\
\hline $\mathrm{C} 1-\mathrm{O} 1$ & $1.23(1.23)$ & $1.23(1.23)$ & $1.27(1.27)$ & $1.27(1.26)$ & $(1.23)$ & (1.27) & 1.19 \\
\hline $\mathrm{C} 1-\mathrm{C} 8$ & $1.47(1.47)$ & $1.47(1.47)$ & $1.44(1.44)$ & $1.45(1.45)$ & $(1.47)$ & (1.44) & 1.52 \\
\hline C8-C9 & $1.42(1.42)$ & $1.43(1.43)$ & $1.44(1.45)$ & $1.44(1.44)$ & $(1.42)$ & $(1.45)$ & 1.38 \\
\hline C9-C10 & $1.39(1.39)$ & $1.39(1.39)$ & $1.38(1.38)$ & $1.38(1.38)$ & (1.39) & (1.38) & 1.38 \\
\hline C10-F2 & $1.36(1.36)$ & $1.36(1.36)$ & $1.37(1.38)$ & $1.37(1.37)$ & $(1.36)$ & (1.38) & 1.36 \\
\hline C10-C11 & $1.40(1.39)$ & $1.40(1.40)$ & $1.39(1.40)$ & $1.40(1.40)$ & $(1.40)$ & $(1.40)$ & 1.39 \\
\hline $\mathrm{C} 11-\mathrm{C} 12$ & $1.42(1.41)$ & $1.41(1.41)$ & $1.42(1.43)$ & $1.42(1.42)$ & $(1.41)$ & $(1.43)$ & 1.39 \\
\hline C12-C18 & $1.39(1.39)$ & $1.39(1.39)$ & $1.38(1.38)$ & $1.38(1.38)$ & (1.39) & (1.38) & 1.38 \\
\hline C18-C8 & $1.39(1.39)$ & $1.39(1.39)$ & $1.40(1.40)$ & $1.39(1.39)$ & (1.39) & (1.40) & 1.38 \\
\hline C12-C13 & $1.48(1.46)$ & $1.48(1.48)$ & $1.45(1.46)$ & $1.45(1.45)$ & $(1.46)$ & (1.46) & 1.38 \\
\hline C13-C14 & $1.38(1.37)$ & $1.38(1.38)$ & $1.38(1.38)$ & $1.38(1.38)$ & $(1.37)$ & (1.38) & 1.38 \\
\hline C14-C15 & $1.39(1.38)$ & $1.39(1.39)$ & $1.38(1.38)$ & $1.38(1.38)$ & (1.39) & (1.38) & 1.38 \\
\hline C15-C16 & $1.40(1.39)$ & $1.39(1.39)$ & $1.40(1.40)$ & $1.40(1.40)$ & (1.39) & (1.40) & 1.39 \\
\hline C16-C17 & $1.39(1.38)$ & $1.39(1.39)$ & $1.38(1.38)$ & $1.39(1.39)$ & $(1.38)$ & (1.38) & 1.39 \\
\hline C17-N3 & $1.36(1.35)$ & $1.36(1.36)$ & $1.36(1.36)$ & $1.36(1.36)$ & $(1.35)$ & (1.36) & 1.32 \\
\hline C13-N3 & $1.34(1.34)$ & $1.34(1.34)$ & $1.35(1.35)$ & $1.35(1.35)$ & $(1.34)$ & (1.35) & 1.32 \\
\hline RMSD & $0.02(0.02)$ & $0.02(0.02)$ & $0.00(0.00)$ & $0.00(0.00)$ & $(0.02)$ & $(0.01)$ & 0.04 \\
\hline MAD & $0.01(0.01)$ & $0.01(0.01)$ & $0.00(0.00)$ & $0.00(0.00)$ & $(0.01)$ & $(0.00)$ & 0.03 \\
\hline
\end{tabular}

Table S30. Strain energy $(\Delta \mathrm{E}, \mathrm{kcal} / \mathrm{mol})$ at $\mathrm{B} 3 \mathrm{LYP} / 6-31 \mathrm{G}(\mathrm{d})$ level for the R1boptimized region of the $\mathrm{FCV}$ and $\mathrm{Zn}$ centers in the histone acetyltransferase 
determined by various quantum refinement schemes with $\omega_{\alpha}$ value of 1.667 including individual B factors for all atoms. The two-center (2C) ONIOM approximation (values given in parenthesis) was also used.

\begin{tabular}{ccc}
\hline & \multicolumn{2}{c}{$\Delta \mathrm{E}$} \\
& FCV & Zn \\
\hline X-ray & 41.1 & 127.2 \\
M1 R1b & $18.1(18.1)$ & $63.1(63.1)$ \\
M1 R2b & $18.1(18.1)$ & $63.1(63.1)$ \\
M5 R1b & $23.7(23.5)$ & $63.2(62.9)$ \\
M5 R3b & $20.8(20.8)$ & $60.6(60.3)$ \\
\hline
\end{tabular}




\section{Quantum refined results of Amyloid- $\beta$}

In addition to the main ONIOM-type QM/MM- or QM/SE/MM-based quantum refinement calculations for the two metalloproteins, a small amyloid- $\beta(20-34)$ protein with L-isoAsp23 (Figure S17a), which contains 223 atoms, was refined by a complete DFT, fragmentation DFT or GFN2-xTB method (M9-M11) briefly. B3LYP-D3/def2$\mathrm{SVP}^{13-14}$ with an efficient RIJCOSX algorithm ${ }^{15}$ implemented in ORCA 4.2.1 $\operatorname{program}^{16}$ (M9) were also used. Moreover, a generalized energy-based fragmentation $(\mathrm{GEBF})^{17-20}$ method at the B3LYP-D3/6-31G(d) level implemented in LSQC 2.3 $\operatorname{code}^{21}$ (M10) was also used. Finally, full SE quantum refinement calculation with an efficient GFN2-xTB implemented in XTB 6.1 code was examined (M11). All atoms were fully relaxed in this monomer cluster model. Moreover, a full geometry optimization for this monomer protein using the GFN2-xTB method in the gas phase without experimental data was performed and the results obtained compared with our quantum refinement results.

Quantum refinement calculations with $\omega_{\alpha}$ value of 0.269 or 1.0 were conducted. Different from our previous two applications, the choice of $\omega_{\alpha}$ values show some influence on $\mathrm{R}$ and $\mathrm{R}_{\text {free }}$ factors (Table S31). When $\omega_{\alpha}$ was set to 1.0, the $\mathrm{R}$ factors are slightly improved. However, R factors increase by $\sim 0.02$, when CNS-suggested value of 0.269 was used. Pleasingly, as shown in Figure S18b, our quantum refinement calculations using the monomer structure and different computational methods (M9M11) were found to give a very similar overall protein structure compared to the $X$ ray crystal structure with a very small RMSD value (0.08-0.09 with $\omega_{\alpha}$ value of 1.0; 0.12-0.14 with $\omega_{\alpha}$ value of 0.269 ). Moreover, slightly reduced $\mathrm{R} / \mathrm{R}_{\text {free }}$ factors by these three methods with $\omega_{\alpha}$ value of 1.0 were also observed (0.245/0.256-0.257 vs. X-ray: $0.246 / 0.263$; see Table S31). While, significantly increased $R / R_{\text {free }}$ factors by the GFN2-xTB method without experimental data was found $(0.560 / 0.569)^{\mathrm{a}}$. From the electron density maps (Figures S19-S20), the reduction of the negative (red contours) difference density closed to the $\mathrm{O}$ atom of IAS23 in the resultant electron density maps indicates that the discrepancy with experimental observations was minimized.

\footnotetext{
${ }^{a}$ The $R / R_{\text {free }}$ factors are different with the PDB database values, because we use CCP4i2 Remac5 for all $\mathrm{R} / \mathrm{R}_{\text {free }}$ factors computations, which is different from the original program.
} 
The RSZD scores (Table S32) are decreased by 0.4-3.2, compared to the X-ray structure. These results suggest that the non-natural amino acid residue L-isoAsp23 can be well described by the experimental refinement approach or quantum refinement approach. In contrast, normal geometry optimization for the monomer protein structure using the GFN2-xTB method in the absence of the experimental data leads to a substantial structural change with a RMSD value of 2.92 (Figure S18a). A few intramolecular H-bonds were also found to be formed without the experimental data (Figure S17b). Alternatively, in the absence of experimental constrains, one may use periodic boundary conditions (PBC) and full optimization of biomolecules to obtain the protein structures. Overall, our quantum refinement calculations are shown to provide an excellent protein structure using the monomer structure (as a cluster model) and afford a flexible combination with different computational methods (such as fragmentation DFT methods).
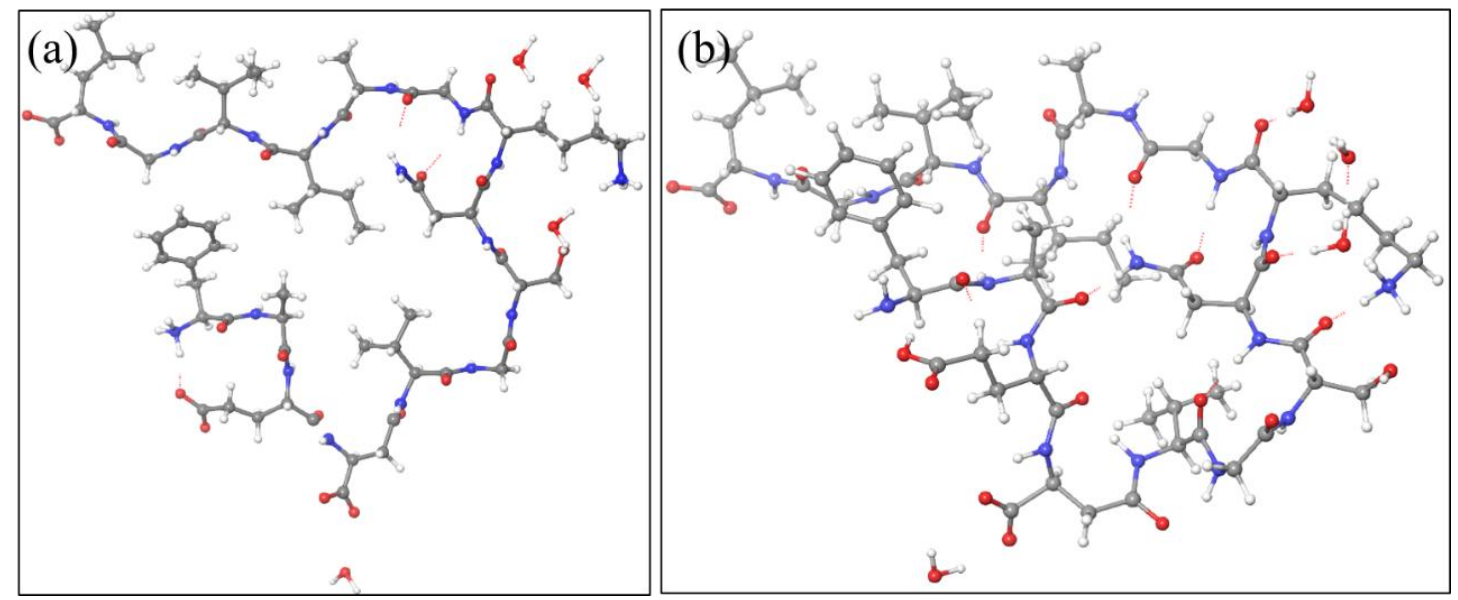

Figure S17. Structures for the amyloid- $\beta(20-34)$ protein from: (a) the X-ray crystal strucutre and (b) the full geometry optimization by the GFN2-xTB method in gas phase. 


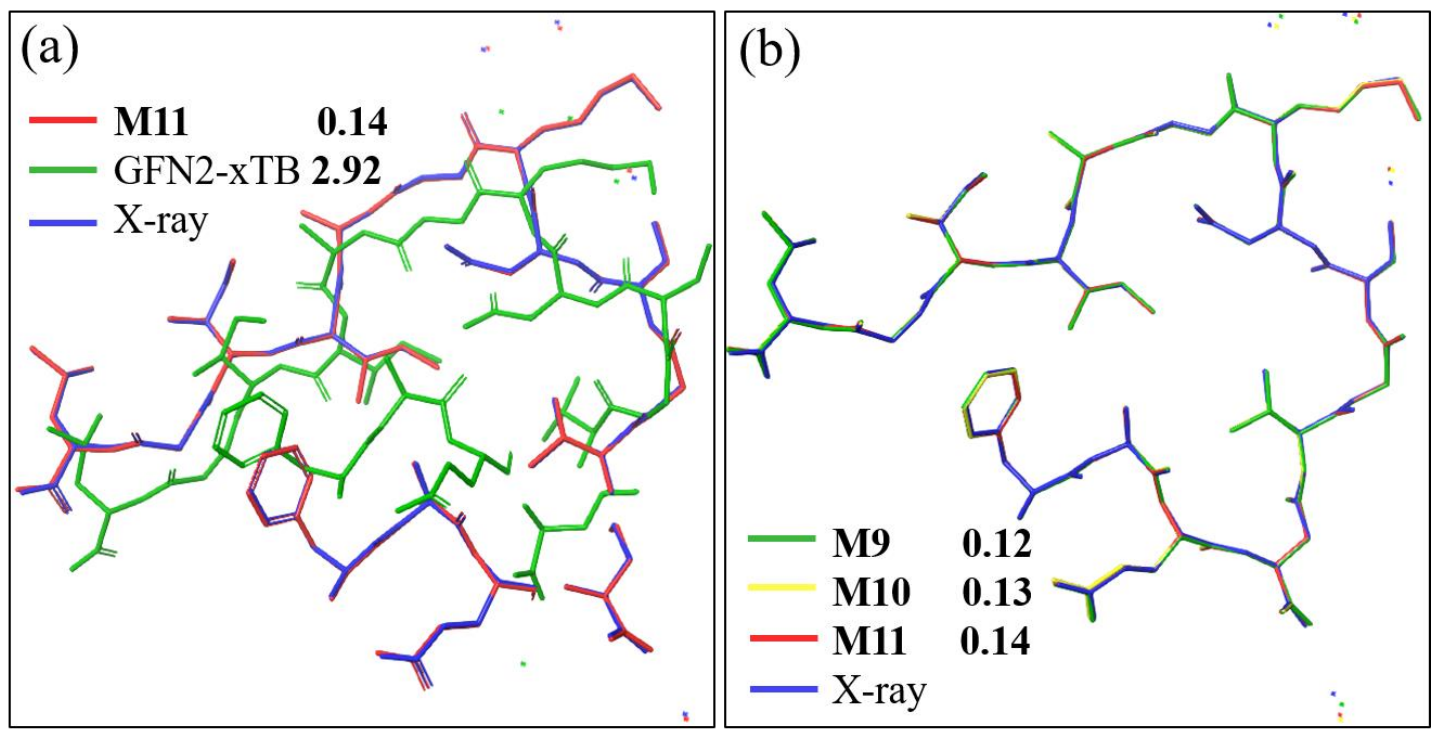

Figure S18. Superposition of the amyloid- $\beta$ structure: (a) X-ray crystal structure (blue line), our quantum refined results obtained by the GFN2-xTB method (M11, red line; normal optimization without experimental data, green line) and (b) X-ray crystal structure (blue line), our quantum refined results with $\omega_{\alpha}$ value of 0.269 obtained by different computational methods (M9, green line; M10, yellow line; M11, red line). RMSD values were determined based on the positions of the heavy atoms (nonhydrogen).

Table S31. Our quantum refined $R$ and $R_{\text {free }}$ factors and RMSD values based on positions of the heavy atoms (non-hydrogen) of the Amyloid- $\beta$ protein using different computational methods with $\omega_{\alpha}$ value of 0.269 and 1.0 (in parenthesis).

\begin{tabular}{cccccc}
\hline & M9 & M10 & M11 & GFN2-xTB & X-ray \\
\hline $\mathrm{R}$ & $0.266(0.245)$ & $0.267(0.245)$ & $0.268(0.245)$ & 0.560 & 0.246 \\
$\mathrm{R}_{\text {free }}$ & $0.280(0.257)$ & $0.279(0.256)$ & $0.282(0.257)$ & 0.569 & 0.263 \\
RMSD & $0.12(0.09)$ & $0.13(0.09)$ & $0.14(0.08)$ & 2.92 & 0.00 \\
\hline
\end{tabular}




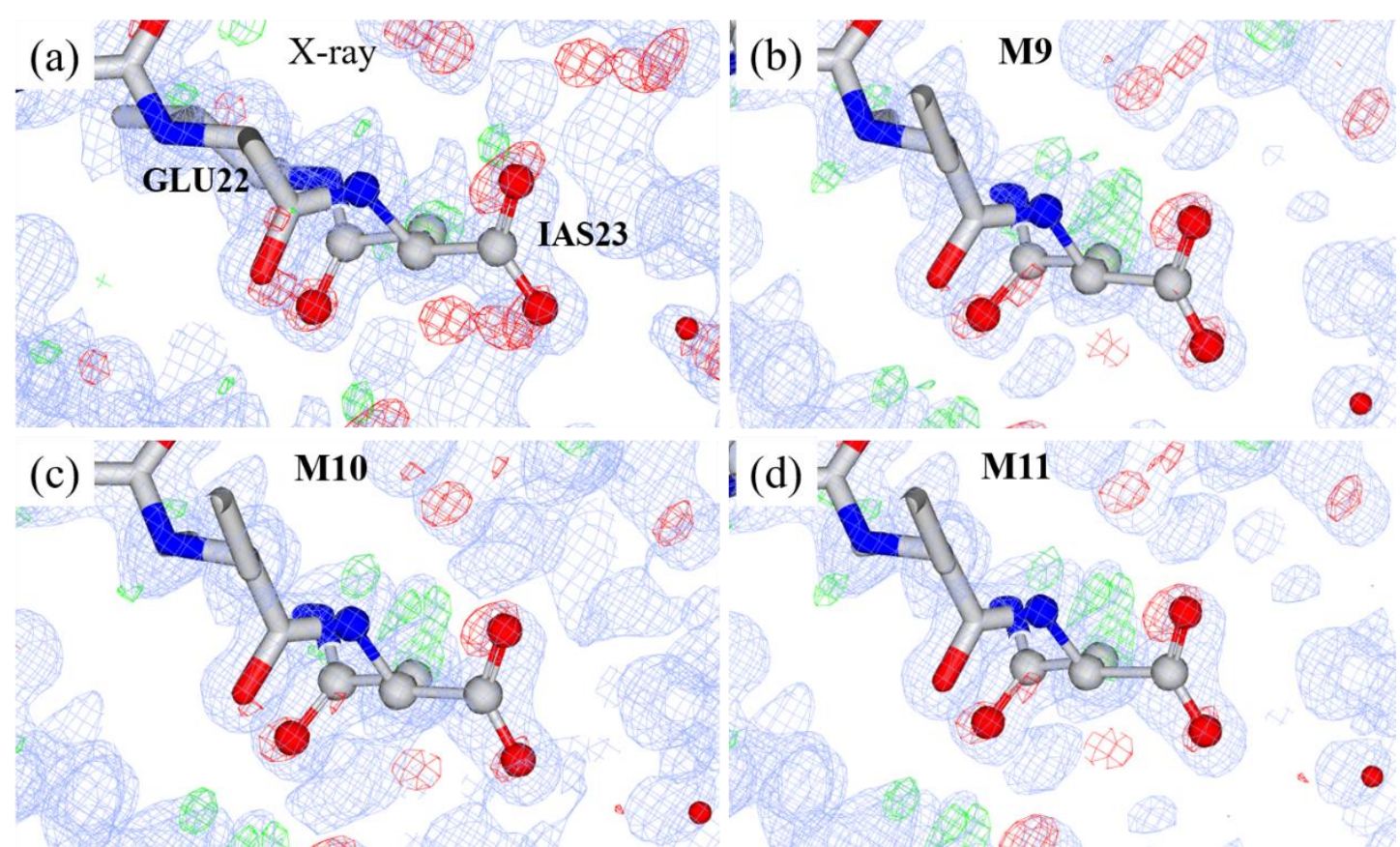

Figure S19. Structures for the L-isoaspartic acid (IAS) in the amyloid- $\beta$ protein from (a) the X-ray crystal structure and various quantum refinement schemes with $\omega_{\alpha}$ value of 0.269 using (b) M9, (c) M10, (d) M11, including the electron density map ( $2 \mathrm{mF}_{\mathrm{o}^{-}}$ $\mathrm{DF}_{\mathrm{c}}$ maps, contoured at $1.0 \sigma$ (blue), $\mathrm{mF}_{\mathrm{o}}-\mathrm{DF}_{\mathrm{c}}$ maps, contoured at $+3.0 \sigma$ (green), and mFo-DFc maps, contoured at $-3.0 \sigma($ red $))$.

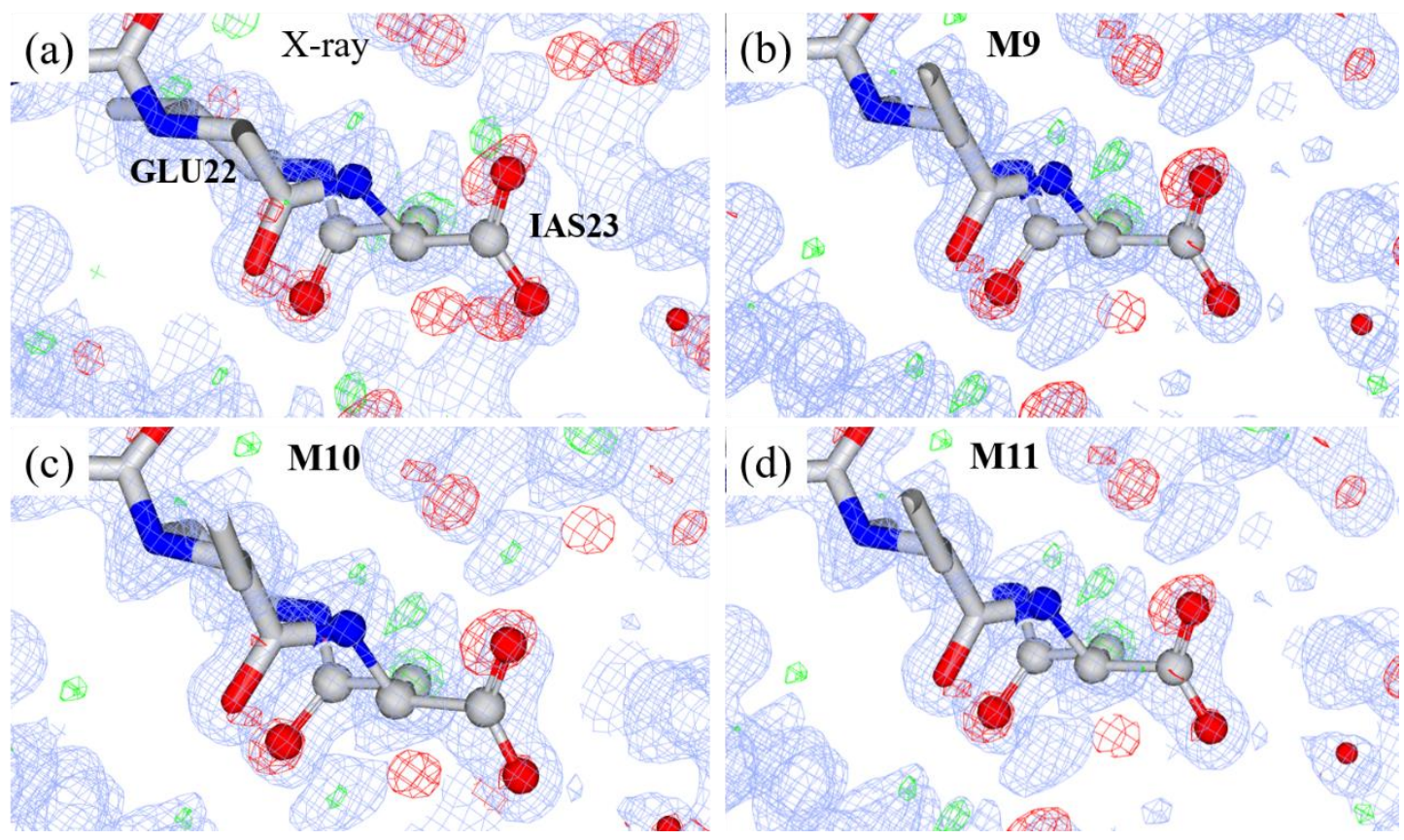

Figure S20. Structures for the L-isoaspartic acid (IAS) in the amyloid- $\beta$ protein from (a) the X-ray crystal structure and various quantum refinement schemes with $\omega_{\alpha}$ value of 1.0 using (b) M9, (c) M10, (d) M11, including the electron density map (2mFoDFc maps, contoured at $1.0 \sigma$ (blue), $\mathrm{mF}_{\mathrm{o}}-\mathrm{DF}_{\mathrm{c}}$ maps, contoured at $+3.0 \sigma$ (green), and $\mathrm{mF}_{\mathrm{o}}-\mathrm{DF}_{\mathrm{c}}$ maps, contoured at $\left.-3.0 \sigma(\mathrm{red})\right)$. 
Table S32. RSZD scores of residues of the Amyloid- $\beta$ protein using different computational methods with $\omega_{\alpha}$ value of 0.269 and 1.0 (in parenthesis).

\begin{tabular}{ccccc}
\hline & X-ray & M9 & M10 & M11 \\
\hline PHE20 & 1.0 & $0.2(0.8)$ & $0.1(0.9)$ & $0.1(0.8)$ \\
ALA21 & 0.6 & $0.8(0.6)$ & $1.1(0.7)$ & $1.0(0.6)$ \\
GLU22 & 1.3 & $0.8(1.2)$ & $1.6(1.1)$ & $0.8(1.2)$ \\
IAS23 & 4.2 & $0.2(3.7)$ & $0.1(3.9)$ & $0.2(3.7)$ \\
VAL24 & 0.7 & $4.0(0.2)$ & $4.0(0.2)$ & $3.9(0.2)$ \\
GLY25 & 3.3 & $0.5(3.4)$ & $0.5(3.6)$ & $0.6(3.4)$ \\
SER26 & 4.8 & $2.8(3.8)$ & $2.9(3.6)$ & $2.7(3.8)$ \\
ASN27 & 2.4 & $2.3(2.4)$ & $2.4(2.4)$ & $2.2(2.4)$ \\
LYS28 & 0.7 & $3.1(0.8)$ & $3.8(0.8)$ & $3.0(0.8)$ \\
GLY29 & 2.9 & $0.7(3.1)$ & $0.5(3.1)$ & $0.8(3.1)$ \\
ALA30 & 3.5 & $3.0(4.3)$ & $2.9(4.4)$ & $2.5(4.3)$ \\
ILE31 & 0.7 & $4.3(1.0)$ & $4.5(1.1)$ & $4.1(1.0)$ \\
ILE32 & 0.7 & $1.3(1.0)$ & $1.0(0.9)$ & $1.3(1.0)$ \\
GLY33 & 0.3 & $0.3(0.1)$ & $0.4(0.1)$ & $0.4(0.1)$ \\
LEU34 & 0.1 & $0.3(0.0)$ & $0.4(0.0)$ & $0.4(0.0)$ \\
Sum & 27.2 & $24.6(26.4)$ & $26.2(26.8)$ & $24.0(26.4)$ \\
\hline
\end{tabular}




\section{Cartesian coordinates of the quantum refinement results}

\section{A. Cartesian coordinates of all optimized regions for Rusticyanin (RC)}

\author{
QM/MM R1a (B3LYP/6-31G(d):Amber) \\ mechanical embedding $\omega_{\alpha}=\mathbf{0 . 6 0 6}$
}

$\mathrm{C}$

$\mathrm{O}$

$\mathrm{N}$

C

$\mathrm{C}$

$\mathrm{O}$

$\mathrm{C}$

C

C

$\mathrm{H}$

$\mathrm{H}$

$\mathrm{H}$

$\mathrm{H}$

$\mathrm{H}$

$\mathrm{H}$

$\mathrm{H}$

$\mathrm{N}$

C

$\mathrm{H}$

C

C

O

$\mathrm{N}$

C

C

$\mathrm{O}$

C

C

C

$\mathrm{N}$

C

$\mathrm{N}$

$\mathrm{H}$

$\mathrm{H}$

$\mathrm{H}$

$\mathrm{H}$

$\mathrm{H}$

$\mathrm{H}$

$\mathrm{H}$

N

C

C

O

C

$\mathrm{O}$

$\mathrm{H}$

H

$\mathrm{H}$

$\mathrm{H}$

$\mathrm{H}$

$\mathrm{N}$

C

$\mathrm{H}$

C

C

7.550043473 .2444015712 .93181343 7.132917984 .1496194411 .77800995 7.981323824 .5597318111 .00097198 5.806593124 .4854880111 .73814437 4.682230213 .9481635712 .53373920 3.991865292 .8250581211 .72627279 3.864295362 .8795613910 .50742461 3.776017885 .1942458912 .68696323 3.920944095 .8661422511 .30927501 5.412538635 .6657465110 .94664964 4.981275233 .5823131413 .52060326 2.749829164 .9165806012 .92657965 4.168011635 .8315588213 .49020505 3.288440215 .3666980810 .57276982 3.650868036 .9238258111 .34348062 5.569503605 .502663279 .87812818 6.025410946 .5233713611 .25146755 3.528389741 .7537717812 .42984722 2.879559760 .6238255611 .74867027 3.720002171 .5960607313 .41365416 $-1.197686006 .6901640521 .93730110$ $-1.982992676 .3832570820 .69201893$ $-3.066485896 .8856868320 .45764332$ $-1.386742375 .4553749619 .90950321$ $-1.995421305 .1243994318 .61600903$ $-3.479193054 .6854427218 .79685831$ $-3.880061224 .2082411719 .84934933$ $-1.203633133 .9783727317 .97948478$ 0.264006344 .2647487817 .90434888 1.291559123 .8024432818 .71340888 0.810297964 .9956887016 .89152703 2.118490314 .9770730317 .03099152 2.449556204 .2665057918 .13488971 $-0.542208874 .9956346820 .22547484$ $-1.974090056 .0100757017 .97475918$ $-1.373307673 .0522774018 .54294320$ $-1.596950813 .8117233916 .97203672$ 1.301942543 .2072286319 .60506979 2.847723405 .4498521816 .39076764 3.402050514 .1158442818 .46241710 $-4.271464294 .83288181 \quad 17.67794654$ $-5.646228124 .3324522217 .67242751$ $-5.737534073 .2390333616 .59034541$ $-4.870290793 .1416133015 .73060064$ $-6.701150855 .4293676917 .38354870$ $-6.482916705 .9742224516 .07575826$ $-3.884257505 .1122122016 .78488151$ $-5.822307633 .9137500418 .66736941$ $-7.700687864 .9815151817 .43316583$ $-6.627761376 .2181776618 .13830975$ $-7.376030105 .9270695215 .64557175$ $-6.812992202 .4128488216 .67379389$ $-7.152767861 .4430543015 .62573488$ $-7.480805382 .4527441917 .44211500$ $-5.929179063 .5096163410 .40358452$ $-5.717883404 .0057689511 .83909879$
$-6.565607983 .8905460712 .70208114$ $-4.511590134 .6150124812 .02207668$ $-4.280691555 .4005226813 .22602998$ $-4.584978546 .8869058012 .94850859$ $-4.188658237 .4487584511 .91925291$ $-2.791022585 .2303941913 .63894729$ $-2.462316836 .2251000615 .12751620$ $-3.889095914 .7410699411 .22954230$ $-4.921854324 .9964994714 .00967774$ $-2.595811104 .1818570813 .87293100$ $-2.137348305 .5527720112 .82373723$ $-5.279148187 .5185314913 .92674936$ $-5.688503088 .9231469313 .78214548$ $-4.668156739 .9196578414 .36584187$ $-4.8483716311 .1232304214 .25064085$ $-5.659510477 .0326513214 .73862163$ $-5.796063699 .1745241412 .72606091$ $-3.553950139 .4544893514 .95059122$ $-2.5577950010 .4416326915 .42081372$ $-2.0603987311 .1861042714 .19245641$ $-1.6398881710 .5187839813 .23494371$ $-1.395907069 .8075883416 .17265622$ $-1.959059159 .1725121017 .44883449$ $-0.2905122610 .8647578716 .47514154$ $-0.857778578 .5959073418 .35098932$ $-3.424790188 .4502217014 .99971654$ $-3.0739517411 .1197689416 .09552435$ $-0.970369799 .0248785415 .53417160$ $-2.518441049 .9213308518 .02517686$ $-2.659316228 .3684387317 .19280288$ 0.1283185811 .3334007915 .57622361 0.5595576710 .4106743016 .99895180 $-0.6678953211 .6664053117 .12156600$ $-0.286304087 .8122976717 .83231431$ $-1.294716268 .1640129519 .24819875$ $-0.145806859 .3657661518 .66456359$ 0.734088698 .4469817011 .22696621 0.244408807 .7911723912 .51408324 1.105351848 .1441074513 .69278346 2.083615149 .1066646113 .79704767 0.998759957 .4629519314 .90272450 1.898272338 .0357925515 .72794610 2.565699949 .0179403315 .09651729 $-0.801851308 .0741773812 .67285930$ 0.267038836 .7087627912 .35804855 2.479646329 .8375205913 .10929464 2.079593997 .7613044516 .75492803 3.292956899 .5950113415 .50045615 0.152983533 .498231999 .52582605 0.277497594 .1998258710 .89855162 $-0.029165903 .1724127511 .97266968$ 0.496732663 .8140156913 .61938975 0.038501442 .3831718614 .62968456 1.305223014 .5608235510 .98736972 $-0.394504575 .0613857710 .93838624$ $-1.099643022 .9532262012 .01099094$ 0.496029562 .2301670911 .80908674 $-1.005259782 .1097816814 .46703063$ 0.674306021 .5310387914 .40110106 0.174071862 .6486124315 .67762650 $-0.231339905 .9919909815 .34708459$ 
QM/MM R2a (B3LYP/6-31G(d):Amber) mechanical embedding $\omega_{\alpha}=\mathbf{0 . 6 0 6}$

$\mathrm{N} \quad 0.23726650-1.9665545917 .88130322$ C $\quad 0.89034138-0.8087131717 .28884248$

C $\quad 2.41106062-0.8797834617 .61084881$

O $\quad 2.79569734-0.8132902518 .79037534$

C $\quad 0.286491010 .4890954217 .83851577$

$\mathrm{H} \quad-0.05441303-1.9647424018 .85231011$

$\mathrm{H} \quad 0.75348008-0.8088277016 .20597799$

$\mathrm{H} \quad-0.446610270 .2726711218 .61756076$

$\mathrm{H} \quad-0.229358321 .0121957217 .03838634$

$\mathrm{H} \quad 1.062882551 .1297948118 .25385230$

$\mathrm{N} \quad 3.20464170-0.9379171316 .55271832$

C $\quad 4.68868613-0.9409471916 .55562067$

C $\quad 5.24674840-1.9088694617 .61332604$

O $\quad 5.61654950-1.5124712818 .72745873$

C $\quad 5.259352340 .5023507916 .74188698$

C $\quad 6.758502720 .5691688316 .34347975$

C $\quad 4.479053871 .5913678415 .98120645$

H $\quad 2.76448129-1.0116050515 .64111136$

$\mathrm{H} \quad 5.01746093-1.2985212815 .57969488$

$\mathrm{H} \quad 5.197416680 .7721516317 .78506339$

$\mathrm{H} \quad 7.358194130 .7004569717 .24346338$

$\mathrm{H} \quad 7.07839961-0.3649068115 .88168547$

$\mathrm{H} \quad 6.974187581 .3828741815 .65458637$

$\mathrm{H} \quad 3.601737551 .8759679316 .56111637$

$\mathrm{H} \quad 4.163102271 .1942956215 .02242674$

$\mathrm{H} \quad 5.096563082 .4688318715 .80872778$

$\mathrm{N} \quad 8.441169072 .1735983512 .55268545$

C $\quad 7.559503713 .2158497713 .04553608$

C $\quad 7.136281484 .0790344811 .83839648$

O $\quad 7.980415724 .4932397411 .05537853$

C $\quad 8.253916834 .0528626614 .12762161$

C $\quad 7.396347205 .1601499714 .73159537$

C $\quad 6.624177804 .9376247915 .88622903$

C $\quad 7.350356536 .4261930314 .10869086$

C $\quad 5.828619215 .9784963116 .40627531$

C $\quad 6.566003307 .4572935814 .62894713$

C $\quad 5.806549037 .2387113715 .78423580$

$\mathrm{H} \quad 9.367056922 .4018688412 .23879235$

$\mathrm{H} \quad 6.697020412 .7471253513 .50381515$

$\mathrm{H} \quad 8.584031293 .3837972414 .92123293$

$\mathrm{H} \quad 9.158531314 .4986144613 .71252722$

$\mathrm{H} \quad 6.641042323 .9735797816 .37419612$

$\mathrm{H} \quad 7.942042396 .6083467513 .22207850$

$\mathrm{H} \quad 5.240259355 .8238127517 .29821620$

$\mathrm{H} \quad 6.553987308 .4180941614 .12822232$

$\mathrm{H} \quad 5.204194028 .0356541216 .19494090$

$\mathrm{N} \quad 5.817475334 .4338177311 .77157046$

C $\quad 4.665839553 .9302474512 .53915250$

C $\quad 3.984293422 .8176991311 .71497125$

O $\quad 3.851467942 .8900689610 .49595115$

C $\quad 3.777182075 .1950196012 .65615400$

C $\quad 3.982251555 .8815101811 .29540901$

C $\quad 5.468579795 .6118213810 .94912985$

$\mathrm{H} \quad 4.934809533 .5739780313 .53741887$

$\mathrm{H} \quad 2.740359724 .9685018612 .90193707$

$\mathrm{H} \quad 4.189940395 .8227594813 .45718493$

$\mathrm{H} \quad 3.349352255 .4106168310 .54007563$

$\mathrm{H} \quad 3.754992766 .9505375911 .31335253$

$\mathrm{H} \quad 5.624782535 .418587219 .88605808$

$\mathrm{H} \quad 6.125703036 .4367080811 .24710639$

$\mathrm{N} \quad 3.530943571 .7383252112 .40533430$
2.923717730 .5984838911 .70978573 $2.09248093-0.2329323812 .67296683$ $2.30890822-0.1833580313 .89294037$ $3.96921506-0.3287130711 .03023589$ $4.78020412-1.0333785111 .97355943$ 3.667345271 .6187014813 .40265694 2.253671650 .9549800110 .92543158 $3.42093511-1.0689368410 .44655501$ 4.603991460 .2465780410 .35455158 $5.48265581-0.4264247312 .29352191$ $1.14754309-0.9835441912 .12190581$ $0.42694709-2.0324041512 .83066605$ $0.99788339-3.4144390912 .52748342$ $1.41191507-3.6908655411 .39018257$ $-1.02795765-2.0220369112 .36610053$ $-1.83244442-0.7827801112 .71360329$ $-2.26422749-0.5592683914 .04103531$ $-2.215097970 .1073704311 .68935422$ $-3.116182980 .5215811214 .31468708$ $-3.019218731 .2086804011 .99393098$ $-3.481021621 .4093464013 .30377696$ $1.01114116-0.9842906511 .11513984$ $0.45821119-1.8528548413 .90476390$ $-1.04296023-2.1610756711 .28485698$ $-1.54425456-2.8825680812 .79373871$ $-1.99033506-1.2539960614 .82270529$ $-1.86973876-0.0431926610 .67559306$ $-3.483395550 .7061828215 .31496827$ $-3.311580441 .8985339511 .21420985$ $-4.124692482 .2490031413 .52810032$ $-6.91786435-4.8817967820 .29884698$ $-5.92585903-3.9130164919 .91731275$ $-5.01609102-3.6161614721 .10925465$ $-5.52078888-3.2291484622 .15302926$ $-6.59535507-2.6210032719 .45719527$ $-5.56050516-1.5697168519 .12799284$ $-4.56224514-1.8216925818 .18507069$ $-5.57877025-0.3585943419 .81151698$ $-3.62437694-0.8234703617 .88719767$ $-4.631195880 .6367882319 .54912916$ $-3.657989410 .3981950718 .55965409$ $-7.57849286-4.6657120621 .04230562$ $-5.32076690-4.2832388619 .09380993$ $-7.18816473-2.8251511518 .56524863$ $-7.27208001-2.2641329420 .23465838$ $-4.54473983-2.7583568917 .64665256$ $-6.34985561-0.1969007620 .54592822$ $-2.86092214-1.0139510617 .14843798$ $-4.666205751 .5808409020 .07326311$ $-2.926653561 .1595654018 .33747289$ $-1.52400130-1.4761852522 .32775536$ $-0.53781893-0.4516221422 .00212962$ $0.75543427-0.7711915922 .76355263$ $0.83233938-0.4821023423 .95728395$ $-1.025426950 .9296864722 .46419236$ 0.036952761 .9677880822 .17913187 $-0.018677423 .1172914922 .81287575$ 0.928012361 .7488059521 .37359206 $-2.03248484-1.4412755923 .20773429$ $-0.33407796-0.4090169620 .93010878$ $-1.915596901 .1893078821 .89362198$ $-1.287510730 .9247046923 .52198286$ $-0.810353243 .3257301523 .40817480$ 0.703782793 .8046595622 .62350536 3.772778980 .5371035021 .87585513 


\begin{tabular}{|c|c|}
\hline $\mathrm{C}$ & 4.712521501 .5648920521 .48926826 \\
\hline $\mathrm{C}$ & 4.817499032 .6545903722 .55246051 \\
\hline $\mathrm{O}$ & 3.914676773 .4459961622 .78194265 \\
\hline $\mathrm{C}$ & 4.304716432 .1139404220 .11861214 \\
\hline $\mathrm{C}$ & 5.361890403 .0049124919 .53185715 \\
\hline $\mathrm{N}$ & 6.638109932 .6692833819 .62131166 \\
\hline $\mathrm{O}$ & 5.041027364 .0116968018 .94158697 \\
\hline $\mathrm{H}$ & 2.797626770 .6794317221 .63717606 \\
\hline $\mathrm{H}$ & 5.682956851 .0909220521 .36603097 \\
\hline $\mathrm{H}$ & 4.109220451 .2839537219 .45232584 \\
\hline $\mathrm{H}$ & 3.376531742 .6610076020 .22796612 \\
\hline $\mathrm{H}$ & 6.955121831 .7668023219 .93755617 \\
\hline $\mathrm{H}$ & 7.281599193 .2933319119 .15181205 \\
\hline $\mathrm{N}$ & 3.604332436 .9419341522 .55672432 \\
\hline $\mathrm{C}$ & 2.542528297 .3400232721 .61272837 \\
\hline $\mathrm{C}$ & 1.224892626 .6628064121 .97293918 \\
\hline $\mathrm{O}$ & 1.249579095 .4901257122 .32312448 \\
\hline $\mathrm{C}$ & 2.930644986 .9218083220 .19549126 \\
\hline $\mathrm{C}$ & 4.185386077 .6096896519 .75711158 \\
\hline $\mathrm{C}$ & 5.444855077 .0440660720 .05830782 \\
\hline $\mathrm{C}$ & 4.089567838 .8520629119 .11090513 \\
\hline $\mathrm{C}$ & 6.598954647 .7588245519 .73336558 \\
\hline $\mathrm{C}$ & 5.253054229 .5597436818 .78621698 \\
\hline $\mathrm{C}$ & 6.505856209 .0062775219 .10523275 \\
\hline $\mathrm{H}$ & 3.726547475 .9583591622 .75674164 \\
\hline $\mathrm{H}$ & 2.363881198 .4169191821 .62748328 \\
\hline $\mathrm{H}$ & 3.064219835 .8413954320 .15092673 \\
\hline $\mathrm{H}$ & 2.115119187 .1854538419 .52357212 \\
\hline $\mathrm{H}$ & 5.522111416 .0858746820 .54980167 \\
\hline $\mathrm{H}$ & 3.128634229 .2902254018 .88613206 \\
\hline $\mathrm{H}$ & 7.574328507 .3668373219 .96544162 \\
\hline $\mathrm{H}$ & 5.1922936110 .5227427718 .29640339 \\
\hline $\mathrm{H}$ & 7.410893679 .5430603418 .85258598 \\
\hline $\mathrm{N}$ & 0.084657987 .3338502021 .80878971 \\
\hline $\mathrm{C}$ & -1.216116686 .7073147922 .07161838 \\
\hline $\mathrm{C}$ & -1.966970306 .3894225620 .76871922 \\
\hline $\mathrm{O}$ & -3.034020156 .9060595520 .48619981 \\
\hline $\mathrm{H}$ & 0.110291968 .2991613121 .51460136 \\
\hline $\mathrm{H}$ & -1.118498415 .7907632022 .65412223 \\
\hline $\mathrm{H}$ & -1.828701657 .3969319722 .65356919 \\
\hline $\mathrm{N}$ & -1.377125785 .4656971519 .96528069 \\
\hline $\mathrm{C}$ & -1.988121035 .1458712618 .66106851 \\
\hline $\mathrm{C}$ & -3.474078134 .6936985018 .82135132 \\
\hline $\mathrm{O}$ & -3.876602664 .1946218619 .86328237 \\
\hline $\mathrm{C}$ & -1.212152683 .9813756018 .02910662 \\
\hline $\mathrm{C}$ & 0.257614864 .2501945617 .92881612 \\
\hline $\mathrm{C}$ & 1.301787193 .7860901618 .70790819 \\
\hline $\mathrm{N}$ & 0.778295584 .9997614816 .90898137 \\
\hline $\mathrm{C}$ & 2.087702424 .9826588817 .01827026 \\
\hline $\mathrm{N}$ & 2.445320844 .2646292018 .10910968 \\
\hline $\mathrm{H}$ & -0.538718994 .9879915320 .27303736 \\
\hline $\mathrm{H}$ & -1.928703686 .0201734818 .00687022 \\
\hline $\mathrm{H}$ & -1.375225673 .0745210318 .62135070 \\
\hline $\mathrm{H}$ & -1.622480113 .7902554717 .03271048 \\
\hline $\mathrm{H}$ & 1.316412943 .1797254319 .59481820 \\
\hline $\mathrm{H}$ & 2.802775515 .4622631916 .36727422 \\
\hline $\mathrm{H}$ & 3.402226974 .1201944518 .42022344 \\
\hline $\mathrm{N}$ & -4.257218914 .8328565817 .69637755 \\
\hline $\mathrm{C}$ & -5.630196904 .3220164817 .66670437 \\
\hline $\mathrm{C}$ & -5.716711013 .2334803116 .58098941 \\
\hline $\mathrm{O}$ & -4.851283883 .1429133815 .71845106 \\
\hline $\mathrm{C}$ & -6.673912705 .4341901417 .38283904 \\
\hline $\mathrm{O}$ & -6.511018895 .9599928816 .06309769 \\
\hline $\mathrm{H}$ & -3.855277955 .1012479716 .80610299 \\
\hline & -5.838986413 .9174341618 .6628802 \\
\hline
\end{tabular}

$\mathrm{H} \quad-7.673911974 .9969495517 .49493693$ $\mathrm{H} \quad-6.559279086 .2316079918 .12460875$ $\mathrm{H} \quad-7.435075505 .9453211815 .69148857$ N $\quad-6.788455492 .4014877516 .66249588$ C $\quad-\quad-7.138296081 .4316167715 .61575821$ C $\quad-8.572519851 .7350889315 .19028600$ O $\quad-9.502625021 .3453982015 .87908870$ C $\quad-6.97203342-0.0079186316 .13585343$ C $\quad-7.08911545-1.0849717915 .08103065$ C $\quad-8.34749735-1.6098047314 .74629071$ C $\quad-5.93592084-1.6175453014 .48417175$ C $\quad-8.42184681-2.6544478713 .80625054$ C $\quad-6.02091278-2.6578141213 .55344552$

C $\mathrm{H}$
$-7.27095379-3.1710005713 .21758459$
-7.472026272 .4481853017 .41735976 $-7.472026272 .4481853017 .41735976$ $-6.497907321 .5603493314 .74142717$ $-5.98654676-0.0828054316 .58711324$ $-7.68857848-0.2052753716 .93277519$ $-9.24420304-1.2110634815 .19988132$ $-4.97004259-1.2247413914 .74624857$ $-9.38719197-3.0616136613 .54033326$ $-5.13173327-3.0502680413 .08045531$ $-7.34790011-3.9739240212 .49732509$ $-8.032030096 .9835731420 .73406336$ $-6.628769937 .0385800721 .19346286$ $-6.400654146 .1600863922 .44032419$ $\begin{array}{ll}-7.14347226 & 6.2237896123 .42495009\end{array}$ $-6.197897418 .4850129221 .45886841$ $-6.197666259 .3305735820 .20817550$ $-5.438523298 .9271883119 .10030122$ $-6.9799897410 .5016991020 .12080107$ $-5.456371669 .6953618217 .91925532$ $-7.0092573311 .2513452818 .92513127$ $-6.2447905010 .8534272417 .81537767$ $-8.568801677 .8452793920 .70641950$ $-5.991910716 .6400675720 .40365666$ $-6.843112898 .9263004122 .21927973$ $-5.185246758 .4798812221 .86347373$ $-4.822557468 .0430895219 .14506518$ $-7.5816795510 .8193964720 .96057337$ $-4.875836159 .3721466817 .07890230$ $-7.6087582912 .1492689818 .86354878$ $-6.2569848711 .4330054816 .90055967$ $-5.359926705 .3335917122 .37284967$ $-4.972222004 .4164455123 .45738366$ $-4.106683085 .1185356724 .49789850$ $-3.500376156 .1465037924 .22739518$ $-4.174007953 .2425788822 .91559744$ $-2.986245063 .6683512822 .28584726$ $-4.788641265 .3661211321 .53978267$ $-5.869168204 .0280980723 .93664524$ $-3.930125872 .5515090823 .72340326$ $-4.774112702 .7099706822 .18070981$ $-3.254447843 .9143608621 .37677225$ $-6.808403762 .3957823110 .37255376$ -5.909722283 .5556992510 .36063105
-5.709449734 .0370315811 .80938314 $-5.709449734 .0370315811 .80938314$ $-6.543581733 .8836915512 .68298605$ $-6.467839544 .690281859 .48232946$ $-6.942501254 .271633968 .06410723$ $-7.619377925 .3957443410 .19143954$ $-7.668703432 .4713199510 .91340277$ $-4.940012603 .271693269 .94985489$ $-5.674269205 .426527579 .35711893$ $-7.930430984 .655842277 .81502510$ 
$-0.3432216712 .6565581211 .22542411$ 0.8213496112 .2472900510 .43429726 1.0441556810 .7506982710 .30875636 1.7169717610 .330861809 .37100795 $-1.2186151612 .7965913810 .72676779$ 0.7031924212 .644914349 .42639661 1.7231733012 .6921480710 .85643641 0.539987319 .9168920211 .23320512 0.806043218 .4692901111 .20451561 0.241553227 .748392359 .95968136 0.980893997 .027655309 .30377016 0.315909467 .7994006212 .49062990 1.151063878 .1576569413 .68183306 2.119392709 .1200573913 .82733776 1.008543727 .4592542414 .88435278 1.872234108 .0340222015 .74289312 2.552641329 .0253493715 .14524292 $-0.0472456210 .3325004011 .95103680$ 1.886703708 .3275573011 .13462664 $-0.737131008 .0617837912 .64834341$ $\begin{array}{llll}0.35791810 & 6.71492681 & 12.34273147\end{array}$ 2.519118629 .8532982913 .14383493 2.014028287 .7538949416 .77482144 3.254239799 .6100944215 .58240855 $-1.049613647 .893968039 .66076084$ $-1.649739617 .323449928 .45081499$ $-0.936441667 .835167917 .19529952$ $-0.675264787 .063123776 .26613667$ $-3.160195367 .625684288 .41514454$ $-1.606889898 .5329319710 .21961282$ $-1.533328726 .238518788 .48660488$ $-3.452009057 .985792937 .43721808$ $-3.452212998 .357925219 .15760234$ $-3.704559106 .698907628 .60143606$ 2.150338278 .566166116 .93234384 3.469494757 .928335386 .80444683 3.354549286 .408290676 .79692723 4.358680225 .724304177 .02184354 4.453184878 .435702507 .86305213 4.898726209 .868912537 .55032428 3.809064428 .479432859 .09628829 1.707734028 .604509157 .84138959 3.924677348 .186681745 .84944690 5.330058327 .790016567 .92637365 3.167697239 .205408669 .04550546 5.1650732610 .403454428 .46293967 4.1315225210 .419969057 .00665167 5.790647849 .826106046 .92447079 0.795722894 .227892178 .48404739 0.149004793 .443937349 .53657605 $-1.322343633 .171074859 .19533431$ $-2.236420323 .826489629 .70451988$ 0.269860804 .1692096710 .89872035 $-0.041753663 .1511035911 .97645031$ 0.494591563 .8102394813 .61485634 0.044059582 .3816333914 .62476908 0.875611185 .237700938 .55682611 0.659838702 .484372319 .63180754 1.304828834 .5112195410 .98801200 $-0.394084275 .0372410210 .92468891$ $-1.109191722 .9338544312 .05286445$ 0.516744822 .2233546211 .83637103 $-1.030044232 .1862902914 .57647166$ 0.584971121 .4935133114 .29622583 0.319202162 .6014686815 .65685364 


$\begin{array}{ll}\mathrm{Cu} & -0.233772425 .9919345115 .34815543 \\ \mathrm{O} & -1.9720989211 .148376988 .89915797 \\ \mathrm{H} & -2.5838619411 .655222148 .33669024 \\ \mathrm{H} & -2.5240947410 .856628999 .64552913 \\ \mathrm{O} & -2.946107869 .7748048811 .08854735 \\ \mathrm{H} & -2.6159193910 .1387127611 .93029723 \\ \mathrm{H} & -3.519565509 .0400231811 .37459735 \\ \mathrm{O} & 2.3915231811 .8191670018 .51223457 \\ \mathrm{H} & 2.1275726712 .5774485717 .98336678 \\ \mathrm{H} & 2.8394080412 .2163441119 .26462415\end{array}$

\section{QM/SE/MM R1a (B3LYP/6-31G(d):xTB:Amber)} mechanical embedding $\omega_{\alpha}=\mathbf{0 . 6 0 6}$

C

C

$\mathrm{O}$

$\mathrm{N}$

C

C

$\mathrm{O}$

C

C

$\mathrm{H}$

$\mathrm{H}$

$\mathrm{H}$

$\mathrm{H}$

$\mathrm{H}$

$\mathrm{H}$

$\mathrm{H}$

$\mathrm{N}$

C

$\mathrm{H}$

C

$\mathrm{C}$
$\mathrm{O}$

$\mathrm{N}$

C

C

$\mathrm{O}$

C

$\mathrm{C}$

C

$\mathrm{N}$
$\mathrm{C}$

$\mathrm{C}$

$\mathrm{H}$

$\mathrm{H}$

$\mathrm{H}$

$\mathrm{H}$

$\mathrm{H}$

$\mathrm{H}$

$\mathrm{H}$

$\mathrm{N}$

C

$\mathrm{C}$

$\mathrm{O}$

C

$\mathrm{O}$

$\mathrm{H}$

$\mathrm{H}$

$\mathrm{H}$

$\mathrm{H}$

$\mathrm{H}$
7.556495943 .2363473512 .94023447 7.137490584 .1466096411 .78335512 7.998441264 .5488879511 .00163089 5.817202744 .4712050911 .72140561 4.698602443 .9289533912 .53233390 3.989318602 .7968422511 .73902971 3.884809552 .8417331910 .51408316 3.795326755 .1804501912 .67943036 3.929620455 .8492119711 .29705758 5.415201685 .6441992810 .92108376 5.018551303 .5743299413 .51553643 2.772657434 .9091780012 .93940300 4.202687975 .8180294513 .47564444 3.292428945 .3518197710 .56410840 3.668754036 .9099516511 .30374440 5.554659635 .475747539 .85173219 6.033049136 .4996771011 .21695264 3.490606451 .7431391412 .44921516 2.870696090 .6285249211 .75726026 3.616088881 .6334783913 .44932308 $-1.185264086 .7000451021 .92732967$ $-1.970341336 .3814775320 .67680488$ $-3.074802416 .8774272420 .46434217$ $-1.395563705 .4617352419 .88774157$ $-2.005122775 .1239491918 .59242981$ $-3.482793754 .6899016718 .78085248$ $-3.876499234 .2079651319 .84655888$ $-1.206094193 .9687622517 .98099188$ 0.265489574 .2495154117 .92218675 1.289564153 .7713041818 .73689672 0.814458944 .9895156616 .91798776 2.118577304 .9633338817 .06565368 2.452845334 .2447941618 .16560872 $-0.526773275 .0247112120 .16220478$ $-1.976264675 .9933487817 .93144287$ $-1.378213143 .0486833218 .55298777$ $-1.588862513 .8001786616 .97042360$ 1.279239233 .1594068419 .62844680 2.846449255 .4411758916 .42893511 3.417616484 .0999713618 .48205845 $-4.272297814 .8269065817 .66843664$ $-5.648602114 .3254746517 .66053394$ $-5.746158153 .2334122416 .57381790$ $-4.874676153 .1192529615 .72102581$ $-6.700811575 .4226184717 .38083987$ $-6.488418725 .9765373516 .08006237$ $-3.871545685 .1246285516 .77666170$ $-5.840907433 .9099488018 .65341540$ $-7.696957444 .9708370917 .44022113$ $-6.632958056 .2111037318 .13614949$ $-7.371940855 .9352306115 .63175733$
$-6.829440892 .4066546616 .65716036$ $-7.148632411 .4327441115 .61510626$ $-7.472297742 .4422511017 .44521494$ $-5.941673453 .4936445410 .39197527$ $-5.736371274 .0136914011 .82643668$ $-6.588293833 .8894283512 .70365465$ $-4.539004834 .6158780012 .02589543$ $-4.286576765 .4056409913 .23370011$ $-4.583331356 .8840755412 .96361759$ $-4.210166797 .4391005511 .90996751$ $-2.788420265 .2292783313 .63029123$ $-2.468957406 .2208748315 .13224844$ $-3.910832214 .7437198211 .24188452$ $-4.906745805 .0005409614 .03167902$ $-2.592873564 .1758998013 .83506878$ $-2.158361965 .5692175012 .80451831$ $-5.271563857 .5334216713 .93296784$ $-5.676361198 .9173715013 .79125671$ $-4.651565479 .9329304814 .36979777$ $-4.8257979111 .1298009314 .25996398$ $-5.570211347 .0439219314 .77454315$ $-5.748002749 .1457805712 .72144573$ $-3.531460079 .4441727114 .93416764$ $-2.5822312310 .4615462615 .41260722$ $-2.0742063611 .1974022314 .19094826$ $-1.6343910010 .5496985113 .23221675$ $-1.416813899 .8247941716 .15416934$ $-1.971469799 .1788983917 .42726385$ $-0.3178433210 .8915020016 .45028268$ $-0.863968088 .6081938718 .32578247$ $-3.309312398 .4507326214 .99966433$ $-3.1336348911 .1404190616 .06548049$ $-0.987762379 .0493894915 .50842115$ $-2.540979599 .9218816118 .00320714$ $-2.662060968 .3723803717 .16138371$ 0.0623553111 .3605680115 .53660814 0.5368458110 .4529491916 .96911941 $-0.7108490211 .6825218117 .10308914$ $-0.290557187 .8353342617 .80069941$ $-1.301688698 .1605064419 .21542280$ $-0.159918329 .3836850818 .64622177$ 0.729040668 .4643610711 .23498037 0.209336817 .7972079112 .50787525 1.095030178 .1547861313 .67595219 2.074319009 .1162073413 .75658200 1.010411957 .4748959614 .88954179 1.914064548 .0514195415 .69774155 2.575436569 .0356221015 .05189909 $-0.830482858 .1008285712 .70643139$ 0.220454466 .7132006012 .34645652 2.454866959 .8424004013 .05677911 2.107563067 .7796120616 .72259031 3.306124579 .6157035115 .44130704 0.160914153 .506999609 .51360956 0.221262494 .2052157310 .89363715 0.003221413 .1516699311 .96510703 0.495754443 .8137181313 .61479810 0.079105282 .3610432614 .61530170 1.209384794 .6735388010 .97545557 $-0.551917034 .9818677010 .94363641$ $-1.045627992 .8405167512 .00467232$ 0.618006202 .2648852011 .79506708 $-0.954667102 .0492085814 .43747218$ 0.760349001 .5345509214 .39449747 0.193406002 .6418153015 .66252977 
QM/SE/MM R3a (B3LYP/6-31G(d):xTB:Amber) mechanical embedding $\omega_{\alpha}=\mathbf{0 . 6 0 6}$

C $\quad-0.54134615-4.2601698317 .89330695$ C $\quad 0.06034749-3.0972159217 .13935656$ $\mathrm{O} \quad 0.31734180-3.1768406015 .94948187$ $\mathrm{N} \quad 0.25960607-2.0038181117 .87172446$ C $\quad 0.88790980-0.8465308017 .27797933$ C $\quad 2.43451036-0.8884310017 .63427370$ O $\quad 2.80285372-0.8140405318 .79626829$ C $\quad 0.326694290 .4711901217 .81302300$ $\mathrm{H} \quad-0.03664570-2.0069488118 .84006625$ H $\quad 0.75468678-0.9223586016 .19709184$ $\mathrm{H} \quad-0.413456260 .2769098418 .58771247$ $\mathrm{H} \quad-0.160655581 .0284507317 .02040290$ $\mathrm{H} \quad 1.108338971 .0823047618 .25250354$ N $\quad 3.19774627-0.9687436416 .55475186$ C $\quad 4.67096226-0.9357476716 .53770984$ C $\quad 5.20583617-1.8998591717 .62761082$ O $\quad 5.61072990-1.5194872718 .70731339$ C $\quad 5.229260910 .4989834416 .78631417$ C $\quad 6.728795930 .5535875716 .36503707$ C $\quad 4.433511251 .5672821016 .02982711$ H $\quad 2.76507017-0.9775602115 .63847629$ $\mathrm{H} \quad 4.96664719-1.2762877215 .54223407$ $\mathrm{H} \quad 5.172502270 .6858423817 .85758545$ $\mathrm{H} \quad 7.369937360 .6752932517 .23491259$ $\mathrm{H} \quad 7.03719967-0.3666597815 .87233308$ $\mathrm{H} \quad 6.922977961 .3702922815 .67884831$ $\mathrm{H} \quad 3.561192631 .8967129716 .58885738$ $\mathrm{H} \quad 4.089846561 .1415970715 .09187034$ $\mathrm{H} \quad 5.061113102 .4278816915 .81840539$ $\mathrm{N} \quad 5.15676301-3.2073787317 .26511968$ C $\quad 5.91744313-4.1636634418 .05094292$ $\mathrm{H} \quad 4.91597246-3.4704493016 .31658044$ C $\quad 8.972059590 .0276307011 .52208058$ C $\quad 7.961922110 .9353195012 .21955064$ O $\quad 6.809704680 .5903573812 .43089041$ $\mathrm{N} \quad 8.457803162 .1285946712 .55338901$ C $\quad 7.568697623 .1595888013 .03727579$ C $\quad 7.140638844 .0503627511 .84122552$ O $\quad 7.996721214 .4458903111 .04645971$ C $\quad 8.268202114 .0398214314 .08727970$ C $\quad 7.422359925 .1358015414 .69187427$ C $\quad 6.672810144 .9110757215 .83642956$ C $\quad 7.345693526 .3984019514 .09991882$ C $\quad 5.874231075 .9066342116 .37991112$ C $\quad 6.558417237 .3910903914 .63823805$ C $\quad 5.818424877 .1550896315 .78222366$ $\mathrm{H} \quad 9.358260522 .3874729712 .19260119$ $\mathrm{H} \quad 6.710761642 .6361373013 .46024886$ $\mathrm{H} \quad 8.624527883 .3879739214 .89128776$ $\mathrm{H} \quad 9.134719384 .4930047313 .59906804$ $\mathrm{H} \quad 6.705454893 .9416885616 .31697752$ $\mathrm{H} \quad 7.923458416 .6061650113 .21197684$ $\mathrm{H} \quad 5.304773395 .7005598417 .27398790$ H $\quad 6.527342658 .3574341514 .15148938$ $\mathrm{H} \quad 5.197728897 .9311986016 .20718771$ $\mathrm{N} \quad 5.834944844 .4197784911 .75888527$ C $\quad 4.684457663 .9274036512 .54483816$ C $\quad 3.983014732 .8056252211 .73331452$ O $\quad 3.881226232 .8655593410 .50850441$
3.810804725 .2048444712 .65199326 4.000801205 .8518708211 .26865986 5.485999565 .5911994010 .92529336 4.978594003 .5657996613 .53390555 2.774698784 .9672934012 .89319448 4.217126625 .8385135913 .45246192 3.364266045 .3655723610 .52730267 3.774026466 .9208072811 .25326559 5.646120175 .402013739 .86251040 6.128585936 .4272413211 .22365429 3.498707321 .7360225312 .42062276 2.921117790 .6169575111 .70197785 $2.10641068-0.2064287412 .68382471$ $2.33439562-0.1663459113 .89101776$ $3.96761741-0.3490672011 .03344395$ $4.75996561-1.0700865411 .94606453$ 3.547603201 .6383772013 .43017172 2.306300001 .0311887810 .89555776 $3.40971045-1.0942539710 .46097471$ 4.573751610 .2515212710 .34792619 $5.46240596-0.4785016612 .28435520$ $1.17189752-0.9687552112 .11886392$ $0.45004107-2.0002724812 .83134991$ $1.01284689-3.3893524312 .48780530$ $1.39683623-3.6770972611 .36645774$ $-1.00796761-2.0103070912 .35364301$ $-1.83152270-0.7889795712 .69918054$ $-2.26821299-0.5798315814 .00879563$ $-2.213647890 .1108563111 .70258224$ $-3.091714450 .4914003814 .30372135$ $-3.013999831 .1904196212 .01994668$ $-3.463155971 .3812186413 .31797957$ $1.06536271-1.0008660511 .10950612$ $0.53548640-1.8029972813 .90201518$ $-0.96168610-2.1072932811 .26861678$ $-1.49767350-2.9046492712 .74721223$ $-1.97931109-1.2810103014 .78010461$ $-1.86395972-0.0346482010 .69099582$ $-3.436376800 .6629135415 .31454476$ $-3.316869841 .8859080911 .25225635$ $-4.098382552 .2171389613 .56816993$ $0.97656468-4.2555563813 .50107519$ $1.33201164-5.6580496913 .31709587$ $0.68550973-3.9969756114 .43904333$ $-8.12161552-6.9679068420 .13343488$ $-6.97223970-6.0927332919 .70411810$ $-6.25977959-6.4208215518 .77622857$ $-6.90796592-4.9023962720 .30970350$ $-5.92697413-3.9401651219 .90801689$ $-5.01651424-3.6003765921 .11005742$ $-5.51928644-3.2384030022 .14972433$ $-6.59098771-2.6253429719 .46833167$ $-5.55465560-1.5864240119 .14609542$ $-4.58369174-1.8224097018 .19613975$ $-5.56952211-0.3729794619 .80326065$ $-3.65992536-0.8370311617 .89584772$ $-4.644059210 .6135962819 .53245713$ $-3.682440230 .3692853418 .55699985$ $-7.54337619-4.6719165421 .06862109$ $-5.36177342-4.3862982619 .09038835$ $-7.20352905-2.8252213518 .58361225$ $-7.24268147-2.2703471420 .26517450$ $-4.55952249-2.7632125017 .66626954$ $-6.33310941-0.2171316720 .54697279$ $-2.89972463-1.0366715517 .15521493$ 


\begin{tabular}{|c|c|c|c|}
\hline $\mathrm{H}$ & -4.659688081 .5564550120 .05260793 & $\mathrm{H}$ & 5.343155096 .0360187220 .57802243 \\
\hline $\mathrm{H}$ & -2.954863311 .1308490318 .31735203 & $\mathrm{H}$ & 3.157442759 .2990986018 .88058027 \\
\hline $\mathrm{N}$ & $-3.69403083-3.7109162620 .89300429$ & $\mathrm{H}$ & 7.465952197 .2258084220 .05318406 \\
\hline $\mathrm{C}$ & $-2.72137692-3.5292341121 .97909571$ & $\mathrm{H}$ & 5.2804567010 .4392871118 .34776713 \\
\hline $\mathrm{C}$ & $-1.72627610-2.4752186121 .53496626$ & $\mathrm{H}$ & 7.415689069 .3918086418 .95227479 \\
\hline $\mathrm{O}$ & $-1.12302440-2.5364872220 .47602539$ & $\mathrm{~N}$ & 0.114950107 .3408655221 .79634842 \\
\hline $\mathrm{C}$ & $-1.97965737-4.8535174322 .27196031$ & $\mathrm{C}$ & -1.167080696 .7183635122 .06921518 \\
\hline $\mathrm{H}$ & $-3.34265033-3.9751443419 .97989607$ & $\mathrm{C}$ & -1.932504516 .3872214020 .76640403 \\
\hline $\mathrm{H}$ & $-3.28493871-3.2027709122 .85230808$ & $\mathrm{O}$ & -3.018703476 .9008332320 .49926841 \\
\hline $\mathrm{N}$ & $-1.52025732-1.4654322922 .36603481$ & $\mathrm{H}$ & 0.112424988 .2732355221 .41385523 \\
\hline $\mathrm{C}$ & $-0.54658485-0.4509426922 .01321597$ & $\mathrm{H}$ & -0.984344765 .7997187622 .62909104 \\
\hline $\mathrm{C}$ & $0.75781753-0.7339940722 .81055163$ & $\mathrm{H}$ & -1.790537327 .3971566622 .65509996 \\
\hline $\mathrm{O}$ & $0.80032848-0.4719816823 .99280322$ & $\mathrm{~N}$ & -1.374523395 .4634544019 .95883833 \\
\hline $\mathrm{C}$ & -1.010121530 .9365912622 .46028719 & $\mathrm{C}$ & -1.994781005 .1479185518 .64871034 \\
\hline $\mathrm{C}$ & 0.051838571 .9665774822 .13862744 & $\mathrm{C}$ & -3.473695444 .6975741918 .81107988 \\
\hline $\mathrm{N}$ & -0.010011673 .1099318422 .83398969 & $\mathrm{O}$ & -3.870333504 .1995255619 .86750422 \\
\hline $\mathrm{O}$ & 0.951250121 .7326393821 .35028256 & $\mathrm{C}$ & -1.208056793 .9909790118 .01283296 \\
\hline $\mathrm{H}$ & $-2.00028429-1.4116600523 .25813271$ & $\mathrm{C}$ & 0.265637774 .2600822317 .93166734 \\
\hline $\mathrm{H}$ & $-0.37139084-0.5011325620 .94114735$ & $\mathrm{C}$ & 1.291976463 .7761227618 .73306414 \\
\hline $\mathrm{H}$ & -1.907974081 .1999805521 .88743481 & $\mathrm{~N}$ & 0.807641944 .9951853416 .91752733 \\
\hline $\mathrm{H}$ & -1.263809110 .9437068723 .51738434 & $\mathrm{C}$ & 2.113003184 .9608206117 .05297741 \\
\hline $\mathrm{H}$ & -0.780484623 .2721215223 .47294761 & $\mathrm{~N}$ & 2.454924544 .2412576618 .15187875 \\
\hline $\mathrm{H}$ & 0.666081293 .8479305922 .68959825 & $\mathrm{H}$ & -0.509745405 .0097123520 .21919020 \\
\hline $\mathrm{N}$ & $1.74708953-1.2733022022 .10239923$ & $\mathrm{H}$ & -1.958907106 .0242798517 .99688105 \\
\hline $\mathrm{C}$ & $3.00908056-1.6619842422 .66961513$ & $\mathrm{H}$ & -1.379788123 .0667029018 .57657617 \\
\hline $\mathrm{C}$ & $4.12891319-0.6195380622 .49668091$ & $\mathrm{H}$ & -1.604409033 .8389870417 .00519097 \\
\hline $\mathrm{O}$ & $5.27002535-0.8049091022 .88222569$ & $\mathrm{H}$ & 1.280748453 .1658112219 .62514045 \\
\hline $\mathrm{C}$ & $3.44205327-3.0041288022 .07446706$ & $\mathrm{H}$ & 2.839913975 .4331402216 .41097181 \\
\hline $\mathrm{H}$ & $1.61062415-1.4763537621 .12325211$ & $\mathrm{H}$ & 3.423520594 .0907919418 .45974459 \\
\hline $\mathrm{H}$ & $2.87095857-1.7919642523 .74137503$ & $\mathrm{~N}$ & -4.256531164 .8262169117 .69003764 \\
\hline $\mathrm{N}$ & 3.744776550 .5307577721 .90613510 & $\mathrm{C}$ & -5.630007804 .3094548017 .66814171 \\
\hline $\mathrm{C}$ & 4.703259751 .5227669521 .51048695 & $\mathrm{C}$ & -5.724833873 .2252872516 .57588252 \\
\hline $\mathrm{C}$ & 4.793774642 .6460937122 .54801269 & $\mathrm{O}$ & -4.853675283 .1183875415 .72064567 \\
\hline $\mathrm{O}$ & 3.926090423 .4450613822 .79950921 & $\mathrm{C}$ & -6.689206465 .4070186317 .38821704 \\
\hline $\mathrm{C}$ & 4.303930742 .0999604820 .14330130 & $\mathrm{O}$ & -6.493858505 .9683862016 .08696031 \\
\hline $\mathrm{C}$ & 5.337931692 .9877591519 .52692133 & $\mathrm{H}$ & -3.847899355 .1292627716 .80349700 \\
\hline $\mathrm{N}$ & 6.630851872 .6327728619 .64096127 & $\mathrm{H}$ & -5.828725703 .8919121818 .65942096 \\
\hline $\mathrm{O}$ & 5.021331034 .0110050718 .94634220 & $\mathrm{H}$ & -7.681784414 .9479262917 .45185560 \\
\hline $\mathrm{H}$ & 2.782684790 .6743938121 .61667645 & $\mathrm{H}$ & -6.626192066 .1956958218 .14508642 \\
\hline $\mathrm{H}$ & 5.680900001 .0372399021 .45447732 & $\mathrm{H}$ & -7.383794155 .9473594515 .64945959 \\
\hline $\mathrm{H}$ & 4.086446771 .2533084119 .48262656 & $\mathrm{~N}$ & -6.807118402 .3951599516 .65067932 \\
\hline $\mathrm{H}$ & 3.376612632 .6547317820 .27621923 & $\mathrm{C}$ & -7.109404251 .4187058515 .60126293 \\
\hline $\mathrm{H}$ & 6.934650061 .7476117420 .00978015 & $\mathrm{C}$ & -8.560715691 .7182969615 .19405455 \\
\hline $\mathrm{H}$ & 7.328855193 .2090310619 .18053769 & $\mathrm{O}$ & -9.487927721 .3431872315 .87659841 \\
\hline $\mathrm{N}$ & 5.964512732 .6578331723 .22949858 & $\mathrm{C}$ & $-6.98894705-0.0206451416 .13152858$ \\
\hline $\mathrm{C}$ & 6.209462173 .6752407124 .26352508 & $\mathrm{C}$ & $-7.09200871-1.0947878715 .08609082$ \\
\hline $\mathrm{H}$ & 6.683439871 .9790627723 .02753304 & $\mathrm{C}$ & $-8.32956936-1.6229946314 .74668386$ \\
\hline $\mathrm{C}$ & 5.402904037 .3773748524 .14635102 & $\mathrm{C}$ & $-5.94931406-1.6115375814 .49007216$ \\
\hline $\mathrm{C}$ & 4.336734527 .8477531523 .17163582 & $\mathrm{C}$ & $-8.39443186-2.6506864413 .81403271$ \\
\hline $\mathrm{O}$ & 4.211251419 .0352987122 .99772054 & $\mathrm{C}$ & $-6.02620485-2.6308350113 .56407256$ \\
\hline $\mathrm{N}$ & 3.630595046 .9177097922 .52500315 & $\mathrm{C}$ & $-7.25770784-3.1497309213 .22861105$ \\
\hline $\mathrm{C}$ & 2.568022417 .3656595621 .63583179 & $\mathrm{H}$ & -7.489361762 .4806900617 .40056888 \\
\hline $\mathrm{C}$ & 1.256477396 .6615843321 .99127452 & $\mathrm{H}$ & -6.430388591 .6096671714 .76713606 \\
\hline $\mathrm{O}$ & 1.283388995 .5134311722 .39590000 & $\mathrm{H}$ & $-6.03048403-0.0962713616 .64715668$ \\
\hline $\mathrm{C}$ & 2.846164756 .9593973920 .17216973 & $\mathrm{H}$ & $-7.79088866-0.1554809516 .85987594$ \\
\hline $\mathrm{C}$ & 4.126255667 .5994786019 .76715644 & $\mathrm{H}$ & $-9.22473008-1.2300642015 .20202476$ \\
\hline $\mathrm{C}$ & 5.339457607 .0005020820 .09927346 & $\mathrm{H}$ & $-4.98863842-1.1967910814 .75590146$ \\
\hline $\mathrm{C}$ & 4.099742118 .8351800919 .13857129 & $\mathrm{H}$ & $-9.35619940-3.0661754013 .54775601$ \\
\hline $\mathrm{C}$ & 6.513487967 .6538976119 .80446596 & $\mathrm{H}$ & $-5.13225074-3.0116912513 .09475737$ \\
\hline $\mathrm{C}$ & 5.286230399 .4798305218 .84483580 & $\mathrm{H}$ & $-7.33051232-3.9519215712 .50794425$ \\
\hline $\mathrm{C}$ & 6.489247328 .8847557419 .18167391 & $\mathrm{~N}$ & -8.698486842 .4690178814 .09811335 \\
\hline $\mathrm{H}$ & 3.652313265 .9275730922 .71648325 & $\mathrm{C}$ & -10.032642472 .8833235813 .64387092 \\
\hline $\mathrm{H}$ & 2.489049618 .4520944921 .73613984 & $\mathrm{H}$ & -7.927854132 .8951006613 .59147955 \\
\hline $\mathrm{H}$ & 2.894836865 .8717296620 .12130808 & $\mathrm{C}$ & -9.906208175 .8814945219 .71415322 \\
\hline $\mathrm{H}$ & 2.025114517 .3092586319 .54008711 & $\mathrm{C}$ & -8.481295245 .8192761020 .27577976 \\
\hline
\end{tabular}


O $\quad-7.877713754 .7689182620 .22761504$

$\mathrm{N} \quad-8.020705906 .9723900520 .77058569$

C $\quad-6.614182797 .0482540821 .17333326$

C $\quad-6.415136116 .1815556322 .44386309$

O $\quad-7.142515786 .2277312023 .41372532$

C $\quad-6.181337698 .4846009121 .45248980$

C $\quad-6.185467399 .3106105620 .19917004$

C $\quad-5.434458688 .9315890819 .09754687$

C $\quad-6.9636924810 .4662880420 .10224975$

C $\quad-5.457258339 .6956423017 .93308376$

C $\quad-6.9939078211 .2188884218 .93499553$

C $\quad-6.2373242510 .8379580617 .83733969$

$\mathrm{H} \quad-8.570429987 .8244155720 .74466284$

$\mathrm{H} \quad-6.013484906 .6164310720 .36734628$

$\mathrm{H} \quad-6.828918598 .9282123322 .21037425$

$\mathrm{H} \quad-5.159314188 .4405582421 .83753463$

$\mathrm{H} \quad-4.808337228 .0541195119 .13639480$

$\mathrm{H} \quad-\quad-7.5632985810 .7803101720 .94517682$

$\mathrm{H} \quad-4.866811129 .3814278517 .08729215$

$\mathrm{H} \quad-7.6015269312 .1108372418 .88801058$

$\mathrm{H} \quad-6.2538364811 .4185104616 .92725247$

$\mathrm{N} \quad-5.349648395 .3784176622 .34649895$

C $\quad-4.971226244 .4778532323 .44445846$

$\mathrm{H} \quad-4.733880975 .3739455021 .54674665$

C $\quad-7.708454040 .297805209 .56451292$

C $\quad-6.559861171 .329297419 .57610493$

O $\quad-5.598960601 .213321258 .84189988$

$\mathrm{N} \quad-6.770992872 .3892498010 .37219110$

C $\quad-5.904064003 .5407383010 .33967314$

C $\quad-5.697462054 .0301138111 .78978186$

O $\quad-6.555596033 .8855214912 .66332621$

C $\quad-6.448545994 .683223889 .46173000$

C $\quad-6.999249154 .176930428 .10656269$

C $\quad-7.529371965 .4419757610 .20870156$

$\mathrm{H} \quad-7.610464622 .4560539410 .94398608$

$\mathrm{H} \quad-4.949113763 .185278219 .94475202$

$\mathrm{H} \quad-5.604586925 .349118439 .26406524$

$\mathrm{H} \quad-7.994152844 .564180317 .91380862$

$\mathrm{H} \quad-6.347330444 .502282007 .30393013$

$\mathrm{H} \quad-7.056931323 .096445448 .05870093$

$\mathrm{H} \quad-8.155487764 .7729171810 .79644905$

$\mathrm{H} \quad-8.161085195 .937009409 .48355521$

$\mathrm{H} \quad-7.110018066 .1841620310 .88532845$

$\mathrm{N} \quad-4.507284824 .6293115912 .01435115$

C $\quad-4.282501945 .4047685013 .23345752$

C $\quad-4.584504896 .8853106212 .97212722$

O $\quad-4.214030897 .4475430611 .92346644$

C $\quad-2.786258515 .2407413213 .62668267$

S $\quad-2.461213646 .2210561115 .13311664$

$\mathrm{H} \quad-3.886630294 .7969776811 .23183674$

$\mathrm{H} \quad-4.895919624 .9769928914 .02537899$

$\mathrm{H} \quad-2.581123204 .1857439913 .81655014$

$\mathrm{H} \quad-2.159707885 .5953541612 .80342706$

$\mathrm{N} \quad-5.257537307 .5388589613 .94929982$

C $\quad-5.654086688 .9256810113 .79552852$

C $\quad-4.632439919 .9294113014 .37468045$

O $\quad-4.8132847311 .1268127714 .27091396$

C $\quad-7.009231779 .1685783114 .47374892$

C $\quad-8.133111078 .5004566713 .67375495$

C $\quad-9.471536539 .1480555814 .05304233$

$\mathrm{N} \quad-10.229654008 .4591040214 .92605150$

O $\quad-9.8030010310 .2183512013 .59932292$

$\mathrm{H} \quad-5.562897857 .0479049414 .78773370$

$\mathrm{H} \quad-5.742063719 .1407813612 .72365713$

H $\quad-6.977088028 .7690958415 .48974282$
$-7.1933818510 .2420555414 .53045621$ $-7.991761198 .6668693912 .60505343$ $-8.146201347 .4271943313 .85262908$ $-11.113890818 .8331478115 .21266141$ $-9.906750777 .5501776715 .26519858$ $-3.519212069 .4281675614 .93465470$ $-2.5825274410 .4353383015 .44520243$ $-2.0863590311 .1969072114 .24428297$ $-1.6614072610 .5874278513 .25299034$ $-1.406822739 .8085752416 .17533086$ $-1.959087439 .1539078217 .44541966$ $-0.3229445810 .8900736116 .46796853$ $-0.847518808 .5905923418 .34543250$ $-3.297089968 .4321303914 .98421246$ $-3.1331494411 .0932793116 .12109585$ $-0.967316909 .0440077115 .52436754$ $-2.540174079 .8863860118 .02274391$ $-2.639193498 .3402833517 .17302911$ 0.0514009711 .3632316015 .55321788 0.5385242410 .4598787616 .98368205 $-0.7232069011 .6740423217 .12556584$ $-0.263380207 .8263788417 .81883526$ $-1.278492448 .1322399719 .23246089$ $-0.151300559 .3709027118 .67283072$ $-2.1511654512 .5180668814 .28470565$ $-1.7456002613 .3152552013 .10720336$ $-0.3695239412 .8829765112 .57313791$ 0.6134797312 .7565937413 .28525870 $-1.7176155414 .7384920213 .66668362$ $-2.8527113214 .7120606914 .68143330$ $-2.6711280713 .3467259615 .34259214$ $-2.5168436813 .2104566012 .34381849$ $-0.7605311014 .9215072214 .15826179$ $-1.8554956715 .4820894312 .88442535$ $-2.8043803615 .5308018815 .39533691$ $-3.8121037514 .7475368114 .16517761$ $-1.9293187613 .4198865716 .14654867$ $-3.6090794712 .9616790315 .73915009$ $-0.3630634012 .6410369011 .24870707$ 0.7856247412 .2312979410 .48077800 1.0265185510 .7444566410 .32348691 1.7016049410 .361078109 .37974949 $-1.2429055812 .7407371510 .75122554$ 0.7284768412 .666732499 .48292723 1.6709748512 .6288276510 .99377489 0.520367209 .9131830211 .24851381 0.826479498 .4939463511 .20726055 0.256512907 .769187909 .95009175 0.968067507 .023022399 .29990770 0.302571707 .8011418412 .46959926 1.140225958 .1581795313 .67166800 2.105074549 .1274822713 .79262738 1.018197337 .4700893114 .87774184 1.892813118 .0441992515 .71778738 2.566750279 .0370611915 .10204968 $-0.0676829810 .2602322111 .99983227$ 1.915153878 .3751518211 .13860059 $-0.747054838 .0892433412 .63721624$ 0.333591916 .7170921512 .30721987 2.501578729 .8622858013 .11063657 2.059525367 .7692057816 .74632288 3.280596389 .6195470615 .51838802 $-1.033558397 .960200709 .66902769$ $-1.626352637 .327767468 .49786147$ $-0.915924667 .778791167 .21520445$ 
O $\quad-0.694403137 .038238916 .27003407$

C $\quad-3.124399037 .672873218 .42283375$

$\mathrm{H} \quad-1.624398428 .5703300810 .23630621$

$\mathrm{H} \quad-1.493019446 .243433728 .58363620$

$\mathrm{H} \quad-3.385292198 .047320747 .44523165$

$\mathrm{H} \quad-3.374217658 .431214389 .15722710$

$\mathrm{H} \quad-3.732399786 .796511978 .62562954$

$\mathrm{N} \quad-0.599893309 .075988457 .19626316$

C $\quad 0.099863889 .655321836 .07055810$

C $\quad 1.482371049 .056898895 .83642362$

O $\quad 1.980331859 .027814364 .72849329$

C $\quad 0.2695615311 .148159476 .27611316$

$\mathrm{H} \quad-0.894756029 .685024807 .95532559$

$\mathrm{H} \quad-0.482422179 .506892565 .15513154$

$\mathrm{N} \quad 2.110269778 .567892396 .91554595$

C $\quad 3.405961807 .958037316 .75440646$

C $\quad 3.336762876 .429363106 .78955745$

O $\quad 4.340529955 .763483856 .98348455$

C $\quad 4.417849718 .447614107 .80963379$

C $\quad 4.976612999 .826457257 .43376914$

O $\quad 3.839134008 .467256919 .07376652$

$\mathrm{H} \quad 1.736940598 .618226037 .85333377$

$\mathrm{H} \quad 3.771756588 .265375155 .76564249$

$\mathrm{H} \quad 5.207967307 .691874507 .86841424$

$\mathrm{H} \quad 3.188364199 .190574359 .10789593$

$\mathrm{H} \quad 5.2370355310 .374227158 .33736074$

$\mathrm{H} \quad 4.2537810810 .408222496 .86816131$

$\mathrm{H} \quad 5.882176429 .720691416 .84038688$

$\mathrm{N} \quad 2.119839885 .884307086 .56597388$

C $\quad 2.017434184 .474489576 .34946914$

C $\quad 1.358963663 .633084017 .43664443$

O $\quad 1.316783532 .413887247 .31418531$

$\mathrm{H} \quad 1.305121486 .453438686 .39384202$

$\mathrm{H} \quad 1.467364364 .276702355 .42294679$

$\mathrm{H} \quad 3.036109204 .086069356 .23690798$

$\mathrm{N} \quad 0.777876374 .254292398 .47605153$

C $\quad 0.168933393 .443809319 .50939594$

C $\quad-1.330088323 .186036709 .20521329$

O $\quad-2.232396643 .829154029 .71247639$

C $\quad 0.268528104 .1495809310 .88255134$

C $\quad-0.027747683 .1399376111 .97156608$

S $\quad 0.487700573 .8122628513 .60787892$

C $\quad 0.070680052 .3698166914 .61082195$

$\mathrm{H} \quad 0.844153805 .259820628 .61196010$

$\mathrm{H} \quad 0.690196772 .481702469 .52398630$

$\mathrm{H} \quad 1.287495014 .5472138010 .97003802$

$\mathrm{H} \quad-0.447546204 .9810496810 .90712558$

$\mathrm{H} \quad-1.094032962 .8961978712 .01636139$

$\mathrm{H} \quad 0.537611442 .2139712111 .83272549$

$\mathrm{H} \quad-0.968362412 .0652113414 .44773609$

$\mathrm{H} \quad 0.739625601 .5339039614 .38666264$

$\mathrm{H} \quad 0.197609572 .6523867315 .65687741$

N $\quad-1.502918092 .234423418 .29644179$

C $\quad-2.803893801 .773961747 .88440923$

$\mathrm{H} \quad-0.719989491 .789588017 .82958979$

$\mathrm{Cu} \quad-0.236855205 .9974539315 .34611360$

O $\quad-1.9562630511 .154927468 .88803984$

$\mathrm{H} \quad-2.5846899911 .682212648 .36439686$

$\mathrm{H} \quad-2.4469049510 .819016859 .65687529$

O $\quad-2.938678159 .7657375711 .07048157$

$\mathrm{H} \quad-2.5385536910 .1546195111 .86840958$

$\mathrm{H} \quad-3.495741259 .0347346711 .39526699$

O $\quad 2.3195391611 .6735702418 .63500212$

$\mathrm{H} \quad 1.9335776012 .4422847318 .20625190$

$\mathrm{H} \quad 2.9446306712 .0009389019 .28557895$

\section{QM/SE R1a (B3LYP/6-31G(d):xTB) mechanical} embedding $\omega_{\alpha}=\mathbf{0 . 6 0 6}$

C $\quad 7.556653153 .2353276812 .94063865$

C $\quad 7.137852254 .1445240811 .78660097$

O $\quad 7.999986944 .5504330111 .00417370$

$\mathrm{N} \quad 5.819327174 .4695223611 .72279930$

C $\quad 4.700328543 .9322270412 .53918521$

C $\quad 3.989980572 .7962927811 .76139098$

O $\quad 3.859362132 .8541244510 .53567098$

C $\quad 3.793161365 .1806581312 .67285803$

C $\quad 3.931020135 .8474243411 .28918072$

C $\quad 5.416844155 .6384024910 .91449313$

$\mathrm{H} \quad 5.024467963 .5826715713 .52249915$

$\mathrm{H} \quad 2.770039304 .9137314012 .93913241$

$\mathrm{H} \quad 4.204566575 .8201343513 .46493010$

$\mathrm{H} \quad 3.291324135 .3507416110 .55622115$

$\mathrm{H} \quad 3.671250336 .9085390811 .28426484$

$\mathrm{H} \quad 5.561597475 .464788449 .84470532$

$\mathrm{H} \quad 6.035975916 .4933521211 .20842142$

$\mathrm{N} \quad 3.501433221 .7368743812 .45583426$

C $\quad 2.875049670 .6308408111 .75535614$

$\mathrm{H} \quad 3.599636921 .6328341313 .45980491$

C $\quad-1.184736486 .7004177721 .92821196$

C $\quad-1.970490856 .3827947420 .68019484$

O $\quad-3.066925626 .8869483020 .45563798$

$\mathrm{N} \quad-1.394159765 .4591186019 .89294363$

C $\quad-1.999375685 .1216737318 .59706271$

C $\quad-3.480739944 .6929116218 .76780164$

O $\quad-3.887408704 .2060603319 .83580785$

C $\quad-1.200816183 .9629208717 .98902544$

C $\quad 0.272010894 .2478281517 .92589511$

C $\quad 1.298002283 .7723160718 .73847097$

$\mathrm{N} \quad 0.814211424 .9911358316 .92147827$

C $\quad 2.118834484 .9679065517 .06562275$

$\mathrm{N} \quad 2.458844564 .2493576618 .16509117$

$\mathrm{H} \quad-0.535372095 .0123068320 .18140184$

$\mathrm{H} \quad-1.959375965 .9848243317 .92873922$

$\mathrm{H} \quad-1.369051043 .0424857318 .56105843$

$\mathrm{H} \quad-1.581731003 .7969118116 .97717391$

$\mathrm{H} \quad 1.294116023 .1608507119 .63096295$

$\mathrm{H} \quad 2.845715705 .4474343616 .42879875$

$\mathrm{H} \quad 3.428030764 .1090351118 .47829305$

$\mathrm{N} \quad-4.270990194 .8284084617 .66806025$

C $\quad-5.653830494 .3237446217 .65819805$

C $\quad-5.747144133 .2250290916 .58206267$

O $\quad-4.882337673 .1251948515 .71724030$

C $\quad-6.688774615 .4371348917 .35537346$

O $\quad-6.471565315 .9828927816 .06714377$

$\mathrm{H} \quad-3.865823115 .1190651716 .77743749$

$\mathrm{H} \quad-5.856769573 .9182538018 .65363374$

$\mathrm{H} \quad-7.691248164 .9968786117 .39909425$

$\mathrm{H} \quad-6.619082426 .2267204618 .11361633$

$\mathrm{H} \quad-7.328863655 .9227805615 .58919029$

$\mathrm{N} \quad-6.832739592 .4069188816 .66578565$

C $\quad-7.147830281 .4381533415 .61850277$

$\mathrm{H} \quad-7.457275792 .4123768817 .47135776$

C $\quad-5.942141943 .4920077410 .39038528$

C $\quad-5.737592764 .0125945311 .82614425$

O $\quad-6.587685463 .8923989812 .70134553$

$\mathrm{N} \quad-4.536946234 .6151942612 .02716740$

C $\quad-4.291949555 .4073209813 .23464606$

C $\quad-4.587116056 .8870795712 .96844462$ 


\section{QM/SE R2a (B3LYP/6-31G(d):xTB) mechanical} embedding $\omega_{\alpha}=\mathbf{0 . 6 0 6}$

$\begin{array}{llll}\mathrm{N} & 0.25508524 & -1.98030858 & 17.90194649 \\ \mathrm{C} & 0.88897420 & -0.84148797 & 17.28292243 \\ \mathrm{C} & 2.43638341 & -0.87995255 & 17.62659659 \\ \mathrm{O} & 2.79821312 & -0.82061819 & 18.79877570\end{array}$

$\begin{array}{llll}\mathrm{C} & 0.32287031 & 0.4810033517 .80801495 \\ \mathrm{H} & -0.03652614-1.95962236 & 18.87183238\end{array}$

$\mathrm{H} \quad 0.75193335-0.9306105416 .20328277$

$\mathrm{H} \quad-0.414447640 .2956314218 .58635263$

$\mathrm{H} \quad-0.167714321 .0296825317 .01311146$

$\mathrm{H} \quad 1.107770131 .0973990918 .23330655$

N $\quad 3.19986601-0.9673345516 .55700452$

C $\quad 4.67943373-0.9464824316 .54647403$

C $\quad 5.23343684-1.9126385117 .63811060$

O $\quad 5.61744952-1.5212904618 .73078366$

C $\quad 5.228721890 .4920007416 .79302082$

C $\quad 6.733597800 .5488408316 .37783055$

C $\quad 4.431198811 .5595347816 .03638348$

$\mathrm{H} \quad 2.75816184-0.9781490115 .64680475$

$\mathrm{H} \quad 4.97279277-1.2967356215 .55650293$

$\mathrm{H} \quad 5.165385990 .6814176017 .86272021$

$\mathrm{H} \quad 7.368580210 .6709690317 .25050935$

$\mathrm{H} \quad 7.04806748-0.3707371115 .89241616$

$\mathrm{H} \quad 6.928660541 .3657339315 .69186967$

$\mathrm{H} \quad 3.554149621 .8864894316 .59035633$

$\mathrm{H} \quad 4.091787031 .1350061715 .09709425$

$\mathrm{H} \quad 5.052765872 .4248045415 .82874648$

$\mathrm{N} \quad 8.444814742 .1855616912 .52299224$

C $\quad 7.547463733 .1815288213 .04723286$

C $\quad 7.112952714 .0666922411 .85341591$

O $\quad 7.979028574 .4682854711 .06845939$

C $\quad 8.260556894 .0605881814 .08844798$

C $\quad 7.410289955 .1487560314 .69857742$

C $\quad 6.663401564 .9145516715 .84300638$

C $\quad 7.334063286 .4146727914 .11274274$

C $\quad 5.860524685 .9062489716 .39027152$

C $\quad 6.544140487 .4019423514 .65825354$

C $\quad 5.803003327 .1594163215 .80015967$

$\mathrm{H} \quad 9.297918332 .4967889312 .09301491$

$\mathrm{H} \quad 6.720209972 .6430370013 .50659333$

$\mathrm{H} \quad 8.641726363 .4002956614 .87324604$

$\mathrm{H} \quad 9.114299804 .5359286913 .59737878$

$\mathrm{H} \quad 6.695050243 .9430532016 .31717957$

$\mathrm{H} \quad 7.908258946 .6368345513 .22681757$

$\mathrm{H} \quad 5.294780295 .6877049417 .28350902$

$\mathrm{H} \quad 6.511176528 .3661200314 .17508244$

$\mathrm{H} \quad 5.181391897 .9292144216 .23090682$

$\mathrm{N} \quad 5.809087044 .4281708611 .76165631$

C $\quad 4.670042343 .9278942412 .55842459$

C $\quad 3.977286362 .7990682711 .76293727$

O $\quad 3.857603792 .8728362910 .53621070$

C $\quad 3.784326895 .1974124512 .66212774$

C $\quad 3.965234285 .8494943111 .28020505$

C $\quad 5.450295605 .5987791310 .92912396$

$\mathrm{H} \quad 4.976349983 .5707412313 .54444608$

$\mathrm{H} \quad 2.751602294 .9560418012 .91150335$

$\mathrm{H} \quad 4.194565825 .8322729513 .45746689$

$\mathrm{H} \quad 3.325894745 .3636096910 .54052768$

$\mathrm{H} \quad 3.733386956 .9167027211 .26170749$

$\mathrm{H} \quad 5.609644945 .409789199 .86479864$

$\mathrm{H} \quad 6.088762366 .4363517711 .22597712$

N $\quad 3.502823201 .7228333012 .43179276$

C $\quad 2.916516370 .6145016211 .70261744$

C $\quad 2.09064582-0.2187213312 .68396990$

O $\quad 2.32777191-0.1787612313 .88993953$

C $\quad 3.97204419-0.3257427211 .03436061$

O $\quad 4.77675566-1.0419119511 .95192741$

$\mathrm{H} \quad 3.553694961 .6078034713 .43945252$

$\mathrm{H} \quad 2.298493581 .0261030210 .89754716$

$\mathrm{H} \quad 3.41483677-1.0774957110 .46979980$ 


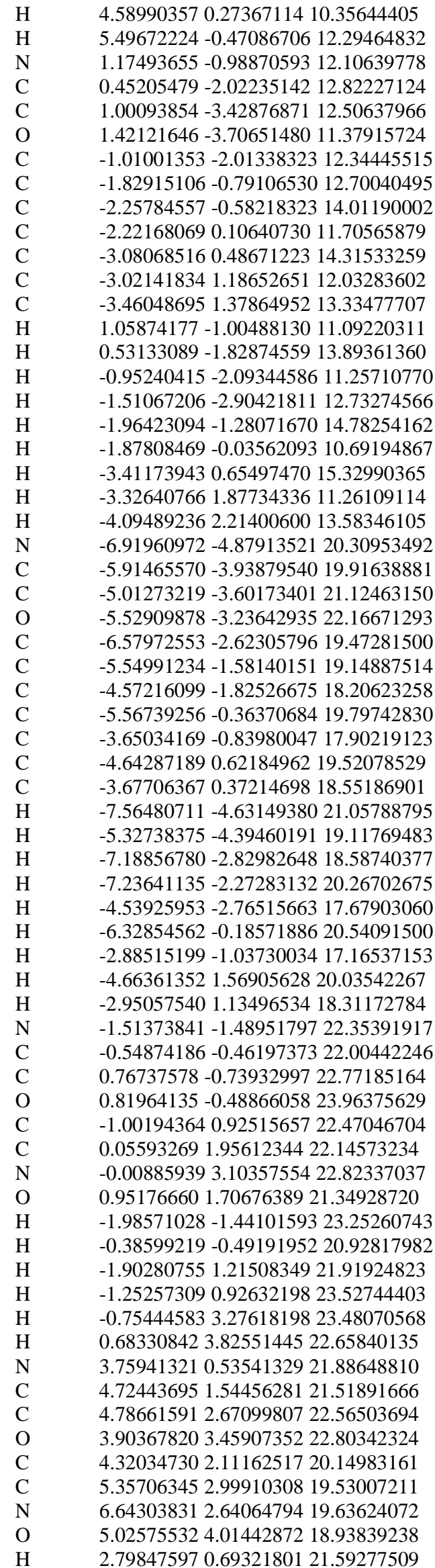

$\mathrm{H} \quad 5.703753131 .0611691521 .47621532$

$\mathrm{H} \quad 4.114959771 .2634801319 .48710696$

$\mathrm{H} \quad 3.390328622 .6596393820 .28173302$

H $\quad 6.942116551 .7594616320 .02209059$

$\mathrm{H} \quad 7.329735153 .2273049419 .17827880$

$\mathrm{N} \quad 3.606391306 .9260982622 .54742336$

C $\quad 2.553846467 .3790617921 .64269068$

C $\quad 1.240733956 .6733388721 .98969242$

O $\quad 1.271137485 .5132805122 .37090840$

C $\quad 2.841379356 .9691694520 .18652204$

C $\quad 4.136530527 .5785805019 .78701831$

C $\quad 5.333954006 .9611038220 .13970901$

C $\quad 4.136119818 .8022939519 .13818860$

C $\quad 6.524258947 .5878232919 .84784218$

C $\quad 5.337102259 .4204049518 .84704777$

C $\quad 6.527189008 .8102792619 .20641262$

$\mathrm{H} \quad 3.640259885 .9340152522 .74468069$

$\mathrm{H} \quad 2.484313258 .4644151121 .74034570$

$\mathrm{H} \quad 2.878733635 .8790060820 .14527718$

$\mathrm{H} \quad 2.025495857 .3231595819 .54787960$

$\mathrm{H} \quad 5.325463836 .0057168520 .63768128$

$\mathrm{H} \quad 3.203608489 .2759790418 .86536570$

$\mathrm{H} \quad 7.458403987 .1310137420 .12414521$

$\mathrm{H} \quad 5.3493207310 .3697937018 .33539565$

$\mathrm{H} \quad 7.464368329 .2951163718 .97631323$

$\mathrm{N} \quad 0.097875377 .3438323021 .80080272$

C $\quad-1.176312066 .7012298422 .06286513$

C $\quad-1.941502766 .3869238420 .75771277$

O $\quad-3.024743856 .9035833720 .49299217$

$\mathrm{H} \quad 0.103678688 .2851926421 .44249468$

$\mathrm{H} \quad-1.001287295 .7647142622 .59310909$

$\mathrm{H} \quad-1.813553927 .3443186522 .67059881$

$\mathrm{N} \quad-1.381494645 .4622901119 .94900006$

C $\quad-1.993369755 .1413082218 .64109356$

C $\quad-3.475963314 .6941069018 .79040118$

O $\quad-3.883871584 .1981754119 .85283269$

C $\quad-1.200050953 .9822499718 .01409624$

C $\quad 0.274204654 .2574589817 .93310912$

C $\quad 1.301698513 .7759600918 .73139733$

$\mathrm{N} \quad 0.809041274 .9979852216 .92106629$

C $\quad 2.114319354 .9708737417 .05558480$

$\mathrm{N} \quad 2.461879384 .2501596218 .15283070$

$\mathrm{H} \quad-0.523783765 .0053867320 .22620470$

$\mathrm{H} \quad-1.949333336 .0083497217 .97855852$

$\mathrm{H} \quad-1.366465903 .0600817618 .58028241$

$\mathrm{H} \quad-1.590498713 .8270999617 .00467045$

$\mathrm{H} \quad 1.293507953 .1627190119 .62175095$

$\mathrm{H} \quad 2.840105845 .4484658916 .41584954$

$\mathrm{H} \quad 3.434220644 .1047510818 .46159451$

$\mathrm{N} \quad-4.260882874 .8258882717 .68217861$

C $\quad-5.641587864 .3083295717 .66180142$

C $\quad-5.736548763 .2131810916 .57872881$

O $\quad-4.872325593 .1196922115 .71217109$

C $\quad-6.679264375 .4249807617 .36027338$

O $\quad-6.475716755 .9752568616 .07050112$

$\mathrm{H} \quad-3.845076025 .1267924916 .79962099$

$\mathrm{H} \quad-5.848778933 .8983389318 .65348061$

$\mathrm{H} \quad-7.679605974 .9834450817 .40872446$

$\mathrm{H} \quad-6.609672326 .2122519718 .12054367$

$\mathrm{H} \quad-\quad-7.339235885 .9344448315 .60236340$

$\mathrm{N} \quad-6.817587462 .3875213316 .66288207$

C $\quad-\quad-7.133008141 .4249557715 .60262450$

C $\quad-8.589753721 .7230180015 .18908984$

O $\quad-9.513069281 .3447748615 .88927072$

C $\quad-6.99889451-0.0195030616 .13097772$ 


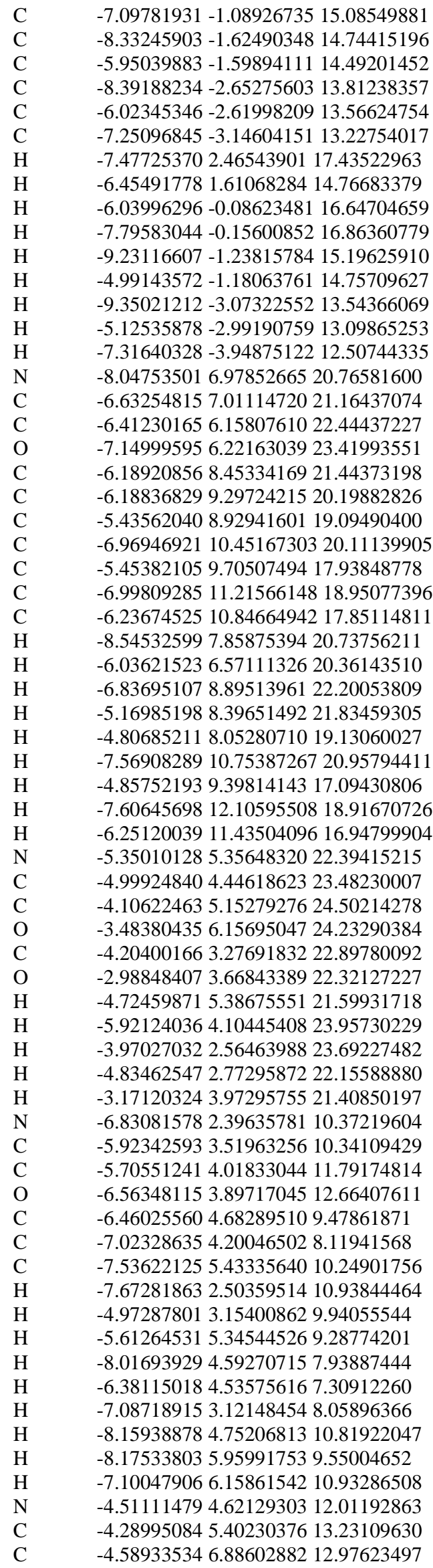

O

C

S

$\mathrm{H}$

$\mathrm{H}$

$\mathrm{H}$

$\mathrm{H}$

$\mathrm{N}$

C

C

O

C

C

C

$\mathrm{N}$

$\mathrm{O}$

$\mathrm{H}$

$\mathrm{H}$

$\mathrm{H}$

$\mathrm{H}$

$\mathrm{H}$

$\mathrm{H}$

$\mathrm{H}$

$\mathrm{H}$

C

C

O

C

C

C

C

$\mathrm{H}$

$\mathrm{H}$

$\mathrm{H}$

$\mathrm{H}$

$\mathrm{H}$

$\mathrm{H}$

$\mathrm{H}$

$\mathrm{H}$

$\mathrm{H}$

$\mathrm{H}$

$\mathrm{H}$

$\mathrm{N}$

C

C

O

C

C

C

$\mathrm{H}$

$\mathrm{H}$

$\mathrm{H}$

$\mathrm{H}$

$\mathrm{H}$

$\mathrm{H}$

$\mathrm{H}$

$\mathrm{N}$

C

C

$\mathrm{O}$

$\mathrm{H}$

$\mathrm{H}$

$\mathrm{H}$

$\mathrm{N}$
$-4.210683757 .4492273611 .92810090$ $-2.795282645 .2346720613 .63226955$ $-2.463691606 .2187468715 .13249845$ $-3.898830404 .7888304511 .22099850$ $-4.903439584 .9680620714 .01838500$ $-2.588434034 .1780719013 .81538877$ $-2.164092585 .5902501112 .81183479$ $-5.264249287 .5339973213 .95160193$ $-5.665528298 .9214683413 .79274947$ $-4.642065899 .9303506214 .36702756$ $-4.8239053511 .1261655614 .26662922$ $-7.020920139 .1692439314 .47241848$ $-8.153174928 .4832719713 .69938869$ $-9.485352769 .1607913714 .05672861$ $-10.249916228 .4985881714 .93900037$ $-9.8035187310 .2246855813 .57213325$ $-5.556616847 .0361877314 .79246819$ $-5.748699629 .1278205812 .71797682$ $-6.990805068 .7880774715 .49351974$ $-7.1957409110 .2455301614 .49909998$ $-8.006861368 .6243286912 .62659387$ $-8.201068137 .4187621513 .92787731$ $-11.135173898 .8944467115 .21138245$ $-9.955412057 .6032604215 .30356072$ $-3.525064419 .4349985114 .93505169$ $-2.5820475410 .4410872815 .43709389$ $-2.0796560611 .1947958014 .22816011$ $-1.6581594010 .5671993313 .24274584$ $-1.411438979 .8121984816 .17164586$ $-1.969315659 .1658065417 .44715698$ $-0.3216435810 .8914583916 .45627158$ $-0.860943708 .6043251018 .35448464$ $-3.300042808 .4442278714 .97827683$ $-3.1247676711 .1000106816 .11719099$ $-0.973913649 .0440336415 .52319222$ $-2.548040899 .9022265618 .01995909$ $-2.653497988 .3536456917 .18014760$ 0.0505836211 .3549162515 .53655464 0.5398229810 .4632990216 .97393384 $-0.7146913411 .6794287617 .11036860$ $-0.275954707 .8350420217 .83665147$ $-1.293130068 .1536293619 .24344002$ $-0.162269489 .3833832918 .67791176$ $-2.1492585112 .5123595014 .25394916$ $-1.7354180113 .3196234913 .08026309$ $-0.3591881112 .8773841212 .55800294$ 0.6177701112 .7452043313 .28126158 $-1.6948015714 .7314008113 .66366631$ $-2.8343113014 .7087013614 .67256211$ $-2.6715275613 .3335828615 .31840352$ $-2.4877187213 .2370217912 .29214007$ $-0.7385900714 .8926986014 .16635595$ $-1.8227696815 .4881932812 .89365594$ $-2.7819320915 .5175295115 .39596523$ $-3.7876194514 .7708066814 .14929670$ $-1.9334212813 .3877220616 .12721660$ $-3.6164739812 .9549353315 .70378562$ $-0.3588082912 .6389426311 .23409579$ 0.7962030612 .2202834910 .48068004 1.0269391810 .7341933910 .32146255 1.7028654410 .341595789 .37904398 $-1.2301916412 .7646961710 .72723479$ 0.7602744512 .657217709 .48181997 1.6867924012 .5959093711 .00428185 0.513935329 .9122559811 .24800352 


\begin{tabular}{|c|c|}
\hline $\mathrm{C}$ & 0.817382058 .4946821111 .21216322 \\
\hline $\mathrm{C}$ & 0.245718177 .776632449 .95357142 \\
\hline $\mathrm{O}$ & 0.961832317 .029467729 .30326584 \\
\hline $\mathrm{C}$ & 0.295364517 .8004504212 .47246279 \\
\hline $\mathrm{C}$ & 1.134619968 .1545317913 .67457457 \\
\hline $\mathrm{C}$ & 2.102827489 .1205688013 .79809511 \\
\hline $\mathrm{N}$ & 1.010090057 .4659262414 .87989601 \\
\hline $\mathrm{C}$ & 1.883623118 .0368529015 .72105967 \\
\hline $\mathrm{N}$ & 2.561524489 .0277760515 .10887000 \\
\hline $\mathrm{H}$ & -0.0849418710 .2716241311 .98506418 \\
\hline $\mathrm{H}$ & 1.905275978 .3692416911 .13350870 \\
\hline $\mathrm{H}$ & -0.751898618 .0964321112 .64067158 \\
\hline $\mathrm{H}$ & 0.325358846 .7170165112 .31034063 \\
\hline $\mathrm{H}$ & 2.497135029 .8517986613 .11163184 \\
\hline $\mathrm{H}$ & 2.050316697 .7626855916 .74997200 \\
\hline $\mathrm{H}$ & 3.275869049 .6090254715 .52514900 \\
\hline $\mathrm{N}$ & -1.041221067 .972730379 .66740674 \\
\hline $\mathrm{C}$ & -1.618458067 .355490898 .48132456 \\
\hline $\mathrm{C}$ & -0.901023157 .829921977 .20730758 \\
\hline $\mathrm{O}$ & -0.692201147 .057986536 .27123690 \\
\hline $\mathrm{C}$ & -3.122940247 .688882678 .40172978 \\
\hline $\mathrm{H}$ & -1.636191678 .5855537810 .23224682 \\
\hline $\mathrm{H}$ & -1.473117816 .270462588 .54826850 \\
\hline $\mathrm{H}$ & -3.383732758 .073187887 .42489036 \\
\hline $\mathrm{H}$ & -3.376984208 .435488929 .14482836 \\
\hline $\mathrm{H}$ & -3.720209236 .803200198 .58838315 \\
\hline $\mathrm{N}$ & 2.116157978 .522953296 .94162234 \\
\hline $\mathrm{C}$ & 3.422728987 .931181656 .77026501 \\
\hline $\mathrm{C}$ & 3.360514776 .399418856 .78993748 \\
\hline $\mathrm{O}$ & 4.363404365 .740728307 .01059669 \\
\hline $\mathrm{C}$ & 4.425525448 .453596247 .81906770 \\
\hline $\mathrm{C}$ & 4.956091759 .838944357 .42910313 \\
\hline $\mathrm{O}$ & 3.840006598 .477075599 .08182318 \\
\hline $\mathrm{H}$ & 1.735227838 .570378147 .87672412 \\
\hline $\mathrm{H}$ & 3.780363678 .241257845 .77931423 \\
\hline $\mathrm{H}$ & 5.230876907 .715439077 .88595987 \\
\hline $\mathrm{H}$ & 3.170604709 .184362619 .10896587 \\
\hline $\mathrm{H}$ & 5.2097316310 .402921218 .32202318 \\
\hline $\mathrm{H}$ & 4.2197301010 .397244346 .85784583 \\
\hline $\mathrm{H}$ & 5.859456889 .743403726 .83267963 \\
\hline $\mathrm{N}$ & 0.769912234 .241897298 .46339101 \\
\hline $\mathrm{C}$ & 0.162675703 .438294679 .50550275 \\
\hline $\mathrm{C}$ & -1.340163923 .180810079 .19261660 \\
\hline $\mathrm{O}$ & -2.236660753 .842393189 .69513805 \\
\hline $\mathrm{C}$ & 0.260033654 .1454915210 .87955740 \\
\hline $\mathrm{C}$ & -0.023764253 .1348360511 .97174940 \\
\hline $\mathrm{S}$ & 0.490522823 .8120846113 .61115310 \\
\hline $\mathrm{C}$ & 0.063882452 .3732890714 .62158292 \\
\hline $\mathrm{H}$ & 0.831963365 .251359938 .57737201 \\
\hline $\mathrm{H}$ & 0.689206192 .478749529 .52928589 \\
\hline $\mathrm{H}$ & 1.276525094 .5491908710 .96096766 \\
\hline $\mathrm{H}$ & -0.462646534 .9692026610 .90207942 \\
\hline $\mathrm{H}$ & -1.087779442 .8886070912 .02742186 \\
\hline $\mathrm{H}$ & 0.551002672 .2155432511 .82918512 \\
\hline $\mathrm{H}$ & $-0.976716092 .07482201 \quad 14.46137368$ \\
\hline $\mathrm{H}$ & 0.726316911 .5322813814 .39828805 \\
\hline $\mathrm{H}$ & 0.194901542 .6608527815 .66536418 \\
\hline $\mathrm{Cu}$ & -0.235882105 .9910986415 .34514680 \\
\hline $\mathrm{O}$ & -1.9218759611 .146949728 .88046181 \\
\hline $\mathrm{H}$ & -2.5131266611 .648867738 .30493389 \\
\hline $\mathrm{H}$ & -2.4511737910 .781518409 .60296523 \\
\hline $\mathrm{O}$ & -2.947655789 .7497682611 .06503445 \\
\hline $\mathrm{H}$ & -2.5247904910 .1209685911 .85888433 \\
\hline $\mathrm{H}$ & -3.483998158 .9964003511 .37557203 \\
\hline$\Omega$ & 2.3192895211 .6635792518 .59119580 \\
\hline
\end{tabular}

\section{QM/MM R1a (B3LYP/6-31G(d):Amber)} mechanical embedding $\omega_{\alpha}=1.0$

C $\quad 7.556017023 .2389557412 .94002613$

C $\quad 7.134940174 .1305455111 .77506278$

O $\quad 7.988093074 .5263491810 .99688364$

$\mathrm{N} \quad 5.806575274 .4639421511 .74325369$

C $\quad 4.680349823 .9188026712 .55002308$

C $\quad 3.990300832 .8117982411 .73549315$

O $\quad 3.859961212 .8744489210 .51749580$

C $\quad 3.774771035 .1551405212 .74270427$

C $\quad 3.891141875 .8347696811 .35804739$

C $\quad 5.391686015 .6589544710 .97808254$

$\mathrm{H} \quad 5.002452613 .5437154113 .51690726$

$\mathrm{H} \quad 2.756645654 .8641359512 .97659720$

$\mathrm{H} \quad 4.163620525 .7894753213 .54197790$

$\mathrm{H} \quad 3.258076975 .3357285510 .62940061$

$\mathrm{H} \quad 3.617535726 .8859816611 .41034267$

$\mathrm{H} \quad 5.525719895 .507352829 .91102810$

$\mathrm{H} \quad 5.983431016 .5233811811 .28502751$

$\mathrm{N} \quad 3.505324391 .7554947912 .44070406$

C $\quad 2.876635640 .6236496311 .75034028$

$\mathrm{H} \quad 3.730935661 .5965657613 .41744426$

C $\quad-1.189016636 .6953281121 .94931742$

C $\quad-1.961035426 .3875194220 .69764450$

O $\quad-3.051542666 .8741091020 .46130613$

$\mathrm{N} \quad-1.367452665 .4499626819 .92875319$

C $\quad-1.991269095 .1332717518 .62856549$

C $\quad-3.470661374 .6815280918 .79314701$

O $\quad-3.887204134 .2163685319 .84729369$

C $\quad-1.205908054 .0010231917 .97009421$

C $\quad 0.267564154 .2738097317 .90636234$

C $\quad 1.282819513 .7854662018 .72448272$

$\mathrm{N} \quad 0.823593394 .9967515716 .91233120$

C $\quad 2.123620864 .9632661117 .05204009$

$\mathrm{N} \quad 2.447566604 .2362710218 .15468220$

$\mathrm{H} \quad-0.523743504 .9894483320 .24240488$

$\mathrm{H} \quad-1.976910116 .0300304318 .00884677$

$\mathrm{H} \quad-1.382288113 .0734601118 .51634711$

$\mathrm{H} \quad-1.594782683 .8533605716 .96579912$

$\mathrm{H} \quad 1.234481003 .1844029719 .60713590$

$\mathrm{H} \quad 2.857729585 .4313039416 .41806289$

$\mathrm{H} \quad 3.394589394 .0662411318 .48617141$

$\mathrm{N} \quad-4.259002054 .8223635817 .65489897$

C $\quad-5.640654814 .3227390117 .66753860$

C $\quad-5.742780573 .2395441616 .58281759$

O $\quad-4.870606403 .1360806215 .72976678$

C $\quad-6.697051105 .4245509317 .37574112$

O $\quad-6.462140105 .9865041416 .08279337$

$\mathrm{H} \quad-3.862260875 .1331448916 .77784189$

$\mathrm{H} \quad-5.801910523 .8799029218 .64981439$

$\mathrm{H} \quad-7.692461864 .9811188917 .40759316$

$\mathrm{H} \quad-6.626023906 .1985346718 .14222503$

$\mathrm{H} \quad-7.332941315 .9389178515 .61675508$

$\mathrm{N} \quad-6.818844682 .4127234416 .67698476$

C $\quad-7.150344271 .4440404315 .62362158$

H $\quad-7.493967652 .4674126317 .43882529$

C $\quad-5.932908113 .5103651310 .39740230$

C $\quad-5.722847024 .0211104511 .83238248$

O $\quad-6.560342363 .8937030012 .69814786$

N $\quad-4.515228454 .6269610312 .01226661$ 


\section{QM/MM R1a (B3LYP/6-31G(d):Amber)} electrostatic embedding $\omega_{\alpha}=1.0$

\section{C $\quad 7.554624683 .2359238112 .93955425$}

7.133577534 .1289740511 .77858418
7.986150484 .5265454510 .99589548 5.806859164 .4650494011 .74256851 4.681406403 .9201875512 .54941299 3.992058792 .8144097511 .73606409 3.859525392 .8739197710 .51980416 3.775361865 .1565637212 .74174889 3.891641775 .8360664111 .35662087 5.391904145 .6600054610 .97669249 5.003413033 .5452282513 .51710934 2.756462474 .8652658112 .97636504 4.164559635 .7912167013 .54149466 3.258036915 .3364535510 .62749445 3.617817456 .8875976311 .40844428 5.526181115 .508034109 .90907660 5.984167126 .5247891411 .28358010 3.507174171 .7539790312 .44205812 2.873584090 .6262934111 .74995446 3.728039581 .5929459413 .41832382 $-1.190788646 .6919194521 .94989849$ $-1.959453776 .3868662920 .69686010$ $-3.052753776 .8762624720 .45904638$ $-1.367161925 .4503173219 .92923130$ $-1.990735435 .1348788918 .62749622$ $-3.469461964 .6844123418 .78945894$ $-3.892027844 .2143185919 .84654891$ $-1.205503164 .0019712517 .96941434$ 0.268149064 .2744961117 .90661767 1.283962083 .7856076118 .72562257 0.824627134 .9978174416 .91333099 2.125216624 .9642263717 .05322021 2.448301124 .2367917218 .15595367 $-0.524664444 .9893338920 .24245062$ $-1.975164286 .0321615018 .00807638$ $-1.383093363 .0732582218 .51578399$ $-1.594177283 .8544775216 .96408823$ 1.236262593 .1837260919 .60891264 2.859392385 .4324916216 .41951959 3.396845454 .0665262218 .48804939 $-4.259246024 .8213857017 .65825695$ $-5.642543564 .3226279317 .66893469$ $-5.744380783 .2388213116 .58358234$ $-4.869697373 .1347827015 .72974707$ $-6.699089805 .4252961417 .37509541$ $-6.463354635 .9860894816 .08217860$ $-3.861629455 .1313726416 .78022705$ $-5.805111903 .8804554518 .65160897$ $-7.694978094 .9820077617 .40640676$ $-6.628244466 .2001065818 .14180233$ $-7.333803345 .9383820115 .61635081$ $-6.820483122 .4170629116 .67825520$ $-7.148514391 .4410504015 .62833398$ $-7.492469192 .4647689217 .44305531$ $-5.931889413 .5093633110 .39607981$ $-5.717803984 .0176008511 .83042527$ $-6.560680793 .8985188512 .69807838$ $-4.518054354 .6264838412 .01426696$ $-4.275353125 .3844221213 .23643207$ $-4.587219976 .8763757612 .96064890$ $-4.194301637 .4534959511 .92360410$ $-2.776571345 .2233506713 .65407884$ $-2.471012456 .2252657615 .13155936$ $-3.878783864 .7032262411 .22912879$ $-4.936868495 .0045879014 .01143223$ $-2.580286754 .1799941313 .90132126$ $-2.135509155 .5529212712 .83279576$ 


\section{QM/MM R1a (B3LYP-D3/6-31G(d):Amber)} mechanical embedding $\omega_{\alpha}=1.0$

\section{$\mathrm{C}$ \\ C \\ $\mathrm{N}$ \\ C \\ C \\ $\mathrm{O}$ \\ C \\ C \\ C}

$\mathrm{O}$

$-5.274250287 .5099696313 .93705114$ $-5.687085878 .9155896913 .78089354$ $-4.673225799 .9273673314 .36594789$ $-4.8408580811 .1281018314 .24877977$ $-5.640830637 .0040014714 .74494910$ $-5.795895049 .1460381812 .72054130$ $-3.556971799 .4591984414 .94191960$ $-2.5634283310 .4498667715 .41977093$ $-2.0608394411 .1886682814 .19877021$ $-1.6480503010 .5227864613 .23682410$ $-1.415488269 .8032793516 .16213724$ $-1.970414419 .1673068317 .44234878$ $-0.2971491510 .8618726716 .47693211$ $-0.865449958 .5823889318 .33812144$ $-3.439789548 .4557576615 .00500556$ $-3.0934116611 .1451405016 .04628512$ $-1.000675459 .0183671515 .52758210$ $-2.520611849 .9184465018 .01398058$ $-2.670082158 .3702478917 .18277231$ 0.0997367111 .3203724915 .56777894 0.5351803410 .3901943716 .99661536 $-0.6928456411 .6523592017 .11977166$ $-0.309957857 .8013388017 .80559385$ $-1.304601988 .1522731819 .23253650$ $-0.156205289 .3557283918 .63901032$ 0.742532848 .4513706211 .22707123 0.254031807 .7901057812 .50361252 1.105709808 .1401593413 .69170674 2.084510899 .1015623213 .79288522 0.981908987 .4572404014 .89899966 1.889581268 .0327002515 .72508743 2.565814939 .0136492015 .09949250 $-0.787201438 .0819170812 .66034586$ 0.260286756 .7123819012 .34078417 2.456520629 .8121658413 .07460117 2.058696157 .7503014916 .75058116 3.294462269 .5832552915 .51093060 0.155864233 .491048199 .51756131 0.265260844 .1794744410 .90037558 $-0.014893693 .1612544411 .98099320$ 0.494854763 .8187855913 .61824151 0.064523312 .3820794014 .62046369 1.277885404 .5727640811 .00154969 $-0.425200665 .0233612610 .94945358$ $-1.078604422 .9061119011 .98583774$ 0.512343712 .2254385211 .79040568 $-0.958542612 .0596648614 .42404578$ 0.743435271 .5574290814 .41549093 0.152375892 .6473249615 .67395085 $-0.234223355 .9894216515 .34483501$

7.553659853 .2355150512 .94007050 7.132871754 .1277327111 .77610426 7.984613884 .5199854410 .99253475 5.804421544 .4576634311 .73678593 4.682052223 .9251278312 .55739271 3.981177992 .8214964311 .74668720 3.875799422 .8683766210 .52465805 3.776389135 .1863350712 .71288011 3.915084585 .8460006211 .32364188 5.413982385 .6392967610 .94466806 4.983037173 .5657034413 .54117223
2.751960084 .9140103312 .95996031 4.175179165 .8264877613 .50963549 3.274474255 .3422462610 .59744901 3.649081446 .9041446611 .35416004 5.556532465 .471693509 .87770567 6.029109526 .4971937311 .23824567 3.497823091 .7603783212 .44442379 2.874732180 .6254506211 .75107674 3.653985241 .6160187013 .43706581 $-1.191782526 .6921346121 .94833465$ $-1.962170006 .3903444820 .69083201$ -3.05556362 6.8765145620 .46086158 $-1.373059925 .4535229619 .91779094$ $-1.986580465 .1428050718 .60297884$ $-3.473200244 .7026819018 .79214837$ -3.87611245 4.21080583 19.83998685 $-1.214620053 .9920973217 .97545491$ 0.254718874 .2686717817 .90980211 1.284835943 .7940940418 .73011562 0.813518004 .9925534816 .91771652 2.115815924 .9670971917 .04590201 2.444887084 .2514399618 .15047245 $-0.530625294 .9877802620 .22669886$ $-1.958462616 .0363811817 .97030362$ $-1.393125793 .0648557118 .53294390$ $-1.601149693 .8357779416 .96644954$ 1.300645663 .1983970219 .61943640 2.842824025 .4373456816 .40378328 3.396263534 .0934756118 .47588323 $-4.266843564 .8244904117 .65790955$ $-5.656009264 .3387539617 .66661565$ $-5.740594943 .2464012116 .58319625$ $-4.868847423 .1426019215 .73176397$ $-6.709122765 .4458635317 .37267654$ $-6.459721765 .9803382816 .06954845$ $-3.894573515 .0998920116 .75712993$ $-5.838224383 .9198875318 .66093007$ $-7.711397735 .0036933017 .41971594$ $-6.635597836 .2363964518 .13120051$ $-7.347003155 .9225053415 .63036415$ $-6.814727552 .4164515316 .67507633$ $-7.148424041 .4421601815 .62595273$ $-7.491333502 .4773252517 .43410858$ $-5.929134193 .5122533510 .39717928$ $-5.710533714 .0158637811 .83490325$ $-6.559889443 .9027801212 .69473379$ $-4.497937304 .6107579812 .02925355$ $-4.282624625 .4006022713 .23719720$ $-4.582546836 .8848733912 .96015289$ $-4.202438357 .4509810711 .91986146$ $-2.773039025 .2350812313 .63856422$ $-2.471343086 .2231905315 .13029820$ $-3.856136024 .7374943111 .25212671$ $-4.915033614 .9924706414 .03116500$ $-2.567757254 .1850766513 .86394033$ $-2.119911495 .5667813512 .82354903$ $-5.275093347 .5211024113 .93726680$ $-5.685955078 .9238619913 .78269362$ $-4.671544559 .9318179414 .37112509$ $-4.8380736411 .1326357114 .24926986$ $-5.657680717 .0495761314 .75574384$ $-5.792080379 .1760395712 .72728633$ $-3.551496149 .4624331714 .94227415$ $-2.5621594710 .4570883715 .42255733$ $-2.0548707311 .1907282214 .20223661$ 


\section{QM/MM R1a (M06L/6-31G(d):Amber)} mechanical embedding $\omega_{\alpha}=1.0$

$-1.6530981310 .5214959213 .23794376$ $-1.398288929 .8021006016 .14452678$ $-1.972654839 .1521691317 .40672488$ $-0.2793754910 .8584360416 .45567511$ $-0.864341488 .5541715418 .29177649$ $-3.421504278 .4586578714 .98186561$ $-3.0714642011 .1236394816 .10786607$ $-0.977072149 .0284716915 .49431510$ $-2.523918429 .8909185417 .99699584$ $-2.674006428 .3548497017 .14318687$ 0.1448286411 .3401080115 .56507976 0.5712173910 .3935004616 .96533315 $-0.6512258311 .6504314017 .11674798$ $-0.297536277 .7750342817 .75106753$ $-1.300588848 .1056852019 .18667025$ $-0.144449089 .3132016418 .61686739$ 0.739826018 .4479536211 .22936163 0.241814447 .7806703912 .50489436 1.103380058 .1400696213 .68314584 2.084805039 .1014162313 .77798590 0.981792337 .4561368114 .89420072 1.887943228 .0377311215 .72262821 2.561265889 .0137367415 .08919187 $-0.804052468 .0643608512 .66471455$ 0.266126226 .6956121112 .35401380 2.497288419 .8325531813 .10041160 2.058426197 .7624295316 .74988151 3.289013499 .5888839915 .49353226 0.156417363 .494011689 .52531575 0.251772934 .2120750710 .90830345 $-0.010625863 .1538122311 .95672520$ 0.498716413 .8163941813 .61212150 0.043304942 .3988425514 .63493044 1.270150384 .6093502510 .99784363 $-0.453515875 .0508189010 .95905463$ $-1.074492582 .9004596312 .00083946$ 0.548614682 .2317313111 .79118856 $-0.996947182 .1182633414 .45436638$ 0.693945111 .5505080214 .43004240 $0.153058922 .68323671 \quad 15.68288529$ $-0.233763235 .9910572715 .34617122$

7.549702303 .2342642512 .93993185 7.125354964 .1353693511 .77967202 7.973342334 .5234966610 .99318629 5.798329104 .4720040911 .75676159 4.668717393 .9262177812 .55341964 3.995636772 .8174950011 .73473929 3.863883082 .8795062910 .51766116 3.765874655 .1425519912 .75815278 3.873521655 .8187279811 .37363274 5.370283285 .6577903411 .00320562 5.002252933 .5442698913 .52991839 2.733974884 .8364091312 .99328233 4.151989095 .7859069113 .56999189 3.236019795 .3078727310 .63697467 3.587828206 .8791347011 .42775853 5.509941175 .506428009 .92221559 5.958589656 .5398329611 .31533465 3.510966451 .7614629712 .43632424 2.877223990 .6278181411 .74960002 3.719089791 .5999392713 .41661297
$-1.190381376 .6931750621 .95066779$ $-1.956323836 .3866470720 .69128057$ $-3.043659236 .8761690820 .45149832$ $-1.359285615 .4504526119 .92307974$ $-1.986490625 .1395447318 .63195603$ $-3.462608244 .6870685218 .78582477$ $-3.882759774 .2253888819 .84079793$ $-1.200373504 .0217532917 .96131175$ 0.269948534 .2878493017 .90508077 1.272490293 .7895500018 .73212881 0.824176525 .0040750716 .92080175 2.123163604 .9665421117 .06055592 2.441042124 .2334363618 .16768182 $-0.514273094 .9824911220 .23442718$ $-1.973583196 .0570845618 .01128459$ $-1.386421673 .0793511118 .50680900$ $-1.596683903 .8759983616 .93940855$ 1.190708433 .1893306519 .61032942 2.865288035 .4317595616 .42884171 3.385503744 .0542255118 .50637784 $-4.259114804 .8428786617 .66069346$ $-5.632508434 .3304836717 .66878267$ $-5.737576473 .2484241216 .58399707$ $-4.875628843 .1390902115 .72448888$ $-6.694393305 .4156284417 .37040715$ $-6.457975115 .9771621516 .08770476$ $-3.863585115 .1679139716 .78408570$ $-5.787517523 .8733378118 .65653082$ $-7.701418074 .9641526817 .39331364$ $-6.631704426 .1914604618 .15023762$ $-7.320625245 .9212650615 .60245571$ $-6.815151972 .4223550316 .67652895$ $-7.148623251 .4471912615 .62421839$ $-7.494085492 .4743004617 .43464279$ $-5.931809233 .5106424110 .39903762$ $-5.721023494 .0153511911 .84076911$ $-6.564525883 .8994945312 .69819803$ $-4.514827674 .6244516412 .02075794$ $-4.259090005 .3726612913 .24106697$ $-4.575110226 .8657739212 .95497555$ $-4.187814587 .4483615011 .92922532$ $-2.760784695 .2180358013 .66634531$ $-2.474003476 .2254448415 .12989855$ $-3.867823214 .6748992811 .23978715$ $-4.936986095 .0092179314 .01950437$ $-2.557874444 .1765863513 .92444708$ $-2.122596085 .5394436412 .84002177$ $-5.266484157 .5020796113 .93922188$ $-5.689953028 .9080532013 .77538567$ $-4.674268469 .9208168014 .36094070$ $-4.8421254411 .1220066614 .24356202$ $-5.625437456 .9890387614 .74345898$ $-5.803674849 .1280836712 .71156264$ $-3.563984129 .4522724714 .94511395$ $-2.5600114510 .4376604015 .42392073$ $-2.0622175311 .1879143914 .20349502$ $-1.6500259710 .5258353113 .23869330$ $-1.420054359 .7846522616 .16649742$ $-1.956268179 .1487374217 .44666329$ $-0.3062504210 .8360617616 .48419891$ $-0.853294598 .5596829918 .32561642$ $-3.452736738 .4469051215 .01263900$ $-3.0999267311 .1521799816 .03263581$ $-1.006518008 .9829023315 .52057050$ $-2.507250799 .9120639518 .02729142$ 

0.0850808011 .2999182115 .56139833 0.5325099310 .3524218717 .00821488 $-0.7090738511 .6337368117 .13384172$ $-0.302039037 .7676084317 .77519993$ $-1.287218798 .1248574319 .23109494$ $-0.131158729 .3397246618 .62224040$ 0.740322238 .4485336111 .22917432 0.266699827 .7876183612 .51954721 1.108060348 .1348222313 .70479703 2.084636159 .1036729113 .80283599 0.986911667 .4564949514 .90173373 1.894609498 .0327735515 .72955210 2.567853009 .0165187515 .10742807 $-0.787945938 .0770181912 .67262517$ 0.260681776 .6999001712 .34778260 2.437636799 .8051838913 .07075902 2.061628877 .7441702516 .75736886 3.295441309 .5867465615 .52047367 0.157130263 .489901519 .52021201 0.273632684 .1616534710 .91367343 $-0.018372723 .1670634511 .99975588$ 0.494395763 .8208681313 .62051061 0.065080902 .3879239914 .61210749 1.295823244 .5552801211 .02159043 $-0.407305955 .0230061110 .95949182$ $-1.085544972 .9125274411 .98433318$ 0.484800972 .2193185211 .80230755 $-0.953370502 .0582712014 .41699583$ 0.742008561 .5645458514 .41523853 0.147309382 .6517608115 .66399273 $-0.233960435 .9890858915 .34491291$

QM/MM R1a (B3LYP/6-31G(d) SDD:Amber) mechanical embedding $\omega_{\alpha}=1.0$

7.554709723 .2364783912 .94046195 7.139652654 .1284761511 .77455820 7.992660444 .5212114710 .99216164 5.812464504 .4615134011 .73390316 4.687234923 .9286813612 .54926356 3.988195762 .8209932011 .73753435 3.873036002 .8676879010 .51635038 3.787030535 .1830942812 .70759401 3.926971145 .8497489011 .32231000 5.426251885 .6437312310 .94585428 4.988223023 .5635157013 .53389555 2.760911784 .9101885912 .95424651 4.187255955 .8204920413 .50731451 3.286382645 .3503698510 .59150676 3.662819986 .9092381211 .35515740 5.570884055 .480903679 .87483664 6.042738226 .5010879511 .24366286 3.505081241 .7617938612 .44049939 2.875950920 .6262671311 .75097617 3.681557171 .6171998313 .42931483 $-1.190848966 .6928919921 .94919570$ $-1.961011586 .3910843120 .69308685$ $-3.055566866 .8747372720 .46357317$ $-1.371688575 .4517679419 .92407671$ $-1.984590955 .1353440718 .61291081$ $-3.473767754 .6954996018 .79839526$ $-3.880863314 .2086026019 .84498780$ $-1.204668043 .9822065517 .98817328$ 0.266698554 .2573081517 .92479347
C

$\mathrm{N}$

C

$\mathrm{N}$

$\mathrm{H}$

$\mathrm{H}$

$\mathrm{H}$

$\mathrm{H}$

H

H

H

N

C

C

$\mathrm{O}$

C

$\mathrm{O}$

H

$\mathrm{H}$

H

H

H

N

C

H

C

O

N

C

$\mathrm{C}$

$\mathrm{O}$

C

H

H

$\mathrm{H}$

H

C

$\mathrm{O}$

H

$\mathrm{H}$

N

C

$\mathrm{C}$

$\mathrm{C}$

$\mathrm{C}$

$\mathrm{C}$

C

$\mathrm{H}$

$\mathrm{H}$

H

H

$\mathrm{H}$

$\mathrm{H}$

$\mathrm{H}$

$\mathrm{H}$

C

$\mathrm{C}$
1.295098093 .7798767518 .74360109 0.823337024 .9809005016 .93689617 2.123089574 .9599072717 .06354605 2.453974014 .2398875718 .16577171 $-0.525186674 .9911912520 .23040873$ $-1.961231636 .0285356417 .98170036$ $-1.381979623 .0539506418 .54815921$ $-1.589404413 .8211374116 .97770456$ 1.315207743 .1824994819 .63001929 2.852549305 .4319289916 .42543679 3.405315874 .0813792718 .49163443 $-4.270833654 .8246196417 .66556956$ $-5.658782434 .3362403217 .67203813$ $-5.745767573 .2443608816 .58928728$ $-4.874426893 .1430456515 .73315189$ $-6.708120385 .4438227217 .37701040$ $-6.466750305 .9798194316 .07506771$ $-3.897129675 .0985199616 .76759638$ $-5.837084213 .9183451718 .66726801$ $-7.712643705 .0044457417 .42290934$ $-6.634414076 .2349000918 .13261934$ $-7.355129875 .9312007415 .63524281$ $-6.818462272 .4135598216 .67805095$ $-7.150187861 .4417973615 .62605701$ $-7.492750272 .4671998917 .44044206$ $-5.930728423 .5106591910 .39694856$ $-5.715445414 .0131959111 .83394648$ $-6.561522183 .9018446912 .69492345$ $-4.502741394 .6082278412 .02456123$ $-4.291834645 .4005186813 .23057528$ $-4.583994186 .8859336312 .95275334$ $-4.196941047 .4565292111 .91952377$ $-2.785194155 .2327859813 .64225982$ $-2.475085236 .2213299215 .12823894$ $-3.863890394 .7259587311 .24402821$ $-4.929906184 .9955550714 .01404682$ $-2.581463844 .1878870213 .87424272$ $-2.128708865 .5619826912 .83671675$ $-5.274298527 .5205097913 .93488337$ $-5.686815508 .9239715713 .78123661$ $-4.670546799 .9297524514 .37046980$ $-4.8388080511 .1305917914 .25132411$ $-5.664055007 .0392305314 .74529701$ $-5.793186989 .1777963812 .72662878$ $-3.552625879 .4603839314 .94556152$ $-2.5634410310 .4568041815 .42501195$ $-2.0566262911 .1901150414 .20197798$ -1.6516468610.5210833513.23839360 $-1.403796609 .8141421916 .15623900$ -1.985481609.17435853 17.42383530 $-0.2856372510 .8735784716 .46421148$ $-0.883430178 .5874033418 .32294318$ $-3.422283808 .4563116114 .98905892$ $-3.0782562511 .1272167716 .10674094$ $-0.975425729 .0385244715 .51305783$ $-2.543121239 .9196459818 .00406798$ -2.68662352 8.37275279 17.16343582 0.1411275311 .3486552615 .56995106 0.5616966910 .4157649316 .98443368 $-0.6615294711 .6725197917 .11767445$ $-0.312739947 .8060618417 .79691866$ - 1.323408348 .1500535319 .21750948 $-0.166145469 .3518497718 .64445644$ 0.740752588 .4504690611 .22721082 0.251104627 .7908678312 .50701660 
C $\quad 1.119628008 .1573517913 .67825827$

C $\quad 2.100320309 .1208040113 .76806422$

$\mathrm{N} \quad 1.009712347 .4788629014 .88794058$

C $\quad 1.915244878 .0613989315 .71244716$

$\mathrm{N} \quad 2.582952219 .0373561615 .07589658$

$\mathrm{H} \quad-0.794203498 .0795053712 .66500841$

$\mathrm{H} \quad 0.276461336 .7079973312 .35631726$

$\mathrm{H} \quad 2.506386539 .8509359613 .08671975$

$\mathrm{H} \quad 2.097529337 .7937431616 .73967544$

$\mathrm{H} \quad 3.311172029 .6160124415 .47352333$

C $\quad 0.155992713 .493051289 .52335567$

C $\quad 0.252731404 .2055784410 .90598104$

C $\quad-0.009903223 .1519643311 .95803594$

S $\quad 0.498179193 .8147665413 .61286048$

C $\quad 0.048477582 .3862629614 .62940337$

$\mathrm{H} \quad 1.268547334 .6007601810 .99324097$

$\mathrm{H} \quad-0.448982035 .0432112410 .95378375$

$\mathrm{H} \quad-1.071039032 .8984150812 .00445339$

$\mathrm{H} \quad 0.547927672 .2317830611 .78966495$

$\mathrm{H} \quad-0.986198392 .0967450014 .44863983$

$\mathrm{H} \quad 0.699968611 .5428075814 .42047168$

$\mathrm{H} \quad 0.159282552 .6614825015 .67639164$

$\mathrm{Cu} \quad-0.232608785 .9920830715 .34562161$

\section{QM/MM R2a (B3LYP/6-31G(d):Amber) \\ mechanical embedding $\omega_{\alpha}=1.0$}

$\mathrm{N} \quad 0.24475949-1.9641903817 .87937025$

C $\quad 0.88823555-0.8147366617 .26619591$

C $\quad 2.41911325-0.8834470717 .60031544$

O $\quad 2.79790622-0.8169925718 .78484097$

C $\quad 0.311072560 .4975977717 .81018696$

$\mathrm{H} \quad-0.05191554-1.9524321818 .84811432$

$\mathrm{H} \quad 0.74332962-0.8422466116 .18323602$

$\mathrm{H} \quad-0.428942130 .2966391918 .58758281$

$\mathrm{H} \quad-0.181144811 .0367596317 .00581633$

$\mathrm{H} \quad 1.094322411 .1178212918 .24698318$

N $\quad 3.20495372-0.9464431716 .54420867$

C $\quad 4.69521406-0.9337458516 .54995634$

C $\quad 5.23988903-1.9117809417 .61547203$

O $\quad 5.60767587-1.5224023218 .73174416$

C $\quad 5.252101010 .5230065716 .75790432$

C $\quad 6.757827380 .5865722416 .34968212$

C $\quad 4.481401781 .6028459915 .97573083$

$\mathrm{H} \quad 2.77498418-1.0094179015 .62722384$

$\mathrm{H} \quad 5.02357376-1.2767295015 .56588425$

$\mathrm{H} \quad 5.183511220 .7561590117 .80997291$

$\mathrm{H} \quad 7.374334580 .7317472217 .23809701$

$\mathrm{H} \quad 7.06618774-0.3547759115 .88372792$

$\mathrm{H} \quad 6.975866341 .3923726415 .65104509$

$\mathrm{H} \quad 3.610192831 .9173711616 .55085238$

$\mathrm{H} \quad 4.161607921 .1658566015 .03296837$

$\mathrm{H} \quad 5.105928142 .4672995015 .76032889$

$\mathrm{N} \quad 8.447375392 .1699470812 .55983843$

C $\quad 7.563809553 .2120210313 .04400961$

C $\quad 7.135151504 .0735770311 .83222440$

O $\quad 7.975752724 .4619002011 .03055712$

C $\quad 8.247093294 .0713773514 .11527744$

C $\quad 7.400304875 .1856888514 .72379651$

C $\quad 6.630085224 .9476713815 .87276276$

C $\quad 7.341219356 .4592438114 .11043579$

C $\quad 5.821798065 .9744904016 .40146429$

C $\quad 6.547758337 .4721033314 .63981471$

C $\quad 5.787716867 .2389045415 .78733943$
$\mathrm{H}$

$\mathrm{H}$

$\mathrm{H}$

$\mathrm{H}$

$\mathrm{H}$

$\mathrm{H}$

$\mathrm{H}$

$\mathrm{H}$

$\mathrm{H}$

$\mathrm{N}$

C

C

$\mathrm{O}$

C

C

C

$\mathrm{H}$

$\mathrm{H}$

$\mathrm{H}$

$\mathrm{H}$

$\mathrm{H}$

H

H

N

C

C

O

C

O

$\mathrm{H}$

$\mathrm{H}$

H

$\mathrm{H}$

H

$\mathrm{N}$

C

C

O

C

C

C

C

C

C

C

H

$\mathrm{H}$

H

$\mathrm{H}$

$\mathrm{H}$

$\mathrm{H}$

$\mathrm{H}$

H

$\mathrm{H}$

N

C

C

$\mathrm{O}$

C

C

C

C

C

C

C
9.346734162 .4104497912 .18549650 6.704000872 .7309676213 .49587118 8.576062343 .4193457514 .92945661 9.132141044 .5235675613 .66322218 6.657347313 .9782729516 .35134132 7.930707766 .6677258513 .22698903 5.227609705 .8039980317 .28775439 6.532465318 .4408743314 .15219580 5.169745818 .0273609916 .19407240 5.812548114 .4230381511 .76646134 4.665849543 .9278516612 .56439629 3.974859862 .8194913311 .74040633 3.875486362 .8748831810 .51855431 3.788132885 .2054127512 .69601976 3.967944575 .8564381111 .31020805 5.464214465 .6085101910 .95510665 4.948915973 .5567622513 .55524924 2.751139514 .9588212812 .93000844 4.191389095 .8384350813 .49997300 3.325394145 .3653775010 .57359961 3.731138256 .9257375811 .32588420 5.621153805 .428599809 .88756125 6.102137336 .4514055911 .25427782 3.504729741 .7409593812 .41845340 2.924985490 .5984695111 .70233795 $2.09175912-0.2238709612 .66783100$ $2.31891059-0.1823180313 .88988575$ $3.96408961-0.3515218211 .02190996$ $4.78593752-1.0353176111 .98035086$ 3.565010771 .6154116213 .42441582 2.270198861 .0072296410 .93134924 $3.42315017-1.1030914210 .44280449$ 4.582011730 .2233441410 .32878932 $5.49379201-0.4267377112 .28673083$ $1.15415100-0.9782134012 .11436715$ $0.44424271-2.0285851712 .83492650$ $1.00243216-3.4166496012 .52630798$ $1.40905552-3.7010849211 .38397330$ $-1.00738899-2.0152722812 .36124384$ $-1.83227774-0.7828886612 .70218256$ $-2.26749425-0.5721565314 .03313866$ $-2.216257920 .1160685111 .68080370$ $-3.104679380 .5109143614 .32312164$ -3.02914781 1.2014511912.01239889 $-3.480184851 .3998465213 .32542506$ $1.04281350-1.0101269711 .10531975$ $0.49705094-1.8542593613 .91055594$ $-0.97782746-2.0937957011 .27487819$ $-1.52461991-2.9021905812 .73746605$ $-1.97971578-1.2683112814 .80969846$ $-1.87736334-0.0214570110 .66169350$ $-3.454762310 .6908857515 .33186134$ $-3.340214641 .8962468011 .24416765$ $-4.119288502 .2431392613 .55393310$ $-6.91350882-4.8822660120 .29882791$ $-5.92326466-3.9204307919 .91722742$ $-5.01191139-3.6074750821 .10916987$ $-5.52553440-3.2412949422 .15313758$ $-6.59918590-2.6252338219 .46587139$ $-5.56188670-1.5792769819 .13718395$ $-4.57856250-1.8330850018 .19154576$ $-5.57831153-0.3677271419 .80723439$ $-3.63941186-0.8408337417 .89253292$ $-4.638730550 .6316969819 .54457121$

$-3.663131140 .3779313018 .55747139$ 
$-7.56866342-4.6598990321 .04524280$ $-5.31744115-4.3089457019 .10227132$ $-7.19390584-2.8216027818 .57039203$ $-7.26927301-2.2697163120 .25188552$ $-4.56297003-2.7753991017 .66064692$ $-6.34923856-0.2067028020 .54334040$ $-2.87899783-1.0420078317 .15252842$ $-4.667083071 .5760764420 .06997807$ $-2.928150801 .1334789018 .32522723$ $-1.52438618-1.4864501622 .33166820$ $-0.53763838-0.4615302122 .00108548$ $0.76457970-0.7615353322 .76053803$ $0.81945646-0.4846166123 .95523889$ $-1.008100320 .9316569022 .45999017$ 0.042449461 .9709804122 .14947352 0.009142043 .1059810622 .82474683 0.934420421 .7331149521 .35849184 $-2.04410458-1.4460857923 .20412330$ $-0.33224312-0.4509315820 .93118310$ $-1.893116231 .1908504521 .87820129$ $-1.282924690 .9446338023 .51537675$ $-0.733870083 .2565703123 .49495168$ 0.722858153 .8087071222 .67021540 3.774042360 .5411302821 .87767285 4.711330391 .5720108421 .49515519 4.816205982 .6617982522 .55193460 3.914752443 .4413893522 .79703662 4.296764662 .1283499420 .12042435 5.354131513 .0023968119 .52917660 6.626988582 .6483970119 .62847388 5.036727054 .0041810718 .93794087 2.805016010 .6806215221 .61514690 5.697376251 .1185277521 .39927798 4.086684241 .2985491619 .45630170 3.369074362 .6726143120 .24009340 6.884027261 .7714951220 .04649537 7.322794873 .2293701119 .17892232 3.622407666 .9438421922 .54025501 2.536578827 .3631214521 .61938092 1.222963536 .6782534921 .97882367 1.255189775 .5068969722 .32928455 2.872060666 .9870670720 .17652342 4.144597917 .6246003819 .75241815 5.370151146 .9965225620 .07788433 4.101355378 .8770787719 .11937151 6.553018667 .6606526419 .77807932 5.304034839 .5288760818 .82183698 6.521546998 .9100092119 .15761457 3.721586905 .9618312322 .75660350 2.375249558 .4412680621 .69041454 2.968626445 .9037186320 .10403506 2.072503297 .3220706619 .51279729 5.392744916 .0298808120 .56054599 3.159899599 .3465214018 .87471900 7.509322327 .2332834420 .01328209 5.3161901010 .4961838718 .33720594 7.454684139 .4089903418 .92724746 0.081028927 .3451349921 .81333044 $-1.205760516 .6973272922 .08187146$ $-1.942428386 .3946783120 .76057506$ $-3.023519816 .8878145420 .48581906$ 0.081611928 .2969972621 .47911625 $-1.097729545 .7571190622 .62494277$ $-1.834474997 .3559257722 .68467162$ $-1.364932385 .4603964019 .96650153$
$-1.985881255 .1592647218 .64720112$ $-3.474431994 .7010292018 .81495741$ $-3.876444554 .1985205419 .85670546$ $-1.216030874 .0004144918 .00836955$ 0.254742824 .2676718217 .92833096 1.281172423 .7882292618 .73939256 0.808082944 .9861660416 .92559269 2.109712294 .9638995017 .04772075 2.438006484 .2447276318 .15237188 $-0.524358184 .9799325720 .26247079$ $-1.956654606 .0547013118 .02079311$ $-1.396913793 .0728513818 .57039396$ $-1.613830423 .8423677516 .99997840$ 1.292544953 .1901167719 .63189844 2.843586475 .4321813316 .40752124 3.389049354 .0857601618 .47350678 $-4.267644284 .8242629517 .67909783$ $-5.654557614 .3261225317 .67517009$ $-5.738379803 .2373017816 .58532986$ $-4.863621473 .1440031515 .73339364$ $-6.709331215 .4392242617 .37354242$ $-6.473900695 .9684500516 .06661124$ $-3.888237315 .0951957916 .78044244$ $-5.835387653 .9090886818 .67111240$ $-7.712767664 .9938775317 .42072635$ $-6.637559396 .2301395718 .12922839$ $-7.366321475 .9316628715 .63581162$ $-6.801617012 .3887035416 .66676367$ $-7.137589181 .4224926715 .60672152$ $-8.572141241 .7225898815 .17422605$ $-9.496231471 .3617234315 .88034508$ $-6.98551396-0.0187914116 .13018249$ $-7.09051522-1.1002230015 .08397725$ $-8.34910119-1.6203644414 .75129135$ $-5.93619890-1.6170584214 .48184716$ $-8.40868746-2.6629955913 .80723723$ $-6.01815832-2.6514876213 .54904204$ $-7.26135315-3.1663059313 .21925020$ $-7.524736082 .4698317317 .38097283$ $-6.482484501 .5675179914 .74516568$ $-6.01802856-0.0984202716 .62269145$ $-7.74978581-0.1959551116 .88784769$ $-9.24786536-1.2286419315 .20955083$ $-4.97357576-1.2134368314 .74315761$ $-9.36877773-3.0821751013 .53846197$ $-5.13330175-3.0477022813 .07130851$ $-7.33514130-3.9685351712 .49648466$ $-8.040799196 .9824652120 .75179999$ $-6.625962447 .0424637921 .19007625$ $-6.395758316 .1630831822 .44114955$ $-7.137347016 .2207100623 .42507871$ $-6.183938288 .4848973421 .46222310$ $-6.188428079 .3230583720 .21307256$ $-5.432781128 .9235730819 .10582385$ $-6.9753418910 .4952531020 .12125703$ $-5.455845819 .6958658017 .92189068$ $-7.0032115811 .2496023918 .92534131$ $-6.2404302310 .8569664817 .81230114$ $-8.581078057 .8416969620 .74498294$ $-6.017157936 .6323936020 .38294634$ $-6.825542148 .9402163522 .22017209$ $-5.160762218 .4682207821 .84592934$ $-4.813518298 .0404104019 .14700264$ $-7.5736683910 .8181365520 .96246424$ $-4.868385519 .3740050617 .08449713$ 
$-7.6037676612 .1473274218 .86081996$ $-6.2564238711 .4392261916 .89806380$ $-5.352641415 .3425323122 .37649876$ $-4.973828224 .4159383623 .46412291$ $-4.102639295 .1137488324 .49914635$ $-3.504356196 .1408374524 .23375134$ $-4.195811333 .2427033122 .90797097$ $-2.994045363 .6740583622 .30430056$ $-4.767033315 .3851156221 .55431491$ $-5.874188444 .0582741423 .96068551$ $-3.967123232 .5354101723 .70709069$ $-4.801556872 .7373592622 .15466161$ $-3.244880703 .9520042121 .40228487$ $-6.793207092 .3988356510 .37703749$ $-5.917056743 .5692324310 .34669720$ $-5.700904854 .0371301811 .80436070$ $-6.541205833 .9030841312 .67470401$ $-6.455862814 .699064129 .45600794$ $-7.001338344 .247825878 .06606957$ $-7.574309875 .4242585810 .18276720$ $-7.627694892 .4775105810 .95702811$ $-4.951764843 .240474719 .96350861$ $-5.619520585 .380241329 .28268425$ $-7.997593764 .627578477 .84804937$ $-6.322179544 .613580497 .29875519$ $-7.058794653 .172458847 .95029334$ $-8.206621424 .7251559610 .73388413$ $-8.197548425 .935968609 .45705545$ $-7.154907486 .1526031910 .87991509$ $-4.488059284 .6223302712 .01530082$ $-4.281905985 .3966037913 .23425118$ $-4.584318036 .8841636212 .96853572$ $-4.210071727 .4544024111 .93258698$ $-2.770183845 .2388286113 .63120810$ $-2.468220656 .2209507615 .12837102$ $-3.861379934 .7850794211 .23242612$ $-4.908089594 .9686730814 .01923023$ $-2.561421534 .1872183113 .84856166$ $-2.126744535 .5849004112 .81433192$ $-5.265037317 .5224195313 .95140095$ $-5.685642068 .9254588513 .79899391$ $-4.664491029 .9232609014 .37924929$ $-4.8336002111 .1225094614 .25171475$ $-7.038403159 .1565680214 .49434244$ $-8.151750048 .4963818513 .67658930$ $-9.501186979 .1283296814 .06654548$ $-10.260495908 .4904611014 .92181186$ $-9.8324466210 .2033666813 .60697524$ $-5.642776417 .0450384614 .76952851$ $-5.803877139 .1594240912 .73985232$ $-7.024790778 .7490961715 .50779532$ $-7.2457047110 .2271195414 .56317871$ $-7.988060378 .6470060112 .61069597$ $-8.162470397 .4248173913 .86124498$ $-11.113320938 .9561246515 .15576726$ $-9.955681597 .5824230715 .27461333$ $-3.541133479 .4453339414 .94011701$ $-2.5655674310 .4348512515 .44990061$ $-2.0659865211 .1936669614 .24962147$ $-1.6710039910 .5500872313 .25950138$ $-1.399338589 .8008017216 .17576959$ $-1.978292999 .1579960517 .43358860$ $-0.2947507610 .8752967616 .48531748$ $-0.871591828 .5819161518 .33661993$ $-3.407487378 .4409970814 .97997261$
$\mathrm{H}$

$\mathrm{H}$

$\mathrm{H}$

$\mathrm{H}$
$-3.0859234611 .0890437816 .14739082$ $-0.960299139 .0317548615 .52741495$ $-2.547043089 .8966997918 .01715942$ $-2.670636478 .3471766617 .16790935$ 0.1316329211 .3526098815 .59307446 0.5588589010 .4216086117 .00695152 $-0.6769744211 .6685649517 .14126225$ $-0.288610987 .8059981417 .81387631$ $-1.304631958 .1358811719 .23091934$ $-0.162731919 .3523029918 .66035367$ $-2.1550103812 .5216205214 .27520845$ $-1.7411796513 .3502752713 .11693238$ $-0.3662943312 .9414398912 .54082149$ 0.6165286712 .7433975613 .27781890 $-1.7092139814 .7660918413 .69778177$ $-2.8542548014 .7314346114 .70624375$ $-2.6713592813 .3536673015 .33889486$ $-2.5090229513 .3115806212 .35098049$ $-0.7613573014 .9493629214 .21145276$ $-1.8634811415 .5222613812 .92706945$ $-2.7763366215 .5387311615 .43699281$ $-3.8147478314 .7757631714 .18674464$ $-1.9249473313 .4256363916 .13340569$ $-3.6265480713 .0064955515 .73368042$ $-0.3441651112 .6558072011 .23826634$ 0.8104178712 .2376974510 .43305867 1.0384228810 .7513933010 .31663676 1.7070068910 .342346219 .37597617 $-1.2238461312 .7715053810 .74253073$ 0.7175180512 .650291069 .42666113 1.7094531512 .6538696010 .88706463 0.538588289 .9143855711 .24644233 0.815591018 .4729723111 .20704822 0.249698737 .752155089 .95174822 0.988427657 .029227019 .30203221 0.308672427 .7989517512 .48268565 1.141112978 .1580914013 .67969863 2.112536589 .1256439313 .79970634 1.003713727 .4660700214 .88595142 1.889924318 .0510011915 .73356469 2.563332529 .0341262815 .11700558 $-0.0222396310 .3235387511 .98864532$ 1.901004948 .3498428011 .15885579 $-0.740152468 .0880741512 .63448629$ 0.334787746 .7160795012 .32009913 2.528408449 .8638265213 .12903115 2.053266717 .7794903116 .76580739 3.278891779 .6133551815 .53981724 $-1.044376107 .889038419 .65638568$ $-1.642801617 .320871928 .44499306$ $-0.925824067 .833227807 .19475466$ $-0.680903097 .061644246 .25868023$ $-3.154259667 .657447488 .41311114$ $-1.634193978 .4696843510 .24489391$ $-1.524898186 .235386768 .47622517$ $-3.423907188 .085122127 .45661502$ $-3.427478278 .377298289 .17471164$ $-3.740733266 .749746288 .56973652$ 2.145819938 .560665556 .93167091 3.468124547 .938913726 .78583449 3.355154226 .418971936 .78285330 4.367531175 .741928127 .00368663 4.453148638 .436255967 .84085327 4.986770909 .826378987 .47147945 3.820589368 .538587809 .06306236 


\section{QM/MM R2a (B3LYP/6-31G(d):Amber)} electrostatic embedding $\omega_{\alpha}=1.0$

$\mathrm{N} \quad 0.24473188-1.9642095917 .87936527$ C $\quad 0.88815294-0.8143356117 .26639471$ C $\quad 2.41870369-0.8827116417 .60017358$ O $\quad 2.79800612-0.8165222918 .78478714$ C $\quad 0.310686270 .4974865617 .81081959$ $\mathrm{H} \quad-0.05189709-1.9529086118 .84825087$

1.727544308 .634039297 .84982775 .909457158.229154615.83144122 3.159978199 .728653747 .94628863 5.2250519710 .403852368 .36486688 4.2795621810 .381575366 .85262201 920821179.684553506 .92021655 0.152195723 .431671359 .53151357 $-1.326958593 .161609979 .19986537$ $-2.234683113 .835864339 .69607856$ (10.90577974 $-0.029978443 .1353528311 .97030016$ .493335403 .8131009713 .60863482 0.841158115 .240583998 .55940681 0.664257602 .471597039 .61969860 1.290532424 .5345958210 .99213595 . 0.505588392 .1946458511 .82417940 $-0.991927362 .1016646214 .45599462$ 0.693463311 .5294470114 .38697579 . $-1.9783562711 .165282118 .89800341$ $-2.5985227811 .662877178 .33712072$ $-2.5192252610 .886063549 .65631506$ $-2.6328426410 .1585453011 .92937167$ $-3.552486499 .0753818811 .37599855$ 2.3462883311 .7324267018 .59694998 1.9580201612 .5583465418 .29908547 2.9372264212 .0032145619 .30443513 $0.74329115-0.8413262116 .18382200$ $-0.428970200 .2961639118 .58786540$ $-0.181711331 .0365480917 .00675083$ 1.093707021 .1175592518 .24723011 $3.20473792-0.9462990116 .54423888$ $4.69478070-0.9341066716 .54996676$ $5.23981948-1.9115643517 .61555052$ $5.60795904-1.5218881318 .73162186$ 5.251785400 .5225334716 .75784530 6.757405940 .5860464616 .34957067 4.481161261 .6024145815 .97606757 $2.77364411-1.0093066015 .62766124$ $5.02297791-1.2773018215 .56617759$ 5.183373550 .7557862217 .80954329 7.373522780 .7310130517 .23790467 $7.06568681-0.3548303015 .88368205$ 6.975300771 .3917670615 .65123559 3.610131271 .9167851216 .55110013 4.161317591 .1660921915 .03354465 5.105482012 .4667234515 .76099558 8.447066922 .1698980212 .55926448
7.563343163 .2114468913 .04413373 7.134622984 .0718151811 .83481084 7.973779564 .4633985511 .03064222 8.246955404 .0704860614 .11560505 7.400244685 .1846579314 .72424394 6.629896154 .9467022115 .87297894 7.341569456 .4581888614 .11095186 5.821816405 .9736104116 .40161266 6.548252357 .4712135814 .64035298 5.788073707 .2380607915 .78772993 9.346748022 .4102991412 .18502582 6.703967672 .7303763213 .49594235 8.576144703 .4183289314 .92917935 9.131778154 .5228100713 .66410298 6.656764623 .9776980716 .35136373 7.931010896 .6663577713 .22800270 5.227776615 .8031653717 .28746363 6.533170828 .4395392714 .15300386 5.170452868 .0261665916 .19447872 5.812566934 .4241398711 .76532890 4.667730523 .9285023012 .56308830 3.977449812 .8227057111 .74076660 3.873254962 .8750391710 .52220392 3.788988835 .2051201512 .69552302 3.967387945 .8571753711 .31020950 5.462870905 .6103450310 .95419308 4.951500253 .5573379913 .55294228 2.752478344 .9579630112 .93007475 4.192141105 .8374258313 .49882029 3.324944045 .3663816410 .57372447 3.730277156 .9252216911 .32632905 5.618975855 .430942749 .88719717 6.100045936 .4525490611 .25333264 3.503839661 .7399010412 .42008643 2.924138990 .5988115011 .70316937 $2.09099178-0.2242835012 .66762041$ $2.31791455-0.1831208913 .88981859$ $3.96410655-0.3501616811 .02199656$ $4.78622348-1.0348043111 .98020079$ 3.563787211 .6133313213 .42494462 2.269580071 .0073522910 .93234052 $3.42349866-1.1011510110 .44232562$ $\begin{array}{llll}4.58207814 & 0.22518821 & 10.32999825\end{array}$ $5.49474131-0.4256459412 .28776495$ $1.15363077-0.9790857712 .11398045$ $0.44363039-2.0289290612 .83461305$ $1.00190248-3.4170325412 .52640407$ $1.40854024-3.7005048011 .38394021$ $-1.00806912-2.0155641012 .36119477$ $-1.83260658-0.7831179412 .70238402$ $-2.26747682-0.5722524814 .03329411$ $-2.216664050 .1157640411 .68111659$ $-3.104597840 .5108539914 .32321530$ $-3.029183281 .2014629812 .01251956$ $-3.479971121 .3998616413 .32552252$ $1.04203677-1.0110726811 .10473351$ $0.49629840-1.8543708813 .90991935$ $-0.97895661-2.0946510911 .27517303$ $-1.52532336-2.9018605812 .73786386$ $-1.97998141-1.2681507314 .80973006$ $-1.87788418-0.0219567610 .66238528$ $-3.454215860 .6905890015 .33162261$ $-3.339908541 .8959289911 .24433184$ $-4.118295532 .2423153313 .55384359$ $-6.91360406-4.8822027120 .29877067$ 
$-5.92311729-3.9204630019 .91730930$ $-5.01185992-3.6076320221 .10932563$ $-5.52558247-3.2409576622 .15298316$ $-6.59881548-2.6250681519 .46589887$ $-5.56170719-1.5790183019 .13713007$ $-4.57826832-1.8327866218 .19165963$ $-5.57832434-0.3673731619 .80705884$ $-3.63926464-0.8403569017 .89243502$ $-4.638837090 .6320351019 .54425129$ $-3.663217500 .3784734618 .55716047$ $-7.56919885-4.6602505821 .04518551$ $-5.31740129-4.3089076819 .10262885$ $-7.19327374-2.8214702618 .57066820$ $-7.26868174-2.2695966920 .25165944$ $-4.56252654-2.7748958017 .66090818$ $-6.34894763-0.2061898720 .54298882$ $-2.87886916-1.0412921117 .15267004$ $-4.667456451 .5762996920 .06947455$ $-2.928428441 .1340584618 .32491977$ $-1.52428251-1.4863125222 .33144100$ $-0.53758405-0.4613777222 .00068334$ $0.76455762-0.7617277822 .76020065$ $0.81972839-0.4846131523 .95478797$ $-1.007802180 .9317326822 .46020463$ 0.042547961 .9715915222 .14993721 0.009125683 .1063826522 .82513196 0.934897581 .7345200021 .35819485 $-2.04379224-1.4464290423 .20419663$ $-0.33263907-0.4502260120 .93102001$ $-1.892724471 .1910534221 .87902016$ $-1.282002470 .9442490423 .51536978$ $-0.734083103 .2571027623 .49541276$ 0.722660763 .8089690422 .67040087 3.774584670 .5406479021 .87776334 4.711715011 .5717251221 .49529435 4.816287822 .6616326522 .55204377 3.913983373 .4407882922 .79618280 4.296715252 .1277504720 .12077010 5.352964933 .0019830919 .52947403 6.626236822 .6487983219 .62849046 5.033813624 .0039662018 .93828370 2.805264310 .6794420221 .61571708 5.697713531 .1184710521 .39933168 4.087056451 .2981012419 .45683130 3.369363352 .6714066120 .24045918 6.884507951 .7717885420 .04598727 7.321592653 .2302763819 .17865635 3.622108896 .9435380922 .54061503 2.536654977 .3625468021 .61948048 1.222724706 .6775878421 .97855708 1.253663445 .5059456522 .32919803 2.872788256 .9861036120 .17691533 4.145313557 .6238883419 .75299666 5.371045306 .9964125020 .07853365 4.101814648 .8760997919 .11979576 6.553827837 .6607984119 .77877343 5.304264639 .5283285418 .82221675 6.522046398 .9100569219 .15812676 3.721899645 .9615784122 .75719852 2.375008678 .4403915221 .68984455 2.969715515 .9031302820 .10470147 2.073271077 .3205837219 .51326673 5.393917996 .0302814520 .56114750 3.160279649 .3449235918 .87521340 7.509860717 .2336871720 .01415717
5.3157201610 .4952053718 .33760143 7.454578929 .4092356118 .92776336 0.081191977 .3441400321 .81301339 $-1.205987176 .6968946222 .08057455$ $-1.941256756 .3944964320 .75995586$ $-3.024705636 .8903298220 .48269370$ 0.083059018 .2962061721 .47853986 $-1.098759905 .7572111022 .62424614$ $-1.834342007 .3562465422 .68256897$ $-1.364603285 .4607441519 .96676994$ $-1.985343295 .1609963618 .64634201$ $-3.472165984 .7041060418 .81017067$ $-3.882348994 .1962328719 .85542558$ $-1.215279544 .0018756118 .00713799$ 0.256138634 .2686957317 .92787008 1.283012483 .7884461418 .73899499 0.809549204 .9881857316 .92533664 2.111991974 .9650361617 .04834443 2.439330424 .2452327718 .15239575 $-0.525678754 .9805736720 .26247056$ $-1.954949366 .0559580018 .02063165$ $-1.396639143 .0741073318 .56848858$ $-1.612444513 .8445934816 .99869909$ 1.292733593 .1896536419 .63106199 2.845017215 .4334125516 .40878181 3.392540234 .0856630818 .47420968 $-4.267160564 .8212640317 .68213790$ $-5.655953784 .3241632417 .67592489$ $-5.739921893 .2356671716 .58513001$ $-4.861785993 .1420800515 .73257361$ $-6.710418945 .4389574817 .37256131$ $-6.475383895 .9688344916 .06677724$ $-3.884717085 .0927376416 .78361875$ $-5.838227563 .9076301318 .67050068$ $\begin{array}{ll}-7.71290721 & 4.99463841 \\ -6.63832126 & 6.41881577\end{array}$ $-6.638321266 .2291259018 .12834953$ $-7.366649275 .9331055915 .63539688$ $-6.801418392 .3895168416 .67015877$ $-7.137028131 .4224472515 .60855024$ $-8.571885391 .7228759815 .17555274$ $-9.496333121 .3614272915 .88055289$ $-6.98499369-0.0190145816 .13122505$ $-7.09015318-1.0999955615 .08446941$ $-8.34883292-1.6201737614 .75158739$ $-5.93593296-1.6167365814 .48227940$ $-8.40857679-2.6626291813 .80719988$ $-6.01803478-2.6509944513 .54915860$ $-7.26129921-3.1657992813 .21909395$ $-7.523993722 .4699054717 .38491865$ $\mathrm{H} \quad-6.482091281 .5681592214 .74721819$ $\mathrm{H} \quad-6.01776219-0.0988691016 .62344612$ $\mathrm{H} \quad-7.74878082-0.1967823016 .88860589$ $\mathrm{H} \quad-9.24759499-1.2290422015 .20946007$ $\mathrm{H} \quad-4.97331701-1.2135948214 .74338225$ $\mathrm{H} \quad-9.36847072-3.0816705813 .53829400$ $\mathrm{H} \quad-5.13334128-3.0469461813 .07137787$ $\mathrm{H} \quad-7.33507770-3.9676671212 .49629671$ N $\quad-8.040466436 .9823763320 .75117630$ C $\quad-6.625742897 .0422885521 .18979826$ C $\quad-6.395560556 .1629257422 .44101749$ O $\quad-7.137712696 .2207234023 .42429015$ C $\quad-6.184224548 .4847769321 .46204334$ C $\quad-6.188403079 .3230590720 .21297675$ C $\quad-5.432353128 .9238063219 .10598480$ C $\quad-6.9754700510 .4949740020 .12102113$ 
$-5.455094049 .6960451217 .92213481$ $-7.0031813511 .2493742518 .92516354$ $-6.2399669410 .8569573217 .81246843$ $-8.581372937 .8415434920 .74422562$ $-6.016667246 .6324722720 .38312831$ $-6.826034388 .9397069422 .21955717$ $-5.161501798 .4683853021 .84602837$ $-4.813013368 .0409281519 .14738854$ $-7.5739805310 .8174744520 .96190420$ $-4.867531389 .3744018117 .08537800$ $-7.6038473012 .1468033418 .86083347$ $-6.2558771611 .4391130716 .89871237$ $-5.352138685 .3425398022 .37602866$ $-4.973761214 .4162284123 .46388574$ $-4.102593325 .1138818324 .49904169$ $-3.503956916 .1409382024 .23326166$ $-4.195385543 .2429141722 .90816667$ $-2.993304593 .6740225622 .30469328$ $-4.766153465 .3850977521 .55359537$ $-5.874135484 .0585653523 .96001656$ $-3.966989602 .5360670723 .70724886$ $-4.800727062 .7374814722 .15486452$ $-3.243621903 .9520960721 .40140692$ $-6.793297772 .3986078310 .37659313$ $-5.916274353 .5685029110 .34597715$ $-5.699250234 .0377333411 .80196427$ $-6.540677323 .9043720212 .67553575$ $-6.455612964 .698513189 .45533722$ $-7.001741114 .247060628 .06596725$ $-7.573768075 .4240612610 .18264796$ $\begin{array}{ll}-7.62788533 & 2.47704131 \\ 10.95674453\end{array}$ $-4.951541883 .240116159 .96205042$ $-5.619854575 .379666659 .28163097$ $-7.997807134 .626969507 .84845447$ $-6.322953504 .612677897 .29877565$ $-7.058968733 .171892507 .95054322$ $-8.205892084 .7253288410 .73394532$ $-8.196993085 .935656649 .45730698$ $-7.154257386 .1524036610 .87923836$ $-4.492015164 .6231643712 .01716416$ $-4.282991615 .3975907413 .23851475$ $-4.585859556 .8845866412 .97454020$ $-4.209249887 .4562500811 .93075919$ $-2.770175615 .2392355513 .63330951$ $-2.466978536 .2217269015 .13111789$ $-3.865954284 .7870222811 .23306950$ $-4.907597204 .9694478214 .02322176$ $-2.562019194 .1884042813 .84852298$ $-2.128637015 .5857800312 .81555331$ $-5.264764987 .5204283413 .95000089$ $-5.685761458 .9245711213 .79859086$ $-4.664446129 .9224639514 .37887247$ $-4.8339729511 .1221064014 .25161330$ $-7.038435429 .1560298714 .49438083$ $-8.152330108 .4965203413 .67659418$ $-9.501670599 .1288417314 .06669451$ $-10.260757858 .4916901414 .92152590$ $-9.8316144710 .2033022013 .60702611$ $-5.642221887 .0404925414 .77004315$ $-5.804380669 .1591109812 .73970867$ $-7.024796228 .7483121915 .50747175$ $-7.2451531510 .2264318114 .56335498$ $-7.988722398 .6475073412 .61105098$ $-8.163504947 .4250300513 .86094150$ $-11.114709818 .9577865915 .15622832$
$-9.957831737 .5841094015 .27453030$ $-3.541370529 .4453247214 .94001877$ $-2.5659634110 .4350314415 .44977564$ $-2.0663402611 .1936050914 .24931866$ $-1.6709526310 .5499100913 .25934351$ $-1.399463849 .8007029116 .17563317$ $-1.978543199 .1582358917 .43385013$ $-0.2945417610 .8750269516 .48495255$ $-0.871976628 .5823546518 .33693950$ $-3.407749098 .4410800714 .98019052$ $-3.0861115411 .0891024016 .14697063$ $-0.960890929 .0318478215 .52758298$ $-2.546852749 .8966999318 .01704589$ $-2.670989888 .3474547117 .16841940$ 0.1307499911 .3514954915 .59312679 0.5577228510 .4215651217 .00606658 $-0.6768802111 .6679141717 .14070130$ $-0.289548757 .8067487817 .81461630$ $-1.304772968 .1368056319 .23083856$ $-0.163739079 .3523106018 .66018654$ $-2.1549309012 .5216919414 .27469898$ $-1.7410335813 .3500246713 .11641815$ $-0.3661974012 .9409890812 .54038510$ 0.6171555812 .7429091313 .27689621 $-1.7090845714 .7658500113 .69698754$ $-2.8538788214 .7314616114 .70551049$ $-2.6715341913 .3537563915 .33837393$ $-2.5087748513 .3107549212 .35072280$ $-0.7615813314 .9491258214 .21054964$ $-1.8634136715 .5217555212 .92644990$ $-2.7759227215 .5385157615 .43598642$ $-3.8140545814 .7757325214 .18613404$ $-1.9255196713 .4255756216 .13279412$ $-3.6265309113 .0068447815 .73296476$ $-0.3441400312 .6557106311 .23755708$ 0.8106323612 .2372612910 .43312421 1.0385407910 .7508917110 .31741931 1.7067475510 .340840949 .37758295 $-1.2241172312 .7713600810 .74204406$ 0.7176322112 .649297119 .42671212 1.7094657312 .6538234310 .88664819 0.538878269 .9146692911 .24681189 0.815971778 .4733881711 .20723245 0.250048477 .752616759 .95173550 0.988512867 .029510909 .30165142 0.308810357 .7988160312 .48273145 1.141117998 .1577791113 .68006056 2.112064069 .1252366213 .80032860 1.003816987 .4649836814 .88676604 1.889705708 .0501818315 .73427687 2.562237899 .0332539515 .11726472 $-0.0223197610 .3239788011 .98862441$ 1.900933498 .3502864411 .15908684 $-0.740337908 .0875199512 .63481466$ 0.335190156 .7158007512 .32007489 2.526113249 .8621550813 .12935919 2.052621097 .7784208016 .76574162 3.277881899 .6129662215 .54036804 $-1.044419277 .888836209 .65677574$ $-1.642953687 .321082038 .44521462$ $-0.925660217 .833289307 .19502839$ $-0.680850967 .061388726 .25938992$ $-3.154210177 .658066548 .41309004$ $-1.633849198 .4700131410 .24528332$ $-1.525600836 .235928618 .47637731$ 


\section{QM/SE/MM R1a (B3LYP/6-31G(d):xTB:Amber)} mechanical embedding $\omega_{\alpha}=1.0$

\begin{tabular}{|c|c|}
\hline $\mathrm{C}$ & 7.5642122 \\
\hline $\mathrm{C}$ & 7.139263124 .1337304111 .77934541 \\
\hline $\mathrm{O}$ & 8.001127524 .5056585910 .98461371 \\
\hline $\mathrm{N}$ & 5.818056314 .4654179311 .73256882 \\
\hline $\mathrm{C}$ & 4.700579163 .9041233412 .55028927 \\
\hline $\mathrm{C}$ & 3.996308222 .7848603211 .74542070 \\
\hline $\mathrm{O}$ & 3.867041162 .8534170210 .52323575 \\
\hline $\mathrm{C}$ & 3.788061085 .1362930012 .75132005 \\
\hline $\mathrm{C}$ & 3.887300375 .8193202211 .35660714 \\
\hline $\mathrm{C}$ & 5.385856465 .6576780810 .97119695 \\
\hline $\mathrm{H}$ & 5.046114833 .5248189713 .51838325 \\
\hline $\mathrm{H}$ & 2.763383234 .8297919212 .98933784 \\
\hline $\mathrm{H}$ & 4.178530255 .7813652013 .55749514 \\
\hline $\mathrm{H}$ & 3.252662885 .3107303310 .62229955 \\
\hline $\mathrm{H}$ & 3.603803036 .8778975811 .40895 \\
\hline $\mathrm{H}$ & $5.519679265 .505065709 .894401 \mathrm{C}$ \\
\hline $\mathrm{H}$ & 5.9729064 \\
\hline
\end{tabular}
$-3.740220876 .750643568 .56958350$ 2.145754618 .560627196 .93164012 3.467953967 .938748356 .78598038 3.355114756 .418844016 .78295390 4.367454985 .741425357 .00450615 4.452906898 .435976667 .84124068 4.985811769 .826527017 .47279440 3.819568808 .537564089 .06362900 1.726810798 .633752317 .84934940 3.909651538 .228815575 .83196428 5.275827817 .728987057 .94639919 3.159126379 .243721618 .98684110 5.2239415410 .403476028 .36612599 4.2785398010 .381568146 .85442602 5.919502749 .685799126 .92134774 0.787145764 .229497478 .48400168 0.152650103 .431665529 .53133346 $-1.326517663 .161651579 .19970656$ $-2.234258463 .835675129 .69652274$ 0.264070314 .1633576710 .90573025 $-0.029713773 .1353295111 .97071265$ 0.493768433 .8130227613 .60892952 0.052757342 .3844588214 .61052677 0.841556315 .240757358 .55902244 0.664820132 .471934069 .61948301 1.290107584 .5341560210 .99182507 $-0.415288785 .0210604510 .93612109$ $-1.098834502 .9084180312 .01792975$ 0.506409232 .1948485111 .82420217 $-0.991510492 .1015959714 .45597215$ 0.693499641 .5288833914 .38588180 0.188256782 .6575851615 .65785981 $-0.234597505 .9906064215 .34509304$ $-1.9781773611 .165180398 .89811960$ $-2.5969785111 .664983328 .33708729$ $-2.5208048510 .882972019 .65425555$ $-2.951706709 .7845599811 .08793453$ $-2.6261746310 .1538238511 .92928544$ $-3.547164129 .0699590811 .37615184$ 2.3467332911 .7357278718 .59522708 1.9579826712 .5617447218 .29812378 2.9362211412 .0054268119 .30465071
3.476888931 .7443605212 .45393845 2.869113860 .6286867511 .75770340 3.731265391 .5602750213 .41706829 $-1.175289526 .7047797421 .94341706$ $-1.946120936 .3840081320 .68522621$ $-3.057120506 .8640745220 .46821866$ $-1.367702815 .4506465019 .92033764$ $-1.994535325 .1328304118 .62050971$ $-3.470802234 .6851633318 .78140182$ $-3.885560174 .2182186719 .84817093$ $-1.203375654 .0013929117 .96645195$ 0.272587984 .2689266017 .91898388 1.279360643 .7649245618 .75343767 0.837957464 .9848191516 .93379591 2.133402764 .9564645617 .08662182 2.453001804 .2221750018 .19220816 $-0.520920854 .9885545220 .22013056$ $-1.982360456 .0377630018 .00043693$ $-1.388565953 .0639698018 .51683844$ $-1.600967653 .8625604916 .95181934$ 1.205546273 .1505851119 .64571759 2.875368575 .4308749516 .45844617 3.408925874 .0525137318 .52957158 $-4.256096774 .8300832817 .65275495$ $-5.641955094 .3289381417 .66450242$ $-5.749008663 .2388279416 .57449266$ $-4.875929573 .1279028215 .72454529$ $-6.706075225 .4214789217 .37134265$ $-6.472049405 .9820759616 .08111454$ $-3.839925335 .1499414216 .77672348$ $-5.795035793 .8820982718 .65690118$ $-7.702106084 .9542944317 .40716709$ $-6.647408786 .2101926618 .13602351$ $-7.337361625 .9297929015 .60223165$ $-6.826895032 .4048899816 .66199827$ $-7.146606291 .4291850615 .61638150$ $-7.447763532 .3993803917 .46403059$ $-5.946689823 .4972189010 .38282336$ $-5.734185574 .0160246411 .82422440$ $-6.584544663 .9024637112 .70029799$ $-4.538278484 .6193660312 .01150442$ $-4.267491455 .3789314413 .23743662$ $-4.579315666 .8690318212 .96994793$ $-4.209361597 .4465867711 .91996302$ $-2.758255225 .2085906713 .64883432$ $-2.476457626 .2223382815 .13202456$ $-3.898086794 .6882157211 .23204250$ $-4.930939475 .0102807414 .02454822$ $-2.560916794 .1655655813 .91824598$ $-2.125914565 .5380960312 .81598576$ $-5.262775377 .5239260213 .94576619$ $-5.680173118 .9065594013 .78518091$ $-4.661096519 .9436513014 .36694976$ $-4.8213984711 .1370356514 .25417027$ $-5.597417247 .0133375014 .75953576$ $-5.762032179 .1027119412 .70814238$ $-3.544994709 .4440371314 .93104297$ $-2.5791246010 .4557740915 .41127462$ $-2.0753137111 .1974200014 .19751553$ $-1.6382185910 .5498347113 .23511081$ $-1.435301859 .8150024216 .15271158$ $-1.973309289 .1668321117 .43337548$ $-0.3231385310 .8867119916 .46879602$ $-0.856961498 .5880630418 .32499406$ $-3.381190608 .4448492915 .02508790$ 

$-1.012722819 .0237858015 .51017043$ $-2.535673509 .9209678418 .01391249$ $-2.670323808 .3579763617 .16637043$ 0.0495096511 .3485534515 .54108352 0.5158029810 .4149678516 .99088070 $-0.7466242011 .6741276017 .11856880$ $-0.299617537 .8120206517 .77723906$ $-1.296646168 .1483887819 .22278896$ $-0.152696909 .3816424018 .62295026$ 0.736212998 .4674480711 .23809242 0.228654007 .7907209012 .50599916 1.101175908 .1518154213 .68692006 2.074837939 .1209449513 .76193306 0.999492947 .4736413014 .89607684 1.915638728 .0523066315 .70571805 2.581563039 .0400975615 .06514373 $-0.822080878 .0918346312 .68760621$ 0.231184356 .7051149712 .33019136 2.416611409 .8254482813 .01728782 2.101797457 .7699054916 .73195980 3.314289059 .6128977515 .46463745 0.164008733 .500274969 .50453223 0.226001184 .1823909410 .89714516 $-0.004112423 .1464819511 .97618766$ 0.493869853 .8173123313 .61359953 0.093538872 .3562148214 .60095612 1.220122254 .6462514211 .00952118 $-0.532784274 .9796927410 .96159668$ $-1.056044322 .8280431711 .95773880$ 0.574552842 .2400933011 .76784886 $-0.920909691 .9998129814 .38303056$ 0.816669561 .5566027714 .39449947 0.160853672 .6325026915 .65691404 $-0.236330435 .9919750615 .34525027$

QM/SE/MM R3a (B3LYP/6-31G(d):xTB:Amber) mechanical embedding $\omega_{\alpha}=1.0$

C $\quad-0.54296368-4.2538659117 .89556527$ C $\quad 0.05664876-3.0989437317 .14700440$ O $\quad 0.31704854-3.1825418015 .95737299$ $\mathrm{N} \quad 0.25019315-1.9986272617 .87133793$ C $\quad 0.88100452-0.8495847317 .27471273$ C $\quad 2.43993984-0.8889412417 .62918807$ O $\quad 2.80377714-0.8100375118 .79324040$ C $\quad 0.327092820 .4769484117 .79669388$ $\mathrm{H} \quad-0.05182618-1.9854978918 .83773517$ H $\quad 0.73932269-0.9121123016 .19391625$ $\mathrm{H} \quad-0.411149700 .2822218718 .57497435$ $\mathrm{H} \quad-0.154809681 .0269719816 .99378196$ $\mathrm{H} \quad 1.107388831 .0903666218 .23283611$ N $\quad 3.18584839-0.9508450616 .54648829$ C $\quad 4.65511275-0.9425407316 .56064460$ C $\quad 5.22902785-1.9115674517 .62794546$ O $\quad 5.59848853-1.5322140818 .72198418$ C $\quad 5.225992310 .5097263516 .78498167$ C $\quad 6.734782520 .5681794516 .37743434$ C $\quad 4.444680411 .5611993016 .00013803$ $\mathrm{H} \quad 2.77087718-0.9859561715 .62162083$ $\mathrm{H} \quad 4.98266950-1.2648731515 .56610773$ $\mathrm{H} \quad 5.167442680 .7157599517 .85547052$ $\mathrm{H} \quad 7.363056370 .7098688817 .25702057$ $\mathrm{H} \quad 7.03237906-0.3679525115 .89676736$ $\mathrm{H} \quad 6.915273031 .3785724315 .68101590$
3.571842491 .9044077416 .55517004 4.113264581 .1183070615 .06337021 5.077139042 .4195133715 .77647004 $5.16351400-3.2173221417 .26348631$ $5.91434919-4.1686917018 .05314870$ $4.93114590-3.4767100816 .31158357$ 8.980760590 .0282340511 .52130770 7.972124450 .9448783012 .20774401 6.815171810 .5943093112 .41500572 8.465712962 .1344519012 .55345194 7.579971863 .1599479313 .04493132 7.141310904 .0548412311 .83476557 7.991969714 .4439209211 .03086010 8.266154464 .0473456614 .09661514 7.423866825 .1455321114 .70353973 6.667646394 .9022644215 .83862544 7.350218276 .4146910314 .12044731 5.865864645 .9002215316 .37798655 6.559201977 .4003340314 .65663579 5.811267207 .1555896215 .79128968 9.370924202 .3932398512 .20525061 6.701880882 .6399216113 .42842656 8.587833763 .3803165114 .91117622 9.132819994 .4926266913 .59829803 6.698193763 .9294798616 .31388607 7.938908686 .6178912213 .23783339 5.288392335 .6910852217 .26566794 6.528713828 .3749870914 .18730295 5.181375367 .9198770816 .22441501 5.831297594 .4212915311 .76355918 4.693435143 .9062014412 .56158395 3.986798442 .8042180811 .73909439 3.872555672 .8682485110 .51559779 3.808278705 .1632722412 .73673739 3.948373195 .8260675911 .34523795 5.445542375 .6170153510 .97849033 5.013493793 .5242877813 .53692490 2.777056114 .8887696512 .96795009 4.204532505 .7974025613 .54395383 3.309809245 .3310858310 .61093541 3.693698536 .8896178111 .38057951 5.584480185 .453290759 .90805348 6.052095646 .4790844111 .28629771 3.515825341 .7410443012 .43432770 2.911836760 .6359097511 .73038451 $2.12299312-0.2205932412 .69062983$ $2.32506062-0.1663579213 .90387371$ $3.92860782-0.3560950811 .01344312$ $4.74778254-1.0542120811 .94109096$ 3.626072351 .6804510313 .43906977 2.264402071 .0887155510 .96770686 $3.36290526-1.1023301110 .45404342$ 4.519919910 .2346719610 .30733789 $5.45908874-0.4396507012 .21124185$ $1.18823738-0.9715459712 .11484809$ $0.44782990-1.9901799312 .83279579$ $1.00841791-3.3872818612 .48605010$ $1.39195895-3.6899220211 .36524415$ $-0.99831194-2.0060040612 .33548530$ $-1.83271808-0.7865652612 .68852448$ $-2.26361534-0.5957257914 .00630374$ $-2.222868260 .1246054011 .70255662$ $\begin{array}{llll}-3.07888579 & 0.47381581 & 14.31598602\end{array}$ $-3.029098531 .1912796812 .04396162$ $-3.467144111 .3717311113 .34895784$ 
$1.05644151-0.9799185511 .10921232$ $0.59065220-1.8067894613 .89903144$ $-0.94163452-2.0690141011 .24648708$ $-1.47772653-2.9135438112 .71880732$ $-1.95878098-1.3023086414 .76689230$ $-1.88866962-0.0064864810 .68381436$ $-3.416059680 .6274426915 .33267597$ $-3.340200901 .8909884511 .28307800$ $-4.101165362 .2020437613 .62031202$ $0.97927976-4.2506316513 .50024227$ $1.32763042-5.6537204113 .30972027$ $0.69578634-4.0031398314 .44362398$ $-8.13067574-6.9675132720 .14503928$ $-6.97793991-6.0975724019 .71633683$ $-6.26913196-6.4219644518 .77763162$ $-6.91167945-4.9070533520 .31391043$ $-5.93770130-3.9499494519 .91212511$ $-5.00760638-3.6011442221 .10957623$ $-5.51376322-3.2356995522 .14583465$ $-6.58768772-2.6278990319 .46984990$ $-5.55838349-1.5823995619 .14787606$ $-4.60450649-1.8193286118 .19032003$ $-5.56277863-0.3764218419 .81109445$ $-3.67321707-0.8428745317 .88760582$ $-4.638948980 .6087243419 .53792945$ $-3.683839790 .3556486118 .55164560$ $-7.56725686-4.6695328921 .05354506$ $-5.35068868-4.4055461419 .11549973$ $-7.19757546-2.8292144218 .57843396$ $-7.22934899-2.2692945920 .27410433$ $-4.58965641-2.7608881317 .65942396$ $-6.32128425-0.2187703720 .56091424$ $-2.92143200-1.0364381017 .13636419$ $-4.643418981 .5494268620 .06042645$ $-2.955862771 .1176618618 .31464630$ $-3.68855527-3.7169187120 .88294892$ $-2.72242426-3.5366289621 .98602427$ $-1.73009837-2.4848972421 .53579803$ $-1.12421373-2.5422886920 .47821850$ $-1.97716217-4.8556369522 .26591509$ $-3.32554473-3.9456620419 .96555614$ $-3.28042360-3.1807884622 .85078040$ $-1.52136212-1.4800242922 .36170227$ $-0.54150588-0.4698433422 .00919488$ $0.76461080-0.7457835922 .81474611$ $0.79826429-0.4702315823 .99121495$ $-1.005677660 .9303465522 .42590998$ 0.059987821 .9581743722 .12902668 0.018338753 .0903057222 .85334083 0.944152081 .7359179021 .32563861 $-2.06177396-1.3892171323 .21474319$ $-0.32600549-0.5647679720 .95091866$ $-1.894325011 .1825276521 .82540527$ $\begin{array}{lll}-1.27745058 & 0.9463115023 .47740727\end{array}$ $-0.716022363 .2202504723 .53920395$ 0.702446793 .8233063822 .73952318 $1.75610545-1.2804885522 .11312427$ $3.01568874-1.6681519822 .68157997$ $4.13996610-0.6291341622 .49964471$ $5.28298974-0.8112505722 .87370256$ $3.43993464-3.0041851422 .07672331$ $1.63537835-1.4574988921 .12703808$ $2.87671163-1.7957643123 .75276951$ 3.753038310 .5140550521 .89653632 4.679063361 .5377841221 .51442585
4.794202142 .6525401222 .54603818 3.929583753 .4475797722 .81094213 4.286949042 .1203460620 .13842934 5.339555142 .9893508519 .53206940 6.624245192 .5976018219 .65000985 5.030171394 .0012772718 .93618936 2.784554230 .6448657521 .62886569 5.665752501 .0715069721 .44596927 4.071421261 .2725466419 .47590016 3.373439302 .6923223920 .26802541 6.883744651 .7306632020 .09156502 7.379876593 .1329221419 .24301960 5.965956142 .6479691523 .23063661 6.206335733 .6746091224 .26228605 6.687504271 .9728704023 .02733531 5.411139027 .3762779824 .14938782 4.348952897 .8401636823 .17689720 4.221416209 .0270431223 .00630168 3.675341996 .9112130722 .50505533 2.586414947 .3514213021 .63672975 1.259031126 .6673957021 .97774034 1.264211335 .5155306022 .37134109 2.881325226 .9777219320 .16989135 4.159629417 .6058346819 .76821096 5.360167116 .9725077820 .09253598 4.157068538 .8494126219 .15907307 6.542449997 .6040079219 .80823001 5.359780139 .4715441518 .87702189 6.547734188 .8421565719 .20510037 3.731826365 .9232158322 .69503876 2.489823458 .4317833721 .78708677 2.921632785 .8842397620 .10599866 2.073865057 .3539410519 .52624289 5.352189206 .0025468520 .56042927 3.226567349 .3383775318 .90471092 7.484879357 .1524930920 .04521114 5.3864967110 .4401548018 .39914480 7.489553129 .3278970318 .98558871 0.128855007 .3672401021 .79481164 $-1.144793746 .7327944222 .07743761$ $-1.908290926 .3915233720 .76631723$ $-3.002164056 .8899189920 .49222445$ 0.136123188 .2980077021 .40906844 $-0.940735665 .8157452122 .63324019$ $-1.780409057 .3905388522 .67445461$ $-1.356147275 .4582458619 .97095822$ $-1.994622195 .1545033218 .65163015$ $-3.471548134 .6956389718 .80824636$ $-3.877392474 .2000265219 .86345390$ $-1.212294754 .0121011417 .98915291$ 0.262671364 .2700223617 .92887915 1.273275623 .7635842218 .75131833 0.830898914 .9890203416 .93873899 2.129497204 .9487298317 .07763062 2.447566444 .2138157218 .18024462 $-0.506280044 .9824621520 .24098164$ $-1.977408926 .0589244018 .04037565$ $-1.400028843 .0755260318 .53625213$ $-1.610884093 .8751277316 .97840935$ 1.215653183 .1515572419 .64108371 2.868977175 .4159802516 .44501687 3.404832584 .0401377818 .50587678 $-4.253192564 .8179938217 .67492265$ $-5.639591944 .3146908317 .66959244$ $-5.733843143 .2344666916 .57165350$ 
$-4.855437683 .1222418415 .72541640$ $-6.709107935 .4102493917 .37342409$ $-6.470378465 .9723909016 .08315756$ $-3.849853055 .1317017716 .79017705$ $-5.810851963 .8720390418 .65478443$ $-7.705117814 .9511902317 .40218675$ $-6.655330446 .1895106618 .14016186$ $-7.337731235 .9222227815 .60854465$ $-6.812885572 .3957602916 .64582371$ $-7.109596091 .4094194115 .59586886$ $-8.562813071 .7156793715 .18380949$ $-9.486449651 .3498706615 .87432604$ $-6.98909007-0.0287647516 .12418682$ $-7.08335794-1.1078675815 .08669198$ $-8.32497942-1.6224819114 .74616993$ $-5.94427222-1.6230349314 .48678339$ $-8.38387697-2.6493271113 .80716550$ $-6.02401220-2.6374738313 .55905628$ $-7.25444497-3.1493031013 .22439728$ $-7.505104292 .4824211717 .38633050$ $-6.452540281 .6295134614 .75087411$ $-6.01792288-0.0978163116 .62434140$ $\begin{array}{llll}-7.80093201 & -0.15899731 & 16.84795527\end{array}$ $-9.22044901-1.2321895715 .20268821$ $-4.97998297-1.2151989114 .75358932$ $-9.34940192-3.0579958513 .54300515$ $-5.13348132-3.0275930613 .09247948$ $-7.32713821-3.9474538912 .49790772$ $-8.702886682 .4730577814 .09990414$ $-10.039535872 .8845712113 .64576792$ $-7.942048792 .9001630013 .57862829$ $-9.913540115 .8846589819 .71956426$ $-8.485717155 .8203957120 .29180660$ $-7.892746864 .7670354120 .22003611$ $-8.027636356 .9799355220 .77104328$ $-6.623476967 .0542044821 .19406075$ $-6.410902566 .1835152622 .45875583$ $-7.135763916 .2197463523 .42925643$ $-6.165650688 .4891424521 .44706571$ $-6.179547289 .3101559820 .19651809$ $-5.433302478 .9315012219 .09681149$ $-6.9608648210 .4699527420 .09899847$ $-5.462457249 .6947897517 .92527872$ $-6.9924503611 .2184603018 .92650084$ $-6.2401071910 .8371339417 .82343342$ $-8.591210717 .8224187220 .76954187$ $-6.027517006 .6287570220 .37959188$ $-6.811799248 .9441245622 .20375871$ $-5.135259188 .4422699421 .81937386$ $-4.813078528 .0496291519 .13723863$ $-7.5546035310 .7841632320 .94616176$ $-4.872691869 .3819145217 .07824487$ $-7.6018574112 .1087723018 .87450715$ $-6.2564251711 .4149924716 .91199169$ $-5.346269785 .3822492222 .35001047$ $-4.971866364 .4716321123 .44718484$ $-4.729152215 .3833028921 .55128719$ $-7.708318980 .299023739 .56519893$ $-6.556078871 .331553309 .57168390$ $-5.594385761 .222449748 .83493743$ $-6.783432702 .3911180710 .36682353$ $-5.905906953 .5259210310 .34321873$ $-5.707930194 .0455320211 .79144698$ $-6.545988563 .8826148012 .67819339$ $-6.404267874 .673347339 .45701409$
$-6.937843374 .173352268 .08723688$ $-7.482235735 .4475908310 .16887740$ $-7.643617702 .4405046310 .90750430$ $-4.939771573 .135444109 .99309427$ $-5.539676405 .318687779 .25794355$ $-7.922711154 .576915787 .88779166$ $-6.267985174 .484741277 .28822833$ $-7.006573903 .089674858 .06334143$ $-8.118855174 .7799067210 .75109803$ $-8.107160235 .957423959 .44192159$ $-7.059746496 .1923992210 .84526457$ $-4.512058784 .6297420512 .01040193$ $-4.264293805 .3858871913 .23846701$ $-4.574735026 .8725054012 .97644094$ $-4.218190477 .4567600411 .93081564$ $-2.754703865 .2212953313 .63318031$ $-2.469170756 .2191446615 .13065842$ $-3.861519514 .7279010911 .24148410$ $-4.911475245 .0025612914 .02552061$ $-2.553846194 .1757475513 .87416974$ $-2.128328615 .5579801912 .80303209$ $-5.254159937 .5175603713 .95566229$ $-5.648317178 .9043371313 .81536388$ $-4.644657949 .9404370214 .37905585$ $-4.8159269011 .1331189814 .26129800$ $-7.008956469 .1646265814 .47576364$ $-8.150616978 .5087857513 .70035611$ $-9.487714819 .1790033214 .05084371$ $-10.267775768 .5458882414 .93577395$ $-9.7897394610 .2354848613 .53869501$ $-5.581122417 .0165213814 .77843170$ $-5.763876339 .0983447812 .74005183$ $-6.992126788 .7823820515 .50160051$ $-7.1804344910 .2418732114 .51767728$ $-7.996077258 .6280730712 .62362258$ $-8.188001287 .4437782713 .92984698$ $-11.138306988 .9844520615 .17896123$ $-9.972293977 .6521112015 .32769304$ $-3.531592269 .4315756214 .93757440$ $-2.5753583210 .4380364015 .44026836$ $-2.0878307011 .1896978514 .24262218$ $-1.6577827010 .5844553713 .24920838$ $-1.423282789 .8023763416 .17053946$ $-1.964991819 .1515851517 .43604830$ $-0.3236680110 .8778773616 .48886221$ $-0.849357818 .5751873618 .32738325$ $-3.355875178 .4310513815 .02006660$ $-3.1399813411 .1357342716 .05986359$ $-0.992062009 .0232718615 .52382203$ $-2.533722839 .8960348218 .01729598$ $-2.654356808 .3422509717 .16424473$ 0.0543439011 .3473421415 .57300021 0.5204121910 .4155321517 .00834061 $-0.7424228711 .6586243017 .14180828$ $-0.284426197 .8076563017 .78402732$ $-1.284112138 .1263293919 .21828990$ $-0.150366959 .3625690418 .63620461$ $-2.1763613512 .5074556014 .28644488$ $-1.7437048613 .3090824913 .11081968$ $-0.3652679812 .8889005212 .56364759$ 0.6191005412 .7637641913 .28073659 $-1.6934169014 .7295791613 .68512571$ $-2.8457690714 .7083846714 .67927807$ $-2.6644769813 .3476330215 .34421512$ $-2.5082246613 .2657271912 .33511339$ 
$-0.7381896914 .9057720314 .18529475$ $-1.8249308615 .4736811912 .89796500$ $-2.7743004815 .5242140115 .39703868$ $-3.8030777214 .7568274614 .15761132$ $-1.9183577713 .4138393516 .14795135$ $-3.6066532012 .9672227415 .74278952$ $-0.3538752012 .6461547711 .24016429$ 0.7979913412 .2229579010 .47939363 1.0383859110 .7444070910 .32928122 1.6959969710 .363076469 .37494025 $-1.2316014912 .7465664610 .73813633$ 0.7651640212 .661390519 .48116592 1.6780775312 .6439520210 .98875037 0.534182049 .9120798411 .26009942 0.818880828 .4906546511 .19874516 0.260296817 .746771309 .93849912 0.986072047 .018773079 .29150361 0.306582647 .7967708412 .46917109 1.143008188 .1595482913 .67245055 2.104714349 .1358578113 .77237732 1.019074017 .4765845314 .87781711 1.910152528 .0607758015 .71172997 2.577216969 .0528124915 .08510125 $-0.0358097210 .2781068412 .01598674$ 1.911317798 .3666246511 .18354796 $-0.745187798 .0841080912 .63804972$ 0.332066556 .7105346312 .31141174 2.473090599 .8526885613 .05622033 2.075085557 .7796916216 .73988688 3.294553419 .6314441815 .50180859 $-1.032370727 .932448939 .66324430$ $-1.637981177 .318069708 .48730777$ $-0.927640237 .780125627 .21206491$ $-0.684277657 .039859256 .26931151$ $-3.136187907 .688374018 .42083923$ $-1.590034898 .5406484810 .26263412$ $-1.528693306 .228127958 .56170041$ $-3.379445258 .092097087 .45096084$ $-3.357207638 .442518899 .16977813$ $-3.758788146 .819162368 .60920517$ $-0.614660739 .075138067 .19611884$ 0.095471109 .654056576 .07181337 1.480503139 .066410455 .84609090 2.006531389 .063854474 .75088101 0.2698390811 .149658396 .27269242 $-0.888419719 .684378927 .96345412$ $-0.495451259 .483807525 .16611673$ 2.095771828 .574424136 .93089271 3.387081017 .959286056 .78233904 3.337085606 .431981716 .78484830 4.354605275 .776076296 .95472981 4.396517738 .399482927 .86082573 4.995235599 .766722697 .50085086 3.792145338 .453537649 .10053066 1.695802088 .619979397 .85758226 3.783624618 .310578685 .81786420 5.175249567 .633730707 .93433571 3.138868149 .176309289 .10216128 5.2272648010 .312076038 .41501107 4.3004499710 .353737656 .90694562 5.919133929 .642659576 .93951862 2.118859705 .882691876 .56175374 2.016448414 .478877566 .34650553 1.367280233 .628511837 .43466375 1.319687402 .408005007 .32159751

\section{QM/SE R1a (B3LYP/6-31G(d):xTB) mechanical embedding $\omega_{\alpha}=\mathbf{1 . 0}$}

C $\quad 7.559806163 .2282860412 .94852878$

C $\quad 7.126399314 .1357852011 .78603690$

O $\quad 7.994187954 .5164713310 .99665585$

$\mathrm{N} \quad 5.807085994 .4666331611 .73248955$

C $\quad 4.698041743 .9175879012 .56337979$

C $\quad 3.984828892 .7936210311 .77711503$

O $\quad 3.863241852 .8585978710 .54879737$

C $\quad 3.795040725 .1624421512 .74815156$

C $\quad 3.894946065 .8279276111 .35169587$

C $\quad 5.383448535 .6503873710 .96083385$

$\mathrm{H} \quad 5.042406363 .5498166913 .53207479$

$\mathrm{H} \quad 2.776509604 .8706170212 .99918257$

$\mathrm{H} \quad 4.196541655 .8070027513 .53899239$

$\mathrm{H} \quad 3.252147775 .3219580410 .63277715$

$\mathrm{H} \quad 3.622892056 .8836864111 .39017506$

$\mathrm{H} \quad 5.507223925 .489186939 .88687980$

$\mathrm{H} \quad 5.977234476 .5220287311 .25517922$

$\mathrm{N} \quad 3.479826271 .7407595012 .46166730$

C $\quad 2.872070250 .6312135611 .75613957$

$\mathrm{H} \quad 3.658834061 .5679884113 .44464536$

C $\quad-1.175962056 .7038442321 .94368457$

C $\quad-1.945229746 .3845493420 .68512363$

O $\quad-3.049025936 .8739325620 .45926553$

$\mathrm{N} \quad-1.370476055 .4511301419 .91328345$

C $\quad-1.997682735 .1347458618 .60925437$

C $\quad-3.475467784 .6910189018 .77405985$

O $\quad-3.893167414 .2099385419 .84114307$

C $\quad-1.206626543 .9926278517 .96459796$ 
C $\quad 0.263210664 .2599713517 .91866218$

C $\quad 1.269588553 .7644440318 .75673144$

$\mathrm{N} \quad 0.836918664 .9761897216 .93448990$

C $\quad 2.134350404 .9462621917 .08208272$

$\mathrm{N} \quad 2.442942194 .2198943818 .19116306$

$\mathrm{H} \quad-0.525598764 .9841160420 .20852738$

$\mathrm{H} \quad-1.980559206 .0281605517 .98981335$

$\mathrm{H} \quad-1.393276573 .0595650218 .51749162$

$\mathrm{H} \quad-1.597808323 .8429390716 .95056453$

$\mathrm{H} \quad 1.209700533 .1574751419 .64985551$

$\mathrm{H} \quad 2.876406615 .4146951116 .45317216$

$\mathrm{H} \quad 3.397536164 .0538730818 .52748572$

$\mathrm{N} \quad-4.260371134 .8185503417 .65720493$

C $\quad-5.653475834 .3328195417 .65633535$

C $\quad-5.749260393 .2425996716 .57007957$

O $\quad-4.871597353 .1235500015 .72384273$

C $\quad-6.705902755 .4420999517 .34460168$

O $\quad-6.450799765 .9849557916 .06457822$

$\mathrm{H} \quad-3.854445705 .1213193016 .77186905$

$\mathrm{H} \quad-5.832648383 .8986615618 .63898882$

$\mathrm{H} \quad-7.706291594 .9990658317 .36637039$

$\mathrm{H} \quad-6.644897786 .2180544418 .11000184$

$\mathrm{H} \quad-7.300505065 .9319622815 .57695812$

$\mathrm{N} \quad-6.828972372 .4127631616 .66710453$

C $\quad-7.146275141 .4345957115 .62191988$

$\mathrm{H} \quad-7.449760512 .4414218317 .47583692$

C $\quad-5.943951043 .4957944610 .38241350$

C $\quad-5.728873674 .0169459211 .82517733$

$\mathrm{O} \quad-6.579258193 .9029009312 .69601672$

$\mathrm{N} \quad-4.531742554 .6215351612 .02204627$

C $\quad-4.268202445 .3929302213 .24197354$

C $\quad-4.573947426 .8773886112 .97526043$

O $\quad-4.216341847 .4495502211 .91656475$

C $\quad-2.750739145 .2127653313 .63860399$

S $\quad-2.475193356 .2193893715 .13214989$

$\mathrm{H} \quad-3.882250634 .6970399111 .24852795$

$\mathrm{H} \quad-4.914811185 .0263247614 .03024458$

$\mathrm{H} \quad-2.553944604 .1715876613 .88387679$

$\mathrm{H} \quad-2.123095975 .5337679912 .80705691$

$\mathrm{N} \quad-5.256332487 .5275280213 .94737197$

C $\quad-5.682325948 .9086997613 .78482253$

C $\quad-4.660149489 .9384828514 .36944955$

O $\quad-4.8229870511 .1316283514 .25182474$

$\mathrm{H} \quad-5.595265127 .0296647514 .76988595$

H $\quad-5.774037559 .1100756912 .71397329$

$\mathrm{N} \quad-3.541828929 .4457923414 .93958453$

C $\quad-2.5782906210 .4618583615 .41848342$

C $\quad-2.0710799411 .2007939314 .20042301$

O $\quad-1.6442030610 .5464431713 .23486780$

C $\quad-1.430138869 .8163447016 .15393292$

C $\quad-1.970068069 .1751385617 .42034546$

C $\quad-0.3184688410 .8843700716 .46597428$

C $\quad-0.856839078 .5951612018 .30877440$

$\mathrm{H} \quad-3.367719708 .4517827315 .03423918$

$\mathrm{H} \quad-3.1393652111 .1702524616 .02498831$

$\mathrm{H} \quad-1.008430599 .0300246615 .51087226$

$\mathrm{H} \quad-2.529831029 .9250970118 .00089570$

$\mathrm{H} \quad-2.666676058 .3716506017 .15472804$

$\mathrm{H} \quad 0.0586400011 .3459031115 .55082280$

$\mathrm{H} \quad 0.5210284810 .4159454816 .98516344$

$\mathrm{H} \quad-0.7272889111 .6690741317 .11361063$

$\mathrm{H} \quad-0.300487247 .8240011317 .77045951$

$\mathrm{H} \quad-1.289862668 .1550442619 .19941385$

$\mathrm{H} \quad-0.153290999 .3749642518 .61171610$

C $\quad 0.735850708 .4680464511 .24130028$
C $\quad 0.222177017 .7864606512 .50978761$

C $\quad 1.103723528 .1571598713 .67969728$

C $\quad 2.079721919 .1236597113 .74224402$

$\mathrm{N} \quad 1.008138057 .4872333414 .89376349$

C $\quad 1.927281318 .0683410715 .69782249$

$\mathrm{N} \quad 2.587040459 .0485205715 .04229911$

$\mathrm{H} \quad-0.818531028 .0936479012 .69516030$

$\mathrm{H} \quad 0.224286886 .7065574212 .34488730$

$\mathrm{H} \quad 2.435815739 .8291672713 .01073671$

$\mathrm{H} \quad 2.114113177 .7908498516 .72357946$

H $\quad 3.320519309 .6202973615 .43694204$

C $\quad 0.165973473 .499738279 .51098301$

C $\quad 0.222117874 .1942830910 .90450743$

C $\quad-0.016408093 .1436530111 .96971516$

S $\quad 0.496642403 .8167583313 .61226143$

C $\quad 0.084286392 .3594888014 .59577268$

$\mathrm{H} \quad 1.210035444 .6499473911 .01507642$

$\mathrm{H} \quad-0.521820404 .9910762710 .96075295$

$\mathrm{H} \quad-1.065201612 .8346865811 .95300492$

$\mathrm{H} \quad 0.554672822 .2337000711 .77731550$

$\mathrm{H} \quad-0.934681712 .0255300214 .39170361$

$\mathrm{H} \quad 0.781184451 .5449734014 .39250190$

$\mathrm{H} \quad 0.167416492 .6336561515 .64468314$

$\mathrm{Cu} \quad-0.233694465 .9914792915 .34497135$

\section{QM/SE R2a (B3LYP/6-31G(d):xTB) mechanical embedding $\omega_{\alpha}=\mathbf{1 . 0}$}

N $\quad 0.25272291-1.9747917117 .89218912$ C $\quad 0.88263274-0.8445192517 .26432926$

C $\quad 2.44563664-0.8896841017 .61429639$

O $\quad 2.79881372-0.8110237518 .78962803$

C $\quad 0.342512530 .4919427017 .77626552$

$\mathrm{H} \quad-0.03864366-1.9249959318 .86045077$

H $\quad 0.73487323-0.9265156616 .18332675$

$\mathrm{H} \quad-0.391313600 .3172542418 .56543497$

$\mathrm{H} \quad-0.149010691 .0371492216 .97357306$

$\mathrm{H} \quad 1.129617811 .1123499918 .20030626$

$\mathrm{N} \quad 3.19773581-0.9620966316 .54659388$

C $\quad 4.67889690-0.9439429016 .55551058$

C $\quad 5.24443196-1.9156640717 .64099257$

O $\quad 5.60163521-1.5285827418 .74319590$

C $\quad 5.226563230 .5100115316 .79729344$

C $\quad 6.737462370 .5768203816 .39212615$

C $\quad 4.438778391 .5691648516 .02497158$

$\mathrm{H} \quad 2.79763253-0.9942695315 .61750531$

$\mathrm{H} \quad 5.00077327-1.2706348815 .56324338$

$\mathrm{H} \quad 5.160917940 .7078189017 .87185384$

$\mathrm{H} \quad 7.366001260 .7133019317 .27252508$

$\mathrm{H} \quad 7.05056567-0.3510185815 .90762732$

$\mathrm{H} \quad 6.921985831 .3955384615 .70153667$

$\mathrm{H} \quad 3.556845651 .9041340716 .57560109$

$\mathrm{H} \quad 4.110158001 .1319563415 .08240861$

$\mathrm{H} \quad 5.064476262 .4358669715 .80902813$

$\mathrm{N} \quad 8.459160272 .1721827112 .55423073$

C $\quad 7.570142833 .1799053113 .05332520$

C $\quad 7.128974924 .0662465011 .85068144$

O $\quad 7.982960394 .4578234111 .04721671$

C $\quad 8.255333824 .0777580014 .10036166$

C $\quad 7.406188345 .1736092414 .70383055$

C $\quad 6.658403114 .9400284915 .84646956$

C $\quad 7.320696876 .4400700714 .11249285$

C $\quad 5.857061315 .9381747316 .38889134$

C $\quad 6.533696087 .4281899514 .64932348$

C $\quad 5.797445037 .1892385215 .79250748$ 


\begin{tabular}{|c|c|}
\hline $\mathrm{H}$ & 429822.4627875412 \\
\hline $\mathrm{H}$ & 6.711582712 .6515008513 .46976801 \\
\hline $\mathrm{H}$ & 8.609744983 .4120142614 .90108450 \\
\hline $\mathrm{H}$ & 9.112380944 .5465838913 .59945457 \\
\hline $\mathrm{H}$ & 6.696363373 .9697088816 .32877094 \\
\hline $\mathrm{H}$ & 7.901244486 .6450908613 .22286347 \\
\hline $\mathrm{H}$ & 5.288503205 .7297647517 .28577629 \\
\hline $\mathrm{H}$ & 6.497334868 .3959339314 .16667299 \\
\hline $\mathrm{H}$ & 5.165997427 .9534886216 .22637205 \\
\hline $\mathrm{N}$ & 5.819555064 .4190458211 .76790013 \\
\hline $\mathrm{C}$ & 4.682959603 .9088330512 .57783043 \\
\hline $\mathrm{C}$ & 3.982177802 .8008967311 .77055728 \\
\hline $\mathrm{O}$ & 3.864191452 .8694696110 .54377665 \\
\hline $\mathrm{C}$ & 3.793958185 .1743341212 .72139127 \\
\hline $\mathrm{C}$ & 3.951905345 .8343571811 .33376917 \\
\hline $\mathrm{C}$ & 5.449116585 .6071432010 .96597700 \\
\hline $\mathrm{H}$ & 4.999253443 .5373617213 .55353763 \\
\hline $\mathrm{H}$ & 2.764765284 .9143525812 .96528907 \\
\hline $\mathrm{H}$ & 4.199579705 .8057136913 .52084526 \\
\hline $\mathrm{H}$ & 3.311792835 .3457013010 .59818950 \\
\hline $\mathrm{H}$ & 3.709061966 .8978771811 .34435023 \\
\hline $\mathrm{H}$ & 5.591641695 .436197929 .89844354 \\
\hline $\mathrm{H}$ & 6.070396666 .4560124211 .26728211 \\
\hline $\mathrm{N}$ & 3.492312821 .7364959712 .44290055 \\
\hline $\mathrm{C}$ & 2.933964600 .6257636111 .71093462 \\
\hline $\mathrm{C}$ & $2.10894241-0.2241257912 .67922209$ \\
\hline $\mathrm{O}$ & $2.32815407-0.1735997113 .89131540$ \\
\hline $\mathrm{C}$ & $3.96219037-0.3645247211 .03286834$ \\
\hline $\mathrm{O}$ & $4.76914892-1.0531097611 .97716762$ \\
\hline $\mathrm{H}$ & 3.613619661 .6067326813 .44243505 \\
\hline $\mathrm{H}$ & 2.303597781 .0440994210 .91768739 \\
\hline $\mathrm{H}$ & $3.38848068-1.1191159610 .48222980$ \\
\hline $\mathrm{H}$ & 4.569488200 .2127809210 .32266558 \\
\hline $\mathrm{H}$ & $5.49653897-0.4685180312 .27947421$ \\
\hline $\mathrm{N}$ & $1.18421019-0.9813472212 .10504766$ \\
\hline $\mathrm{C}$ & $0.46692466-2.0146531412 .83398980$ \\
\hline $\mathrm{C}$ & $1.01337829-3.42630001 \quad 12.51000439$ \\
\hline $\mathrm{O}$ & $1.41016847-3.7157292411 .37401156$ \\
\hline $\mathrm{C}$ & $-0.98752383-2.0170780812 .34632138$ \\
\hline $\mathrm{C}$ & $-1.82197506-0.7957523212 .69213277$ \\
\hline $\mathrm{C}$ & $-2.25924831-0.5927274414 .00752987$ \\
\hline $\mathrm{C}$ & -2.218121590 .1110658211 .70211557 \\
\hline $\mathrm{C}$ & -3.077796770 .4762793014 .31330757 \\
\hline $\mathrm{C}$ & -3.024219251 .1814604512 .04085110 \\
\hline $\mathrm{C}$ & -3.463942091 .3720822113 .34434473 \\
\hline $\mathrm{H}$ & $1.03204722-1.0076774011 .09694961$ \\
\hline $\mathrm{H}$ & $0.59660443-1.8296012513 .90639683$ \\
\hline $\mathrm{H}$ & $-0.92551806-2.0837751811 .25258938$ \\
\hline $\mathrm{H}$ & $-1.47550386-2.9217758512 .73003949$ \\
\hline $\mathrm{H}$ & $-1.95509309-1.3007174714 .77021435$ \\
\hline $\mathrm{H}$ & $-1.88077031-0.0202912410 .68214080$ \\
\hline $\mathrm{H}$ & -3.413047110 .6435477315 .32935453 \\
\hline $\mathrm{H}$ & $-3.33824671 \quad 1.8791470111 .27559300$ \\
\hline $\mathrm{H}$ & -4.100380672 .2040027513 .60918027 \\
\hline $\mathrm{N}$ & $-6.90789783-4.8830301220 .31493078$ \\
\hline $\mathrm{C}$ & $-5.91840854-3.9424089519 .91034719$ \\
\hline $\mathrm{C}$ & $-5.00897164-3.5889041021 .12277862$ \\
\hline $\mathrm{O}$ & $-5.53577257-3.2451687622 .16250143$ \\
\hline $\mathrm{C}$ & $-6.58621597-2.6228356219 .47311046$ \\
\hline $\mathrm{C}$ & $-5.55565214-1.5847632619 .14748539$ \\
\hline $\mathrm{C}$ & $-4.59614276-1.8280761118 .19676823$ \\
\hline $\mathrm{C}$ & $-5.56051778-0.3726181919 .80179881$ \\
\hline $\mathrm{C}$ & $-3.66599919-0.8497518917 .89709138$ \\
\hline $\mathrm{C}$ & -4.637199180 .6126806519 .52929453 \\
\hline $\mathrm{C}$ & -3.675254370 .3523557618 .55141205 \\
\hline
\end{tabular}

$\mathrm{H}$

$\mathrm{H}$

$\mathrm{H}$

$\mathrm{H}$

$\mathrm{H}$

$\mathrm{H}$

$\mathrm{H}$

$\mathrm{H}$

$\mathrm{H}$

$\mathrm{N}$

C

C

$\mathrm{O}$

C

C

$\mathrm{N}$

$\mathrm{O}$

$\mathrm{H}$

$\mathrm{H}$

$\mathrm{H}$

$\mathrm{H}$

$\mathrm{H}$

$\mathrm{H}$

$\mathrm{N}$

C

C

$\mathrm{O}$

C

C

$\mathrm{N}$

O

$\mathrm{H}$

$\mathrm{H}$

$\mathrm{H}$

$\mathrm{H}$

$\mathrm{H}$

$\mathrm{H}$

$\mathrm{N}$

C

C

O

C

C

C

C

C

C

C

$\mathrm{H}$

$\mathrm{H}$

$\mathrm{H}$

$\mathrm{H}$

$\mathrm{H}$

$\mathrm{H}$

$\mathrm{H}$

$\mathrm{H}$

$\mathrm{H}$

$\mathrm{N}$

C

C

O

$\mathrm{H}$

$\mathrm{H}$

$\mathrm{H}$

$\mathrm{N}$
$-7.55287806-4.6175052221 .05729791$ $-5.31869012-4.3907954319 .11130904$ $-7.19522986-2.8265885818 .58106545$ $-7.24525266-2.2707739920 .27168822$ $-4.58086105-2.7725737017 .66948578$ $-6.32050826-0.2005344020 .55304716$ $-2.90667605-1.0530096917 .15145263$ $-4.639482931 .5616758120 .04313841$ $-2.942122851 .1127029218 .31128818$ $-1.51243172-1.4960748022 .35279525$ $-0.54509511-0.4700719921 .99577481$ $0.77422291-0.7391428522 .77217334$ $0.81035902-0.4858682523 .96088330$ $-0.998598850 .9238929922 .45942201$ 0.059166181 .9498669922 .13110451 0.015955843 .0897467922 .83335605 0.944560851 .7041677121 .33022803 $-2.01814659-1.4038255623 .22865659$ $-0.35401294-0.5336097120 .92519468$ $-1.901754941 .1969616021 .89305560$ $-1.257118490 .9293409423 .51754657$ $-0.704406153 .2375706023 .52353015$ 0.711289523 .8110134022 .68880535 3.755980550 .5356554321 .89115960 4.700753831 .5542943721 .52397103 4.785392392 .6797570022 .56101161 3.909266723 .4654532622 .80959318 4.302644312 .1279889720 .14524536 5.349877072 .9976418519 .53366695 6.634436962 .6222804819 .64467946 5.026960784 .0068260518 .93285300 2.798423940 .6970601821 .59250552 5.689721281 .0846861021 .46964785 4.089637971 .2763913519 .48318167 3.377485112 .6883058320 .27664176 6.902517581 .7558521620 .08049520 7.367296753 .1722950519 .21842191 3.651019226 .9228755122 .51436930 2.574680377 .3754206721 .63147451 1.251056966 .6811428121 .97519755 1.263247815 .5196304522 .34686387 2.849022306 .9752809120 .16633850 4.144714777 .5847187019 .77679977 5.336385606 .9441178020 .12644425 4.154078048 .8176474919 .14706195 6.530217197 .5592364319 .84481044 5.363401929 .4257949018 .86700444 6.540771958 .7880735919 .22013658 3.683227895 .9296631022 .70295065 2.499936578 .4597191521 .76935728 2.870963725 .8807139320 .11561864 2.044059417 .3450753319 .51824965 5.315821345 .9801698220 .61163056 3.224624659 .3048134518 .87767909 7.469191107 .0974844220 .10646055 5.4054448510 .3870978318 .37377877 7.487291209 .2684087619 .00080083 0.116074187 .3653065321 .79244900 $-1.157452426 .7287301922 .07450356$ $-1.917415866 .3953415920 .75952256$ $-3.013842956 .8868844220 .49384423$ 0.105412108 .2994601521 .41763207 $-0.988888045 .8044296122 .62904410$ $-1.799869107 .3868569622 .66530962$ $-1.364335155 .4621899419 .96039205$ 


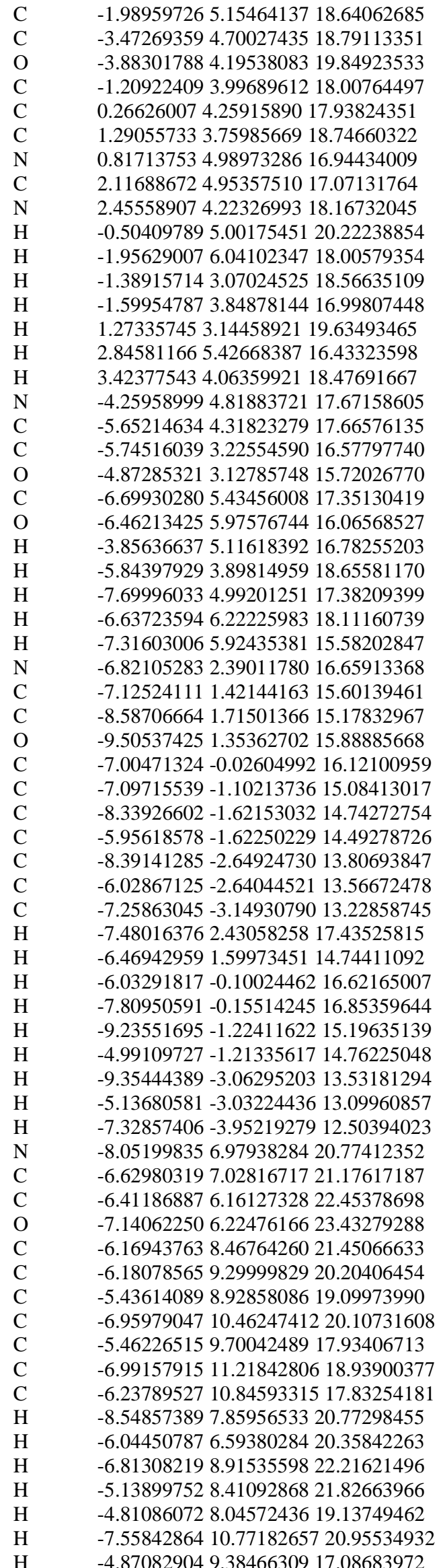

H

$\mathrm{H}$

$\mathrm{N}$

C

C

O

C

O

$\mathrm{H}$

$\mathrm{H}$

$\mathrm{H}$

$\mathrm{H}$

$\mathrm{H}$

$\mathrm{N}$

C

C

O

C

C

C

$\mathrm{H}$

$\mathrm{H}$

$\mathrm{H}$

$\mathrm{H}$

$\mathrm{H}$

$\mathrm{H}$

$\mathrm{H}$

$\mathrm{H}$

$\mathrm{H}$

$\mathrm{N}$

C

C

O

C

S

$\mathrm{H}$

$\mathrm{H}$

$\mathrm{H}$

$\mathrm{H}$

$\mathrm{N}$

C

C

O

C

C

C

$\mathrm{N}$

O

$\mathrm{H}$

$\mathrm{H}$

$\mathrm{H}$

$\mathrm{H}$

$\mathrm{H}$

$\mathrm{H}$

$\mathrm{H}$

$\mathrm{N}$

C

C

C

C

C

C

$\mathrm{H}$
$-7.5950662712 .1141013018 .89573195$ $-6.2527715111 .4278882016 .92207228$ $-5.350798295 .3605248922 .39181200$ $-4.989846954 .4374014323 .47416529$ $-4.096810175 .1396121824 .49873822$ $-3.496844136 .1555234724 .24081747$ $-4.201472793 .2705109322 .89870311$ $-2.985418753 .6717547122 .32368665$ $-4.734179595 .3942258221 .59216963$ $-5.915197644 .1150359823 .96437982$ $-3.972231952 .5518717123 .69586633$ $-4.829264072 .7698569522 .14561794$ $-3.172807933 .9780450321 .41482829$ $-6.819058732 .3952397710 .38355235$ $-5.929987023 .5248214810 .32961312$ $-5.703630684 .0362978811 .78466546$ $-6.547253783 .8951995712 .66750959$ $-6.440134274 .675172709 .44819112$ $-7.030162844 .180065438 .09906596$ $-7.497742355 .44642071 \quad 10.20710824$ $-7.655950192 .5005087610 .95810595$ $-4.973578173 .137206189 .95294703$ $-5.579658435 .321598169 .21936090$ $-8.025581394 .580368817 .93312488$ $-6.394018364 .497710107 .27010101$ $-7.099867333 .094898248 .06560933$ $-8.113440894 .7765802410 .80636807$ $-8.153356655 .963327359 .50730527$ $-7.047748916 .1894637710 .86991841$ $-4.502647974 .6210426512 .01013503$ $-4.279209245 .3924844713 .23578609$ $-4.580630216 .8770431212 .98414719$ $-4.218527327 .4557709411 .93372929$ $-2.768110665 .2299594313 .62990010$ $-2.469403056 .2176452515 .13223415$ $-3.866857054 .7624679711 .23306461$ $-4.902478984 .9751999614 .02411431$ $-2.558568504 .1780427713 .83201389$ $-2.142767975 .5848580912 .80534161$ $-5.253723767 .5218834813 .96140758$ $-5.662904728 .9049118413 .80208060$ $-4.648037099 .9351806014 .37307808$ $-4.8185795011 .1273637314 .25932030$ $-7.013349389 .1590187314 .48897553$ $-8.151011228 .4870780213 .71439617$ $-9.492871249 .1731380314 .05377469$ $-10.262510578 .5261438714 .94224999$ $-9.8017964710 .2273383313 .54924531$ $-5.561967987 .0261900214 .79733666$ $-5.753178449 .1089505012 .72938390$ $-6.991074058 .7740277415 .51384154$ $-7.1783059110 .2396687414 .53025391$ $-8.002202458 .6094299412 .63668344$ $-8.195796287 .4204455613 .95220466$ $-11.136569488 .9636911315 .18076286$ $-9.983474407 .6397329515 .34020729$ $-3.525912859 .4331954414 .93226693$ $-2.5822585210 .4455078515 .44207353$ $-2.0822914011 .1909223814 .24001214$ $-1.6639692610 .5688519813 .24859439$ $-1.419016489 .8089839216 .16667299$ $-1.981529189 .1568032717 .42911152$ $-0.3176521710 .8862252416 .47543186$ $-0.869965478 .5845315318 .32870878$ $-3.327097598 .4396881515 .01140278$ 


\begin{tabular}{|c|c|}
\hline $\mathrm{H}$ & -3.1368970811 .1177486316 .09394388 \\
\hline $\mathrm{H}$ & -0.982421189 .0416348915 .51685631 \\
\hline $\mathrm{H}$ & -2.555840589 .8920514318 .00720654 \\
\hline $\mathrm{H}$ & -2.664004068 .3479035417 .15278826 \\
\hline $\mathrm{H}$ & 0.0620729211 .3570697615 .56317600 \\
\hline $\mathrm{H}$ & 0.5340449310 .4401897416 .99455992 \\
\hline $\mathrm{H}$ & -0.7212448711 .6674040417 .13217492 \\
\hline $\mathrm{H}$ & -0.290720737 .8185759717 .79742974 \\
\hline $\mathrm{H}$ & -1.305582468 .1295449219 .21578364 \\
\hline $\mathrm{H}$ & -0.171074739 .3632139018 .65186221 \\
\hline $\mathrm{N}$ & -2.1709255612 .5060980814 .2719707 \\
\hline $\mathrm{C}$ & -1.7417580413 .3176613213 .09107492 \\
\hline $\mathrm{C}$ & -0.3588886012 .8924425512 .56552969 \\
\hline $\mathrm{O}$ & 0.6221982012 .7512997313 .28648145 \\
\hline $\mathrm{C}$ & -1.6925657614 .7290728013 .69371675 \\
\hline $\mathrm{C}$ & -2.8405555714 .7028028914 .6923413 \\
\hline $\mathrm{C}$ & -2.6703070413 .3296030315 .3369289 \\
\hline $\mathrm{H}$ & -2.4970786813 .2757487112 .30303401 \\
\hline $\mathrm{H}$ & -0.7356503114 .8901740614 .20271139 \\
\hline $\mathrm{H}$ & -1.8177095915 .4882827312 .92003221 \\
\hline $\mathrm{H}$ & -2.7719151015 .5104695415 .42307196 \\
\hline $\mathrm{H}$ & -3.7975616814 .7703245414 .16760652 \\
\hline $\mathrm{H}$ & -1.9237288013 .3859787616 .14538457 \\
\hline $\mathrm{H}$ & -3.6169774612 .9511867715 .73106495 \\
\hline $\mathrm{N}$ & -0.3596967612 .6475021011 .24475569 \\
\hline $\mathrm{C}$ & 0.7843275512 .2173070410 .47610462 \\
\hline $\mathrm{C}$ & 1.0293126510 .7402721210 .33094945 \\
\hline $\mathrm{O}$ & 1.6956547610 .351866169 .38299517 \\
\hline $\mathrm{H}$ & -1.2208508312 .7634612610 .71786862 \\
\hline $\mathrm{H}$ & 0.7448802112 .642597619 .47003001 \\
\hline $\mathrm{H}$ & 1.6750680312 .6191647010 .98767615 \\
\hline $\mathrm{N}$ & 0.522091319 .9116424711 .26170675 \\
\hline $\mathrm{C}$ & 0.824464498 .4987168611 .20253223 \\
\hline $\mathrm{C}$ & 0.253053467 .7 \\
\hline $\mathrm{O}$ & 0.980514017 .0 \\
\hline $\mathrm{C}$ & $0.304025087 .79555763 \quad 12.46208624$ \\
\hline $\mathrm{C}$ & 1.142949818 .1593560913 .66440874 \\
\hline $\mathrm{C}$ & 2.109481199 .1298035913 .77555616 \\
\hline $\mathrm{N}$ & 1.011671017 .4725212714 .87383605 \\
\hline $\mathrm{C}$ & 1.894488988 .0580742415 .71343326 \\
\hline $\mathrm{N}$ & 2.567614519 .0454454715 .09284181 \\
\hline $\mathrm{H}$ & -0.0558154610 .2647828812 .0180368 \\
\hline $\mathrm{H}$ & 1.914363468 .3662717111 .13395983 \\
\hline $\mathrm{H}$ & -0.744785898 .0816278212 .63399123 \\
\hline $\mathrm{H}$ & 0.338108156 .7120109312 .29757660 \\
\hline $\mathrm{H}$ & 2.501942299 .8558212713 .08294231 \\
\hline $\mathrm{H}$ & $2.056877357 .78497151 \quad 16.74338389$ \\
\hline $\mathrm{H}$ & 3.281532899 .6270721115 .50966259 \\
\hline $\mathrm{N}$ & -1.037965037 .940470409 .66540343 \\
\hline $\mathrm{C}$ & -1.618398397 .339432848 .47099210 \\
\hline $\mathrm{C}$ & -0.902663567 .831306867 .20323877 \\
\hline $\mathrm{O}$ & -0.689571617 .058040296 .26580474 \\
\hline $\mathrm{C}$ & -3.126195377 .693555288 .39886095 \\
\hline $\mathrm{H}$ & -1.629317418 .5330998110 .25099488 \\
\hline $\mathrm{H}$ & -1.491430406 .247566208 .52667029 \\
\hline $\mathrm{H}$ & -3.374771658 .109218767 .42790902 \\
\hline $\mathrm{H}$ & -3.366257558 .433405529 .15791257 \\
\hline $\mathrm{H}$ & -3.740314046 .812071658 .56907518 \\
\hline $\mathrm{N}$ & 2.109393068 .530045986 .94474006 \\
\hline $\mathrm{C}$ & 3.408722017 .940116496 .76830535 \\
\hline $\mathrm{C}$ & 3.360108836 .408326106 .78728560 \\
\hline $\mathrm{O}$ & 4.376966025 .761990586 .98279099 \\
\hline $\mathrm{C}$ & 4.424168298 .423131137 .81893754 \\
\hline $\mathrm{C}$ & 4.980252999 .806625387 .43699219 \\
\hline $\mathrm{O}$ & 3.845426438 .467980009 .07249044 \\
\hline
\end{tabular}

$\mathrm{H} \quad 1.734227468 .576976467 .88137778$

$\mathrm{H} \quad 3.773082038 .279925885 .78620465$

$\mathrm{H} \quad 5.221420877 .673017837 .88466525$

$\mathrm{H} \quad 3.176898199 .175552299 .08166226$

$\mathrm{H} \quad 5.2289687510 .361205188 .34104095$

$\mathrm{H} \quad 4.2608461510 .379744256 .85382210$

$\mathrm{H} \quad 5.896227599 .697198616 .85470127$

$\mathrm{N} \quad 0.777530604 .241604588 .47210867$

C $\quad 0.160482703 .436211409 .50164163$

C $\quad-1.348948043 .175362679 .19421104$

O $\quad-2.238683813 .846919729 .69170440$

C $\quad 0.227915454 .1408817010 .89699194$

C $\quad-0.003400993 .1080358311 .97488777$

S $\quad 0.492663303 .8154441113 .60503925$

C $\quad 0.070232712 .3767568614 .60545517$

$\mathrm{H} \quad 0.827748815 .251551278 .58237376$

$\mathrm{H} \quad 0.672927042 .469868399 .54732468$

$\mathrm{H} \quad 1.223620394 .5931710410 .98688114$

$\mathrm{H} \quad-0.530703214 .9313324910 .93983194$

$\mathrm{H} \quad-1.056153152 .8095461912 .00977651$

$\mathrm{H} \quad 0.587818322 .2030356811 .80987615$

$\mathrm{H} \quad-0.959875302 .0579541814 .41921635$

$\mathrm{H} \quad 0.754231931 .5481627314 .40046316$

$\mathrm{H} \quad 0.171434542 .6624647615 .65300330$

$\mathrm{Cu} \quad-0.234306625 .9911040015 .34520580$

O $\quad-1.9463315111 .164735618 .88883144$

$\mathrm{H} \quad-2.5743991311 .644748318 .33693443$

$\mathrm{H} \quad-2.4353025710 .822985549 .64968891$

O $\quad-2.948299329 .7692417011 .07179424$

$\mathrm{H} \quad-2.5294922610 .1690551011 .85364689$

$\mathrm{H} \quad-3.488885259 .0433716011 .43137516$

O $\quad 2.3184814711 .7396534718 .63290822$

$\mathrm{H} \quad 1.9529539212 .5852464418 .36720239$

$\mathrm{H} \quad 2.9530856511 .9331737219 .32618000$ 
B. Cartesian coordinates of all optimized regions for MYST histone acetyltransferase KAT6A/B in complex with WM-1119

\section{C QM/MM R1b (B3LYP/6-31G(d):Amber)} mechanical embedding $\omega_{\alpha}=1.667$ including $B$ factors

C

$\quad 21.41952270-8.01360077-4.34741474$

$\mathrm{N} \quad 23.52996977-8.66526497-4.77018317$

C $\quad 23.90058430-7.38390579-5.36880287$

C $\quad 23.06594641-7.23776731-6.66608127$

O $\quad 23.13422709-8.10399219-7.53808421$

C $\quad 25.39047247-7.37436441-5.83033538$

S $\quad 25.81305002-5.71741486-6.50323110$

$\mathrm{H} \quad 24.14347636-9.46732353-4.90655694$

$\mathrm{H} \quad 23.71433881-6.57919738-4.65694980$

$\mathrm{H} \quad 25.58275533-8.18348918-6.53751208$

$\mathrm{H} \quad 25.98596062-7.56832590-4.92767633$

$\mathrm{N} \quad 22.36986906-6.08586207-6.79301186$

C $\quad 21.45646356-5.93158302-7.92156576$

$\mathrm{H} \quad 22.15885922-5.52481623-5.97314360$

C $\quad 24.31464426-4.85997693-10.21425324$

C $\quad 25.25336528-5.90171697-10.73698499$

O $\quad 25.25373829-6.08482209-11.95030613$

$\mathrm{N} \quad 26.00908195-6.59718043-9.82452832$

C $\quad 26.84244165-7.67002402-10.30689395$

C $\quad 26.23521058-9.07205678-10.12487182$

O $\quad 26.77423595-10.09028589-10.57260122$

C $\quad 28.24684731-7.59498332-9.72360581$

S $\quad 28.23655950-8.13574302-7.88059322$

$\mathrm{H} \quad 25.99026924-6.32500688-8.83902927$

H $\quad 26.90939709-7.53808048-11.39042380$

$\mathrm{H} \quad 28.63143703-6.57859683-9.82395200$

$\mathrm{H} \quad 28.89435646-8.28298561-10.27102847$

$\mathrm{N} \quad 25.07706370-9.11595236-9.41687339$

C $\quad 24.41120315-10.40462451-9.18060668$

H $\quad 24.60144550-8.30389824-9.04658929$

C $\quad 29.87618767-4.491399042 .80734460$

C $\quad 30.08809079-4.513042511 .25997262$

O $\quad 30.68177758-3.651896880 .65538702$

$\mathrm{N} \quad 29.50878361-5.570566620 .64034629$

C $\quad 29.56468735-5.63294728-0.82963224$

C $\quad 29.06034466-4.33597995-1.43967688$

O $\quad 29.79331547-3.73646848-2.21400992$

C $\quad 28.84073746-6.85845777-1.37065211$

C $\quad 28.94490551-6.94732431-2.85614620$

$\mathrm{H} \quad 29.27409099-7.75211230-0.91376376$

$\mathrm{H} \quad 28.96143463-6.247354631 .16406704$

$\mathrm{H} \quad 30.62154031-5.65715345-1.11010517$

$\mathrm{H} \quad 27.78226465-6.81356902-1.10188711$

C $\quad 28.13636501-6.45547340-3.86218308$

$\mathrm{H} \quad 30.87958019-7.83143239-3.02821072$

$\mathrm{H} \quad 27.22424926-5.88777328-3.77790857$

C $\quad 29.81360910-7.47099245-4.82354680$

$\mathrm{N} \quad 30.00621728-7.59005678-3.49266547$

$\mathrm{N} \quad 28.65468046-6.87895189-5.03036549$

$\mathrm{H} \quad 30.47767511-7.84755967-5.58167924$

$\mathrm{N} \quad 27.83963988-3.88198010-1.05152279$

C $\quad 27.28004636-2.64030923-1.54927257$

$\mathrm{H} \quad 27.30420088-4.36721450-0.33308501$

C $\quad 32.66577007-1.85125542-2.42098860$

C $\quad 32.41206854-1.96660021-3.93852705$
2C QM/MM R1b (B3LYP/6-31G(d): Amber) mechanical embedding $\omega_{\alpha}=1.667$ including $\mathbf{B}$ factors

C $\quad 21.94065383-10.35660226-4.21796255$

C $\quad 22.25676507-8.89284205-4.41784677$

O $\quad 21.41823759-8.01610196-4.34941772$

$\mathrm{N} \quad 23.52907014-8.66573171-4.76820555$

$33.16291283-1.50130576-4.79179252$ (1) $.87574310-1.72773794-6.40635342$ $30.13257839-0.83792937-6.06463419$ $29.67282759-3.82665333-5.59210692$ $29.19530776-4.40310987-7.28267219$ $30.76041128-3.03843701-3.47109740$ $31.78348960-3.67165832-6.01940026$ $29.81214063-4.67407763-4.91775721$ $31.60211060-1.61416253-7.51677801$ $31.62350322-0.46811741-8.37660949$ $32.39354180-2.18616113-7.66946925$ $7.68880347-15.97673983-16.35325433$ $7.13257647-16.36225543-17.19389092$ $8.07429751-16.79164897-15.30030228$ $7.86120392-17.85116936-15.32508937$ $.75144409-16.18649120-14.24483267$ $9.12710908-16.74867484-13.41609152$ .97153808 -14.81993887-14.27158004 $8.49562773-13.99473752-15.23625285$ $7.91284040-14.62527482-16.26793244$ $7.39481130-13.77933435-17.35077773$ $7.52223061-14.20235981-18.69358602$ $7.00241290-13.37208453-19.69119257$ $6.41159701-12.14712662-19.40456826$ $6.01918558-11.54881197-20.19725982$ $7.07150359-13.77176911-20.99464842$ $8.05665654-15.12083328-18.92672037$ $6.71249257-12.31380973-15.98116706$ $6.26512854-11.77640741-18.04021655$ $5.46015755-10.60241534-17.66963750$ $5.69970977-9.85429347-16.72119726$ $4.37078163-10.33592303-18.51532000$ $3.97189627-11.04969422-19.13329522$ $3.37743513-9.17809524-17.19840049$ $3.59506388-7.95862163-19.05943988$ $4.97173062-7.56611062-19.42231035$ $2.81214898-6.88746735-18.42152319$ $2.76969810-8.39279158-20.68427117$ $1.62025016-7.67156497-21.07773761$ $1.13308883-6.98731600-20.39278126$ $0.10994536-7.47349478-22.61951817$ $1.61488253-8.88265105-23.19477280$ $1.16039281-9.12052380-24.15482562$ $2.78757877-9.53083345-22.84397326$ $3.27404571-10.22876551-23.52268338$ $3.35393759-9.28994509-21.58860604$ $4.52475627-9.96797300-21.28809894$ $28.08282134-6.23748601-6.90668687$ 

$2.76424923-8.38377803-20.69464331$ $1.61229815-7.67344220-21.08687147$ $1.12110719-6.99617528-20.40242164$ $1.04370381-7.93997574-22.34978018$ H $\quad 0.09654219-7.49677972-22.61980748$ C $\quad 1.60863587-8.89277528-23.19608508$ $\mathrm{H} \quad 1.14799064-9.13746602-24.15024171$ C $\quad 2.78322836-9.53444350-22.84544575$

$\mathrm{H} \quad 3.26757874-10.23574609-23.51968610$

C $\quad 3.34877393-9.28422144-21.59426917$

F $\quad 4.51959999-9.96019119-21.29063924$

$\mathrm{Zn} \quad 28.08268870-6.23738754-6.90737083$

\section{C QM/MM R2b (B3LYP/6-31G(d):Amber) mechanical embedding $\omega_{\alpha}=1.667$ including $B$ factors}

C $\quad 21.94167691-10.35519930-4.21859036$

C $\quad 22.25983100-8.89540303-4.43123794$

O $\quad 21.41754299-8.02191682-4.37097362$

$\mathrm{N} \quad 23.53323118-8.67210414-4.78342570$

C $\quad 23.90738725-7.38675209-5.37377066$

C $\quad 23.07318658-7.23956770-6.67545341$

O $\quad 23.14234653-8.10904867-7.54407867$

C $\quad 25.39078096-7.37739787-5.84412114$

S $\quad 25.81668630-5.70905714-6.49731704$

$\mathrm{H} \quad 24.13609555-9.47571038-4.94817929$

$\mathrm{H} \quad 23.71837296-6.58409871-4.65941943$

$\mathrm{H} \quad 25.54853764-8.15773425-6.59118636$

$\mathrm{H} \quad 26.01121939-7.61715515-4.97067929$

$\mathrm{N} \quad 22.36952236-6.09351754-6.79912171$

C $\quad 21.45700819-5.93566445-7.92165106$

$\mathrm{H} \quad 22.15498981-5.53901509-5.97510605$ 


\begin{tabular}{|c|c|c|c|}
\hline $\mathrm{N}$ & $23.47295214-5.18831370-9.11997185$ & $\mathrm{H}$ & $32.37203537-11.89093638-3.58095052$ \\
\hline $\mathrm{C}$ & $24.31494423-4.77397945-10.22385210$ & $\mathrm{H}$ & $32.00373095-10.74137713-2.43182943$ \\
\hline $\mathrm{C}$ & $25.23766801-5.84776435-10.74079815$ & $\mathrm{H}$ & $29.77903556-10.07076149-4.07125991$ \\
\hline $\mathrm{O}$ & $25.23010262-6.04725132-11.95162548$ & $\mathrm{H}$ & $30.74787482-11.19174090-5.06954607$ \\
\hline $\mathrm{C}$ & $25.10745261-3.55311358-9.87564921$ & $\mathrm{H}$ & $26.58567746-9.91304229-7.82256431$ \\
\hline $\mathrm{C}$ & $24.25522616-2.40544710-9.40905184$ & $\mathrm{H}$ & $27.72035127-9.98501005-5.53420485$ \\
\hline $\mathrm{C}$ & $23.30436766-1.81904462-10.27291759$ & $\mathrm{H}$ & $28.82212948-10.74652393-6.70838195$ \\
\hline $\mathrm{C}$ & $24.38825346-1.93902790-8.06705125$ & $\mathrm{C}$ & $29.87591104-4.491379922 .80645452$ \\
\hline $\mathrm{C}$ & $22.52831043-0.72919118-9.82347592$ & $\mathrm{C}$ & $30.09430088-4.505174961 .26450543$ \\
\hline $\mathrm{C}$ & $23.56639692-0.89706190-7.60836707$ & $\mathrm{O}$ & $30.68568111-3.637515730 .66727599$ \\
\hline $\mathrm{C}$ & $22.65765774-0.27486757-8.48379664$ & $\mathrm{~N}$ & $29.51771259-5.559368020 .63847406$ \\
\hline $\mathrm{O}$ & $22.032364830 .85331702-8.08206294$ & $\mathrm{C}$ & $29.56115057-5.62401838-0.82920478$ \\
\hline $\mathrm{H}$ & $23.66560853-4.49818318-11.05706113$ & $\mathrm{C}$ & $29.05755224-4.32280789-1.43089064$ \\
\hline $\mathrm{H}$ & $21.81632371-0.27242553-10.48629283$ & $\mathrm{O}$ & $29.78401633-3.70678765-2.20050699$ \\
\hline $\mathrm{H}$ & $23.67403557-0.52663365-6.60489994$ & $\mathrm{C}$ & $28.82795731-6.85294982-1.36262333$ \\
\hline $\mathrm{H}$ & $23.87319790-5.24341541-8.18897781$ & $\mathrm{C}$ & $28.93187171-6.94209242-2.84898909$ \\
\hline $\mathrm{H}$ & $25.11916058-2.37814950-7.40209276$ & $\mathrm{H}$ & $29.25653154-7.74803324-0.89715749$ \\
\hline $\mathrm{H}$ & $23.17857980-2.19625695-11.27892994$ & $\mathrm{H}$ & $28.98954343-6.248580731 .16605231$ \\
\hline $\mathrm{H}$ & $25.65907881-3.22328152-10.75523230$ & $\mathrm{H}$ & $30.61338104-5.65889562-1.12812328$ \\
\hline $\mathrm{H}$ & $25.83085006-3.79806332-9.09734872$ & $\mathrm{H}$ & $27.76936894-6.81392760-1.08501938$ \\
\hline $\mathrm{H}$ & $22.526436251 .63816308-8.33886430$ & $\mathrm{C}$ & $28.12949099-6.45457775-3.86532085$ \\
\hline $\mathrm{N}$ & $25.99450566-6.55216065-9.83465233$ & $\mathrm{H}$ & $30.88117821-7.79116171-3.00685464$ \\
\hline $\mathrm{C}$ & $26.81962035-7.63550409-10.31095909$ & $\mathrm{H}$ & $27.20523403-5.89884654-3.81411296$ \\
\hline $\mathrm{C}$ & $26.21133639-9.03977492-10.12496609$ & $\mathrm{C}$ & $29.82032608-7.46628331-4.81586358$ \\
\hline $\mathrm{O}$ & $26.75966075-10.05222167-10.57679630$ & $\mathrm{~N}$ & $30.00501753-7.57584394-3.48054208$ \\
\hline $\mathrm{C}$ & $28.23262999-7.58046108-9.73532001$ & $\mathrm{~N}$ & $28.65928423-6.88102902-5.02816286$ \\
\hline S & $28.23999870-8.13303510-7.87983661$ & $\mathrm{H}$ & $30.47872588-7.84665326-5.58176318$ \\
\hline $\mathrm{H}$ & $25.98630736-6.27759949-8.84953693$ & $\mathrm{~N}$ & $27.83446428-3.87961894-1.04431477$ \\
\hline $\mathrm{H}$ & $26.88303809-7.50509826-11.39600067$ & $\mathrm{C}$ & $27.27814911-2.64032047-1.54809659$ \\
\hline $\mathrm{H}$ & $28.64388502-6.57299399-9.83121709$ & $\mathrm{H}$ & $27.27585561-4.39147351-0.36314407$ \\
\hline $\mathrm{H}$ & $28.86708716-8.28032370-10.28329555$ & $\mathrm{C}$ & $32.66516908-1.85323837-2.42115263$ \\
\hline $\mathrm{N}$ & $25.04614188-9.09283702-9.42384802$ & $\mathrm{C}$ & $32.41187520-1.98047778-3.93686602$ \\
\hline $\mathrm{C}$ & $24.40259677-10.39348430-9.16297329$ & $\mathrm{O}$ & $33.13227588-1.47002388-4.79194406$ \\
\hline $\mathrm{C}$ & $25.21052292-11.35454305-8.28077132$ & $\mathrm{~N}$ & $31.34192100-2.72781943-4.23159740$ \\
\hline $\mathrm{O}$ & $24.84435477-12.53761347-8.14496630$ & $\mathrm{C}$ & $31.00859041-3.06238024-5.62625189$ \\
\hline $\mathrm{C}$ & $23.88522427-11.10256706-10.45031938$ & $\mathrm{C}$ & $30.89610268-1.73800516-6.40168923$ \\
\hline $\mathrm{C}$ & $22.95421353-10.20038672-11.28376053$ & $\mathrm{O}$ & $30.12594540-0.86740831-6.06856208$ \\
\hline $\mathrm{C}$ & $22.45363208-10.98794729-12.50950635$ & $\mathrm{C}$ & $29.69451717-3.84222943-5.58974697$ \\
\hline $\mathrm{C}$ & $21.70932963-9.78233642-10.49320917$ & $\mathrm{~S}$ & $29.17250026-4.38931062-7.26601997$ \\
\hline $\mathrm{H}$ & $22.01292473-9.14799586-9.66570020$ & $\mathrm{H}$ & $30.81973626-3.15174869-3.47981665$ \\
\hline $\mathrm{H}$ & $21.04370659-9.21411387-11.13215838$ & $\mathrm{H}$ & $31.79931952-3.69170579-6.04942574$ \\
\hline $\mathrm{H}$ & $21.21092708-10.66888513-10.10433870$ & $\mathrm{H}$ & $28.92915314-3.20281691-5.14123039$ \\
\hline $\mathrm{H}$ & $24.59914343-8.28641210-9.00769030$ & $\mathrm{H}$ & $29.85139478-4.69955203-4.92970530$ \\
\hline $\mathrm{H}$ & $21.92145914-11.88084689-12.18698796$ & $\mathrm{~N}$ & $31.63520892-1.62354122-7.51256366$ \\
\hline $\mathrm{H}$ & $21.80452234-10.35600270-13.10969400$ & $\mathrm{C}$ & $31.62546221-0.47299261-8.37018127$ \\
\hline $\mathrm{H}$ & $23.31289804-11.28394191-13.11221979$ & $\mathrm{H}$ & $32.54229877-2.05883599-7.52611168$ \\
\hline $\mathrm{H}$ & $23.50712541-10.19830272-8.58159029$ & $\mathrm{~N}$ & $30.16842381-1.81793007-9.80390227$ \\
\hline $\mathrm{H}$ & $23.48409423-9.31172527-11.61757725$ & $\mathrm{C}$ & $29.14130685-2.02548930-10.83883268$ \\
\hline $\mathrm{H}$ & $23.33095928-11.99659740-10.16566169$ & $\mathrm{C}$ & $27.90716792-1.20088291-10.52995894$ \\
\hline $\mathrm{H}$ & $24.72204825-11.40676200-11.07441439$ & $\mathrm{O}$ & $27.41568401-1.15007622-9.40609524$ \\
\hline $\mathrm{N}$ & $26.26908910-10.85666386-7.61463046$ & $\mathrm{C}$ & $28.76861840-3.49995628-10.96251026$ \\
\hline $\mathrm{C}$ & $26.90504805-11.69600630-6.57679792$ & $\mathrm{C}$ & $29.83226543-4.33582038-11.57107039$ \\
\hline $\mathrm{C}$ & $25.85594978-12.11796167-5.50344122$ & $\mathrm{C}$ & $31.17756958-4.21618616-11.41577218$ \\
\hline $\mathrm{O}$ & $25.16897443-11.26446731-4.97219345$ & $\mathrm{C}$ & $29.64037152-5.46024549-12.46812868$ \\
\hline $\mathrm{C}$ & $28.06428617-10.93433503-5.94686879$ & $\mathrm{~N}$ & $31.82517754-5.17465626-12.16832480$ \\
\hline $\mathrm{C}$ & $28.70066730-11.78429741-4.84461713$ & $\mathrm{C}$ & $30.92457301-6.02976994-12.75378842$ \\
\hline $\mathrm{C}$ & $29.97916986-11.11124278-4.29981334$ & $\mathrm{C}$ & $28.51108254-6.12188185-12.97934915$ \\
\hline $\mathrm{C}$ & $30.38716836-11.87329261-3.01750948$ & $\mathrm{C}$ & $31.07222564-7.25411604-13.41913732$ \\
\hline $\mathrm{N}$ & $31.81021727-11.68756219-2.75207249$ & $\mathrm{C}$ & $28.65070819-7.37527476-13.59147455$ \\
\hline $\mathrm{H}$ & $27.26809120-12.59865248-7.07298761$ & $\mathrm{C}$ & $29.91163977-7.94486591-13.81893124$ \\
\hline $\mathrm{H}$ & $29.78959834-11.49489041-2.18208617$ & $\mathrm{H}$ & $29.98928255-8.88663862-14.32958055$ \\
\hline $\mathrm{H}$ & $30.17391241-12.93708253-3.15192152$ & $\mathrm{H}$ & $29.54152319-1.69425944-11.79852887$ \\
\hline $\mathrm{H}$ & $27.98990970-11.87124980-4.02242807$ & $\mathrm{H}$ & $32.82552581-5.27823564-12.24457642$ \\
\hline $\mathrm{H}$ & $28.93777124-12.78066354-5.21844794$ & $\mathrm{H}$ & $27.53151129-5.70081208-12.81889368$ \\
\hline $\mathrm{H}$ & $32.13012202-12.32119699-2.03297719$ & $\mathrm{H}$ & $27.76462175-7.89592385-13.88603350$ \\
\hline
\end{tabular}




\begin{tabular}{|c|c|c|c|}
\hline $\mathrm{H}$ & $32.05860331-7.63940501-13.60544812$ & $\mathrm{C}$ & $11.94982306-12.72577914-16.24955118$ \\
\hline $\mathrm{H}$ & $31.68783988-3.47828376-10.81663038$ & $\mathrm{O}$ & $12.89327467-12.00806455-16.54929253$ \\
\hline $\mathrm{H}$ & $30.30127201-2.54542761-9.11149029$ & $\mathrm{C}$ & $13.02885142-14.92484166-15.78346764$ \\
\hline $\mathrm{H}$ & $27.89425383-3.58615323-11.60784316$ & $\mathrm{C}$ & $12.69407588-15.14802586-14.23356365$ \\
\hline $\mathrm{H}$ & $28.50912889-3.91213042-9.99044738$ & $\mathrm{~S}$ & $12.77746230-13.59122166-13.36685055$ \\
\hline $\mathrm{N}$ & $12.43760285-18.61015020-10.12989502$ & $\mathrm{C}$ & $14.53425330-13.20047999-13.56261389$ \\
\hline $\mathrm{C}$ & $11.14927026-18.22813795-10.71353477$ & $\mathrm{H}$ & $11.68409636-15.54302105-14.16687920$ \\
\hline $\mathrm{C}$ & $10.79162991-19.01224024-11.95305098$ & $\mathrm{H}$ & $14.87502686-12.57313173-12.74031668$ \\
\hline $\mathrm{O}$ & $9.71889136-18.79906286-12.50460078$ & $\mathrm{H}$ & $15.11193261-14.12476314-13.56622431$ \\
\hline $\mathrm{C}$ & $11.06263146-16.73014025-10.95660656$ & $\mathrm{H}$ & $14.70104717-12.68091186-14.50486294$ \\
\hline $\mathrm{C}$ & $11.55127050-15.94249233-9.76661837$ & $\mathrm{H}$ & $13.17461210-15.88441776-16.24025741$ \\
\hline $\mathrm{C}$ & $10.76483495-15.89671328-8.58369868$ & $\mathrm{H}$ & $13.39422568-15.86288622-13.80498848$ \\
\hline $\mathrm{C}$ & $12.80275542-15.29410649-9.82447964$ & $\mathrm{H}$ & $12.91032495-13.63394249-18.44346735$ \\
\hline $\mathrm{C}$ & $11.26022764-15.19965969-7.46010141$ & $\mathrm{H}$ & $11.00265396-14.61548780-16.34744829$ \\
\hline $\mathrm{C}$ & $13.28568704-14.61821779-8.68924300$ & $\mathrm{H}$ & $13.96688045-14.38496915-15.86624627$ \\
\hline $\mathrm{C}$ & $12.51105905-14.56104612-7.50733690$ & $\mathrm{~N}$ & $10.81451071-12.25332682-15.73042189$ \\
\hline $\mathrm{H}$ & $12.90737561-14.06024278-6.63857528$ & $\mathrm{C}$ & $10.67496373-10.89654774-15.17947324$ \\
\hline $\mathrm{H}$ & $10.36503637-18.47361073-10.00025635$ & $\mathrm{C}$ & $10.54752714-11.07510693-13.64238812$ \\
\hline $\mathrm{H}$ & $10.68000293-15.17812734-6.56666916$ & $\mathrm{O}$ & $9.84483591-11.95850338-13.14124406$ \\
\hline $\mathrm{H}$ & $14.26368857-14.16550755-8.73297548$ & $\mathrm{C}$ & $9.42522889-10.21454329-15.73368043$ \\
\hline $\mathrm{H}$ & $13.26870780-18.49973464-10.70161243$ & $\mathrm{C}$ & $9.40077770-10.13608941-17.29031189$ \\
\hline $\mathrm{H}$ & $13.40989868-15.36820623-10.71541791$ & $\mathrm{C}$ & $9.21996275-8.81369053-15.13746936$ \\
\hline $\mathrm{H}$ & $9.81449340-16.40736474-8.53363594$ & $\mathrm{C}$ & $10.49099844-9.19418511-17.79391327$ \\
\hline $\mathrm{H}$ & $10.02813304-16.44683676-11.14259482$ & $\mathrm{H}$ & $11.54829683-10.28505554-15.40394446$ \\
\hline $\mathrm{H}$ & $11.66536334-16.46702741-11.82397126$ & $\mathrm{H}$ & $8.33284063-8.34825114-15.55736266$ \\
\hline $\mathrm{N}$ & $11.59294182-19.98753691-12.34502887$ & $\mathrm{H}$ & $9.07779080-8.90819081-14.06466284$ \\
\hline $\mathrm{C}$ & $11.30761406-20.78002042-13.51949050$ & $\mathrm{H}$ & $8.56762816-10.81798604-15.44811519$ \\
\hline $\mathrm{C}$ & $11.28976328-22.23558713-13.16712658$ & $\mathrm{H}$ & $9.53867986-11.12567160-17.72060531$ \\
\hline $\mathrm{O}$ & $12.19757462-22.71958889-12.49733336$ & $\mathrm{H}$ & $8.43297510-9.74878868-17.59227769$ \\
\hline $\mathrm{C}$ & $12.31942718-20.46363607-14.63710330$ & $\mathrm{H}$ & $11.46398753-9.56104591-17.46969650$ \\
\hline $\mathrm{C}$ & $12.27719350-18.99574065-15.06684648$ & $\mathrm{H}$ & $10.05440475-12.89991298-15.57775674$ \\
\hline $\mathrm{C}$ & $13.37928605-18.84783014-16.11583750$ & $\mathrm{H}$ & $10.49245502-9.19062073-18.87679148$ \\
\hline $\mathrm{C}$ & $10.92687141-18.62484304-15.68496887$ & $\mathrm{H}$ & $10.09790275-8.19015292-15.29896264$ \\
\hline $\mathrm{H}$ & $10.14329772-18.73158918-14.94826305$ & $\mathrm{H}$ & $10.34535884-8.17860213-17.43977512$ \\
\hline $\mathrm{H}$ & $10.96771856-17.59822206-16.03369524$ & $\mathrm{~N}$ & $6.48946163-7.96393440-12.69525710$ \\
\hline $\mathrm{H}$ & $10.72213087-19.29578718-16.51907945$ & $\mathrm{C}$ & $5.61983303-8.75486234-13.50214578$ \\
\hline $\mathrm{H}$ & $12.43090521-20.19657384-11.82129357$ & $\mathrm{C}$ & $4.32108905-7.98651249-13.75924609$ \\
\hline $\mathrm{H}$ & $13.22737535-19.53968817-16.94561041$ & $\mathrm{O}$ & $3.92803732-7.12764423-12.94759979$ \\
\hline $\mathrm{H}$ & $13.38116945-17.85519001-16.52813870$ & $\mathrm{C}$ & $5.35520021-10.11617377-12.90155449$ \\
\hline $\mathrm{H}$ & $14.34975765-19.04003555-15.66146102$ & $\mathrm{C}$ & $6.64382927-10.96584692-12.90234847$ \\
\hline $\mathrm{H}$ & $10.31503296-20.55873915-13.90686380$ & $\mathrm{C}$ & $6.43304413-12.29845481-12.27232950$ \\
\hline $\mathrm{H}$ & $12.47398457-18.32542442-14.23657997$ & $\mathrm{H}$ & $6.94799585-11.14192502-13.93181001$ \\
\hline $\mathrm{H}$ & $12.09500642-21.09668332-15.49369613$ & $\mathrm{H}$ & $6.11417412-8.89610749-14.46276142$ \\
\hline $\mathrm{H}$ & $13.32038849-20.70657926-14.28089178$ & $\mathrm{H}$ & $7.44361547-10.45979811-12.38723336$ \\
\hline $\mathrm{N}$ & $12.74878125-16.68116726-20.49461818$ & $\mathrm{H}$ & $6.76281767-8.28279471-11.77415499$ \\
\hline $\mathrm{C}$ & $12.41693504-15.30708890-20.23390742$ & $\mathrm{H}$ & $4.59292947-10.62296566-13.49363895$ \\
\hline $\mathrm{C}$ & $11.78064841-15.23561386-18.81857674$ & $\mathrm{H}$ & $4.99193910-9.99984155-11.88037950$ \\
\hline $\mathrm{O}$ & $10.91918117-16.04870938-18.44593115$ & $\mathrm{H}$ & $8.35300132-12.84299419-12.48827391$ \\
\hline $\mathrm{C}$ & $11.51540431-14.74659171-21.34918098$ & $\mathrm{O}$ & $5.31880981-12.59848283-11.86014745$ \\
\hline $\mathrm{C}$ & $11.23799528-13.27841477-21.07502253$ & $\mathrm{~N}$ & $7.44852129-13.12178267-12.10930924$ \\
\hline $\mathrm{C}$ & $10.22653783-15.57579187-21.50212111$ & $\mathrm{H}$ & $7.27961726-14.03815405-11.72201863$ \\
\hline $\mathrm{C}$ & $10.56568888-12.54168062-22.21770287$ & $\mathrm{~N}$ & $3.72803815-8.28923128-14.95039830$ \\
\hline $\mathrm{H}$ & $13.33441080-14.72028368-20.21841173$ & $\mathrm{C}$ & $2.44823828-7.76727821-15.43244632$ \\
\hline $\mathrm{H}$ & $9.66072756-15.19676504-22.34874144$ & $\mathrm{C}$ & $2.47020732-6.24319468-15.46552634$ \\
\hline $\mathrm{H}$ & $10.47201518-16.61470050-21.70351003$ & $\mathrm{O}$ & $1.45262345-5.63261682-15.16927589$ \\
\hline $\mathrm{H}$ & $12.06804177-14.80623552-22.28496745$ & $\mathrm{C}$ & $1.31780991-8.29581954-14.50906386$ \\
\hline $\mathrm{H}$ & $10.58380338-13.19799200-20.20645303$ & $\mathrm{C}$ & $1.27836416-9.83530902-14.45683393$ \\
\hline $\mathrm{H}$ & $12.16862519-12.76622868-20.84514587$ & $\mathrm{C}$ & $0.09035997-10.24877315-13.60408940$ \\
\hline $\mathrm{H}$ & $9.59559826-12.98624206-22.43064184$ & $\mathrm{~N}$ & $0.06544808-11.69709897-13.41140368$ \\
\hline $\mathrm{H}$ & $12.10424591-17.39960557-20.20707426$ & $\mathrm{C}$ & $-0.67888644-12.56461166-14.08485472$ \\
\hline $\mathrm{H}$ & $10.42079858-11.50356899-21.93146913$ & $\mathrm{~N}$ & $-1.55023665-12.14006155-14.96754944$ \\
\hline $\mathrm{H}$ & $9.62415902-15.49007137-20.60329080$ & $\mathrm{~N}$ & $-0.53090297-13.86105132-13.88904236$ \\
\hline $\mathrm{H}$ & $11.19803335-12.59884819-23.10312497$ & $\mathrm{H}$ & $2.18617137-10.23722194-14.01095981$ \\
\hline $\mathrm{N}$ & $12.20516028-14.25251726-18.04075216$ & $\mathrm{H}$ & $2.20470124-8.11995210-16.43019184$ \\
\hline $\mathrm{C}$ & $11.97241278-14.17200609-16.57377804$ & $\mathrm{H}$ & $0.72561138-12.07124324-12.73168304$ \\
\hline
\end{tabular}




\begin{tabular}{|c|c|}
\hline $\mathrm{H}$ & $1.15686331-10.22779611-15.46442179$ \\
\hline $\mathrm{H}$ & $-1.08643410-14.54516098-14.37718656$ \\
\hline $\mathrm{H}$ & $0.08623161-14.19026682-13.16219720$ \\
\hline $\mathrm{H}$ & $4.29660204-8.90500984-15.52214055$ \\
\hline $\mathrm{H}$ & $-0.83673817-9.90688764-14.06619695$ \\
\hline $\mathrm{H}$ & $0.16541059-9.77218322-12.62519896$ \\
\hline $\mathrm{H}$ & $-2.10962813-12.79689372-15.47580922$ \\
\hline $\mathrm{H}$ & $-1.59978866-11.15446864-15.13851820$ \\
\hline $\mathrm{H}$ & $0.35977998-7.92567852-14.87904503$ \\
\hline $\mathrm{H}$ & $1.46044653-7.91295792-13.49637213$ \\
\hline $\mathrm{N}$ & $3.58981012-5.59961787-15.80912913$ \\
\hline $\mathrm{C}$ & $3.71055359-4.12378069-15.85744413$ \\
\hline $\mathrm{C}$ & $4.28070221-3.61789063-17.18992443$ \\
\hline $\mathrm{O}$ & $4.54503264-2.43409955-17.35710474$ \\
\hline $\mathrm{C}$ & $4.51461529-3.60907741-14.66410332$ \\
\hline $\mathrm{C}$ & $3.67996175-3.51913241-13.39626438$ \\
\hline $\mathrm{C}$ & $4.55988897-3.72093090-12.17200092$ \\
\hline $\mathrm{N}$ & $3.86586994-3.26616862-10.94699700$ \\
\hline $\mathrm{C}$ & $3.43079460-4.05344255-9.96389335$ \\
\hline $\mathrm{N}$ & $3.43931691-5.35689599-10.08700644$ \\
\hline $\mathrm{N}$ & $2.98764622-3.55278092-8.83879241$ \\
\hline $\mathrm{H}$ & $2.88495423-4.26135984-13.38451188$ \\
\hline $\mathrm{H}$ & $2.72343403-3.66746550-15.77191285$ \\
\hline $\mathrm{H}$ & $3.82562032-2.27238757-10.79341324$ \\
\hline $\mathrm{H}$ & $3.22840625-2.52422206-13.35230768$ \\
\hline $\mathrm{H}$ & $2.73629655-4.18532431-8.10434404$ \\
\hline $\mathrm{H}$ & $2.94706171-2.56027907-8.71566942$ \\
\hline $\mathrm{H}$ & $4.40459331-6.17721092-15.98393107$ \\
\hline $\mathrm{H}$ & $5.48098729-3.14522955-12.27919997$ \\
\hline $\mathrm{H}$ & $4.83638426-4.77408944-12.11834377$ \\
\hline $\mathrm{H}$ & $3.22790254-6.01491216-9.35914696$ \\
\hline $\mathrm{H}$ & $3.84835521-5.71563739-10.92950729$ \\
\hline $\mathrm{H}$ & $4.90782464-2.61279772-14.87929098$ \\
\hline $\mathrm{H}$ & $5.36182600-4.26591004-14.49531835$ \\
\hline $\mathrm{N}$ & $4.30064308-4.47962400-18.21177477$ \\
\hline $\mathrm{C}$ & $4.75877851-4.11038899-19.55840082$ \\
\hline $\mathrm{C}$ & $6.23992972-4.46370539-19.87435470$ \\
\hline $\mathrm{O}$ & $6.59957835-4.44196491-21.06429323$ \\
\hline $\mathrm{H}$ & $4.05582109-5.44034459-18.00079887$ \\
\hline $\mathrm{H}$ & $4.14867764-4.64381207-20.28386616$ \\
\hline $\mathrm{H}$ & $4.64343758-3.03695328-19.71663867$ \\
\hline $\mathrm{N}$ & $7.04829456-4.82520663-18.86341009$ \\
\hline $\mathrm{C}$ & $8.46012100-5.18551616-19.00922833$ \\
\hline $\mathrm{C}$ & $8.61199660-6.50291348-19.75154921$ \\
\hline $\mathrm{O}$ & $9.39848884-6.55780118-20.69264177$ \\
\hline $\mathrm{C}$ & $9.16741453-5.24769942-17.62140107$ \\
\hline $\mathrm{C}$ & $9.11580952-3.93439106-16.87067782$ \\
\hline $\mathrm{C}$ & $9.98808683-2.87187713-17.14705733$ \\
\hline $\mathrm{C}$ & $8.16517299-3.79567221-15.85132563$ \\
\hline $\mathrm{C}$ & $9.90935031-1.67387715-16.40720256$ \\
\hline $\mathrm{C}$ & $8.06375365-2.60461345-15.10271168$ \\
\hline $\mathrm{C}$ & $8.95865526-1.54987722-15.35507549$ \\
\hline $\mathrm{O}$ & $8.77407331-0.40369907-14.67274430$ \\
\hline $\mathrm{H}$ & $8.96140130-4.40827920-19.58949629$ \\
\hline $\mathrm{H}$ & $10.57716783-0.85396602-16.61972799$ \\
\hline $\mathrm{H}$ & $7.32312705-2.48681390-14.33657352$ \\
\hline $\mathrm{H}$ & $6.67343007-4.80481278-17.92661212$ \\
\hline $\mathrm{H}$ & $7.49248272-4.60448665-15.64933405$ \\
\hline $\mathrm{H}$ & $10.73737676-2.95297995-17.91659440$ \\
\hline $\mathrm{H}$ & $10.20577858-5.48679115-17.80234693$ \\
\hline $\mathrm{H}$ & $8.74006130-6.04067376-17.01073312$ \\
\hline $\mathrm{H}$ & $9.606949150 .14303920-14.75070205$ \\
\hline $\mathrm{N}$ & $7.83959594-7.54112482-19.36879074$ \\
\hline $\mathrm{C}$ & $7.78123756-8.82029137-20.07302001$ \\
\hline $\mathrm{C}$ & $7.38947946-8.60308259-21.58124964$ \\
\hline
\end{tabular}

$7.96263129-9.25462500-22.45643239$ $7.18107973-7.35675720-18.62480469$ $8.75305377-9.30909833-20.05882777$ $7.04613458-9.47052171-19.61079811$ $6.46475090-7.64297887-21.83711474$ $5.95481992-7.33045157-23.17246746$ $7.07449945-6.70957215-23.99514298$ $7.18544662-6.98349945-25.19327597$ $4.74944368-6.39054033-23.10161984$ $4.14782040-6.04821729-24.48997660$ $2.94491655-5.14513133-24.21910753$ $2.22735563-4.75093634-25.42941240$ $1.13200401-3.97974348-25.46486261$ $0.59407640-3.53510537-24.33638411$ $0.61029844-3.59568708-26.60352542$ $3.81128159-6.95105008-24.99924922$ $5.67341771-8.25345970-23.68515424$ $2.60498134-5.05606585-26.32760974$ $4.86666907-5.51403273-25.10670798$ $-0.23066343-3.05372333-26.66540647$ $0.96247770-3.99806752-27.46429726$ $6.05792193-7.20846372-21.01941849$ $3.28813400-4.25004820-23.70283900$ $2.24680254-5.68595394-23.58011870$ $-0.21490625-2.93744085-24.38721913$ $0.82531900-4.08097569-23.54001559$ $5.02227691-5.46465532-22.60847991$ $3.97954361-6.86593269-22.50746095$ $9.61936298-9.43389908-24.94587706$ $9.06755820-10.21476520-26.06942840$ $9.19593860-9.39456355-27.37046148$ $9.79515723-9.85452119-28.31719708$ $7.61321336-10.69854880-25.80379685$ $7.58364221-11.69376434-24.63670437$ $7.09985832-11.41863590-27.05777834$ $6.17715438-11.89193306-24.08004224$ $9.68840501-11.10393492-26.20018368$ $6.08350253-11.76716491-26.89642377$ $7.07914242-10.73765039-27.90452563$ $6.98665950-9.83387788-25.58389192$ $8.00979855-12.64393380-24.94877860$ $8.20257137-11.32429154-23.82498030$ $5.56092321-12.35378735-24.84941206$ $9.04860957-9.21542365-24.14190611$ $6.23127790-12.55968813-23.22714658$ $7.74149625-12.26406539-27.28938872$ $5.74709790-10.93477890-23.79411663$ $6.59458876-20.99416394-19.57814182$ $6.07973526-20.91129351-18.20217754$ $4.58264804-20.57014641-18.30864809$ $4.08684181-20.17330694-19.39312001$ $6.81295769-19.87080409-17.36917815$ $6.65471973-18.58733374-17.94208603$ $7.17155101-20.27342602-19.99373638$ $6.18649828-21.88301132-17.71649197$ $7.87308601-20.12472513-17.32392930$ $6.41106305-19.86735308-16.35675015$ $7.53735474-18.35475484-18.30140053$ $3.14368968-17.89155130-16.99693399$ $3.07655669-16.45537429-17.25528794$ $3.61589077-16.04056613-18.63488551$ $3.11334640-15.09615174-19.27350531$ $3.79650678-15.71804639-16.14358211$ $3.77683219-14.18657033-16.26484554$ $2.34979305-13.64939304-16.44622365$ 

$6.99756791-13.34662518-19.70505897$ $6.40399920-12.12154063-19.43320356$ $6.02688916-11.52841880-20.24320974$ $7.07778935-13.75426067-21.00247348$ 8.00287377 -15.13033845 -18.94081651 $6.80408760-12.54011535-17.04562442$ $6.74265955-12.27617676-15.99835540$ $6.25759165-11.75974875-18.05790110$ $5.45226808-10.59708269-17.67016401$ $5.67597494-9.86211510-16.71187415$ $4.38199608-10.33323708-18.53935227$ $4.00665522-11.07339288-19.14022392$ $3.42714061-9.38405177-18.22593209$ $3.25148941-9.28653940-17.22544401$ $3.59379036-7.96688514-19.04528500$ $4.96931746-7.56138834-19.40715402$ $2.80881680-6.89809568-18.41054810$ $2.78607286-8.38227776-20.67408130$ $1.64593317-7.64569283-21.06435983$ $1.17170620-6.95202690-20.37821386$ $1.07358633-7.88378621-22.33349814$ $0.15921788-7.38257203-22.63464236$ $1.63357214-8.83452357-23.19078126$ $1.18842291-9.04441091-24.16161542$ $2.79230375-9.50827149-22.83511712$ $3.27740724-10.19865055-23.52149482$ $3.36356095-9.27955902-21.57972048$ $4.53311517-9.95627548-21.29071444$ $28.08268935-6.23448893-6.91302500$ $-2.11527736-6.04377722-23.95473260$ $-2.83007724-5.48175674-23.60202835$ $-2.40989432-6.92424179-23.70380989$ $7.34983672-19.81026870-13.53927724$ $8.21324415-19.44094857-13.27909423$ $7.38033829-20.69780536-13.16480218$ $9.24741488-17.93730153-18.97676673$ $9.88421399-17.25349130-18.67822376$ $9.81854590-18.65377930-19.27087758$ $5.98289016-7.01589569-16.77139544$ $6.45295527-6.72469305-15.96720670$ $6.21441585-7.95223672-16.83545876$ $26.49495109-0.90541342-4.84861016$ $27.11735688-0.81702934-4.11379321$ $26.76825147-1.71331058-5.29255427$ $0.73178084-10.02638257-18.02563115$ $-0.04571784-10.48651076-18.41128892$ $1.46686462-10.39511780-18.52315865$ $4.78278329-18.68727467-14.47372451$ $5.70302548-18.92201538-14.25667490$ $4.28474777-19.33604181-13.96459517$ $-0.46055749-6.70292695-25.85970037$ $-0.90518386-7.26736228-26.50274164$ $-1.17817376-6.40045096-25.27247000$

\section{C QM /MM R2b (B3LYP/6-31G(d): Amber) mechanical embedding $\omega_{\alpha}=1.667$ including $\mathbf{B}$ factors \\ C $\quad 21.94167461-10.35518864-4.21854163$ \\ C $\quad 22.25979855-8.89534213-4.43112188$}




\begin{tabular}{|c|c|c|c|}
\hline $\mathrm{O}$ & $21.41762524-8.02167206-4.37087021$ & $\mathrm{C}$ & $26.90453359-11.69629907-6.57764479$ \\
\hline $\mathrm{N}$ & $23.53313716-8.67214123-4.78349300$ & $\mathrm{C}$ & $25.85599502-12.11798262-5.50352263$ \\
\hline $\mathrm{C}$ & $23.90761191-7.38685079-5.37390014$ & $\mathrm{O}$ & $25.16938133-11.26445725-4.97193183$ \\
\hline $\mathrm{C}$ & $23.07347719-7.23945520-6.67555613$ & $\mathrm{C}$ & $28.06479523-10.93515932-5.94893123$ \\
\hline $\mathrm{O}$ & $23.14247307-8.10889252-7.54421057$ & $\mathrm{C}$ & $28.69991447-11.78371164-4.84480523$ \\
\hline $\mathrm{C}$ & $25.39075096-7.37800903-5.84510323$ & $\mathrm{C}$ & $29.97900155-11.11095901-4.30085552$ \\
\hline S & $25.81634250-5.70941938-6.49719735$ & $\mathrm{C}$ & $30.38601522-11.87196352-3.01732702$ \\
\hline $\mathrm{H}$ & $24.13560222-9.47587346-4.94905230$ & $\mathrm{~N}$ & $31.80943206-11.68799161-2.75239055$ \\
\hline $\mathrm{H}$ & $23.71911531-6.58411783-4.65949683$ & $\mathrm{H}$ & $27.26636273-12.59913335-7.07466282$ \\
\hline $\mathrm{H}$ & $25.54739777-8.15729093-6.59356243$ & $\mathrm{H}$ & $29.78902079-11.49192496-2.18209270$ \\
\hline $\mathrm{H}$ & $26.01218508-7.61943843-4.97278277$ & $\mathrm{H}$ & $30.17120924-12.93576100-3.14994300$ \\
\hline $\mathrm{N}$ & $22.36953134-6.09350524-6.79919407$ & $\mathrm{H}$ & $27.98860054-11.86821380-4.02277955$ \\
\hline $\mathrm{C}$ & $21.45700457-5.93568542-7.92166495$ & $\mathrm{H}$ & $28.93585356-12.78134176-5.21630776$ \\
\hline $\mathrm{H}$ & $22.15485440-5.53928275-5.97506132$ & $\mathrm{H}$ & $32.12903807-12.32166336-2.03309018$ \\
\hline $\mathrm{N}$ & $23.47286122-5.18786322-9.12007771$ & $\mathrm{H}$ & $32.37089949-11.89212146-3.58134140$ \\
\hline $\mathrm{C}$ & $24.31490255-4.77367371-10.22401684$ & $\mathrm{H}$ & $32.00409280-10.74191815-2.43255894$ \\
\hline $\mathrm{C}$ & $25.23765296-5.84765340-10.74053746$ & $\mathrm{H}$ & $29.78006170-10.06979057-4.07402209$ \\
\hline $\mathrm{O}$ & $25.22963783-6.04775687-11.95134015$ & $\mathrm{H}$ & $30.74773924-11.19294924-5.07053332$ \\
\hline $\mathrm{C}$ & $25.10753181-3.55284733-9.87598697$ & $\mathrm{H}$ & $26.58463417-9.91293430-7.82279579$ \\
\hline $\mathrm{C}$ & $24.25522913-2.40559881-9.40916495$ & $\mathrm{H}$ & $27.72256342-9.98421651-5.53854322$ \\
\hline $\mathrm{C}$ & $23.30414667-1.81945285-10.27307548$ & $\mathrm{H}$ & $28.82302196-10.74984141-6.71092345$ \\
\hline $\mathrm{C}$ & $24.38856104-1.93928525-8.06703310$ & $\mathrm{C}$ & $29.87593044-4.491462012 .80642625$ \\
\hline $\mathrm{C}$ & $22.52810245-0.72950100-9.82359357$ & $\mathrm{C}$ & $30.09432805-4.505409191 .26441155$ \\
\hline $\mathrm{C}$ & $23.56639382-0.89753275-7.60811213$ & $\mathrm{O}$ & $30.68553445-3.637680360 .66704640$ \\
\hline $\mathrm{C}$ & $22.65755883-0.27529006-8.48371323$ & $\mathrm{~N}$ & $29.51768121-5.559637690 .63838927$ \\
\hline $\mathrm{O}$ & $22.032513070 .85315845-8.08212629$ & $\mathrm{C}$ & $29.56102198-5.62452724-0.82922201$ \\
\hline $\mathrm{H}$ & $23.66572914-4.49831546-11.05759752$ & $\mathrm{C}$ & $29.05745901-4.32328474-1.43117961$ \\
\hline $\mathrm{H}$ & $21.81593210-0.27263866-10.48653198$ & $\mathrm{O}$ & $29.78431096-3.70724927-2.20037479$ \\
\hline $\mathrm{H}$ & $23.67423916-0.52683191-6.60452639$ & $\mathrm{C}$ & $28.82794159-6.85377095-1.36192317$ \\
\hline $\mathrm{H}$ & $23.87353273-5.24331982-8.18934208$ & $\mathrm{C}$ & $28.93185286-6.94256681-2.84807001$ \\
\hline $\mathrm{H}$ & $25.12003914-2.37812978-7.40215528$ & $\mathrm{H}$ & $29.25636739-7.74893816-0.89613743$ \\
\hline $\mathrm{H}$ & $23.17804076-2.19690443-11.27916539$ & $\mathrm{H}$ & $28.98958820-6.249118501 .16565425$ \\
\hline $\mathrm{H}$ & $25.65902997-3.22267632-10.75560912$ & $\mathrm{H}$ & $30.61322881-5.65919115-1.12832024$ \\
\hline $\mathrm{H}$ & $25.83127923-3.79766451-9.09783436$ & $\mathrm{H}$ & $27.76923576-6.81519933-1.08427725$ \\
\hline $\mathrm{H}$ & $22.526345791 .63784222-8.33995841$ & $\mathrm{C}$ & $28.12909230-6.45445495-3.86378145$ \\
\hline $\mathrm{N}$ & $25.99460225-6.55159849-9.83428190$ & $\mathrm{H}$ & $30.88146549-7.79057592-3.00696302$ \\
\hline $\mathrm{C}$ & $26.81957378-7.63505743-10.31079108$ & $\mathrm{H}$ & $27.20458649-5.89913421-3.81153818$ \\
\hline $\mathrm{C}$ & $26.21146740-9.03961580-10.12492387$ & $\mathrm{C}$ & $29.81998632-7.46486160-4.81546637$ \\
\hline $\mathrm{O}$ & $26.75996093-10.05186997-10.57686873$ & $\mathrm{~N}$ & $30.00504613-7.57541846-3.48028204$ \\
\hline $\mathrm{C}$ & $28.23276709-7.58007493-9.73577618$ & $\mathrm{~N}$ & $28.65871356-6.87972095-5.02702422$ \\
\hline S & $28.23993316-8.13281325-7.88005566$ & $\mathrm{H}$ & $30.47824733-7.84427232-5.58221803$ \\
\hline $\mathrm{H}$ & $25.98669214-6.27665718-8.84917409$ & $\mathrm{~N}$ & $27.83439001-3.87987735-1.04465393$ \\
\hline $\mathrm{H}$ & $26.88202874-7.50442857-11.39586086$ & $\mathrm{C}$ & $27.27817555-2.64034426-1.54812135$ \\
\hline $\mathrm{H}$ & $28.64464991-6.57274851-9.83188914$ & $\mathrm{H}$ & $27.27582042-4.39166685-0.36339927$ \\
\hline $\mathrm{H}$ & $28.86723715-8.28004353-10.28380646$ & $\mathrm{C}$ & $32.66518545-1.85319854-2.42123075$ \\
\hline $\mathrm{N}$ & $25.04591736-9.09292289-9.42437086$ & $\mathrm{C}$ & $32.41194715-1.98039091-3.93710811$ \\
\hline $\mathrm{C}$ & $24.40236513-10.39355137-9.16352744$ & $\mathrm{O}$ & $33.13347586-1.47208912-4.79258683$ \\
\hline $\mathrm{C}$ & $25.20997565-11.35452825-8.28122924$ & $\mathrm{~N}$ & $31.34070699-2.72593388-4.23154195$ \\
\hline $\mathrm{O}$ & $24.84375330-12.53751515-8.14546345$ & $\mathrm{C}$ & $31.00778740-3.06160763-5.62590557$ \\
\hline $\mathrm{C}$ & $23.88531798-11.10266781-10.45092130$ & $\mathrm{C}$ & $30.89593171-1.73755174-6.40184296$ \\
\hline $\mathrm{C}$ & $22.95479465-10.19973797-11.28396151$ & $\mathrm{O}$ & $30.12626656-0.86652096-6.06867620$ \\
\hline $\mathrm{C}$ & $22.45404727-10.98760184-12.50935123$ & $\mathrm{C}$ & $29.69323188-3.84089908-5.58960419$ \\
\hline $\mathrm{C}$ & $21.71013185-9.78152584-10.49316571$ & $\mathrm{~S}$ & $29.17248053-4.38926318-7.26607062$ \\
\hline $\mathrm{H}$ & $22.01391066-9.14712523-9.66566132$ & $\mathrm{H}$ & $30.81594114-3.14623916-3.47961654$ \\
\hline $\mathrm{H}$ & $21.04425618-9.21332963-11.13193251$ & $\mathrm{H}$ & $31.79876854-3.69132098-6.04808561$ \\
\hline $\mathrm{H}$ & $21.21176736-10.66793708-10.10406742$ & $\mathrm{H}$ & $28.92766054-3.20056427-5.14259211$ \\
\hline $\mathrm{H}$ & $24.59934739-8.28691733-9.00705987$ & $\mathrm{H}$ & $29.84840146-4.69756749-4.92824478$ \\
\hline $\mathrm{H}$ & $21.92218919-11.88059530-12.18660827$ & $\mathrm{~N}$ & $31.63496024-1.62351408-7.51275180$ \\
\hline $\mathrm{H}$ & $21.80471836-10.35592828-13.10955242$ & $\mathrm{C}$ & $31.62546530-0.47297432-8.37024818$ \\
\hline $\mathrm{H}$ & $23.31321665-11.28369966-13.11217008$ & $\mathrm{H}$ & $32.54190367-2.05895353-7.52647254$ \\
\hline $\mathrm{H}$ & $23.50699350-10.19832389-8.58195704$ & $\mathrm{~N}$ & $30.16831183-1.81792488-9.80404713$ \\
\hline $\mathrm{H}$ & $23.48505255-9.31112737-11.61783914$ & $\mathrm{C}$ & $29.14114173-2.02548603-10.83891611$ \\
\hline $\mathrm{H}$ & $23.33089607-11.99678405-10.16653350$ & $\mathrm{C}$ & $27.90713544-1.20082787-10.52994116$ \\
\hline $\mathrm{H}$ & $24.72220257-11.40724636-11.07503342$ & $\mathrm{O}$ & $27.41553389-1.15002242-9.40611817$ \\
\hline $\mathrm{N}$ & $26.26831159-10.85662977-7.61499582$ & $\mathrm{C}$ & $28.76819059-3.49986472-10.96270250$ \\
\hline
\end{tabular}


C $\quad 29.83168977-4.33575877-11.57113548$

C $\quad 31.17697401-4.21672521-11.41527013$

C $\quad 29.63951361-5.46011134-12.46825865$

$\mathrm{N} \quad 31.82437254-5.17468427-12.16869298$

C $\quad 30.92363414-6.02977927-12.75408215$

C $\quad 28.51008182-6.12174524-12.97928003$

C $\quad 31.07117587-7.25422554-13.41940250$

C $\quad 28.64957251-7.37549743-13.59093326$

C $\quad 29.91046106-7.94533449-13.81857379$

$\mathrm{H} \quad 29.98798877-8.88735510-14.32916323$

$\mathrm{H} \quad 29.54131380-1.69415012-11.79866705$

$\mathrm{H} \quad 32.82466745-5.27858756-12.24526761$

$\mathrm{H} \quad 27.53042465-5.70022812-12.81910028$

$\mathrm{H} \quad 27.76319736-7.89602920-13.88539064$

$\mathrm{H} \quad 32.05780999-7.63904205-13.60617218$

$\mathrm{H} \quad 31.68743514-3.47932951-10.81539791$

$\mathrm{H} \quad 30.30081630-2.54544942-9.11165577$

$\mathrm{H} \quad 27.89391323-3.58591630-11.60836534$

$\mathrm{H} \quad 28.50808284-3.91224946-9.99087249$

$\mathrm{N} \quad 12.43764312-18.61065256-10.13011905$

C $\quad 11.14947367-18.22865420-10.71416098$

C $\quad 10.79198427-19.01281106-11.95363753$

O $\quad 9.71895060-18.79998187-12.50461755$

C $\quad 11.06302634-16.73049712-10.95721401$

C $\quad 11.55134249-15.94313698-9.76692825$

C $\quad 10.76433777-15.89752438-8.58425476$

C $\quad 12.80278305-15.29441617-9.82465063$

C $\quad 11.25933324-15.20040506-7.46032716$

C $\quad 13.28543872-14.61860738-8.68905187$

C $\quad 12.51033647-14.56168419-7.50712474$

$\mathrm{H} \quad 12.90625038-14.06059602-6.63809019$

$\mathrm{H} \quad 10.36490782-18.47425118-10.00115787$

$\mathrm{H} \quad 10.67835739-15.17866010-6.56712624$

H $\quad 14.26347098-14.16550941-8.73279113$

H $\quad 13.26868133-18.50073663-10.70200854$

$\mathrm{H} \quad 13.40984536-15.36806942-10.71598928$

$\mathrm{H} \quad 9.81367871-16.40822801-8.53458620$

$\mathrm{H} \quad 10.02861995-16.44679386-11.14349751$

$\mathrm{H} \quad 11.66607448-16.46699643-11.82442283$

$\mathrm{N} \quad 11.59350152-19.98769787-12.34607397$

C $\quad 11.30814908-20.78042423-13.52024088$

C $\quad 11.28980975-22.23579891-13.16699354$

O $\quad 12.19712261-22.71990577-12.49654476$

C $\quad 12.31991557-20.46456302-14.63824064$

C $\quad 12.27822347-18.99640320-15.06707414$

C $\quad 13.37980387-18.84839428-16.11659682$

C $\quad 10.92756469-18.62439823-15.68399410$

$\mathrm{H} \quad 10.14438227-18.73104961-14.94679873$

$\mathrm{H} \quad 10.96885240-17.59752309-16.03207092$

$\mathrm{H} \quad 10.72175104-19.29458310-16.51837995$

$\mathrm{H} \quad 12.43154140-20.19670946-11.82262901$

$\mathrm{H} \quad 13.22697154-19.53899910-16.94727701$

$\mathrm{H} \quad 13.38228570-17.85517534-16.52743610$

$\mathrm{H} \quad 14.35049521-19.04199261-15.66315950$

$\mathrm{H} \quad 10.31547950-20.55912632-13.90756958$

$\mathrm{H} \quad 12.47594665-18.32663032-14.23650503$

$\mathrm{H} \quad 12.09484503-21.09711352-15.49516828$

$\mathrm{H} \quad 13.32107603-20.70865504-14.28290723$

$\mathrm{N} \quad 12.74862333-16.68086813-20.49489799$

C $\quad 12.41669036-15.30668987-20.23405681$

C $\quad 11.78017822-15.23553155-18.81861937$

O $\quad 10.91919892-16.04915514-18.44570833$

C $\quad 11.51582119-14.74568945-21.34963256$

C $\quad 11.23833039-13.27776550-21.07497334$

C $\quad 10.22663524-15.57423629-21.50308171$
$\mathrm{C}$

$\mathrm{H}$

$\mathrm{H}$

$\mathrm{H}$

$\mathrm{H}$

$\mathrm{H}$

$\mathrm{H}$

$\mathrm{H}$

$\mathrm{H}$

$\mathrm{H}$

$\mathrm{H}$

$\mathrm{H}$

C

C

O

C

C

S

C

$\mathrm{H}$

$\mathrm{H}$

$\mathrm{H}$

$\mathrm{H}$

$\mathrm{H}$

$\mathrm{H}$

$\mathrm{H}$

$\mathrm{H}$

$\mathrm{H}$

$\mathrm{N}$

C

C

O

C

C

C

C

$\mathrm{H}$

$\mathrm{H}$

$\mathrm{H}$

$\mathrm{H}$

$\mathrm{H}$

$\mathrm{H}$

$\mathrm{H}$

$\mathrm{H}$

$\mathrm{H}$

$\mathrm{H}$

$\mathrm{H}$

$\mathrm{N}$

C

C

$\mathrm{O}$

C

C

$\mathrm{H}$

$\mathrm{H}$

$\mathrm{H}$

$\mathrm{H}$

$\mathrm{H}$

$\mathrm{H}$

$\mathrm{H}$

$\mathrm{O}$

$\mathrm{N}$

$\mathrm{H}$
$10.56518678-12.54131896-22.21738766$ $13.33430134-14.71992766-20.21828278$ $9.66079153-15.19487494-22.34973976$ $10.47157840-16.61323685-21.70485123$ $12.06886332-14.80504150-22.28541940$ $10.58455481-13.19771791-20.20605030$ $12.16897090-12.76526462-20.84528760$ $9.59500221-12.98584679-22.42986934$ $12.10391325-17.39899492-20.20703867$ $10.42004855-11.50326643-21.93103459$ $9.62421090-15.48861919-20.60433152$ $11.19689990-12.59812191-23.10323719$ $12.20440806-14.25226150-18.04089875$ $11.97249802-14.17221394-16.57359903$ $11.94979759-12.72610269-16.24920693$ $12.89324334-12.00837881-16.54898294$ $13.02964400-14.92462389-15.78403814$ $12.69459700-15.14833225-14.23410260$ $12.77729451-13.59151308-13.36704907$ $14.53424280-13.20030864-13.56199042$ $11.68461254-15.54354924-14.16807368$ $14.87506765-12.57337583-12.73924035$ $15.11194211-14.12456825-13.56598852$ $14.70107506-12.68047584-14.50408752$ $13.17630141-15.88383955-16.24158744$ $13.39457892-15.86325424-13.80505911$ $12.90932366-13.63358957-18.44380780$ $11.00302518-14.61616284-16.34655217$ $13.96728959-14.38396278-15.86659155$ $10.81444059-12.25364631-15.73026804$ $10.67487421-10.89665656-15.17961269$ $10.54753791-11.07520642-13.64238038$ $9.84497160-11.95880516-13.14123290$ $9.42504934-10.21478168-15.73380241$ $9.40097477-10.13605103-17.29038683$ $9.21912678-8.81400870-15.13751763$ $10.49069189-9.19294306-17.79307215$ $11.54801852-10.28497846-15.40465044$ $8.33148922-8.34907416-15.55711903$ $9.07716078-8.90863085-14.06469811$ $8.56754000-10.81875263-15.44846040$ $9.53983269-11.12554820-17.72089237$ $8.43280482-9.74968692-17.59248717$ $11.46384874-9.55924264-17.46871954$ $10.05407489-12.89985614-15.57778396$ $10.49267912-9.18852987-18.87595209$ $10.09641964-8.18960027-15.29901873$ $10.34439034-8.17757057-17.43860065$ $6.48957562-7.96398138-12.69518061$ $5.61996779-8.75522840-13.50191364$ $4.32117590-7.98687433-13.75919655$ $3.92818563-7.12803681-12.94753989$ $5.35537484-10.11630791-12.90081054$ $6.64391383-10.96635682-12.90260411$ $6.43317044-12.29871653-12.27210531$ $6.94712026-11.14300459-13.93228808$ $6.11435953-8.89680624-14.46251261$ $7.44479438-10.46056494-12.38875013$ $6.76258124-8.28227741-11.77374658$ $4.59214494-10.62302868-13.49185931$ $4.99287592-9.99975572-11.87934428$ $8.35298512-12.84326686-12.48831521$ $5.31895780-12.59855512-11.85974270$ $7.44858964-13.12218843-12.10923695$ $7.27963093-14.03868056-11.72224759$ 
$\mathrm{N} \quad 3.72822256-8.28941451-14.95046748$

C $\quad 2.44841168-7.76728091-15.43257526$

C $\quad 2.47050666-6.24299068-15.46576864$

O $\quad 1.45291616-5.63242121-15.16937960$

C $\quad 1.31797097-8.29586693-14.50898712$

C $\quad 1.27848966-9.83548827-14.45696843$
C

O
$8.95866826-1.54943009-15.35492083$ $8.77383431-0.40306220-14.67304516$ $8.96139588-4.40840455-19.58953922$ $10.57768906-0.85342299-16.61964980$ $7.32231910-2.48604670-14.33677803$ $6.67348209-4.80385306-17.92669887$ $7.49108116-4.60367463-15.65056261$ $10.73791072-2.95266813-17.91650477$ $10.20594757-5.48530499-17.80209723$ $8.74081024-6.04011626-17.00988592$ $9.606829420 .14349071-14.75087454$ $7.83952927-7.54134762-19.36836046$ $7.78102806-8.82048827-20.07272893$ $7.38955437-8.60319583-21.58118412$ $7.96252166-9.25485566-22.45642584$ $7.18084291-7.35719666-18.62446159$ $8.75260375-9.30980902-20.05810204$ $7.04543533-9.47033257-19.61063377$ $6.46506456-7.64287211-21.83707712$ $5.95473878-7.33020417-23.17227775$ $7.07450779-6.70960647-23.99515282$ $7.18533774-6.98362151-25.19329156$ $4.74941206-6.38990055-23.10126553$ $4.14710030-6.04849233-24.48970117$ $2.94402527-5.14570545-24.21853022$ $2.22718116-4.75101895-25.42905695$ $1.13198790-3.97963354-25.46477999$ $0.59437089-3.53423375-24.33640005$ $0.61007354-3.59636058-26.60360234$ $3.81055949-6.95163761-24.99865792$ $5.67302723-8.25317076-23.68491078$ $2.60461121-5.05668195-26.32713141$ $4.86540987-5.51453712-25.10739880$ $-0.23082661-3.05433528-26.66564412$ $0.96180726-3.99962225-27.46417785$ $6.05841298-7.20806559-21.01946190$ $3.28676332-4.25093507-23.70134838$ $2.24546206-5.68679656-23.58007065$ $-0.21446180-2.93629702-24.38744903$ $0.82571363-4.08021292-23.53995961$ $5.02219643-5.46356270-22.60874642$ $3.97982967-6.86451729-22.50593274$ $9.61930907-9.43391052-24.94600453$ $9.06730048-10.21469515-26.06961163$ $9.19588883-9.39457679-27.37060193$ $9.79528165-9.85451920-28.31723117$ $7.61294367-10.69826543-25.80389854$ $7.58437527-11.69399394-24.63716609$ $7.09907439-11.41797837-27.05795194$ $6.17805508-11.89230143-24.08038461$ $9.68783571-11.10407632-26.20030879$ $6.08247051-11.76593096-26.89662006$ $7.07868116-10.73701204-27.90482636$ $6.98655143-9.83344827-25.58329962$ $8.01095900-12.64393128-24.94960370$ $8.20358376-11.32477641-23.82535714$ $5.56174474-12.35394636-24.84978206$ $9.04824798-9.21506303-24.14240686$ $6.23228386-12.56020251-23.22760353$ $7.73994068-12.26389121-27.28982476$ $5.74787593-10.93532743-23.79409717$ $6.59450517-20.99399810-19.57814771$ $6.07987226-20.91097797-18.20205194$ $4.58281412-20.56968892-18.30855374$ $4.08718745-20.17253374-19.39297612$ 

$8.49305590-13.98899110-15.23843801$ $7.90795703-14.61606575-16.27141143$ $7.38541760-13.76706991-17.36012338$ $7.49736467-14.19346226-18.70239944$ $6.99734349-13.34713120-19.70531189$ $6.40368075-12.12196859-19.43318485$ $6.02530008-11.52967468-20.24326563$ $7.07755072-13.75417788-21.00260079$ $8.00276903-15.13117759-18.94076543$ $6.80399425-12.54005590-17.04577244$ $6.74251856-12.27659394-15.99830329$ $6.25697771-11.75982097-18.05807012$ $5.45147351-10.59672681-17.67078108$ $5.67652423-9.86168743-16.71240015$ $4.38146984-10.33272342-18.53897362$ $4.00570133-11.07225282-19.14050780$ $3.42712604-9.38323484-18.22493344$ $3.25223102-9.28582572-17.22419667$ $3.59320842-7.96692403-19.04576016$ $4.96950286-7.56269044-19.40692671$ $2.80959309-6.89733379-18.41067294$ $2.78554652-8.38296249-20.67449869$ $1.64482594-7.64696539-21.06486693$ $1.16905685-6.95421383-20.37872013$ $1.07331188-7.88523925-22.33448252$ $0.15831025-7.38516024-22.63556717$ $1.63464418-8.83474948-23.19223338$ $1.19021596-9.04450648-24.16349882$ $2.79367842-9.50795909-22.83626664$ $3.27945183-10.19794971-23.52281776$ $3.36373997-9.27976969-21.58022235$ $4.53375268-9.95655528-21.29022520$ $\mathrm{Zn} \quad 28.08310060-6.23441893-6.91254238$

$\mathrm{H}$ $-2.10866911-6.04256302-23.95331848$ $-2.82318363-5.47642944-23.60749244$ $-2.40863869-6.92076016-23.69985219$ $7.34923536-19.80969014-13.53894323$ $8.21335279-19.44180396-13.27932953$ $7.37769770-20.69639980-13.16246258$ $9.24761494-17.93744864-18.98035543$ $9.88002556-17.25031981-18.68045914$ $9.82421427-18.65692144-19.25857282$ $5.98280948-7.01653550-16.77148116$ $6.45367046-6.72544003-15.96780510$ $6.21328141-7.95313267-16.83508129$ $26.49407347-0.90809781-4.84887327$ $27.11630126-0.82001480-4.11396664$ $26.76582713-1.71735874-5.29123432$ $0.73163336-10.02469854-18.02520022$ $-0.04357004-10.48686893-18.41286294$ $1.46921835-10.39186484-18.52008374$ 


$\begin{array}{ll}\mathrm{O} & 4.78284773-18.68717480-14.47386265 \\ \mathrm{H} & 5.70325743-18.92087106-14.25649973 \\ \mathrm{H} & 4.28543008-19.33738352-13.96613729 \\ \mathrm{O} & -0.45920945-6.70337312-25.86136120 \\ \mathrm{H} & -0.90434201-7.26849694-26.50326302 \\ \mathrm{H} & -1.17567613-6.40192276-25.27242006\end{array}$

1C QM/SE/MM R1b (B3LYP/6-

31G(d):xTB:Amber) mechanical embedding $\omega_{\alpha}=\mathbf{1 . 6 6 7}$ including $B$ factors

C $\quad 22.27282083-8.92436286-4.42227427$

O $\quad 21.42203731-8.05673249-4.33817943$

$\mathrm{N} \quad 23.53837507-8.67713073-4.77428101$

C $\quad 23.88304980-7.39000885-5.37305674$

C $\quad 23.06228446-7.24443947-6.68301598$

O $\quad 23.12358015-8.11387364-7.55527362$

C $\quad 25.38118965-7.35371153-5.80320518$

S $\quad 25.81328984-5.71118521-6.50623255$

$\mathrm{H} \quad 24.17088853-9.46491764-4.89901889$

$\mathrm{H} \quad 23.67499577-6.58504626-4.66699980$

$\mathrm{H} \quad 25.61219351-8.18796499-6.47116225$

$\mathrm{H} \quad 25.94870544-7.50149457-4.87250544$

$\mathrm{N} \quad 22.37015149-6.08667505-6.82285062$

$\mathrm{H} \quad 22.18530785-5.53217017-5.99245907$

C $\quad 25.24718822-5.90687421-10.75098074$

O $\quad 25.31336158-6.06205639-11.97150991$

$\mathrm{N} \quad 26.00186049-6.60828800-9.83420844$

C $\quad 26.81625749-7.70200019-10.30136128$

C $\quad 26.19822323-9.10300030-10.09931884$

O $\quad 26.73394933-10.11837609-10.56596256$

C $\quad 28.22155186-7.61440034-9.73143668$

S $\quad 28.24190562-8.13569583-7.88272360$

$\mathrm{H} \quad 25.94545417-6.37904354-8.83797259$

$\mathrm{H} \quad 26.90496134-7.58879721-11.38476573$

H $\quad 28.58639509-6.59407556-9.83674701$

$\mathrm{H} \quad 28.86962921-8.30351285-10.27529691$

$\mathrm{N} \quad 25.05588455-9.15130820-9.36319046$

$\mathrm{H} \quad 24.58981962-8.33017431-9.00240178$

C $\quad 30.09073227-4.490793791 .28432223$

O $\quad 30.68396685-3.641590890 .66424795$

$\mathrm{N} \quad 29.50389409-5.549821580 .66329845$

C $\quad 29.56120574-5.60344284-0.80621393$

C $\quad 29.05295275-4.31805256-1.43663139$

O $\quad 29.79475730-3.74610328-2.22221497$

C $\quad 28.84631869-6.83038305-1.35760628$

C $\quad 28.95200900-6.93540881-2.83369861$

$\mathrm{H} \quad 29.28815715-7.71803202-0.89805243$

$\mathrm{H} \quad 28.93597557-6.215418971 .18004028$

$\mathrm{H} \quad 30.61925720-5.63025863-1.08354373$

$\mathrm{H} \quad 27.78848636-6.78259297-1.09771759$

C $\quad 28.15360160-6.45332726-3.85128152$

$\mathrm{H} \quad 30.87747561-7.86993980-2.99690440$

H $\quad 27.24715365-5.88434272-3.75490344$

C $\quad 29.81876104-7.49090502-4.79874186$

$\mathrm{N} \quad 30.00328871-7.61672100-3.45913414$

$\mathrm{N} \quad 28.66877072-6.88230657-5.01554641$

$\mathrm{H} \quad 30.49980038-7.87662278-5.53189687$

$\mathrm{N} \quad 27.82914279-3.85266364-1.06935742$

$\mathrm{H} \quad 27.30385568-4.30862731-0.32511636$

C $\quad 32.42369638-1.94868818-3.92966428$

O $\quad 33.17702618-1.49391053-4.78339235$

$\mathrm{N} \quad 31.31296847-2.64457324-4.22686395$

C $\quad 30.98152882-3.02620492-5.61507439$
$\mathrm{C}$

$\mathrm{O}$

$\mathrm{C}$

\section{C QM/SE/MM R1b (B3LYP/6-}

31G(d):xTB:Amber) mechanical embedding $\omega_{\alpha}=\mathbf{1 . 6 6 7}$ including $\mathrm{B}$ factors

C $\quad 22.27376086-8.92401909-4.42405813$

O $\quad 21.42461151-8.05582481-4.33631712$

$\mathrm{N} \quad 23.54034655-8.67759477-4.77800900$

C $\quad 23.88937278-7.39052448-5.37514304$

C $\quad 23.06297665-7.24353414-6.68179139$

O $\quad 23.12546412-8.11355236-7.55362070$

C $\quad 25.38497484-7.36376479-5.82153118$

S $\quad 25.81703024-5.71280535-6.50591722$ 


\begin{tabular}{|c|c|}
\hline $\mathrm{H}$ & $24.15900389-9.46928969-4.93862774$ \\
\hline $\mathrm{H}$ & $23.68918464-6.58519808-4.66677282$ \\
\hline $\mathrm{H}$ & $25.59496241-8.18059185-6.51648312$ \\
\hline $\mathrm{H}$ & $25.97125889-7.54265887-4.90966546$ \\
\hline $\mathrm{N}$ & $22.36938213-6.08815422-6.82047407$ \\
\hline $\mathrm{H}$ & $22.17566048-5.54100761-5.98682763$ \\
\hline $\mathrm{C}$ & $25.24773667-5.90755002-10.75006497$ \\
\hline $\mathrm{O}$ & $25.31471763-6.06130246-11.97071284$ \\
\hline $\mathrm{N}$ & $26.00079286-6.61150979-9.83386652$ \\
\hline $\mathrm{C}$ & $26.81881899-7.70219860-10.30415291$ \\
\hline $\mathrm{C}$ & $26.20097959-9.10504290-10.10073757$ \\
\hline $\mathrm{O}$ & $26.73449642-10.12178899-10.56613248$ \\
\hline $\mathrm{C}$ & $28.22711433-7.61391126-9.73513758$ \\
\hline $\mathrm{S}$ & $28.24193601-8.13476475-7.88507891$ \\
\hline $\mathrm{H}$ & $25.94532653-6.38213969-8.83736586$ \\
\hline $\mathrm{H}$ & $26.89699205-7.58463266-11.38807391$ \\
\hline $\mathrm{H}$ & $28.60284417-6.59754011-9.84252653$ \\
\hline $\mathrm{H}$ & $28.87504188-8.30687187-10.27352100$ \\
\hline $\mathrm{N}$ & $25.05664557-9.15201435-9.36423206$ \\
\hline $\mathrm{H}$ & $24.59372175-8.32858386-9.00524261$ \\
\hline $\mathrm{C}$ & $30.09147482-4.491052991 .28289158$ \\
\hline $\mathrm{O}$ & $30.68571979-3.641516300 .66505304$ \\
\hline $\mathrm{N}$ & $29.50535786-5.549611270 .65952982$ \\
\hline $\mathrm{C}$ & $29.55728365-5.60836494-0.81203715$ \\
\hline $\mathrm{C}$ & $29.05045499-4.31949962-1.44055792$ \\
\hline $\mathrm{O}$ & $29.79488422-3.73545363-2.21558149$ \\
\hline $\mathrm{C}$ & $28.83867103-6.83884381-1.35669089$ \\
\hline $\mathrm{C}$ & $28.94380793-6.93701111-2.83721442$ \\
\hline $\mathrm{H}$ & $29.27505698-7.72962412-0.89566706$ \\
\hline $\mathrm{H}$ & $28.94480430-6.222198121 .17469172$ \\
\hline $\mathrm{H}$ & $30.61340130-5.63355602-1.09575597$ \\
\hline $\mathrm{H}$ & $27.78021213-6.79654024-1.09335674$ \\
\hline $\mathrm{C}$ & $28.14475046-6.45016877-3.85410615$ \\
\hline $\mathrm{H}$ & $30.87888328-7.84787925-3.00357367$ \\
\hline $\mathrm{H}$ & $27.23631331-5.87952836-3.76907133$ \\
\hline $\mathrm{C}$ & $29.81357413-7.48535989-4.80721906$ \\
\hline $\mathrm{N}$ & $29.99994395-7.61247032-3.46626923$ \\
\hline $\mathrm{N}$ & $28.66263664-6.87729364-5.01748947$ \\
\hline $\mathrm{H}$ & $30.48339685-7.87048296-5.55298867$ \\
\hline $\mathrm{N}$ & $27.82703598-3.85418433-1.07090545$ \\
\hline $\mathrm{H}$ & $27.28543437-4.33191098-0.35234398$ \\
\hline $\mathrm{C}$ & $32.42067854-1.95009883-3.92931273$ \\
\hline $\mathrm{O}$ & $33.17274397-1.49090806-4.78085563$ \\
\hline $\mathrm{N}$ & $31.31251101-2.64901593-4.22817084$ \\
\hline $\mathrm{C}$ & $30.98196696-3.02927033-5.61608048$ \\
\hline $\mathrm{C}$ & $30.87469587-1.72220190-6.41715338$ \\
\hline $\mathrm{O}$ & $30.16564873-0.80814908-6.06769466$ \\
\hline $\mathrm{C}$ & $29.67005631-3.81466158-5.59213057$ \\
\hline S & $29.19030220-4.40133969-7.28474687$ \\
\hline $\mathrm{H}$ & $30.76059826-3.01877855-3.47086145$ \\
\hline $\mathrm{H}$ & $31.78302433-3.66621409-6.01264769$ \\
\hline $\mathrm{H}$ & $28.88946380-3.16295139-5.19076003$ \\
\hline $\mathrm{H}$ & $29.80628489-4.66214112-4.91612583$ \\
\hline $\mathrm{N}$ & $31.60841649-1.59977940-7.52279749$ \\
\hline $\mathrm{H}$ & $32.12995217-2.37270204-7.85802255$ \\
\hline $\mathrm{C}$ & $7.68135978-15.98286114-16.35596184$ \\
\hline $\mathrm{H}$ & $7.12312775-16.37117369-17.19605006$ \\
\hline $\mathrm{C}$ & $8.06534423-16.79711722-15.30741963$ \\
\hline $\mathrm{H}$ & $7.84775264-17.85456029-15.32258423$ \\
\hline $\mathrm{C}$ & $8.74624951-16.20553379-14.23976785$ \\
\hline $\mathrm{H}$ & $9.11492094-16.80790262-13.43236881$ \\
\hline $\mathrm{C}$ & $8.98376836-14.84181903-14.26688271$ \\
\hline $\mathrm{H}$ & $9.56474477-14.35982168-13.48979655$ \\
\hline $\mathrm{N}$ & $8.51872528-14.00438168-15.23324954$ \\
\hline $\mathrm{C}$ & $7.91107569-14.62515009-16.26862814$ \\
\hline
\end{tabular}

C $\quad 7.39887418-13.77971386-17.33920017$ C $\quad 7.52061587-14.21296579-18.69289436$ C $\quad 6.99625299-13.37294943-19.67956574$ C $\quad 6.40888684-12.15533434-19.39974519$ $\mathrm{H} \quad 6.02487910-11.54312886-20.17955044$ F $\quad 7.06937273-13.77711203-20.99268897$ $\mathrm{H} \quad 8.07779925-15.11323972-18.92378817$ C $\quad 6.82388890-12.56693472-17.01562358$ $\mathrm{H} \quad 6.71750344-12.31814478-15.97030979$ C $\quad 6.26877395-11.76096129-18.01660346$ C $\quad 5.46708418-10.61835485-17.66049753$ O $\quad 5.69652227-9.83850761-16.67972258$

$\mathrm{N} \quad 4.38507064-10.33602372-18.52299379$

$\mathrm{H} \quad 3.96115907-11.04581327-19.11582277$ $\mathrm{N} \quad 3.45702203-9.34164259-18.19323517$

$\mathrm{H} \quad 3.52504456-9.08373163-17.20086298$

S $\quad 3.60679041-7.95764818-19.07873042$

O $\quad 5.01318583-7.55701701-19.41727381$

O $\quad 2.84628534-6.86135057-18.41667979$

C $\quad 2.78993308-8.37320243-20.66486725$

C $\quad 1.62050896-7.64709063-21.06303678$

$\mathrm{H} \quad 1.14030847-6.97371530-20.36684392$

C $\quad 1.05357347-7.91454334-22.31653842$

$\mathrm{H} \quad 0.10640380-7.45834281-22.58078472$

C $\quad 1.60104830-8.87866394-23.17815093$

$\mathrm{H} \quad 1.12179875-9.12820622-24.11755439$

C $\quad 2.78146988-9.53951153-22.82776844$

$\mathrm{H} \quad 3.22237221-10.26796712-23.49727054$

C $\quad 3.36109572-9.28411777-21.59186344$

F $\quad 4.52822008-9.98732933-21.29327171$

$\mathrm{Zn} \quad 28.08231401-6.24165852-6.90615848$

\section{C QM/SE/MM R3b (B3L YP/6- \\ 31G(d):xTB:Amber) mechanical embedding $\omega_{\alpha}=\mathbf{1 . 6 6 7}$ including $\mathrm{B}$ factors}

$\begin{array}{lll}\mathrm{C} & 21.94235593-10.35441323-4.21923405 \\ \mathrm{C} & 22.26213096-8.89486943-4.42683948 \\ \mathrm{O} & 21.42258707-8.01927272-4.34633861 \\ \mathrm{~N} & 23.53417164-8.67665758-4.78455333 \\ \mathrm{C} & 23.89355583-7.39534152-5.38780835 \\ \mathrm{C} & 23.07461744-7.24669929-6.69724290 \\ \mathrm{O} & 23.12874591-8.13033821-7.55514783 \\ \mathrm{C} & 25.38353526-7.36442034-5.81668671 \\ \mathrm{~S} & 25.81678803-5.71693765-6.50245878 \\ \mathrm{H} & 24.14804832-9.47563419-4.92435699 \\ \mathrm{H} & 23.68607490-6.59087292-4.68517898 \\ \mathrm{H} & 25.59703691-8.17908706-6.50867519 \\ \mathrm{H} & 25.96653131-7.54172180-4.90466163 \\ \mathrm{~N} & 22.39336607-6.08868686-6.84980670 \\ \mathrm{C} & 21.47687488-5.96277464-7.96253623 \\ \mathrm{C} & 22.17619051-5.64169626-9.30394485 \\ \mathrm{O} & 21.56568092-5.75563631-10.34942644 \\ \mathrm{C} & 20.49320556-4.87081719-7.64678233 \\ \mathrm{H} & 20.93182619-6.90049926-8.10598104 \\ \mathrm{H} & 22.23909599-5.51084407-6.02737978 \\ \mathrm{~N} & 23.43905266-5.18534741-9.15261499 \\ \mathrm{C} & 24.28962152-4.79077336-10.23530244 \\ \mathrm{C} & 25.23403167-5.87009104-10.73709497 \\ \mathrm{O} & 25.27754317-6.06812498-11.95352447 \\ \mathrm{C} & 25.10063870-3.57070430-9.88367227 \\ \mathrm{C} & 24.25439009-2.42767539-9.42218032 \\ \mathrm{C} & 23.30333541-1.84842535-10.24981436 \\ \mathrm{C} & 24.39152719-1.92964815-8.10914418\end{array}$


C

$\mathrm{H}$

C

C

$\mathrm{O}$

$\mathrm{N}$

C

C

O

C

C

$\mathrm{H}$

$\mathrm{H}$

$\mathrm{H}$

$\mathrm{H}$

C

$\mathrm{H}$

$\mathrm{H}$

C

$\mathrm{N}$

N

$\mathrm{H}$

$\mathrm{N}$

C

$\mathrm{H}$

C

C

$\mathrm{O}$

N

C

$\mathrm{C}$

O

C

S

$\mathrm{H}$

$\mathrm{H}$

$\mathrm{H}$

$\mathrm{H}$

N

C

C

O

C

$\mathrm{H}$

$\mathrm{H}$

N

C

C

O

C

C

$\mathrm{C}$

C

$\mathrm{N}$

C

C

$\mathrm{C}$

$\mathrm{C}$

$\mathrm{C}$

$\mathrm{H}$

$\mathrm{H}$

H

$\mathrm{H}$

$\mathrm{H}$

H
$24.89854097-13.85121236-4.12911153$ $26.23738580-14.13969646-5.72856318$ $29.87642389-4.489018042 .80743862$ $30.09409099-4.502036361 .26424678$ $30.68533311-3.642032650 .65773434$ $29.51313746-5.560888470 .64599284$ $29.56560303-5.61738194-0.81829936$ $29.05575303-4.32482432-1.43543876$ $29.77753803-3.72980162-2.22384976$ $28.84329301-6.84365062-1.35804838$ $28.95276614-6.94895573-2.83491434$ $29.27349521-7.73259600-0.89300725$ $28.95436180-6.227003891 .17253586$ $30.62079218-5.64665631-1.10080977$ $27.78828711-6.79527640-1.09509363$ $28.15785125-6.46365474-3.85191454$ $30.88046317-7.86906768-2.99363656$ $27.24861073-5.89797997-3.77312821$ $29.82598062-7.50169774-4.79758267$ $30.00659313-7.62439621-3.45936394$ $28.67999219-6.89115311-5.01440459$ $30.49663694-7.88792675-5.54064594$ $27.83683733-3.87712793-1.04286821$ $27.27826719-2.63905941-1.54909625$ $27.30871224-4.36214470-0.31965026$ $32.66455425-1.85341088-2.42329706$ $32.41679646-1.97471158-3.94347622$ $33.16520052-1.50026733-4.78981450$ $31.32156575-2.68955511-4.24301479$ $30.98415104-3.04274693-5.63310056$ $30.87159399-1.72697755-6.42265572$ $30.15712492-0.82216724-6.05682126$ $29.67107760-3.82331547-5.60688110$ $29.16662296-4.39060570-7.28692503$ $30.76898837-3.05395481-3.48251648$ $31.77633577-3.67652076-6.04249569$ $28.89905608-3.17682503-5.18697322$ $29.81178133-4.67680390-4.94300042$ $31.56870806-1.58802679-7.55078818$ $31.55431489-0.40545664-8.34621571$ $30.64913733-0.54442507-9.57634755$ $30.289047590 .42106668-10.22164774$ $33.00361561-0.10007440-8.72663592$ $31.162034520 .42053820-7.75231988$ $32.16725484-2.32082790-7.85615908$ $30.22845600-1.79093586-9.83296395$ $29.18139741-2.01141876-10.83623999$ $27.94090666-1.18720416-10.49688796$ $27.41032801-1.15225293-9.41278282$ $28.78207754-3.48486865-10.96224891$ $29.83575661-4.33427168-11.57927163$ $31.18566806-4.22444691-11.42443492$ $29.62522732-5.45223757-12.44911435$ $31.83711878-5.20005082-12.13436608$ $30.91144133-5.98340572-12.75479850$ $28.49876700-6.11696686-12.92615107$ $31.06755656-7.17469178-13.45571152$ $28.66698844-7.31552349-13.58145682$ $29.92517245-7.83859087-13.85773023$ $30.01469478-8.73470657-14.43453295$ $29.58543529-1.64454741-11.78700329$ $32.83300722-5.31150070-12.22183890$ $27.51761956-5.71942452-12.75718426$ $27.80097042-7.85475960-13.89000732$ $32.05255088-7.52829820-13.70055019$ 


\begin{tabular}{|c|c|c|c|}
\hline $\mathrm{H}$ & $31.72733562-3.49021255-10.87319211$ & $\mathrm{H}$ & $10.50509788-16.58567105-21.68084298$ \\
\hline $\mathrm{H}$ & $30.36933090-2.51708812-9.14032557$ & $\mathrm{H}$ & $12.06861525-14.80253562-22.27307493$ \\
\hline $\mathrm{H}$ & $27.89901016-3.55753914-11.59018200$ & $\mathrm{H}$ & $10.59429288-13.21631549-20.17305783$ \\
\hline $\mathrm{H}$ & $28.54876757-3.88166221-9.97686129$ & $\mathrm{H}$ & $12.17112845-12.78010042-20.81236459$ \\
\hline $\mathrm{N}$ & $27.43419175-0.49464490-11.53579607$ & $\mathrm{H}$ & $9.61327151-13.01663972-22.41227078$ \\
\hline $\mathrm{C}$ & $26.127310970 .15140242-11.47717085$ & $\mathrm{H}$ & $12.11621601-17.42993727-20.18966812$ \\
\hline $\mathrm{H}$ & $27.94595285-0.38065280-12.40983798$ & $\mathrm{H}$ & $10.40484159-11.53551682-21.91262214$ \\
\hline $\mathrm{C}$ & $14.00742517-19.32486299-8.43976762$ & $\mathrm{H}$ & $9.68291781-15.48482234-20.55030515$ \\
\hline $\mathrm{C}$ & $12.58256894-19.02992183-8.88598750$ & $\mathrm{H}$ & $11.19672370-12.60367297-23.06898900$ \\
\hline $\mathrm{O}$ & $11.66498721-19.12743371-8.10885034$ & $\mathrm{~N}$ & $12.19423490-14.21944363-18.07171520$ \\
\hline $\mathrm{N}$ & $12.46556699-18.62168110-10.17117789$ & $\mathrm{C}$ & $11.97177877-14.17529900-16.59864227$ \\
\hline $\mathrm{C}$ & $11.18009709-18.24156650-10.70873880$ & $\mathrm{C}$ & $11.95024653-12.72332505-16.24657886$ \\
\hline $\mathrm{C}$ & $10.79943164-19.00365582-11.95432770$ & $\mathrm{O}$ & $12.89227871-12.00820951-16.532195$ \\
\hline $\mathrm{O}$ & $9.74657124-18.76806506-12.53107111$ & $\mathrm{C}$ & $13.02951965-14.91022050-15.81251601$ \\
\hline $\mathrm{C}$ & $11.04914652-16.73553234-10.93086434$ & $\mathrm{C}$ & $12.65419862-15.13111956-14.25927938$ \\
\hline $\mathrm{C}$ & $11.55465377-15.97157996-9.74015679$ & $\mathrm{~S}$ & $12.77736683-13.58098547-13.37542930$ \\
\hline $\mathrm{C}$ & $10.78578981-15.89929601-8.57201897$ & $\mathrm{C}$ & $14.55316298-13.26157453-13.56946083$ \\
\hline $\mathrm{C}$ & $12.77819158-15.31079826-9.80101241$ & $\mathrm{H}$ & $11.61261483-15.42854832-14.22762074$ \\
\hline $\mathrm{C}$ & $11.26999082-15.19375936-7.47446240$ & $\mathrm{H}$ & $14.89084601-12.68282178-12.72333910$ \\
\hline $\mathrm{C}$ & $13.25165167-14.61960751-8.70220601$ & $\mathrm{H}$ & $15.08236967-14.20910462-13.58695876$ \\
\hline $\mathrm{C}$ & $12.49358593-14.55482496-7.53366051$ & $\mathrm{H}$ & $14.73144031-12.72292158-14.49019235$ \\
\hline $\mathrm{H}$ & $12.88681677-14.03684034-6.68319419$ & $\mathrm{H}$ & $13.21171056-15.88849931-16.23407205$ \\
\hline $\mathrm{H}$ & $10.45246175-18.55162824-9.94919988$ & $\mathrm{H}$ & $13.30067555-15.90417149-13.87060492$ \\
\hline $\mathrm{H}$ & $10.67951444-15.15532406-6.59098841$ & $\mathrm{H}$ & $12.91894526-13.61704196-18.4672582$ \\
\hline $\mathrm{H}$ & $14.21362246-14.14719022-8.76190784$ & $\mathrm{H}$ & $11.00145665-14.65198741-16.44540765$ \\
\hline $\mathrm{H}$ & $13.28125153-18.50054266-10.76317160$ & $\mathrm{H}$ & $13.95141056-14.33853804-15.83764195$ \\
\hline $\mathrm{H}$ & $13.37024081-15.38170908-10.69622091$ & $\mathrm{~N}$ & $10.79188799-12.27637061-15.72637028$ \\
\hline $\mathrm{H}$ & $9.83810003-16.39839549-8.52301050$ & $\mathrm{C}$ & $10.67213557-10.91582242-15.21766043$ \\
\hline $\mathrm{H}$ & $10.00727588-16.48615490-11.08128586$ & $\mathrm{C}$ & $10.55507679-11.05241025-13.66286864$ \\
\hline $\mathrm{H}$ & $11.63143927-16.44131847-11.79634541$ & $\mathrm{O}$ & $9.89466765-11.93276815-13.13398094$ \\
\hline $\mathrm{N}$ & $11.60708901-19.99221460-12.32201911$ & $\mathrm{C}$ & $9.41848979-10.22391496-15.72801847$ \\
\hline $\mathrm{C}$ & $11.31282624-20.75750784-13.49626782$ & $\mathrm{C}$ & $9.40970277-10.11986293-17.27296998$ \\
\hline $\mathrm{C}$ & $11.32401728-22.22060932-13.13491779$ & $\mathrm{C}$ & $9.24442583-8.84794583-15.11003662$ \\
\hline $\mathrm{O}$ & $12.21712147-22.72153854-12.48898374$ & $\mathrm{C}$ & $10.50271888-9.19318712-17.74919598$ \\
\hline $\mathrm{C}$ & $12.30779731-20.45759605-14.63698049$ & $\mathrm{H}$ & $11.56649298-10.36050733-15.47363862$ \\
\hline $\mathrm{C}$ & $12.27014534-18.99636567-15.04859582$ & $\mathrm{H}$ & $8.32955580-8.39701415-15.46431387$ \\
\hline $\mathrm{C}$ & $13.37958147-18.76325298-16.05997016$ & $\mathrm{H}$ & $9.17806102-8.94805425-14.03272342$ \\
\hline $\mathrm{C}$ & $10.91852540-18.63784139-15.64500117$ & $\mathrm{H}$ & $8.55973681-10.83338802-15.45476642$ \\
\hline $\mathrm{H}$ & $10.15441698-18.76600912-14.89899129$ & $\mathrm{H}$ & $9.54855374-11.11052364-17.67804501$ \\
\hline $\mathrm{H}$ & $10.93629809-17.61837552-15.98563922$ & $\mathrm{H}$ & $8.44472233-9.72646859-17.55716564$ \\
\hline $\mathrm{H}$ & $10.72019954-19.29468767-16.48581948$ & $\mathrm{H}$ & $11.46723757-9.59258000-17.45071396$ \\
\hline $\mathrm{H}$ & $12.45066384-20.19910393-11.80587103$ & $\mathrm{H}$ & $9.98761334-12.89899077-15.60436814$ \\
\hline $\mathrm{H}$ & $13.23651741-19.38555711-16.93714017$ & $\mathrm{H}$ & $10.49544104-9.14115363-18.82276761$ \\
\hline $\mathrm{H}$ & $13.36166460-17.73282433-16.38377246$ & $\mathrm{H}$ & $10.08645010-8.21220628-15.33477531$ \\
\hline $\mathrm{H}$ & $14.34838474-18.97842462-15.63478385$ & $\mathrm{H}$ & $10.39792454-8.19699891-17.35006034$ \\
\hline $\mathrm{H}$ & $10.31742159-20.46895260-13.83393893$ & $\mathrm{~N}$ & $11.34221567-10.26731419-12.92484405$ \\
\hline $\mathrm{H}$ & $12.42940591-18.35084562-14.19093141$ & $\mathrm{C}$ & $11.28864112-10.40336918-11.44852483$ \\
\hline $\mathrm{H}$ & $12.04779401-21.07317630-15.48904735$ & $\mathrm{H}$ & $11.90992981-9.51937914-13.31097481$ \\
\hline $\mathrm{H}$ & $13.30443223-20.71642415-14.30145543$ & $\mathrm{C}$ & $7.89723641-6.08054616-12.13765453$ \\
\hline $\mathrm{N}$ & $10.25844732-22.88867415-13.58376683$ & $\mathrm{C}$ & $6.87342730-6.76386502-13.06382365$ \\
\hline $\mathrm{C}$ & $10.13903180-24.32453866-13.36906361$ & $\mathrm{O}$ & $6.61435865-6.34122948-14.16601876$ \\
\hline $\mathrm{H}$ & $9.60954543-22.49116235-14.25134166$ & $\mathrm{~N}$ & $6.46145803-7.97249715-12.66679384$ \\
\hline $\mathrm{C}$ & $14.10220081-18.55266917-21.17926353$ & $\mathrm{C}$ & $5.62409353-8.76736822-13.50038770$ \\
\hline $\mathrm{C}$ & $13.90748450-17.06526733-21.07347251$ & $\mathrm{C}$ & $4.31797681-7.97123801-13.75258140$ \\
\hline $\mathrm{O}$ & $14.72751837-16.29812514-21.48179236$ & $\mathrm{O}$ & $3.94822679-7.13526555-12.94249910$ \\
\hline $\mathrm{N}$ & $12.75188925-16.70567943-20.49041722$ & $\mathrm{C}$ & $5.35242328-10.12883664-12.91920291$ \\
\hline $\mathrm{C}$ & $12.43239063-15.33322783-20.24416053$ & $\mathrm{C}$ & $6.62030552-10.99297106-12.92892645$ \\
\hline $\mathrm{C}$ & $11.79356022-15.24499108-18.82536374$ & $\mathrm{C}$ & $6.38425427-12.30971986-12.27247455$ \\
\hline $\mathrm{O}$ & $10.92776780-16.03839069-18.44966508$ & $\mathrm{H}$ & $6.89222824-11.19092295-13.96849541$ \\
\hline $\mathrm{C}$ & $11.51981139-14.73771896-21.33696622$ & $\mathrm{H}$ & $6.12622063-8.87240282-14.47080322$ \\
\hline $\mathrm{C}$ & $11.24258532-13.28714658-21.04947916$ & $\mathrm{H}$ & $7.45328865-10.48776083-12.47018417$ \\
\hline $\mathrm{C}$ & $10.24589562-15.55962503-21.47061672$ & $\mathrm{H}$ & $6.67278913-8.26363445-11.71971562$ \\
\hline $\mathrm{C}$ & $10.57009273-12.56557179-22.18696007$ & $\mathrm{H}$ & $4.57954264-10.61669696-13.50386816$ \\
\hline $\mathrm{H}$ & $13.37524360-14.78848068-20.25018237$ & $\mathrm{H}$ & $4.99871105-10.03982904-11.89597061$ \\
\hline $\mathrm{H}$ & $9.65451937-15.16119448-22.27762786$ & $\mathrm{H}$ & $8.31831919-12.84886885-12.58058751$ \\
\hline
\end{tabular}


$5.27789231-12.59940807-11.83738377$ $7.45758955-13.10008524-12.11072109$ $7.34242565-14.04652083-11.77478464$ $3.75225366-8.26980790-14.95851841$ $2.46535941-7.77517901-15.40458505$ $2.46298067-6.24573991-15.44582113$ $1.45705738-5.62499680-15.16162511$ $1.34184259-8.28979279-14.47704178$ $1.27311976-9.82035696-14.45706037$ $0.08299750-10.24788909-13.61622272$ $0.02829764-11.65247909-13.33007155$ $-0.67124735-12.54412669-14.08295577$ $-1.63835558-12.08734554-14.94481319$ $-0.41381812-13.90328440-13.94886854$ $2.16835417-10.24390177-14.01861080$ $2.22507139-8.14566761-16.39726436$ $0.73058817-12.03528102-12.70027020$ $1.15236449-10.19137923-15.47113375$ $-1.18006377-14.50972163-14.20077574$ $-0.01467260-14.15486506-13.05137301$ $4.31314688-8.89808856-15.53781588$ $-0.84260745-9.95988271-14.10756098$ $0.10621528-9.73605382-12.65654789$ $-2.02204400-12.79535082-15.55032186$ $-1.46279294-11.19254626-15.36867516$ $0.40302931-7.88059200-14.82950697$ $1.52181394-7.92975153-13.46874000$ $3.60032025-5.64779205-15.82085043$ $3.69651604-4.20295905-15.85186848$ $4.27668222-3.65707761-17.17116907$ $4.54834999-2.48113564-17.32443700$ $4.48953241-3.65987956-14.66709320$ $3.66210889-3.54199221-13.40129631$ $4.52427865-3.78245528-12.17986761$ $3.87287389-3.32625623-10.96509886$ $3.44369585-4.14251422-9.97875138$ $3.30735556-5.47609174-10.19668043$ $3.04777283-3.61942444-8.77544450$ $2.84751534-4.25752041-13.40709689$ $2.67743647-3.81742674-15.76259244$ $3.79679822-2.33752665-10.81138114$ $3.23540263-2.54262790-13.33609037$ $2.55443044-4.26876920-8.18332530$ $2.69570600-2.67981193-8.79139648$ $4.42385334-6.20874201-16.02343395$ $5.47055438-3.25323176-12.26896270$ $4.73339782-4.84513202-12.12062740$ $3.31731755-6.10783818-9.40091230$ $3.91606899-5.84706970-10.91217018$ $4.88650682-2.68586363-14.93091434$ $5.32042495-4.33716606-14.47851264$ $4.26311323-4.52898497-18.20432803$ $4.75210785-4.12731387-19.49338963$ $6.23307210-4.48733512-19.85215466$ $6.57281087-4.41776548-21.03762524$ $4.00918625-5.49897151-18.04559357$ $4.14248720-4.59368985-20.26583056$ $4.68867696-3.03791220-19.55050206$ $7.02475790-4.84712388-18.83192172$ $8.42321153-5.18366417-19.00405069$ $8.58757238-6.49896477-19.76991452$ $9.39624784-6.56345835-20.67844001$ $9.17301007-5.24928478-17.63034740$ $9.10479968-3.95039483-16.88709144$ $9.95706253-2.89300632-17.13792456$
$8.17470385-3.80152663-15.87483106$ $9.88924259-1.72591993-16.40625176$ $8.06870913-2.63060808-15.14077108$ $8.95625385-1.58456483-15.37275415$ $8.78740380-0.44407819-14.66735615$ $8.89667294-4.40133460-19.60250146$ $10.58475448-0.92620029-16.58635280$ $7.33533231-2.54675367-14.36808811$ $6.63594151-4.90045712-17.89719383$ $7.51429577-4.61677125-15.64438639$ $10.71145929-2.97074823-17.89439419$ $10.20408375-5.45584638-17.87097358$ $8.75759956-6.04807446-17.03359687$ $9.633699610 .11489916-14.78253315$ $7.79283722-7.52350216-19.37867234$ $7.78724951-8.77947498-20.07467355$ $7.39554707-8.59584242-21.61568199$ $7.96875701-9.24250999-22.46759724$ $7.07667629-7.34813430-18.68414320$ $8.78292156-9.21955165-20.09861384$ $7.08016666-9.46601189-19.62393701$ $6.50884562-7.58568733-21.82798055$ $5.96261183-7.33201555-23.13701800$ $7.07461432-6.70077442-24.00186611$ $7.19178644-6.98134865-25.18538026$ $4.75074957-6.41022260-23.07735234$ $4.14727534-6.05129998-24.45575802$ $2.93736865-5.16148538-24.20154743$ $2.27419048-4.74386794-25.40765293$ $1.14767004-4.00458088-25.43300783$ $0.66059481-3.50419364-24.23754758$ $0.68968834-3.51809886-26.60469066$ $3.81489158-6.94108567-24.97895368$ $5.67505098-8.26945073-23.63373458$ $2.64704728-5.05711167-26.30395136$ $4.86974927-5.51904396-25.05442179$ $-0.25360163-3.17154908-26.66940184$ $1.00367375-3.96717418-27.45890957$ $5.97745122-7.31389990-21.00558003$ $3.26391934-4.28054603-23.65303349$ $2.22796016-5.72805383-23.59045683$ $-0.24768823-3.05943869-24.33198709$ $0.77980564-4.16447846-23.49025897$ $5.01446005-5.48954348-22.57195186$ $3.98136039-6.92543951-22.50850035$ $7.75295045-5.75323565-23.33588734$ $8.91836581-5.13418363-23.92948295$ $7.60965508-5.58244563-22.34886260$ $11.29349146-8.06844140-23.87322662$ $10.87734164-8.98618658-25.03428394$ $11.70422723-9.33618536-25.85963819$ $9.58690308-9.38655820-24.95071404$ $9.07872790-10.20121468-26.04456406$ $9.21067295-9.41242393-27.36708875$ $9.78567508-9.87370159-28.31897524$ $7.63598877-10.68263510-25.78878673$ $7.61547761-11.64940491-24.61516927$ $7.11137944-11.36735067-27.03427386$ $6.21247407-11.91084435-24.10991036$ $9.73607220-11.07057492-26.15858773$ $6.08754708-11.66860713-26.86236106$ $7.13513771-10.68441734-27.86887744$ $7.03438662-9.80522253-25.56137705$ $8.09007702-12.57631563-24.91756244$ $8.19201024-11.22095538-23.80328163$ 
C

O

$\mathrm{H}$

$\mathrm{H}$

$\mathrm{H}$

$\mathrm{H}$

$\mathrm{H}$

N

C

$\mathrm{H}$

C

C

O

$\mathrm{N}$

C

C

$\mathrm{O}$

C

$\mathrm{H}$

$\mathrm{H}$

$\mathrm{N}$

C

C

O

C

C

C

C

$\mathrm{C}$

C

C

$\mathrm{O}$

$\mathrm{H}$

$\mathrm{H}$

$\mathrm{H}$

$\mathrm{H}$

H

$\mathrm{H}$

$\mathrm{H}$

$\mathrm{H}$

$\mathrm{H}$

$\mathrm{N}$

C

$\mathrm{H}$

C

$\mathrm{H}$

C

H

C

$\mathrm{H}$

C

$\mathrm{H}$

N

C

$\mathrm{C}$

C

C

C

$\mathrm{H}$

F

$\mathrm{H}$

C

$\mathrm{H}$

C

C
$3.51954258-12.77210518-21.41657431$ $3.31490622-12.54962691-19.98886377$ $2.07880920-14.79775319-20.97954828$ $1.50303120-12.19296863-22.01210096$ $3.98388300-11.89780162-21.83967752$ $4.16826912-13.63382874-21.56258610$ $3.23661647-13.42277495-19.57603321$ $2.80815732-14.34969680-24.17219442$ $3.06995157-14.51817564-25.60615293$ $3.15884971-15.08622077-23.57026567$ $-0.57229731-15.19309579-26.42161212$ $-1.14754107-13.78981731-26.54072064$ $-1.63917614-13.37340808-27.56256981$ $-0.98556150-13.04394436-25.45607329$ $-1.46042718-11.68225734-25.51504203$ $-0.67209727-10.83859561-26.52205591$ $-1.20311946-9.99126911-27.21821770$ $-1.41026865-11.11052582-24.10578027$ $-0.61210549-13.39488646-24.58817473$ $-2.50204113-11.66566453-25.85477428$ $0.66727376-11.06495430-26.51540215$ $1.53711502-10.23423554-27.32426875$ $1.17944509-10.47687902-28.83037156$ $0.98537215-9.54867152-29.56330233$ $3.04449445-10.49176386-27.07568200$ $3.93524659-9.89567114-28.14950788$ $4.25963443-8.55324374-28.12542795$ $4.33015645-10.65935488-29.23316634$ $5.07466402-8.01821636-29.10122145$ $5.15870654-10.15397823-30.21807096$ $5.55402273-8.82929803-30.13029377$ $6.49542681-8.33993983-30.96421010$ $1.33272624-9.17315724-27.16456468$ $5.38755746-6.99174359-29.05338138$ $5.48818529-10.75765784-31.03799770$ $1.08313882-11.65483840-25.80421807$ $4.03080285-11.68942846-29.28997542$ $3.97128339-7.93342684-27.29548198$ $3.21155107-11.56055633-27.05062266$ $3.31382205-10.05625551-26.12216226$ $6.34751007-7.38914990-31.14301300$ $1.15651169-11.75997685-29.18333189$ $0.80304740-12.22317106-30.51051451$ $1.37760223-12.50738026-28.53555360$ $7.68217861-15.97690732-16.36418098$ $7.12254397-16.36750228-17.20069989$ $8.07648392-16.79276338-15.31945612$ $7.85729247-17.84996134-15.33694786$ $8.75870063-16.20278752-14.25415311$ $9.12625807-16.80048497-13.44339763$ $8.99098528-14.83677642-14.28168290$ $9.57161257-14.35467151-13.50364123$ $8.52668672-14.00136957-15.24899978$ $7.91016055-14.61832578-16.27942232$ $7.39897561-13.77681812-17.34860638$ $7.52094206-14.20655789-18.69851394$ $7.00240332-13.36486238-19.68679697$ $6.39885219-12.15579967-19.40196969$ $6.01778979-11.54289200-20.18354837$ $7.08320044-13.76044700-20.99553112$ $8.06991252-15.11031458-18.93179642$ $6.81294964-12.56768026-17.02342955$ $6.70574911-12.31950291-15.97808578$ $6.25559135-11.76943478-18.02212993$ $5.45274879-10.61749401-17.66717616$ 
O $\quad 5.68049024-9.84683430-16.69046124$

$\mathrm{N} \quad 4.37515977-10.33800879-18.52887656$

$\mathrm{H} \quad 3.95733629-11.05109354-19.12511478$

$\mathrm{N} \quad 3.43847409-9.34605338-18.20283370$

$\mathrm{H} \quad 3.49825919-9.09857489-17.20693623$

S $\quad 3.59526720-7.95835410-19.08027287$

O $\quad 5.00108548-7.56576977-19.41142831$

O $\quad 2.82997325-6.86383473-18.43404836$

C $\quad 2.78758594-8.36641159-20.68669780$

C $\quad 1.62516936-7.64881915-21.07927957$

$\mathrm{H} \quad 1.14545226-6.97075632-20.38748318$

C $\quad 1.05439765-7.91521619-22.33475487$

$\mathrm{H} \quad 0.11718173-7.44999895-22.60680032$

C $\quad 1.60756145-8.87418179-23.19269341$

$\mathrm{H} \quad 1.13442018-9.11688301-24.13621255$

C $\quad 2.78242724-9.53452409-22.84147425$

$\mathrm{H} \quad 3.22515469-10.26486496-23.50766607$

C $\quad 3.35749555-9.27715018-21.59813207$

F $\quad 4.51983647-9.97387957-21.28333426$

$\mathrm{Zn} \quad 28.08414942-6.24470381-6.90523499$

O $\quad-2.07835974-5.83308129-23.88819308$

$\mathrm{H} \quad-2.90110967-5.38314707-23.59452560$

$\mathrm{H} \quad-2.05926289-6.61691143-23.33362697$

O $\quad 7.33600506-19.80968297-13.54026121$

$\mathrm{H} \quad 8.19373291-19.42990339-13.28679194$

$\mathrm{H} \quad 7.26754191-20.64546085-13.06701320$

O $\quad 9.22341642-17.93344830-18.98515666$

$\mathrm{H} \quad 9.90840138-17.29887037-18.70730840$

$\mathrm{H} \quad 9.57944128-18.42484022-19.72908332$

O $\quad 5.96117265-7.07148173-16.80234511$

$\mathrm{H} \quad 6.35400673-6.81970684-15.94665669$

$\mathrm{H} \quad 5.98120640-8.04765835-16.82013910$

O $\quad 26.49123228-0.94026836-4.85369520$

$\mathrm{H} \quad 27.13565902-0.82278198-4.13850104$

$\mathrm{H} \quad 26.58808403-1.83889971-5.18566907$

O $\quad 0.68191358-10.01569531-18.05760280$

$\mathrm{H} \quad-0.10165910-10.49906162-18.42755163$

$\mathrm{H} \quad 1.40564056-10.25888941-18.63658931$

O $\quad 4.77648370-18.69792216-14.48880416$

$\mathrm{H} \quad 5.69990975-18.93292012-14.29061171$

$\mathrm{H} \quad 4.26881323-19.21992187-13.85714012$

O $\quad-0.45133746-6.71260190-25.79702711$

$\mathrm{H} \quad-0.81737428-7.44210354-26.30379010$

$\mathrm{H} \quad-1.12760029-6.42922260-25.17604645$

\section{C QM/SE/MM R3b (B3LYP/6-}

31G(d):xTB:Amber) mechanical embedding $\omega_{\alpha}=\mathbf{1 . 6 6 7}$ including $B$ factors

$\begin{array}{ll}\mathrm{C} & 21.94221254-10.35494139-4.21911262 \\ \mathrm{C} & 22.26240038-8.89560787-4.42634652 \\ \mathrm{O} & 21.42303712-8.01960655-4.34485608 \\ \mathrm{~N} & 23.53424087-8.67743090-4.78427732 \\ \mathrm{C} & 23.89215258-7.39599253-5.38749846 \\ \mathrm{C} & 23.07359739-7.24778315-6.69673150 \\ \mathrm{O} & 23.12829742-8.13091714-7.55514740 \\ \mathrm{C} & 25.38268323-7.36337760-5.81516597 \\ \mathrm{~S} & 25.81188667-5.71585913-6.50274049 \\ \mathrm{H} & 24.14957123-9.47583484-4.92118669 \\ \mathrm{H} & 23.68390764-6.59224778-4.68519771 \\ \mathrm{H} & 25.59802074-8.17849443-6.50489386 \\ \mathrm{H} & 25.96451838-7.53800515-4.90274208 \\ \mathrm{~N} & 22.39314334-6.08960028-6.84954899 \\ \mathrm{C} & 21.47664358-5.96327970-7.96234276\end{array}$

$22.17633835-5.64203603-9.30351978$ $21.56591351-5.75490382-10.34925780$ $20.49295809-4.87114348-7.64664162$ $20.93161184-6.90021634-8.10586206$ $22.24043742-5.51093834-6.02725907$ $23.43932276-5.18673572-9.15262337$ $24.28942884-4.79172627-10.23576557$ $25.23363007-5.87142295-10.73780907$ $25.27697219-6.07006873-11.95429022$ $25.10056510-3.57159998-9.88369979$ $24.25457077-2.42835164-9.42214754$ $23.30333065-1.84921793-10.24969395$ $24.39253144-1.92938740-8.10983448$ $22.54582249-0.76839495-9.82372384$ $23.59701166-0.89502082-7.66681659$ $22.68981366-0.28546243-8.52525642$ $22.015673920 .82768499-8.08634469$ $23.62097473-4.54426542-11.06742681$ $21.80976174-0.35088619-10.48308306$ $23.71773282-0.49514843-6.68050064$ $23.85985464-5.31187405-8.23427514$ $25.13004573-2.34477071-7.45350111$ $23.14301282-2.23914380-11.24029681$ $25.66574009-3.24991647-10.75309829$ $25.80233991-3.81439618-9.08884804$ $22.413987991 .63983169-8.42232974$ $26.00001082-6.57935743-9.83191763$ $26.81854055-7.67002752-10.30758716$ $26.20490948-9.07481661-10.11222125$ $26.74549848-10.08932642-10.57425667$ $28.22362871-7.59849453-9.73449075$ $28.24795174-8.14705956-7.89564145$ $25.96439295-6.34214470-8.83636203$ $26.90542506-7.54808188-11.38855763$ $28.60730678-6.58681949-9.82706267$ $28.86727175-8.28347470-10.28239851$ $25.05680527-9.11695991-9.38517385$ $24.39707807-10.38675549-9.15544660$ $25.18353767-11.36536312-8.28326975$ $24.83902878-12.53013236-8.12762681$ $23.92569156-11.10356514-10.43667574$ $22.97211392-10.22930734-11.25174649$ $22.49500866-11.00671989-12.48510801$ $21.75369908-9.82115059-10.43877940$ $22.06908623-9.22738302-9.59522148$ $21.08598811-9.24241372-11.04910910$ $21.25358205-10.71128699-10.07200047$ $24.64901804-8.28854637-8.97220565$

$21.98373197-11.90884189-12.17014735$ $21.82370484-10.39041366-13.05882522$ $23.34583692-11.28594913-13.09191428$ $23.48358681-10.16278950-8.60130440$ $23.49125350-9.33328786-11.57404052$ $23.41671907-12.01151794-10.14049769$ $24.78670867-11.33469780-11.04526070$ $26.23735971-10.83475556-7.62011196$ $26.89251755-11.70550022-6.64584343$ $25.84840223-12.11241036-5.53716182$ $25.17569682-11.27215562-4.98967778$ $28.05817047-10.98710801-5.99264583$ $28.65372822-11.78313378-4.84496277$ $29.95516040-11.13119197-4.32817683$ $30.34667117-11.81875272-3.00923100$ $31.78609910-11.67555826-2.76457269$ $27.23314335-12.61963308-7.14052387$ 
C

$\mathrm{N}$

C

C

C

C

C

$\mathrm{H}$

$\mathrm{H}$

$\mathrm{H}$

$\mathrm{H}$

$\mathrm{H}$

$\mathrm{H}$

$\mathrm{H}$

$\mathrm{H}$

$\mathrm{H}$

$\mathrm{H}$

$\mathrm{N}$

C

$\mathrm{H}$

C

C

O

$\mathrm{N}$

C

C

O

C

C

C

C

C

C

C

$\mathrm{H}$

$\mathrm{H}$

$\mathrm{H}$

$\mathrm{H}$
$29.62592172-5.45299057-12.44848993$ $31.83769725-5.20013214-12.13426979$ $30.91203949-5.98358546-12.75462424$ $28.49947808-6.11763274-12.92563779$ $31.06836250-7.17414249-13.45665168$ $28.66780367-7.31514049-13.58277632$ $29.92622982-7.83749914-13.85962508$ $30.01586073-8.73234065-14.43730103$ $29.58539533-1.64436791-11.78577361$ $32.83355446-5.31174794-12.22128421$ $27.51886576-5.72079611-12.75609233$ $27.80230373-7.85410253-13.89130533$ $32.05292459-7.52715032-13.70165480$ $31.72672017-3.49016198-10.87353820$ $30.36985854-2.51762028-9.13969477$ $27.90024722-3.55766295-11.59037656$ $28.54945650-3.88198892-9.97782179$ $27.43373068-0.49529999-11.53606901$ $26.126997230 .15112271-11.47693681$ $27.94591596-0.38211788-12.41006170$ $14.00733264-19.32514235-8.43975218$ $12.58243994-19.03077971-8.88601322$ $11.66470664-19.12905292-8.10898536$ $12.46517480-18.62210093-10.17099121$ $11.17946360-18.24195904-10.70825860$ $10.79889988-19.00337163-11.95442944$ $9.74619043-18.76747798-12.53130693$ $11.04914590-16.73575005-10.92997468$ $11.55466627-15.97174974-9.73949185$ $10.78587597-15.89904018-8.57156393$ $12.77793624-15.31090373-9.80071890$ $11.26999347-15.19320099-7.47420653$ $13.25148234-14.61938885-8.70215016$ $12.49364902-14.55449177-7.53360946$ $12.88666026-14.03650064-6.68377013$ $10.45218228-18.55197321-9.94928500$ $10.67995669-15.15470427-6.59105029$ $14.21303003-14.14718343-8.76198619$ $13.28127500-18.50123363-10.76272075$ $13.36979435-15.38201046-10.69532240$ $9.83882052-16.39787844-8.52227046$ $10.00792421-16.48629764-11.07994271$ $11.63122112-16.44206200-11.79477042$ $11.60684251-19.99167517-12.32243902$ $11.31272161-20.75706009-13.49680608$ $11.32385834-22.22038087-13.13572028$ $12.21710774-22.72122966-12.48966182$ $12.30788420-20.45702553-14.63731898$ $12.27049516-18.99595760-15.04912461$ $13.37995405-18.76210691-16.06019035$ $10.91901744-18.63767374-15.64539586$ $10.15533308-18.76638255-14.89995065$ $10.93669099-17.61872561-15.98561671$ $10.72115134-19.29400695-16.48611067$ $12.45032390-20.19790606-11.80545302$ $13.23729020-19.38380518-16.93708032$ $13.36153125-17.73205435-16.38330673$ $14.34811891-18.97688783-15.63487205$ $10.31794491-20.46886253-13.83458592$ $12.42928453-18.35072720-14.19181215$ $12.04799951-21.07208491-15.48879094$ $13.30372442-20.71518891-14.30143593$ $10.25843481-22.88882417-13.58434024$ $10.13919696-24.32458736-13.36889572$ $9.60921364-22.49046177-14.25120101$ 


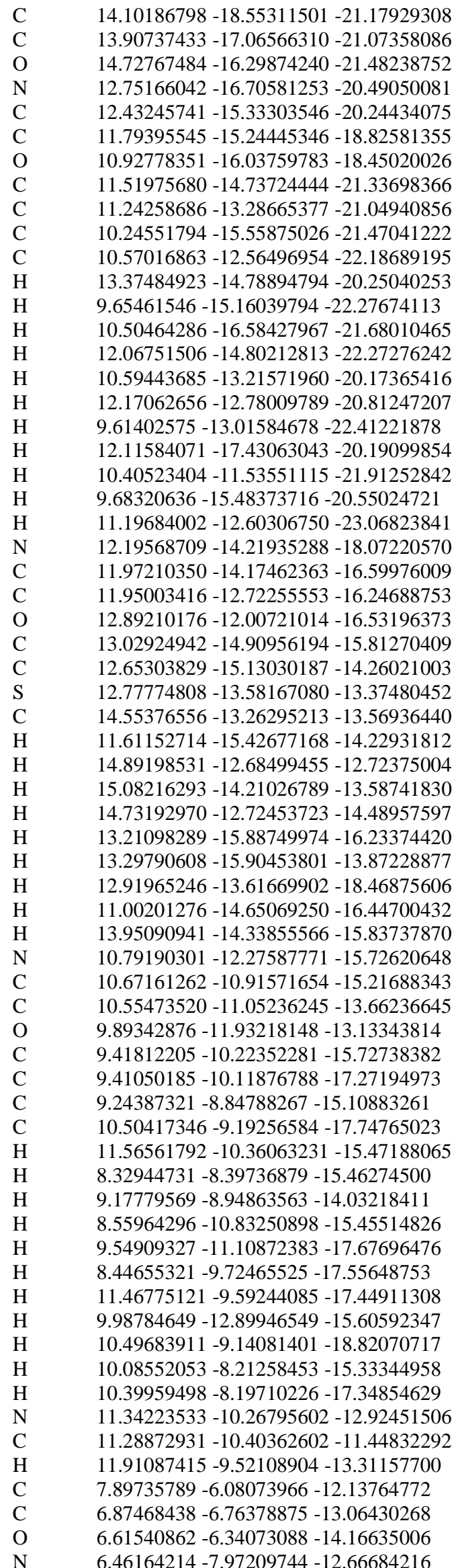

C $\quad 5.62428525-8.76718849-13.50080254$

C $\quad 4.31797992-7.97190308-13.75320216$

O $\quad 3.94736861-7.13640131-12.94285215$

C $\quad 5.35274386-10.12878634-12.91934202$

C $\quad 6.62001118-10.99356777-12.92954912$

C $\quad 6.38418635-12.30987135-12.27169570$

$\mathrm{H} \quad 6.89088122-11.19196601-13.96852979$

$\mathrm{H} \quad 6.12618204-8.87184579-14.47062478$

$\mathrm{H} \quad 7.45278007-10.48840821-12.47149487$

$\mathrm{H} \quad 6.67372655-8.26313125-11.71972708$

$\mathrm{H} \quad 4.57987539-10.61603315-13.50336040$

$\mathrm{H} \quad 4.99999688-10.03939131-11.89652548$

$\mathrm{H} \quad 8.31842167-12.84820948-12.57932561$

O $\quad 5.27784562-12.59977751-11.83648931$

$\mathrm{N} \quad 7.45738729-13.10013245-12.11041920$

$\mathrm{H} \quad 7.34294041-14.04587873-11.77219428$

$\mathrm{N} \quad 3.75186257-8.27036284-14.95831353$

C $\quad 2.46468811-7.77561042-15.40419920$

C $\quad 2.46217358-6.24609080-15.44563332$

O $\quad 1.45648759-5.62503042-15.16112566$

C $\quad 1.34134639-8.29009159-14.47647622$

C $\quad 1.27204837-9.82047697-14.45647507$

C $\quad 0.08185316-10.24820206-13.61597771$

$\mathrm{N} \quad 0.02806759-11.65301754-13.33001535$

C $\quad-0.67160442-12.54474621-14.08236919$

$\mathrm{N} \quad-1.63778899-12.08809801-14.94461946$

$\mathrm{N} \quad-0.41494117-13.90357328-13.94760871$

$\mathrm{H} \quad 2.16652857-10.24380650-14.01798992$

$\mathrm{H} \quad 2.22424914-8.14607078-16.39616732$

$\mathrm{H} \quad 0.72886263-12.03555454-12.69863623$

$\mathrm{H} \quad 1.15114812-10.19122483-15.46990185$

$\mathrm{H} \quad-1.18057124-14.50993035-14.20075503$

$\mathrm{H} \quad-0.01535394-14.15520016-13.05046511$

$\mathrm{H} \quad 4.31269959-8.89873315-15.53797460$

$\mathrm{H} \quad-0.84290560-9.96073917-14.10771919$

$\mathrm{H} \quad 0.10462193-9.73658201-12.65698116$

$\mathrm{H} \quad-2.02295310-12.79594117-15.54954201$

$\mathrm{H} \quad-1.46482856-11.19200825-15.36642956$

$\mathrm{H} \quad 0.40306168-7.88112729-14.82862882$

$\mathrm{H} \quad 1.52153135-7.93054790-13.46875655$

$\mathrm{N} \quad 3.59974646-5.64810983-15.82044046$

C $\quad 3.69616396-4.20337243-15.85145885$

C $\quad 4.27669299-3.65770409-17.17058624$

O $\quad 4.54953835-2.48185956-17.32311921$

C $\quad 4.48937261-3.66061676-14.66655695$

C $\quad 3.66198436-3.54277966-13.40063604$

C $\quad 4.52391349-3.78447461-12.17925499$

$\mathrm{N} \quad 3.87263675-3.32908933-10.96411231$

C $\quad 3.44300791-4.14618513-9.97890004$

$\mathrm{N} \quad 3.30874561-5.47959470-10.19714949$

$\mathrm{N} \quad 3.04616484-3.62427167-8.77598284$

$\mathrm{H} \quad 2.84756182-4.25744788-13.40691263$

$\mathrm{H} \quad 2.67794918-3.81728841-15.76222470$

$\mathrm{H} \quad 3.79473541-2.34050354-10.81015195$

$\mathrm{H} \quad 3.23651944-2.54368578-13.33493381$

$\mathrm{H} \quad 2.55329530-4.27360270-8.18342723$

$\mathrm{H} \quad 2.69553800-2.68398907-8.79050789$

$\mathrm{H} \quad 4.42262171-6.20995730-16.02389028$

$\mathrm{H} \quad 5.46968642-3.25575510-12.26796819$

$\mathrm{H} \quad 4.73223882-4.84667640-12.12083380$

$\mathrm{H} \quad 3.31676372-6.11142199-9.40163622$

$\mathrm{H} \quad 3.91837596-5.84971067-10.91220975$

$\mathrm{H} \quad 4.88586939-2.68701392-14.92978840$

$\mathrm{H} \quad 5.31962378-4.33765465-14.47832101$

$\mathrm{N} \quad 4.26317538-4.52927202-18.20385083$ 


\begin{tabular}{|c|c|}
\hline $\mathrm{C}$ & $4.75201207-4.12781691-19.49316648$ \\
\hline $\mathrm{C}$ & $6.23287836-4.48726199-19.85147129$ \\
\hline $\mathrm{O}$ & $6.57306115-4.41780481-21.03680711$ \\
\hline $\mathrm{H}$ & $4.00851337-5.49921126-18.04460324$ \\
\hline $\mathrm{H}$ & $4.14264761-4.59443769-20.26500750$ \\
\hline $\mathrm{H}$ & $4.68790009-3.03897766-19.55088622$ \\
\hline $\mathrm{N}$ & $7.02453343-4.84709384-18.83136849$ \\
\hline $\mathrm{C}$ & $8.42301083-5.18349333-19.00446347$ \\
\hline $\mathrm{C}$ & $8.58724952-6.49871921-19.77029055$ \\
\hline $\mathrm{O}$ & $9.39615002-6.56371214-20.67856015$ \\
\hline $\mathrm{C}$ & $9.17326408-5.24896352-17.63134878$ \\
\hline $\mathrm{C}$ & $9.10444573-3.95046505-16.88755183$ \\
\hline $\mathrm{C}$ & $9.95684320-2.89311688-17.13799208$ \\
\hline $\mathrm{C}$ & $8.17431172-3.80198299-15.87535482$ \\
\hline $\mathrm{C}$ & $9.88904090-1.72636969-16.40605764$ \\
\hline $\mathrm{C}$ & $8.06875701-2.63151395-15.14072106$ \\
\hline $\mathrm{C}$ & $8.95614720-1.58539787-15.37264553$ \\
\hline $\mathrm{O}$ & $8.78789555-0.44472888-14.66708915$ \\
\hline $\mathrm{H}$ & $8.89563330-4.40136724-19.60272265$ \\
\hline $\mathrm{H}$ & $10.58420562-0.92708182-16.58594386$ \\
\hline $\mathrm{H}$ & $7.33578473-2.54814691-14.36836212$ \\
\hline $\mathrm{H}$ & $6.63520330-4.90100336-17.89680150$ \\
\hline $\mathrm{H}$ & $7.51443923-4.61698125-15.64517568$ \\
\hline $\mathrm{H}$ & $10.71095811-2.97102570-17.89400551$ \\
\hline $\mathrm{H}$ & $10.20390030-5.45464698-17.87200283$ \\
\hline $\mathrm{H}$ & $8.75861916-6.04766214-17.03507856$ \\
\hline $\mathrm{H}$ & $9.634121720 .11395237-14.78284091$ \\
\hline $\mathrm{N}$ & $7.79253653-7.52348532-19.37929116$ \\
\hline $\mathrm{C}$ & $7.78709069-8.77917971-20.07603082$ \\
\hline $\mathrm{C}$ & $7.39496442-8.59555302-21.61603831$ \\
\hline $\mathrm{O}$ & $7.96878417-9.24161004-22.46851835$ \\
\hline $\mathrm{H}$ & $7.07732840-7.34813508-18.68373192$ \\
\hline $\mathrm{H}$ & $8.78276706-9.21835834-20.10012598$ \\
\hline $\mathrm{H}$ & $7.08139209-9.46628877-19.62533366$ \\
\hline $\mathrm{N}$ & $6.50761377-7.58610034-21.82844437$ \\
\hline $\mathrm{C}$ & $5.96204095-7.33209767-23.13782544$ \\
\hline $\mathrm{C}$ & $7.07410616-6.70047117-24.00209075$ \\
\hline $\mathrm{O}$ & $7.19170238-6.98127264-25.18564706$ \\
\hline $\mathrm{C}$ & $4.74979917-6.41087627-23.07817218$ \\
\hline $\mathrm{C}$ & $4.14651973-6.05115254-24.45610624$ \\
\hline $\mathrm{C}$ & $2.93693989-5.16107754-24.20191452$ \\
\hline $\mathrm{N}$ & $2.27400786-4.74323272-25.40823891$ \\
\hline $\mathrm{C}$ & $1.14791509-4.00364758-25.43365755$ \\
\hline $\mathrm{N}$ & $0.66067367-3.50291327-24.23894312$ \\
\hline $\mathrm{N}$ & $0.68988240-3.51756329-26.60520359$ \\
\hline $\mathrm{H}$ & $3.81409579-6.93997422-24.97928909$ \\
\hline $\mathrm{H}$ & $5.67486801-8.26885834-23.63463219$ \\
\hline $\mathrm{H}$ & $2.64741798-5.05570777-26.30446958$ \\
\hline $\mathrm{H}$ & $4.86884036-5.51887482-25.05380301$ \\
\hline $\mathrm{H}$ & $-0.25275814-3.16955624-26.66943542$ \\
\hline $\mathrm{H}$ & $1.00436686-3.96579190-27.45952942$ \\
\hline $\mathrm{H}$ & $5.97677942-7.31387588-21.00575452$ \\
\hline $\mathrm{H}$ & $3.26402234-4.28074552-23.65385332$ \\
\hline $\mathrm{H}$ & $2.22779881-5.72698883-23.59135602$ \\
\hline $\mathrm{H}$ & $-0.24787746-3.05879359-24.33279741$ \\
\hline $\mathrm{H}$ & $0.78311510-4.15962171-23.48924551$ \\
\hline $\mathrm{H}$ & $5.01295693-5.49100410-22.57226961$ \\
\hline $\mathrm{H}$ & $3.98084769-6.92670337-22.51045774$ \\
\hline $\mathrm{N}$ & $7.75366634-5.75397196-23.33606150$ \\
\hline $\mathrm{C}$ & $8.91851202-5.13407263-23.92996000$ \\
\hline $\mathrm{H}$ & $7.60877129-5.58253842-22.34922060$ \\
\hline $\mathrm{C}$ & $11.29384618-8.06821768-23.87305515$ \\
\hline $\mathrm{C}$ & $10.87738344-8.98568545-25.03405831$ \\
\hline $\mathrm{O}$ & $11.70382290-9.33556849-25.85993609$ \\
\hline $\mathrm{N}$ & $9.58709193-9.38611921-24.95100128$ \\
\hline
\end{tabular}

C $\quad 9.07890781-10.20087136-26.04480191$ C $\quad 9.21030583-9.41250271-27.36735603$ O $\quad 9.78426696-9.87427562-28.31977647$ C $\quad 7.63638009-10.68204743-25.78862501$ C $\quad 7.61604574-11.64910026-24.61517875$ C $\quad 7.11067332-11.36615139-27.03392773$ C $\quad 6.21314958-11.91123547-24.11010172$ $\mathrm{H} \quad 9.73619478-11.06956101-26.15866254$ $\mathrm{H} \quad 6.08738376-11.66648950-26.86136700$ $\mathrm{H} \quad 7.13463010-10.68322649-27.86768550$ $\mathrm{H} \quad 7.03544288-9.80516634-25.56081237$ $\mathrm{H} \quad 8.09053835-12.57528342-24.91757033$ $\mathrm{H} \quad 8.19185951-11.22062480-23.80374577$ $\mathrm{H} \quad 5.62880647-12.36002118-24.90602943$ $\mathrm{H} \quad 9.00293916-9.11998278-24.17601952$ $\mathrm{H} \quad 6.26696264-12.59581202-23.28242142$ $\mathrm{H} \quad 7.71173515-12.23118984-27.26155986$ $\mathrm{H} \quad 5.74768197-10.98461377-23.81384121$ $\mathrm{N} \quad 8.75190519-8.16432274-27.37131658$ C $\quad 8.84974772-7.32505707-28.54791177$ $\mathrm{H} \quad 8.25521650-7.76544748-26.58692100$ C $\quad 6.58969445-21.92076189-21.79764284$ C $\quad 6.21540378-22.03059166-20.30638962$ O $\quad 5.51742192-22.93016760-19.88486193$ $\mathrm{N} \quad 6.58455511-20.95531600-19.60359106$ C $\quad 6.10546422-20.90583963-18.22475257$ C $\quad 4.59899375-20.55885810-18.34683873$ O $\quad 4.09718101-20.19880454-19.40726471$ C $\quad 6.80683719-19.84459839-17.39066246$ O $\quad 6.64642324-18.56324279-17.96410978$ $\mathrm{H} \quad 7.28456015-20.30668918-19.93919926$ $\mathrm{H} \quad 6.22296583-21.88909740-17.76546483$ $\mathrm{H} \quad 7.86187899-20.10257741-17.30425637$ $\mathrm{H} \quad 6.36236690-19.82931587-16.39573779$ $\mathrm{H} \quad 7.52886386-18.29538766-18.30043730$ $\mathrm{N} \quad 3.93277000-20.54849896-17.16592309$ C $\quad 2.51749596-20.23127823-17.17061910$ C $\quad 2.35146447-18.74427647-17.58203836$ O $\quad 1.52121486-18.35294357-18.38421386$ C $\quad 1.92455245-20.50876666-15.78876816$ $\mathrm{H} \quad 4.31552931-21.06287699-16.38161653$ $\mathrm{H} \quad 1.98253687-20.83347106-17.90702865$ $\mathrm{N} \quad 3.21194487-17.89294014-17.00509015$ C $\quad 3.09744250-16.46653607-17.24614572$ C $\quad 3.61774115-16.06345665-18.63318006$ O $\quad 3.11006578-15.15228759-19.30116711$ C $\quad 3.82244759-15.70126208-16.16717955$ C $\quad 3.77594916-14.17528542-16.25195858$ C $\quad 2.35309390-13.68084238-16.46413389$ C $\quad 4.33086928-13.62784346-14.92217811$ $\mathrm{H} \quad 5.34002205-13.97129668-14.76522594$ $\mathrm{H} \quad 4.30006934-12.54960326-14.93594230$ $\mathrm{H} \quad 3.71311171-13.97592199-14.10100476$ $\mathrm{H} \quad 3.88274111-18.24922958-16.32296063$ $\mathrm{H} \quad 1.72545124-14.03069152-15.64937551$ $\mathrm{H} \quad 2.35136313-12.59791856-16.44315168$ $\mathrm{H} \quad 1.97124360-14.02482153-17.40619831$ $\mathrm{H} \quad 2.03811002-16.20017759-17.22398523$ $\mathrm{H} \quad 4.41303909-13.80845219-17.05089122$ $\mathrm{H} \quad 4.87018449-15.98564025-16.17582305$ $\mathrm{H} \quad 3.40260526-15.99044614-15.20735662$ $\mathrm{N} \quad 4.66504390-16.72690771-19.06830569$ C $\quad 5.13651209-16.53095228-20.42165363$ C $\quad 4.04893395-16.92969396-21.45447692$ O $\quad 3.82217986-16.18345211-22.37794833$ 

$5.68220533-9.84636191-16.69140233$ $4.37403241-10.33787751-18.52710235$ $3.95683930-11.04989063-19.12499129$ $3.43804837-9.34470101-18.20169696$ $\mathrm{H} \quad 3.50045150-9.09559483-17.20669738$ $5.00081626-7.56585976-19.41069218$ $2.83107821-6.86212871-18.43551215$ $2.78705807-8.36602848-20.68731931$ $1.62426440-7.64954686-21.07977421$ $1.14442043-6.97163037-20.38818657$ $1.05352084-7.91649525-22.33500584$ $0.11609799-7.45185416-22.60683411$ $1.60740459-8.87482589-23.19295217$ $1.13404897-9.11800220-24.13613806$ $2.78272057-9.53438033-22.84169565$ $3.22524353-10.26482436-23.50756905$ $3.35710523-9.27690089-21.59835950$ $4.51955847-9.97303422-21.28309148$ $28.08448875-6.24451569-6.90568749$ $-2.08112447-5.82863740-23.88786699$ $-2.90462471-5.38071291-23.59335653$ $-2.05728239-6.61107165-23.33123895$ $7.33632982-19.80957570-13.54025489$ $8.19406527-19.42987300-13.28587547$ $7.26599469-20.64490332-13.06612822$ $9.22368062-17.93419302-18.98595054$ $9.90921659-17.29938161-18.70924440$ $9.57363264-18.41921146-19.73692957$ $5.95958765-7.07211106-16.80178679$ $6.35248172-6.81966767-15.94617283$ $5.98223949-8.04810784-16.82136906$ $26.49193796-0.94137612-4.85440485$ $27.13594336-0.82382673-4.13866384$ $26.58627895-1.84080295-5.18498544$ $0.68042045-10.01634455-18.05996251$ $-0.10372856-10.49922237-18.42940454$ $1.40361060-10.25704209-18.64086349$ $4.77644016-18.69621731-14.48879252$ $5.69966673-18.93392304-14.29198063$ $4.26828731-19.21695909-13.85579806$ $-0.45009236-6.71248062-25.79476169$ $-0.81725293-7.44246602-26.30075918$ $-1.12326918-6.43143876-25.16921924$

\section{C WF-in-DFT/MM R1b (2C-CCSD-in-B3LYP/6- 31G(d):Amber) mechanical embedding $\omega_{\alpha}=1.667$ including $B$ factors}

C $\quad 21.94051230-10.35799734-4.21780018$

C $\quad 22.25637491-8.88878619-4.41745086$ 
O $\quad 21.42063857-8.01790473-4.34986592$

$\mathrm{N} \quad 23.52792260-8.66482181-4.76907916$

C $\quad 23.89464868-7.38700718-5.36713765$

C $\quad 23.06546307-7.23964510-6.66944239$

O $\quad 23.13089562-8.10209151-7.53968613$

C $\quad 25.38728585-7.35691680-5.80949028$

S $\quad 25.80835503-5.71665459-6.50477240$

$\mathrm{H} \quad 24.15961566-9.46176574-4.86297495$

$\mathrm{H} \quad 23.69485059-6.58058026-4.65760738$

$\mathrm{H} \quad 25.60709337-8.19504793-6.47872976$

$\mathrm{H} \quad 25.95931350-7.51781168-4.88051707$

$\mathrm{N} \quad 22.37200360-6.08981625-6.79345017$

C $\quad 21.45687274-5.93119478-7.92202629$

$\mathrm{H} \quad 22.17694716-5.51297751-5.97838188$

C $\quad 24.31358558-4.85876801-10.21384602$

C $\quad 25.25416471-5.90418948-10.74149895$

O $\quad 25.25342018-6.08404794-11.94889138$

$\mathrm{N} \quad 26.00892366-6.59737626-9.82808700$

C $\quad 26.83990208-7.67152183-10.30490730$

C $\quad 26.23427013-9.07339612-10.12878016$

O $\quad 26.77205430-10.08672623-10.57681326$

C $\quad 28.23894960-7.59886081-9.71256138$

$\mathrm{S} \quad 28.23838425-8.15068800-7.88539896$

$\mathrm{H} \quad 25.98439894-6.33120828-8.83851371$

H $\quad 26.91923773-7.54489791-11.38920545$

$\mathrm{H} \quad 28.61075072-6.57576419-9.82134117$

$\mathrm{H} \quad 28.88901548-8.27706703-10.27818352$

$\mathrm{N} \quad 25.08019504-9.11687977-9.41712108$

C $\quad 24.41083148-10.40521118-9.18036592$

$\mathrm{H} \quad 24.60384509-8.30516936-9.04000924$

C $\quad 29.87597387-4.490832142 .81070020$

C $\quad 30.09002231-4.510019111 .26044428$

O $\quad 30.68173359-3.654399510 .65890257$

$\mathrm{N} \quad 29.50663000-5.566348610 .64586121$

C $\quad 29.56754018-5.62491396-0.82000874$

C $\quad 29.06408619-4.33207150-1.43544634$

O $\quad 29.78910189-3.74765535-2.22284014$

C $\quad 28.85029517-6.84559719-1.37008616$

C $\quad 28.95396443-6.94583529-2.84964089$

$\mathrm{H} \quad 29.28937817-7.73608798-0.91404919$

$\mathrm{H} \quad 28.94887046-6.236088211 .17188168$

$\mathrm{H} \quad 30.62842998-5.64867156-1.09391679$

$\mathrm{H} \quad 27.79219433-6.79546190-1.10562895$

C $\quad 28.14917649-6.46027068-3.85853782$

$\mathrm{H} \quad 30.87816429-7.85969650-3.01925125$

$\mathrm{H} \quad 27.23946599-5.88896973-3.75944666$

C $\quad 29.81084277-7.47678653-4.81330066$

$\mathrm{N} \quad 30.01258289-7.59612577-3.48257776$

$\mathrm{N} \quad 28.66842539-6.88753734-5.02553612$

$\mathrm{H} \quad 30.49677466-7.85966970-5.55473717$

$\mathrm{N} \quad 27.84491702-3.87907740-1.04813792$

C $\quad 27.28002262-2.63940483-1.54939069$

$\mathrm{H} \quad 27.32568509-4.34231316-0.30086210$

C $\quad 32.66620370-1.85100711-2.41941337$

C $\quad 32.41393324-1.96564375-3.94238416$

O $\quad 33.16443939-1.50658488-4.79020278$

$\mathrm{N} \quad 31.30906487-2.66271416-4.22921402$

C $\quad 30.97885828-3.03444167-5.61169838$

C $\quad 30.86941394-1.72413939-6.40332914$

O $\quad 30.13311942-0.83496361-6.05927666$

C $\quad 29.67130921-3.82536030-5.60463377$

S $\quad 29.20147092-4.39242911-7.29064155$

$\mathrm{H} \quad 30.75543372-3.02022576-3.46213470$

$\mathrm{H} \quad 31.78053971-3.66749618-6.01687860$

H $\quad 28.88643207-3.17462405-5.19544363$
$\mathrm{H} \quad 29.80984801-4.67253907-4.92630369$

$\mathrm{N} \quad 31.59783875-1.60620356-7.50550317$

C $\quad 31.62322509-0.46738690-8.37684343$

$\mathrm{H} \quad 32.31487521-2.24660412-7.72732145$

C $\quad 7.67619198-15.97266621-16.35304340$

$\mathrm{H} \quad 7.10526556-16.36263485-17.18046685$

C $\quad 8.06731548-16.78754457-15.30378601$

$\mathrm{H} \quad 7.85391872-17.84653647-15.32404306$

C $\quad 8.75144245-16.18777121-14.25140810$

$\mathrm{H} \quad 9.13298667-16.75572854-13.43152528$

C $\quad 8.97630035-14.82356635-14.27721491$

$\mathrm{H} \quad 9.55533975-14.35515025-13.49255791$

$\mathrm{N} \quad 8.50225172-13.99826135-15.23970186$

C $\quad 7.91137399-14.62447960-16.26856921$

C $\quad 7.39831418-13.77953614-17.34618528$

C $\quad 7.53124109-14.19826321-18.68698826$

C $\quad 7.00632224-13.37431105-19.68278373$

C $\quad 6.41197946-12.15473448-19.38901899$

$\mathrm{H} \quad 6.00814585-11.55428302-20.17006057$

F $\quad 7.07357741-13.77345765-20.98580234$

$\mathrm{H} \quad 8.07403550-15.10672591-18.92253443$

C $\quad 6.79924090-12.57368881-17.01762132$

$\mathrm{H} \quad 6.68201540-12.33338809-15.97425771$

C $\quad 6.26164428-11.78445712-18.02781126$

C $\quad 5.45569233-10.60524001-17.66527197$

O $\quad 5.70456368-9.85440229-16.72092926$

$\mathrm{N} \quad 4.36201270-10.33320608-18.50057629$

$\mathrm{H} \quad 3.96074663-11.03439266-19.13067887$

$\mathrm{N} \quad 3.43274272-9.33456730-18.19185065$

$\mathrm{H} \quad 3.43920989-9.11626004-17.19420350$

S $\quad 3.59207829-7.94958049-19.06621681$

O $\quad 4.97596990-7.57192890-19.41696461$

O $\quad 2.80962003-6.87165081-18.43476784$

C $\quad 2.76466956-8.38431891-20.69399149$

C $\quad 1.61276225-7.67328634-21.08631276$

$\mathrm{H} \quad 1.12171617-6.99567731-20.40174915$

C $\quad 1.04415999-7.93932627-22.34950428$

$\mathrm{H} \quad 0.09729161-7.49551505-22.61980249$

C $\quad 1.60894577-8.89226475-23.19603283$

$\mathrm{H} \quad 1.14873348-9.13656939-24.15056512$

C $\quad 2.78343449-9.53429576-22.84540977$

$\mathrm{H} \quad 3.26806935-10.23535590-23.51995370$

C $\quad 3.34916899-9.28453171-21.59400757$

F $\quad 4.52000965-9.96072343-21.29062810$

$\mathrm{Zn} \quad 28.08092433-6.23401021-6.90730545$

\section{C WF-in-DFT/SE/MM R1b (2C-CCSD-in- B3LYP/6-31G(d):xTB:Amber) mechanical embedding $\omega_{\alpha}=1.667$ including $B$ factors}

C $\quad 22.27401658-8.92022241-4.42330374$

O $\quad 21.42733192-8.05536294-4.33582911$

$\mathrm{N} \quad 23.54044048-8.67556138-4.78315713$

C $\quad 23.89241636-7.39442869-5.37702940$

C $\quad 23.06361428-7.24364703-6.68469664$

O $\quad 23.12674562-8.11162044-7.55190567$

C $\quad 25.38772754-7.35257865-5.83505796$

$\mathrm{S} \quad 25.80846324-5.70999815-6.50521253$

$\mathrm{H} \quad 24.15589591-9.47059527-4.95025578$

$\mathrm{H} \quad 23.69067369-6.58186507-4.66724850$

$\mathrm{H} \quad 25.58574938-8.18187292-6.53099025$

$\mathrm{H} \quad 25.97656715-7.55818967-4.91991863$

$\mathrm{N} \quad 22.36684002-6.09183529-6.81790638$

H $\quad 22.18174874-5.54150746-5.98171035$ 
C $\quad 25.25096854-5.91047805-10.75127341$

O $\quad 25.31840868-6.06224332-11.96725416$

$\mathrm{N} \quad 26.00198104-6.61621594-9.83459703$

C $\quad 26.82149949-7.70297790-10.30260957$

C $\quad 26.20227646-9.10908824-10.10612773$

O $\quad 26.73110230-10.12270230-10.56834487$

C $\quad 28.22846060-7.62201394-9.71649631$

$\mathrm{S} \quad 28.24443861-8.16262148-7.89800247$

$\mathrm{H} \quad 25.94441315-6.38946290-8.83424360$

$\mathrm{H} \quad 26.89782582-7.58478883-11.39213759$

$\mathrm{H} \quad 28.60790014-6.59958662-9.85007660$

$\mathrm{H} \quad 28.87762798-8.30616065-10.28539637$

$\mathrm{N} \quad 25.05988406-9.15251783-9.36542652$

$\mathrm{H} \quad 24.59609337-8.32620532-9.00622246$

C $\quad 30.09490135-4.488749881 .27999165$

O $\quad 30.68508105-3.641072950 .66440396$

$\mathrm{N} \quad 29.50541141-5.546573720 .65843343$

C $\quad 29.55446074-5.61025308-0.80941921$

C $\quad 29.05412728-4.31824181-1.44211508$

O $\quad 29.79565467-3.73331304-2.21278393$

C $\quad 28.83821823-6.83862599-1.35411792$

C $\quad 28.94246427-6.94057146-2.83277260$

H $\quad 29.27511552-7.73444595-0.88917386$

$\mathrm{H} \quad 28.94959221-6.223992671 .17663915$

$\mathrm{H} \quad 30.61565358-5.63412665-1.09938744$

$\mathrm{H} \quad 27.77611815-6.79979924-1.08792538$

C $\quad 28.14643133-6.45826869-3.85223103$

$\mathrm{H} \quad 30.88489769-7.84125393-3.00079998$

$\mathrm{H} \quad 27.23282742-5.87859369-3.77011509$

C $\quad 29.80014173-7.48459562-4.80430773$

$\mathrm{N} \quad 30.00484660-7.61318323-3.46296810$

$\mathrm{N} \quad 28.67164716-6.88846234-5.01116411$

$\mathrm{H} \quad 30.48076568-7.87142480-5.55694993$

$\mathrm{N} \quad 27.82942410-3.85491270-1.07210843$

$\mathrm{H} \quad 27.28168546-4.34365411-0.36174909$

C $\quad 32.41914184-1.95042669-3.93183107$

O $\quad 33.16672750-1.49092330-4.78081610$

$\mathrm{N} \quad 31.31131119-2.65242182-4.22934957$

C $\quad 30.98000832-3.02598536-5.61127276$

C $\quad 30.87825210-1.71778939-6.41655249$

O $\quad 30.17665092-0.80434488-6.06524190$

C $\quad 29.66889114-3.81869669-5.61331106$

S $\quad 29.20429000-4.37799384-7.29667101$

$\mathrm{H} \quad 30.75757213-3.02762882-3.47089103$

$\mathrm{H} \quad 31.78689967-3.66441485-6.01448904$

$\mathrm{H} \quad 28.89167735-3.16140425-5.18566862$

$\mathrm{H} \quad 29.80842443-4.66005060-4.91539096$

$\mathrm{N} \quad 31.60765078-1.60307658-7.52526058$

$\mathrm{H} \quad 32.10302936-2.39139668-7.87494003$

C $\quad 7.68460720-15.98404754-16.35653576$

H $\quad 7.13081347-16.37159102-17.20030424$

C $\quad 8.06703263-16.79860430-15.30709063$

$\mathrm{H} \quad 7.84931414-17.85623247-15.32361054$

C $\quad 8.74618073-16.20680597-14.23796221$

$\mathrm{H} \quad 9.11321109-16.80961028-13.42916944$

C $\quad 8.98224033-14.84245938-14.26480068$

$\mathrm{H} \quad 9.56034223-14.35978146-13.48526342$

$\mathrm{N} \quad 8.51714565-14.00442748-15.23168467$

C $\quad 7.91127307-14.62536177-16.26871556$

C $\quad 7.39807665-13.77922213-17.34040785$

C $\quad 7.51798033-14.21351622-18.69493393$

C $\quad 6.99501959-13.37122908-19.68185653$

C $\quad 6.40856619-12.15203676-19.40391364$

$\mathrm{H} \quad 6.02806015-11.54044248-20.18722898$

F $\quad 7.06900551-13.77563570-20.99507306$
H $\quad 8.07207037-15.11722032-18.92519171$

C $\quad 6.82580787-12.56399122-17.01815806$

$\mathrm{H} \quad 6.72709407-12.31192216-15.97198963$

C $\quad 6.26958496-11.75813311-18.01944748$

C $\quad 5.46771162-10.61725083-17.66111899$

O $\quad 5.69467564-9.83746351-16.67936049$

$\mathrm{N} \quad 4.38755872-10.33680712-18.52707656$

$\mathrm{H} \quad 3.96398325-11.05063454-19.11518942$

$\mathrm{N} \quad 3.45870117-9.34680318-18.19616704$

$\mathrm{H} \quad 3.51533038-9.09722107-17.20141739$

S $\quad 3.60790504-7.95990892-19.07742365$

O $\quad 5.01245898-7.55395440-19.41859862$

O $\quad 2.84691037-6.86630593-18.41266477$

C $\quad 2.79103689-8.37642175-20.66150838$

C $\quad 1.62310837-7.64527344-21.05991338$

$\mathrm{H} \quad 1.14471625-6.96960246-20.36345822$

C $\quad 1.05576188-7.91010247-22.31410065$

$\mathrm{H} \quad 0.11051064-7.44957712-22.57905127$

C $\quad 1.60122000-8.87552024-23.17644187$

$\mathrm{H} \quad 1.12252912-9.12333676-24.11681634$

C $\quad 2.78106093-9.53919454-22.82648558$

$\mathrm{H} \quad 3.22140998-10.26772493-23.49686466$

C $\quad 3.36183826-9.28637375-21.59005159$

F $\quad 4.52942354-9.99018740-21.29258692$

Zn $\quad 28.08229227-6.23943430-6.90760889$

\section{C QM/MM R1b (B3LYP/6-31G(d):Amber) \\ mechanical embedding $\omega_{\alpha}=1.667$}

C $\quad 21.93944613-10.35331763-4.22240600$

C $\quad 22.24711678-8.89009438-4.43052047$

O $\quad 21.40507677-8.01104709-4.35332935$

$\mathrm{N} \quad 23.51659206-8.66762391-4.80148808$

C $\quad 23.89512661-7.38418327-5.38185053$

C $\quad 23.07111559-7.22670851-6.68330161$

O $\quad 23.13859188-8.07986952-7.57063711$

C $\quad 25.39655539-7.36389985-5.80518457$

S $\quad 25.83226407-5.73231388-6.52612871$

$\mathrm{H} \quad 24.15640266-9.45877900-4.86323560$

$\mathrm{H} \quad 23.69715714-6.58462769-4.66647169$

$\mathrm{H} \quad 25.61906533-8.20853974-6.46303163$

$\mathrm{H} \quad 25.95387648-7.50611250-4.86711745$

N $\quad 22.36560731-6.08516244-6.79319254$

C $\quad 21.45927119-5.92812617-7.91957611$

$\mathrm{H} \quad 22.17853706-5.50864658-5.97892831$

C $\quad 24.31486653-4.85811935-10.21474579$

C $\quad 25.24487132-5.91039522-10.73133102$

O $\quad 25.23447530-6.11408071-11.94258079$

$\mathrm{N} \quad 26.00545653-6.59576077-9.80983388$

C $\quad 26.82900289-7.67672881-10.28695168$

C $\quad 26.22641367-9.06824193-10.09786026$

O $\quad 26.74788981-10.09032721-10.56097945$

C $\quad 28.23959229-7.58526367-9.73395265$

S $\quad 28.26866263-8.13034854-7.89379493$

$\mathrm{H} \quad 25.98388834-6.32308543-8.82540577$

$\mathrm{H} \quad 26.89595249-7.55724581-11.37274839$

$\mathrm{H} \quad 28.59855887-6.56162439-9.84548621$

$\mathrm{H} \quad 28.87880172-8.27007088-10.29680699$

N $\quad 25.07419824-9.10777799-9.38506322$

C $\quad 24.40941007-10.40627415-9.17346176$

H $\quad 24.59659740-8.29911484-9.01208674$

C $\quad 29.87806132-4.489388312 .81336424$

C $\quad 30.09617890-4.497565911 .26552990$

O $\quad 30.70774033-3.647749750 .66713150$ 


\begin{tabular}{|c|c|}
\hline $\mathrm{N}$ & $29.50287700-5.543035270 .64512067$ \\
\hline $\mathrm{C}$ & $29.58115060-5.61575548-0.82671960$ \\
\hline $\mathrm{C}$ & $29.08165379-4.33058108-1.44422563$ \\
\hline $\mathrm{O}$ & $29.80985180-3.76532285-2.23876622$ \\
\hline $\mathrm{C}$ & $28.85244121-6.83503526-1.37959194$ \\
\hline $\mathrm{C}$ & $28.96235323-6.94978561-2.85235514$ \\
\hline $\mathrm{H}$ & $29.28777090-7.72325528-0.91589446$ \\
\hline $\mathrm{H}$ & $28.95214502-6.217078861 .16993931$ \\
\hline $\mathrm{H}$ & $30.64250915-5.65475385-1.08942737$ \\
\hline $\mathrm{H}$ & $27.79569262-6.77271342-1.11508206$ \\
\hline $\mathrm{C}$ & $28.16631640-6.46504410-3.85966823$ \\
\hline $\mathrm{H}$ & $30.87327756-7.88805277-3.01483347$ \\
\hline $\mathrm{H}$ & $27.26452667-5.88606597-3.76207004$ \\
\hline $\mathrm{C}$ & $29.81904232-7.49670741-4.80782501$ \\
\hline $\mathrm{N}$ & $30.00823956-7.61872889-3.48021855$ \\
\hline $\mathrm{N}$ & $28.69099757-6.86507214-5.03619340$ \\
\hline $\mathrm{H}$ & $30.50408091-7.87628581-5.54307418$ \\
\hline $\mathrm{N}$ & $27.85638155-3.88129930-1.06097509$ \\
\hline $\mathrm{C}$ & $27.28380742-2.64304231-1.55372964$ \\
\hline $\mathrm{H}$ & $27.35763462-4.33155985-0.29529816$ \\
\hline $\mathrm{C}$ & $32.66419561-1.85224232-2.42418191$ \\
\hline $\mathrm{C}$ & $32.40878240-1.95377860-3.94355374$ \\
\hline $\mathrm{O}$ & $33.14156575-1.46673312-4.79934562$ \\
\hline $\mathrm{N}$ & $31.31010309-2.66000521-4.23301941$ \\
\hline $\mathrm{C}$ & $30.97287175-3.04547743-5.62208245$ \\
\hline $\mathrm{C}$ & $30.85234139-1.73647708-6.40549865$ \\
\hline $\mathrm{O}$ & $30.11803936-0.84830271-6.04912029$ \\
\hline $\mathrm{C}$ & $29.67040767-3.84552132-5.59058817$ \\
\hline $\mathrm{S}$ & $29.20125526-4.43224089-7.28319292$ \\
\hline $\mathrm{H}$ & $30.76719012-3.02011585-3.46379387$ \\
\hline $\mathrm{H}$ & $31.77563902-3.67394720-6.02446601$ \\
\hline $\mathrm{H}$ & $28.88343825-3.19461686-5.19678412$ \\
\hline $\mathrm{H}$ & $29.82229438-4.68969955-4.91713562$ \\
\hline $\mathrm{N}$ & $31.59429283-1.60668725-7.50406685$ \\
\hline $\mathrm{C}$ & $31.62342357-0.46992573-8.37288408$ \\
\hline $\mathrm{H}$ & $32.28496998-2.26719635-7.73610876$ \\
\hline $\mathrm{C}$ & $7.66112807-15.95912726-16.33722496$ \\
\hline $\mathrm{H}$ & $7.09269744-16.35283475-17.16529337$ \\
\hline $\mathrm{C}$ & $8.05905716-16.77351510-15.29254538$ \\
\hline $\mathrm{H}$ & $7.84943650-17.83284517-15.31302005$ \\
\hline $\mathrm{C}$ & $8.75872354-16.17313229-14.24796094$ \\
\hline $\mathrm{H}$ & $9.14772966-16.74328557-13.43553768$ \\
\hline $\mathrm{C}$ & $8.98709124-14.80736071-14.27589457$ \\
\hline $\mathrm{H}$ & $9.57052972-14.33615956-13.49812254$ \\
\hline $\mathrm{N}$ & $8.48957362-13.98236106-15.22670878$ \\
\hline $\mathrm{C}$ & $7.90094299-14.60943055-16.25647274$ \\
\hline $\mathrm{C}$ & $7.40080550-13.76544055-17.33915142$ \\
\hline $\mathrm{C}$ & $7.53588377-14.18364039-18.67812452$ \\
\hline $\mathrm{C}$ & $7.00453167-13.36550326-19.67477654$ \\
\hline $\mathrm{C}$ & $6.39758995-12.15539427-19.37477015$ \\
\hline $\mathrm{H}$ & $5.99772420-11.55221150-20.15513405$ \\
\hline $\mathrm{F}$ & $7.06983418-13.75290887-20.97787938$ \\
\hline $\mathrm{H}$ & $8.08290121-15.08815082-18.91344867$ \\
\hline $\mathrm{C}$ & $6.79010081-12.56687197-17.01199100$ \\
\hline $\mathrm{H}$ & $6.66379745-12.33125523-15.96928160$ \\
\hline $\mathrm{C}$ & $6.24761321-11.78568997-18.01936223$ \\
\hline $\mathrm{C}$ & $5.43974503-10.60122510-17.65989032$ \\
\hline $\mathrm{O}$ & $5.67979319-9.85719318-16.70914472$ \\
\hline $\mathrm{N}$ & $4.35078147-10.33177817-18.48634362$ \\
\hline $\mathrm{H}$ & $3.95301326-11.02283075-19.12974450$ \\
\hline $\mathrm{N}$ & $3.43822624-9.31177760-18.19930396$ \\
\hline $\mathrm{H}$ & $3.45344396-9.08514157-17.20133061$ \\
\hline $\mathrm{S}$ & $3.59592249-7.93548628-19.07447330$ \\
\hline $\mathrm{O}$ & $4.97759343-7.56019184-19.42295116$ \\
\hline $\mathrm{O}$ & $2.80703095-6.86715170-18.43424114$ \\
\hline
\end{tabular}

$\begin{array}{ll}\mathrm{C} & 2.75431326-8.38332375-20.70053234 \\ \mathrm{C} & 1.60603816-7.67298834-21.10112254 \\ \mathrm{H} & 1.11580113-6.99601737-20.41558402 \\ \mathrm{C} & 1.03594705-7.94132802-22.35677485 \\ \mathrm{H} & 0.08598619-7.50413107-22.62408103 \\ \mathrm{C} & 1.60981938-8.88626241-23.20181104 \\ \mathrm{H} & 1.14968291-9.13551761-24.15497861 \\ \mathrm{C} & 2.78560953-9.52960299-22.85327074 \\ \mathrm{H} & 3.27181703-10.23193583-23.52346931 \\ \mathrm{C} & 3.34183700-9.28397922-21.59482275 \\ \mathrm{~F} & 4.51725485-9.94328282-21.28798891 \\ \mathrm{Zn} & 28.06990887-6.24304492-6.91170965\end{array}$

\section{C QM/MM R1b (B3LYP/6-31G(d): Amber) mechanical embedding $\omega_{\alpha}=1.667$}

C $\quad 21.93944622-10.35329974-4.22237745$

C $\quad 22.24717835-8.89008879-4.43052378$

O $\quad 21.40516476-8.01101034-4.35339125$

$\mathrm{N} \quad 23.51663859-8.66769137-4.80154789$

C $\quad 23.89525393-7.38429646-5.38189581$

C $\quad 23.07120864-7.22673793-6.68328072$

O $\quad 23.13860014-8.07981385-7.57066171$

C $\quad 25.39657274-7.36398197-5.80542425$

S $\quad 25.83218339-5.73232215-6.52612117$

$\mathrm{H} \quad 24.15640412-9.45888589-4.86343186$

$\mathrm{H} \quad 23.69737696-6.58472489-4.66652979$

$\mathrm{H} \quad 25.61888241-8.20837936-6.46358928$

$\mathrm{H} \quad 25.95418557-7.50659652-4.86760814$

$\mathrm{N} \quad 22.36569622-6.08523364-6.79312682$

C $\quad 21.45935026-5.92814472-7.91955872$

$\mathrm{H} \quad 22.17864604-5.50874509-5.97879246$

C $\quad 24.31479667-4.85829407-10.21466420$

C $\quad 25.24494439-5.91042224-10.73131640$

O $\quad 25.23428314-6.11405317-11.94272960$

$\mathrm{N} \quad 26.00545890-6.59578414-9.80989119$

C $\quad 26.82907555-7.67675768-10.28695977$

C $\quad 26.22651617-9.06828917-10.09783317$

O $\quad 26.74798990-10.09029834-10.56101019$

C $\quad 28.23962366-7.58529044-9.73393130$

S $\quad 28.26863888-8.13036839-7.89380117$

$\mathrm{H} \quad 25.98397137-6.32296140-8.82551439$

$\mathrm{H} \quad 26.89593711-7.55727112-11.37277618$

$\mathrm{H} \quad 28.59881687-6.56175029-9.84556715$

$\mathrm{H} \quad 28.87879428-8.27012323-10.29677974$

$\mathrm{N} \quad 25.07428931-9.10776281-9.38516089$

C $\quad 24.40942232-10.40625627-9.17349946$

$\mathrm{H} \quad 24.59703464-8.29909178-9.01174130$

C $\quad 29.87805571-4.489288542 .81334833$

C $\quad 30.09618778-4.497652761 .26543452$

O $\quad 30.70773776-3.647732950 .66708754$

$\mathrm{N} \quad 29.50286465-5.542921060 .64508300$

C $\quad 29.58113565-5.61573565-0.82674267$

C $\quad 29.08164706-4.33056148-1.44429983$

O $\quad 29.80972178-3.76519882-2.23888708$

C $\quad 28.85242594-6.83501833-1.37958345$

C $\quad 28.96232847-6.94977163-2.85235748$

$\mathrm{H} \quad 29.28758159-7.72332409-0.91588576$

$\mathrm{H} \quad 28.95239562-6.217184421 .16993892$

$\mathrm{H} \quad 30.64248678-5.65477943-1.08949509$

$\mathrm{H} \quad 27.79569286-6.77275417-1.11503666$

C $\quad 28.16629140-6.46503846-3.85967864$

$\mathrm{H} \quad 30.87325170-7.88796948-3.01486427$

$\mathrm{H} \quad 27.26450446-5.88602894-3.76212752$ 


\begin{tabular}{|c|c|}
\hline $\mathrm{C}$ & $29.81902853-7.49664207-4.80786695$ \\
\hline $\mathrm{N}$ & $30.00825497-7.61858845-3.48026315$ \\
\hline $\mathrm{N}$ & $28.69096002-6.86503760-5.03619436$ \\
\hline $\mathrm{H}$ & $30.50402263-7.87620633-5.54319101$ \\
\hline $\mathrm{N}$ & $27.85637192-3.88136069-1.06099211$ \\
\hline $\mathrm{C}$ & $27.28387922-2.64301718-1.55367409$ \\
\hline $\mathrm{H}$ & $27.35770026-4.33168861-0.29529576$ \\
\hline $\mathrm{C}$ & $32.66420188-1.85217980-2.42419056$ \\
\hline $\mathrm{C}$ & $32.40871777-1.95394187-3.94353821$ \\
\hline $\mathrm{O}$ & $33.14157200-1.46687712-4.79935685$ \\
\hline $\mathrm{N}$ & $31.31010926-2.66008055-4.23300159$ \\
\hline $\mathrm{C}$ & $30.97290830-3.04544144-5.62207325$ \\
\hline $\mathrm{C}$ & $30.85228002-1.73641041-6.40541404$ \\
\hline $\mathrm{O}$ & $30.11795301-0.84834532-6.04921432$ \\
\hline $\mathrm{C}$ & $29.67050361-3.84550204-5.59063133$ \\
\hline S & $29.20124914-4.43223459-7.28315264$ \\
\hline $\mathrm{H}$ & $30.76722999-3.02038537-3.46383014$ \\
\hline $\mathrm{H}$ & $31.77564142-3.67388641-6.02451255$ \\
\hline $\mathrm{H}$ & $28.88354501-3.19468465-5.19672190$ \\
\hline $\mathrm{H}$ & $29.82234090-4.68965110-4.91716009$ \\
\hline $\mathrm{N}$ & $31.59436960-1.60679279-7.50426318$ \\
\hline $\mathrm{C}$ & $31.62338604-0.46992919-8.37291724$ \\
\hline $\mathrm{H}$ & $32.28578413-2.26665338-7.73542324$ \\
\hline $\mathrm{C}$ & $7.66110544-15.95918612-16.33719272$ \\
\hline $\mathrm{H}$ & $7.09241853-16.35285774-17.16507728$ \\
\hline $\mathrm{C}$ & $8.05894735-16.77378231-15.29270215$ \\
\hline $\mathrm{H}$ & $7.84886502-17.83302646-15.31297229$ \\
\hline $\mathrm{C}$ & $8.75846140-16.17339312-14.24790709$ \\
\hline $\mathrm{H}$ & $9.14650876-16.74365204-13.43505021$ \\
\hline $\mathrm{C}$ & $8.98671883-14.80776520-14.27595491$ \\
\hline $\mathrm{H}$ & $9.56942834-14.33658507-13.49745903$ \\
\hline $\mathrm{N}$ & $8.48999357-13.98241365-15.22685751$ \\
\hline $\mathrm{C}$ & $7.90089015-14.60937149-16.25662288$ \\
\hline $\mathrm{C}$ & $7.40080713-13.76541088-17.33926226$ \\
\hline $\mathrm{C}$ & $7.53583783-14.18371532-18.67822447$ \\
\hline $\mathrm{C}$ & $7.00446657-13.36555224-19.67495162$ \\
\hline $\mathrm{C}$ & $6.39767782-12.15530050-19.37478358$ \\
\hline $\mathrm{H}$ & $5.99718318-11.55254492-20.15521717$ \\
\hline $\mathrm{F}$ & $7.06991621-13.75276216-20.97780989$ \\
\hline $\mathrm{H}$ & $8.08192686-15.08882850-18.91352648$ \\
\hline $\mathrm{C}$ & $6.79004419-12.56689538-17.01204173$ \\
\hline $\mathrm{H}$ & $6.66314443-12.33164691-15.96926354$ \\
\hline $\mathrm{C}$ & $6.24736103-11.78577171-18.01941370$ \\
\hline $\mathrm{C}$ & $5.43915867-10.60120416-17.66011842$ \\
\hline $\mathrm{O}$ & $5.68052764-9.85717058-16.70934093$ \\
\hline $\mathrm{N}$ & $4.35073094-10.33189822-18.48605329$ \\
\hline $\mathrm{H}$ & $3.95292062-11.02285240-19.12969271$ \\
\hline $\mathrm{N}$ & $3.43855050-9.31173115-18.19925405$ \\
\hline $\mathrm{H}$ & $3.45227777-9.08587246-17.20118728$ \\
\hline S & $3.59560356-7.93555360-19.07494153$ \\
\hline $\mathrm{O}$ & $4.97777293-7.56026268-19.42263930$ \\
\hline $\mathrm{O}$ & $2.80765830-6.86715280-18.43389273$ \\
\hline $\mathrm{C}$ & $2.75434125-8.38327682-20.70054572$ \\
\hline $\mathrm{C}$ & $1.60599423-7.67285010-21.10091413$ \\
\hline $\mathrm{H}$ & $1.11555503-6.99606387-20.41534507$ \\
\hline $\mathrm{C}$ & $1.03591969-7.94116941-22.35660243$ \\
\hline $\mathrm{H}$ & $0.08603516-7.50385204-22.62394105$ \\
\hline $\mathrm{C}$ & $1.60975165-8.88606399-23.20173095$ \\
\hline $\mathrm{H}$ & $1.14952032-9.13532214-24.15481030$ \\
\hline $\mathrm{C}$ & $2.78553822-9.52954722-22.85320168$ \\
\hline $\mathrm{H}$ & $3.27151337-10.23202206-23.52340383$ \\
\hline $\mathrm{C}$ & $3.34167691-9.28399659-21.59484121$ \\
\hline $\mathrm{F}$ & $4.51753761-9.94335655-21.28784901$ \\
\hline $\mathrm{Zn}$ & $28.06978765-6.24300435-6.91161499$ \\
\hline
\end{tabular}

\section{C QM/MM R2b (B3LYP/6-31G(d):Amber) mechanical embedding $\omega_{\alpha}=1.667$}

C $\quad 21.93966067-10.35383512-4.22263429$

C $\quad 22.25008786-8.89404235-4.43464929$

O $\quad 21.40679393-8.01662041-4.35798531$

$\mathrm{N} \quad 23.51810333-8.67352726-4.81053851$

C $\quad 23.89957060-7.38713607-5.38603167$

C $\quad 23.07839871-7.22991711-6.68764304$

O $\quad 23.15003720-8.08555410-7.57272592$

C $\quad 25.40062597-7.36565891-5.80445120$

$\mathrm{S} \quad 25.83733548-5.73704570-6.52277826$

$\mathrm{H} \quad 24.15429720-9.46589494-4.88255968$

$\mathrm{H} \quad 23.69507764-6.59085372-4.66991110$

$\mathrm{H} \quad 25.62180275-8.20093341-6.47175194$

$\mathrm{H} \quad 25.96333201-7.52030786-4.87409099$

$\mathrm{N} \quad 22.36630037-6.09352000-6.79580487$

C $\quad 21.46142823-5.93202511-7.92004894$

$\mathrm{H} \quad 22.17298557-5.52317886-5.97789701$

$\mathrm{N} \quad 23.47776561-5.21751011-9.14223368$

C $\quad 24.31571012-4.81272101-10.25452396$

C $\quad 25.23696899-5.89817082-10.75600185$

O $\quad 25.22565418-6.13742887-11.96174898$

C $\quad 25.10431644-3.59641681-9.91020746$

C $\quad 24.27231485-2.43752392-9.45177934$

C $\quad 23.30035041-1.87294645-10.29602249$

C $\quad 24.43499124-1.93904078-8.12566421$

C $\quad 22.52409144-0.78693738-9.83881938$

C $\quad 23.62010985-0.89973806-7.66315274$

C $\quad 22.67637978-0.30578452-8.51732371$

O $\quad 22.050152840 .81586013-8.11233242$

$\mathrm{H} \quad 23.66431372-4.53090953-11.08261658$

$\mathrm{H} \quad 21.79274299-0.37006896-10.49237550$

$\mathrm{H} \quad 23.77513565-0.49492710-6.68629207$

$\mathrm{H} \quad 23.88612445-5.31254764-8.21737336$

$\mathrm{H} \quad 25.19996359-2.33736039-7.48454039$

$\mathrm{H} \quad 23.15086733-2.27014388-11.28235876$

H $\quad 25.66421636-3.26140161-10.77790476$

$\mathrm{H} \quad 25.80799246-3.84014467-9.11831721$

$\mathrm{H} \quad 22.479908371 .61978618-8.42298224$

N $\quad 25.99689458-6.57387441-9.82716176$

C $\quad 26.81708954-7.66552675-10.29049041$

C $\quad 26.20732371-9.05532332-10.09680378$

O $\quad 26.73573690-10.07337858-10.56006327$

C $\quad 28.23161189-7.57566694-9.73507159$

S $\quad 28.27247037-8.13414031-7.90041096$

$\mathrm{H} \quad 25.98043914-6.29039438-8.84561461$

$\mathrm{H} \quad 26.88550699-7.55174240-11.37617823$

$\mathrm{H} \quad 28.59798034-6.55482353-9.84201145$

$\mathrm{H} \quad 28.87245357-8.25486079-10.30010733$

$\mathrm{N} \quad 25.04277381-9.09194785-9.39702376$

C $\quad 24.37958892-10.39358003-9.16000734$

C $\quad 25.17764597-11.36482586-8.29634104$

O $\quad 24.79990365-12.54554007-8.16495142$

C $\quad 23.88425097-11.09192153-10.45114109$

C $\quad 22.95075832-10.19346939-11.26933093$

C $\quad 22.45248170-10.96243486-12.50470454$

C $\quad 21.71526816-9.81017953-10.46598859$

$\mathrm{H} \quad 22.04085697-9.19877906-9.63507706$

$\mathrm{H} \quad 21.06423393-9.21586464-11.08159298$

$\mathrm{H} \quad 21.22944879-10.70634748-10.08643036$

$\mathrm{H} \quad 24.61852375-8.28061785-8.96741362$

$\mathrm{H} \quad 21.94430426-11.87325627-12.19179077$

$\mathrm{H} \quad 21.79025032-10.32325544-13.07128872$ 

$31.83958390-5.18564120-12.15919214$ $30.93995537-6.01885154-12.75035594$ $28.52761748-6.13234261-12.95253561$ $31.09221427-7.20863080-13.45059806$ $28.67501479-7.35935746-13.60191472$ $29.93968179-7.88462105-13.87321022$ $30.03140153-8.77189923-14.44625063$ $29.55610901-1.66346911-11.81320067$ $32.83809818-5.30581422-12.22693613$ $27.54639699-5.74255070-12.76379351$ $27.79715229-7.87793940-13.89947584$ $32.07705413-7.55931048-13.67392761$ $31.69211826-3.48773954-10.83709910$ $30.33132633-2.54311597-9.12775757$ $27.92832673-3.57152190-11.63503855$ $28.53877882-3.90369026-10.01630937$ $12.44898487-18.60440877-10.13055902$ $11.16164132-18.22621457-10.70006264$ $10.80430302-18.99870972-11.94185656$ $9.73997619-18.77422741-12.49889184$ $11.06801296-16.72939676-10.91898024$ $11.56338972-15.94141481-9.74060649$ $10.78333009-15.87877734-8.55914307$ $12.81209028-15.30027193-9.81430527$ $11.28258391-15.17380068-7.45514184$ $13.29974740-14.61637980-8.69628152$ $12.53253727-14.55109170-7.51282099$ $12.92592017-14.03992241-6.66012848$ $10.38162636-18.48613260-9.98832035$ $10.70439206-15.14105764-6.56781428$ $14.26770129-14.16127302-8.75366156$ $13.27791460-18.51855711-10.71071567$ $13.40670579-15.38420426-10.70450804$ $9.83788857-16.38145037-8.50366338$ $10.03346554-16.44358891-11.07196565$ $11.66313383-16.45306022-11.77991997$ $11.59873241-19.98139503-12.32854723$ $11.29344809-20.77178613-13.50367545$ $11.28198595-22.22846420-13.15176451$ $12.20973120-22.71567724-12.50944642$ $12.28891215-20.45570818-14.63944161$ $12.25661504-19.00178468-15.06918141$ $13.35521850-18.80415538-16.10397458$ $10.90869357-18.65917845-15.68317476$ $10.14421402-18.78414417-14.93655265$ $10.94724390-17.63021578-16.00672483$ $10.71933059-19.32710669-16.52118667$ $12.43932585-20.18067303-11.80591847$ $13.21411434-19.45224307-16.96817861$ $13.33620115-17.79569096-16.46008660$ $14.32519362-18.99011397-15.65084139$ $10.30339103-20.54629544-13.88878319$ $12.42948826-18.34126627-14.23257385$ $12.04677072-21.08289380-15.49205477$ $13.28912014-20.68846833-14.29177297$ $\mathrm{N} \quad 12.73602677-16.67261300-20.53240655$ 
C $\quad 12.42014525-15.29071504-20.26103610$

C $\quad 11.79139629-15.20353639-18.84816349$

O $\quad 10.93973795-16.02980626-18.47058184$

C $\quad 11.51306274-14.70909810-21.35304175$

C $\quad 11.23376125-13.25368327-21.07460540$

C $\quad 10.22333669-15.52361806-21.48241422$

C $\quad 10.56900929-12.52192333-22.21407323$

$\mathrm{H} \quad 13.34373065-14.71855993-20.24854116$

$\mathrm{H} \quad 9.64904015-15.13212200-22.31079146$

$\mathrm{H} \quad 10.47646868-16.55245348-21.69207319$

$\mathrm{H} \quad 12.03910131-14.77054192-22.29807828$

$\mathrm{H} \quad 10.57267964-13.17621020-20.21292043$

$\mathrm{H} \quad 12.16276354-12.74815495-20.84626029$

$\mathrm{H} \quad 9.60499980-12.97373838-22.42951909$

$\mathrm{H} \quad 12.10524382-17.40716536-20.24972046$

$\mathrm{H} \quad 10.41452411-11.49206263-21.91276849$

$\mathrm{H} \quad 9.65060144-15.43267541-20.56700438$

$\mathrm{H} \quad 11.21116597-12.57332180-23.09037829$

N $\quad 12.20367552-14.21053712-18.07567159$

C $\quad 11.97792923-14.14780962-16.60576158$

C $\quad 11.95434465-12.71409541-16.25084497$

O $\quad 12.90718638-12.00084638-16.52891829$

C $\quad 13.02817181-14.88640001-15.80516367$

C $\quad 12.65624236-15.11475404-14.27838871$

S $\quad 12.78726272-13.58755586-13.36049197$

C $\quad 14.55608004-13.25366093-13.56412973$

$\mathrm{H} \quad 11.62617824-15.44690037-14.25134658$

$\mathrm{H} \quad 14.92561172-12.66613205-12.73030778$

$\mathrm{H} \quad 15.10114904-14.19825442-13.59440908$

$\mathrm{H} \quad 14.73849482-12.72517490-14.49612357$

$\mathrm{H} \quad 13.18812121-15.84099262-16.24825899$

$\mathrm{H} \quad 13.29930431-15.88914410-13.87563226$

$\mathrm{H} \quad 12.90839051-13.60067382-18.49456617$

$\mathrm{H} \quad 11.00517041-14.59448152-16.39999934$

$\mathrm{H} \quad 13.96722753-14.34863464-15.85874670$

$\mathrm{N} \quad 10.80836658-12.24631333-15.75149834$

C $\quad 10.67240660-10.88974215-15.19118860$

C $\quad 10.53682481-11.05903418-13.65154684$

O $\quad 9.80327408-11.92152342-13.16182518$

C $\quad 9.42709059-10.20498496-15.72709053$

C $\quad 9.40609694-10.09738852-17.26587684$

C $\quad 9.21330853-8.82409033-15.10373336$

C $\quad 10.51507416-9.17585528-17.73831503$

$\mathrm{H} \quad 11.54193313-10.27711756-15.40859579$

$\mathrm{H} \quad 8.29840692-8.38446681-15.48219242$

$\mathrm{H} \quad 9.11581384-8.94461508-14.03294220$

$\mathrm{H} \quad 8.56302935-10.80402236-15.46596490$

$\mathrm{H} \quad 9.54905475-11.07913222-17.69081440$

$\mathrm{H} \quad 8.45600586-9.67666262-17.55921511$

$\mathrm{H} \quad 11.47690576-9.57479889-17.42674158$

$\mathrm{H} \quad 10.04372524-12.89883953-15.64990096$

$\mathrm{H} \quad 10.50384399-9.15280930-18.81520579$

$\mathrm{H} \quad 10.07410332-8.19213351-15.29103246$

$\mathrm{H} \quad 10.39446756-8.17009138-17.35716345$

$\mathrm{N} \quad 6.47228844-7.95105864-12.67290569$

C $\quad 5.62320154-8.75913455-13.49196373$

C $\quad 4.31096541-8.00869062-13.75423053$

O $\quad 3.90515148-7.16842292-12.93107560$

C $\quad 5.36097573-10.11998871-12.88579239$

C $\quad 6.62591771-10.98715086-12.90118855$

C $\quad 6.41040223-12.31003196-12.27822773$

$\mathrm{H} \quad 6.89393777-11.17775649-13.93888462$

$\mathrm{H} \quad 6.11929650-8.88126268-14.45403531$

$\mathrm{H} \quad 7.44301697-10.48492481-12.41259463$

H $\quad 6.73860643-8.24788324-11.74136311$
$4.58505097-10.60871260-13.47175879$ $5.02644014-10.00439777-11.85808545$ $8.32577340-12.82861950-12.48940541$ $5.28227159-12.61337922-11.87924144$ $7.42970289-13.13123287-12.10998335$ $7.28865919-14.04291329-11.69915346$ $3.73055358-8.28477750-14.96226455$ $2.43429650-7.77600699-15.41529834$ $2.45150159-6.25282015-15.45207887$ $1.43903168-5.63317424-15.15674646$ $1.32184050-8.29901687-14.47051301$ $1.24365469-9.82854878-14.44241365$ $0.05021459-10.25800206-13.61171887$ $0.03689671-11.70119669-13.39524373$ $-0.68484305-12.57114615-14.08244643$ $-1.51091496-12.16316249-15.01018992$ $-0.56771898-13.85983102-13.85708105$ $2.13863618-10.24261560-13.99355875$ $2.16633405-8.13844288-16.40387651$ $0.67154938-12.06231781-12.68515533$ $1.11740295-10.19573162-15.45682131$ $-1.13220263-14.54236605-14.33476551$ $0.01414003-14.18116206-13.09708547$ $4.29298358-8.90964191-15.52866537$ $-0.86525111-9.94865170-14.11229731$ $0.08806224-9.76477657-12.64454532$ $-2.06299286-12.82803194-15.51445806$ $-1.55673235-11.18302942-15.21027167$ $0.36660937-7.90828440-14.81761303$ $1.49214344-7.94527901-13.45585963$ $3.57152535-5.60973652-15.81015630$ $3.68893369-4.13131734-15.85247500$ $4.27787288-3.62543251-17.16470812$ $4.56370306-2.44444775-17.31067602$ $4.48241488-3.62529534-14.65945628$ $3.64789257-3.52016571-13.39528253$ $4.50830216-3.75952744-12.17138383$ $3.81092377-3.32110125-10.94217144$ $3.38976120-4.13766762-9.98438729$ $3.46664836-5.42491112-10.12370598$ $2.88333144-3.69888864-8.86815943$ $2.83641008-4.23938624-13.40010870$ $2.70385240-3.67427647-15.76867006$ $3.72681076-2.33268808-10.78415657$ $3.23625911-2.51324812-13.33048379$ $2.60706613-4.35434695-8.16106037$ $2.77658412-2.71354054-8.74303063$ $4.37455475-6.19243177-16.01992987$ $5.44365663-3.21209738-12.25656699$ $4.74911542-4.82013108-12.14039477$ $3.25850450-6.09999562-9.41112219$ $3.96701211-5.77577494-10.91742702$ $4.88397380-2.63514908-14.87209440$ $5.31576143-4.29361788-14.48290579$ $4.29948335-4.47860344-18.19210607$ $4.76212947-4.10103801-19.53774244$ $6.24107431-4.44550896-19.85617556$ $6.61461788-4.42577849-21.04827437$ $4.03275966-5.43556234-17.99224046$ $4.15428195-4.63105696-20.26717095$ $4.64486024-3.02723208-19.68483331$ $7.02990162-4.81469984-18.83168615$ $8.44459442-5.17389028-18.98340560$ $8.59330179-6.48784013-19.72000028$ $9.39230032-6.53797036-20.65351720$ 


\begin{tabular}{|c|c|}
\hline $\mathrm{C}$ & $9.17335236-5.23182337-17.60906926$ \\
\hline $\mathrm{C}$ & $9.10601470-3.92522684-16.86362658$ \\
\hline $\mathrm{C}$ & $9.97550161-2.87172907-17.12785780$ \\
\hline $\mathrm{C}$ & $8.15713602-3.79609037-15.85255240$ \\
\hline $\mathrm{C}$ & $9.87788609-1.67305963-16.40427912$ \\
\hline $\mathrm{C}$ & $8.06288562-2.62655744-15.08515857$ \\
\hline $\mathrm{C}$ & $8.94607590-1.56387099-15.32973192$ \\
\hline $\mathrm{O}$ & $8.77991572-0.42415730-14.63356375$ \\
\hline $\mathrm{H}$ & $8.93512856-4.39595658-19.56981033$ \\
\hline $\mathrm{H}$ & $10.54395294-0.86182987-16.61321965$ \\
\hline $\mathrm{H}$ & $7.32027901-2.54063554-14.32871161$ \\
\hline $\mathrm{H}$ & $6.63659275-4.83497370-17.90125256$ \\
\hline $\mathrm{H}$ & $7.48399852-4.60146099-15.65991411$ \\
\hline $\mathrm{H}$ & $10.73017158-2.95990729-17.88133754$ \\
\hline $\mathrm{H}$ & $10.21400249-5.42933881-17.81347282$ \\
\hline $\mathrm{H}$ & $8.77049978-6.02958074-16.99965191$ \\
\hline $\mathrm{H}$ & $9.614681190 .11155116-14.74175214$ \\
\hline $\mathrm{N}$ & $7.81544889-7.52777948-19.35613414$ \\
\hline $\mathrm{C}$ & $7.79893247-8.79841846-20.06748993$ \\
\hline $\mathrm{C}$ & $7.39169835-8.56783141-21.57629533$ \\
\hline $\mathrm{O}$ & $7.96324320-9.21670655-22.45349291$ \\
\hline $\mathrm{H}$ & $7.15006685-7.35569777-18.61500004$ \\
\hline $\mathrm{H}$ & $8.79260014-9.24236892-20.07344865$ \\
\hline $\mathrm{H}$ & $7.09692574-9.49229721-19.61623948$ \\
\hline $\mathrm{N}$ & $6.46093904-7.61569683-21.83778049$ \\
\hline $\mathrm{C}$ & $5.95175110-7.32811501-23.17689419$ \\
\hline $\mathrm{C}$ & $7.07056376-6.70333979-23.99581059$ \\
\hline $\mathrm{O}$ & $7.18832245-6.96900686-25.19195812$ \\
\hline $\mathrm{C}$ & $4.73758970-6.41178747-23.10944516$ \\
\hline $\mathrm{C}$ & $4.13840379-6.05982846-24.49425728$ \\
\hline $\mathrm{C}$ & $2.93543517-5.16034719-24.23281458$ \\
\hline $\mathrm{N}$ & $2.23886572-4.76529214-25.44949669$ \\
\hline $\mathrm{C}$ & $1.14175213-4.00218984-25.48679022$ \\
\hline $\mathrm{N}$ & $0.60510712-3.54965380-24.36115002$ \\
\hline $\mathrm{N}$ & $0.62615551-3.61997808-26.62564957$ \\
\hline $\mathrm{H}$ & $3.79218635-6.95272662-25.00526301$ \\
\hline $\mathrm{H}$ & $5.67678239-8.25479334-23.68241144$ \\
\hline $\mathrm{H}$ & $2.63205481-5.05994588-26.34363661$ \\
\hline $\mathrm{H}$ & $4.85780691-5.51042727-25.08716707$ \\
\hline $\mathrm{H}$ & $-0.21741880-3.08394059-26.68450212$ \\
\hline $\mathrm{H}$ & $1.00172201-3.98447487-27.49346940$ \\
\hline $\mathrm{H}$ & $6.03160404-7.20497504-21.01833924$ \\
\hline $\mathrm{H}$ & $3.28170527-4.27048317-23.71346067$ \\
\hline $\mathrm{H}$ & $2.22649572-5.70485923-23.61208083$ \\
\hline $\mathrm{H}$ & $-0.22369764-2.98570599-24.41631593$ \\
\hline $\mathrm{H}$ & $0.92333947-3.96383353-23.51980622$ \\
\hline $\mathrm{H}$ & $4.98657179-5.48910555-22.60209643$ \\
\hline $\mathrm{H}$ & $3.97105472-6.92269820-22.54904504$ \\
\hline $\mathrm{N}$ & $9.61278947-9.42238143-24.96073307$ \\
\hline $\mathrm{C}$ & $9.07647739-10.21479416-26.07938057$ \\
\hline $\mathrm{C}$ & $9.19621842-9.39523911-27.37744508$ \\
\hline $\mathrm{O}$ & $9.77811860-9.86141827-28.32161187$ \\
\hline $\mathrm{C}$ & $7.62812380-10.67942458-25.82200088$ \\
\hline $\mathrm{C}$ & $7.58088042-11.66455588-24.66011450$ \\
\hline $\mathrm{C}$ & $7.09529299-11.37208712-27.06456921$ \\
\hline $\mathrm{C}$ & $6.18135245-11.90034443-24.14100261$ \\
\hline $\mathrm{H}$ & $9.70356227-11.10070614-26.19942042$ \\
\hline $\mathrm{H}$ & $6.06593571-11.65405426-26.89190775$ \\
\hline $\mathrm{H}$ & $7.10056548-10.67654239-27.89316363$ \\
\hline $\mathrm{H}$ & $7.02210733-9.80896522-25.59299745$ \\
\hline $\mathrm{H}$ & $8.04601230-12.59904384-24.95492343$ \\
\hline $\mathrm{H}$ & $8.15961036-11.26433815-23.83995888$ \\
\hline $\mathrm{H}$ & $5.60748998-12.37184467-24.93419851$ \\
\hline $\mathrm{H}$ & $9.03257327-9.16841973-24.17474847$ \\
\hline $\mathrm{H}$ & $6.25567903-12.58494843-23.30804498$ \\
\hline
\end{tabular}

H

$\mathrm{H}$

$\mathrm{N}$

C

C

O

C

$\mathrm{O}$

$\mathrm{H}$

$\mathrm{H}$

$\mathrm{H}$

$\mathrm{H}$

$\mathrm{H}$

$\mathrm{N}$

C

C

O

C

C

C

C

$\mathrm{H}$

$\mathrm{H}$

$\mathrm{H}$

$\mathrm{H}$

$\mathrm{H}$

$\mathrm{H}$

$\mathrm{H}$

$\mathrm{H}$

$\mathrm{H}$

$\mathrm{H}$

$\mathrm{H}$

$\mathrm{N}$

C

C

$\mathrm{O}$

$\mathrm{H}$

$\mathrm{H}$

$\mathrm{H}$

$\mathrm{N}$

C

C

$\mathrm{O}$

C

$\mathrm{O}$

$\mathrm{H}$

$\mathrm{H}$

$\mathrm{H}$

$\mathrm{H}$

C

C

$\mathrm{O}$

$\mathrm{H}$

$\mathrm{H}$

$\mathrm{N}$

C

C

O

C

C

C

C

C
$7.69645801-12.24402791-27.29353376$ $5.72387467-10.95977437-23.85258621$ $6.60489128-20.99262056-19.57946497$ $6.08698552-20.90021387-18.19635702$ $4.59230711-20.56673105-18.29994476$ $4.09754145-20.18360238-19.39319820$ $6.81882549-19.84989659-17.38644973$ $6.67836860-18.56824025-17.98085131$ $7.22649068-20.29765823-19.97323043$ $6.20431890-21.86254197-17.70024042$ $7.87236401-20.11918300-17.32832688$ $6.39614872-19.82403952-16.38519970$ $7.57558026-18.33554912-18.31085594$ $3.13703895-17.88591693-16.99640177$ $3.06861621-16.44875502-17.26283036$ $3.60880981-16.05448658-18.64900008$ $3.11631643-15.12011044-19.30632020$ $3.80487875-15.70366989-16.16980255$ $3.77319901-14.18006247-16.23850620$ $2.35353512-13.65655163-16.42536321$ $4.31276587-13.63473233-14.90907262$ $5.32794461-13.99340869-14.75960415$ $4.29062398-12.55213850-14.92131090$ $3.69723409-13.99738234-14.08475208$ $3.82648097-18.26780674-16.34765532$ $1.72766065-14.02534584-15.61502943$ $2.37953745-12.57265394-16.41041480$ $1.96839950-13.99103540-17.37677587$ $2.02130699-16.15797657-17.22591823$ $4.39950124-13.81041915-17.03866973$ $4.84849635-15.99024232-16.19317725$ $3.38112588-16.00325158-15.21461257$ $4.66750163-16.69636180-19.07807133$ $5.15872491-16.55743988-20.44933457$ $4.05749748-16.96495263-21.45608743$ $3.86015243-16.19700264-22.36796251$ $5.07523478-17.42316192-18.48993190$ $5.44539759-15.52551181-20.63448057$ $6.02496269-17.20148994-20.60284065$ $1.58537750-14.26601807-21.66196010$ $2.20634884-13.05059872-22.16694176$ $2.48571209-13.18955234-23.69393614$ $2.32485871-12.22368171-24.40200686$ $3.55133406-12.79919400-21.41598328$ $3.32370698-12.58737820-19.97855649$ $2.09737008-14.87469689-21.03080597$ $1.55984463-12.19293838-21.99812684$ $4.05332763-11.97180858-21.88851833$ $4.19359841-13.66966602-21.54947066$ $3.22718100-13.49122684-19.60910476$ $-1.01159788-13.04812973-25.46768621$ $-1.44927147-11.65649444-25.47474985$ $-0.64096804-10.85219831-26.48941993$ $-1.19375409-10.01046090-27.20228019$ $-0.54931491-13.44926665-24.66487832$ $-2.49428571-11.62041319-25.79075822$ $0.70099172-11.00905438-26.47875278$ $1.56268028-10.21692082-27.35997792$ $1.19563023-10.53442968-28.83529222$ $1.01087378-9.60653716-29.60411546$ $3.06911725-10.47171160-27.09829798$ $3.97008095-9.89445267-28.17760641$ $4.28939825-8.53703600-28.14255170$ $4.34281841-10.68852292-29.26702993$ $5.11325918-8.00363044-29.13915202$ 


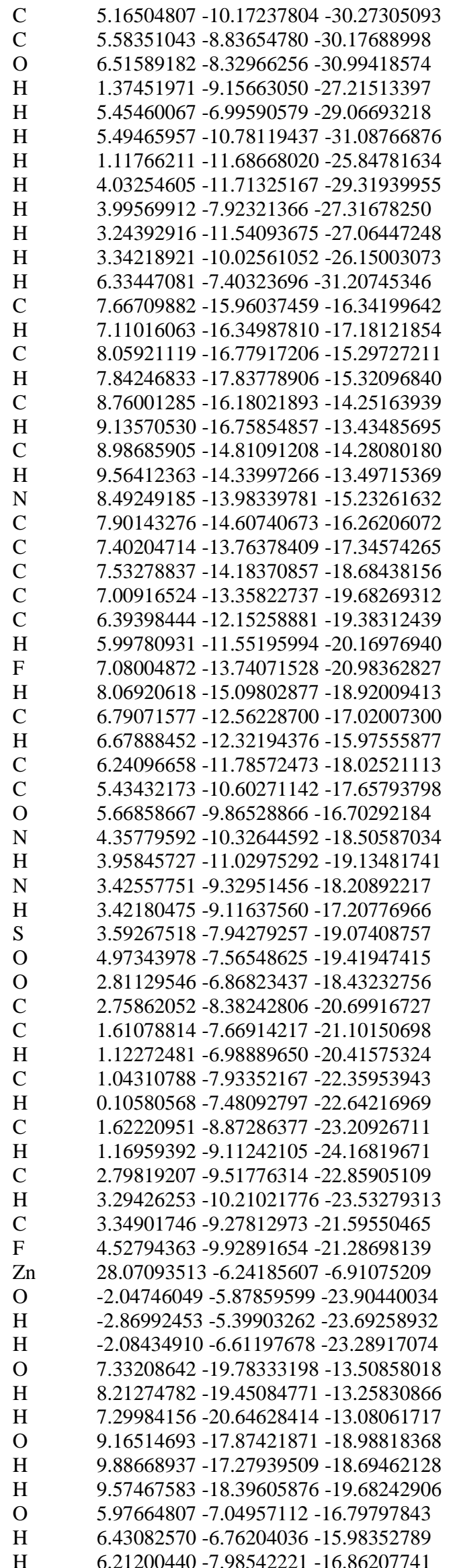

O $\quad 26.47616157-0.94802544-4.85810727$

$\mathrm{H} \quad 27.12677038-0.86124674-4.14852531$

$\mathrm{H} \quad 26.61677208-1.83516396-5.20000483$

O $\quad 0.70091978-10.01415199-18.09922285$

$\mathrm{H} \quad-0.07133587-10.50402632-18.46274884$

$\mathrm{H} \quad 1.42693052-10.31182645-18.65238519$

O $\quad 4.77435874-18.65168310-14.46129455$

$\mathrm{H} \quad 5.69040634-18.93410874-14.28751665$

$\mathrm{H} \quad 4.27505573-19.20314871-13.85041419$

O $\quad-0.45094987-6.74209485-25.79729544$

$\mathrm{H} \quad-0.89886810-7.39810716-26.34249537$

$\mathrm{H} \quad-1.15065925-6.40281755-25.20834522$

\section{C QM /MM R2b (B3LYP/6-31G(d): Amber) mechanical embedding $\omega_{\alpha}=\mathbf{1 . 6 6 7}$}

C $\quad 21.93966067-10.35383512-4.22263429$

C $\quad 22.25008786-8.89404235-4.43464929$

$\mathrm{O} \quad 21.40679393-8.01662041-4.35798531$

$\mathrm{N} \quad 23.51810333-8.67352726-4.81053851$

C $\quad 23.89957060-7.38713607-5.38603167$

C $\quad 23.07839871-7.22991711-6.68764304$

O $\quad 23.15003720-8.08555410-7.57272592$

C $\quad 25.40062597-7.36565891-5.80445120$

S $\quad 25.83733548-5.73704570-6.52277826$

$\mathrm{H} \quad 24.15429720-9.46589494-4.88255968$

$\mathrm{H} \quad 23.69507764-6.59085372-4.66991110$

$\mathrm{H} \quad 25.62180275-8.20093341-6.47175194$

$\mathrm{H} \quad 25.96333201-7.52030786-4.87409099$

N $\quad 22.36630037-6.09352000-6.79580487$

C $\quad 21.46142823-5.93202511-7.92004894$

$\mathrm{H} \quad 22.17298557-5.52317886-5.97789701$

$\mathrm{N} \quad 23.47776561-5.21751011-9.14223368$

C $\quad 24.31571012-4.81272101-10.25452396$

C $\quad 25.23696899-5.89817082-10.75600185$

O $\quad 25.22565418-6.13742887-11.96174898$

C $\quad 25.10431644-3.59641681-9.91020746$

C $\quad 24.27231485-2.43752392-9.45177934$

C $\quad 23.30035041-1.87294645-10.29602249$

C $\quad 24.43499124-1.93904078-8.12566421$

C $\quad 22.52409144-0.78693738-9.83881938$

C $\quad 23.62010985-0.89973806-7.66315274$

C $\quad 22.67637978-0.30578452-8.51732371$

$\mathrm{O} \quad 22.050152840 .81586013-8.11233242$

$\mathrm{H} \quad 23.66431372-4.53090953-11.08261658$

H $\quad 21.79274299-0.37006896-10.49237550$

$\mathrm{H} \quad 23.77513565-0.49492710-6.68629207$

$\mathrm{H} \quad 23.88612445-5.31254764-8.21737336$

$\mathrm{H} \quad 25.19996359-2.33736039-7.48454039$

$\mathrm{H} \quad 23.15086733-2.27014388-11.28235876$

$\mathrm{H} \quad 25.66421636-3.26140161-10.77790476$

$\mathrm{H} \quad 25.80799246-3.84014467-9.11831721$

$\mathrm{H} \quad 22.479908371 .61978618-8.42298224$

$\mathrm{N} \quad 25.99689458-6.57387441-9.82716176$

C $\quad 26.81708954-7.66552675-10.29049041$

C $\quad 26.20732371-9.05532332-10.09680378$

O $\quad 26.73573690-10.07337858-10.56006327$

C $\quad 28.23161189-7.57566694-9.73507159$

S $\quad 28.27247037-8.13414031-7.90041096$

$\mathrm{H} \quad 25.98043914-6.29039438-8.84561461$

H $\quad 26.88550699-7.55174240-11.37617823$

$\mathrm{H} \quad 28.59798034-6.55482353-9.84201145$

$\mathrm{H} \quad 28.87245357-8.25486079-10.30010733$

N $\quad 25.04277381-9.09194785-9.39702376$ 
C $\quad 24.37958892-10.39358003-9.16000734$

C $\quad 25.17764597-11.36482586-8.29634104$

O $\quad 24.79990365-12.54554007-8.16495142$

C $\quad 23.88425097-11.09192153-10.45114109$

C $\quad 22.95075832-10.19346939-11.26933093$

C $\quad 22.45248170-10.96243486-12.50470454$

C $\quad 21.71526816-9.81017953-10.46598859$

$\mathrm{H} \quad 22.04085697-9.19877906-9.63507706$

$\mathrm{H} \quad 21.06423393-9.21586464-11.08159298$

$\mathrm{H} \quad 21.22944879-10.70634748-10.08643036$

H $\quad 24.61852375-8.28061785-8.96741362$

$\mathrm{H} \quad 21.94430426-11.87325627-12.19179077$

H $\quad 21.79025032-10.32325544-13.07128872$

$\mathrm{H} \quad 23.31861051-11.22927544-13.10665296$

$\mathrm{H} \quad 23.46906376-10.21102380-8.60186155$

$\mathrm{H} \quad 23.46885488-9.29980676-11.58401012$

$\mathrm{H} \quad 23.34771124-11.99832373-10.18551255$

$\mathrm{H} \quad 24.72696401-11.34394040-11.07454647$

$\mathrm{N} \quad 26.23568758-10.87029760-7.62318217$

C $\quad 26.87042941-11.71864300-6.60110422$

C $\quad 25.83731789-12.12049593-5.51263440$

O $\quad 25.15935370-11.25864781-4.97697308$

C $\quad 28.05346077-10.98903084-5.98286788$

C $\quad 28.65998982-11.80110160-4.84510073$

C $\quad 29.96340657-11.14569069-4.34246289$

C $\quad 30.36439144-11.82357364-3.01387880$

$\mathrm{N} \quad 31.79356245-11.68638184-2.77711721$

$\mathrm{H} \quad 27.21068565-12.62642028-7.10026736$

$\mathrm{H} \quad 29.80109409-11.34992623-2.20694144$

$\mathrm{H} \quad 30.10592668-12.88148685-3.06911505$

$\mathrm{H} \quad 27.94845323-11.79448094-4.02250613$

$\mathrm{H} \quad 28.86128300-12.82272394-5.15203157$

$\mathrm{H} \quad 32.10241061-12.30183626-2.03879612$

$\mathrm{H} \quad 32.33315829-11.93166816-3.60884250$

$\mathrm{H} \quad 32.02442162-10.73803303-2.49203674$

$\mathrm{H} \quad 29.80305873-10.09079212-4.17485183$

$\mathrm{H} \quad 30.72221433-11.31367340-5.10179414$

$\mathrm{H} \quad 26.54536193-9.92474305-7.82967810$

$\mathrm{H} \quad 27.75269943-10.00926802-5.62237497$

H $\quad 28.82175477-10.88860051-6.74710089$

C $\quad 29.87757315-4.488602962 .81212480$

C $\quad 30.09718423-4.493823701 .26665390$

$\mathrm{O} \quad 30.70655520-3.640365690 .67003933$

$\mathrm{N} \quad 29.50446268-5.536670550 .64275641$

C $\quad 29.57852237-5.61173130-0.82734585$

C $\quad 29.07538681-4.32576772-1.44281398$

O $\quad 29.79714274-3.74895345-2.23717528$

C $\quad 28.84726441-6.83531260-1.37542552$

C

$\mathrm{H}$

$\mathrm{H}$

$\mathrm{H}$

$\mathrm{H}$

$\mathrm{C}$

$\mathrm{H}$

$\mathrm{H}$

C

$\mathrm{N}$

N

$\mathrm{H}$

N

C

$\mathrm{H}$

C

C
O

$\mathrm{N}$

C

C
$33.12070622-1.44133847-4.79491727$ $31.32599480-2.68954537-4.23512615$ $30.98113492-3.05533260-5.62544812$ $30.86064314-1.73893004-6.40308369$ $30.12300950-0.85356089-6.04455227$ $29.67358513-3.84919878-5.58708565$ $29.18342601-4.41970199-7.27787047$ $30.78330097-3.05691076-3.46944825$ $31.77860100-3.68259265-6.03667525$ $28.89340772-3.20296481-5.17710837$ $29.82294888-4.69768903-4.91973100$ $31.59204726-1.60840153-7.50933168$ $31.62021256-0.47004896-8.37149217$ $32.29816886-2.25483907-7.74036585$ $30.18797031-1.81784940-9.82127554$ $29.15952385-2.01467326-10.86015879$ $27.91529759-1.20367518-10.54020050$ $27.41915245-1.15064906-9.41742645$ $28.79628701-3.48969292-10.98457459$ $29.85210627-4.34134957-11.57330772$ $31.19122831-4.23248093-11.41895524$ $29.65794237-5.46412839-12.45558266$ $31.83958390-5.18564120-12.15919214$ $30.93995537-6.01885154-12.75035594$ $28.52761748-6.13234261-12.95253561$ $31.09221427-7.20863080-13.45059806$ $28.67501479-7.35935746-13.60191472$ $29.93968179-7.88462105-13.87321022$ $30.03140153-8.77189923-14.44625063$ $29.55610901-1.66346911-11.81320067$ $32.83809818-5.30581422-12.22693613$ $27.54639699-5.74255070-12.76379351$ $27.79715229-7.87793940-13.89947584$ $32.07705413-7.55931048-13.67392761$ $31.69211826-3.48773954-10.83709910$ $30.33132633-2.54311597-9.12775757$ $27.92832673-3.57152190-11.63503855$ $28.53877882-3.90369026-10.01630937$ $12.44898487-18.60440877-10.13055902$ $11.16164132-18.22621457-10.70006264$ $10.80430302-18.99870972-11.94185656$ $9.73997619-18.77422741-12.49889184$ $11.06801296-16.72939676-10.91898024$ $11.56338972-15.94141481-9.74060649$ $10.78333009-15.87877734-8.55914307$ $12.81209028-15.30027193-9.81430527$ $11.28258391-15.17380068-7.45514184$ $13.29974740-14.61637980-8.69628152$ $12.53253727-14.55109170-7.51282099$ $12.92592017-14.03992241-6.66012848$ $10.38162636-18.48613260-9.98832035$ $10.70439206-15.14105764-6.56781428$ $14.26770129-14.16127302-8.75366156$ $13.27791460-18.51855711-10.71071567$ $13.40670579-15.38420426-10.70450804$ $9.83788857-16.38145037-8.50366338$ $10.03346554-16.44358891-11.07196565$ $11.66313383-16.45306022-11.77991997$ $11.59873241-19.98139503-12.32854723$ $11.29344809-20.77178613-13.50367545$ $11.28198595-22.22846420-13.15176451$ $12.20973120-22.71567724-12.50944642$ $12.28891215-20.45570818-14.63944161$ $12.25661504-19.00178468-15.06918141$ $13.35521850-18.80415538-16.10397458$ 


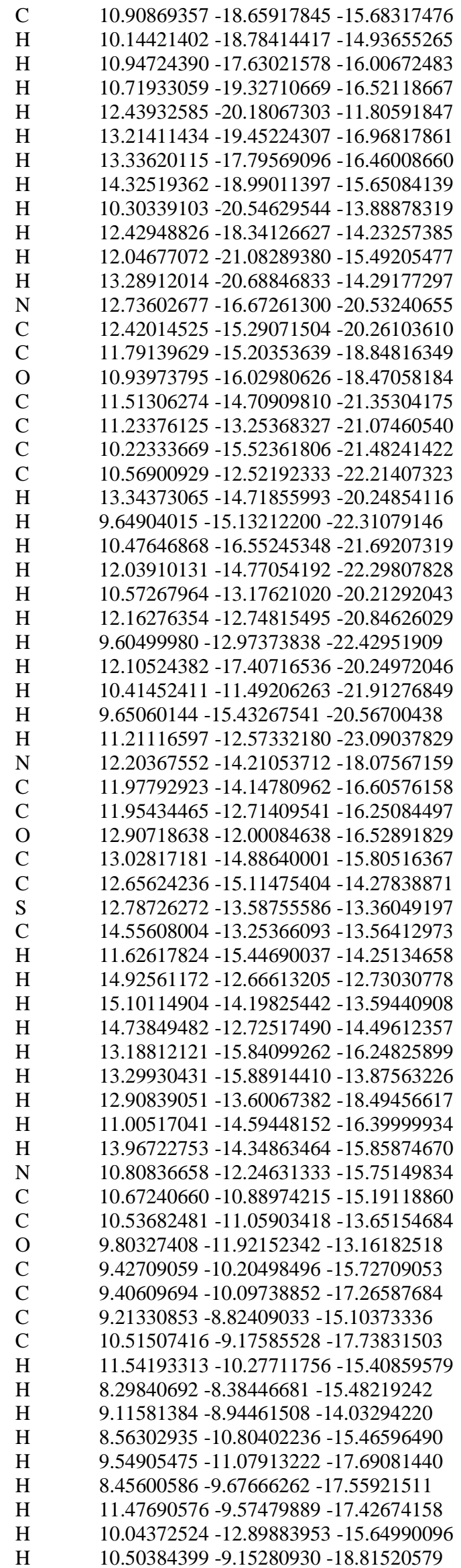

$10.07410332-8.19213351-15.29103246$ $10.39446756-8.17009138-17.35716345$ $6.47228844-7.95105864-12.67290569$ $5.62320154-8.75913455-13.49196373$ $4.31096541-8.00869062-13.75423053$ $3.90515148-7.16842292-12.93107560$ $5.36097573-10.11998871-12.88579239$ $6.62591771-10.98715086-12.90118855$ $6.41040223-12.31003196-12.27822773$ $6.89393777-11.17775649-13.93888462$ $6.11929650-8.88126268-14.45403531$ $7.44301697-10.48492481-12.41259463$ $6.73860643-8.24788324-11.74136311$ $4.58505097-10.60871260-13.47175879$ $5.02644014-10.00439777-11.85808545$ $8.32577340-12.82861950-12.48940541$ $5.28227159-12.61337922-11.87924144$ $7.42970289-13.13123287-12.10998335$ $7.28865919-14.04291329-11.69915346$ $3.73055358-8.28477750-14.96226455$ $2.43429650-7.77600699-15.41529834$ $2.45150159-6.25282015-15.45207887$ $1.43903168-5.63317424-15.15674646$ $1.32184050-8.29901687-14.47051301$ $1.24365469-9.82854878-14.44241365$ $0.05021459-10.25800206-13.61171887$ $0.03689671-11.70119669-13.39524373$ $-0.68484305-12.57114615-14.08244643$ $-1.51091496-12.16316249-15.01018992$ $-0.56771898-13.85983102-13.85708105$ $2.13863618-10.24261560-13.99355875$ $2.16633405-8.13844288-16.40387651$ $0.67154938-12.06231781-12.68515533$ $1.11740295-10.19573162-15.45682131$ $-1.13220263-14.54236605-14.33476551$ $0.01414003-14.18116206-13.09708547$ $4.29298358-8.90964191-15.52866537$ $-0.86525111-9.94865170-14.11229731$ $0.08806224-9.76477657-12.64454532$ $-2.06299286-12.82803194-15.51445806$ $-1.55673235-11.18302942-15.21027167$ $0.36660937-7.90828440-14.81761303$ $1.49214344-7.94527901-13.45585963$ $3.57152535-5.60973652-15.81015630$ $3.68893369-4.13131734-15.85247500$ $4.27787288-3.62543251-17.16470812$ $4.56370306-2.44444775-17.31067602$ $4.48241488-3.62529534-14.65945628$ $3.64789257-3.52016571-13.39528253$ $4.50830216-3.75952744-12.17138383$ $3.81092377-3.32110125-10.94217144$ $3.38976120-4.13766762-9.98438729$ $3.46664836-5.42491112-10.12370598$ $2.88333144-3.69888864-8.86815943$ $2.83641008-4.23938624-13.40010870$ $2.70385240-3.67427647-15.76867006$ $3.72681076-2.33268808-10.78415657$ $3.23625911-2.51324812-13.33048379$ $2.60706613-4.35434695-8.16106037$ $2.77658412-2.71354054-8.74303063$ $4.37455475-6.19243177-16.01992987$ $5.44365663-3.21209738-12.25656699$ $4.74911542-4.82013108-12.14039477$ $3.25850450-6.09999562-9.41112219$

$3.96701211-5.77577494-10.91742702$ 


\begin{tabular}{|c|c|}
\hline $\mathrm{H}$ & $4.88397380-2.63514908-14.87209440$ \\
\hline $\mathrm{H}$ & $5.31576143-4.29361788-14.48290579$ \\
\hline $\mathrm{N}$ & $4.29948335-4.47860344-18.19210607$ \\
\hline $\mathrm{C}$ & $4.76212947-4.10103801-19.53774244$ \\
\hline $\mathrm{C}$ & $6.24107431-4.44550896-19.85617556$ \\
\hline $\mathrm{O}$ & $6.61461788-4.42577849-21.04827437$ \\
\hline $\mathrm{H}$ & $4.03275966-5.43556234-17.99224046$ \\
\hline $\mathrm{H}$ & $4.15428195-4.63105696-20.26717095$ \\
\hline $\mathrm{H}$ & $4.64486024-3.02723208-19.68483331$ \\
\hline $\mathrm{N}$ & $7.02990162-4.81469984-18.83168615$ \\
\hline $\mathrm{C}$ & $8.44459442-5.17389028-18.98340560$ \\
\hline $\mathrm{C}$ & $8.59330179-6.48784013-19.72000028$ \\
\hline $\mathrm{O}$ & $9.39230032-6.53797036-20.65351720$ \\
\hline $\mathrm{C}$ & $9.17335236-5.23182337-17.60906926$ \\
\hline $\mathrm{C}$ & $9.10601470-3.92522684-16.86362658$ \\
\hline $\mathrm{C}$ & $9.97550161-2.87172907-17.12785780$ \\
\hline $\mathrm{C}$ & $8.15713602-3.79609037-15.85255240$ \\
\hline $\mathrm{C}$ & $9.87788609-1.67305963-16.40427912$ \\
\hline $\mathrm{C}$ & $8.06288562-2.62655744-15.08515857$ \\
\hline $\mathrm{C}$ & $8.94607590-1.56387099-15.32973192$ \\
\hline $\mathrm{O}$ & $8.77991572-0.42415730-14.63356375$ \\
\hline $\mathrm{H}$ & $8.93512856-4.39595658-19.56981033$ \\
\hline $\mathrm{H}$ & $10.54395294-0.86182987-16.61321965$ \\
\hline $\mathrm{H}$ & $7.32027901-2.54063554-14.32871161$ \\
\hline $\mathrm{H}$ & $6.63659275-4.83497370-17.90125256$ \\
\hline $\mathrm{H}$ & $7.48399852-4.60146099-15.65991411$ \\
\hline $\mathrm{H}$ & $10.73017158-2.95990729-17.88133754$ \\
\hline $\mathrm{H}$ & $10.21400249-5.42933881-17.81347282$ \\
\hline $\mathrm{H}$ & $8.77049978-6.02958074-16.99965191$ \\
\hline $\mathrm{H}$ & $9.614681190 .11155116-14.74175214$ \\
\hline $\mathrm{N}$ & $7.81544889-7.52777948-19.35613414$ \\
\hline $\mathrm{C}$ & $7.79893247-8.79841846-20.06748993$ \\
\hline $\mathrm{C}$ & $7.39169835-8.56783141-21.57629533$ \\
\hline $\mathrm{O}$ & $7.96324320-9.21670655-22.45349291$ \\
\hline $\mathrm{H}$ & $7.15006685-7.35569777-18.61500004$ \\
\hline $\mathrm{H}$ & $8.79260014-9.24236892-20.07344865$ \\
\hline $\mathrm{H}$ & $7.09692574-9.49229721-19.61623948$ \\
\hline $\mathrm{N}$ & $6.46093904-7.61569683-21.83778049$ \\
\hline $\mathrm{C}$ & $5.95175110-7.32811501-23.17689419$ \\
\hline $\mathrm{C}$ & $7.07056376-6.70333979-23.99581059$ \\
\hline $\mathrm{O}$ & $7.18832245-6.96900686-25.19195812$ \\
\hline $\mathrm{C}$ & $4.73758970-6.41178747-23.10944516$ \\
\hline $\mathrm{C}$ & $4.13840379-6.05982846-24.49425728$ \\
\hline $\mathrm{C}$ & $2.93543517-5.16034719-24.23281458$ \\
\hline $\mathrm{N}$ & $2.23886572-4.76529214-25.44949669$ \\
\hline $\mathrm{C}$ & $1.14175213-4.00218984-25.48679022$ \\
\hline $\mathrm{N}$ & $0.60510712-3.54965380-24.36115002$ \\
\hline $\mathrm{N}$ & $0.62615551-3.61997808-26.62564957$ \\
\hline $\mathrm{H}$ & $3.79218635-6.95272662-25.00526301$ \\
\hline $\mathrm{H}$ & $5.67678239-8.25479334-23.68241144$ \\
\hline $\mathrm{H}$ & $2.63205481-5.05994588-26.34363661$ \\
\hline $\mathrm{H}$ & $4.85780691-5.51042727-25.08716707$ \\
\hline $\mathrm{H}$ & $-0.21741880-3.08394059-26.68450212$ \\
\hline $\mathrm{H}$ & $1.00172201-3.98447487-27.49346940$ \\
\hline $\mathrm{H}$ & $6.03160404-7.20497504-21.01833924$ \\
\hline $\mathrm{H}$ & $3.28170527-4.27048317-23.71346067$ \\
\hline $\mathrm{H}$ & $2.22649572-5.70485923-23.61208083$ \\
\hline $\mathrm{H}$ & $-0.22369764-2.98570599-24.41631593$ \\
\hline $\mathrm{H}$ & $0.92333947-3.96383353-23.51980622$ \\
\hline $\mathrm{H}$ & $4.98657179-5.48910555-22.60209643$ \\
\hline $\mathrm{H}$ & $3.97105472-6.92269820-22.54904504$ \\
\hline $\mathrm{N}$ & $9.61278947-9.42238143-24.96073307$ \\
\hline $\mathrm{C}$ & $9.07647739-10.21479416-26.07938057$ \\
\hline $\mathrm{C}$ & $9.19621842-9.39523911-27.37744508$ \\
\hline $\mathrm{O}$ & $9.77811860-9.86141827-28.32161187$ \\
\hline
\end{tabular}

C $\quad 7.62812380-10.67942458-25.82200088$ C $\quad 7.58088042-11.66455588-24.66011450$ C $\quad 7.09529299-11.37208712-27.06456921$ C $\quad 6.18135245-11.90034443-24.14100261$ $\mathrm{H} \quad 9.70356227-11.10070614-26.19942042$ $\mathrm{H} \quad 6.06593571-11.65405426-26.89190775$ $\mathrm{H} \quad 7.10056548-10.67654239-27.89316363$ $\mathrm{H} \quad 7.02210733-9.80896522-25.59299745$ $\mathrm{H} \quad 8.04601230-12.59904384-24.95492343$ $\mathrm{H} \quad 8.15961036-11.26433815-23.83995888$ $\mathrm{H} \quad 5.60748998-12.37184467-24.93419851$ $\mathrm{H} \quad 9.03257327-9.16841973-24.17474847$

$\mathrm{H} \quad 6.25567903-12.58494843-23.30804498$ $\mathrm{H} \quad 7.69645801-12.24402791-27.29353376$ $\mathrm{H} \quad 5.72387467-10.95977437-23.85258621$ $\mathrm{N} \quad 6.60489128-20.99262056-19.57946497$ C $\quad 6.08698552-20.90021387-18.19635702$ C $\quad 4.59230711-20.56673105-18.29994476$ O $\quad 4.09754145-20.18360238-19.39319820$ C $\quad 6.81882549-19.84989659-17.38644973$ O $\quad 6.67836860-18.56824025-17.98085131$ $\mathrm{H} \quad 7.22649068-20.29765823-19.97323043$ $\mathrm{H} \quad 6.20431890-21.86254197-17.70024042$ $\mathrm{H} \quad 7.87236401-20.11918300-17.32832688$ $\mathrm{H} \quad 6.39614872-19.82403952-16.38519970$ $\mathrm{H} \quad 7.57558026-18.33554912-18.31085594$ $\mathrm{N} \quad 3.13703895-17.88591693-16.99640177$ C $\quad 3.06861621-16.44875502-17.26283036$ C $\quad 3.60880981-16.05448658-18.64900008$ O $\quad 3.11631643-15.12011044-19.30632020$ C $\quad 3.80487875-15.70366989-16.16980255$ C $\quad 3.77319901-14.18006247-16.23850620$ C $\quad 2.35353512-13.65655163-16.42536321$ C $\quad 4.31276587-13.63473233-14.90907262$ $\mathrm{H} \quad 5.32794461-13.99340869-14.75960415$ $\mathrm{H} \quad 4.29062398-12.55213850-14.92131090$ $\mathrm{H} \quad 3.69723409-13.99738234-14.08475208$ $\mathrm{H} \quad 3.82648097-18.26780674-16.34765532$ H $\quad 1.72766065-14.02534584-15.61502943$ $\mathrm{H} \quad 2.37953745-12.57265394-16.41041480$ $\mathrm{H} \quad 1.96839950-13.99103540-17.37677587$ $\mathrm{H} \quad 2.02130699-16.15797657-17.22591823$ $\mathrm{H} \quad 4.39950124-13.81041915-17.03866973$ $\mathrm{H} \quad 4.84849635-15.99024232-16.19317725$ $\mathrm{H} \quad 3.38112588-16.00325158-15.21461257$ $\mathrm{N} \quad 4.66750163-16.69636180-19.07807133$ C $\quad 5.15872491-16.55743988-20.44933457$ C $\quad 4.05749748-16.96495263-21.45608743$ O $\quad 3.86015243-16.19700264-22.36796251$ $\mathrm{H} \quad 5.07523478-17.42316192-18.48993190$ $\mathrm{H} \quad 5.44539759-15.52551181-20.63448057$ $\mathrm{H} \quad 6.02496269-17.20148994-20.60284065$ $\mathrm{N} \quad 1.58537750-14.26601807-21.66196010$ C $\quad 2.20634884-13.05059872-22.16694176$ C $\quad 2.48571209-13.18955234-23.69393614$ O $\quad 2.32485871-12.22368171-24.40200686$ C $\quad 3.55133406-12.79919400-21.41598328$ O $\quad 3.32370698-12.58737820-19.97855649$ $\mathrm{H} \quad 2.09737008-14.87469689-21.03080597$ $\mathrm{H} \quad 1.55984463-12.19293838-21.99812684$ $\mathrm{H} \quad 4.05332763-11.97180858-21.88851833$ $\mathrm{H} \quad 4.19359841-13.66966602-21.54947066$ $\mathrm{H} \quad 3.22718100-13.49122684-19.60910476$ $\mathrm{N} \quad-1.01159788-13.04812973-25.46768621$ C $\quad-1.44927147-11.65649444-25.47474985$ 
C $\quad-0.64096804-10.85219831-26.48941993$

O $\quad-1.19375409-10.01046090-27.20228019$

$\mathrm{H} \quad-0.54931491-13.44926665-24.66487832$

$\mathrm{H} \quad-2.49428571-11.62041319-25.79075822$

$\mathrm{N} \quad 0.70099172-11.00905438-26.47875278$

C $\quad 1.56268028-10.21692082-27.35997792$

C $\quad 1.19563023-10.53442968-28.83529222$

O $\quad 1.01087378-9.60653716-29.60411546$

C $\quad 3.06911725-10.47171160-27.09829798$

C $\quad 3.97008095-9.89445267-28.17760641$

C $\quad 4.28939825-8.53703600-28.14255170$

C $\quad 4.34281841-10.68852292-29.26702993$

C $\quad 5.11325918-8.00363044-29.13915202$

C $\quad 5.16504807-10.17237804-30.27305093$

C $\quad 5.58351043-8.83654780-30.17688998$

O $\quad 6.51589182-8.32966256-30.99418574$

$\mathrm{H} \quad 1.37451971-9.15663050-27.21513397$

H $\quad 5.45460067-6.99590579-29.06693218$

H $\quad 5.49465957-10.78119437-31.08766876$

$\mathrm{H} \quad 1.11766211-11.68668020-25.84781634$

$\mathrm{H} \quad 4.03254605-11.71325167-29.31939955$

$\mathrm{H} \quad 3.99569912-7.92321366-27.31678250$

$\mathrm{H} \quad 3.24392916-11.54093675-27.06447248$

$\mathrm{H} \quad 3.34218921-10.02561052-26.15003073$

$\mathrm{H} \quad 6.33447081-7.40323696-31.20745346$

C $\quad 7.66709882-15.96037459-16.34199642$

$\mathrm{H} \quad 7.11016063-16.34987810-17.18121854$

C $\quad 8.05921119-16.77917206-15.29727211$

$\mathrm{H} \quad 7.84246833-17.83778906-15.32096840$

C $\quad 8.76001285-16.18021893-14.25163939$

$\mathrm{H} \quad 9.13570530-16.75854857-13.43485695$

C $\quad 8.98685905-14.81091208-14.28080180$

$\mathrm{H} \quad 9.56412363-14.33997266-13.49715369$

$\mathrm{N} \quad 8.49249185-13.98339781-15.23261632$

C $\quad 7.90143276-14.60740673-16.26206072$

C $\quad 7.40204714-13.76378409-17.34574265$

C $\quad 7.53278837-14.18370857-18.68438156$

C $\quad 7.00916524-13.35822737-19.68269312$

C $\quad 6.39398444-12.15258881-19.38312439$

H $\quad 5.99780931-11.55195994-20.16976940$

F $\quad 7.08004872-13.74071528-20.98362827$

$\mathrm{H} \quad 8.06920618-15.09802877-18.92009413$

C $\quad 6.79071577-12.56228700-17.02007300$

H $\quad 6.67888452-12.32194376-15.97555877$

C $\quad 6.24096658-11.78572473-18.02521113$

C $\quad 5.43432173-10.60271142-17.65793798$

O $\quad 5.66858667-9.86528866-16.70292184$

$\mathrm{N} \quad 4.35779592-10.32644592-18.50587034$

$\mathrm{H} \quad 3.95845727-11.02975292-19.13481741$

$\mathrm{N} \quad 3.42557751-9.32951456-18.20892217$

$\mathrm{H} \quad 3.42180475-9.11637560-17.20776966$

S $\quad 3.59267518-7.94279257-19.07408757$

O $\quad 4.97343978-7.56548625-19.41947415$

O $\quad 2.81129546-6.86823437-18.43232756$

C $\quad 2.75862052-8.38242806-20.69916727$

C $\quad 1.61078814-7.66914217-21.10150698$

$\mathrm{H} \quad 1.12272481-6.98889650-20.41575324$

C $\quad 1.04310788-7.93352167-22.35953943$

$\mathrm{H} \quad 0.10580568-7.48092797-22.64216969$

C $\quad 1.62220951-8.87286377-23.20926711$

$\mathrm{H} \quad 1.16959392-9.11242105-24.16819671$

C $\quad 2.79819207-9.51776314-22.85905109$

$\mathrm{H} \quad 3.29426253-10.21021776-23.53279313$

C $\quad 3.34901746-9.27812973-21.59550465$

F $\quad 4.52794363-9.92891654-21.28698139$
Zn $\quad 28.07093513-6.24185607-6.91075209$

O $\quad-2.04746049-5.87859599-23.90440034$

$\mathrm{H} \quad-2.86992453-5.39903262-23.69258932$

$\mathrm{H} \quad-2.08434910-6.61197678-23.28917074$

O $\quad 7.33208642-19.78333198-13.50858018$

$\mathrm{H} \quad 8.21274782-19.45084771-13.25830866$

$\mathrm{H} \quad 7.29984156-20.64628414-13.08061717$

O $\quad 9.16514693-17.87421871-18.98818368$

H $\quad 9.88668937-17.27939509-18.69462128$

$\mathrm{H} \quad 9.57467583-18.39605876-19.68242906$

O $\quad 5.97664807-7.04957112-16.79797843$

$\mathrm{H} \quad 6.43082570-6.76204036-15.98352789$

$\mathrm{H} \quad 6.21200440-7.98542221-16.86207741$

O $\quad 26.47616157-0.94802544-4.85810727$

$\mathrm{H} \quad 27.12677038-0.86124674-4.14852531$

$\mathrm{H} \quad 26.61677208-1.83516396-5.20000483$

O $\quad 0.70091978-10.01415199-18.09922285$

$\mathrm{H} \quad-0.07133587-10.50402632-18.46274884$

$\mathrm{H} \quad 1.42693052-10.31182645-18.65238519$

O $\quad 4.77435874-18.65168310-14.46129455$

$\mathrm{H} \quad 5.69040634-18.93410874-14.28751665$

H $\quad 4.27505573-19.20314871-13.85041419$

O $\quad-0.45094987-6.74209485-25.79729544$

$\mathrm{H} \quad-0.89886810-7.39810716-26.34249537$

$\mathrm{H} \quad-1.15065925-6.40281755-25.20834522$

\section{C QM/SE/MM R1b (B3LYP/6- \\ 31G(d):xTB:Amber) mechanical embedding $\omega_{\alpha}=\mathbf{1 . 6 6 7}$}

C $\quad 22.26398727-8.92349891-4.44015221$

$\mathrm{O} \quad 21.41840878-8.04711262-4.33346493$

$\mathrm{N} \quad 23.53157430-8.68327051-4.82073223$

C $\quad 23.90641664-7.39114463-5.39137152$

C $\quad 23.07124037-7.22622293-6.69062700$

O $\quad 23.14087898-8.08816267-7.57295073$

C $\quad 25.40213469-7.39194661-5.87984048$

S $\quad 25.83595707-5.72739895-6.52282016$

$\mathrm{H} \quad 24.09271276-9.47991308-5.10980900$

$\mathrm{H} \quad 23.72600753-6.59131060-4.66750071$

$\mathrm{H} \quad 25.53307376-8.15243902-6.65732264$

$\mathrm{H} \quad 26.04482406-7.67368866-5.03464319$

N $\quad 22.35704803-6.09132866-6.81341678$

$\mathrm{H} \quad 22.12861836-5.57635732-5.96713108$

C $\quad 25.23825673-5.92306597-10.73965244$

$\mathrm{O} \quad 25.28578279-6.10499498-11.95948101$

$\mathrm{N} \quad 25.99930975-6.61414388-9.81628814$

C $\quad 26.81765614-7.70487659-10.29479263$

C $\quad 26.20250142-9.10638696-10.08074933$

O $\quad 26.70691547-10.13412986-10.55595702$

C $\quad 28.24958617-7.59722574-9.76548321$

S $\quad 28.27169470-8.12541028-7.90638618$

$\mathrm{H} \quad 25.94236485-6.38040463-8.82022503$

$\mathrm{H} \quad 26.84084006-7.58463439-11.38471857$

$\mathrm{H} \quad 28.64978444-6.58671792-9.88241011$

$\mathrm{H} \quad 28.89776148-8.29906559-10.29766496$

$\mathrm{N} \quad 25.05753235-9.14518129-9.34164075$

$\mathrm{H} \quad 24.58781224-8.32293151-8.98951884$

C $\quad 30.10264491-4.479479401 .27635010$

$\mathrm{O} \quad 30.71095117-3.631318660 .67418764$

$\mathrm{N} \quad 29.51429892-5.527208810 .64296642$

C $\quad 29.55464404-5.62051059-0.84066168$

C $\quad 29.05888215-4.32380441-1.46457323$

O $\quad 29.81527701-3.71687205-2.20372933$ 


\section{C QM/SE/MM R1b (B3LYP/6- 31G(d):xTB:Amber) mechanical embedding $\omega_{\alpha}=\mathbf{1 . 6 6 7}$}

C $\quad 22.26634521-8.92097895-4.44045206$ O $\quad 21.41684666-8.04754192-4.33322122$

$\mathrm{N} \quad 23.53123575-8.68329832-4.81992676$

C $\quad 23.90501927-7.39158538-5.39153555$

C $\quad 23.07168655-7.22697262-6.69079320$

O $\quad 23.14118526-8.08920704-7.57348104$

C $\quad 25.39957852-7.38956707-5.87751731$

$\mathrm{S} \quad 25.83579805-5.72714899-6.52249581$

$\mathrm{H} \quad 24.09635486-9.48032030-5.09952940$

$\mathrm{H} \quad 23.72225531-6.59228111-4.66959167$

$\mathrm{H} \quad 25.52698977-8.15215209-6.65155742$

$\mathrm{H} \quad 26.04122062-7.66739406-5.03129179$

$\mathrm{N} \quad 22.35635313-6.08942602-6.81497931$

$\mathrm{H} \quad 22.13644213-5.57021646-5.96994870$

C $\quad 25.23571633-5.92237677-10.74287403$

O $\quad 25.28302947-6.10726814-11.96015493$

$\mathrm{N} \quad 25.99906879-6.61421796-9.81681068$

C $\quad 26.81773257-7.70566186-10.29382064$

C $\quad 26.20179883-9.10587380-10.07943435$

O $\quad 26.70938916-10.13360571-10.55614872$

C $\quad 28.24742788-7.59925247-9.76167090$

S $\quad 28.27178241-8.12618334-7.90553162$

$\mathrm{H} \quad 25.94011562-6.37852531-8.82190164$

$\mathrm{H} \quad 26.84718714-7.58747721-11.38308239$

$\mathrm{H} \quad 28.64530106-6.58863497-9.87714579$

$\mathrm{H} \quad 28.89532592-8.29986637-10.29469068$

$\mathrm{N} \quad 25.05981542-9.14383200-9.34294823$

$\mathrm{H} \quad 24.58788550-8.32292109-8.99225333$

C $\quad 30.10315640-4.478995021 .27866508$

O $\quad 30.71022208-3.632608060 .67480766$

$\mathrm{N} \quad 29.51480876-5.525532000 .64350510$

C $\quad 29.55622347-5.61784511-0.83945324$

C $\quad 29.06055445-4.32162613-1.46409452$

O $\quad 29.81465771-3.71716718-2.20696859$

C $\quad 28.81640028-6.85870226-1.36012990$

C $\quad 28.92859053-6.95436193-2.84703124$

$\mathrm{H} \quad 29.22980153-7.75559833-0.87264079$

$\mathrm{H} \quad 28.99501759-6.229803881 .15911598$

$\mathrm{H} \quad 30.60796323-5.65994498-1.14313298$

$\mathrm{H} \quad 27.75519243-6.82239996-1.08303153$

C $\quad 28.14098307-6.45529406-3.86225832$

$\mathrm{H} \quad 30.88690889-7.79172127-3.01796887$

$\mathrm{H} \quad 27.22318168-5.88421990-3.82668892$

C $\quad 29.80245219-7.49161834-4.82780493$

$\mathrm{N} \quad 29.99135663-7.63044600-3.48282772$

$\mathrm{N} \quad 28.67626963-6.85093335-5.03137277$

$\mathrm{H} \quad 30.43918738-7.88218895-5.61101187$

$\mathrm{N} \quad 27.83596870-3.86087715-1.08869254$

$\mathrm{H} \quad 27.25132959-4.39979644-0.45154476$

C $\quad 32.40930414-1.93988779-3.92930672$

O $\quad 33.12265333-1.41928868-4.77955319$

$\mathrm{N} \quad 31.32133300-2.66857180-4.24031135$

C $\quad 30.98595505-3.04775972-5.63301070$

C $\quad 30.87050873-1.73816568-6.43135947$ 
O $\quad 30.19502942-0.80952393-6.06206935$

C $\quad 29.68648101-3.85831103-5.57782549$

$\mathrm{S} \quad 29.19833737-4.42621914-7.28077307$

$\mathrm{H} \quad 30.80990728-3.12220077-3.50243068$

$\mathrm{H} \quad 31.79217936-3.67388955-6.03772730$

$\mathrm{H} \quad 28.90443641-3.22675648-5.14674518$

$\mathrm{H} \quad 29.85118818-4.70587590-4.90589448$

$\mathrm{N} \quad 31.60997185-1.62040818-7.54497831$

$\mathrm{H} \quad 31.99970269-2.44138867-7.97352894$

C $\quad 7.70084550-15.97490091-16.33175113$

H $\quad 7.18906768-16.36084449-17.20597125$

C $\quad 8.08001002-16.78953443-15.27691093$

H $\quad 7.86182978-17.84869086-15.30029578$

C $\quad 8.76790038-16.19264612-14.20863745$

$\mathrm{H} \quad 9.13671887-16.79460812-13.39142392$

C $\quad 8.99275348-14.81940626-14.24444788$

$\mathrm{H} \quad 9.55181789-14.32523426-13.45231670$

$\mathrm{N} \quad 8.49557490-13.98284883-15.20600495$

C $\quad 7.90198550-14.60284262-16.25426977$

C $\quad 7.38723076-13.75191496-17.34486221$

C $\quad 7.49095605-14.19505302-18.70596409$

C $\quad 6.97209371-13.33725170-19.69683790$

C $\quad 6.37751735-12.11576164-19.43137430$

$\mathrm{H} \quad 6.03345779-11.51468454-20.25205177$

F $\quad 7.05594905-13.72348045-21.01123832$

$\mathrm{H} \quad 8.01584528-15.13090363-18.92750868$

C $\quad 6.82837311-12.51951365-17.03935673$

$\mathrm{H} \quad 6.80200656-12.23835815-15.98832193$

C $\quad 6.25716731-11.72508040-18.04234532$

C $\quad 5.45958278-10.59393298-17.66465200$

O $\quad 5.65932570-9.82363130-16.67174654$

$\mathrm{N} \quad 4.39925006-10.34994652-18.54406375$

$\mathrm{H} \quad 3.99675416-11.09159658-19.11148687$

$\mathrm{N} \quad 3.48022216-9.37727515-18.23515741$

$\mathrm{H} \quad 3.40994618-9.21529824-17.22858219$

S $\quad 3.62126604-7.97052766-19.07255935$

O $\quad 4.99851277-7.49855613-19.43655806$

O $\quad 2.85622697-6.92406045-18.36019659$

C $\quad 2.79084652-8.41155933-20.63653005$

C $\quad 1.64115623-7.63571813-21.04784754$

$\mathrm{H} \quad 1.18423755-6.93267505-20.35339452$

C $\quad 1.06497616-7.87428119-22.30270218$

$\mathrm{H} \quad 0.14376993-7.36277945-22.57513972$

C $\quad 1.60712408-8.83591664-23.17223609$

$\mathrm{H} \quad 1.14009626-9.06338949-24.12472231$

C $\quad 2.78337053-9.52734142-22.82437159$

$\mathrm{H} \quad 3.22286702-10.25260834-23.50206072$

C $\quad 3.36250638-9.31033254-21.57122230$

F $\quad 4.54123397-9.99482832-21.28044120$

\section{C QM/SE/MM R3b (B3LYP/6-}

\section{G(d):xTB:Amber) mechanical embedding}

$\omega_{o}=\mathbf{1 . 6 6 7}$

C $\quad 21.94126857-10.35051432-4.22419597$

C $\quad 22.25418007-8.89143491-4.44250367$

O $\quad 21.41128062-8.01405868-4.34952080$

$\mathrm{N} \quad 23.52430939-8.67546724-4.81768198$

C $\quad 23.89728852-7.39005715-5.40131637$

C $\quad 23.07798669-7.23041366-6.70796290$

O $\quad 23.13850600-8.10371004-7.58059725$

C $\quad 25.39582866-7.36565773-5.83015769$

S $\quad 25.84285697-5.72614468-6.52176980$

$\mathrm{H} \quad 24.13180624-9.47618782-4.97205996$

$\mathrm{H} \quad 23.69347277-6.58960090-4.68959628$

$\mathrm{H} \quad 25.58844563-8.17777121-6.53555508$

$\mathrm{H} \quad 25.98198499-7.56262610-4.92257637$

$\mathrm{N} \quad 22.38653397-6.08325395-6.84704626$

C $\quad 21.47882281-5.95725563-7.96224494$

C $\quad 22.16868981-5.65805541-9.31493421$

O $\quad 21.56126554-5.81377111-10.35561503$

C $\quad 20.48982790-4.86935086-7.64942759$

$\mathrm{H} \quad 20.93080333-6.89639795-8.10223553$

$\mathrm{H} \quad 22.22012269-5.51427624-6.02087443$

$\mathrm{N} \quad 23.42373924-5.18636493-9.17084674$

C $\quad 24.28423108-4.80921829-10.25049448$

C $\quad 25.22231151-5.89207672-10.73766789$

O $\quad 25.25803221-6.09736350-11.95407903$

C $\quad 25.10230282-3.59804592-9.91359396$

C $\quad 24.26595193-2.44638123-9.45869581$

C $\quad 23.30438460-1.87274038-10.28009723$

C $\quad 24.40519987-1.95613698-8.13461128$

C $\quad 22.54107504-0.79234848-9.83903639$

C $\quad 23.60900065-0.92908604-7.68326603$

C $\quad 22.69427924-0.31664053-8.53958796$

O $\quad 22.032098630 .79423664-8.09026480$

$\mathrm{H} \quad 23.62160506-4.57225785-11.09312573$

$\mathrm{H} \quad 21.80241366-0.36357596-10.49322118$

$\mathrm{H} \quad 23.71584750-0.53350671-6.69028143$

$\mathrm{H} \quad 23.84810458-5.31143851-8.25431642$

$\mathrm{H} \quad 25.13855899-2.38350073-7.47700542$

$\mathrm{H} \quad 23.13998544-2.25506677-11.27478322$

$\mathrm{H} \quad 25.66670316-3.28524578-10.79145620$

$\mathrm{H} \quad 25.81173316-3.83630150-9.11906283$

$\mathrm{H} \quad 22.411150661 .60808608-8.44361474$

N $\quad 25.99243867-6.58497505-9.82231425$

C $\quad 26.80756085-7.67869769-10.28985018$

C $\quad 26.19299035-9.07539503-10.08436967$

O $\quad 26.71533445-10.09522104-10.55757856$

C $\quad 28.22494173-7.58273837-9.74519135$

S $\quad 28.28080452-8.13998382-7.90917320$

$\mathrm{H} \quad 25.95265609-6.34221875-8.82838000$

$\mathrm{H} \quad 26.87527753-7.56427475-11.37582724$

$\mathrm{H} \quad 28.59933171-6.56564616-9.84688226$

$\mathrm{H} \quad 28.86692407-8.26709928-10.30239926$

$\mathrm{N} \quad 25.04233598-9.11412382-9.36278008$

C $\quad 24.38212836-10.39721946-9.15958384$

C $\quad 25.17375424-11.37620893-8.30029251$

O $\quad 24.82191060-12.53928822-8.13572670$

C $\quad 23.90727053-11.10091438-10.45259295$

C $\quad 22.96327734-10.19935453-11.26402395$

C $\quad 22.48150417-10.95379965-12.51772927$

C $\quad 21.72909333-9.80528379-10.45694716$

$\mathrm{H} \quad 22.03339167-9.21747870-9.59999540$

$\mathrm{H} \quad 21.06477142-9.21574263-11.06588060$

$\mathrm{H} \quad 21.22947103-10.70440545-10.10516970$

$\mathrm{H} \quad 24.61361593-8.29612563-8.95297739$

$\mathrm{H} \quad 21.96223450-11.85826325-12.21113336$

$\mathrm{H} \quad 21.81955803-10.32521608-13.09306548$

$\mathrm{H} \quad 23.33618394-11.24089878-13.12254017$

$\mathrm{H} \quad 23.46863037-10.17928144-8.59872245$

$\mathrm{H} \quad 23.49312112-9.30119966-11.56663305$

$\mathrm{H} \quad 23.38784394-12.00912763-10.16348708$

$\mathrm{H} \quad 24.77100430-11.33826497-11.06196604$

$\mathrm{N} \quad 26.21479056-10.83606763-7.61515320$

C $\quad 26.87196561-11.71087502-6.65449577$

C $\quad 25.83123016-12.12185126-5.54080263$

O $\quad 25.17137709-11.27703134-4.98105559$ 


\begin{tabular}{|c|c|}
\hline $\mathrm{C}$ & $28.02659892-10.97923928-5.98621901$ \\
\hline $\mathrm{C}$ & $28.65049542-11.80912961-4.87825927$ \\
\hline $\mathrm{C}$ & $29.93814849-11.13000786-4.34349044$ \\
\hline $\mathrm{C}$ & $30.33720351-11.83279277-3.03024472$ \\
\hline $\mathrm{N}$ & $31.77163792-11.69641969-2.78424944$ \\
\hline $\mathrm{H}$ & $27.22060315-12.62234003-7.15472242$ \\
\hline $\mathrm{H}$ & $29.78763748-11.39105147-2.20427438$ \\
\hline $\mathrm{H}$ & $30.10457756-12.89232236-3.10337608$ \\
\hline $\mathrm{H}$ & $27.93822558-11.87730583-4.05677436$ \\
\hline $\mathrm{H}$ & $28.88865128-12.81081532-5.21791553$ \\
\hline $\mathrm{H}$ & $32.10883772-12.32839401-2.04954672$ \\
\hline $\mathrm{H}$ & $32.32668149-11.91923216-3.63569952$ \\
\hline $\mathrm{H}$ & $32.00772426-10.73322031-2.49127957$ \\
\hline $\mathrm{H}$ & $29.76619246-10.07458263-4.18170788$ \\
\hline $\mathrm{H}$ & $30.71199964-11.26914845-5.09283931$ \\
\hline $\mathrm{H}$ & $26.59874773-9.92823171-7.85009723$ \\
\hline $\mathrm{H}$ & $27.69572469-10.02046049-5.60374961$ \\
\hline $\mathrm{H}$ & $28.78991570-10.79103916-6.74152331$ \\
\hline $\mathrm{N}$ & $25.76353801-13.42704580-5.19586379$ \\
\hline $\mathrm{C}$ & $24.89697945-13.85116827-4.13083612$ \\
\hline $\mathrm{H}$ & $26.19958655-14.15312837-5.74710110$ \\
\hline $\mathrm{C}$ & $29.87738705-4.487260812 .81045623$ \\
\hline $\mathrm{C}$ & $30.09721461-4.491083111 .26276390$ \\
\hline $\mathrm{O}$ & $30.70300399-3.638092010 .66485736$ \\
\hline $\mathrm{N}$ & $29.50808323-5.540163830 .63874008$ \\
\hline $\mathrm{C}$ & $29.57335687-5.61539075-0.83129121$ \\
\hline $\mathrm{C}$ & $29.07166490-4.32333917-1.44876909$ \\
\hline $\mathrm{O}$ & $29.79780904-3.72859220-2.22663739$ \\
\hline $\mathrm{C}$ & $28.83612195-6.83938290-1.37074588$ \\
\hline $\mathrm{C}$ & $28.95235999-6.94916480-2.84375844$ \\
\hline $\mathrm{H}$ & $29.25637154-7.73136126-0.89383927$ \\
\hline $\mathrm{H}$ & $28.97011033-6.224466141 .16317149$ \\
\hline $\mathrm{H}$ & $30.62964891-5.65986555-1.11339056$ \\
\hline $\mathrm{H}$ & $27.77939349-6.78242369-1.10554757$ \\
\hline $\mathrm{C}$ & $28.16685666-6.46398289-3.85958416$ \\
\hline $\mathrm{H}$ & $30.87713081-7.87236939-2.99936919$ \\
\hline $\mathrm{H}$ & $27.25849011-5.89121425-3.79276168$ \\
\hline $\mathrm{C}$ & $29.81951672-7.50651354-4.80641633$ \\
\hline $\mathrm{N}$ & $30.00201557-7.63348774-3.46720399$ \\
\hline $\mathrm{N}$ & $28.70021232-6.86306231-5.02989642$ \\
\hline $\mathrm{H}$ & $30.48990381-7.89529246-5.55302184$ \\
\hline $\mathrm{N}$ & $27.84715013-3.88409634-1.06035184$ \\
\hline $\mathrm{C}$ & $27.28148377-2.64221502-1.55395437$ \\
\hline $\mathrm{H}$ & $27.31862204-4.37524016-0.34172896$ \\
\hline $\mathrm{C}$ & $32.66140136-1.85458605-2.42719094$ \\
\hline $\mathrm{C}$ & $32.40766150-1.95967538-3.94895724$ \\
\hline $\mathrm{O}$ & $33.11897403-1.42609269-4.79128125$ \\
\hline $\mathrm{N}$ & $31.33818599-2.70695064-4.25979159$ \\
\hline $\mathrm{C}$ & $30.99027370-3.05794725-5.65523822$ \\
\hline $\mathrm{C}$ & $30.86810618-1.73812419-6.43581989$ \\
\hline $\mathrm{O}$ & $30.17056615-0.83136567-6.04635806$ \\
\hline $\mathrm{C}$ & $29.68155796-3.85083468-5.60397254$ \\
\hline $\mathrm{S}$ & $29.16818726-4.42420672-7.28683546$ \\
\hline $\mathrm{H}$ & $30.80580566-3.11172852-3.50594726$ \\
\hline $\mathrm{H}$ & $31.78242087-3.68885889-6.07330063$ \\
\hline $\mathrm{H}$ & $28.91101300-3.20548284-5.17580783$ \\
\hline $\mathrm{H}$ & $29.84250547-4.70139989-4.93946357$ \\
\hline $\mathrm{N}$ & $31.56531652-1.59529583-7.57232578$ \\
\hline $\mathrm{C}$ & $31.55426755-0.40753148-8.35957459$ \\
\hline $\mathrm{C}$ & $30.67042173-0.52796828-9.61470639$ \\
\hline $\mathrm{O}$ & $30.327938120 .43905355-10.27072995$ \\
\hline $\mathrm{C}$ & $33.00663004-0.10090071-8.72718175$ \\
\hline $\mathrm{H}$ & $31.156559110 .41463990-7.76306921$ \\
\hline $\mathrm{H}$ & $32.08301154-2.36443439-7.94570272$ \\
\hline 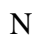 & $30.24789027-1.77275307-9.87735420$ \\
\hline
\end{tabular}

C

C

O

C

C

C

C

$\mathrm{N}$

C

$\mathrm{C}$

C

C

C

$\mathrm{H}$

$\mathrm{H}$

$\mathrm{H}$

$\mathrm{H}$

$\mathrm{H}$

$\mathrm{H}$

$\mathrm{H}$

$\mathrm{H}$

$\mathrm{H}$

$\mathrm{H}$

$\mathrm{N}$

C

$\mathrm{H}$

C

C

O

$\mathrm{N}$

C

C

O

C

C

$\mathrm{C}$

C

C

C

C

$\mathrm{H}$

$\mathrm{H}$

$\mathrm{H}$

$\mathrm{H}$

$\mathrm{H}$

$\mathrm{H}$

$\mathrm{H}$

$\mathrm{H}$

$\mathrm{H}$

N

C

C

O

C

C

C

C

$\mathrm{H}$

$\mathrm{H}$

$\mathrm{H}$

$\mathrm{H}$

$\mathrm{H}$

$\mathrm{H}$

$\mathrm{H}$

$\mathrm{H}$
$29.18716940-1.99505020-10.87355588$ $27.95109674-1.17026211-10.51960763$ $27.42023676-1.12544384-9.43351178$ $28.78349923-3.46932969-10.97470645$ $29.83638022-4.32947094-11.57993536$ $31.18532665-4.22640485-11.41993813$ $29.62507583-5.44674441-12.45258056$ $31.83324713-5.20332465-12.12375233$ $30.91079895-5.98951982-12.74349523$ $28.49577955-6.11079522-12.93885392$ $31.06352977-7.19555959-13.42149721$ $28.66243285-7.32240391-13.57594967$ $29.92049576-7.85955261-13.83241598$ $30.00851764-8.77276551-14.38549095$ $29.58402720-1.64257885-11.83302267$ $32.82998318-5.31609808-12.20997788$ $27.51347124-5.70333820-12.78304828$ $27.79327882-7.85911451-13.88609705$ $32.04945124-7.56464997-13.65078892$ $31.73659234-3.49360479-10.86982821$ $30.36501340-2.48952169-9.17086298$ $27.89600967-3.54899445-11.60135897$ $28.54567672-3.85133723-9.98129678$ $27.43870512-0.47357451-11.55082024$ $26.130403390 .15449985-11.47770303$ $27.93938186-0.35846061-12.43089118$ $14.00414517-19.32631751-8.43486537$ $12.58045847-19.03032025-8.87981331$ $11.65566567-19.11972444-8.10230809$ $12.47688478-18.59478114-10.15752490$ $11.19537141-18.22692070-10.69905820$ $10.81545245-18.99708218-11.93611428$ $9.76643452-18.75949310-12.51582975$ $11.05541139-16.72390793-10.93307647$ $11.57063706-15.96115257-9.73972147$ $10.80301316-15.89118335-8.56472998$ $12.80130452-15.30586365-9.80038187$ $11.28916734-15.18066294-7.47466709$ $13.28379683-14.62511446-8.69694785$ $12.52171409-14.55818957-7.52597738$ $12.91419049-14.03848211-6.67334785$ $10.46527188-18.53190411-9.93637499$ $10.69850692-15.13791266-6.58835516$ $14.24953370-14.15610812-8.75195836$ $13.29144140-18.49140570-10.75317264$ $13.39053338-15.37428632-10.69970150$ $9.85102764-16.38663493-8.51837270$ $10.01007583-16.47785500-11.08503355$ $11.63563722-16.43077348-11.80422450$ $11.61766351-19.99395965-12.30000587$ $11.29861419-20.75060583-13.47619499$ $11.31825989-22.21139649-13.11715337$ $12.23375423-22.71795511-12.50834127$ $12.28201411-20.45189335-14.63520386$ $12.23577006-18.99645646-15.05671778$ $13.33878261-18.76788286-16.07845755$ $10.87410606-18.65127439-15.64995359$ $10.11417083-18.76781193-14.89439471$ $10.88461472-17.63210452-16.00259407$ $10.67164865-19.32115939-16.48209529$ $12.47507588-20.19990562-11.81150385$ $13.19392586-19.40473428-16.94771310$ $13.31980696-17.73873009-16.41459492$ $14.31382416-18.97622007-15.65508975$ $10.29880151-20.45698985-13.79867076$ 


\begin{tabular}{|c|c|c|c|}
\hline $\mathrm{H}$ & $12.39919473-18.34459853-14.20179275$ & $\mathrm{C}$ & $11.29029442-10.40657471-11.44556413$ \\
\hline $\mathrm{H}$ & $12.01954097-21.07973475-15.48078457$ & $\mathrm{H}$ & $11.90658477-9.53007626-13.31270721$ \\
\hline $\mathrm{H}$ & $13.28511143-20.71043926-14.30591245$ & $\mathrm{C}$ & $7.89832056-6.08053557-12.13455221$ \\
\hline $\mathrm{N}$ & $10.24494756-22.87619158-13.56381045$ & $\mathrm{C}$ & $6.86560589-6.75262238-13.05176581$ \\
\hline $\mathrm{C}$ & $10.13342367-24.32164228-13.36917201$ & $\mathrm{O}$ & $6.61048240-6.32322761-14.14931732$ \\
\hline $\mathrm{H}$ & $9.59930757-22.47925367-14.23359036$ & $\mathrm{~N}$ & $6.44559762-7.96150420-12.65396183$ \\
\hline $\mathrm{C}$ & $14.09735237-18.54641718-21.17681653$ & $\mathrm{C}$ & $5.62004516-8.76975256-13.48890197$ \\
\hline $\mathrm{C}$ & $13.89434970-17.05586811-21.07073759$ & $\mathrm{C}$ & $4.30252775-7.98011041-13.74786687$ \\
\hline $\mathrm{O}$ & $14.71224501-16.27339792-21.45967844$ & $\mathrm{O}$ & $3.92559800-7.15152188-12.93321317$ \\
\hline $\mathrm{N}$ & $12.72264838-16.68782138-20.52828465$ & $\mathrm{C}$ & $5.34924969-10.13028313-12.89761416$ \\
\hline $\mathrm{C}$ & $12.42615044-15.30977775-20.26711283$ & $\mathrm{C}$ & $6.62232452-10.97838325-12.89003772$ \\
\hline $\mathrm{C}$ & $11.79957693-15.21999829-18.83911403$ & $\mathrm{C}$ & $6.37486070-12.30378927-12.28276060$ \\
\hline $\mathrm{O}$ & $10.95257558-16.03804871-18.45983280$ & $\mathrm{H}$ & $6.92758679-11.15191603-13.92842122$ \\
\hline $\mathrm{C}$ & $11.50595452-14.71415596-21.34670791$ & $\mathrm{H}$ & $6.12667189-8.87992795-14.45724140$ \\
\hline $\mathrm{C}$ & $11.23739965-13.26361425-21.06440315$ & $\mathrm{H}$ & $7.44155146-10.47787958-12.40007509$ \\
\hline $\mathrm{C}$ & $10.22764551-15.54378919-21.47304254$ & $\mathrm{H}$ & $6.65838130-8.26016017-11.71041638$ \\
\hline $\mathrm{C}$ & $10.56527049-12.54428763-22.20496689$ & $\mathrm{H}$ & $4.58760008-10.62628512-13.49475557$ \\
\hline $\mathrm{H}$ & $13.37629119-14.77409635-20.27842997$ & $\mathrm{H}$ & $4.97786593-10.03828867-11.87905452$ \\
\hline $\mathrm{H}$ & $9.62997888-15.14584557-22.27948318$ & $\mathrm{H}$ & $8.29994198-12.86960801-12.58981216$ \\
\hline $\mathrm{H}$ & $10.48509161-16.57166182-21.69181685$ & $\mathrm{O}$ & $5.25367307-12.59156594-11.86632569$ \\
\hline $\mathrm{H}$ & $12.05120727-14.78731300-22.28716887$ & $\mathrm{~N}$ & $7.43303523-13.11777087-12.12981168$ \\
\hline $\mathrm{H}$ & $10.59336620-13.18998677-20.18256528$ & $\mathrm{H}$ & $7.30300810-14.07067556-11.81982412$ \\
\hline $\mathrm{H}$ & $12.17182029-12.75999225-20.83124739$ & $\mathrm{~N}$ & $3.76075619-8.24843857-14.97710985$ \\
\hline $\mathrm{H}$ & $9.60349670-12.99434624-22.42424922$ & $\mathrm{C}$ & $2.46577627-7.76232743-15.40685893$ \\
\hline $\mathrm{H}$ & $12.09435256-17.40117846-20.19020274$ & $\mathrm{C}$ & $2.45955070-6.23686887-15.44537879$ \\
\hline $\mathrm{H}$ & $10.40179079-11.50947120-21.93554277$ & $\mathrm{O}$ & $1.45383013-5.61792660-15.15874832$ \\
\hline $\mathrm{H}$ & $9.66777290-15.47278169-20.54895501$ & $\mathrm{C}$ & $1.35161478-8.28179708-14.46727440$ \\
\hline $\mathrm{H}$ & $11.18827569-12.58924955-23.09234220$ & $\mathrm{C}$ & $1.27623319-9.81053798-14.45625899$ \\
\hline $\mathrm{N}$ & $12.17193331-14.18316253-18.08229979$ & $\mathrm{C}$ & $0.09082473-10.24225624-13.60795157$ \\
\hline $\mathrm{C}$ & $11.97728689-14.17591262-16.59670958$ & $\mathrm{~N}$ & $0.01804383-11.64632988-13.31720343$ \\
\hline $\mathrm{C}$ & $11.95897988-12.72772786-16.23948468$ & $\mathrm{C}$ & $-0.66998434-12.53283202-14.08515142$ \\
\hline $\mathrm{O}$ & $12.90607953-12.01997145-16.52588712$ & $\mathrm{~N}$ & $-1.62975169-12.08083031-14.95696278$ \\
\hline $\mathrm{C}$ & $13.03562273-14.91284573-15.81829599$ & $\mathrm{~N}$ & $-0.41144386-13.89200117-13.96433793$ \\
\hline $\mathrm{C}$ & $12.66134559-15.13140249-14.26684312$ & $\mathrm{H}$ & $2.17926252-10.23617388-14.02615783$ \\
\hline $\mathrm{S}$ & $12.77568913-13.57684125-13.37367587$ & $\mathrm{H}$ & $2.22124832-8.12570604-16.40296383$ \\
\hline $\mathrm{C}$ & $14.54486711-13.23281308-13.56949898$ & $\mathrm{H}$ & $0.75264416-12.03123448-12.72540845$ \\
\hline $\mathrm{H}$ & $11.62382028-15.44454270-14.22085670$ & $\mathrm{H}$ & $1.15533716-10.17741623-15.47436500$ \\
\hline $\mathrm{H}$ & $14.87160917-12.64792942-12.72027876$ & $\mathrm{H}$ & $-1.19013313-14.49479635-14.19054476$ \\
\hline $\mathrm{H}$ & $15.08615151-14.17661346-13.58577310$ & $\mathrm{H}$ & $-0.00354967-14.14810181-13.07118747$ \\
\hline $\mathrm{H}$ & $14.71803417-12.68981708-14.49136368$ & $\mathrm{H}$ & $4.31335790-8.88842967-15.55025634$ \\
\hline $\mathrm{H}$ & $13.22159730-15.89091125-16.24418284$ & $\mathrm{H}$ & $-0.83909056-9.94477100-14.09040891$ \\
\hline $\mathrm{H}$ & $13.32443036-15.88878757-13.86602835$ & $\mathrm{H}$ & $0.13014230-9.73027313-12.64609168$ \\
\hline $\mathrm{H}$ & $12.90963717-13.58432492-18.45639996$ & $\mathrm{H}$ & $-1.99656556-12.79243807-15.56705089$ \\
\hline $\mathrm{H}$ & $11.00550208-14.65432149-16.44070373$ & $\mathrm{H}$ & $-1.42561619-11.20285425-15.40481141$ \\
\hline $\mathrm{H}$ & $13.95680317-14.33675325-15.84412761$ & $\mathrm{H}$ & $0.40926173-7.86735161-14.81275067$ \\
\hline $\mathrm{N}$ & $10.79217278-12.27563194-15.74117997$ & $\mathrm{H}$ & $1.54072346-7.92237159-13.45864564$ \\
\hline $\mathrm{C}$ & $10.67774556-10.91353427-15.23015858$ & $\mathrm{~N}$ & $3.59632534-5.63766339-15.83105067$ \\
\hline $\mathrm{C}$ & $10.54248616-11.05188782-13.67116604$ & $\mathrm{C}$ & $3.68689600-4.18784202-15.86311357$ \\
\hline $\mathrm{O}$ & $9.85893873-11.92207497-13.15699586$ & $\mathrm{C}$ & $4.28282460-3.65115153-17.17031498$ \\
\hline $\mathrm{C}$ & $9.42506025-10.22289655-15.73345080$ & $\mathrm{O}$ & $4.57100497-2.47689406-17.31505230$ \\
\hline $\mathrm{C}$ & $9.39228469-10.12525151-17.27810303$ & $\mathrm{C}$ & $4.47077576-3.65149825-14.67535123$ \\
\hline $\mathrm{C}$ & $9.25068479-8.84063693-15.11213458$ & $\mathrm{C}$ & $3.63836254-3.54547895-13.41157015$ \\
\hline $\mathrm{C}$ & $10.48796243-9.19222596-17.74895688$ & $\mathrm{C}$ & $4.50706689-3.77302395-12.18863765$ \\
\hline $\mathrm{H}$ & $11.57716149-10.36110550-15.48037490$ & $\mathrm{~N}$ & $3.84104380-3.32816571-10.97701358$ \\
\hline $\mathrm{H}$ & $8.33781617-8.38426392-15.47445471$ & $\mathrm{C}$ & $3.44504713-4.14993742-9.97995162$ \\
\hline $\mathrm{H}$ & $9.17463630-8.93841516-14.03271966$ & $\mathrm{~N}$ & $3.25904681-5.47465764-10.21395789$ \\
\hline $\mathrm{H}$ & $8.56835903-10.83424701-15.44463373$ & $\mathrm{~N}$ & $3.08007386-3.63692518-8.76264341$ \\
\hline $\mathrm{H}$ & $9.53369754-11.11555262-17.69077746$ & $\mathrm{H}$ & $2.83292365-4.27269326-13.41497026$ \\
\hline $\mathrm{H}$ & $8.42315356-9.73512718-17.56045230$ & $\mathrm{H}$ & $2.66377504-3.80821081-15.77731497$ \\
\hline $\mathrm{H}$ & $11.45769676-9.58606168-17.45597361$ & $\mathrm{H}$ & $3.81924719-2.34090372-10.80188443$ \\
\hline $\mathrm{H}$ & $9.98941215-12.89409209-15.59578989$ & $\mathrm{H}$ & $3.19958145-2.54914978-13.34254589$ \\
\hline $\mathrm{H}$ & $10.48528530-9.13325747-18.82820178$ & $\mathrm{H}$ & $2.57110646-4.28261263-8.18110413$ \\
\hline $\mathrm{H}$ & $10.09730008-8.20482724-15.33220556$ & $\mathrm{H}$ & $2.72575682-2.69715625-8.77831408$ \\
\hline $\mathrm{H}$ & $10.37630155-8.19221770-17.35521550$ & $\mathrm{H}$ & $4.42245519-6.19343775-16.03265901$ \\
\hline $\mathrm{N}$ & $11.34353764-10.28296028-12.92883591$ & $\mathrm{H}$ & $5.44769263-3.22785584-12.27823856$ \\
\hline
\end{tabular}




\begin{tabular}{|c|c|c|c|}
\hline $\mathrm{H}$ & $4.73868644-4.83428495-12.12546687$ & $\mathrm{C}$ & $8.92289118-5.13448542-23.92742657$ \\
\hline $\mathrm{H}$ & $3.29670391-6.11346437-9.42048622$ & $\mathrm{H}$ & $7.63390371-5.59811314-22.34063117$ \\
\hline $\mathrm{H}$ & $3.83157305-5.85797162-10.95060566$ & $\mathrm{C}$ & $11.29232766-8.07323074-23.87669680$ \\
\hline $\mathrm{H}$ & $4.87129224-2.67393152-14.93397242$ & $\mathrm{C}$ & $10.87106095-8.98204082-25.04540229$ \\
\hline $\mathrm{H}$ & $5.30515463-4.32880567-14.48617729$ & $\mathrm{O}$ & $11.69831828-9.32138987-25.87437940$ \\
\hline $\mathrm{N}$ & $4.27750185-4.52556229-18.20130919$ & $\mathrm{~N}$ & $9.57893484-9.39763848-24.96655606$ \\
\hline $\mathrm{C}$ & $4.76556935-4.11607990-19.49464841$ & $\mathrm{C}$ & $9.07304659-10.21584321-26.06274925$ \\
\hline $\mathrm{C}$ & $6.24240496-4.47763568-19.85798845$ & $\mathrm{C}$ & $9.21621777-9.41695842-27.38471017$ \\
\hline $\mathrm{O}$ & $6.59494802-4.42275328-21.04221378$ & $\mathrm{O}$ & $9.80573429-9.86693761-28.32662855$ \\
\hline $\mathrm{H}$ & $4.01770531-5.49460783-18.05045529$ & $\mathrm{C}$ & $7.62723062-10.69454377-25.83041284$ \\
\hline $\mathrm{H}$ & $4.14960461-4.57418867-20.26897577$ & $\mathrm{C}$ & $7.57868896-11.65146398-24.64732936$ \\
\hline $\mathrm{H}$ & $4.70568920-3.02407968-19.53979965$ & $\mathrm{C}$ & $7.12477116-11.38542811-27.08138028$ \\
\hline $\mathrm{N}$ & $7.01483766-4.84180992-18.82451290$ & $\mathrm{C}$ & $6.17613618-11.89074327-24.13992644$ \\
\hline $\mathrm{C}$ & $8.42272940-5.17731971-18.97813713$ & $\mathrm{H}$ & $9.73005907-11.08801627-26.17421695$ \\
\hline $\mathrm{C}$ & $8.58096059-6.48567374-19.74019750$ & $\mathrm{H}$ & $6.09972987-11.70167210-26.92092930$ \\
\hline $\mathrm{O}$ & $9.38776986-6.54200766-20.65493153$ & $\mathrm{H}$ & $7.14735899-10.70567900-27.92102035$ \\
\hline $\mathrm{C}$ & $9.16341097-5.24567296-17.59052400$ & $\mathrm{H}$ & $7.02000829-9.81441529-25.61622611$ \\
\hline $\mathrm{C}$ & $9.09697137-3.92852880-16.86886483$ & $\mathrm{H}$ & $8.04717722-12.58784270-24.93832097$ \\
\hline $\mathrm{C}$ & $9.95321321-2.87550387-17.12173409$ & $\mathrm{H}$ & $8.16056331-11.22498003-23.83288431$ \\
\hline $\mathrm{C}$ & $8.17050305-3.77585969-15.85253583$ & $\mathrm{H}$ & $5.58169274-12.33400948-24.93581296$ \\
\hline $\mathrm{C}$ & $9.86574071-1.69247108-16.41411483$ & $\mathrm{H}$ & $8.99182558-9.14501367-24.18962776$ \\
\hline $\mathrm{C}$ & $8.07546830-2.61678259-15.09928289$ & $\mathrm{H}$ & $6.21577620-12.57534865-23.30537390$ \\
\hline $\mathrm{C}$ & $8.95166984-1.56465504-15.34652368$ & $\mathrm{H}$ & $7.74019414-12.24778405-27.29535574$ \\
\hline $\mathrm{O}$ & $8.78583811-0.43369619-14.62862441$ & $\mathrm{H}$ & $5.72395353-10.95299354-23.84597674$ \\
\hline $\mathrm{H}$ & $8.90537445-4.39512328-19.57221892$ & $\mathrm{~N}$ & $8.74037513-8.17543930-27.38530276$ \\
\hline $\mathrm{H}$ & $10.55502423-0.88570126-16.59834836$ & $\mathrm{C}$ & $8.84823536-7.32536146-28.54605145$ \\
\hline $\mathrm{H}$ & $7.35190802-2.53547380-14.31491262$ & $\mathrm{H}$ & $8.24007907-7.78120829-26.60100054$ \\
\hline $\mathrm{H}$ & $6.62672746-4.86459311-17.88905209$ & $\mathrm{C}$ & $6.59359662-21.92030832-21.79991568$ \\
\hline $\mathrm{H}$ & $7.51059209-4.59419622-15.61809572$ & $\mathrm{C}$ & $6.23320887-22.03540259-20.30803359$ \\
\hline $\mathrm{H}$ & $10.70877667-2.95535847-17.87961130$ & $\mathrm{O}$ & $5.53516755-22.93761182-19.89153921$ \\
\hline $\mathrm{H}$ & $10.19375395-5.46858554-17.82950007$ & $\mathrm{~N}$ & $6.59122873-20.95112292-19.60925902$ \\
\hline $\mathrm{H}$ & $8.73067938-6.03692052-16.99243140$ & $\mathrm{C}$ & $6.12493903-20.90759504-18.21528849$ \\
\hline $\mathrm{H}$ & $9.627931740 .13254065-14.74926466$ & $\mathrm{C}$ & $4.62235295-20.55895977-18.32237909$ \\
\hline $\mathrm{N}$ & $7.78518391-7.50863844-19.35412496$ & $\mathrm{O}$ & $4.11880132-20.20885651-19.39091833$ \\
\hline $\mathrm{C}$ & $7.80179964-8.76290418-20.04539229$ & $\mathrm{C}$ & $6.83905527-19.84828129-17.39445127$ \\
\hline $\mathrm{C}$ & $7.39932665-8.57387689-21.59482046$ & $\mathrm{O}$ & $6.65131777-18.55912319-17.96047430$ \\
\hline $\mathrm{O}$ & $7.95929554-9.22994988-22.44842874$ & $\mathrm{H}$ & $7.27639844-20.29179200-19.95379103$ \\
\hline $\mathrm{H}$ & $7.06676172-7.33959089-18.66129762$ & $\mathrm{H}$ & $6.24900271-21.89770812-17.76606828$ \\
\hline $\mathrm{H}$ & $8.80369234-9.19158485-20.07423003$ & $\mathrm{H}$ & $7.90211005-20.09437895-17.32999349$ \\
\hline $\mathrm{H}$ & $7.09721731-9.45956586-19.60121786$ & $\mathrm{H}$ & $6.41565554-19.83794359-16.38615124$ \\
\hline $\mathrm{N}$ & $6.51988010-7.56335565-21.81345889$ & $\mathrm{H}$ & $7.52360523-18.28077032-18.31171475$ \\
\hline $\mathrm{C}$ & $5.97192391-7.33841234-23.12821086$ & $\mathrm{~N}$ & $3.96169984-20.53940105-17.13706003$ \\
\hline $\mathrm{C}$ & $7.08274887-6.70744636-23.99363085$ & $\mathrm{C}$ & $2.53983278-20.23847232-17.16141710$ \\
\hline $\mathrm{O}$ & $7.18978190-6.96338994-25.18270725$ & $\mathrm{C}$ & $2.34899152-18.74772659-17.57470089$ \\
\hline $\mathrm{C}$ & $4.76344179-6.41494954-23.07590328$ & $\mathrm{O}$ & $1.51078111-18.38207085-18.37786804$ \\
\hline $\mathrm{C}$ & $4.15983211-6.07476763-24.46620052$ & $\mathrm{C}$ & $1.92425287-20.51093143-15.79033630$ \\
\hline $\mathrm{C}$ & $2.93813073-5.18934003-24.22158707$ & $\mathrm{H}$ & $4.33088520-21.06968234-16.35712783$ \\
\hline $\mathrm{N}$ & $2.26103228-4.80057494-25.43011320$ & $\mathrm{H}$ & $2.01884017-20.84725768-17.90556727$ \\
\hline $\mathrm{C}$ & $1.14294583-4.04865778-25.45796288$ & $\mathrm{~N}$ & $3.20108104-17.88281396-17.00488573$ \\
\hline $\mathrm{N}$ & $0.65219044-3.56522475-24.25579770$ & $\mathrm{C}$ & $3.08540505-16.45950995-17.25091122$ \\
\hline $\mathrm{N}$ & $0.68315102-3.56645255-26.63009739$ & $\mathrm{C}$ & $3.61137118-16.06472689-18.64094409$ \\
\hline $\mathrm{H}$ & $3.83406000-6.97551175-24.97915549$ & $\mathrm{O}$ & $3.11583308-15.15828965-19.32268324$ \\
\hline $\mathrm{H}$ & $5.68751492-8.28487225-23.61205609$ & $\mathrm{C}$ & $3.82088225-15.69521227-16.17645980$ \\
\hline $\mathrm{H}$ & $2.64893688-5.09877280-26.32455664$ & $\mathrm{C}$ & $3.76832007-14.17079334-16.26313885$ \\
\hline $\mathrm{H}$ & $4.88082447-5.54201423-25.07041324$ & $\mathrm{C}$ & $2.34236205-13.67436963-16.47995485$ \\
\hline $\mathrm{H}$ & $-0.26089422-3.22046384-26.70250416$ & $\mathrm{C}$ & $4.31718240-13.61366886-14.93993431$ \\
\hline $\mathrm{H}$ & $1.00462553-4.01026887-27.48412828$ & $\mathrm{H}$ & $5.33014578-13.95703864-14.77544754$ \\
\hline $\mathrm{H}$ & $5.98143651-7.29075529-20.99647316$ & $\mathrm{H}$ & $4.29710353-12.53135338-14.95995726$ \\
\hline $\mathrm{H}$ & $3.25345642-4.29541640-23.68195168$ & $\mathrm{H}$ & $3.69829719-13.95453558-14.11320862$ \\
\hline $\mathrm{H}$ & $2.23540921-5.75530607-23.59822530$ & $\mathrm{H}$ & $3.87548069-18.22784950-16.32092166$ \\
\hline $\mathrm{H}$ & $-0.23948739-3.08572187-24.35910675$ & $\mathrm{H}$ & $1.70862993-14.02495872-15.66715167$ \\
\hline $\mathrm{H}$ & $0.69472296-4.28826219-23.55589528$ & $\mathrm{H}$ & $2.34037680-12.58812575-16.46485407$ \\
\hline $\mathrm{H}$ & $5.03085781-5.48317235-22.58823595$ & $\mathrm{H}$ & $1.95909907-14.01527176-17.42733108$ \\
\hline $\mathrm{H}$ & $3.98968609-6.91556078-22.49570252$ & $\mathrm{H}$ & $2.02419769-16.19280958-17.23122868$ \\
\hline $\mathrm{N}$ & $7.76259243-5.76164997-23.33109627$ & $\mathrm{H}$ & $4.40319933-13.80888855-17.07009158$ \\
\hline
\end{tabular}




\begin{tabular}{|c|c|}
\hline $\mathrm{H}$ & $4.86979422-15.98585191-16.18455652$ \\
\hline $\mathrm{H}$ & $3.40146147-15.98455720-15.21248516$ \\
\hline $\mathrm{N}$ & $4.65537708-16.73486120-19.07261934$ \\
\hline $\mathrm{C}$ & $5.14180426-16.51624933-20.42608227$ \\
\hline $\mathrm{C}$ & $4.05841420-16.91476328-21.46661347$ \\
\hline $\mathrm{O}$ & $3.84923570-16.16581413-22.39107965$ \\
\hline $\mathrm{H}$ & $5.12009519-17.43964078-18.50214508$ \\
\hline $\mathrm{H}$ & $5.38142388-15.46508449-20.58733370$ \\
\hline $\mathrm{H}$ & $6.03141112-17.12463583-20.58579793$ \\
\hline $\mathrm{N}$ & $3.38352890-18.06172085-21.29160805$ \\
\hline $\mathrm{C}$ & $2.28384429-18.49266053-22.16789878$ \\
\hline $\mathrm{H}$ & $3.64212271-18.70772617-20.54795757$ \\
\hline $\mathrm{C}$ & $-0.21892821-15.82577368-21.26966975$ \\
\hline $\mathrm{C}$ & $0.32352223-14.61223263-21.99414654$ \\
\hline $\mathrm{O}$ & $-0.42818053-14.00176774-22.73305432$ \\
\hline $\mathrm{N}$ & $1.57848723-14.27836127-21.69164625$ \\
\hline $\mathrm{C}$ & $2.16698122-13.05286187-22.16646747$ \\
\hline $\mathrm{C}$ & $2.47927115-13.16859561-23.70473794$ \\
\hline $\mathrm{O}$ & $2.34407780-12.19790700-24.40952622$ \\
\hline $\mathrm{C}$ & $3.51813944-12.76564563-21.40897811$ \\
\hline $\mathrm{O}$ & $3.30811911-12.56384110-19.98032550$ \\
\hline $\mathrm{H}$ & $2.08517851-14.79723489-20.98257054$ \\
\hline $\mathrm{H}$ & $1.49279036-12.20519798-22.02601642$ \\
\hline $\mathrm{H}$ & $3.97236235-11.87854133-21.82109402$ \\
\hline $\mathrm{H}$ & $4.17823184-13.62040156-21.56420353$ \\
\hline $\mathrm{H}$ & $3.24432025-13.44122414-19.57416509$ \\
\hline $\mathrm{N}$ & $2.80538747-14.36202041-24.17406703$ \\
\hline $\mathrm{C}$ & $3.07333290-14.52082505-25.60718477$ \\
\hline $\mathrm{H}$ & $3.15801941-15.09928222-23.57483640$ \\
\hline $\mathrm{C}$ & $-0.57268130-15.19133509-26.42371091$ \\
\hline $\mathrm{C}$ & $-1.14229331-13.78420788-26.53940716$ \\
\hline $\mathrm{O}$ & $-1.61404594-13.34839781-27.56210842$ \\
\hline $\mathrm{N}$ & $-1.00278424-13.05119161-25.44466150$ \\
\hline $\mathrm{C}$ & $-1.45430259-11.68250874-25.51800713$ \\
\hline $\mathrm{C}$ & $-0.65105844-10.86041809-26.53044330$ \\
\hline $\mathrm{O}$ & $-1.17487589-10.02253965-27.24143092$ \\
\hline $\mathrm{C}$ & $-1.40822698-11.10856079-24.10459023$ \\
\hline $\mathrm{H}$ & $-0.63863818-13.40097615-24.57259342$ \\
\hline $\mathrm{H}$ & $-2.49543641-11.65311713-25.86433910$ \\
\hline $\mathrm{N}$ & $0.69581293-11.06858221-26.51346532$ \\
\hline $\mathrm{C}$ & $1.55393702-10.22149829-27.33010115$ \\
\hline $\mathrm{C}$ & $1.18147721-10.47189713-28.84088390$ \\
\hline $\mathrm{O}$ & $1.00332452-9.55725145-29.59585264$ \\
\hline $\mathrm{C}$ & $3.06850914-10.48497198-27.09111505$ \\
\hline $\mathrm{C}$ & $3.95296129-9.89826561-28.17763912$ \\
\hline $\mathrm{C}$ & $4.27708175-8.55427890-28.15042788$ \\
\hline $\mathrm{C}$ & $4.33917250-10.66565064-29.26187939$ \\
\hline $\mathrm{C}$ & $5.08525006-8.01993835-29.13207423$ \\
\hline $\mathrm{C}$ & $5.16004665-10.16184108-30.25309224$ \\
\hline $\mathrm{C}$ & $5.56250049-8.83603996-30.16360389$ \\
\hline $\mathrm{O}$ & $6.50708365-8.34298420-30.99129206$ \\
\hline $\mathrm{H}$ & $1.34638790-9.16152818-27.16275448$ \\
\hline $\mathrm{H}$ & $5.39103022-6.98926547-29.09682171$ \\
\hline $\mathrm{H}$ & $5.49584365-10.76486146-31.07415027$ \\
\hline $\mathrm{H}$ & $1.12382680-11.64789092-25.80225462$ \\
\hline $\mathrm{H}$ & $4.04077745-11.69892219-29.30718017$ \\
\hline $\mathrm{H}$ & $3.97487573-7.93058855-27.32619618$ \\
\hline $\mathrm{H}$ & $3.22678993-11.55836963-27.06189257$ \\
\hline $\mathrm{H}$ & $3.34266643-10.05177819-26.13543624$ \\
\hline $\mathrm{H}$ & $6.36331517-7.39102257-31.16943210$ \\
\hline $\mathrm{N}$ & $1.14872638-11.75736678-29.18264821$ \\
\hline $\mathrm{C}$ & $0.80767637-12.22207600-30.51402524$ \\
\hline $\mathrm{H}$ & $1.36762523-12.50474155-28.53441989$ \\
\hline $\mathrm{C}$ & $7.67541671-15.96790468-16.34402422$ \\
\hline $\mathrm{H}$ & $7.12990383-16.36009959-17.19034476$ \\
\hline
\end{tabular}

C $\quad 8.06755817-16.78455571-15.29825222$ $\mathrm{H} \quad 7.84966255-17.84211670-15.31831820$ C $\quad 8.76599736-16.19219847-14.24044088$ $\mathrm{H} \quad 9.14418350-16.78848605-13.43256603$ C $\quad 9.00020640-14.82277410-14.27569025$ $\mathrm{H} \quad 9.58498305-14.33698303-13.50240840$ $\mathrm{N} \quad 8.51109295-13.98725245-15.23213099$ C $\quad 7.89942045-14.60389735-16.26580291$ C $\quad 7.39748140-13.76224704-17.34596174$ C $\quad 7.51363701-14.19641292-18.69673633$ C $\quad 6.99412680-13.35303448-19.68685056$ C $\quad 6.38223008-12.14750740-19.40103933$ $\mathrm{H} \quad 6.01271373-11.53626433-20.19313025$ F $\quad 7.07584112-13.73271730-20.99624863$ $\mathrm{H} \quad 8.05426253-15.10792165-18.92685732$ C $\quad 6.81209566-12.54986303-17.02831594$ $\mathrm{H} \quad 6.72331246-12.29253420-15.98182758$ C $\quad 6.24600728-11.76075818-18.02563239$ C $\quad 5.44533866-10.60764399-17.66541763$ O $\quad 5.65846180-9.84718628-16.67821024$

$\mathrm{N} \quad 4.37769129-10.33958100-18.53064185$ $\mathrm{H} \quad 3.96612984-11.06111230-19.12164729$ $\mathrm{N} \quad 3.44970861-9.34558582-18.22418524$ $\mathrm{H} \quad 3.45685674-9.12794346-17.21864019$ S $\quad 3.60348575-7.95621867-19.08511453$ O $\quad 4.99603390-7.54729729-19.42635593$ O $\quad 2.83098030-6.88325246-18.41284485$ C $\quad 2.78028160-8.38113782-20.68181547$ C $\quad 1.62794516-7.64834650-21.08531796$ $\mathrm{H} \quad 1.16076751-6.95751495-20.39559699$ C $\quad 1.05243540-7.90760889-22.33613394$ $\mathrm{H} \quad 0.12666018-7.42364401-22.61524279$ C $\quad 1.60879500-8.86311649-23.19395916$ $\mathrm{H} \quad 1.14056643-9.09812634-24.14213033$ C $\quad 2.78475454-9.53097554-22.84570051$ $\mathrm{H} \quad 3.22863705-10.25974922-23.51235102$ C $\quad 3.35219815-9.28770928-21.59195620$ F $\quad 4.52778694-9.95964346-21.28182431$ $\mathrm{Zn} \quad 28.07132634-6.25160132-6.90925493$ O $\quad-2.06705675-5.89948902-23.83673221$ $\mathrm{H} \quad-2.87323080-5.44535044-23.50842197$ $\mathrm{H}$ $\mathrm{O}$ $-2.11288899-6.75734414-23.40726437$ $7.33725916-19.80118092-13.52143235$ $8.19331807-19.41810423-13.27051545$ $7.29701616-20.65473130-13.07814108$ $9.18410687-17.88858389-19.01570740$ $9.86622799-17.26128921-18.71380237$ $9.64402678-18.54676417-19.54668865$ $5.96598586-7.06764494-16.81658114$ $6.35861116-6.81000850-15.96283348$ $5.97115905-8.04329963-16.81488540$ $26.48523063-0.93217449-4.86024112$ $27.12928731-0.80483508-4.14697169$ $26.62183567-1.81691568-5.21244991$ $0.68555472-10.03041521-18.09130439$ $-0.10246744-10.50957000-18.45411549$ $1.41583820-10.32735908-18.63521269$ $4.76710353-18.66100665-14.47075280$ $5.69118099-18.88976355-14.27119402$ $4.26345726-19.22098287-13.86874755$ $-0.47226573-6.69947401-25.81498839$ $-0.80849900-7.43194339-26.33754685$ $-1.21107482-6.36800178-25.29499693$ 
2C QM/SE/MM R3b (B3L YP/6-

31G(d):xTB:Amber) mechanical embedding $\omega_{\alpha}=\mathbf{1 . 6 6 7}$

C $\quad 21.94130761-10.34954627-4.22487254$ C $\quad 22.25461464-8.89027182-4.44514589$

$\mathrm{O} \quad 21.41396197-8.01173075-4.34532705$

$\mathrm{N} \quad 23.52570241-8.67724078-4.82196312$

C $\quad 23.90375455-7.38918091-5.40121120$

C $\quad 23.07602608-7.22919465-6.70303396$

O $\quad 23.13708388-8.10704002-7.57180597$

C $\quad 25.39603153-7.37961569-5.86446560$

S $\quad 25.84263162-5.72334884-6.51833425$

$\mathrm{H} \quad 24.09847449-9.48259013-5.05860835$

$\mathrm{H} \quad 23.71084711-6.58904055-4.68339788$

$\mathrm{H} \quad 25.53580429-8.15563246-6.62225983$

$\mathrm{H} \quad 26.02011960-7.64033217-4.99985265$

$\mathrm{N} \quad 22.37969204-6.08519454-6.84405890$

C $\quad 21.47241666-5.96053282-7.95950307$

C $\quad 22.16479535-5.65878565-9.30775236$

O $\quad 21.56647754-5.83072786-10.35160761$

C $\quad 20.48843465-4.86950567-7.64841733$

$\mathrm{H} \quad 20.92391516-6.89894120-8.09907486$

$\mathrm{H} \quad 22.19881847-5.52272505-6.01700176$

$\mathrm{N} \quad 23.40820679-5.15658465-9.15867295$

C $\quad 24.27022963-4.80672747-10.24146340$

C $\quad 25.21211050-5.88210203-10.71632569$

O $\quad 25.25402771-6.06041394-11.93636093$

C $\quad 25.10799767-3.59881895-9.93791527$

C $\quad 24.26912648-2.45029608-9.47430378$

C $\quad 23.30665357-1.87097972-10.29648443$

C $\quad 24.39192499-1.99568951-8.13278856$

C $\quad 22.54487928-0.78965010-9.84649314$

C $\quad 23.59080331-0.97488502-7.66997709$

C $\quad 22.69815418-0.33663205-8.53665672$

O $\quad 22.052395820 .78016332-8.07890883$

H $\quad 23.61377522-4.60491006-11.09954372$

$\mathrm{H} \quad 21.81458488-0.33296297-10.49935091$

$\mathrm{H} \quad 23.65418929-0.61211349-6.65795032$

$\mathrm{H} \quad 23.83288752-5.24826681-8.24016751$

$\mathrm{H} \quad 25.09807864-2.46376315-7.46786614$

$\mathrm{H} \quad 23.14859614-2.23676040-11.30331124$

$\mathrm{H} \quad 25.65551222-3.31366938-10.83897772$

$\mathrm{H} \quad 25.83934046-3.83106307-9.15935991$

$\mathrm{H} \quad 22.350474311 .57737318-8.53153104$

$\mathrm{N} \quad 25.98364161-6.58325313-9.81128009$

C $\quad 26.80137777-7.67079596-10.29036229$

C $\quad 26.18842592-9.07176428-10.08337023$

O $\quad 26.71386352-10.09170482-10.55411729$

C $\quad 28.22627642-7.56961194-9.75284514$

S $\quad 28.28111822-8.13913324-7.91551027$

$\mathrm{H} \quad 25.93844080-6.35030525-8.81427755$

$\mathrm{H} \quad 26.84915046-7.54598227-11.37746123$

$\mathrm{H} \quad 28.60524416-6.55099957-9.84689287$

$\mathrm{H} \quad 28.87534925-8.24701863-10.31194204$

$\mathrm{N} \quad 25.03575394-9.11860607-9.36135570$

C $\quad 24.38237809-10.40842361-9.16339165$

C $\quad 25.18314637-11.38146910-8.30267743$

O $\quad 24.83459065-12.54477440-8.12304241$

C $\quad 23.88917334-11.11023621-10.46035789$

C $\quad 22.95500502-10.18497326-11.27819926$

C $\quad 22.46800790-10.92130506-12.55485706$

C $\quad 21.70364048-9.77503946-10.48414144$

$\mathrm{H} \quad 21.97808186-9.17928474-9.61928794$

$\mathrm{H} \quad 21.04302645-9.18533764-11.10702807$
$21.18582913-10.66949809-10.14458355$ $24.57523605-8.30960057-8.96905505$ $21.91726059-11.81107218-12.26155190$ $21.83000614-10.27820394-13.14935702$ $23.31545604-11.23370057-13.16253884$ $23.48591524-10.18809667-8.57300452$ $23.50455288-9.28790791-11.56815379$ $23.35659310-12.01120620-10.16268845$ $24.74836357-11.38232005-11.07056084$ $26.22122226-10.83554533-7.62021982$ $26.87627503-11.71090770-6.65946367$ $25.82913924-12.13054699-5.54800442$ $25.18702176-11.28128184-4.97606563$ $27.99104855-10.93956245-5.96186725$ $28.67907738-11.82167356-4.92618018$ $29.92192657-11.09836600-4.32866371$ $30.33083815-11.86002385-3.04358707$ $31.76257672-11.70785267-2.78060358$ $27.24577444-12.61332858-7.16449974$ $29.76609621-11.48628228-2.19145018$ $30.12165294-12.92132624-3.17548207$ $27.97512546-12.02648181-4.11751045$ $28.98700988-12.76853522-5.36378311$ $32.11305548-12.35231046-2.05968877$ $32.33002256-11.90364120-3.63122840$ $31.98236659-10.74825920-2.46247404$ $29.68749756-10.05970226-4.11784319$ $30.71788994-11.14076660-5.07012308$ $26.64561985-9.94709090-7.86255504$ $27.58087489-10.04360378-5.50048759$ $28.71943112-10.61792788-6.70853662$ $25.76124203-13.43537866-5.20032686$ $24.89579973-13.85206304-4.13102706$ $26.12148689-14.16573727-5.79890149$ $29.87748201-4.488037372 .80690417$ $30.10035068-4.492406571 .25698900$ $30.70167128-3.633224010 .66426089$ $29.52069447-5.543913860 .62600964$ $29.56978920-5.62467021-0.84827892$ $29.07188569-4.32232781-1.45709810$ $29.80200665-3.70366577-2.21459754$ $28.81931822-6.85235243-1.37004465$ $28.93512975-6.95421769-2.85034655$ $29.23341705-7.74655831-0.88397137$ $29.00643210-6.247569021 .14777100$ $30.62064283-5.67901671-1.15223663$ $27.76079551-6.80115493-1.09556521$ $28.15231834-6.46505748-3.86972549$ $30.87341497-7.84273112-2.99549090$ $27.23342378-5.89889365-3.83525036$ $29.81902846-7.50245892-4.81566324$ $29.99738788-7.62630441-3.47320523$ $28.69756624-6.86343433-5.03587103$ $30.47266066-7.89881288-5.57901501$ $27.84738419-3.88583063-1.06296289$ $27.28130414-2.64276140-1.55364598$ $27.29054266-4.41164540-0.39175382$ $32.66055773-1.85561942-2.42762130$ $32.40578989-1.95891996-3.94920940$ $33.09176713-1.38239246-4.78329940$ $31.36229715-2.74149744-4.27107845$ $31.00603417-3.07270796-5.66808588$ $30.87944865-1.74499729-6.44448231$ $30.19634800-0.82936034-6.04718919$ $29.70480619-3.88011560-5.59721236$ 
.

$30.87504248-3.22477516-3.53218820$

$\mathrm{H} \quad 31.79402190-3.69970212-6.10334715$

$\mathrm{H} \quad 28.93809606-3.26422453-5.11996573$

$\mathrm{H} \quad 29.90053475-4.74270964-4.95442721$

$\mathrm{N} \quad 31.56843486-1.60414226-7.58908416$

C $\quad 31.55132365-0.41028684-8.36619369$

C $\quad 30.66320080-0.52992202-9.61861943$

O $\quad 30.310078990 .43981190-10.26472901$

C $\quad 33.00620198-0.10092957-8.72772702$

$\mathrm{H} \quad 31.153094500 .40604004-7.76195913$

$\mathrm{H} \quad 32.03104297-2.38666821-8.01498257$

$\mathrm{N} \quad 30.25089823-1.77701651-9.89853856$

C $\quad 29.17864846-1.99427298-10.88382287$

C $\quad 27.95131251-1.16002092-10.52107346$

O $\quad 27.42378161-1.11553858-9.43307885$

C $\quad 28.75819708-3.46647806-10.96062673$

C $\quad 29.81358268-4.32825873-11.56946589$

C $\quad 31.16637251-4.22959370-11.40714657$

C $\quad 29.59935658-5.44155415-12.45540760$

$\mathrm{N} \quad 31.80866945-5.20850083-12.11648532$

C $\quad 30.88569918-5.99558629-12.74002751$

C $\quad 28.46772129-6.10021937-12.95754170$

C $\quad 31.03856783-7.21917655-13.39757739$

C $\quad 28.63553411-7.32462461-13.57791731$

C $\quad 29.89314399-7.88642837-13.80710687$

H $\quad 29.97199998-8.83424758-14.31360656$

$\mathrm{H} \quad 29.56785586-1.66279565-11.85527555$

$\mathrm{H} \quad 32.80638465-5.31697997-12.19928048$

$\mathrm{H} \quad 27.48193574-5.68024202-12.83291491$

$\mathrm{H} \quad 27.76413256-7.86586999-13.88554723$

$\mathrm{H} \quad 32.02524161-7.61344189-13.59770504$

$\mathrm{H} \quad 31.74448502-3.51482619-10.85336420$

$\mathrm{H} \quad 30.34505810-2.49657878-9.19302677$

$\mathrm{H} \quad 27.85921234-3.54950059-11.57484582$

$\mathrm{H} \quad 28.51670694-3.82380614-9.95564175$

N $\quad 27.43581660-0.46877931-11.55348197$

C $\quad 26.128559650 .15627431-11.47779627$

$\mathrm{H} \quad 27.94229445-0.33485178-12.42735072$

C $\quad 14.00436796-19.32586490-8.43496656$

C $\quad 12.58211272-19.02586321-8.87908725$

O $\quad 11.65725180-19.11740084-8.10157287$

$\mathrm{N} \quad 12.48061093-18.57774626-10.15191975$

C $\quad 11.19964861-18.21418751-10.69585307$

C $\quad 10.82330227-18.99938320-11.92406482$

O $\quad 9.77704860-18.75915802-12.50998054$

C $\quad 11.05113618-16.71508558-10.95895481$

C $\quad 11.56597930-15.95415586-9.75548389$

C $\quad 10.79545879-15.89859048-8.57629828$

C $\quad 12.80466839-15.30417593-9.80186343$

C $\quad 11.28085430-15.19615786-7.47615542$

C $\quad 13.28822937-14.63498431-8.68558136$

C $\quad 12.51917880-14.57416180-7.51363728$

$\mathrm{H} \quad 12.91370456-14.06745714-6.64792929$

H $\quad 10.47394352-18.50540035-9.92393257$

$\mathrm{H} \quad 10.68766905-15.16063064-6.58755723$

$\mathrm{H} \quad 14.26334493-14.17522866-8.72790089$

$\mathrm{H} \quad 13.29371072-18.46390428-10.74692393$

$\mathrm{H} \quad 13.40219272-15.36586106-10.70114068$

$\mathrm{H} \quad 9.83887773-16.39502448-8.53576895$

$\mathrm{H} \quad 10.00222540-16.48635013-11.13557130$

$\mathrm{H} \quad 11.63082746-16.42905863-11.83853606$

$\mathrm{N} \quad 11.62042146-20.00238779-12.28671774$

C $\quad 11.28952337-20.75544267-13.46182613$

C $\quad 11.31636596-22.21542490-13.10939945$
$12.23432310-22.71798360-12.50209092$ $12.27031373-20.46172925-14.62677420$ $12.21556822-19.00124199-15.05887185$ $13.32867453-18.80294796-16.08475678$ $10.84607899-18.64438170-15.65324657$ $10.07944673-18.73023854-14.89498741$ $10.85400275-17.63191749-16.03672723$ $10.61882531-19.32991334-16.46920013$ $12.48538336-20.22077173-11.81755954$ $13.18135710-19.46964850-16.93292156$ $13.33221606-17.78677395-16.46226604$ $14.30195324-19.01499053-15.65402158$ $10.28374404-20.45914276-13.76609912$ $12.38539867-18.34379464-14.20473902$ $12.01688704-21.10474286-15.46709030$ $13.27728849-20.72368554-14.29809491$ $10.24628811-22.88053216-13.56300203$ $10.13337546-24.32351581-13.36865524$ $9.60318253-22.48463905-14.23557363$ $14.09520548-18.54495523-21.17642129$ $13.88867037-17.05366251-21.06660925$ $14.70997018-16.27313088-21.45524183$ $12.71877675-16.67825815-20.52377676$ $12.42371838-15.29919097-20.26583222$ $11.79956481-15.21960207-18.83170714$ $10.95828315-16.04500852-18.45044829$ $11.49796366-14.71676260-21.35425551$ $11.23272082-13.25709191-21.07862310$ $10.22174592-15.56684657-21.48951754$ $10.54639610-12.53863919-22.21924585$ $13.37315748-14.75858113-20.27661836$ $9.61488636-15.17519897-22.29672238$ $10.48189981-16.59545983-21.71882833$ $12.05927402-14.79472411-22.28967479$ $10.60458931-13.17803616-20.18342774$ $12.17281270-12.75166731-20.85603357$ $9.57935450-12.98600983-22.42876706$ $12.07783148-17.37052774-20.16785036$ $10.38600450-11.49662065-21.96611760$ $9.65184363-15.52145243-20.56886421$ $11.15450019-12.58726072-23.11902876$ $12.16294423-14.18236235-18.06970971$ $11.97265915-14.19191354-16.58094288$ $11.96104568-12.74172602-16.22798511$ $12.91013563-12.03843899-16.51968642$ $13.04019922-14.93736759-15.81770665$ $12.69571647-15.15214703-14.24949411$ $12.76978426-13.57793670-13.37844741$ $14.52012388-13.15720091-13.57597283$ $11.67909650-15.52222331-14.15897200$ $14.81577749-12.55497726-12.72520191$ $15.10166508-14.07524629-13.59052974$ $14.66595288-12.59881136-14.49570372$ $13.22107066-15.91465281-16.25853682$ $13.41255143-15.85545485-13.83201269$ $12.90865917-13.57515564-18.41040741$ $10.99900111-14.66528369-16.41489030$ $13.95883641-14.35487617-15.86202690$ $10.79643883-12.27942392-15.73297598$ $10.69320870-10.91277381-15.23094306$ $10.54630281-11.05213273-13.67021269$ $9.86066644-11.92449665-13.16200409$ $9.43427423-10.22748645-15.73775124$ $9.38566655-10.15298615-17.29234943$ $9.25739491-8.83088820-15.12778325$ 
H $\quad 11.60285152-10.37344568-15.48506650$

$\mathrm{H} \quad 8.37182531-8.35472724-15.53435006$

$\mathrm{H} \quad 9.13063534-8.91139583-14.04940730$

$\mathrm{H} \quad 8.59057404-10.85003900-15.42239443$

$\mathrm{H} \quad 9.55223385-11.14410644-17.70850575$

$\mathrm{H} \quad 8.39535870-9.81199247-17.58088966$

$\mathrm{H} \quad 11.43692297-9.52488457-17.46281977$

H $\quad 9.99573643-12.88407924-15.53427034$

$\mathrm{H} \quad 10.46663513-9.11809195-18.85201843$

$\mathrm{H} \quad 10.12041349-8.20003154-15.31225727$

$\mathrm{H} \quad 10.29182154-8.17649117-17.38964372$

$\mathrm{N} \quad 11.34179784-10.28010388-12.92645048$

C $\quad 11.28964191-10.40596041-11.44458442$

$\mathrm{H} \quad 11.88418055-9.50991761-13.30605965$

C $\quad 7.89755205-6.08277138-12.13481470$

C $\quad 6.86467368-6.75989285-13.05034484$

O $\quad 6.61129003-6.32618945-14.14669803$

$\mathrm{N} \quad 6.44592087-7.97320542-12.65665850$

C $\quad 5.61129617-8.77816693-13.48714668$

$\mathrm{C}$

$\mathrm{O}$

C

$\mathrm{C}$

C

$\mathrm{H}$

$\mathrm{H}$

$\mathrm{H}$

$\mathrm{H}$

$\mathrm{H}$

$\mathrm{H}$

$\mathrm{H}$

$\mathrm{O}$

$\mathrm{N}$

$\mathrm{H}$

N

C

C

C

C

N

C

$\mathrm{N}$

$\mathrm{N}$

$\mathrm{H}$

$\mathrm{H}$

$\mathrm{H}$

$\mathrm{H}$

$\mathrm{H}$

$\mathrm{H}$

$\mathrm{H}$

$\mathrm{H}$

$\mathrm{H}$

$\mathrm{H}$

$\mathrm{H}$

$\mathrm{H}$

$\mathrm{H}$

$\mathrm{N}$

C

C

O

C
$5.61129617-8.77816693-13.48714668$
$4.30346137-7.97180812-13.74632122$ $3.92589992-7.14258168-12.93013429$ $5.33945108-10.14130514-12.89737146$ $6.63757191-10.95421353-12.86118143$ $6.38588297-12.29430891-12.28826821$ $7.00906050-11.08511281-13.88266150$ $6.12018833-8.90670596-14.45224631$ $7.41688664-10.45152739-12.31085886$ $6.62927264-8.27117046-11.70745980$ $4.60821629-10.66525969-13.51095059$ $4.92811042-10.05745834-11.89046496$ $8.29440404-12.90657409-12.62806395$ $5.26340070-12.57303384-11.87080433$ $7.43191308-13.12790283-12.14507314$ $7.26594120-14.09439920-11.90076381$ $3.76789285-8.23982835-14.97556041$ $2.48420012-7.74694861-15.42109589$ $1.46550214-5.61800672-15.16212473$ $1.36161415-8.26125464-14.48595181$ $1.30082983-9.79434287-14.46308348$ $0.11752243-10.21697671-13.60015907$ $0.02690741-11.62563179-13.32010134$ $-0.67260119-12.50854987-14.08886140$ $-1.60747640-12.05575196-14.99232169$ $-0.40656188-13.87037689-13.98067619$ $2.21701568-10.20948112-14.03745264$ $2.26312505-8.09456836-16.42967231$ $0.80087780-12.00958814-12.77842428$ $1.18975698-10.17087213-15.48228913$ $-1.18070380-14.48653872-14.19078668$ $0.03207123-14.12958539-13.10379871$ $4.32059180-8.87569028-15.55077029$ $-0.82251415-9.90272126-14.05514010$ $0.19175892-9.71321422-12.63132820$ $-1.97033890-12.78562871-15.58486711$ $-1.34324241-11.22425622-15.49948576$ $0.41965847-7.84893424-14.84048850$ $1.54348663-7.88120491-13.48077347$ $3.60878844-5.61930063-15.84383071$ $3.68823066-4.16955914-15.87937619$ $4.28593863-3.63691361-17.18822507$ $4.57223703-2.46185656-17.33825751$ $4.47219330-3.62564889-14.69394527$ $3.63936063-3.53933378-13.42500333$ $2.47743038-6.22519178-15.45382087$
C

$\mathrm{N}$

C

$\mathrm{N}$

$\mathrm{N}$

$\mathrm{H}$

$\mathrm{H}$

$\mathrm{H}$

$\mathrm{H}$

$\mathrm{H}$

$\mathrm{H}$

$\mathrm{H}$

$\mathrm{H}$

$\mathrm{H}$

$\mathrm{H}$

$\mathrm{H}$

$\mathrm{H}$

$\mathrm{H}$

$\mathrm{N}$

C

C

O

$\mathrm{H}$

$\mathrm{H}$

$\mathrm{H}$

$\mathrm{N}$

C

C

O

C

C

C

C

C

C

C

O

$\mathrm{H}$

$\mathrm{H}$

$\mathrm{H}$

$\mathrm{H}$

$\mathrm{H}$

$\mathrm{H}$

$\mathrm{H}$

$\mathrm{H}$

$\mathrm{H}$

$\mathrm{N}$

C

C

O

$\mathrm{H}$

$\mathrm{H}$

$\mathrm{H}$

N

C

C

O

C

C

C

$\mathrm{N}$

C

$\mathrm{N}$

$\mathrm{N}$

H
$4.52065505-3.76359707-12.20431727$ $3.85447667-3.30706117-10.98967129$ $3.46691872-4.12780531-9.97996632$ $3.14114807-5.43255484-10.21000736$ $3.23285715-3.60950192-8.72960947$ $2.83783908-4.27545379-13.42974347$ $2.65820629-3.80457212-15.80062864$ $3.99379066-2.33836963-10.75638983$ $3.18043657-2.54963191-13.34716715$ $2.70789294-4.21182215-8.11882898$ $3.00318943-2.63243007-8.68954650$ $4.45006264-6.15816703-16.02629636$ $5.45772573-3.20682348-12.30096175$ $4.76915025-4.82321848-12.12894547$ $3.30325755-6.10445174-9.46231045$ $3.42925191-5.80719824-11.09886732$ $4.86924808-2.64546173-14.96314565$ $5.31717612-4.29540672-14.51022330$ $4.28507989-4.51402907-18.21899078$ $4.77851695-4.09107956-19.50712344$ $6.24764628-4.47070696-19.87411471$ $6.59851324-4.42515348-21.05829071$ $4.05118955-5.48924848-18.07081503$ $4.15437399-4.52028708-20.29082583$ $4.73497234-2.99699879-19.52743232$ $7.01597263-4.84659181-18.84148251$ $8.42762963-5.17820994-18.97413590$ $8.58439720-6.48420791-19.74368626$ $9.38827959-6.53585417-20.66091247$ $9.14329386-5.25332607-17.56820497$ $9.09585932-3.91913314-16.85610576$ $9.95190381-2.86253683-17.12363859$ $8.17420866-3.75576744-15.82748720$ $9.87035311-1.67223119-16.41717224$ $8.08036292-2.58740316-15.08225820$ $8.95927000-1.53847507-15.34341606$ $8.78119958-0.41057762-14.62060794$ $8.92645501-4.39793365-19.55974509$ $10.55497958-0.85794982-16.61390239$ $7.37096789-2.48227979-14.28114671$ $6.63948307-4.82641074-17.90286434$ $7.52000690-4.57492733-15.57131485$ $10.70263560-2.93510839-17.89262662$ $10.16632284-5.53595769-17.78586272$ $8.66978026-6.02357407-16.96364662$ $9.618567120 .17056425-14.72415801$ $7.79124138-7.50701963-19.35506273$ $7.80566881-8.76804985-20.03528463$ $7.40808708-8.58363051-21.58660713$ $7.95922237-9.23588275-22.44949687$ $7.06651912-7.33478741-18.66955305$ $8.80043391-9.21152486-20.04597278$ $7.08843589-9.44449128-19.57962515$ $6.53327855-7.56898693-21.80001770$ $5.98242769-7.34701064-23.11095953$ $7.08878525-6.71637622-23.98801452$ $7.17866674-6.95948131-25.18122927$ $4.78874730-6.39986955-23.05772720$ $4.18841828-6.08031376-24.46008984$ $2.94164573-5.21804133-24.22349216$ $2.26613677-4.82090216-25.43424386$ $1.12947348-4.09404244-25.45528550$ $0.61053620-3.68330861-24.23006179$ $0.66421694-3.58209867-26.61866375$ $3.89518373-6.99716914-24.97085355$ 
C

C

C

C

$\mathrm{H}$

$\mathrm{H}$

$\mathrm{H}$

$\mathrm{H}$

$\mathrm{H}$

$\mathrm{H}$

$\mathrm{H}$

$\mathrm{H}$

$\mathrm{H}$

$\mathrm{H}$

$\mathrm{H}$

$\mathrm{N}$

C

C

O

$\mathrm{H}$

$\mathrm{H}$

$\mathrm{H}$

$\mathrm{N}$

C

$\mathrm{H}$

C

C

$\mathrm{O}$

N

C

$\mathrm{C}$

O

C

O

$\mathrm{H}$

$\mathrm{H}$

$\mathrm{H}$

$\mathrm{H}$

$\mathrm{H}$

$\mathrm{N}$

C

$\mathrm{H}$

C

C

$\mathrm{O}$

N

C

C

O

C

$\mathrm{H}$

$\mathrm{H}$

$\mathrm{N}$

C

C

O

C

C

C

C

C

C

C

$\mathrm{O}$

$\mathrm{H}$
$3.81136364-15.69824479-16.17042178$ $3.75656741-14.17056815-16.29309911$ $2.32164074-13.67443095-16.52185094$ $4.32469989-13.57615674-14.98624651$ $5.33715006-13.92748331-14.81597343$ $4.33252751-12.49204271-15.03590215$ $3.71082494-13.88094508-14.14227976$ $3.87166255-18.19111190-16.30247928$ $1.67608042-14.01622638-15.71349921$ $2.31249777-12.58517728-16.51689110$ $1.93125735-14.01365223-17.47219007$ $2.01313871-16.20551216-17.24493717$ $4.38381484-13.83899180-17.12517614$ $4.85987392-16.00618076-16.16129543$ $3.38387992-15.97967126-15.20489329$ $4.65145988-16.72877166-19.07657430$ $5.14174535-16.50911974-20.42904024$ $4.06143127-16.91623052-21.46960538$ $3.84733425-16.16793449-22.39332403$ $5.11615085-17.43551258-18.51045225$ $5.36670096-15.45494217-20.59365448$ $6.04358970-17.10176955-20.57804033$ $3.38619071-18.06297159-21.29268937$ $2.28482958-18.49285846-22.16783711$ $3.66876026-18.72501878-20.57198642$ $-0.21876534-15.82604133-21.26908101$ $0.32402936-14.61255411-21.99288123$ $-0.42764888-14.00808658-22.73740245$ $1.57840409-14.27285206-21.69131975$ $2.16076724-13.04476035-22.16974568$ $2.47789257-13.16537647-23.70757792$ $2.34216536-12.19383456-24.41137087$ $3.51200244-12.75245185-21.40702947$ $3.30904615-12.56374395-19.97326843$ $2.08560774-14.75926211-20.96057721$ $1.46955654-12.20462522-22.04836211$ $3.96180118-11.85992968-21.81014544$ $4.17923963-13.60045396-21.56899741$ $3.27498468-13.44092745-19.55923363$ $2.80654810-14.35875177-24.17474198$ $3.07342400-14.52049493-25.60720069$ $3.15906246-15.09576660-23.57510567$ $-0.57326155-15.19062050-26.42305799$ $-1.14291407-13.78336961-26.53714942$ $-1.60720579-13.34357683-27.56208114$ $-1.00458116-13.04998886-25.44257733$ $-1.45634833-11.68193642-25.51722008$ $-0.65226633-10.86301530-26.52978782$ $-1.17612716-10.02321417-27.23919406$ $-1.40821738-11.10871127-24.10441019$ $-0.67878110-13.40124764-24.55669178$ $-2.49749051-11.65046936-25.86387023$ $0.69352533-11.07520894-26.51696535$ $1.55703972-10.22092714-27.32134531$ $1.18451104-10.46265418-28.84020285$ $1.00395150-9.54595777-29.59234428$ $3.07575288-10.49711740-27.07939228$ $3.95654864-9.90841089-28.17812772$ $4.25554582-8.55505030-28.16006545$ $4.35009857-10.67942840-29.26294385$ $5.06495283-8.01705955-29.14356474$ $5.17181370-10.17242806-30.25752008$ $5.55937263-8.83950217-30.16486642$ $6.50951600-8.34895020-30.99002851$ $1.33776277-9.16443160-27.13406528$ 


\section{C QM/MM R1b (B3LYP/6-31G(d):Amber) mechanical embedding $\omega_{\alpha}=1.0$}

C $\quad 21.94250567-10.35340956-4.21741037$ C $\quad 22.25689860-8.88622348-4.43368751$

O $\quad 21.41108921-8.00960815-4.34545664$

$\mathrm{N} \quad 23.53503612-8.67605689-4.80411180$

C $\quad 23.91787549-7.39753976-5.40113065$

C $\quad 23.07991681-7.24086502-6.68265989$

O $\quad 23.14898444-8.09330856-7.56888026$

C $\quad 25.40001678-7.38945164-5.85267950$

S $\quad 25.82711505-5.73153736-6.51886608$

$\mathrm{H} \quad 24.09838922-9.49284652-5.03161321$

$\mathrm{H} \quad 23.72815056-6.59755706-4.68415430$

$\mathrm{H} \quad 25.55967682-8.16438892-6.60544609$

$\mathrm{H} \quad 26.02084062-7.63484327-4.98353118$

N $\quad 22.35028720-6.11441320-6.78418845$

C $\quad 21.46135612-5.93336637-7.92126503$

$\mathrm{H} \quad 22.13271485-5.56811383-5.95676126$

C $\quad 24.31831765-4.87394999-10.22379946$

C $\quad 25.23593845-5.94242589-10.76212339$

O $\quad 25.21136996-6.17372885-11.96656667$

N $\quad 26.00118413-6.60734773-9.83795050$

C $\quad 26.84889883-7.68707897-10.30258804$

C $\quad 26.24353331-9.08592472-10.11518146$

O $\quad 26.78270146-10.10228366-10.56105122$

C $\quad 28.26139703-7.61737820-9.71812790$

S $\quad 28.26814310-8.15190141-7.90587389$

$\mathrm{H} \quad 25.98727394-6.30701549-8.86060562$

$\mathrm{H} \quad 26.90747822-7.56019609-11.38855418$

$\mathrm{H} \quad 28.67075954-6.60973129-9.82890548$

$\mathrm{H} \quad 28.90015293-8.30962168-10.27203540$

N $\quad 25.08432739-9.12689643-9.41752925$

C $\quad 24.40766283-10.41278491-9.17844111$

$\mathrm{H} \quad 24.60594096-8.31043135-9.06007984$

C $\quad 29.87817528-4.494980732 .80826416$

C $\quad 30.09495677-4.499414191 .27080674$

O $\quad 30.69198448-3.629392680 .67802758$

$\mathrm{N} \quad 29.50992954-5.549679950 .63980357$

C $\quad 29.57580524-5.63518937-0.82450341$

C $\quad 29.07141300-4.33858643-1.44720761$

O $\quad 29.78645085-3.74086622-2.23131576$

C $\quad 28.83772894-6.87263135-1.35155167$

C $\quad 28.93908926-6.97301092-2.83647659$

H $\quad 29.25802528-7.76569522-0.87049419$

H $\quad 29.02141136-6.266402751 .16732984$

$\mathrm{H} \quad 30.62882155-5.67370122-1.11904521$

$\mathrm{H} \quad 27.78019921-6.83262440-1.06738308$

C $\quad 28.12967692-6.49791188-3.83830895$

$\mathrm{H} \quad 30.89581728-7.78280282-3.01749562$

$\mathrm{H} \quad 27.20252683-5.94618956-3.78164896$

C $\quad 29.81148913-7.46633668-4.81715753$ 
$\mathrm{N} \quad 30.00920109-7.59464100-3.48152903$

$\mathrm{N} \quad 28.66940535-6.85670018-5.03035008$

$\mathrm{H} \quad 30.46574610-7.83157908-5.59721035$

$\mathrm{N} \quad 27.85256765-3.88440279-1.05061726$

C $\quad 27.28078540-2.64087560-1.55470601$

$\mathrm{H} \quad 27.29623966-4.39952405-0.37025262$

C $\quad 32.65987311-1.85137456-2.42376192$

C $\quad 32.40663147-1.96154267-3.93672340$

O $\quad 33.11166590-1.41052363-4.77871949$

$\mathrm{N} \quad 31.34477120-2.71796787-4.23776177$

C $\quad 30.99983839-3.05349699-5.62158799$

C $\quad 30.85116691-1.73901573-6.39585931$

O $\quad 30.04745770-0.89899051-6.06390044$

C $\quad 29.69265709-3.85210770-5.59576024$

$\mathrm{S} \quad 29.22370031-4.40819248-7.29232904$

$\mathrm{H} \quad 30.83215305-3.15950052-3.49070593$

$\mathrm{H} \quad 31.79263897-3.67219546-6.05570252$

$\mathrm{H} \quad 28.91173772-3.21808036-5.16844368$

$\mathrm{H} \quad 29.84460050-4.70358580-4.93012070$

$\mathrm{N} \quad 31.61471285-1.61221805-7.49283714$

C $\quad 31.62667725-0.47282199-8.36952449$

$\mathrm{H} \quad 32.50774013-2.07814804-7.50439093$

C $\quad 7.68471435-15.96989805-16.32764272$

$\mathrm{H} \quad 7.15410770-16.35667439-17.18644805$

C $\quad 8.08536547-16.77971068-15.27354528$

$\mathrm{H} \quad 7.87951488-17.84269107-15.29525238$

C $\quad 8.76398032-16.16023852-14.22394487$

H $\quad 9.13719193-16.70956087-13.37949096$

C $\quad 8.96795810-14.78789153-14.25756802$

H $\quad 9.51947526-14.32007988-13.44624653$

$\mathrm{N} \quad 8.48626943-13.97796192-15.22581159$

C $\quad 7.90434255-14.60670895-16.26092031$

C $\quad 7.40277191-13.75597248-17.36064833$

C $\quad 7.51273934-14.18908385-18.69887828$

C $\quad 6.99604958-13.35966213-19.69944024$

C $\quad 6.38256285-12.14413535-19.41833831$

H $\quad 5.99881512-11.56548972-20.24337581$

F $\quad 7.06727109-13.73808013-21.00009127$

$\mathrm{H} \quad 8.02334834-15.12779879-18.92749323$

C $\quad 6.81114471-12.53265474-17.04978993$

H $\quad 6.76851441-12.25637067-16.00218431$

C $\quad 6.25366979-11.75970980-18.06547028$

C $\quad 5.44751656-10.58564582-17.68069822$

O $\quad 5.66404679-9.85659889-16.71829348$

$\mathrm{N} \quad 4.36683542-10.34635916-18.52338226$

$\mathrm{H} \quad 3.99643427-11.08324051-19.13085777$

$\mathrm{N} \quad 3.45262550-9.37030267-18.22558534$

$\mathrm{H} \quad 3.22503624-9.29878698-17.23684728$

S

$\mathrm{O}$

$\mathrm{O}$

C

C

$\mathrm{H}$

C

$\mathrm{H}$

C

$\mathrm{H}$

C

$\mathrm{H}$

C

$\mathrm{F}$

$\mathrm{Zn}$

\section{C-QM/MM R1b (2C-B3LYP/6-31G(d):Amber)} mechanical embedding $\omega_{\alpha}=1.0$

C $\quad 21.94153684-10.35320693-4.21756948$

C $\quad 22.25471536-8.88531244-4.43369794$

O $\quad 21.40914360-8.00855989-4.34590485$

$\mathrm{N} \quad 23.53342678-8.67456013-4.80286262$

C $\quad 23.91777213-7.39549912-5.39944653$

C $\quad 23.07838414-7.23967113-6.68185299$

O $\quad 23.14740770-8.09274325-7.56836132$

C $\quad 25.40288719-7.39172046-5.85119977$

S $\quad 25.82725919-5.73133967-6.51897700$

$\mathrm{H} \quad 24.09428878-9.49078397-5.03708802$

$\mathrm{H} \quad 23.73028308-6.59310961-4.68088592$

$\mathrm{H} \quad 25.57082418-8.16859346-6.60377872$

$\mathrm{H} \quad 26.02153264-7.63518097-4.97798151$

$\mathrm{N} \quad 22.34896726-6.11347621-6.78402544$

C $\quad 21.46105368-5.93350598-7.92167542$

$\mathrm{H} \quad 22.11944617-5.57439277-5.95523701$

C $\quad 24.31761457-4.87366287-10.22317811$

C $\quad 25.23736041-5.94100951-10.75872829$

O $\quad 25.21322500-6.17194809-11.96300208$

$\mathrm{N} \quad 26.00206088-6.60566837-9.83397913$

C $\quad 26.84957788-7.68419968-10.30219385$

C $\quad 26.24295675-9.08429002-10.11502957$

O $\quad 26.78125665-10.10108675-10.56210454$

C $\quad 28.26509189-7.61359530-9.71993029$

S $\quad 28.26876748-8.15131350-7.90527335$

$\mathrm{H} \quad 25.99378651-6.30492145-8.85643304$

$\mathrm{H} \quad 26.90204568-7.55402063-11.38859443$

$\mathrm{H} \quad 28.67643906-6.60451782-9.82941207$

$\mathrm{H} \quad 28.90484328-8.30725562-10.27369497$

$\mathrm{N} \quad 25.08353838-9.12655179-9.41709690$

C $\quad 24.40735329-10.41278517-9.17857558$

H $\quad 24.60230921-8.31300549-9.05751317$

C $\quad 29.87723156-4.495356412 .80773540$

C $\quad 30.09335957-4.502216281 .26996096$

O $\quad 30.68908627-3.631621950 .67620185$

$\mathrm{N} \quad 29.50999256-5.553562900 .63950184$

C $\quad 29.57428371-5.64034596-0.82487516$

C $\quad 29.07007086-4.34245774-1.44581137$

O $\quad 29.78978205-3.74419167-2.22531331$

C $\quad 28.83267098-6.87824669-1.35197999$

C $\quad 28.93281576-6.97145764-2.83856055$

$\mathrm{H} \quad 29.25179160-7.77623199-0.87494573$

$\mathrm{H} \quad 29.02070960-6.271320701 .16445235$

$\mathrm{H} \quad 30.62870145-5.67197208-1.11985138$

$\mathrm{H} \quad 27.77257179-6.83854130-1.06775663$

C $\quad 28.12227099-6.48772077-3.83646170$

$\mathrm{H} \quad 30.88845178-7.78512169-3.02718673$

$\mathrm{H} \quad 27.19957211-5.92453459-3.77730644$

C $\quad 29.79927591-7.46275325-4.82347147$

$\mathrm{N} \quad 30.00023736-7.59574331-3.48813182$

$\mathrm{N} \quad 28.65870257-6.84759006-5.03024310$

$\mathrm{H} \quad 30.44768750-7.82845661-5.61061236$

$\mathrm{N} \quad 27.85132390-3.88584304-1.05100556$

C $\quad 27.28140227-2.64110886-1.55452871$

$\mathrm{H} \quad 27.29099532-4.40017163-0.37356287$

C $\quad 32.65969250-1.85107410-2.42408467$

C $\quad 32.40712144-1.95862993-3.93732062$

O $\quad 33.11350098-1.40832313-4.77956887$

$\mathrm{N} \quad 31.34502743-2.71463971-4.23852519$

C $\quad 31.00038888-3.05577505-5.62086580$

C $\quad 30.85377988-1.74001722-6.39664761$

O $\quad 30.05064107-0.90002179-6.06352850$ 


\section{C-WF-in-DFT/MM R1b (2C-CCSD-in-B3LYP/6-} 31G(d):Amber) mechanical embedding $\omega_{\alpha}=1.0$

$29.68875355-3.85246734-5.58802782$ $29.22360688-4.40790256-7.28946680$ $30.82404056-3.14753004-3.49328999$ $31.79333421-3.67868385-6.05354688$ $28.90599054-3.21652519-5.16174600$ $29.83660272-4.70339492-4.91735052$ $31.61777182-1.61215246-7.49366311$ $31.62683512-0.47299928-8.36903534$ $32.51991410-2.06393278-7.49896153$ $7.68332993-15.97174981-16.33122188$ $7.14982769-16.35494452-17.19101879$ $8.08064354-16.78259614-15.27785984$ $7.86918838-17.84489098-15.30123491$ $8.76172203-16.16396061-14.22800682$ $9.13650211-16.71541053-13.38591399$ $8.96702678-14.79155597-14.25823841$ $9.51927723-14.32445097-13.44713104$ $8.48486308-13.97787309-15.22478193$ $7.90697816-14.60895147-16.26033494$ $7.40510073-13.75539083-17.36061006$ $7.51726651-14.19121360-18.69863513$ $6.99729990-13.36220487-19.69815401$ $6.38442861-12.14623242-19.41557371$ $5.99878549-11.56725043-20.23926874$ $7.06493418-13.74088625-20.99817065$ $8.03122066-15.13012989-18.92706716$ $6.81310529-12.53210898-17.04725107$ $6.77150253-12.25813190-15.99812263$ $6.25456578-11.75932134-18.06293531$ $5.44520963-10.58732458-17.67856141$ $5.65577097-9.86225190-16.71080406$ $4.37097918-10.33965810-18.53158030$ $3.98660573-11.07775991-19.12895437$ $3.45543884-9.36754320-18.22268146$ $3.23843607-9.28920835-17.23052379$ $3.58856762-7.95218915-19.07068835$ $4.95011461-7.55298709-19.43530274$ $2.78859080-6.93435894-18.38670917$ $2.76206430-8.40221895-20.67186151$ $1.60555054-7.69232510-21.07255243$ $1.11793504-7.00278980-20.38486395$ $1.03976770-7.93134877-22.33892121$ $0.10599404-7.45714708-22.62271565$ $1.62948984-8.85573117-23.20665591$ $1.20219583-9.07617849-24.18419817$ $2.80094016-9.51517090-22.84848521$ $3.30300783-10.19877379-23.53506603$ $3.35016717-9.29503611-21.58164535$ $4.52148162-9.93805435-21.27856923$ $28.07125793-6.23642753-6.91435390$

C

C $\quad 22.25555358-8.88196799-4.43220532$

O $\quad 21.41091266-8.01019941-4.34629469$

$\mathrm{N} \quad 23.53226225-8.67376975-4.80590608$

C $\quad 23.91733414-7.40137134-5.40033495$

C $\quad 23.07775355-7.24204234-6.68506926$

O $\quad 23.14765101-8.09151013-7.56757283$

C $\quad 25.40208560-7.37640936-5.85766426$

S $\quad 25.81557519-5.72671803-6.51801373$

$\mathrm{H} \quad 24.09663836-9.49165726-5.03460135$

$\mathrm{H} \quad 23.72588269-6.59221213-4.68144432$
$25.56710674-8.17134488-6.60187659$ $26.01855220-7.63576913-4.97594764$ $22.34926215-6.11772076-6.78325111$ $21.46107778-5.93400548-7.92160564$ $22.12661595-5.57106475-5.95434124$ $24.31697550-4.87176880-10.22349076$ $25.23891351-5.94393673-10.75987268$ $25.21526296-6.17191167-11.96039134$ $26.00353336-6.60824076-9.83591008$ $26.85061237-7.68462313-10.30002319$ $26.24407831-9.08807467-10.12101136$ $26.77853777-10.10073378-10.56549025$ $28.26205922-7.62128201-9.70281209$ $28.27180418-8.17554679-7.91605271$ $25.99209424-6.31166646-8.85359415$ $26.90487071-7.55591419-11.39133778$ $28.67533945-6.60606167-9.83434721$ $28.90467826-8.30610450-10.28426844$ $25.08690327-9.12707435-9.41800245$ $24.40777631-10.41315851-9.17868336$ $24.60255627-8.31200184-9.05747485$ $29.87705108-4.495231122 .80969280$ $30.09573656-4.500041051 .26721912$ $30.68892693-3.631864900 .67694100$ $29.51096325-5.550409140 .64057750$ $29.57219206-5.63926260-0.82056224$ $29.07345689-4.34011494-1.44669622$ $29.78956844-3.74506925-2.22598485$ $28.83558230-6.87371900-1.34980388$ $28.93529031-6.97378027-2.83321706$ $29.25599307-7.77478906-0.86911282$ $29.02120446-6.269262701 .16829047$ $30.63189261-5.67162151-1.11854870$ $27.77318554-6.83628673-1.06419559$ $28.12832077-6.49751914-3.83508693$ $30.89402783-7.78546873-3.02075629$ $27.19986696-5.92897142-3.77422823$ $29.79003083-7.46374073-4.81824750$ $30.00618407-7.59829614-3.48142016$ $28.66938610-6.86180680-5.02281850$ $30.45536019-7.83083373-5.60467344$ $27.85496631-3.88571094-1.05177038$ $27.28090200-2.64052465-1.55529056$ $27.29304673-4.40216641-0.37312482$ $32.66085911-1.85121830-2.42163830$ $32.40688180-1.95887349-3.93921789$ $33.10972177-1.41031308-4.77826264$ $31.34554339-2.71637518-4.23798560$ $30.99874808-3.05077710-5.61423331$ $30.85402125-1.73522569-6.39420581$ $30.05468585-0.89684033-6.06054019$ $29.69000357-3.85447573-5.60957038$ $29.23614043-4.38821137-7.30051318$ $30.82119323-3.15160925-3.49047231$ $31.79666326-3.67469621-6.05392230$ $28.90849768-3.21442129-5.16093527$ $29.84045080-4.70138039-4.92051548$ $31.61655780-1.61027503-7.48996139$ $31.62695260-0.47130737-8.37094229$ $32.51569234-2.06849007-7.50289230$ $7.68376341-15.97129562-16.33049861$ $7.15068171-16.35532479-17.18984958$ $8.08173986-16.78194089-15.27686254$ $7.87139376-17.84440438-15.29987505$ $8.76216096-16.16320436-14.22708814$ 


$\begin{array}{ll}\mathrm{H} & 9.13603244-16.71415581-13.38417633 \\ \mathrm{C} & 8.96706886-14.79096673-14.25813471 \\ \mathrm{H} & 9.51891234-14.32357359-13.44669215 \\ \mathrm{~N} & 8.48535396-13.97782179-15.22499822 \\ \mathrm{C} & 7.90630014-14.60843707-16.26054549 \\ \mathrm{C} & 7.40468077-13.75545198-17.36071873 \\ \mathrm{C} & 7.51615589-14.19079303-18.69874111 \\ \mathrm{C} & 6.99693550-13.36171449-19.69850515 \\ \mathrm{C} & 6.38401312-12.14574173-19.41625649 \\ \mathrm{H} & 5.99851919-11.56700825-20.24022991 \\ \mathrm{~F} & 7.06523294-13.74042531-20.99858470 \\ \mathrm{H} & 8.02914962-15.12973010-18.92720127 \\ \mathrm{C} & 6.81274145-12.53221530-17.04794801 \\ \mathrm{H} & 6.77048082-12.25791304-15.99911553 \\ \mathrm{C} & 6.25410448-11.75956249-18.06353925 \\ \mathrm{C} & 5.44527978-10.58722388-17.67922779 \\ \mathrm{O} & 5.65779485-9.86100420-16.71252316 \\ \mathrm{~N} & 4.37028002-10.34098591-18.52944449 \\ \mathrm{H} & 3.98866564-11.07916257-19.12945383 \\ \mathrm{~N} & 3.45474571-9.36822212-18.22350654 \\ \mathrm{H} & 3.23483793-9.29160455-17.23192864 \\ \mathrm{~S} & 3.58873962-7.95253985-19.07111799 \\ \mathrm{O} & 4.95014540-7.55231270-19.43490435 \\ \mathrm{O} & 2.78868469-6.93510111-18.38660357 \\ \mathrm{C} & 2.76249322-8.40217109-20.67233069 \\ \mathrm{C} & 1.60662467-7.69259770-21.07266538 \\ \mathrm{H} & 1.11952479-7.00292475-20.38537045 \\ \mathrm{C} & 1.04064599-7.93081244-22.33873341 \\ \mathrm{H} & 0.10780722-7.45476260-22.62285431 \\ \mathrm{C} & 1.62954188-8.85542092-23.20612816 \\ \mathrm{H} & 1.20257512-9.07423843-24.18405232 \\ \mathrm{C} & 2.80059641-9.51547402-22.84766239 \\ \mathrm{H} & 3.30180755-10.19877890-23.53448864 \\ \mathrm{C} & 3.35031194-9.29554201-21.58137957 \\ \mathrm{~F} & 4.52175309-9.93819659-21.27784714 \\ \mathrm{Zn} & 28.07258036-6.23429361-6.91766063 \\ & \end{array}$

\section{C QM/MM R2b (B3LYP/6-31G(d):Amber)} mechanical embedding $\omega_{\alpha}=1.0$

$\begin{array}{lll}\mathrm{C} & 21.94185370-10.35375643-4.21758034 \\ \mathrm{C} & 22.25696289-8.88934834-4.43527578 \\ \mathrm{O} & 21.40814789-8.01462162-4.35628552 \\ \mathrm{~N} & 23.53407859-8.67720612-4.80583719 \\ \mathrm{C} & 23.91686267-7.39692789-5.39949079 \\ \mathrm{C} & 23.08386511-7.24132542-6.68510190 \\ \mathrm{O} & 23.15089081-8.09656849-7.56893949 \\ \mathrm{C} & 25.39924974-7.38485628-5.83625718 \\ \mathrm{~S} & 25.83105628-5.73362717-6.51267081 \\ \mathrm{H} & 24.12034945-9.48956991-4.98588720 \\ \mathrm{H} & 23.72111774-6.59673785-4.68245718 \\ \mathrm{H} & 25.56752469-8.17053103-6.57702437 \\ \mathrm{H} & 26.00870941-7.61944836-4.95414607 \\ \mathrm{~N} & 22.35367944-6.11685816-6.78747870 \\ \mathrm{C} & 21.46377060-5.93668491-7.92132242 \\ \mathrm{H} & 22.15221446-5.55452475-5.96619203 \\ \mathrm{~N} & 23.48148494-5.22794029-9.13438795 \\ \mathrm{C} & 24.31277337-4.81369759-10.25508263 \\ \mathrm{C} & 25.22365277-5.90330681-10.77104221 \\ \mathrm{O} & 25.20205221-6.14820013-11.97321694 \\ \mathrm{C} & 25.11800409-3.59133269-9.91476130 \\ \mathrm{C} & 24.27374958-2.43586398-9.44694650 \\ \mathrm{C} & 23.30924557-1.86501877-10.30510301 \\ \mathrm{C} & 24.42356024-1.95871033-8.11571697 \\ \mathrm{C} & 22.52674618-0.78103108-9.84954406\end{array}$

$23.61504559-0.90589461-7.65656323$ $22.68293059-0.30360269-8.52648393$ $22.037227280 .81740117-8.13444581$ $23.65860063-4.53984485-11.08461328$ $21.79883969-0.33076982-10.50187088$ $23.73389609-0.52787759-6.65594680$ $23.88872583-5.26493966-8.20580832$ $25.15943199-2.39786053-7.45528482$ $23.17187580-2.24797479-11.30803718$ $25.66635519-3.26926047-10.79950016$ $25.84560186-3.83784549-9.14038443$ $22.550676081 .60070308-8.35330727$ $25.98348437-6.56970431-9.84417904$ $26.81962769-7.66345588-10.29663846$ $26.20984309-9.05933626-10.10766651$ $26.75801753-10.06859071-10.55840420$ $28.23486554-7.60446985-9.71669915$ $28.27225437-8.15048248-7.90687533$ $25.96762231-6.27305229-8.86596570$ $26.88897391-7.54305731-11.38267681$ $28.64325536-6.59629322-9.83105108$ $28.86208532-8.29707875-10.28426067$ $25.04175885-9.10837517-9.41736236$ $24.38128432-10.40554587-9.16695514$ $25.18683591-11.37176245-8.29442724$ $24.80718774-12.54541311-8.15497978$ $23.86808066-11.08723596-10.46770206$ $22.93819424-10.17336486-11.29132315$ $22.44705973-10.93778457-12.53410135$ $21.68893462-9.77798062-10.49510874$ $21.98223438-9.14621675-9.66069786$ $21.01863599-9.20689064-11.12825394$ $21.19600692-10.67246551-10.11914525$ $24.58912316-8.30150254-9.00747738$ $21.91042876-11.83311104-12.22585657$ $21.80403793-10.29720830-13.13203163$ $23.30892463-11.23290526-13.13365606$ $23.48857374-10.20901237-8.58102089$ $23.46657094-9.27633339-11.60777970$ $23.31276020-11.98656806-10.20124091$ $24.70709066-11.38812897-11.09250861$ $26.24831828-10.89165072-7.62177712$ $26.88622675-11.71893248-6.58639583$ $25.84788684-12.11724484-5.51134447$ $25.16897360-11.25053858-4.97586041$ $28.05644214-10.95830957-5.96085620$ $28.69082389-11.80326864-4.85404526$ $29.96927174-11.13052878-4.32209735$ $30.38085379-11.86240762-3.02790092$ $31.80610665-11.67057308-2.74469226$ $27.24351936-12.62864755-7.07389283$ $29.77393661-11.48397910-2.19866754$ $30.17326171-12.92856180-3.15388870$ $27.98266447-11.88670290-4.02951016$ $28.93002378-12.80057688-5.22372107$ $32.11917786-12.30273642-2.02164879$ $32.37274179-11.87615391-3.56978921$ $31.99120597-10.72182357-2.42818052$ $29.77458512-10.08456879-4.10755726$ $30.74039753-11.21960372-5.08889621$ $26.57113918-9.95145373-7.83401370$ $27.71745696-10.00358137-5.55523733$ $28.81078838-10.77595666-6.72817050$ $29.87489511-4.495932702 .80736160$ $30.09415344-4.498048381 .27254654$ 
O $\quad 30.68696542-3.625889680 .67779277$

$\mathrm{N} \quad 29.50965901-5.547497760 .64277706$

C $\quad 29.57843303-5.62600211-0.81879431$

C $\quad 29.07135609-4.33202110-1.44024929$

O $\quad 29.77693791-3.73896354-2.23788717$

C $\quad 28.83931621-6.85830790-1.35152019$

C $\quad 28.94062805-6.96321030-2.83174642$

$\mathrm{H} \quad 29.26208194-7.75108993-0.87361100$

$\mathrm{H} \quad 29.01240745-6.258560791 .17083538$

$\mathrm{H} \quad 30.63249888-5.66597103-1.11131096$

$\mathrm{H} \quad 27.78176048-6.81180607-1.07079808$

C $\quad 28.13288527-6.49345149-3.83581614$

$\mathrm{H} \quad 30.89400612-7.79190591-3.00585136$

$\mathrm{H} \quad 27.20648040-5.94136123-3.77384354$

C $\quad 29.81914137-7.45661885-4.80680595$

$\mathrm{N} \quad 30.01313083-7.58102643-3.47202000$

$\mathrm{N} \quad 28.67612711-6.85041124-5.02662322$

$\mathrm{H} \quad 30.48615239-7.82032197-5.57520456$

$\mathrm{N} \quad 27.85276465-3.88163713-1.04379781$

C $\quad 27.28106579-2.64032827-1.55445441$

$\mathrm{H} \quad 27.30492799-4.38274257-0.34581991$

C $\quad 32.66053881-1.85303693-2.42403705$

C $\quad 32.40925740-1.96050996-3.93706992$

O $\quad 33.09520487-1.38501429-4.77830458$

$\mathrm{N} \quad 31.36623155-2.74272114-4.23979597$

C $\quad 31.00878674-3.06375316-5.62442637$

C $\quad 30.85333419-1.74351928-6.39154694$

O $\quad 30.03853419-0.91308812-6.05909226$

C $\quad 29.69905117-3.85643357-5.59276770$

S $\quad 29.20191732-4.39360050-7.28668304$

$\mathrm{H} \quad 30.87001972-3.19950236-3.48935233$

$\mathrm{H} \quad 31.79468497-3.68317354-6.07137698$

$\mathrm{H} \quad 28.92719723-3.22507156-5.14525224$

H $\quad 29.85963859-4.71730957-4.93970252$

$\mathrm{N} \quad 31.61272302-1.61232493-7.49231817$

C $\quad 31.62447834-0.47262436-8.36740246$

$\mathrm{H} \quad 32.50902944-2.07135369-7.50536975$

$\mathrm{N} \quad 30.17010424-1.81923390-9.80822218$

C $\quad 29.15725419-2.01665589-10.85173943$

C $\quad 27.91548368-1.19912669-10.53119935$

O $\quad 27.43083833-1.14614031-9.39992206$

C $\quad 28.79468333-3.49105999-10.97855124$

C $\quad 29.86281642-4.33665491-11.57226262$

C $\quad 31.20444359-4.21339382-11.41577397$

C $\quad 29.66911260-5.47526812-12.44893406$

$\mathrm{N} \quad 31.84688185-5.18175898-12.15429520$

C $\quad 30.94863917-6.04055267-12.73603856$

C $\quad 28.53623564-6.13937431-12.95738310$

C $\quad 31.09788231-7.24770374-13.43016060$

C $\quad 28.67705140-7.37861478-13.60216570$

C $\quad 29.94242996-7.93389600-13.85131570$

$\mathrm{H} \quad 30.02225164-8.86752385-14.37952829$

$\mathrm{H} \quad 29.56068829-1.68173565-11.80851328$

$\mathrm{H} \quad 32.84768569-5.27679018-12.23598454$

$\mathrm{H} \quad 27.55469814-5.72232396-12.78971981$

$\mathrm{H} \quad 27.79076417-7.89908429-13.89986369$

$\mathrm{H} \quad 32.08499029-7.62868025-13.62560187$

$\mathrm{H} \quad 31.71325029-3.46393538-10.82763654$

$\mathrm{H} \quad 30.29476500-2.54850918-9.11588390$

$\mathrm{H} \quad 27.92633106-3.57633327-11.63273046$

$\mathrm{H} \quad 28.51902551-3.90063673-10.00939961$

$\mathrm{N} \quad 12.42974512-18.59710066-10.12393062$

C $\quad 11.14127630-18.21542252-10.69896756$

C $\quad 10.77937988-19.00140916-11.94208747$

O $\quad 9.70889400-18.78179359-12.49491685$
$11.07361451-16.71311541-10.94584060$ $11.56639914-15.91713654-9.75917968$ $10.78894956-15.86300028-8.57596983$ $12.82727089-15.28884451-9.81642372$ $11.29421039-15.17631317-7.45686208$ $13.32284986-14.61632935-8.68702385$ $12.55681128-14.56027455-7.50109437$ $12.96071523-14.06999166-6.62891911$ $10.35764018-18.45096740-9.98154764$ $10.71813206-15.15712619-6.55893682$ $14.30638431-14.17451728-8.73229927$ $13.25836434-18.48601571-10.69909219$ $13.43204069-15.36853688-10.70938691$ $9.83495702-16.36871203-8.52512683$ $10.04201541-16.42311695-11.14127641$ $11.67757429-16.46239672-11.81735731$ $11.58568355-19.96978811-12.34907685$ $11.29505474-20.76625973-13.52715536$ $11.29036576-22.22512809-13.16845670$ $12.20190186-22.69969467-12.49485753$ $12.30752384-20.45804361-14.64391354$ $12.26027669-19.00472263-15.11232690$ $13.35059516-18.85861541-16.17223733$ $10.90213942-18.65414935-15.72868935$ $10.12406428-18.73932336-14.98090854$ $10.93427459-17.63382157-16.09904965$ $10.68948894-19.34245659-16.54625032$ $12.43567919-20.16986470-11.84257455$ $13.19852908-19.57066379-16.98434023$ $13.33880664-17.86869845-16.59793625$ $14.32483566-19.03765018-15.71954902$ $10.29905216-20.55057395-13.90896427$ $12.45962127-18.31974003-14.29260754$ $12.09594111-21.11086096-15.48932267$ $13.31045951-20.68769685-14.28256763$ $12.73499080-16.67201117-20.52173198$ $12.40760054-15.28580098-20.25338338$ $11.78577170-15.20822118-18.84309442$ $10.93985854-16.03451039-18.46242709$ $11.50261339-14.71445891-21.35995826$ $11.22067478-13.24251125-21.07790389$ $10.20703168-15.53647186-21.51605747$ $10.56125552-12.50909971-22.23538632$ $13.32714587-14.70227996-20.24401731$ $9.64004869-15.15816609-22.36265343$ $10.44496466-16.57776710-21.72003209$ $12.05436980-14.77127891-22.29704074$ $10.56268699-13.16476583-20.21208106$ $12.15163116-12.73141376-20.84200841$ $9.59101895-12.95002947-22.45497249$ $12.07715995-17.38200619-20.24471693$ $10.41555082-11.46913732-21.95498904$ $9.60440266-15.45434412-20.61671955$ $11.20054746-12.56806481-23.11613971$ $12.21812264-14.23116716-18.05994875$ $11.97525726-14.15540794-16.60468234$ $11.95407548-12.70222141-16.25936692$ $12.91380959-11.98986933-16.52778926$ $13.04166142-14.90332240-15.81592204$ $12.69370310-15.12689146-14.29871607$ $12.78161151-13.59809163-13.35644138$ $14.53700086-13.19387336-13.55666399$ $11.68625418-15.53197403-14.23391276$ $14.87526996-12.56913195-12.73153687$ $15.11976218-14.11486581-13.56806303$ 


\begin{tabular}{|c|c|}
\hline $\mathrm{H}$ & $14.69593844-12.66675494-14.49619981$ \\
\hline $\mathrm{H}$ & $13.19795584-15.86526200-16.27378782$ \\
\hline $\mathrm{H}$ & $13.38746779-15.85303665-13.87441620$ \\
\hline $\mathrm{H}$ & $12.92407719-13.60981440-18.45721525$ \\
\hline $\mathrm{H}$ & $11.00712295-14.60192812-16.37911011$ \\
\hline $\mathrm{H}$ & $13.97581807-14.35554297-15.88905457$ \\
\hline $\mathrm{N}$ & $10.81793951-12.23356661-15.74240586$ \\
\hline $\mathrm{C}$ & $10.67102565-10.88752187-15.17679158$ \\
\hline $\mathrm{C}$ & $10.54232506-11.07342000-13.64866289$ \\
\hline $\mathrm{O}$ & $9.78917672-11.92449206-13.16519045$ \\
\hline $\mathrm{C}$ & $9.41982193-10.20319059-15.73009603$ \\
\hline $\mathrm{C}$ & $9.40214460-10.10867868-17.27662732$ \\
\hline $\mathrm{C}$ & $9.20992347-8.80744972-15.11319156$ \\
\hline $\mathrm{C}$ & $10.51276591-9.18456742-17.77812340$ \\
\hline $\mathrm{H}$ & $11.54490748-10.27284495-15.39074562$ \\
\hline $\mathrm{H}$ & $8.33307525-8.33175324-15.54382558$ \\
\hline $\mathrm{H}$ & $9.05404619-8.90783115-14.04306969$ \\
\hline $\mathrm{H}$ & $8.56289369-10.81239796-15.45191908$ \\
\hline $\mathrm{H}$ & $9.52334691-11.09738976-17.71689229$ \\
\hline $\mathrm{H}$ & $8.43963906-9.70889761-17.58233281$ \\
\hline $\mathrm{H}$ & $11.48288073-9.55928254-17.45695206$ \\
\hline $\mathrm{H}$ & $10.06216877-12.88613419-15.58847918$ \\
\hline $\mathrm{H}$ & $10.50844837-9.17460105-18.86217151$ \\
\hline $\mathrm{H}$ & $10.08947391-8.18167805-15.26497053$ \\
\hline $\mathrm{H}$ & $10.37643886-8.16619624-17.42373093$ \\
\hline $\mathrm{N}$ & $6.48738439-7.95818863-12.67390151$ \\
\hline $\mathrm{C}$ & $5.62339941-8.75768482-13.49936091$ \\
\hline $\mathrm{C}$ & $4.31779590-8.00023380-13.75838654$ \\
\hline $\mathrm{O}$ & $3.89612963-7.17792983-12.92793768$ \\
\hline $\mathrm{C}$ & $5.35447559-10.11724691-12.88168198$ \\
\hline $\mathrm{C}$ & $6.62576895-10.97924245-12.89847652$ \\
\hline $\mathrm{C}$ & $6.39813630-12.31569482-12.27685706$ \\
\hline $\mathrm{H}$ & $6.92678196-11.14612447-13.93049399$ \\
\hline $\mathrm{H}$ & $6.12345206-8.90510844-14.45576559$ \\
\hline $\mathrm{H}$ & $7.43183479-10.48390767-12.37982917$ \\
\hline $\mathrm{H}$ & $6.76696138-8.28545968-11.75772673$ \\
\hline $\mathrm{H}$ & $4.57892372-10.61969809-13.46051539$ \\
\hline $\mathrm{H}$ & $5.00343723-9.99334761-11.85686979$ \\
\hline $\mathrm{H}$ & $8.32115940-12.85880447-12.49893060$ \\
\hline $\mathrm{O}$ & $5.27661144-12.61890459-11.87439291$ \\
\hline $\mathrm{N}$ & $7.41590322-13.13783408-12.12123599$ \\
\hline $\mathrm{H}$ & $7.24535850-14.05837185-11.74514526$ \\
\hline $\mathrm{N}$ & $3.73047146-8.28091551-14.94873110$ \\
\hline $\mathrm{C}$ & $2.43492820-7.75689336-15.40039129$ \\
\hline $\mathrm{C}$ & $2.44100309-6.22763533-15.44421776$ \\
\hline $\mathrm{O}$ & $1.41603433-5.61712309-15.17150176$ \\
\hline $\mathrm{C}$ & $1.31455395-8.29494962-14.47459150$ \\
\hline $\mathrm{C}$ & $1.26140449-9.82861616-14.43302489$ \\
\hline $\mathrm{C}$ & $0.07697897-10.25919372-13.57497930$ \\
\hline $\mathrm{N}$ & $0.06140002-11.71389174-13.39106330$ \\
\hline $\mathrm{C}$ & $-0.65680304-12.58790588-14.07780456$ \\
\hline $\mathrm{N}$ & $-1.49042065-12.18922337-15.00040360$ \\
\hline $\mathrm{N}$ & $-0.52654361-13.87355790-13.86479547$ \\
\hline $\mathrm{H}$ & $2.17434143-10.23212632-13.99674832$ \\
\hline $\mathrm{H}$ & $2.18559923-8.10364499-16.39923885$ \\
\hline $\mathrm{H}$ & $0.72362721-12.07935659-12.70956964$ \\
\hline $\mathrm{H}$ & $1.14114717-10.21525621-15.44393720$ \\
\hline $\mathrm{H}$ & $-1.06176089-14.56306216-14.36742325$ \\
\hline $\mathrm{H}$ & $0.07449327-14.19154566-13.12034031$ \\
\hline $\mathrm{H}$ & $4.28681984-8.89899352-15.52892241$ \\
\hline $\mathrm{H}$ & $-0.85212487-9.91917685-14.03462734$ \\
\hline $\mathrm{H}$ & $0.15561502-9.78478203-12.59574714$ \\
\hline $\mathrm{H}$ & $-2.04171941-12.85526501-15.50633165$ \\
\hline $\mathrm{H}$ & $-1.55040905-11.20569684-15.18041502$ \\
\hline & $0.35354761-7.92638758-14.83793165$ \\
\hline
\end{tabular}

$\mathrm{H} \quad 1.45578816-7.91781873-13.46031193$ $\mathrm{N} \quad 3.56281979-5.58472693-15.80164174$ C $\quad 3.69327280-4.10683217-15.85992881$ C $\quad 4.27399449-3.60784403-17.18163877$ O $\quad 4.54216552-2.42514705-17.34186641$ C $\quad 4.49919818-3.60034676-14.66481468$ C $\quad 3.65341214-3.49978826-13.39826272$ C $\quad 4.54629569-3.66330520-12.17411190$ $\mathrm{N} \quad 3.84597655-3.23143455-10.94590112$ C $\quad 3.42031588-4.02503538-9.97360917$ $\mathrm{N} \quad 3.48402304-5.31828295-10.08999056$ $\mathrm{N} \quad 2.92657905-3.54639375-8.86679113$ $\mathrm{H} \quad 2.87668891-4.26177949-13.37667249$ $\mathrm{H}$ $\mathrm{H}$ $\mathrm{H}$
$2.70806635-3.64478862-15.78241365$ $3.76819747-2.23881110-10.80033702$ $3.17958448-2.51506219-13.37203706$ $2.68816838-4.18303532-8.13031757$ $2.84611231-2.55571808-8.73719798$ $4.37499320-6.16639818-15.97691519$ $5.44676612-3.05793848-12.29010350$ $4.86089293-4.70655269-12.11674565$ $3.26128143-5.97739826-9.36461462$ $3.93685428-5.66746078-10.91326889$ $4.90205079-2.60696475-14.87536174$ $5.34172918-4.26416074-14.49434780$ $4.32187670-4.47063143-18.19849096$ $4.77966197-4.10399109-19.54682314$ $6.24960274-4.45186587-19.85933055$ $6.61335852-4.43814196-21.04609736$ $4.08123785-5.43270161-17.98791518$ $4.16985949-4.63952745-20.27083977$ $4.66265759-3.03097241-19.70665590$ $7.04433280-4.80590677-18.84221955$ $8.45848626-5.17372018-18.98374119$ $8.61272918-6.48865224-19.72836396$ $9.39640569-6.54682244-20.67146567$ $9.15814717-5.24011930-17.60318400$ $9.10000440-3.92667224-16.85020496$ $9.97551799-2.86553230-17.12745175$ $8.14006719-3.79035344-15.83849358$ $9.87911023-1.66328679-16.39694636$ $8.04878920-2.61274434-15.07672652$ $8.93259310-1.55098482-15.33725722$ $8.76865683-0.41775666-14.62405886$ $8.96400777-4.39991648-19.56509477$ $10.54536220-0.83868697-16.60425748$ $7.32062516-2.49664489-14.29584322$ $6.66340565-4.78316476-17.90837392$ $7.46728112-4.60022906-15.64072899$ $10.73319572-2.94647078-17.89062267$ $10.19676324-5.48339845-17.78120448$ $8.72683311-6.03443076-16.99548037$ $9.595829640 .13506237-14.71073566$ $7.83701802-7.52156470-19.35817234$ $7.78131431-8.78997824-20.07994009$ $7.38861421-8.56577503-21.57445212$ $7.96115281-9.20254183-22.45708731$ $7.17865741-7.34537944-18.61152423$ $8.75424334-9.27766316-20.06660166$ $7.04783636-9.44720528-19.62344759$ $5.94455227-7.32897024-23.17823884$ $7.06380587-6.70078649-23.98947250$ $7.17899369-6.96040635-25.18467763$ $4.73248668-6.39476754-23.11661528$ $6.44207078-7.63788252-21.83590338$ 
C $\quad 4.12330519-6.08130023-24.50634821$

C $\quad 2.90441417-5.19015094-24.25040787$

$\mathrm{N} \quad 2.19408413-4.81334735-25.47495817$

C $\quad 1.11564296-4.03452677-25.54090860$

$\mathrm{N} \quad 0.55397632-3.59562658-24.43372100$

$\mathrm{N} \quad 0.62199349-3.65031662-26.68525267$

$\mathrm{H} \quad 3.80162104-6.99771624-25.00227169$

$\mathrm{H} \quad 5.67616011-8.25616425-23.69105294$

$\mathrm{H} \quad 2.59136449-5.11291032-26.36594517$

$\mathrm{H} \quad 4.83696596-5.54885309-25.13218705$

$\mathrm{H} \quad-0.21403422-3.10104961-26.76269353$

$\mathrm{H} \quad 0.98270587-4.05638875-27.54010969$

$\mathrm{H} \quad 6.02459421-7.20865428-21.02079215$

$\mathrm{H} \quad 3.23543137-4.28677654-23.73931968$

$\mathrm{H} \quad 2.21008801-5.73299570-23.60893668$

$\mathrm{H} \quad-0.25272025-2.99658037-24.49782697$

$\mathrm{H} \quad 0.75442402-4.15841915-23.63884437$

$\mathrm{H} \quad 5.00542959-5.45691552-22.64300158$

$\mathrm{H} \quad 3.96567127-6.85817162-22.50785987$

$\mathrm{N} \quad 9.62219680-9.43434783-24.97407583$

C $\quad 9.06979610-10.22008463-26.09608305$

C $\quad 9.19801828-9.39882958-27.38974852$

O $\quad 9.78390577-9.85295166-28.34603147$

C $\quad 7.61650672-10.68935178-25.84160704$

C $\quad 7.56632341-11.67276476-24.66054531$

C $\quad 7.08807924-11.40136237-27.09286640$

C $\quad 6.15458336-11.87954217-24.12406165$

$\mathrm{H} \quad 9.68470050-11.11305661-26.22553620$

$\mathrm{H} \quad 6.07055641-11.74835637-26.93495470$

$\mathrm{H} \quad 7.06995652-10.72194897-27.94253378$

$\mathrm{H} \quad 6.99663067-9.81813721-25.62485157$

H $\quad 7.99848019-12.62476995-24.96081468$

$\mathrm{H} \quad 8.17209485-11.29542498-23.84134355$

$\mathrm{H} \quad 5.54577723-12.35000580-24.89395887$

$\mathrm{H} \quad 9.04764597-9.21643843-24.17220098$

$\mathrm{H} \quad 6.20083366-12.54047031-23.26382820$

$\mathrm{H} \quad 7.72651716-12.24874056-27.32924335$

$\mathrm{H} \quad 5.71862771-10.92247115-23.84580488$

$\mathrm{N} \quad 6.60485371-21.00037575-19.56962887$

C $\quad 6.08297352-20.89410648-18.19657192$

C $\quad 4.59081648-20.55526849-18.29800609$

O $\quad 4.09286429-20.16362222-19.38020378$

C $\quad 6.83389423-19.84386677-17.38812770$

O $\quad 6.67840705-18.56794021-17.98617970$

$\mathrm{H} \quad 7.17382260-20.27891823-19.99519660$

$\mathrm{H} \quad 6.18940722-21.85912033-17.69748456$

$\mathrm{H} \quad 7.89282308-20.10464193-17.34680689$

$\mathrm{H} \quad 6.44166867-19.81941105-16.37158943$

$\mathrm{H} \quad 7.56300171-18.33014270-18.34275595$

$\mathrm{N} \quad 3.12460443-17.88054579-16.98005810$

C $\quad 3.06596904-16.44249717-17.25122487$

C $\quad 3.61175111-16.05156410-18.63644128$

O $\quad 3.10314461-15.13018750-19.29397475$

C $\quad 3.80319536-15.70529383-16.14045077$

C $\quad 3.76939278-14.17465609-16.25133892$

C $\quad 2.34437058-13.63097754-16.42645505$

C $\quad 4.33715138-13.59543775-14.94586000$

$\mathrm{H} \quad 5.35122934-13.96379877-14.78865272$

$\mathrm{H} \quad 4.34629162-12.50984007-14.98707770$

H $\quad 3.72693005-13.91988999-14.10167244$

$\mathrm{H} \quad 3.80398392-18.23656528-16.30792488$

$\mathrm{H} \quad 1.71943890-13.99817163-15.61436631$

$\mathrm{H} \quad 2.36633858-12.54346912-16.40869311$

$\mathrm{H} \quad 1.92776980-13.94997118-17.37745265$

H $\quad 2.01726541-16.14936085-17.22497080$
$4.38098072-13.83967529-17.08649814$ $4.84486871-16.02499600-16.13106532$ $3.35945270-15.99477713-15.18705869$ $4.67008682-16.70498804-19.07463459$ $5.16272012-16.60121430-20.44627600$ $4.06094224-16.97275843-21.45297829$ $3.85898433-16.19795968-22.36670919$ $5.07780954-17.42833688-18.48505968$ $5.49403130-15.58556382-20.64678696$ $6.00255351-17.28262682-20.58560382$ $1.58184496-14.25813908-21.64944951$ $2.22920471-13.05926914-22.16751779$ $2.48827486-13.18498145-23.68937319$ $2.29844243-12.21294026-24.39529672$ $3.56565434-12.83081039-21.41431675$ $3.32645072-12.59537310-19.99740503$ $2.09529793-14.85334851-21.00819750$ $1.58731851-12.19392423-22.00433875$ $4.09415301-12.01156132-21.88121973$ $4.19573157-13.71437807-21.52508632$ $3.21959630-13.49121567-19.61095198$ $-1.00993012-13.04449886-25.46453089$ $-1.45158940-11.65340688-25.47120248$ $-0.64176923-10.82937604-26.47384719$ $-1.20155169-9.99563650-27.18895200$ $-0.54513310-13.44222283-24.66242186$ $-2.49639766-11.61737287-25.78714574$ $0.69539976-10.98479622-26.47253003$ $1.56590737-10.21488499-27.36273996$ $1.20583869-10.53049722-28.83231993$ $0.99779932-9.59955928-29.59921389$ $3.06543417-10.48265270-27.08732711$ $3.96133796-9.89933499-28.17063110$ $4.27708215-8.53098257-28.14105573$ $4.35810997-10.70701501-29.25314011$ $5.10635168-7.99683222-29.14074395$ $5.18437501-10.18324136-30.26207579$ $5.58832262-8.83735894-30.16939389$ $6.51832872-8.33785420-31.00103057$ $1.38118484-9.15060372-27.22007625$ $5.42763154-6.97193425-29.10914430$ $5.54981726-10.78874867-31.08031655$ $1.12253656-11.68420057-25.87569854$ $4.06777990-11.74620972-29.29394888$ $3.93821560-7.89770557-27.33914694$ $3.24325083-11.55649427-27.04203179$ $3.34350165-10.04714117-26.12874211$ $6.36444786-7.41030964-31.23886866$ $7.67922251-15.96650089-16.33541268$ $7.14721853-16.35420750-17.19311823$ $8.07600953-16.78164452-15.28465031$ $7.86178630-17.84313175-15.30671183$ $8.76430250-16.17424333-14.23509516$ $9.13176382-16.74259527-13.39866863$ $8.97635933-14.80073613-14.27022821$ $9.53620084-14.32992733-13.46843034$ $8.49610726-13.98437264-15.23270184$ $7.90347969-14.60414964-16.26707212$ $7.40322976-13.75372881-17.36199243$ $7.51183637-14.18344571-18.69867403$ $6.99888860-13.35384538-19.69820717$ $6.37952437-12.14422791-19.41237506$ $5.99310971-11.56021401-20.23040595$ $7.07600173-13.73258329-20.99559229$ $8.01737575-15.12056838-18.93441465$ 
C $\quad 6.80323738-12.53658987-17.04784541$

$\mathrm{H} \quad 6.74529384-12.26513103-16.00180003$

C $\quad 6.24247321-11.76493285-18.05895589$

C $\quad 5.43662660-10.58693338-17.67298681$

O $\quad 5.65241725-9.86503290-16.70494922$

$\mathrm{N} \quad 4.36269150-10.33811474-18.52221680$

$\mathrm{H} \quad 4.00045418-11.07376421-19.13652759$

$\mathrm{N} \quad 3.42619877-9.36968655-18.22967700$

$\mathrm{H} \quad 3.26057376-9.25381910-17.22989013$

S $\quad 3.58854133-7.94851896-19.07791810$

O $\quad 4.96002983-7.56885580-19.42620990$

O $\quad 2.79564618-6.91161290-18.40806253$

C $\quad 2.76463659-8.39070937-20.67913239$

C $\quad 1.60990864-7.68677169-21.07774827$

$\mathrm{H} \quad 1.12469270-7.00062846-20.39060570$

C $\quad 1.04855984-7.92075447-22.34373262$

$\mathrm{H} \quad 0.12987771-7.42684962-22.64652263$

C $\quad 1.64142612-8.83960962-23.21052187$

$\mathrm{H} \quad 1.21507414-9.04031805-24.19167706$

C $\quad 2.81001287-9.50084860-22.84964491$

$\mathrm{H} \quad 3.31406753-10.17686552-23.53672765$

C $\quad 3.35576937-9.28075687-21.58496299$

F $\quad 4.52771949-9.91807298-21.27906737$

$\mathrm{Zn} \quad 28.07144298-6.23496514-6.91408115$

O $\quad-2.05725138-5.98967309-23.84076449$

$\mathrm{H} \quad-2.79439568-5.46045571-23.47893997$

$\mathrm{H} \quad-2.36862493-6.88932790-23.70031895$

O $\quad 7.32087790-19.76439622-13.50313411$

$\mathrm{H} \quad 8.19106793-19.41119722-13.24128614$

$\mathrm{H} \quad 7.33741011-20.65608492-13.13736679$

O $\quad 9.17126424-17.89504744-18.97389360$

$\mathrm{H} \quad 9.82373496-17.22036865-18.68978243$

$\mathrm{H} \quad 9.72839550-18.65030984-19.19588326$

O $\quad 5.98044414-7.03636801-16.78865955$

$\mathrm{H} \quad 6.45196895-6.74112652-15.98718845$

$\mathrm{H} \quad 6.19655100-7.97766715-16.83723469$

O $\quad 26.47835443-0.92809580-4.84577741$

$\mathrm{H} \quad 27.10343032-0.82221445-4.11541006$

$\mathrm{H} \quad 26.79458875-1.70671123-5.31322887$

O $\quad 0.75721898-10.02278606-18.06627959$

$\mathrm{H} \quad-0.01561438-10.49249547-18.44910977$

$\mathrm{H} \quad 1.50236488-10.41523657-18.52976283$

O $\quad 4.77965723-18.65786537-14.46007781$

$\mathrm{H} \quad 5.69963490-18.88029127-14.22792482$

$\mathrm{H} \quad 4.28355923-19.32509351-13.97317065$

O $\quad-0.44512913-6.73864212-25.82630342$

$\mathrm{H} \quad-0.89828595-7.28273293-26.48152038$

H $\quad-1.15913615-6.39922782-25.26053028$

2C-QM/MM R2b (2C-B3LYP/6-31G(d):Amber) mechanical embedding $\omega_{\alpha}=1.0$

C $\quad 21.94244773-10.35579316-4.21611358$

C $\quad 22.25661889-8.89214453-4.42812496$

O $\quad 21.40470357-8.01936316-4.36145027$

$\mathrm{N} \quad 23.53078254-8.67541999-4.79871559$

C $\quad 23.90021583-7.39389279-5.39120247$

C $\quad 23.08410173-7.24150154-6.68765963$

O $\quad 23.14890457-8.09225187-7.57468677$

C $\quad 25.39466408-7.35124959-5.76296326$

S $\quad 25.83049357-5.73803568-6.51866858$

$\mathrm{H} \quad 24.16899587-9.46813888-4.85372572$

$\mathrm{H} \quad 23.67086177-6.59924581-4.68107348$

$\mathrm{H} \quad 25.64779156-8.19718616-6.40509421$

$\mathrm{H} \quad 25.93639057-7.47261855-4.81617145$
$\mathrm{N} \quad 22.35993016-6.11336053-6.79151268$

C $\quad 21.46443029-5.93499814-7.92153366$

$\mathrm{H} \quad 22.18940528-5.52451274-5.98159568$

$\mathrm{N} \quad 23.48822018-5.25471365-9.13859550$

C $\quad 24.31628707-4.83567927-10.25972633$

C $\quad 25.23074998-5.92588945-10.78721953$

O $\quad 25.20994599-6.17748116-11.98859551$

C $\quad 25.10609597-3.60893452-9.90628850$

C $\quad 24.27776831-2.44674697-9.44972413$

C $\quad 23.30915589-1.88819984-10.29129253$

C $\quad 24.45175034-1.92954337-8.14736190$

C $\quad 22.53169751-0.80920589-9.84432117$

C $\quad 23.65215517-0.87676742-7.69650846$

C $\quad 22.69436060-0.30143310-8.54207106$

O $\quad 22.027219730 .80112454-8.14296504$

$\mathrm{H} \quad 23.65729819-4.54590873-11.07664866$

$\mathrm{H} \quad 21.78795141-0.41163981-10.49073770$

$\mathrm{H} \quad 23.81248835-0.47444478-6.72435676$

$\mathrm{H} \quad 23.89349698-5.33107652-8.21041373$

$\mathrm{H} \quad 25.21212917-2.32980526-7.50760119$

$\mathrm{H} \quad 23.15079357-2.29514075-11.26814283$

$\mathrm{H} \quad 25.66580586-3.27895050-10.77411023$

$\mathrm{H} \quad 25.80457643-3.85884204-9.11568275$

$\mathrm{H} \quad 22.457673091 .60988990-8.43643780$

$\mathrm{N} \quad 25.99281261-6.58751838-9.85819643$

C $\quad 26.82867846-7.68300980-10.30003577$

C $\quad 26.22013516-9.07298061-10.10980061$

O $\quad 26.76662321-10.08213150-10.56016488$

C $\quad 28.22928818-7.60518321-9.70804137$

S $\quad 28.27170238-8.15574686-7.90575882$

$\mathrm{H} \quad 25.97081353-6.29980326-8.87897894$

$\mathrm{H} \quad 26.92455410-7.57532276-11.38329058$

$\mathrm{H} \quad 28.58928014-6.58185461-9.81690685$

$\mathrm{H} \quad 28.87496739-8.27055212-10.28405222$

$\mathrm{N} \quad 25.05238219-9.10815815-9.42027515$

C $\quad 24.37499697-10.39386286-9.16258666$

C $\quad 25.16696867-11.36908236-8.29830248$

O $\quad 24.78163138-12.54106079-8.16911662$

C $\quad 23.88290616-11.08379178-10.44760332$

C $\quad 22.93824881-10.21470189-11.26511357$

C $\quad 22.45027861-10.98728364-12.48871017$

C $\quad 21.72157686-9.82707646-10.44972112$

$\mathrm{H} \quad 22.05338050-9.20777986-9.62983106$

$\mathrm{H} \quad 21.07071231-9.23161999-11.06173612$

$\mathrm{H} \quad 21.23282629-10.71807604-10.06544126$

$\mathrm{H} \quad 24.64724824-8.28671824-8.98994051$

$\mathrm{H} \quad 21.94364925-11.89815612-12.17627140$

$\mathrm{H} \quad 21.78304815-10.35268075-13.05427576$

$\mathrm{H} \quad 23.31326479-11.24656893-13.09708222$

$\mathrm{H} \quad 23.46648361-10.20347460-8.60715211$

$\mathrm{H} \quad 23.44769285-9.32218021-11.58615769$

$\mathrm{H} \quad 23.36078878-11.99702007-10.18185513$

$\mathrm{H} \quad 24.72897540-11.32262687-11.06895356$

$\mathrm{N} \quad 26.23441483-10.90143166-7.62648839$

C $\quad 26.86926450-11.73988450-6.59846329$

C $\quad 25.84206235-12.11908743-5.51517835$

O $\quad 25.16303383-11.24767175-4.98539006$

C $\quad 28.06723657-11.02340161-5.99401077$

C $\quad 28.65434242-11.79542690-4.82155089$

C $\quad 29.96279678-11.16374688-4.33716226$

C $\quad 30.36954737-11.80251494-3.00372594$

$\mathrm{N} \quad 31.80489478-11.66034494-2.76608722$

$\mathrm{H} \quad 27.20475601-12.65551342-7.08239720$

$\mathrm{H} \quad 29.80997387-11.30926262-2.20843367$

H $\quad 30.10791390-12.85863699-3.03843701$ 


\begin{tabular}{|c|c|}
\hline $\mathrm{H}$ & $27.94100077-11.75472130-4.00547946$ \\
\hline $\mathrm{H}$ & $28.84167085-12.82554912-5.10070815$ \\
\hline $\mathrm{H}$ & $32.10709396-12.26933367-2.02132829$ \\
\hline $\mathrm{H}$ & $32.33644787-11.92006512-3.59807206$ \\
\hline $\mathrm{H}$ & $32.03221894-10.70773971-2.49675675$ \\
\hline $\mathrm{H}$ & $29.81436390-10.10511127-4.19279059$ \\
\hline $\mathrm{H}$ & $30.71708538-11.35770294-5.09174558$ \\
\hline $\mathrm{H}$ & $26.55262949-9.96111756-7.84285581$ \\
\hline $\mathrm{H}$ & $27.77701643-10.03338414-5.66138460$ \\
\hline $\mathrm{H}$ & $28.83762653-10.95716789-6.75697160$ \\
\hline $\mathrm{C}$ & $29.87674766-4.493773842 .81235662$ \\
\hline $\mathrm{C}$ & $30.09308327-4.491572231 .28035446$ \\
\hline $\mathrm{O}$ & $30.69419949-3.624909430 .68496706$ \\
\hline $\mathrm{N}$ & $29.50362289-5.538720000 .65398206$ \\
\hline $\mathrm{C}$ & $29.59118929-5.60282513-0.80368343$ \\
\hline $\mathrm{C}$ & $29.07254338-4.32370687-1.43108136$ \\
\hline $\mathrm{O}$ & $29.76237152-3.76575688-2.26422437$ \\
\hline $\mathrm{C}$ & $28.87383642-6.83119546-1.35995769$ \\
\hline $\mathrm{C}$ & $28.98035456-6.95675264-2.82904324$ \\
\hline $\mathrm{H}$ & $29.31320084-7.71434828-0.89437397$ \\
\hline $\mathrm{H}$ & $28.95809765-6.214864211 .18203488$ \\
\hline $\mathrm{H}$ & $30.64971427-5.63308864-1.07386206$ \\
\hline $\mathrm{H}$ & $27.81784484-6.77900568-1.09761778$ \\
\hline $\mathrm{C}$ & $28.17794342-6.49512905-3.83566715$ \\
\hline $\mathrm{H}$ & $30.89675833-7.88084312-2.99373666$ \\
\hline $\mathrm{H}$ & $27.27015081-5.92725220-3.74205614$ \\
\hline $\mathrm{C}$ & $29.83956035-7.49516415-4.78861203$ \\
\hline $\mathrm{N}$ & $30.03110810-7.61017248-3.45660011$ \\
\hline $\mathrm{N}$ & $28.70800048-6.87148650-5.02692773$ \\
\hline $\mathrm{H}$ & $30.52658342-7.87673545-5.52006208$ \\
\hline $\mathrm{N}$ & $27.85326896-3.87308487-1.03582656$ \\
\hline $\mathrm{C}$ & $27.27964017-2.63817985-1.55419694$ \\
\hline $\mathrm{H}$ & $27.35484212-4.31835583-0.26704314$ \\
\hline $\mathrm{C}$ & $32.66069192-1.85163624-2.42492288$ \\
\hline $\mathrm{C}$ & $32.41151740-1.96201614-3.94007392$ \\
\hline $\mathrm{O}$ & $33.14086144-1.45557067-4.78714716$ \\
\hline $\mathrm{N}$ & $31.32215817-2.68332784-4.23159385$ \\
\hline $\mathrm{C}$ & $30.97320230-3.03797785-5.61333304$ \\
\hline $\mathrm{C}$ & $30.82756978-1.73041947-6.39389439$ \\
\hline $\mathrm{O}$ & $30.04387481-0.87594483-6.04966691$ \\
\hline $\mathrm{C}$ & $29.67021058-3.83890468-5.60273395$ \\
\hline $\mathrm{S}$ & $29.20555387-4.40148700-7.29444796$ \\
\hline $\mathrm{H}$ & $30.77454232-3.03668157-3.46191610$ \\
\hline $\mathrm{H}$ & $31.77206234-3.65659843-6.03322210$ \\
\hline $\mathrm{H}$ & $28.88011780-3.20519511-5.19489794$ \\
\hline $\mathrm{H}$ & $29.82040231-4.69438498-4.94490466$ \\
\hline $\mathrm{N}$ & $31.57721325-1.59866115-7.48992750$ \\
\hline $\mathrm{C}$ & $31.62028693-0.46959806-8.37112929$ \\
\hline $\mathrm{H}$ & $32.30603356-2.24197613-7.67783693$ \\
\hline $\mathrm{N}$ & $30.17699418-1.81932053-9.80388186$ \\
\hline $\mathrm{C}$ & $29.16674615-2.01473196-10.85041900$ \\
\hline $\mathrm{C}$ & $27.91440629-1.21136265-10.53754394$ \\
\hline $\mathrm{O}$ & $27.42070436-1.17744529-9.41170888$ \\
\hline $\mathrm{C}$ & $28.81829379-3.48997597-10.98817218$ \\
\hline $\mathrm{C}$ & $29.87407410-4.34343703-11.57587617$ \\
\hline $\mathrm{C}$ & $31.21050853-4.22792343-11.42713683$ \\
\hline $\mathrm{C}$ & $29.68135457-5.48067924-12.43266416$ \\
\hline $\mathrm{N}$ & $31.86078472-5.19543951-12.14455466$ \\
\hline $\mathrm{C}$ & $30.96009191-6.02257283-12.73884685$ \\
\hline $\mathrm{C}$ & $28.55433932-6.14839497-12.92538270$ \\
\hline $\mathrm{C}$ & $31.11593766-7.18202878-13.47549549$ \\
\hline $\mathrm{C}$ & $28.70551037-7.35045587-13.60752942$ \\
\hline $\mathrm{C}$ & $29.96993596-7.84929914-13.90694924$ \\
\hline $\mathrm{H}$ & $30.06336327-8.71196332-14.51163854$ \\
\hline $\mathrm{H}$ & $29.56875073-1.65682428-11.79676440$ \\
\hline
\end{tabular}

H $\quad 32.85960300-5.30860722-12.21237449$ $\mathrm{H} \quad 27.57109477-5.76854349-12.73443875$ $\mathrm{H} \quad 27.83274147-7.86689236-13.91600954$ $\mathrm{H} \quad 32.09850039-7.51885806-13.71772721$ $\mathrm{H} \quad 31.70350634-3.47386072-10.85465710$ $\mathrm{H} \quad 30.34252891-2.54781161-9.11761592$ $\mathrm{H} \quad 27.95110848-3.56904566-11.63837814$ $\mathrm{H} \quad 28.55996659-3.90733699-10.02245238$ $\mathrm{N} \quad 12.43199267-18.61250802-10.12816964$ C $\quad 11.14049177-18.23250249-10.68636881$ C $\quad 10.77817933-18.99210309-11.94160266$ O $\quad 9.71242706-18.76253823-12.49647161$ C $\quad 11.06087182-16.73174479-10.89695559$ C $\quad 11.55658023-15.93693640-9.73085425$ C $\quad 10.78764487-15.86027428-8.55678289$ C $\quad 12.80173942-15.30482804-9.80822358$ C $\quad 11.28613748-15.15494217-7.46182598$ C $\quad 13.29024458-14.60897521-8.70490767$ C $\quad 12.53257508-14.53553431-7.52749778$ $\mathrm{H} \quad 12.92641935-14.02399971-6.67984516$ $\mathrm{H} \quad 10.36470871-18.48965082-9.97076093$ $\mathrm{H} \quad 10.71317545-15.12198676-6.57349110$ $\mathrm{H} \quad 14.25641334-14.15860995-8.76726173$ H $\quad 13.25892065-18.52806194-10.71236658$ H $\quad 13.39472006-15.39746412-10.69441469$ $\mathrm{H} \quad 9.84695005-16.36367371-8.49336431$ $\mathrm{H} \quad 10.02803234-16.44442612-11.05171426$ $\mathrm{H} \quad 11.65115558-16.46385145-11.76040349$ $\mathrm{N} \quad 11.58079754-19.96101484-12.34707494$ C $\quad 11.29432413-20.75831575-13.52626932$ C $\quad 11.29098303-22.22224313-13.17447866$ O $\quad 12.20729169-22.70006589-12.50971972$ C $\quad 12.29609070-20.44245446-14.64415661$ C $\quad 12.27418599-18.99660308-15.08907675$ C $\quad 13.37697417-18.78557945-16.11052602$ C $\quad 10.93662262-18.65702843-15.70757874$ $\mathrm{H} \quad 10.17063833-18.77766717-14.96263910$ H $\quad 10.97165355-17.62748810-16.02462687$ $\mathrm{H} \quad 10.74869031-19.32422117-16.54497956$ $\mathrm{H} \quad 12.41529050-20.15548634-11.81196587$ $\mathrm{H} \quad 13.24303668-19.42934957-16.97646603$ $\mathrm{H} \quad 13.35690115-17.77343615-16.45304270$ $\mathrm{H} \quad 14.34077483-18.97099814-15.64767754$ $\mathrm{H} \quad 10.30853809-20.53469627-13.92000886$ $\mathrm{H} \quad 12.43343854-18.33764049-14.25175130$ $\mathrm{H} \quad 12.06168587-21.07076182-15.49579312$ $\mathrm{H} \quad 13.29089043-20.66916153-14.28571869$ $\mathrm{N} \quad 12.73319636-16.68441134-20.52464432$ C $\quad 12.41430610-15.29647426-20.25628504$ C $\quad 11.79487870-15.19595396-18.85620354$ O $\quad 10.93182175-16.00320041-18.48189422$ C $\quad 11.51072578-14.70920827-21.34611671$ C $\quad 11.22260813-13.25021084-21.06666312$ C $\quad 10.22282254-15.51086482-21.47407003$ C $\quad 10.56843390-12.52200882-22.21491089$ $\mathrm{H} \quad 13.33858403-14.72815767-20.24743127$ $\mathrm{H} \quad 9.65055292-15.12252921-22.30232459$ $\mathrm{H} \quad 10.47517298-16.53808867-21.68431769$ $\mathrm{H} \quad 12.03102249-14.76994419-22.29052898$ $\mathrm{H} \quad 10.55992871-13.17165824-20.20919635$ $\mathrm{H} \quad 12.15158481-12.74764741-20.84111823$ $\mathrm{H} \quad 9.60757310-12.97458469-22.43423077$ $\mathrm{H} \quad 12.09917521-17.42008605-20.25385127$ $\mathrm{H} \quad 10.41316232-11.49312745-21.91426194$ H $\quad 9.65126970-15.42163623-20.55908996$ 
$\mathrm{H} \quad 11.21795923-12.57414385-23.08478656$

$\mathrm{N} \quad 12.24007501-14.21792984-18.08303960$

C $\quad 11.97578847-14.12347843-16.63365453$

C $\quad 11.95185338-12.67912415-16.27169499$

O $\quad 12.91152783-11.96209515-16.52482874$

C $\quad 13.01546823-14.86260064-15.81073478$

C $\quad 12.62839750-15.08499106-14.31533680$

S $\quad 12.79493592-13.58959306-13.34575128$

C $\quad 14.56750474-13.28834367-13.56520734$

$\mathrm{H} \quad 11.59224562-15.39578739-14.28734743$

$\mathrm{H} \quad 14.94643476-12.70972572-12.73329987$

$\mathrm{H} \quad 15.10060626-14.23806280-13.60955320$

$\mathrm{H} \quad 14.74817930-12.74796141-14.48999613$

$\mathrm{H} \quad 13.17118222-15.82202654-16.24315530$

$\mathrm{H} \quad 13.24172985-15.88595460-13.91809387$

$\mathrm{H} \quad 12.93930883-13.60769795-18.51416508$

$\mathrm{H} \quad 11.00019330-14.56549889-16.44279982$

$\mathrm{H} \quad 13.95824815-14.33520319-15.85618581$

$\mathrm{N} \quad 10.81027830-12.22464692-15.75895432$

C $\quad 10.66057643-10.88763628-15.17489576$

C $\quad 10.53790291-11.06678588-13.65132758$

O $\quad 9.77790968-11.90782765-13.16767918$

C $\quad 9.42055367-10.19402025-15.71917201$

C $\quad 9.43395682-10.06037955-17.24371759$

C $\quad 9.20721592-8.82725722-15.08287995$

C $\quad 10.55118458-9.15783226-17.71519327$

$\mathrm{H} \quad 11.53061346-10.27558747-15.38117275$

$\mathrm{H}$

$\mathrm{H}$

$\mathrm{H}$

$\mathrm{H}$

$\mathrm{H}$

$\mathrm{H}$

$\mathrm{H}$

$\mathrm{H}$

$\mathrm{H}$

$\mathrm{H}$

$\mathrm{N}$

C

$\mathrm{C}$

$\mathrm{O}$

C

C

C

$\mathrm{H}$

$\mathrm{H}$

$\mathrm{H}$

$\mathrm{H}$

$\mathrm{H}$

$\mathrm{H}$

$\mathrm{H}$

O

N

$\mathrm{H}$

$\mathrm{N}$

C

C

O

C

C

C

$\mathrm{N}$

C

$\mathrm{N}$
$\mathrm{H}$

$\mathrm{H}$

$\mathrm{H}$

$\mathrm{H}$

$\mathrm{H}$

$\mathrm{H}$

$\mathrm{H}$

$\mathrm{H}$

$\mathrm{H}$

$\mathrm{H}$

$\mathrm{H}$

$\mathrm{H}$

$\mathrm{H}$

$\mathrm{N}$

C

C

$\mathrm{O}$

C

C

C

$\mathrm{N}$

C

$\mathrm{N}$

$\mathrm{N}$

$\mathrm{H}$

$\mathrm{H}$

$\mathrm{H}$

$\mathrm{H}$

$\mathrm{H}$

$\mathrm{H}$

$\mathrm{H}$

$\mathrm{H}$

$\mathrm{H}$

$\mathrm{H}$

$\mathrm{H}$

$\mathrm{H}$

$\mathrm{H}$

$\mathrm{N}$

C

C

O

$\mathrm{H}$

$\mathrm{H}$

$\mathrm{H}$

$\mathrm{N}$

C

C

O

C

C

C

C

C

C

C

O

$\mathrm{H}$

$\mathrm{H}$

$\mathrm{H}$

$\mathrm{H}$

$\mathrm{H}$

$\mathrm{H}$

$\mathrm{H}$

$\mathrm{H}$

$\mathrm{H}$
$2.11484151-10.24368501-13.97780730$ $2.15781079-8.15149568-16.38087443$ $0.66170127-12.06567401-12.65742098$ $1.09979171-10.20126125-15.44303138$ $-1.13853140-14.55624193-14.28742085$ $0.00529883-14.18586512-13.04678307$ $4.28438605-8.91894571-15.51592155$ $-0.87909006-9.97423664-14.11357295$ $0.06178157-9.77153914-12.64142439$ $-2.05396996-12.86035494-15.50053910$ $-1.54961332-11.21674433-15.21196329$ $0.35853195-7.92251699-14.81183742$ $1.47910619-7.95885887-13.44786356$ $3.54283590-5.62058219-15.78960474$ $3.67736866-4.14659805-15.83890565$ $4.26423247-3.64156603-17.14948659$ $4.54743654-2.46296461-17.29132737$ $4.48024109-3.64348172-14.64687046$ $3.65167751-3.52398824-13.37862504$ $4.50425216-3.78307695-12.15451251$ $3.81135744-3.35348494-10.92372687$ $3.38149101-4.16688285-9.97510447$ $3.47187562-5.44657714-10.11752503$ $2.85069617-3.73487715-8.87046166$ $2.83424553-4.23407846-13.38899985$ $2.69770076-3.68169500-15.75152627$ $3.72290614-2.36632081-10.76547511$ $3.25711443-2.51396822-13.30945395$ $2.57476802-4.38894108-8.15989570$ $2.75183178-2.74989477-8.73654953$ $4.33826524-6.20874464-16.01405891$ $5.44138321-3.24415045-12.23928427$ $4.73275995-4.84474449-12.13317525$ $3.26188416-6.12697799-9.40765104$ $3.98280109-5.80330589-10.90299184$ $4.88394255-2.65788921-14.86273264$ $5.30610287-4.31610680-14.47157184$ $4.30385317-4.49212249-18.17587951$ $4.76291601-4.11857938-19.51939084$ $6.23301619-4.45822742-19.83607941$ $6.59593250-4.42824501-21.02394671$ $4.03009620-5.44720579-17.97616752$ $4.15367845-4.64634276-20.24927863$ $4.64653483-3.04499306-19.66485885$ $7.02928234-4.82459075-18.82305370$ $8.44407758-5.17307338-18.99538066$ $8.60941823-6.49021315-19.73301101$ $9.40179371-6.55120485-20.66873397$ $9.17797273-5.22782090-17.64146823$ $9.10314476-3.94323859-16.87108240$ $9.96994409-2.88677686-17.12550510$ $8.15069729-3.82305247-15.86594312$ $9.87405532-1.70186293-16.38886750$ $8.05453958-2.65763458-15.10319652$ $8.92961487-1.59013552-15.34162979$ $8.77804140-0.45674709-14.63249651$ $8.92049404-4.39113236-19.58548186$ $10.54524423-0.89593601-16.58420976$ $7.31900729-2.57601452-14.34263104$ $6.63156859-4.87089986-17.89510684$ $7.48414312-4.62872463-15.67163260$ $10.72436503-2.96772406-17.87546741$ $10.21832658-5.41767641-17.85025682$ $8.78698481-6.03417538-17.03948110$ $9.610283080 .08211761-14.73711348$ 
C $\quad 3.80267814-15.70002437-16.15235236$ C $\quad 3.76619805-14.17941687-16.20306943$ C $\quad 2.35463582-13.65684172-16.39487748$ C $\quad 4.29783182-13.64378828-14.87794582$ $\mathrm{H} \quad 5.31347329-13.99949770-14.73242424$ $\mathrm{H} \quad 4.27428530-12.56265285-14.88743948$ $\mathrm{H} \quad 3.68202248-14.01242051-14.05743160$ $\mathrm{H} \quad 3.81870185-18.27557194-16.33582309$ H $\quad 1.72190929-14.02958275-15.59351839$ $\mathrm{H} \quad 2.38104828-12.57544125-16.38705175$ $\mathrm{H} \quad 1.98056389-13.99115726-17.34962323$ $\mathrm{H} \quad 2.02292172-16.15488304-17.21520914$ $\mathrm{H} \quad 4.39373038-13.80601752-16.99628718$ $\mathrm{H} \quad 4.84473160-15.98058685-16.18397385$ $\mathrm{H} \quad 3.38066785-16.00706711-15.20131305$ $\mathrm{N} \quad 4.66801142-16.72034560-19.07136397$ C $\quad 5.15663089-16.58727527-20.44266299$ C $\quad 4.06158475-16.97203557-21.45450981$ O $\quad 3.86413347-16.20255887-22.37443326$ $\mathrm{H} \quad 5.07374793-17.44719027-18.48051313$ $\mathrm{H} \quad 5.45157713-15.55600450-20.62035365$ $\mathrm{H} \quad 6.01871250-17.23446868-20.60222455$ $\mathrm{N} \quad 1.58283076-14.26728760-21.65116310$ C $\quad 2.22809150-13.06564716-22.16687434$ C $\quad 2.48729835-13.18683675-23.68887237$ O $\quad 2.29658767-12.21569613-24.39566433$ C $\quad 3.56147884-12.82580701-21.42423307$ O $\quad 3.33224211-12.59375618-20.00650828$ $\mathrm{H} \quad 2.08519023-14.88589016-21.02180911$ $\mathrm{H} \quad 1.59378689-12.20092341-21.99185372$ $\mathrm{H} \quad 4.06167539-11.99220394-21.89190904$ $\mathrm{H} \quad 4.19938748-13.69834849-21.55647183$ $\mathrm{H} \quad 3.24007395-13.48955218-19.61818830$ $\mathrm{N} \quad-1.01067879-13.04637658-25.46535426$ C $\quad-1.45312750-11.65474427-25.47067300$ C $\quad-0.64503479-10.83126868-26.47442651$ O $\quad-1.20314929-9.99822300-27.19084987$ $\mathrm{H} \quad-0.54423777-13.44687391-24.66445381$ $\mathrm{H} \quad-2.49784515-11.62058753-25.78702606$ $\mathrm{N} \quad 0.69198006-10.98250850-26.46737891$ C $\quad 1.55326964-10.21341117-27.36994212$ C $\quad 1.20007612-10.53220519-28.83603973$ O $\quad 0.99679422-9.60608056-29.60782601$

C $\quad 3.04681931-10.46584521-27.10468179$ C $\quad 3.95226981-9.88824657-28.16914394$ C $\quad 4.30654220-8.54225193-28.11864490$ C $\quad 4.33888509-10.67724228-29.25372105$ C $\quad 5.13218109-8.00938621-29.10881262$ C $\quad 5.16184020-10.15498532-30.25169460$ C $\quad 5.59134934-8.82670302-30.15666553$ O $\quad 6.51086750-8.32679390-30.99250739$ $\mathrm{H} \quad 1.36634952-9.15289849-27.22871705$ $\mathrm{H} \quad 5.47786473-7.00747490-29.02698718$ $\mathrm{H} \quad 5.48515173-10.76077368-31.06680259$ $\mathrm{H} \quad 1.09938108-11.66520054-25.83447223$ $\mathrm{H} \quad 4.02630097-11.69731462-29.31785926$ $\mathrm{H} \quad 4.01851989-7.93469621-27.29080847$

$\mathrm{H} \quad 3.21986347-11.53348753-27.07141270$ $\mathrm{H} \quad 3.31418476-10.02137485-26.15663343$ $\mathrm{H} \quad 6.32973190-7.39693093-31.19115373$

C $\quad 7.64203998-15.95684337-16.33518483$ H $\quad 7.07557932-16.35254175-17.16337478$ C $\quad 8.05328423-16.77143694-15.29657248$ $\mathrm{H} \quad 7.84081715-17.83086140-15.31300614$ C $\quad 8.75835749-16.17361678-14.25706736$ 
H $\quad 9.13806210-16.75275487-13.44135991$

C $\quad 8.98540777-14.80742960-14.28438494$

$\mathrm{H} \quad 9.56433152-14.33783913-13.50038976$

$\mathrm{N} \quad 8.51242270-13.99043779-15.24272096$

C $\quad 7.90240108-14.60637538-16.26586258$

C $\quad 7.41565807-13.75770558-17.34362203$

C $\quad 7.54813520-14.17184235-18.67550402$

C $\quad 7.01666499-13.36187750-19.67011070$

C $\quad 6.38632232-12.16670547-19.36138427$

$\mathrm{H} \quad 5.97958017-11.56248490-20.14319321$

F $\quad 7.08852127-13.74690264-20.96608018$

H $\quad 8.08334488-15.08238797-18.91340512$

C $\quad 6.78499382-12.57019578-17.00900378$

$\mathrm{H} \quad 6.65170410-12.33511794-15.96739313$

C $\quad 6.23109319-11.79494338-18.01440709$

C $\quad 5.42568280-10.59895420-17.65483434$

O $\quad 5.67610766-9.85744310-16.70857147$

$\mathrm{N} \quad 4.32962863-10.33202623-18.46892061$

$\mathrm{H} \quad 3.94407126-11.01818728-19.12399327$

$\mathrm{N} \quad 3.41268009-9.30802572-18.18560305$

$\mathrm{H} \quad 3.47759669-9.05389800-17.19802990$

S $\quad 3.58389557-7.91994044-19.09890586$

O $\quad 4.97428237-7.57461518-19.41224764$

O $\quad 2.78334698-6.86285749-18.45838153$

C $\quad 2.75231356-8.36984986-20.70842111$

C $\quad 1.59005901-7.68877240-21.10584664$

$\mathrm{H} \quad 1.09925330-7.00923570-20.42353182$

C $\quad 1.02234547-7.96153775-22.35648635$

$\mathrm{H} \quad 0.08074956-7.51493280-22.63818210$

C $\quad 1.61161910-8.89047005-23.20946753$

$\mathrm{H} \quad 1.15837920-9.13437017-24.16627078$

C $\quad 2.79233421-9.52557972-22.85363897$

$\mathrm{H} \quad 3.28686398-10.21845518-23.52640328$

C $\quad 3.34331792-9.27232915-21.60084474$

F $\quad 4.51493068-9.90438495-21.27942875$

$\mathrm{Zn} \quad 28.07230211-6.23863472-6.91176801$

O $\quad-2.02926157-5.78114103-23.88547456$

$\mathrm{H} \quad-2.87411954-5.34636510-23.65829683$

$\mathrm{H} \quad-2.03688759-6.53875609-23.30051002$

O $\quad 7.30982453-19.76597207-13.49803681$

$\mathrm{H} \quad 8.19338840-19.43788431-13.24998837$

$\mathrm{H} \quad 7.27623060-20.62997438-13.07204287$

O $\quad 9.13910144-17.86134361-18.99294155$

$\mathrm{H} \quad 9.88288929-17.28788272-18.71466321$

$\mathrm{H} \quad 9.50008309-18.34921432-19.73754749$

O $\quad 5.97571714-7.04440088-16.78762548$

$\mathrm{H} \quad 6.41721261-6.77013845-15.96143335$

$\mathrm{H} \quad 6.20839505-7.98044699-16.86168025$

O $\quad 26.46596446-0.95621527-4.84736431$

$\mathrm{H} \quad 27.13195739-0.86675617-4.15259835$

$\mathrm{H} \quad 26.59955286-1.84471639-5.18853373$

O $\quad 0.72643947-9.98511228-18.10446721$

$\mathrm{H} \quad-0.04964111-10.47356391-18.45822506$

$\mathrm{H} \quad 1.44127990-10.26944985-18.67871309$

O $\quad 4.77996249-18.65505289-14.46165944$

$\mathrm{H} \quad 5.69295239-18.95023299-14.29038808$

$\mathrm{H} \quad 4.27769995-19.18079025-13.83123476$

O $\quad-0.39396870-6.77788977-25.72226987$

$\mathrm{H} \quad-0.85427526-7.39842631-26.29750220$

$\mathrm{H} \quad-1.09708092-6.40096866-25.16396787$

1C QM/SE/MM R1b (B3LYP/6-

31G(d):xTB:Amber) mechanical embedding

$\omega_{\alpha}=\mathbf{1 . 0}$
C

O

$\mathrm{N}$

C

C

O

C

$\mathrm{S}$

$\mathrm{H}$

$\mathrm{H}$

$\mathrm{H}$

$\mathrm{H}$

$\mathrm{N}$

$\mathrm{H}$

C

O

$\mathrm{N}$

C

C

O

C

S

$\mathrm{H}$

$\mathrm{H}$

$\mathrm{H}$

$\mathrm{H}$

$\mathrm{N}$

$\mathrm{H}$

C

O

$\mathrm{N}$

C

C

O

C

C

$\mathrm{H}$

$\mathrm{H}$

$\mathrm{H}$

$\mathrm{H}$

C

$\mathrm{H}$

$\mathrm{H}$

C

$\mathrm{N}$

$\mathrm{N}$

$\mathrm{H}$

$\mathrm{N}$

$\mathrm{H}$

C

$\mathrm{O}$

$\mathrm{N}$

C

C

O

C

S

$\mathrm{H}$

$\mathrm{H}$

$\mathrm{H}$

$\mathrm{H}$

$\mathrm{N}$

$\mathrm{N}$

C

$\mathrm{H}$
$22.27484563-8.92226598-4.43701792$ $21.41898472-8.05412142-4.32981081$ $23.54490886-8.68977876-4.81724373$ $23.90497039-7.40225217-5.40690613$ $23.07432975-7.24325915-6.69717227$ $23.13578968-8.10267385-7.58070980$ $25.39039227-7.37905919-5.84903155$ $25.83087033-5.72561906-6.51896265$ $24.10611457-9.49243194-5.08961625$ $23.70013683-6.60603809-4.69039038$ $25.54964914-8.15480546-6.60033714$ $26.01258191-7.62465751-4.98145484$ $22.34659427-6.11679007-6.81293970$ $22.16348303-5.56719052-5.97725226$ $25.22419259-5.94081586-10.77059107$ $25.26245552-6.14288986-11.98457208$ $25.98744748-6.62061454-9.84687074$ $26.82314846-7.71684737-10.30136369$ $26.20781588-9.11701122-10.09205231$ $26.73237025-10.13484507-10.55818589$ $28.24087133-7.63563001-9.73058277$ $28.27386602-8.15001739-7.91298948$ $25.92791166-6.37156873-8.85560390$ $26.88983669-7.60443740-11.38693262$ $28.64584047-6.63005567-9.85113885$ $28.87947837-8.33372439-10.27496178$ $25.06542222-9.15793989-9.35987174$ $24.60023019-8.32974708-9.01586894$ $30.09957728-4.472532651 .29410590$ $30.69758479-3.610222270 .69277879$ $29.51142437-5.524657240 .65899723$ $29.56975929-5.60871973-0.80797718$ $29.06326230-4.31812808-1.44803172$ $29.78857327-3.72665374-2.22669439$ $28.83544843-6.85206758-1.33207342$ $28.93683844-6.96747271-2.81522515$ $29.25983452-7.73813842-0.83950145$ $29.01847050-6.242575321 .18112364$ $30.61992502-5.65371920-1.11078122$ $27.78021439-6.81587064-1.04690303$ $28.14607735-6.48913481-3.83195190$ $30.89642888-7.79297044-2.98684830$ $27.22490503-5.92998668-3.78734207$ $29.81277837-7.49604652-4.79902130$ $29.99912396-7.63573302-3.44956499$ $28.68462521-6.85940272-5.01801270$ $30.46205236-7.88567542-5.56921301$ $27.83944663-3.85833922-1.07199100$ $27.27116835-4.37697892-0.40390902$ $32.41852766-1.93865759-3.92514332$ $33.11555639-1.37701699-4.76125953$ $31.35495812-2.70315290-4.23901115$ $30.99693344-3.04312847-5.62527266$ $30.84501959-1.73116174-6.40876463$ $30.09822587-0.85070778-6.04908317$ $29.69412668-3.84623710-5.59608986$ $29.21630693-4.40597635-7.29410108$ $30.86238817-3.17166571-3.49505593$ $31.79072673-3.66225461-6.05856899$ $28.91429454-3.21893424-5.16314277$ $29.85516378-4.70255034-4.93678029$ $31.59025076-1.59516201-7.51311181$ $32.08605064-2.38990395-7.85735911$ $7.68142283-15.97792447-16.32942998$ $7.15826662-16.37298690-17.19119503$ 
C $\quad 8.08071707-16.79025954-15.28073365$

H $\quad 7.87317228-17.85203503-15.29812842$

C $\quad 8.76398552-16.19630507-14.21112000$

H $\quad 9.13112636-16.79884199-13.39273539$

C $\quad 8.98628502-14.82443997-14.24352691$

$\mathrm{H} \quad 9.54323093-14.33359934-13.44925336$

$\mathrm{N} \quad 8.51825819-14.00026621-15.21494348$

C $\quad 7.90572311-14.60649633-16.26009979$

C $\quad 7.40856549-13.75168837-17.34770666$

C $\quad 7.49772254-14.19550789-18.70017988$

C $\quad 6.97860420-13.35107346-19.68998706$

C $\quad 6.37130981-12.13927307-19.41831128$

$\mathrm{H} \quad 6.00833917-11.54408945-20.23772479$

F $\quad 7.05904083-13.73564954-20.99990650$

$\mathrm{H} \quad 8.01541241-15.12784299-18.92717909$

C $\quad 6.84243681-12.52328253-17.03971404$

$\mathrm{H} \quad 6.80622865-12.23929870-15.99238810$

C $\quad 6.26117880-11.73280922-18.04209496$

C $\quad 5.46124603-10.59075302-17.66979585$

O $\quad 5.65278610-9.83206485-16.66988328$

$\mathrm{N} \quad 4.39420576-10.34681112-18.53799961$

$\mathrm{H} \quad 4.00149754-11.09781362-19.09932164$

$\mathrm{N} \quad 3.46918520-9.36836875-18.22665610$

$\mathrm{H} \quad 3.46756529-9.17500617-17.22256724$

S $\quad 3.61584822-7.95335808-19.09423347$

O $\quad 5.00063123-7.52216306-19.42904790$

O $\quad 2.83529196-6.92679238-18.37139492$

C $\quad 2.79581191-8.38546127-20.64394101$

C $\quad 1.63077178-7.65113350-21.04193172$

$\mathrm{H} \quad 1.17172651-6.95461790-20.34728747$

C $\quad 1.05409854-7.88832103-22.28954473$

$\mathrm{H} \quad 0.13024008-7.38010742-22.56028449$

C $\quad 1.60624917-8.83580306-23.16857177$

H $\quad 1.14153477-9.05665763-24.12262422$

C $\quad 2.78046751-9.52452238-22.81507938$

$\mathrm{H} \quad 3.22261847-10.24668955-23.49146426$

C $\quad 3.36006454-9.29732601-21.57434268$

F $\quad 4.52861288-9.96530307-21.28016985$

Zn $\quad 28.07209169-6.24260600-6.91164462$

\section{C-QM/SE/MM R1b (2C-B3LYP/6-}

31G(d):xTB:Amber) mechanical embedding $\omega_{\alpha}=\mathbf{1 . 0}$

C $\quad 22.27368070-8.92165207-4.43761382$

O $\quad 21.41696811-8.05327573-4.33124994$

$\mathrm{N} \quad 23.54393240-8.68959135-4.81701209$

C $\quad 23.90400734-7.40169816-5.40623619$

C $\quad 23.07301050-7.24438028-6.69830865$

O $\quad 23.13382398-8.10272164-7.58264334$

C $\quad 25.39389884-7.37628187-5.84057692$

S $\quad 25.83082000-5.72400942-6.51830370$

$\mathrm{H} \quad 24.10531162-9.49063061-5.09443742$

$\mathrm{H} \quad 23.69665804-6.60302907-4.68847966$

$\mathrm{H} \quad 25.57355933-8.16092871-6.58322181$

$\mathrm{H} \quad 26.00949983-7.60905463-4.96176661$

$\mathrm{N} \quad 22.34554624-6.11615779-6.81128456$

$\mathrm{H} \quad 22.14539900-5.58245449-5.97022543$

C $\quad 25.22682718-5.93874075-10.76857827$

$\mathrm{O} \quad 25.26683441-6.13806164-11.98331988$

$\mathrm{N} \quad 25.98810115-6.61976039-9.84406238$

C $\quad 26.82404637-7.71505654-10.29967156$

C $\quad 26.20688779-9.11612376-10.09144317$

O $\quad 26.72907727-10.13484061-10.55837716$

C $\quad 28.24409949-7.62953863-9.73040358$
$28.27466540-8.14957189-7.91097628$ $25.93313152-6.37121826-8.85207215$ $26.88516531-7.60047230-11.38746127$ $28.64784905-6.62031570-9.84589060$ $28.88864589-8.32398376-10.27593806$ $25.06417517-9.15659231-9.35856702$ $24.59457749-8.32960501-9.01713533$ $30.09921506-4.474137601 .29410880$ $30.69465478-3.611622370 .69073819$ $29.51154596-5.526970810 .66026147$ $29.56710440-5.61212210-0.80616548$ $29.06020590-4.32215233-1.44597827$ $29.78986158-3.73274886-2.22259449$ $28.83152352-6.85646307-1.33130659$ $28.93028074-6.96264399-2.81559709$ $29.25441886-7.74965919-0.84583792$ $29.01589003-6.244866651 .17910821$ $30.61943999-5.64555556-1.11006753$ $27.77264716-6.82394094-1.04706935$ $28.13799251-6.47412173-3.82707334$ $30.88473102-7.80260904-2.99757175$ $27.22217490-5.90085119-3.77769048$ $29.79431103-7.49216062-4.80507478$ $29.98703664-7.63600748-3.45631310$ $28.66996622-6.84695020-5.01513673$ $30.43368768-7.88643162-5.58453326$ $27.83697533-3.85952123-1.07140162$ $27.26639620-4.37530780-0.40301778$ $32.41949790-1.93727423-3.92579588$ $33.12451484-1.38827063-4.76423363$ $31.34822821-2.69033237-4.23620559$ $30.99301093-3.03884911-5.61980011$ $30.84002922-1.72649399-6.40459657$ $30.08533152-0.85161591-6.05024783$ $29.68885829-3.84537729-5.58825941$ $29.21643361-4.40699311-7.29024595$ $30.83881989-3.13962094-3.49248959$ $31.79294271-3.65935224-6.04757112$ $28.90367070-3.21607316-5.15844518$ $29.84212945-4.70015397-4.92148669$ $31.58266722-1.58985911-7.50913841$ $32.16296089-2.34410941-7.78816349$ $7.67863735-15.97937419-16.33488336$ $7.15240696-16.36820728-17.19918602$ $8.07162905-16.79161749-15.28549548$ $7.85632065-17.85223449-15.30219147$ $8.75850729-16.19672703-14.21748425$ $9.12510546-16.80253639-13.40244181$ $8.98566573-14.82586293-14.24652251$ $9.54641624-14.33566960-13.45415776$ $8.51702700-13.99830788-15.21637914$ $7.90904930-14.60910133-16.26044981$ $7.41317040-13.75352576-17.34780672$ $7.50933005-14.20161667-18.69880500$ $6.98301611-13.35901220-19.68643607$ $6.37411307-12.14821694-19.41086110$ $6.00888142-11.55193250-20.22741729$ $7.05911373-13.74302388-20.99650371$ $8.03794106-15.13042558-18.92488290$ $6.84353462-12.52807859-17.03438045$ $6.80618865-12.24694388-15.98540864$ $6.25990810-11.73781801-18.03525816$ $5.45903360-10.59690637-17.66229366$ $5.64769546-9.84208290-16.65631435$

$4.40014507-10.33473838-18.54482226$ 


$\begin{array}{ll}\mathrm{H} & 3.98451177-11.07898311-19.09937240 \\ \mathrm{~N} & 3.47438723-9.36054049-18.22211886 \\ \mathrm{H} & 3.47357044-9.15702153-17.21994070 \\ \mathrm{~S} & 3.61339690-7.94908075-19.09359746 \\ \mathrm{O} & 5.00356632-7.53383510-19.42822362 \\ \mathrm{O} & 2.83506433-6.91516566-18.37493222 \\ \mathrm{C} & 2.78968321-8.38454020-20.64311304 \\ \mathrm{C} & 1.61918481-7.65066250-21.04412188 \\ \mathrm{H} & 1.15302239-6.95940487-20.34517801 \\ \mathrm{C} & 1.04585814-7.89819566-22.29265661 \\ \mathrm{H} & 0.11217349-7.40808687-22.55975726 \\ \mathrm{C} & 1.60336023-8.84388374-23.17215741 \\ \mathrm{H} & 1.13774077-9.07865392-24.12318948 \\ \mathrm{C} & 2.78252168-9.52574593-22.82030818 \\ \mathrm{H} & 3.22854572-10.25037734-23.49535565 \\ \mathrm{C} & 3.35755949-9.29388560-21.57714533 \\ \mathrm{~F} & 4.52742866-9.96335344-21.28379828 \\ \mathrm{Zn} & 28.07141853-6.24244516-6.91290544\end{array}$

2C-WF-in-DFT/SE/MM R1b (2C-CCSD-inB3LYP/6-31G(d):xTB:Amber) mechanical embedding $\omega_{\alpha}=1.0$

C $\quad 22.27396529-8.91579513-4.43615126$

O $\quad 21.41993970-8.05462177-4.33226126$

$\mathrm{N} \quad 23.54223392-8.68827411-4.81908027$

C $\quad 23.90313792-7.40639765-5.40643779$

C $\quad 23.07119189-7.24609028-6.70070267$

O $\quad 23.13218084-8.10093772-7.58113311$

C $\quad 25.39219778-7.36381633-5.84596673$

$\mathrm{S} \quad 25.81817685-5.71904104-6.51740637$

$\mathrm{H} \quad 24.10704260-9.49106793-5.09263927$

H $\quad 23.69299142-6.60212614-4.68796419$

$\mathrm{H} \quad 25.57060854-8.16255439-6.58297151$

$\mathrm{H} \quad 26.00702729-7.60973454-4.96082454$

$\mathrm{N} \quad 22.34593472-6.12072428-6.80941120$

$\mathrm{H} \quad 22.15103104-5.57994425-5.96850357$

C $\quad 25.23119697-5.94355012-10.77121905$

O $\quad 25.26861222-6.13840508-11.97900333$

$\mathrm{N} \quad 25.98963730-6.62207639-9.84479619$

C $\quad 26.82449292-7.71506512-10.29852429$

C $\quad 26.20699735-9.11935080-10.09808161$

O $\quad 26.72402520-10.13401213-10.56340576$

C $\quad 28.24172191-7.63693303-9.71701669$

S $\quad 28.27863884-8.17498789-7.92208727$

$\mathrm{H} \quad 25.93431840-6.37669650-8.84823436$

$\mathrm{H} \quad 26.88662068-7.60052306-11.38975779$

$\mathrm{H} \quad 28.64784102-6.62258589-9.84994263$

$\mathrm{H} \quad 28.88824418-8.32471195-10.28632865$

$\mathrm{N} \quad 25.06798478-9.15681486-9.36042736$

$\mathrm{H} \quad 24.59538062-8.32851024-9.01606038$

C $\quad 30.10168118-4.472389151 .28927478$

$\mathrm{O} \quad 30.69297711-3.613792720 .69115380$

$\mathrm{N} \quad 29.51243366-5.524688690 .66046565$

C $\quad 29.56517554-5.61210681-0.80327339$

C $\quad 29.06327553-4.32061502-1.44692485$

O $\quad 29.78914639-3.73355295-2.22226033$

C $\quad 28.83340472-6.85355057-1.33027916$

C $\quad 28.93122854-6.96442270-2.81226441$

$\mathrm{H} \quad 29.25702140-7.74931446-0.84223885$

$\mathrm{H} \quad 29.01588771-6.244163261 .18142116$

$\mathrm{H} \quad 30.62195135-5.64573947-1.10935669$

H $\quad 27.77249240-6.82241034-1.04490592$

C $\quad 28.14185655-6.48050661-3.82626422$

$\mathrm{H} \quad 30.88799923-7.80305556-2.99400091$
$27.22202176-5.90256733-3.77554347$ $29.78251315-7.49114526-4.80318541$ $29.99159450-7.63760017-3.45221268$ $28.67977642-6.85822602-5.01019078$ $30.43986801-7.88771738-5.58084559$ $27.84070860-3.86052545-1.07198706$ $27.26782623-4.37775236-0.40221448$ $32.41899065-1.93696776-3.93114870$ $33.12068244-1.38972389-4.76358393$ $31.35034014-2.69210826-4.23683933$ $30.99252089-3.03460162-5.61460953$ $30.84254172-1.72058282-6.40172699$ $30.09211990-0.84869046-6.04774297$ $29.69040275-3.84647670-5.60586797$ $29.22745773-4.38693556-7.30164601$ $30.83639091-3.14286090-3.49123041$ $31.79608518-3.65709915-6.04854482$ $28.90564585-3.21311004-5.15785655$ $29.84445907-4.69813618-4.92446938$ $31.58045666-1.59059679-7.50701043$ $32.15701228-2.35002717-7.79106107$ $7.67867217-15.97919319-16.33439409$ $7.15247750-16.36853476-17.19828696$ $8.07217037-16.79141610-15.28507132$ $7.85745591-17.85211588-15.30177800$ $8.75886359-16.19662585-14.21701337$ $9.12547972-16.80214073-13.40174406$ $8.98575494-14.82579486-14.24643575$ $9.54626801-14.33554536-13.45393648$ $8.51733272-13.99835861-15.21635069$ $7.90878761-14.60885432-16.26044575$ $7.41289304-13.75340574-17.34773311$ $7.50850915-14.20119394-18.69882192$ $6.98265702-13.35857099-19.68655668$ $6.37387718-12.14775832-19.41126487$ $6.00860998-11.55149571-20.22780616$ $7.05897898-13.74274998-20.99658487$ $8.03634031-15.13011134-18.92494950$ $6.84335285-12.52788386-17.03467159$ $6.80556807-12.24674656-15.98585740$ $6.25970535-11.73773554-18.03557685$ $5.45898667-10.59662901-17.66295030$ $5.64815838-9.84137693-16.65735407$ $4.39937296-10.33579212-18.54400178$ $3.98560844-11.08018971-19.09940531$ $3.47377556-9.36091792-18.22233415$ $3.47328667-9.15782983-17.21993646$ $3.61337087-7.94938326-19.09371472$ $5.00350456-7.53316343-19.42831348$ $2.83480678-6.91596679-18.37479094$ $2.79004699-8.38447061-20.64333362$ $1.61990285-7.65085381-21.04414940$ $1.15422934-6.95923247-20.34562412$ $1.04632735-7.89769568-22.29250265$ $0.11329384-7.40639273-22.55990031$ $1.60341773-8.84354453-23.17187450$ $1.13782040-9.07731761-24.12309250$ $2.78230056-9.52573077-22.81991493$ $3.22797895-10.25016925-23.49502585$ $3.35760708-9.29416663-21.57703060$ $4.52753550-9.96330267-21.28346445$ $28.07196175-6.23909730-6.91200497$ 
1C QM/SE/MM R3b (B3L YP/6-

31G(d):xTB:Amber) mechanical embedding $\omega_{\alpha}=\mathbf{1 . 0}$

C $\quad 21.94416514-10.35261693-4.21816128$ C $\quad 22.26071489-8.88920541-4.43779550$

O $\quad 21.41083976-8.01620802-4.34259202$

$\mathrm{N} \quad 23.53584780-8.67829995-4.81431451$

C $\quad 23.90099292-7.39893842-5.41448999$

C $\quad 23.08534360-7.24524264-6.71445028$

O $\quad 23.13658553-8.11712469-7.58656283$

C $\quad 25.39084292-7.36225324-5.81101427$

S $\quad 25.83764933-5.72826370-6.51510108$

$\mathrm{H} \quad 24.12571997-9.48599447-4.99853877$

$\mathrm{H} \quad 23.67905432-6.59897804-4.70932510$

$\mathrm{H} \quad 25.59837035-8.16664108-6.51797637$

$\mathrm{H} \quad 25.97470463-7.55913953-4.90544281$

$\mathrm{N} \quad 22.38148138-6.10770756-6.85118091$

C $\quad 21.48689612-5.96761924-7.97307391$

C $\quad 22.18167626-5.66010338-9.31513788$

O $\quad 21.57673340-5.77860537-10.36249617$

C $\quad 20.50050654-4.87452871-7.65170459$

$\mathrm{H} \quad 20.93229387-6.90031430-8.12039820$

$\mathrm{H} \quad 22.22509151-5.52813091-6.03027809$

$\mathrm{N} \quad 23.44359343-5.21493372-9.16998857$

C $\quad 24.28829663-4.81512780-10.25805120$

C $\quad 25.21703868-5.90678445-10.76393505$

O $\quad 25.24195960-6.12672118-11.97577039$

C $\quad 25.11123739-3.59831527-9.90777648$

C $\quad 24.26838608-2.44907773-9.44899110$

C $\quad 23.31303790-1.87737387-10.27872950$

C $\quad 24.41028737-1.95378694-8.14162399$

C $\quad 22.54852042-0.80191896-9.84470836$

C $\quad 23.62942740-0.90803791-7.69910536$

C $\quad 22.70993588-0.30965249-8.55451527$

O $\quad 22.010308340 .79211650-8.11842525$

$\mathrm{H} \quad 23.62055280-4.57670905-11.09251544$

$\mathrm{H} \quad 21.80461271-0.37675031-10.49497873$

$\mathrm{H} \quad 23.73369742-0.51974835-6.70462145$

$\mathrm{H} \quad 23.86851326-5.31187114-8.25124219$

$\mathrm{H} \quad 25.13093861-2.39168094-7.47757187$

$\mathrm{H} \quad 23.14894010-2.26364128-11.27147420$

$\mathrm{H} \quad 25.67521234-3.29165740-10.78547422$

$\mathrm{H} \quad 25.82013031-3.84296215-9.11953259$

$\mathrm{H} \quad 22.419040251 .60825154-8.42984172$

$\mathrm{N} \quad 25.98174544-6.59331801-9.84701475$

C $\quad 26.81302542-7.69099092-10.29884087$

C $\quad 26.20034712-9.08913154-10.09371071$

O $\quad 26.73777594-10.10051391-10.55859026$

C $\quad 28.22453503-7.61498973-9.72607987$

S $\quad 28.28193298-8.15977070-7.92063083$

$\mathrm{H} \quad 25.94347398-6.33894295-8.85643805$

$\mathrm{H} \quad 26.89546295-7.57739807-11.38263677$

$\mathrm{H} \quad 28.61523625-6.60508596-9.83395364$

$\mathrm{H} \quad 28.86096867-8.29658913-10.28912722$

$\mathrm{N} \quad 25.05032114-9.13254206-9.37557122$

C $\quad 24.37960893-10.40388945-9.16040546$

C $\quad 25.16883817-11.37688193-8.29657269$

O $\quad 24.81557501-12.53644510-8.12962428$

C $\quad 23.90964308-11.09779220-10.45255441$

C $\quad 22.95770750-10.21054785-11.26414640$

C $\quad 22.48698184-10.96782309-12.51019487$

C $\quad 21.73250131-9.81153663-10.44768668$

$\mathrm{H} \quad 22.02885190-9.21541068-9.59700765$

$\mathrm{H} \quad 21.05832958-9.23117714-11.05455428$
$21.23092762-10.70511417-10.09319338$ $24.62470683-8.30783927-8.97353446$ $21.96464523-11.86837487-12.20760675$ $21.82612686-10.34923802-13.09639917$ $23.33588225-11.25797319-13.11766990$ $23.47636152-10.17483268-8.59117769$ $23.48309310-9.30993737-11.56952251$ $23.40051521-12.01109863-10.16654832$ $24.77373427-11.33934928-11.05788040$ $26.21475961-10.85134807-7.61446505$ $26.87063960-11.71757796-6.65170885$ $25.83542793-12.10959873-5.55199594$ $25.17801757-11.26091414-4.98936815$ $28.03714739-10.98954936-5.99254861$ $28.64761825-11.80193498-4.86293985$ $29.93731687-11.14157101-4.33810026$ $30.34082281-11.82796460-3.02927889$ $31.78320760-11.67005112-2.76712677$ $27.21819058-12.63143948-7.14585561$ $29.78571326-11.40044961-2.20097550$ $30.12298168-12.88870252-3.09975282$ $27.93284475-11.86078959-4.04662358$ $28.87421848-12.80984132-5.19044305$ $32.11903095-12.30198089-2.03414603$ $32.33752726-11.88844179-3.61852133$ $32.00006291-10.70454869-2.47397740$ $29.77576922-10.08266825-4.19056797$ $30.70940965-11.28289519-5.08685552$ $26.61210259-9.94909475-7.85333318$ $27.71010797-10.02488641-5.62453578$ $28.79864331-10.80852302-6.74710936$ $25.77611996-13.41325879-5.19777715$ $24.90341771-13.85114353-4.12701567$ $26.18725482-14.13881398-5.76914757$ $29.87682768-4.493766162 .80922188$ $30.09523653-4.490545721 .27488652$ $30.69142113-3.621545070 .68139302$ $29.50827727-5.541097190 .64497406$ $29.57945665-5.61449261-0.81419357$ $29.06826642-4.32476653-1.44347067$ $29.76991488-3.73626113-2.24726420$ $28.84605339-6.84563102-1.34977178$ $28.95498973-6.96628674-2.82325956$ $29.26386566-7.73458082-0.86785594$ $28.99034152-6.238485361 .17189153$ $30.63255432-5.65544784-1.10332827$ $27.79140927-6.79428107-1.08040040$ $28.16673056-6.49319003-3.83786068$ $30.88962979-7.85968319-2.98438729$ $27.25500561-5.92571381-3.77585080$ $29.82643807-7.50761447-4.79266215$ $30.00933560-7.63695180-3.44903277$ $28.70528023-6.86797341-5.02304471$ $30.49560424-7.89329980-5.54129473$ $27.84999969-3.87853084-1.04295391$ $27.27872556-2.63885795-1.55432205$ $27.31617141-4.37640973-0.33264901$ $32.65840262-1.85421683-2.42773560$ $32.41313859-1.96264564-3.94614449$ $33.11993469-1.40268568-4.77490916$ $31.35666227-2.72858941-4.26438189$ $30.99820427-3.04963741-5.65330601$ $30.84076969-1.73029035-6.42525968$ $30.08569115-0.86591516-6.04065106$ $29.69075505-3.84482105-5.62091529$ 
$29.18837348-4.40094742-7.30323411$ $30.82909485-3.14911354-3.51449919$ $31.78482146-3.67070589-6.09183488$ $28.91938518-3.21256087-5.18088205$ $29.84812387-4.70170363-4.96552151$ $31.53702755-1.57061511-7.56011587$ $31.54567556-0.38677655-8.35728411$ $30.66169023-0.52230526-9.60864128$ $30.325236240 .43557695-10.27922829$ $33.00291971-0.09742282-8.72657759$ $31.154351010 .44698665-7.77449429$ $32.15906389-2.29331801-7.86056679$ $30.23931661-1.77112926-9.86257942$ $29.20032572-1.98953948-10.86734508$ $27.95857758-1.16915794-10.51378969$ $27.43874760-1.13333211-9.42227445$ $28.80055258-3.46253081-10.97790258$ $29.85894909-4.32026251-11.58201637$ $31.20827381-4.20697601-11.43143809$ $29.64449301-5.45591389-12.42927709$ $31.85288029-5.19909515-12.12055526$ $30.92618172-5.99383859-12.72587102$ $28.51277479-6.11611276-12.91255330$ $31.08254425-7.18212301-13.43166139$ $28.68049008-7.31426402-13.57223295$ $29.94113912-7.84184038-13.84343055$ $30.02584914-8.75014996-14.40581640$ $29.60073253-1.63663645-11.82332954$ $32.84911080-5.30182327-12.21984769$ $27.53031901-5.70966222-12.76126951$ $27.81484902-7.85189151-13.89010921$ $32.06682884-7.54653699-13.67005643$ $31.76355554-3.47229288-10.89039388$ $30.37073642-2.49125713-9.16238162$ $27.91283526-3.54274064-11.60051340$ $28.55882894-3.84208685-9.98605058$ $27.43456223-0.49428917-11.55505081$ $26.127763500 .14944088-11.47965451$ $27.94636062-0.36596350-12.42666464$ $14.00102427-19.32361722-8.43946266$ $12.57086379-19.03375222-8.87936662$ $11.65373970-19.13543199-8.09851162$ $12.45480875-18.59606202-10.15418916$ $11.17467821-18.22307915-10.69388591$ $10.79306062-18.99211344-11.93768886$ $9.74284395-18.74667457-12.51488348$ $11.05592577-16.71419381-10.93228129$ $11.56903994-15.94886478-9.74201507$ $10.80967507-15.87225506-8.57067215$ $12.80459675-15.31073281-9.79774297$ $11.29908396-15.17110473-7.47959382$ $13.28895524-14.62198143-8.70049391$ $12.53428418-14.55155806-7.53315228$ $12.92814809-14.03566532-6.67935758$ $10.44404019-18.51601011-9.93163483$ $10.71339238-15.13191210-6.58939359$ $14.25837994-14.16091135-8.75867322$ $13.26897179-18.49187870-10.75178404$ $13.39629713-15.38529421-10.69489700$ $9.85484069-16.36268944-8.52225683$ $10.01471836-16.46525042-11.09732117$ $11.63548217-16.43357130-11.80569243$ $11.60270781-19.97717547-12.31846233$ $11.30209310-20.74346825-13.49728747$ $11.33014847-22.20918932-13.13169615$
$12.23063782-22.69855162-12.48927594$ $12.29473972-20.45053732-14.63671527$ $12.24882566-18.99792906-15.08005069$ $13.35436803-18.77065872-16.09986494$ $10.88882842-18.65660920-15.67688519$ $10.12238166-18.76104387-14.92750958$ $10.89266962-17.64348814-16.04263703$ $10.68180230-19.33194184-16.50039053$ $12.45890548-20.18516861-11.82619282$ $13.21250273-19.41553882-16.95961214$ $13.33915376-17.74753041-16.44327882$ $14.32517494-18.97918524-15.67315904$ $10.30278740-20.46181153-13.82484951$ $12.40684506-18.34139989-14.22818994$ $12.04955182-21.08616340-15.47977310$ $13.29404857-20.70463583-14.29928188$ $10.26624290-22.88598107-13.58605268$ $10.13968128-24.32346701-13.37334067$ $9.61879838-22.48794845-14.25478569$ $14.10025550-18.55351859-21.17853964$ $13.89447449-17.05801493-21.07059621$ $14.72290199-16.27891518-21.45045060$ $12.72473014-16.69524626-20.51798590$ $12.42222716-15.31191571-20.25816412$ $11.80289965-15.22117058-18.84563994$ $10.95775601-16.03266697-18.45813238$ $11.50559347-14.71696732-21.34436692$ $11.22824522-13.25933340-21.05656051$ $10.22704904-15.53949556-21.47558278$ $10.56308765-12.54306346-22.20811262$ $13.37138300-14.77840603-20.27242445$ $9.62726080-15.14545851-22.27923311$ $10.47397941-16.56832744-21.69477303$ $12.05641226-14.78463249-22.27979971$ $10.58315443-13.19120669-20.17924602$ $12.15847591-12.75457441-20.81756525$ $9.60545626-12.99229191-22.43238648$ $12.08700653-17.40445357-20.19020067$ $10.39813192-11.50893475-21.94846986$ $9.66512732-15.47707697-20.55499638$ $11.18952340-12.58996971-23.08944370$ $12.20753003-14.20386366-18.08084530$ $11.97046486-14.16725727-16.62140094$ $11.95311630-12.70717134-16.25449733$ $12.90942296-12.00044721-16.51840231$ $13.03439532-14.89910365-15.82181370$ $12.65746085-15.11913182-14.31220636$ $12.77826829-13.58182024-13.36664149$ $14.54789284-13.23895672-13.56730824$ $11.62258510-15.43831941-14.24861036$ $14.87445985-12.65788914-12.71946407$ $15.08675155-14.17939200-13.58962132$ $14.71622803-12.68923116-14.48342315$ $13.22961999-15.87042702-16.26208988$ $13.31091633-15.87527203-13.88854719$ $12.93245773-13.59022811-18.45637654$ $11.00080133-14.64101211-16.46340943$ $13.95026089-14.31744579-15.85553717$ $10.79417509-12.26217867-15.74093722$ $10.67215653-10.91291731-15.21304592$ $10.55151459-11.06625152-13.67389432$ $9.84760820-11.92291135-13.16562585$ $9.41818042-10.21891501-15.73149322$ $9.40896015-10.11250635-17.26858735$ $9.24661033-8.84143211-15.10187453$ 
$11.56928748-10.35833646-15.46671801$

$\mathrm{H} \quad 8.34703136-8.37297942-15.47337533$

$\mathrm{H} \quad 9.16130587-8.93641955-14.02704591$

$\mathrm{H} \quad 8.56512717-10.83464268-15.44944298$

$\mathrm{H} \quad 9.53529253-11.10184298-17.68938017$

$\mathrm{H} \quad 8.44384118-9.72281084-17.56455398$

$\mathrm{H} \quad 11.47686808-9.59040706-17.45863871$

$\mathrm{H} \quad 9.99506813-12.88599545-15.59122266$

$\mathrm{H} \quad 10.50343942-9.12919525-18.82735567$

$\mathrm{H} \quad 10.09575132-8.20836034-15.31495008$

$\mathrm{H} \quad 10.40836939-8.19451050-17.35643999$

$\mathrm{N} \quad 11.34530817-10.29017166-12.92588479$

C $\quad 11.28671741-10.39946683-11.45181386$

$\mathrm{H} \quad 11.90852496-9.54030044-13.31542609$

C $\quad 7.90878795-6.08280570-12.13535706$

C $\quad 6.89151246-6.76307365-13.06318957$

O $\quad 6.61539858-6.31823093-14.15473594$

$\mathrm{N} \quad 6.45743708-7.95794318-12.65074849$

C $\quad 5.62180309-8.75758284-13.49641934$

C $\quad 4.31258448-7.97185515-13.75015328$

O $\quad 3.90904653-7.16769478-12.92524255$

C $\quad 5.35017308-10.12130312-12.90139135$

C $\quad 6.60729018-10.98680821-12.91568633$

C $\quad 6.35180130-12.31149331-12.27744241$

$\mathrm{H} \quad 6.89721869-11.17048070-13.95164132$

$\mathrm{H} \quad 6.13153227-8.87655318-14.45840138$

$\mathrm{H} \quad 7.43979701-10.49723044-12.43756095$

$\mathrm{H} \quad 6.67593681-8.26817686-11.71306440$

$\mathrm{H} \quad 4.57640785-10.61281761-13.48372425$

$\mathrm{H} \quad 4.98735546-10.02896983-11.88171025$

$\mathrm{H} \quad 8.28306124-12.86960350-12.60753080$

O $\quad 5.23868642-12.59320428-11.84955049$

$\mathrm{N} \quad 7.42240066-13.10962108-12.13109829$

$\mathrm{H} \quad 7.30069057-14.06488681-11.82137888$

$\mathrm{N} \quad 3.76616996-8.24866261-14.96271131$

C $\quad 2.46542115-7.76257954-15.38551005$

C $\quad 2.44430105-6.23286998-15.43312940$

O $\quad 1.43317258-5.61529671-15.16701179$

C $\quad 1.35415505-8.28526295-14.45607318$

C $\quad 1.27222629-9.81107126-14.44579298$

C $\quad 0.09063728-10.24546326-13.58624824$

$\mathrm{N} \quad 0.02545683-11.65451385-13.30018962$

C $\quad-0.65019318-12.54473032-14.07298937$

$\mathrm{N} \quad-1.61600154-12.10112267-14.94458937$

$\mathrm{N} \quad-0.38793908-13.89831427-13.94852557$

$\mathrm{H} \quad 2.17999248-10.23632357-14.02744533$

$\mathrm{H} \quad 2.23061714-8.12144691-16.38470276$

$\mathrm{H} \quad 0.77681987-12.03140069-12.72420779$

H $\quad 1.15127498-10.17486339-15.46260395$

$\mathrm{H} \quad-1.15800346-14.51071000-14.17984231$

$\mathrm{H} \quad 0.02267816-14.15566862-13.05765291$

$\mathrm{H} \quad 4.31357593-8.88536706-15.54479297$

$\mathrm{H} \quad-0.84123127-9.94861566-14.05854687$

$\mathrm{H} \quad 0.14217094-9.73552621-12.62695616$

$\mathrm{H} \quad-1.97294865-12.81433403-15.55863609$

$\mathrm{H} \quad-1.40599629-11.22927429-15.40335557$

$\mathrm{H} \quad 0.41415757-7.86943390-14.80027378$

$\mathrm{H} \quad 1.53821462-7.92366935-13.44941940$

$\mathrm{N} \quad 3.58241844-5.63911737-15.82578829$

C $\quad 3.68764672-4.19256674-15.85779640$

C $\quad 4.27563919-3.65758995-17.16508583$

O $\quad 4.56122384-2.48465105-17.31414803$

C $\quad 4.48546253-3.66383402-14.66961242$

C $\quad 3.65502060-3.53933414-13.40178678$
C $\quad 4.52462052-3.78858140-12.17999240$

$\mathrm{N} \quad 3.87927590-3.34987194-10.95967432$

C $\quad 3.43017021-4.16301325-9.98298895$

$\mathrm{N} \quad 3.19460005-5.47696437-10.22966524$

$\mathrm{N} \quad 3.07530246-3.63970973-8.77065203$

$\mathrm{H} \quad 2.83931092-4.25282564-13.40872789$

$\mathrm{H} \quad 2.66920219-3.80422628-15.77049665$

$\mathrm{H} \quad 3.86226935-2.36016203-10.79070962$

$\mathrm{H} \quad 3.22967839-2.54029153-13.33540808$

$\mathrm{H} \quad 2.55920910-4.26312135-8.17308578$

$\mathrm{H} \quad 2.77608885-2.68091308-8.76198997$

$\mathrm{H} \quad 4.40698347-6.19902933-16.02409964$

$\mathrm{H} \quad 5.46732161-3.25203834-12.27371256$

$\mathrm{H} \quad 4.74853084-4.84973498-12.12232577$

H $\quad 3.22016468-6.11742506-9.43732642$

$\mathrm{H} \quad 3.75192491-5.87349756-10.97072753$

$\mathrm{H} \quad 4.89951796-2.69627220-14.93415659$

$\mathrm{H} \quad 5.30789184-4.35067762-14.48199465$

$\mathrm{N} \quad 4.29077109-4.53507782-18.19071981$

C $\quad 4.77293413-4.13031152-19.48399559$

C $\quad 6.23716861-4.48862468-19.84153826$

O $\quad 6.58685641-4.42510662-21.02170064$

$\mathrm{H} \quad 4.02519932-5.50247891-18.03677376$

$\mathrm{H} \quad 4.15123582-4.58160195-20.25582490$

$\mathrm{H} \quad 4.70906843-3.03955448-19.53097147$

$\mathrm{N} \quad 7.01570205-4.85002164-18.81723020$

C $\quad 8.42221803-5.17549305-18.98092442$

C $\quad 8.58933845-6.48392155-19.75105229$

O $\quad 9.38814798-6.55310072-20.66852388$

C $\quad 9.15790014-5.24983330-17.61250583$

C $\quad 9.09209091-3.94525145-16.86800083$

C $\quad 9.94701820-2.88851005-17.12354422$

C $\quad 8.15968745-3.79810362-15.85417950$

C $\quad 9.86389979-1.71214168-16.40398768$

C $\quad 8.06793722-2.63906023-15.10723280$

C $\quad 8.93935303-1.58414241-15.35890918$

O $\quad 8.78189030-0.45830886-14.62611000$

$\mathrm{H} \quad 8.89430477-4.39269582-19.57980544$

$\mathrm{H} \quad 10.55265362-0.90352800-16.58327229$

$\mathrm{H} \quad 7.35099988-2.54677191-14.31764170$

$\mathrm{H} \quad 6.62911053-4.88862855-17.88219023$

$\mathrm{H} \quad 7.50783622-4.61827644-15.61420555$

$\mathrm{H} \quad 10.70547764-2.96377315-17.87825308$

H $\quad 10.18714711-5.48038831-17.84341914$

$\mathrm{H} \quad 8.73084860-6.04614512-17.01641854$

$\mathrm{H} \quad 9.618082040 .11578504-14.74186616$

$\mathrm{N} \quad 7.79426493-7.50171014-19.35857608$

C $\quad 7.78876280-8.74901484-20.07337577$

C $\quad 7.40133679-8.55784617-21.58939319$

O $\quad 7.95629938-9.19796800-22.45821892$

$\mathrm{H} \quad 7.07501787-7.33173648-18.66637872$

$\mathrm{H} \quad 8.78107998-9.19835779-20.08220958$

$\mathrm{H} \quad 7.07605451-9.43221596-19.62341709$

$\mathrm{N} \quad 6.49292565-7.57756890-21.80797136$

C $\quad 5.96238227-7.33964999-23.13093185$

C $\quad 7.06832790-6.70650677-23.98847120$

O $\quad 7.18484231-6.95507756-25.17722096$

C $\quad 4.74760961-6.41807764-23.08188082$

C $\quad 4.14119704-6.08244773-24.46109326$

C $\quad 2.91232985-5.20901163-24.22269032$

$\mathrm{N} \quad 2.24154770-4.82013364-25.43867405$

C $\quad 1.13566186-4.06384648-25.48216687$

$\mathrm{N} \quad 0.62240544-3.61319615-24.28665453$

$\mathrm{N} \quad 0.69498939-3.56894812-26.65445824$

$\mathrm{H} \quad 3.83051208-6.98520044-24.97634379$ 
C

C

C

C
$3.82236839-15.70442366-16.16348368$ $3.76743201-14.17805283-16.24924207$ $2.34479159-13.67483816-16.46885104$ $4.31695395-13.61963979-14.93390516$ $5.32681904-13.96385009-14.76475720$ $4.30638574-12.53980959-14.95383119$ $3.70003883-13.95359095-14.10676778$ $3.86149074-18.23197045-16.31651046$ $1.70578912-14.02495831-15.66388368$ $2.34084545-12.59177096-16.45420700$ $1.95732074-14.01087050-17.41558015$ $2.02717161-16.19475216-17.23313955$ $4.39969900-13.82374374-17.06142952$ $4.86788531-16.00171774-16.16732685$ $3.40088788-15.99740772-15.20381277$ $4.65331397-16.75811746-19.07529664$ $5.14081833-16.55191352-20.42666116$ $4.06373559-16.92918748-21.46504040$ $3.84738252-16.18122422-22.39496599$ $5.12460683-17.44972928-18.49489130$ $5.40173107-15.50715601-20.58894700$ $6.02418998-17.16805578-20.57928031$ $3.37894375-18.06689106-21.27562164$ $2.28365537-18.49269403-22.16593579$ $3.64672840-18.71814475-20.53959648$ $-0.21963856-15.81823294-21.26327502$ $0.31586415-14.59420910-21.98947795$ $-0.42742893-13.98657651-22.73936014$ $1.57378143-14.27168435-21.68694709$ $2.18654335-13.05824726-22.17232024$ $2.47319884-13.15646192-23.70041587$ $2.30827300-12.18480990-24.40633287$ $3.52075168-12.79152672-21.41319092$ $3.30779368-12.56078568-20.00815926$ $2.07532900-14.79334367-20.97616518$ $1.51678725-12.20712161-22.02717120$ $3.99633420-11.91764419-21.83162024$ $4.17295248-13.65185087-21.55580246$ $3.23654018-13.42526309-19.57256267$ $2.82152352-14.34765328-24.16961373$ $3.07087121-14.52027273-25.60544992$ $3.17288384-15.08133853-23.56498613$ $-0.57173096-15.19394068-26.42670318$ $-1.14641716-13.78620054-26.55929904$ $-1.63187446-13.37868458-27.58724239$ $-1.00416204-13.04718648-25.46400264$ $-1.46029470-11.68031034-25.51900846$ $-0.65376577-10.84050726-26.52148449$ $-1.18074703-10.01404756-27.24252926$ $-1.41118900-11.11772464-24.10332768$ $-0.64173484-13.40307508-24.59383880$ $-2.49868790-11.64731031-25.86522403$ $0.68619252-11.04596111-26.50531434$ $1.54972693-10.22137778-27.33815857$ $1.19139312-10.46974610-28.83524618$ $0.99013575-9.55755162-29.59320714$ $3.05222819-10.48751555-27.08852364$ $3.93241514-9.89532115-28.17029461$ $4.27646274-8.55439829-28.13641619$ $4.33623391-10.66534959-29.24910176$ $5.08885097-8.02065026-29.11400145$ $5.15934468-10.15561791-30.23440493$ $5.56572054-8.83078380-30.14459663$ $6.50182274-8.34918773-30.99100873$ $1.34011366-9.16214811-27.16962399$ 
$\mathrm{H} \quad 27.12649775-0.79373425-4.14208290$ $\mathrm{H} \quad 26.64600107-1.80073857-5.22945851$ O $\quad 0.70283604-10.03131645-18.06300518$ $\mathrm{H} \quad-0.08250492-10.50519701-18.44050155$ $\mathrm{H} \quad 1.44742354-10.33450873-18.58418951$ O $\quad 4.77033485-18.66309689-14.46998383$ $\mathrm{H} \quad 5.69536407-18.87939529-14.25900118$ $\mathrm{H} \quad 4.26498891-19.24786803-13.89201617$ O $\quad-0.46308964-6.67797317-25.79993359$ $\mathrm{H} \quad-0.79028920-7.40779136-26.33371945$ $\mathrm{H} \quad-1.20934373-6.33574736-25.29513499$

\section{C-QM/SE/MM R3b (2C-B3LYP/6-}

31G(d):xTB:Amber) mechanical embedding $\omega_{\alpha}=\mathbf{1 . 0}$

C $\quad 21.94390771-10.35124677-4.21908860$ C $\quad 22.25724949-8.88641206-4.44180042$

O $\quad 21.40868871-8.01345226-4.33936867$

$\mathrm{N} \quad 23.53329510-8.67654103-4.81976788$

C $\quad 23.90467719-7.39492302-5.41642651$

C $\quad 23.08228484-7.24424569-6.71390902$

O $\quad 23.13214844-8.12415848-7.57956273$

C $\quad 25.39363080-7.37457272-5.84052430$

S $\quad 25.84049047-5.72249151-6.51141229$

$\mathrm{H} \quad 24.08604120-9.48599487-5.08781023$

$\mathrm{H} \quad 23.69450355-6.59083825-4.70522521$

$\mathrm{H} \quad 25.57153536-8.15533657-6.58853715$

$\mathrm{H} \quad 26.00376537-7.61708505-4.96003485$

N $\quad 22.37796008-6.10818512-6.85715880$

C $\quad 21.48524681-5.96974610-7.97973904$

C $\quad 22.17976631-5.65683209-9.32189195$

O $\quad 21.57696604-5.78789014-10.36854098$

C $\quad 20.50094242-4.87559248-7.65208298$

H $\quad 20.93282852-6.90477868-8.12942899$

$\mathrm{H} \quad 22.20474722-5.53077650-6.03888159$

$\mathrm{N} \quad 23.43311681-5.18654954-9.17473004$

C $\quad 24.28650873-4.79723095-10.25788022$

C $\quad 25.21510294-5.89094892-10.74750588$

O $\quad 25.24041825-6.10518202-11.96021519$

C $\quad 25.12202426-3.58488685-9.91105789$

C $\quad 24.26812898-2.44117035-9.45060685$

C $\quad 23.31330663-1.86907281-10.28775364$

C $\quad 24.39663092-1.96829497-8.12843942$

C $\quad 22.54607094-0.79231071-9.84954610$

C $\quad 23.60969759-0.92511190-7.68265498$

C $\quad 22.70588934-0.31181303-8.55071813$

O $\quad 22.014866670 .79800463-8.11338559$

$\mathrm{H} \quad 23.62772507-4.57628751-11.10916000$

$\mathrm{H} \quad 21.80606901-0.34720968-10.50387199$

H $\quad 23.68718593-0.54584178-6.67453116$

$\mathrm{H} \quad 23.86858931-5.28712374-8.26362185$

$\mathrm{H} \quad 25.10600206-2.42562493-7.45440272$

$\mathrm{H} \quad 23.15504887-2.24641057-11.29295023$

$\mathrm{H} \quad 25.69140401-3.28656587-10.79564044$

$\mathrm{H} \quad 25.83997968-3.82865014-9.12107586$

$\mathrm{H} \quad 22.430621871 .61124619-8.42394499$

$\mathrm{N} \quad 25.97832031-6.58086386-9.83336792$

C $\quad 26.80809055-7.67722199-10.29459706$

C $\quad 26.19173037-9.07924514-10.08962173$

O $\quad 26.72748374-10.09204785-10.55554538$

C $\quad 28.23133524-7.60606216-9.73278382$

S $\quad 28.28534491-8.15668511-7.92001098$

$\mathrm{H} \quad 25.94317571-6.32881401-8.84095120$

$\mathrm{H} \quad 26.87022422-7.55666177-11.38121841$ 

$29.70102503-3.85302094-5.61147407$ $29.18143555-4.39739296-7.29859069$ $30.86994602-3.21389547-3.54178109$ $31.79501433-3.68891756-6.12138130$ $28.93727019-3.21815965-5.15179910$ $29.86594398-4.71258161-4.95340880$ $31.53344893-1.56453591-7.57497502$ $31.53862658-0.37384652-8.36014447$ $30.64848283-0.50931952-9.61019616$ $30.288412350 .44998658-10.26647686$ $33.00116496-0.09666683-8.72622501$ $31.149195750 .46025568-7.77334407$ $32.16928693-2.26627131-7.89514539$ $30.25155833-1.76502824-9.88325361$ $29.20074295-1.98060545-10.87848904$ $27.96501260-1.15319134-10.51417050$ $27.44661302-1.11596464-9.42127166$ $28.78494994-3.45403843-10.97192741$ $29.85004475-4.30908235-11.57592247$ $31.20357535-4.19326744-11.42961918$ $29.63066398-5.44435666-12.42785296$ $31.84162993-5.18899543-12.12337323$ $30.91296971-5.98875461-12.72306182$ $28.49329222-6.10242795-12.91424624$ $31.06960169-7.19406489-13.40807529$ $28.66144089-7.31623410-13.55298557$ $29.92347677-7.86260708-13.80673609$ $30.00280855-8.80289165-14.33544596$ $29.59936981-1.63970169-11.84502812$ $32.83806538-5.28860810-12.23244198$ $27.50573947-5.68270035-12.78088281$ $27.79164925-7.86295326-13.86682033$ $32.05897175-7.57295090-13.63641528$ $31.78273993-3.46463529-10.89087340$ $30.35053076-2.47929634-9.17329255$ $27.88732413-3.53936860-11.59149429$ $28.53742862-3.82168192-9.97050226$ $27.43329702-0.48847580-11.55783252$ $26.126629310 .15081626-11.47975879$ $27.94854619-0.33439128-12.42271711$ $14.00097850-19.32302807-8.44015270$ $12.57232933-19.02596705-8.88090465$ $11.65540515-19.12213985-8.09873782$ $12.45735796-18.58381746-10.15408560$ $11.17933680-18.21180342-10.69937137$ $10.80261630-18.99676583-11.93425429$ $9.75162959-18.75520849-12.51285939$ $11.05620074-16.70167500-10.96121715$ $11.57016937-15.94078523-9.75992738$ $10.80737599-15.87562567-8.58384729$ $12.81336130-15.30760400-9.80932555$ $11.30160420-15.18192652-7.48415025$

C $\quad 13.30176700-14.62816163-8.70237235$ 


\begin{tabular}{|c|c|c|c|}
\hline $\mathrm{H}$ & $12.94206901-14.04950589-6.66668819$ & $\mathrm{H}$ & $13.26333436-15.88568557-16.27953172$ \\
\hline $\mathrm{H}$ & $10.44678955-18.49794064-9.93227392$ & $\mathrm{H}$ & $13.37760771-15.86195484-13.85712582$ \\
\hline $\mathrm{H}$ & $10.71458239-15.14526737-6.58784430$ & $\mathrm{H}$ & $12.92198579-13.59009816-18.41458830$ \\
\hline $\mathrm{H}$ & $14.27960329-14.16822077-8.75457548$ & $\mathrm{H}$ & $10.99495991-14.67305864-16.43427021$ \\
\hline $\mathrm{H}$ & $13.26525220-18.47491021-10.75756704$ & $\mathrm{H}$ & $13.95797525-14.31167565-15.86697504$ \\
\hline $\mathrm{H}$ & $13.41005211-15.37991632-10.71214736$ & $\mathrm{~N}$ & $10.79768093-12.27079149-15.73149149$ \\
\hline $\mathrm{H}$ & $9.84362851-16.36597581-8.54072884$ & $\mathrm{C}$ & $10.68566967-10.91257232-15.22310521$ \\
\hline $\mathrm{H}$ & $10.00921806-16.45781012-11.14301763$ & $\mathrm{C}$ & $10.55967932-11.06417416-13.67803731$ \\
\hline $\mathrm{H}$ & $11.63852133-16.41985552-11.84353534$ & $\mathrm{O}$ & $9.86128897-11.92835864-13.17281164$ \\
\hline $\mathrm{N}$ & $11.60813388-19.98666494-12.31414263$ & $\mathrm{C}$ & $9.41970007-10.23028436-15.74037299$ \\
\hline $\mathrm{C}$ & $11.29635075-20.75239659-13.48952826$ & $\mathrm{C}$ & $9.38745287-10.14378561-17.28777616$ \\
\hline $\mathrm{C}$ & $11.32692183-22.21484264-13.11973138$ & $\mathrm{C}$ & $9.25339429-8.83732227-15.12249534$ \\
\hline $\mathrm{O}$ & $12.22595720-22.70226336-12.47396894$ & $\mathrm{C}$ & $10.48710734-9.21234838-17.78480152$ \\
\hline $\mathrm{C}$ & $12.29164376-20.47203044-14.63584014$ & $\mathrm{H}$ & $11.58493368-10.36075962-15.49831160$ \\
\hline $\mathrm{C}$ & $12.23494404-19.01307502-15.08257023$ & $\mathrm{H}$ & $8.35497037-8.36074631-15.50344310$ \\
\hline $\mathrm{C}$ & $13.34104668-18.80470463-16.11470261$ & $\mathrm{H}$ & $9.16255954-8.91360116-14.03980198$ \\
\hline $\mathrm{C}$ & $10.86360299-18.66641542-15.67743566$ & $\mathrm{H}$ & $8.57469090-10.85687877-15.43067751$ \\
\hline $\mathrm{H}$ & $10.09046000-18.75728697-14.92343202$ & $\mathrm{H}$ & $9.50983577-11.14277862-17.70985437$ \\
\hline $\mathrm{H}$ & $10.86069909-17.65265008-16.06294202$ & $\mathrm{H}$ & $8.40587251-9.77182778-17.58077367$ \\
\hline $\mathrm{H}$ & $10.64357915-19.35362041-16.49548317$ & $\mathrm{H}$ & $11.46169611-9.60094889-17.49652939$ \\
\hline $\mathrm{H}$ & $12.47155092-20.21094204-11.84465323$ & $\mathrm{H}$ & $9.99182944-12.87139281-15.53848795$ \\
\hline $\mathrm{H}$ & $13.18869832-19.46427501-16.96834076$ & $\mathrm{H}$ & $10.48114712-9.14236170-18.86968213$ \\
\hline $\mathrm{H}$ & $13.33765210-17.78359169-16.47965224$ & $\mathrm{H}$ & $10.10685948-8.20026480-15.33875131$ \\
\hline $\mathrm{H}$ & $14.31942728-19.01989800-15.69339765$ & $\mathrm{H}$ & $10.37975470-8.20465744-17.39754598$ \\
\hline $\mathrm{H}$ & $10.29022846-20.46563774-13.80516371$ & $\mathrm{~N}$ & $11.34612728-10.28454389-12.92615580$ \\
\hline $\mathrm{H}$ & $12.40184931-18.34899735-14.22944639$ & $\mathrm{C}$ & $11.28664197-10.39876111-11.45214966$ \\
\hline $\mathrm{H}$ & $12.04961267-21.11986092-15.47921988$ & $\mathrm{H}$ & $11.88761726-9.51390169-13.30644488$ \\
\hline $\mathrm{H}$ & $13.29930356-20.73665662-14.30131111$ & $\mathrm{C}$ & $7.90771684-6.08429564-12.13543070$ \\
\hline $\mathrm{N}$ & $10.26608604-22.89083398-13.58214432$ & $\mathrm{C}$ & $6.88798574-6.76805873-13.06091813$ \\
\hline $\mathrm{C}$ & $10.13928017-24.32581054-13.37274059$ & $\mathrm{O}$ & $6.60922130-6.32036888-14.15079060$ \\
\hline $\mathrm{H}$ & $9.62570638-22.49882261-14.26028309$ & $\mathrm{~N}$ & $6.45840698-7.96692333-12.65283964$ \\
\hline $\mathrm{C}$ & $14.09906616-18.55215873-21.17842093$ & $\mathrm{C}$ & $5.61855757-8.76536068-13.49543678$ \\
\hline $\mathrm{C}$ & $13.89082662-17.05614810-21.06878383$ & $\mathrm{C}$ & $4.31720587-7.96135670-13.74877759$ \\
\hline $\mathrm{O}$ & $14.71880605-16.27719752-21.45179009$ & $\mathrm{O}$ & $3.91880877-7.15587257-12.92285602$ \\
\hline $\mathrm{N}$ & $12.72360144-16.68855822-20.51406520$ & $\mathrm{C}$ & $5.34085609-10.12618739-12.89184279$ \\
\hline $\mathrm{C}$ & $12.42298848-15.30529929-20.25515519$ & $\mathrm{C}$ & $6.61558529-10.97516138-12.90217799$ \\
\hline $\mathrm{C}$ & $11.79751951-15.22692970-18.83906598$ & $\mathrm{C}$ & $6.35507474-12.30743806-12.28281536$ \\
\hline $\mathrm{O}$ & $10.96174317-16.04862531-18.44915920$ & $\mathrm{H}$ & $6.92771131-11.14703238-13.93798372$ \\
\hline $\mathrm{C}$ & $11.50870205-14.71877728-21.35216926$ & $\mathrm{H}$ & $6.13213791-8.89815023-14.45745263$ \\
\hline $\mathrm{C}$ & $11.22617751-13.25747248-21.05766824$ & $\mathrm{H}$ & $7.44714838-10.49228201-12.40597834$ \\
\hline $\mathrm{C}$ & $10.22725851-15.55332333-21.49212405$ & $\mathrm{H}$ & $6.65945596-8.28298476-11.71416462$ \\
\hline $\mathrm{C}$ & $10.55525263-12.54477974-22.21568943$ & $\mathrm{H}$ & $4.56895833-10.63060103-13.47595024$ \\
\hline $\mathrm{H}$ & $13.37540672-14.76670556-20.26569787$ & $\mathrm{H}$ & $4.95987440-10.03421259-11.87154080$ \\
\hline $\mathrm{H}$ & $9.62050258-15.16187155-22.30121277$ & $\mathrm{H}$ & $8.27290271-12.89209902-12.64891613$ \\
\hline $\mathrm{H}$ & $10.47095464-16.59049879-21.71257269$ & $\mathrm{O}$ & $5.24158693-12.58403702-11.85403794$ \\
\hline $\mathrm{H}$ & $12.07865281-14.78417651-22.28503428$ & $\mathrm{~N}$ & $7.41981352-13.11809387-12.15102752$ \\
\hline $\mathrm{H}$ & $10.58110415-13.19626906-20.17214875$ & $\mathrm{H}$ & $7.27720188-14.08686084-11.89909985$ \\
\hline $\mathrm{H}$ & $12.15849158-12.74393838-20.81146087$ & $\mathrm{~N}$ & $3.77915294-8.23188295-14.96552035$ \\
\hline $\mathrm{H}$ & $9.59479487-12.99911411-22.44321140$ & $\mathrm{C}$ & $2.48011087-7.75076206-15.39568309$ \\
\hline $\mathrm{H}$ & $12.08107602-17.37883082-20.15940379$ & $\mathrm{C}$ & $2.45940905-6.22217874-15.44060796$ \\
\hline $\mathrm{H}$ & $10.38250627-11.50264835-21.96802553$ & $\mathrm{O}$ & $1.44429324-5.60925970-15.17707772$ \\
\hline $\mathrm{H}$ & $9.65624912-15.49944562-20.57085427$ & $\mathrm{C}$ & $1.36685711-8.27404025-14.46122551$ \\
\hline $\mathrm{H}$ & $11.18214591-12.59136736-23.10344208$ & $\mathrm{C}$ & $1.29508280-9.80718312-14.45571721$ \\
\hline $\mathrm{N}$ & $12.18627262-14.20638871-18.06953286$ & $\mathrm{C}$ & $0.11529482-10.23251765-13.57590310$ \\
\hline $\mathrm{C}$ & $11.96449944-14.19202493-16.60316825$ & $\mathrm{~N}$ & $0.03500031-11.64561676-13.29540985$ \\
\hline $\mathrm{C}$ & $11.95240776-12.72956052-16.24038390$ & $\mathrm{C}$ & $-0.63612271-12.53204315-14.08404669$ \\
\hline $\mathrm{O}$ & $12.91058411-12.02824709-16.51392098$ & $\mathrm{~N}$ & $-1.60932526-12.08830600-14.95415799$ \\
\hline $\mathrm{C}$ & $13.05194655-14.91737779-15.82389947$ & $\mathrm{~N}$ & $-0.36172569-13.88803906-13.96847684$ \\
\hline $\mathrm{C}$ & $12.69035692-15.14066086-14.30198277$ & $\mathrm{H}$ & $2.21865784-10.23693122-14.05182102$ \\
\hline $\mathrm{S}$ & $12.77114117-13.58437995-13.37477342$ & $\mathrm{H}$ & $2.25416201-8.10652760-16.40210019$ \\
\hline $\mathrm{C}$ & $14.53454399-13.19595809-13.56456652$ & $\mathrm{H}$ & $0.81788944-12.01994608-12.75719614$ \\
\hline $\mathrm{H}$ & $11.66364311-15.49184863-14.22078975$ & $\mathrm{H}$ & $1.17264955-10.17049789-15.48080079$ \\
\hline $\mathrm{H}$ & $14.84627500-12.60063934-12.71203370$ & $\mathrm{H}$ & $-1.11139509-14.52160083-14.21392267$ \\
\hline $\mathrm{H}$ & $15.09053163-14.13008830-13.57836769$ & $\mathrm{H}$ & $0.05981567-14.15491171-13.08455048$ \\
\hline $\mathrm{H}$ & $14.69215952-12.64344937-14.48631678$ & $\mathrm{H}$ & $4.32191333-8.87449211-15.54274274$ \\
\hline
\end{tabular}




\begin{tabular}{|c|c|c|c|}
\hline $\mathrm{H}$ & $-0.82969046-9.92275171-14.02574371$ & $\mathrm{~N}$ & $6.51242778-7.57498095-21.79261947$ \\
\hline $\mathrm{H}$ & $0.19110667-9.72591267-12.60708739$ & $\mathrm{C}$ & $5.97406455-7.34605453-23.11215911$ \\
\hline $\mathrm{H}$ & $-1.96112512-12.79656741-15.57672462$ & $\mathrm{C}$ & $7.07726047-6.71627888-23.98184352$ \\
\hline $\mathrm{H}$ & $-1.39390374-11.22016600-15.42089732$ & $\mathrm{O}$ & $7.17821090-6.95619412-25.17380992$ \\
\hline $\mathrm{H}$ & $0.42234504-7.85082704-14.80295747$ & $\mathrm{C}$ & $4.76600265-6.40710466-23.06342352$ \\
\hline $\mathrm{H}$ & $1.55266428-7.90512064-13.44978814$ & $\mathrm{C}$ & $4.15393994-6.10043544-24.45654439$ \\
\hline $\mathrm{N}$ & $3.59647507-5.62315620-15.82960064$ & $\mathrm{C}$ & $2.91119549-5.23529857-24.21492690$ \\
\hline $\mathrm{C}$ & $3.68817747-4.17620851-15.86815062$ & $\mathrm{~N}$ & $2.23673890-4.85891372-25.43550511$ \\
\hline $\mathrm{C}$ & $4.27262384-3.64526326-17.18117802$ & $\mathrm{C}$ & $1.12431059-4.11086511-25.48759507$ \\
\hline $\mathrm{O}$ & $4.54950326-2.47111327-17.34723148$ & $\mathrm{~N}$ & $0.60620886-3.67119189-24.28621562$ \\
\hline $\mathrm{C}$ & $4.49016342-3.62949056-14.68789762$ & $\mathrm{~N}$ & $0.67809826-3.62839904-26.66522861$ \\
\hline $\mathrm{C}$ & $3.64798014-3.49003554-13.42398622$ & $\mathrm{H}$ & $3.85531844-7.02068714-24.96577882$ \\
\hline $\mathrm{C}$ & $4.54227770-3.66866703-12.19948710$ & $\mathrm{H}$ & $5.69556883-8.30497580-23.57909825$ \\
\hline $\mathrm{N}$ & $3.89562333-3.19313339-10.99106394$ & $\mathrm{H}$ & $2.63067014-5.17257075-26.32343752$ \\
\hline $\mathrm{C}$ & $3.44341874-3.97523617-9.98871217$ & $\mathrm{H}$ & $4.87391469-5.58091559-25.08716629$ \\
\hline $\mathrm{N}$ & $3.26240462-5.31031002-10.18903350$ & $\mathrm{H}$ & $-0.27237971-3.29908488-26.76448424$ \\
\hline $\mathrm{N}$ & $3.07785042-3.41624157-8.78833997$ & $\mathrm{H}$ & $1.00049131-4.07904858-27.51664156$ \\
\hline $\mathrm{H}$ & $2.85073581-4.23435344-13.40483163$ & $\mathrm{H}$ & $5.96920973-7.28507493-20.98693380$ \\
\hline $\mathrm{H}$ & $2.65798844-3.80842398-15.78359846$ & $\mathrm{H}$ & $3.19588212-4.32788529-23.67399443$ \\
\hline $\mathrm{H}$ & $3.86357763-2.19526479-10.86704238$ & $\mathrm{H}$ & $2.21420289-5.80866743-23.58546468$ \\
\hline $\mathrm{H}$ & $3.17789382-2.50063043-13.39256367$ & $\mathrm{H}$ & $-0.26236052-3.14743774-24.38972603$ \\
\hline $\mathrm{H}$ & $2.58251211-4.03103368-8.16478716$ & $\mathrm{H}$ & $0.56240584-4.44972952-23.64429635$ \\
\hline $\mathrm{H}$ & $2.74468213-2.46671034-8.79588718$ & $\mathrm{H}$ & $5.05163164-5.46390350-22.59454356$ \\
\hline $\mathrm{H}$ & $4.44112068-6.16534396-15.98252655$ & $\mathrm{H}$ & $3.99601099-6.87172717-22.44151214$ \\
\hline $\mathrm{H}$ & $5.47764762-3.10839374-12.31707150$ & $\mathrm{~N}$ & $7.76786500-5.77830766-23.32435289$ \\
\hline $\mathrm{H}$ & $4.80064968-4.72438682-12.10314162$ & $\mathrm{C}$ & $8.91761303-5.13912649-23.93491531$ \\
\hline $\mathrm{H}$ & $3.26728768-5.92138015-9.38301156$ & $\mathrm{H}$ & $7.66439974-5.62113346-22.33032465$ \\
\hline $\mathrm{H}$ & $3.81563969-5.72309246-10.92186991$ & $\mathrm{C}$ & $11.29018670-8.07871783-23.87765334$ \\
\hline $\mathrm{H}$ & $4.91265544-2.66418218-14.98041285$ & $\mathrm{C}$ & $10.86930798-8.99625664-25.03940270$ \\
\hline $\mathrm{H}$ & $5.31881125-4.31556180-14.48611208$ & $\mathrm{O}$ & $11.67918427-9.31160333-25.88658445$ \\
\hline $\mathrm{N}$ & $4.29479873-4.52895433-18.20269478$ & $\mathrm{~N}$ & $9.57875803-9.40304812-24.97901116$ \\
\hline $\mathrm{C}$ & $4.77815655-4.11877951-19.49441291$ & $\mathrm{C}$ & $9.06573413-10.21627105-26.07492973$ \\
\hline $\mathrm{C}$ & $6.24157861-4.48390735-19.85324754$ & $\mathrm{C}$ & $9.21547189-9.41028447-27.38493926$ \\
\hline $\mathrm{O}$ & $6.58983986-4.42798096-21.03338701$ & $\mathrm{O}$ & $9.81456946-9.84315029-28.33368000$ \\
\hline $\mathrm{H}$ & $4.05580304-5.50231096-18.05044116$ & $\mathrm{C}$ & $7.61862811-10.69034381-25.84116000$ \\
\hline $\mathrm{H}$ & $4.15350253-4.56457356-20.26952352$ & $\mathrm{C}$ & $7.57344594-11.64900318-24.64652973$ \\
\hline $\mathrm{H}$ & $4.72082249-3.02475561-19.52800904$ & $\mathrm{C}$ & $7.11859415-11.38857922-27.10177936$ \\
\hline $\mathrm{N}$ & $7.01818572-4.84528532-18.82740439$ & $\mathrm{C}$ & $6.15711443-11.89226921-24.14977630$ \\
\hline $\mathrm{C}$ & $8.42670082-5.17315027-18.97130134$ & $\mathrm{H}$ & $9.71483657-11.09589427-26.19392559$ \\
\hline $\mathrm{C}$ & $8.58874419-6.48143409-19.74418857$ & $\mathrm{H}$ & $6.09699526-11.73572238-26.96184537$ \\
\hline $\mathrm{O}$ & $9.38408637-6.54567366-20.66478153$ & $\mathrm{H}$ & $7.13588328-10.72181549-27.95881739$ \\
\hline $\mathrm{C}$ & $9.14828689-5.25739637-17.58880357$ & $\mathrm{H}$ & $7.00357614-9.80742691-25.62875469$ \\
\hline $\mathrm{C}$ & $9.09285303-3.93803012-16.85746005$ & $\mathrm{H}$ & $8.04562750-12.58837202-24.93826949$ \\
\hline $\mathrm{C}$ & $9.95030922-2.88029002-17.12683906$ & $\mathrm{H}$ & $8.15585220-11.22911992-23.82375165$ \\
\hline $\mathrm{C}$ & $8.15707792-3.78350386-15.84148909$ & $\mathrm{H}$ & $5.55254005-12.33050450-24.94359184$ \\
\hline $\mathrm{C}$ & $9.86827428-1.69567300-16.41204085$ & $\mathrm{H}$ & $8.98853096-9.18966516-24.19177533$ \\
\hline $\mathrm{C}$ & $8.06554897-2.61692355-15.10036061$ & $\mathrm{H}$ & $6.16493800-12.57335042-23.30549063$ \\
\hline $\mathrm{C}$ & $8.94323906-1.56535995-15.36157040$ & $\mathrm{H}$ & $7.75225312-12.24207623-27.32150796$ \\
\hline $\mathrm{O}$ & $8.77544175-0.44119415-14.62733570$ & $\mathrm{H}$ & $5.70389655-10.95035452-23.85588968$ \\
\hline $\mathrm{H}$ & $8.91414839-4.39282504-19.56883304$ & $\mathrm{~N}$ & $8.72031964-8.16884152-27.37913515$ \\
\hline $\mathrm{H}$ & $10.55599515-0.87651148-16.59759444$ & $\mathrm{C}$ & $8.84782531-7.31826203-28.54817843$ \\
\hline $\mathrm{H}$ & $7.34873510-2.50608222-14.30328323$ & $\mathrm{H}$ & $8.20154273-7.78027460-26.60525017$ \\
\hline $\mathrm{H}$ & $6.63976449-4.86926772-17.89009255$ & $\mathrm{C}$ & $6.58566031-21.92424236-21.79283698$ \\
\hline $\mathrm{H}$ & $7.50262169-4.60638185-15.59306961$ & $\mathrm{C}$ & $6.21027400-22.04007861-20.31223566$ \\
\hline $\mathrm{H}$ & $10.71169678-2.95378199-17.88790115$ & $\mathrm{O}$ & $5.48692369-22.92357942-19.90477003$ \\
\hline $\mathrm{H}$ & $10.17721524-5.51714789-17.81367226$ & $\mathrm{~N}$ & $6.60186106-20.97550544-19.59792138$ \\
\hline $\mathrm{H}$ & $8.70261560-6.04932113-16.98499897$ & $\mathrm{C}$ & $6.12371708-20.90716527-18.21962173$ \\
\hline $\mathrm{H}$ & $9.607748550 .14271095-14.73087936$ & $\mathrm{C}$ & $4.62574494-20.54782200-18.33140017$ \\
\hline $\mathrm{N}$ & $7.79619275-7.50002422-19.35066214$ & $\mathrm{O}$ & $4.11887701-20.19128773-19.39091485$ \\
\hline $\mathrm{C}$ & $7.78532733-8.75451162-20.05369611$ & $\mathrm{C}$ & $6.84960494-19.84487565-17.39554950$ \\
\hline $\mathrm{C}$ & $7.40755986-8.56627951-21.57457389$ & $\mathrm{O}$ & $6.64893664-18.56074354-17.96202159$ \\
\hline $\mathrm{O}$ & $7.95450868-9.20727412-22.44824541$ & $\mathrm{H}$ & $7.24527226-20.29131896-19.97350759$ \\
\hline $\mathrm{H}$ & $7.06161169-7.32728857-18.67595021$ & $\mathrm{H}$ & $6.23399492-21.90137364-17.76572987$ \\
\hline $\mathrm{H}$ & $8.77173020-9.22067064-20.04674417$ & $\mathrm{H}$ & $7.91805278-20.08133665-17.33386527$ \\
\hline $\mathrm{H}$ & $7.05368737-9.41676703-19.59520726$ & $\mathrm{H}$ & $6.44250095-19.83720593-16.37746330$ \\
\hline
\end{tabular}




\begin{tabular}{|c|c|}
\hline $\mathrm{H}$ & $7.51528095-18.26962180-18.32277113$ \\
\hline $\mathrm{N}$ & $3.95425236-20.55442266-17.15653954$ \\
\hline $\mathrm{C}$ & $2.53598165-20.24220507-17.17094728$ \\
\hline $\mathrm{C}$ & $2.34409239-18.75660923-17.58519073$ \\
\hline $\mathrm{O}$ & $1.49983691-18.39727935-18.38580089$ \\
\hline $\mathrm{C}$ & $1.93127079-20.51418856-15.79220228$ \\
\hline $\mathrm{H}$ & $4.31404816-21.09083100-16.37730317$ \\
\hline $\mathrm{H}$ & $2.01195614-20.85704892-17.90843954$ \\
\hline $\mathrm{N}$ & $3.19187825-17.88634600-17.00993540$ \\
\hline $\mathrm{C}$ & $3.08479917-16.46015830-17.25162013$ \\
\hline $\mathrm{C}$ & $3.61353317-16.06287454-18.64001820$ \\
\hline $\mathrm{O}$ & $3.09675671-15.17407021-19.32382588$ \\
\hline $\mathrm{C}$ & $3.82793790-15.70606631-16.16026454$ \\
\hline $\mathrm{C}$ & $3.77189240-14.17475126-16.27523327$ \\
\hline $\mathrm{C}$ & $2.33858464-13.66961659-16.49724676$ \\
\hline $\mathrm{C}$ & $4.33282240-13.59732417-14.96480474$ \\
\hline $\mathrm{H}$ & $5.34772020-13.94432937-14.78887259$ \\
\hline $\mathrm{H}$ & $4.33444008-12.51130379-14.99368763$ \\
\hline $\mathrm{H}$ & $3.71636829-13.91870420-14.12707601$ \\
\hline $\mathrm{H}$ & $3.85992387-18.20083352-16.30752013$ \\
\hline $\mathrm{H}$ & $1.69534746-14.01514083-15.68691209$ \\
\hline $\mathrm{H}$ & $2.32814171-12.57886554-16.48612259$ \\
\hline $\mathrm{H}$ & $1.93717194-14.00389489-17.44726315$ \\
\hline $\mathrm{H}$ & $2.02042047-16.19591279-17.24235811$ \\
\hline $\mathrm{H}$ & $4.40152712-13.83391889-17.10597216$ \\
\hline $\mathrm{H}$ & $4.87752830-16.01908728-16.15108445$ \\
\hline $\mathrm{H}$ & $3.40346973-15.99335651-15.19363253$ \\
\hline $\mathrm{N}$ & $4.65498627-16.74278222-19.07981297$ \\
\hline $\mathrm{C}$ & $5.14858593-16.54549681-20.43104497$ \\
\hline $\mathrm{C}$ & $4.06718183-16.92832799-21.46520514$ \\
\hline $\mathrm{O}$ & $3.83416029-16.17163204-22.38374731$ \\
\hline $\mathrm{H}$ & $5.13071774-17.43251393-18.50329858$ \\
\hline $\mathrm{H}$ & $5.40647281-15.49869594-20.60413114$ \\
\hline $\mathrm{H}$ & $6.03706726-17.16265486-20.56747380$ \\
\hline $\mathrm{N}$ & $3.38791052-18.07037437-21.28012603$ \\
\hline $\mathrm{C}$ & $2.28564491-18.49275775-22.16526491$ \\
\hline $\mathrm{H}$ & $3.68607119-18.74557128-20.57830566$ \\
\hline $\mathrm{C}$ & $-0.21943339-15.81773670-21.26310561$ \\
\hline $\mathrm{C}$ & $0.31438779-14.59290223-21.98894983$ \\
\hline $\mathrm{O}$ & $-0.42977829-13.98976191-22.74171966$ \\
\hline $\mathrm{N}$ & $1.57039330-14.26410134-21.68726591$ \\
\hline $\mathrm{C}$ & $2.17832152-13.04840437-22.17289218$ \\
\hline $\mathrm{C}$ & $2.47263504-13.15349870-23.70162123$ \\
\hline $\mathrm{O}$ & $2.31283649-12.18080478-24.40713610$ \\
\hline $\mathrm{C}$ & $3.51481624-12.78300493-21.40477484$ \\
\hline $\mathrm{O}$ & $3.30117385-12.55403094-19.99613374$ \\
\hline $\mathrm{H}$ & $2.07637466-14.75474874-20.95903076$ \\
\hline $\mathrm{H}$ & $1.49500572-12.19906292-22.04209668$ \\
\hline $\mathrm{H}$ & $3.99636000-11.90644342-21.81840624$ \\
\hline $\mathrm{H}$ & $4.17000663-13.64655495-21.54420097$ \\
\hline $\mathrm{H}$ & $3.23796956-13.41636050-19.55409053$ \\
\hline $\mathrm{N}$ & $2.81969114-14.34528708-24.16914378$ \\
\hline $\mathrm{C}$ & $3.07041008-14.52002625-25.60474806$ \\
\hline $\mathrm{H}$ & $3.17893006-15.07694907-23.56732844$ \\
\hline $\mathrm{C}$ & $-0.57219411-15.19315392-26.42634253$ \\
\hline $\mathrm{C}$ & $-1.14576412-13.78430378-26.55894658$ \\
\hline $\mathrm{O}$ & $-1.62121737-13.37287830-27.59037257$ \\
\hline $\mathrm{N}$ & $-1.00867471-13.04384842-25.46369338$ \\
\hline $\mathrm{C}$ & $-1.46471875-11.67762252-25.52229866$ \\
\hline $\mathrm{C}$ & $-0.65284036-10.84223954-26.52472762$ \\
\hline $\mathrm{O}$ & $-1.17648657-10.01358163-27.24603502$ \\
\hline $\mathrm{C}$ & $-1.41161540-11.11828313-24.10459953$ \\
\hline $\mathrm{H}$ & $-0.68458578-13.39426060-24.57765882$ \\
\hline $\mathrm{H}$ & $-2.50488266-11.64013551-25.87076764$ \\
\hline $\mathrm{N}$ & $0.68593808-11.05519667-26.51114528$ \\
\hline
\end{tabular}

C $\quad 1.55683850-10.22248172-27.32912318$ C $\quad 1.19859355-10.46219540-28.83340862$ O $\quad 0.99436928-9.54634074-29.58597793$ C $\quad 3.06555917-10.49610999-27.07347932$ C $\quad 3.93573878-9.90007929-28.16865893$ C $\quad 4.25377930-8.54839378-28.14808389$ C $\quad 4.33880800-10.67958419-29.24660715$ C $\quad 5.06294686-8.01597658-29.13387074$ C $\quad 5.16177407-10.17282065-30.23901658$ C $\quad 5.55681470-8.84050499-30.14965321$ O $\quad 6.50713480-8.36817066-30.98656821$ $\mathrm{H} \quad 1.33685955-9.16195457-27.14987037$ $\mathrm{H} \quad 5.34326734-6.97126838-29.13664591$ $\mathrm{H} \quad 5.52426950-10.77067486-31.06564418$ $\mathrm{H} \quad 1.12848416-11.63349499-25.80923659$ $\mathrm{H} \quad 4.05200793-11.72207663-29.28438062$ $\mathrm{H} \quad 3.91778396-7.90488522-27.34533936$

$\mathrm{H} \quad 3.23478783-11.57360553-27.03534905$ $\mathrm{H} \quad 3.34904121-10.07426988-26.10775450$ $\mathrm{H} \quad 6.39579871-7.41580577-31.18793295$

$\mathrm{N} \quad 1.15156443-11.75309159-29.17748189$ C $\quad 0.80024855-12.21887625-30.50951013$ $\mathrm{H} \quad 1.39982593-12.50165952-28.54193281$ C $\quad 7.67957880-15.97905749-16.34618183$ $\mathrm{H} \quad 7.15115054-16.36793587-17.20799409$ C $\quad 8.07415855-16.79614752-15.29940524$ $\mathrm{H} \quad 7.85175255-17.85575060-15.32132884$ C $\quad 8.76521151-16.20473276-14.23281450$ $\mathrm{H} \quad 9.13381254-16.80656286-13.41552524$ C $\quad 8.99161396-14.83184249-14.26397570$ $\mathrm{H} \quad 9.56331341-14.34403263-13.47817859$ $\mathrm{N} \quad 8.52025985-14.00022696-15.22932349$ C $\quad 7.90778370-14.60789568-16.27176353$ C $\quad 7.41236634-13.75469113-17.36177216$ C $\quad 7.50530806-14.20244263-18.71022537$ C $\quad 6.98793631-13.35539296-19.69911756$ C $\quad 6.37444450-12.14582599-19.42037502$ $\mathrm{H} \quad 6.01480956-11.54671444-20.23848595$ F $\quad 7.06605592-13.73111551-21.00523787$ $\mathrm{H} \quad 8.02288273-15.13593703-18.93995746$ C $\quad 6.84011219-12.52832343-17.04840563$ $\mathrm{H} \quad 6.80113552-12.24760296-15.99976681$ C $\quad 6.25770436-11.74228826-18.04813990$ C $\quad 5.45381700-10.59512722-17.67240491$ O $\quad 5.63366028-9.85259846-16.66435302$

$\mathrm{N} \quad 4.40164902-10.33847857-18.55751495$ $\mathrm{H} \quad 3.99741582-11.08877843-19.11605980$ $\mathrm{N} \quad 3.46221593-9.37377675-18.23808551$

$\mathrm{H} \quad 3.43512867-9.18687957-17.23110224$

S $\quad 3.60420384-7.95518789-19.09578253$

O $\quad 4.99102079-7.54885754-19.43114128$

O $\quad 2.81589187-6.92735510-18.39037771$

C $\quad 2.78978884-8.38052414-20.66664439$

C $\quad 1.62752731-7.65499906-21.05960499$

$\mathrm{H} \quad 1.16484230-6.95823457-20.36491479$

C $\quad 1.05285576-7.89644272-22.31320453$

$\mathrm{H} \quad 0.13652050-7.38893629-22.59755220$

C $\quad 1.61352450-8.84198683-23.18604227$

$\mathrm{H} \quad 1.15509347-9.06019445-24.14482575$

C $\quad 2.78535921-9.52423132-22.83532445$

$\mathrm{H} \quad 3.23216384-10.25025334-23.50880295$

C $\quad 3.35815333-9.28742592-21.58616313$

F $\quad 4.52928714-9.94559305-21.28211478$

Zn $\quad 28.07416727-6.24769366-6.90846372$

O $\quad-1.95835999-5.96496652-23.75209058$ 
$\mathrm{H} \quad-2.74194756-5.45714988-23.46877009$

$\mathrm{H} \quad-2.15028290-6.86177371-23.46172743$

O $\quad 7.32156795-19.78476582-13.51828775$

$\mathrm{H} \quad 8.17191660-19.38204756-13.27923609$

$\mathrm{H} \quad 7.32976343-20.65755949-13.11203421$

O $\quad 9.17839363-17.90824242-18.98747195$

$\mathrm{H} \quad 9.77160270-17.18586457-18.71028771$

$\mathrm{H} \quad 9.73875801-18.69038830-19.00472617$

O $\quad 5.96397648-7.07740115-16.81563360$

$\mathrm{H} \quad 6.39064489-6.81513263-15.98042594$

$\mathrm{H} \quad 5.93729278-8.05257029-16.79060695$

O $\quad 26.47598059-0.91749748-4.84371469$

$\mathrm{H} \quad 27.11430053-0.76503795-4.12976886$

$\mathrm{H} \quad 26.71839120-1.74429338-5.27004538$

O $\quad 0.71482878-10.03211959-18.01746331$

$\mathrm{H} \quad-0.04178552-10.52299476-18.41678492$

$\mathrm{H} \quad 1.50057062-10.36720590-18.45210423$

O $\quad 4.77145554-18.66691849-14.46809945$

$\mathrm{H} \quad 5.69821551-18.83642923-14.22777577$

$\mathrm{H} \quad 4.28916669-19.33600368-13.96641260$

O $\quad-0.44907659-6.69329790-25.87239281$

$\mathrm{H} \quad-0.74605932-7.40127280-26.45186754$

$\mathrm{H} \quad-1.21590815-6.40309900-25.36931002$ 
C. Cartesian coordinates for Amyloid-Beta (20-34) with L-isoaspartate 23

\section{Full optimization with xTB}

$\mathrm{N} \quad-0.984843925 .8846976711 .40894590$

C $\quad-0.389819544 .7524920410 .71964109$

C $\quad 0.957098755 .2318348910 .18002412$

O $\quad 1.284146026 .4038823110 .20898657$

C $\quad-1.277410884 .095743329 .63044235$

C $\quad-1.922335845 .097634578 .71242809$

C $\quad-3.307190085 .162560288 .60970122$

C $\quad-1.154435975 .976251907 .95590419$

C $\quad-3.913853186 .078832307 .76927846$

C $\quad-1.760066686 .911983127 .13569751$

C $\quad-3.140967076 .963023997 .03666304$

$\mathrm{H} \quad-0.171905923 .9847241611 .47473864$

$\mathrm{H} \quad-0.669319443 .398899789 .04881138$

$\mathrm{H} \quad-2.058510963 .5177831610 .12921911$

$\mathrm{H} \quad-3.923765334 .482874989 .17848447$

$\mathrm{H} \quad-0.077353255 .934473128 .01084775$

$\mathrm{H} \quad-4.988421066 .089265657 .68567245$

$\mathrm{H} \quad-1.149391327 .603989406 .57253629$

$\mathrm{H} \quad-3.610582087 .687566576 .38759908$

$\mathrm{H} \quad-1.862142045 .6239183611 .84402389$

$\mathrm{H} \quad 0.155833977 .0997420712 .48430022$

$\mathrm{H} \quad-1.174500976 .6259999310 .74034203$

$\mathrm{N} \quad 1.800783954 .237250099 .81433890$

C $\quad 3.129077784 .524653269 .30440932$

C $\quad 4.004499075 .2393498610 .34863577$

O $\quad 5.062523095 .7616694610 .01972779$

C $\quad 3.075226735 .324454267 .99926757$

$\mathrm{H} \quad 1.399330293 .363699499 .50102856$

$\mathrm{H} \quad 3.611002673 .556824209 .12039062$

$\mathrm{H} \quad 4.069566835 .462689437 .58608609$

$\mathrm{H} \quad 2.448126554 .794257097 .28498817$

$\mathrm{H} \quad 2.640831246 .301504878 .19470783$

$\mathrm{N} \quad 3.545026745 .1960577111 .59531481$

C $\quad 4.170437965 .7976090912 .75458690$

C $\quad 4.863469234 .6619235313 .53628771$

O $\quad 5.501163973 .8196586312 .89467960$

C $\quad 3.126009136 .6030357313 .52948735$

C $\quad 2.563905167 .7450782012 .67054096$

C $\quad 1.373692048 .3841230413 .36826388$

O $\quad 1.480626439 .3389743914 .08980491$

O $\quad 0.186788367 .8213778613 .15155469$

$\mathrm{H} \quad 2.700936994 .6738070911 .77757903$

$\mathrm{H} \quad 4.963348336 .4530950212 .37238006$

$\mathrm{H} \quad 2.316379715 .9373904613 .84145137$

$\mathrm{H} \quad 3.574038837 .0316903714 .42601246$

$\mathrm{H} \quad 3.331978178 .5078589112 .53640625$

$\mathrm{H} \quad 2.256118047 .3747223811 .69252624$

$\mathrm{N} \quad 7.600924693 .0927190114 .44433911$

C $\quad 8.755726292 .7569175913 .63625447$

C $\quad 9.475120404 .0833977013 .37552740$

O $\quad 9.317875884 .7555861812 .36509119$

C $\quad 8.324348912 .0496087412 .34872923$

C $\quad 9.553554741 .6496329311 .53473545$

C $\quad 7.479542780 .8187111412 .66367449$

$\mathrm{H} \quad 6.732933853 .2713016313 .91795699$

$\mathrm{H} \quad 9.414472362 .1073543614 .22882762$

$\mathrm{H} \quad 7.711711912 .7528149011 .77728495$

H $\quad 9.247630891 .2293292110 .57968844$

H $\quad 10.195118522 .5068691511 .34570429$
10.131087370 .8982277912 .07082692 7.232851150 .2955832911 .74281940 8.022175440 .1376150613 .31618873 6.553035961 .1030182613 .15152192 10.298353524 .4363120114 .38385050 10.845414295 .7753304914 .47193460 11.625852226 .2279282213 .24864211 11.796409717 .3979753912 .98600685 10.058204963 .9830221515 .28341715 10.058735476 .5233647014 .63175327 11.519500005 .8086514015 .33172995 12.174853005 .2402694612 .49289468 12.808288685 .5548095211 .23413491 12.099689994 .8777341310 .06034073 12.727033444 .370187639 .11722269 14.323608405 .2644053711 .25052545 15.072908986 .2475852710 .56855178 11.989303964 .2777027112 .72659353 12.668071526 .6422985111 .11534033 14.655115585 .1842031712 .29227209 14.535029024 .3228147810 .73785283 14.937389717 .0939612111 .01192647 10.777304634 .8937917110 .10477237 10.001348524 .349873359 .01585307 10.412122045 .040072827 .70925349 10.765555166 .208311857 .66638822 8.510769654 .575347499 .25842448 7.669452103 .809565948 .25684812 8.157697222 .960711587 .50303593 6.388308204 .127730428 .23753036 10.267109435 .2060849310 .93130304 10.201285633 .274313958 .92238679 $8.255413494 .25815288 \quad 10.27161802$ 8.271899185 .637465639 .18414673 5.824884763 .734337747 .49775096 5.982330414 .824226138 .87223267 10.334899714 .241034676 .63906805 10.518663324 .682801925 .28084323 9.418004684 .244399464 .31406604 9.502622634 .594078153 .13762839 11.892670584 .210421574 .76075433 13.014052855 .048138475 .39104133 14.134378314 .217003136 .00972122 15.156984195 .101699196 .71889527 14.519183765 .816903427 .83828280 9.997809713 .299060676 .79158229 10.507442815 .780333315 .28713179 12.008751403 .156823235 .02093808 11.909606164 .291821403 .67530310 13.430370895 .729925274 .65072223 12.566870175 .643738906 .17956309 13.709322573 .525710596 .73918361 14.643038513 .633873745 .24122673 15.967905174 .481387077 .10617335 15.577503005 .829318986 .02154433 13.934040316 .663138437 .51544161 13.841164905 .172431048 .34066530 15.178182926 .137514488 .55433908 8.433977123 .468287214 .76831355 7.400070832 .993028873 .88204582 6.009087633 .480852884 .28433154 5.731175113 .859019885 .40855843 8.281264433 .282065225 .76470465 7.645387563 .332562302 .87218544 7.382950901 .895925633 .90179440 


\begin{tabular}{|c|c|}
\hline $\mathrm{N}$ & 5.112970293 .432024323 .28830183 \\
\hline $\mathrm{C}$ & 3.716554463 .807494013 .45850200 \\
\hline $\mathrm{C}$ & 2.952701542 .610995074 .06664577 \\
\hline $\mathrm{O}$ & 2.287150551 .850708523 .39198585 \\
\hline $\mathrm{C}$ & 3.124135854 .201380212 .11632967 \\
\hline $\mathrm{H}$ & 5.363188593 .031833612 .39760311 \\
\hline $\mathrm{H}$ & 3.689707384 .644465894 .16487059 \\
\hline $\mathrm{H}$ & 2.119765524 .588544812 .25785297 \\
\hline $\mathrm{H}$ & 3.734275574 .963986181 .63870810 \\
\hline $\mathrm{H}$ & 3.058046733 .326353451 .47360726 \\
\hline $\mathrm{N}$ & 3.136619382 .477304335 .38715189 \\
\hline $\mathrm{C}$ & 2.495586521 .419745136 .15366899 \\
\hline $\mathrm{C}$ & 1.139928311 .999419876 .60678581 \\
\hline $\mathrm{O}$ & 1.000757282 .542031287 .69420875 \\
\hline $\mathrm{C}$ & 3.336341710 .971522507 .35395850 \\
\hline $\mathrm{C}$ & 4.793395440 .697396286 .96711785 \\
\hline $\mathrm{C}$ & $2.67302117-0.255961237 .97585967$ \\
\hline $\mathrm{C}$ & 5.690982940 .475098958 .18064011 \\
\hline $\mathrm{H}$ & 3.684190333 .163117805 .88890490 \\
\hline $\mathrm{H}$ & 2.326167450 .584363685 .46677614 \\
\hline $\mathrm{H}$ & 3.315092371 .784057258 .08798140 \\
\hline $\mathrm{H}$ & $4.83296622-0.178286846 .31493821$ \\
\hline $\mathrm{H}$ & 5.186419681 .545134796 .40282830 \\
\hline $\mathrm{H}$ & $3.16044543-0.523937528 .90841802$ \\
\hline $\mathrm{H}$ & $1.62611331-0.053353798 .18658478$ \\
\hline $\mathrm{H}$ & $2.72741960-1.104023187 .29654726$ \\
\hline $\mathrm{H}$ & 6.734378720 .463447667 .87491311 \\
\hline $\mathrm{H}$ & 5.563512591 .277615738 .90418478 \\
\hline $\mathrm{H}$ & $5.46627440-0.468448788 .67004060$ \\
\hline $\mathrm{N}$ & 0.207338171 .977678895 .65957760 \\
\hline $\mathrm{C}$ & -1.118971152 .532568185 .84208925 \\
\hline $\mathrm{C}$ & -2.037558601 .407764996 .35785060 \\
\hline $\mathrm{O}$ & -1.855314530 .253768596 .01752281 \\
\hline $\mathrm{C}$ & -1.659996933 .079106164 .50718848 \\
\hline $\mathrm{C}$ & -0.600986063 .901232553 .75120579 \\
\hline $\mathrm{C}$ & -2.930508263 .896196104 .73258941 \\
\hline $\mathrm{C}$ & 0.006472345 .039047094 .56444927 \\
\hline $\mathrm{H}$ & 0.349012371 .419056134 .83034802 \\
\hline $\mathrm{H}$ & -1.049860573 .331433246 .58516438 \\
\hline $\mathrm{H}$ & -1.909553682 .215974863 .87796736 \\
\hline $\mathrm{H}$ & 0.196300973 .239221903 .40812729 \\
\hline $\mathrm{H}$ & -1.080049604 .317532842 .86201814 \\
\hline $\mathrm{H}$ & -3.274368274 .309470553 .78699170 \\
\hline $\mathrm{H}$ & -3.734171403 .290002525 .13874992 \\
\hline $\mathrm{H}$ & -2.753290434 .718638505 .42074599 \\
\hline $\mathrm{H}$ & 0.678223855 .629649503 .94356998 \\
\hline $\mathrm{H}$ & -0.768017445 .697739224 .94498994 \\
\hline $\mathrm{H}$ & 0.576174974 .656427025 .40737047 \\
\hline $\mathrm{N}$ & -2.980506011 .811330317 .21812583 \\
\hline $\mathrm{C}$ & -4.132386261 .002496707 .54504456 \\
\hline $\mathrm{C}$ & -5.379812321 .864455697 .25042968 \\
\hline $\mathrm{O}$ & -5.235666683 .077444637 .11576551 \\
\hline $\mathrm{H}$ & -3.200140162 .803738147 .30054224 \\
\hline $\mathrm{H}$ & -4.124318540 .091068626 .94529487 \\
\hline $\mathrm{H}$ & -4.122007220 .731510358 .60610747 \\
\hline $\mathrm{N}$ & -6.500861051 .185666877 .12040872 \\
\hline $\mathrm{C}$ & -7.827854981 .732869677 .01355595 \\
\hline $\mathrm{C}$ & -8.759106620 .655753397 .77345717 \\
\hline $\mathrm{O}$ & $-8.15418973-0.391285838 .05307536$ \\
\hline $\mathrm{C}$ & -8.321876851 .840130795 .57220770 \\
\hline $\mathrm{C}$ & -7.490701282 .786236614 .68795034 \\
\hline $\mathrm{C}$ & -6.421090602 .016840563 .91405636 \\
\hline $\mathrm{C}$ & -8.390113953 .540035863 .70904605 \\
\hline $\mathrm{O}$ & -9.921815550 .989507747 .95794274 \\
\hline $\mathrm{H}$ & -6.617433950 .203455557 .44400611 \\
\hline
\end{tabular}

$\mathrm{H} \quad-7.887157272 .706672477 .50850879$

$\mathrm{H} \quad-8.336938190 .840407815 .13054226$

$\mathrm{H} \quad-9.354635742 .188360985 .63201705$

$\mathrm{H} \quad-6.989759273 .514673045 .33289808$

$\mathrm{H} \quad-5.802710972 .699388193 .33193488$

$\mathrm{H} \quad-5.776772381 .465690474 .59340449$

$\mathrm{H} \quad-6.885755291 .306635213 .23118098$

$\mathrm{H} \quad-7.800330084 .200324223 .07404992$

$\mathrm{H} \quad-8.925974232 .838527943 .07153008$

$\mathrm{H} \quad-9.120076424 .141887334 .24555680$

$\mathrm{N} \quad 4.774153494 .6758182514 .85304836$

C $\quad 5.111603273 .5851021715 .73667994$

C $\quad 3.956202463 .6438936116 .86289552$

O $\quad 3.195222374 .6125783516 .73332937$

C $\quad 6.437131553 .7464014516 .47270046$

C $\quad 7.709374933 .4006887215 .74082524$

O $\quad 8.799641313 .3962036716 .32213674$

O $\quad 3.985695772 .7512201117 .70171926$

$\mathrm{H} \quad 4.030839635 .2081274815 .35974552$

$\mathrm{H} \quad 5.059240232 .6187440815 .22554588$

$\mathrm{H} \quad 6.393617043 .1105988317 .36362177$

$\mathrm{H} \quad 6.530783954 .7765620916 .83004093$

O $\quad 12.952539457 .832709497 .21169626$

$\mathrm{H} \quad 12.871229828 .014048076 .25229792$

$\mathrm{H} \quad 12.084545497 .492162117 .48394462$

O $\quad 1.990581793 .7122482119 .40871919$

$\mathrm{H} \quad 1.842888574 .4238337318 .77849602$

H $\quad 2.690402513 .1892227218 .97735404$

O $\quad 10.953640146 .887920472 .80436662$

$\mathrm{H} \quad 10.436738386 .061616742 .89578044$

$\mathrm{H} \quad 10.898608007 .131200081 .87936157$

O $\quad 12.606003188 .239335444 .54121025$

$\mathrm{H} \quad 12.031905467 .687920503 .97799209$

H $\quad 12.339333949 .145833644 .37842388$

\section{Quantum refinement with $\mathrm{xTB} \omega_{\alpha}=\mathbf{0 . 2 6 9}$}

$\mathrm{N} \quad-2.7141094 .03140013 .983455$

C $\quad-1.6964733 .17295113 .370232$

C $\quad-0.4151074 .00035413 .225711$

O $\quad-0.4461405 .21398913 .322587$

C $\quad-2.1675442 .62684611 .982303$

C $\quad-2.2982873 .66096910 .876195$

C $\quad-3.4920894 .34454410 .659857$

C $\quad-1.2299213 .91769810 .019607$

C $\quad-3.6078515 .2730749 .633130$

C $\quad-1.3317334 .8769429 .017382$

C $\quad-2.5285595 .5394948 .812826$

$\mathrm{H} \quad-1.5113642 .31393314 .024583$

$\mathrm{H} \quad-1.4610301 .88671611 .621684$

$\mathrm{H} \quad-3.1411802 .14981412 .122119$

$\mathrm{H} \quad-4.3430614 .14851011 .298238$

$\mathrm{H} \quad-0.3124163 .35648310 .128167$

$\mathrm{H} \quad-4.5409625 .7964879 .488366$

$\mathrm{H} \quad-0.4805005 .0985628 .390613$

$\mathrm{H} \quad-2.6154486 .2631218 .015308$

$\mathrm{H} \quad-3.5783523 .52439514 .123045$

$\mathrm{H} \quad-2.2775354 .65424015 .702124$

$\mathrm{H} \quad-2.8634364 .84196313 .391951$

$\mathrm{N} \quad 0.7223533 .29699113 .014213$

C $\quad 2.0371663 .93742512 .904603$

C $\quad 2.3273194 .79322114 .174901$

O $\quad 2.4412376 .00058714 .156127$

C $\quad 2.1054404 .80984411 .648394$ 


\begin{tabular}{|c|c|}
\hline $\mathrm{H}$ & 0.6765602 .29324912$. \\
\hline $\mathrm{H}$ & 2.7844753 .13903612 .858512 \\
\hline $\mathrm{H}$ & 3.0749545 .29742611 .598190 \\
\hline $\mathrm{H}$ & 1.9603474 .20734210 .755579 \\
\hline $\mathrm{H}$ & 1.3364515 .57350411 .703479 \\
\hline $\mathrm{N}$ & 2.4366224 .06109915 .295957 \\
\hline $\mathrm{C}$ & 2.4987294 .59802016 .650637 \\
\hline $\mathrm{C}$ & 3.6622853 .94382417 .432414 \\
\hline $\mathrm{O}$ & 3.7035582 .72184217 .495472 \\
\hline $\mathrm{C}$ & 1.2310834 .26167817 .460485 \\
\hline $\mathrm{C}$ & -0.0508814 .88529416 .908729 \\
\hline $\mathrm{C}$ & -1.2908844 .12799217 .359490 \\
\hline $\mathrm{O}$ & -1.3314673 .44047818 .346786 \\
\hline $\mathrm{O}$ & -2.3885264 .29189716 .619596 \\
\hline $\mathrm{H}$ & 2.4522443 .05132515 .263005 \\
\hline $\mathrm{H}$ & 2.6315545 .67722716 .538662 \\
\hline $\mathrm{H}$ & 1.1202633 .17551217 .489422 \\
\hline $\mathrm{H}$ & 1.3876834 .59790818 .485517 \\
\hline $\mathrm{H}$ & -0.1540135 .91463717 .262659 \\
\hline $\mathrm{H}$ & -0.0186804 .93293015 .816137 \\
\hline $\mathrm{N}$ & 7.6370814 .99710116 .017213 \\
\hline $\mathrm{C}$ & 8.1262724 .53899414 .724063 \\
\hline $\mathrm{C}$ & 9.5224375 .14803914 .494005 \\
\hline $\mathrm{O}$ & 9.6967596 .36068614 .506246 \\
\hline $\mathrm{C}$ & 7.1896774 .95411813 .558947 \\
\hline $\mathrm{C}$ & 7.7600454 .46286812 .226717 \\
\hline $\mathrm{C}$ & 5.7711434 .41931913 .756217 \\
\hline $\mathrm{H}$ & 7.5991055 .99661916 .142967 \\
\hline $\mathrm{H}$ & 8.1768453 .44618514 .783618 \\
\hline $\mathrm{H}$ & 7.1617606 .04883313 .552818 \\
\hline $\mathrm{H}$ & 7.0277404 .59640711 .433910 \\
\hline $\mathrm{H}$ & 8.6638325 .00818111 .960363 \\
\hline $\mathrm{H}$ & 8.0005413 .40206012 .282440 \\
\hline $\mathrm{H}$ & 5.1429254 .71817612 .921630 \\
\hline $\mathrm{H}$ & 5.7872913 .33183713 .807376 \\
\hline $\mathrm{H}$ & 5.3326924 .79090014 .676516 \\
\hline $\mathrm{N}$ & 10.5554164 .29346514 .317571 \\
\hline $\mathrm{C}$ & 11.9082374 .78643214 .157397 \\
\hline $\mathrm{C}$ & 12.1607435 .41261912 .766676 \\
\hline $\mathrm{O}$ & 12.3724826 .60830012 .642324 \\
\hline $\mathrm{H}$ & 10.3939063 .29939614 .328957 \\
\hline $\mathrm{H}$ & 12.0996075 .57988014 .884229 \\
\hline $\mathrm{H}$ & 12.5955713 .95347214 .313171 \\
\hline $\mathrm{N}$ & 12.1529304 .55247011 .699113 \\
\hline $\mathrm{C}$ & 12.4777374 .92544010 .342337 \\
\hline $\mathrm{C}$ & 11.5268214 .2588559 .324151 \\
\hline $\mathrm{O}$ & 11.3646023 .0458729 .289155 \\
\hline $\mathrm{C}$ & 13.9279374 .47335810 .004774 \\
\hline $\mathrm{O}$ & 14.2752144 .7029138 .656931 \\
\hline $\mathrm{H}$ & 11.9119223 .58156911 .835017 \\
\hline $\mathrm{H}$ & 12.3978516 .01593010 .270080 \\
\hline $\mathrm{H}$ & 14.6232204 .99324010 .675880 \\
\hline $\mathrm{H}$ & 14.0162613 .39578210 .155097 \\
\hline $\mathrm{H}$ & 14.3017705 .6566778 .499870 \\
\hline $\mathrm{N}$ & 10.8913095 .1070358 .468072 \\
\hline $\mathrm{C}$ & 10.0412424 .6619207 .379967 \\
\hline $\mathrm{C}$ & 10.5575325 .1589326 .033256 \\
\hline $\mathrm{O}$ & 10.6330386 .3656725 .747576 \\
\hline $\mathrm{C}$ & 8.5797685 .1428937 .566866 \\
\hline $\mathrm{C}$ & 7.7219074 .5845376 .410140 \\
\hline $\mathrm{O}$ & 7.7111273 .3940066 .121558 \\
\hline $\mathrm{N}$ & 7.0342845 .4877455 .694318 \\
\hline $\mathrm{H}$ & 11.0023756 .1041468 .566116 \\
\hline $\mathrm{H}$ & 10.0589673 .5677477 .402723 \\
\hline 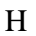 & 8.2045544 .7750288 .520454 \\
\hline
\end{tabular}

H

$\mathrm{H}$

$\mathrm{H}$

$\mathrm{N}$

C

C

O

C

C

C

C

$\mathrm{N}$

$\mathrm{H}$

$\mathrm{H}$

$\mathrm{H}$

$\mathrm{H}$

$\mathrm{H}$

$\mathrm{H}$

$\mathrm{H}$

$\mathrm{H}$

$\mathrm{H}$

$\mathrm{H}$

$\mathrm{H}$

$\mathrm{H}$

$\mathrm{H}$

$\mathrm{N}$

C

C

O

$\mathrm{H}$

$\mathrm{H}$

$\mathrm{H}$

$\mathrm{N}$

C

C

O

C

$\mathrm{H}$

$\mathrm{H}$

$\mathrm{H}$

$\mathrm{H}$

$\mathrm{H}$

$\mathrm{N}$

C

C

O

C

C

$\mathrm{C}$

C

$\mathrm{H}$

$\mathrm{H}$

$\mathrm{H}$

$\mathrm{H}$

$\mathrm{H}$

$\mathrm{H}$

$\mathrm{H}$

$\mathrm{H}$

$\mathrm{H}$

$\mathrm{H}$

$\mathrm{H}$

$\mathrm{N}$

C

C
8.5472936 .2321927 .554438 6.5034875 .1903684 .889532 7.0967076 .4720875 .886026 10.9600184 .2064565 .145000 11.2913394 .4766873 .771693 10.2703793 .9149472 .757492 10.4009764 .1631551 .574209 12.6889203 .9320333 .427148 13.8007784 .7409794 .085997 15.1660574 .3287493 .518251 16.2184165 .3593563 .876957 16.3322495 .4477955 .368483 11.0157993 .2498985 .460362 11.2950905 .5672933 .656086 12.7476302 .8933583 .762547 12.8147583 .9435172 .343655 13.6345975 .8014443 .897585 13.7726244 .5632465 .162621 15.4543103 .3554383 .918361 15.0999654 .2556942 .434595 17.1913225 .0879463 .466395 15.9286556 .3337453 .486801 15.4726815 .8712175 .779014 16.4265874 .5031155 .761607 17.1394976 .0114985 .649466 9.3173573 .1179923 .267675 8.3116462 .4491782 .470165 6.9575493 .1640532 .555314 6.8823294 .3800822 .662187 9.1676173 .0832904 .268177 8.6607642 .4435731 .433274 8.2107911 .4220792 .831007 5.8776442 .3549672 .527937 4.5201932 .8310812 .612193 3.8346642 .1920743 .856983 3.8556540 .9798014 .007430 3.7373162 .4510701 .350621 5.9774591 .3527872 .469359 4.5482713 .9198452 .716170 2.7099562 .7936061 .434501 4.1917322 .9186650 .480718 3.7323141 .3709671 .226712 3.1827173 .0241214 .684661 2.3379782 .5872895 .779117 0.9848763 .2923965 .540379 0.9260424 .5197115 .633111 2.8889722 .9191077 .186459 4.3495712 .4555397 .379045 1.9592482 .3319028 .242897 4.9817172 .8389968 .722357 3.2213094 .0249864 .553720 2.2350521 .5005555 .689910 2.8798714 .0124267 .288033 4.3860651 .3685007 .281022 4.9613702 .8863466 .584362 2.2942922 .6125729 .236681 0.9452352 .7012428 .108355 1.9526381 .2445948 .176880 6.0373772 .5820038 .704400 4.8806773 .9079148 .901915 4.5101302 .3103959 .545730 $-0.0466292 .5255955 .155622$ $-1.2877723 .0906724 .623923$ $-2.4885802 .4336345 .331774$ $-2.5789881 .2060865 .370275$ 


\begin{tabular}{|c|c|}
\hline $\mathrm{C}$ & -1.4355672 .8045533 .106790 \\
\hline $\mathrm{C}$ & -0.1545263 .0767922 .299553 \\
\hline $\mathrm{C}$ & -2.6401083 .5701192 .554746 \\
\hline $\mathrm{C}$ & 0.2047644 .5447212 .121088 \\
\hline $\mathrm{H}$ & -0.0251621 .5198525 .230883 \\
\hline $\mathrm{H}$ & -1.2456864 .1632654 .827395 \\
\hline $\mathrm{H}$ & -1.6271841 .7282123 .018213 \\
\hline $\mathrm{H}$ & 0.6776642 .5687372 .796887 \\
\hline $\mathrm{H}$ & -0.2737942 .6154531 .315550 \\
\hline $\mathrm{H}$ & -2.6630893 .5391951 .470442 \\
\hline $\mathrm{H}$ & -3.5620683 .1325862 .927250 \\
\hline $\mathrm{H}$ & -2.6083954 .6123812 .864185 \\
\hline $\mathrm{H}$ & 1.1910844 .6368071 .669151 \\
\hline $\mathrm{H}$ & -0.5143785 .0433391 .476860 \\
\hline $\mathrm{H}$ & 0.2229275 .0569023 .079873 \\
\hline $\mathrm{N}$ & -3.3812843 .2594535 .898117 \\
\hline $\mathrm{C}$ & -4.6154082 .8049126 .510328 \\
\hline $\mathrm{C}$ & -5.8229333 .3763835 .719605 \\
\hline $\mathrm{O}$ & -5.9212654 .5909515 .572283 \\
\hline $\mathrm{H}$ & -3.2626304 .2602455 .858144 \\
\hline $\mathrm{H}$ & -4.6032141 .7164186 .551799 \\
\hline $\mathrm{H}$ & -4.6681213 .2092687 .526680 \\
\hline $\mathrm{N}$ & -6.6907142 .4846595 .238982 \\
\hline $\mathrm{C}$ & -7.8989612 .8427104 .542612 \\
\hline $\mathrm{C}$ & -9.2344442 .2875925 .308289 \\
\hline $\mathrm{O}$ & -9.0007911 .4462176 .169972 \\
\hline $\mathrm{C}$ & -7.9638122 .2326553 .145073 \\
\hline $\mathrm{C}$ & -6.8365512 .6173292 .165167 \\
\hline $\mathrm{C}$ & -5.7512741 .5381912 .177399 \\
\hline $\mathrm{C}$ & -7.4131672 .7746420 .757123 \\
\hline $\mathrm{O}$ & -10.2775022 .7575214 .870855 \\
\hline $\mathrm{H}$ & -6.5319011 .4951445 .358149 \\
\hline $\mathrm{H}$ & -7.9144303 .9324804 .481365 \\
\hline $\mathrm{H}$ & -7.9790951 .1424813 .243312 \\
\hline $\mathrm{H}$ & -8.9271992 .5345892 .732319 \\
\hline $\mathrm{H}$ & -6.3895483 .5713452 .463570 \\
\hline $\mathrm{H}$ & -4.8875171 .8345721 .585410 \\
\hline $\mathrm{H}$ & -5.4144101 .3448913 .193257 \\
\hline $\mathrm{H}$ & -6.1431170 .6103621 .763518 \\
\hline $\mathrm{H}$ & -6.6216282 .9428430 .027752 \\
\hline $\mathrm{H}$ & -7.9529311 .8738500 .470581 \\
\hline $\mathrm{H}$ & -8.1027663 .6143670 .722555 \\
\hline $\mathrm{N}$ & 4.5227464 .77602518 .021853 \\
\hline $\mathrm{C}$ & 5.6014934 .35613818 .907317 \\
\hline $\mathrm{C}$ & 5.3913155 .03921020 .387956 \\
\hline $\mathrm{O}$ & 4.6489486 .01873020 .379073 \\
\hline $\mathrm{C}$ & 6.9578404 .82280718 .361845 \\
\hline $\mathrm{C}$ & 7.3495564 .15578417 .055878 \\
\hline $\mathrm{O}$ & 7.4238162 .94428916 .918174 \\
\hline $\mathrm{O}$ & 6.0126054 .50511721 .301819 \\
\hline $\mathrm{H}$ & 4.3864575 .77558417 .977599 \\
\hline $\mathrm{H}$ & 5.5907223 .26731118 .965070 \\
\hline $\mathrm{H}$ & 7.7038444 .53976119 .108968 \\
\hline $\mathrm{H}$ & 6.9646195 .91090318 .257536 \\
\hline $\mathrm{O}$ & 13.9834816 .9578596 .781074 \\
\hline $\mathrm{H}$ & 14.0219717 .7789477 .278887 \\
\hline $\mathrm{H}$ & 13.0682096 .8691006 .485644 \\
\hline $\mathrm{O}$ & 5.6984475 .19157024 .712998 \\
\hline $\mathrm{H}$ & 6.3083215 .76088324 .238737 \\
\hline $\mathrm{H}$ & 5.2356964 .72352424 .011536 \\
\hline $\mathrm{O}$ & 12.3977346 .5630630 .105674 \\
\hline $\mathrm{H}$ & 12.2476396 .6971351 .044501 \\
\hline $\mathrm{H}$ & $11.6112786 .110681-0.210294$ \\
\hline $\mathrm{O}$ & 15.0502916 .6001101 .137817 \\
\hline $\mathrm{H}$ & 14.9230887 .5512601 .11629 \\
\hline
\end{tabular}

\section{Quantum refinement with $\mathrm{ORCA} \omega_{\alpha}=\mathbf{0 . 2 6 9}$}

N $\quad-2.7224164 .03417514 .000294$

C $\quad-1.7040403 .17606313 .377281$

C $\quad-0.4164523 .99686713 .237756$

O $\quad-0.4467605 .21927513 .323098$

C $\quad-2.1710712 .62657111 .986666$

C $\quad-2.3025633 .65698110 .868536$

C $\quad-3.5054504 .35094710 .645402$

C $\quad-1.2202863 .91856910 .006803$

C $\quad-3.6193925 .2890209 .607346$

C $\quad-1.3198184 .8805838 .988336$

C $\quad-2.5270115 .5530708 .780440$

$\mathrm{H} \quad-1.5188732 .31175014 .038229$

$\mathrm{H} \quad-1.4694841 .86783211 .622431$

$\mathrm{H} \quad-3.1452392 .13062212 .130689$

$\mathrm{H} \quad-4.3671584 .14915211 .289387$

$\mathrm{H} \quad-0.2900263 .35716810 .125800$

$\mathrm{H} \quad-4.5613165 .8200519 .449777$

$\mathrm{H} \quad-0.4597455 .0992078 .349438$

$\mathrm{H} \quad-2.6158256 .2853677 .972809$

$\mathrm{H} \quad-3.5896523 .52061314 .146012$

$\mathrm{H} \quad-2.3054174 .53840915 .694376$

$\mathrm{H} \quad-2.8918404 .84585313 .404107$

$\mathrm{N} \quad 0.7306143 .29309313 .012783$

C $\quad 2.0419893 .95301412 .892281$

C $\quad 2.3393814 .79910214 .174039$

O $\quad 2.4455096 .01454114 .144923$

C $\quad 2.1188714 .81620611 .630296$

$\mathrm{H} \quad 0.6840952 .28345312 .956819$

$\mathrm{H} \quad 2.7903823 .14456412 .850104$

$\mathrm{H} \quad 3.0983495 .31142311 .567502$

$\mathrm{H} \quad 1.9712654 .19477110 .735673$

$\mathrm{H} \quad 1.3498245 .59648311 .673774$

$\mathrm{N} \quad 2.4439654 .06158515 .305757$

C $\quad 2.4967924 .59809816 .667056$

C $\quad 3.6677743 .95055517 .427441$

O $\quad 3.7045672 .72044817 .479670$

C $\quad 1.2244724 .26925717 .479308$

C $\quad-0.0663904 .87124916 .926688$

C $\quad-1.3095894 .11543817 .389013$

O $\quad-1.3351493 .45284018 .401206$

O $\quad-2.4123254 .25488916 .646501$

$\mathrm{H} \quad 2.4663903 .04706215 .258990$

$\mathrm{H} \quad 2.6261865 .68302916 .546558$

$\mathrm{H} \quad 1.1303953 .17402917 .527225$

$\mathrm{H} \quad 1.3883314 .60699018 .513693$

$\mathrm{H} \quad-0.1873335 .91898317 .256171$

$\mathrm{H} \quad-0.0455544 .91824015 .825294$

$\mathrm{N} \quad 7.6405885 .01427316 .022841$

C $\quad 8.1218884 .55411214 .725685$

C $\quad 9.5237125 .14908514 .500824$

O $\quad 9.7010456 .36866614 .498002$

C $\quad 7.1861944 .96114113 .551028$

C $\quad 7.7566064 .47921912 .205336$

C $\quad 5.7583134 .43203713 .755540$

$\mathrm{H} \quad 7.6126636 .01717216 .168633$

$\mathrm{H} \quad 8.1589083 .45439314 .789563$

$\mathrm{H} \quad 7.1681026 .06515013 .544956$

$\mathrm{H} \quad 7.0155994 .61824011 .399994$

$\mathrm{H} \quad 8.6753495 .01546611 .916707$

$\mathrm{H} \quad 7.9958243 .40160112 .241697$ 


\begin{tabular}{|c|c|}
\hline $\mathrm{H}$ & 5.1133894 .73180112 .914583 \\
\hline $\mathrm{H}$ & 5.7568523 .32984513 .806473 \\
\hline $\mathrm{H}$ & 5.3095074 .79996914 .687308 \\
\hline $\mathrm{N}$ & 10.5648764 .28686114 .328818 \\
\hline $\mathrm{C}$ & 11.9107594 .80101514 .168971 \\
\hline $\mathrm{C}$ & 12.1645805 .42215012 .772245 \\
\hline $\mathrm{O}$ & 12.3781546 .62023812 .640447 \\
\hline $\mathrm{H}$ & 10.4069823 .28868214 .395864 \\
\hline $\mathrm{H}$ & 12.1008805 .61555014 .880567 \\
\hline $\mathrm{H}$ & 12.6200803 .97802914 .347660 \\
\hline $\mathrm{N}$ & 12.1546844 .54719011 .696256 \\
\hline $\mathrm{C}$ & 12.4877054 .91994710 .341451 \\
\hline $\mathrm{C}$ & 11.5336174 .2608869 .327597 \\
\hline $\mathrm{O}$ & 11.3642193 .0477999 .299586 \\
\hline $\mathrm{C}$ & 13.9366154 .4795729 .995855 \\
\hline $\mathrm{O}$ & 14.2659834 .6967068 .634698 \\
\hline $\mathrm{H}$ & 11.8976293 .57596511 .843953 \\
\hline $\mathrm{H}$ & 12.4139976 .01764810 .272399 \\
\hline $\mathrm{H}$ & 14.6251035 .00269010 .685221 \\
\hline $\mathrm{H}$ & 14.0270003 .39627810 .170522 \\
\hline $\mathrm{H}$ & 14.2718925 .6462148 .442241 \\
\hline $\mathrm{N}$ & 10.8971715 .1174658 .464702 \\
\hline $\mathrm{C}$ & 10.0390804 .6725977 .383320 \\
\hline $\mathrm{C}$ & 10.5780745 .1687146 .032929 \\
\hline $\mathrm{O}$ & 10.6371976 .3736855 .766672 \\
\hline $\mathrm{C}$ & 8.5751915 .1497897 .560160 \\
\hline $\mathrm{C}$ & 7.7095354 .5914616 .404013 \\
\hline $\mathrm{O}$ & 7.7144683 .3877156 .123844 \\
\hline $\mathrm{N}$ & 7.0178035 .4892905 .675063 \\
\hline $\mathrm{H}$ & 11.0512326 .1152188 .558768 \\
\hline $\mathrm{H}$ & 10.0411103 .5747137 .410217 \\
\hline $\mathrm{H}$ & 8.1949764 .7792198 .523246 \\
\hline $\mathrm{H}$ & 8.5448116 .2503077 .559200 \\
\hline $\mathrm{H}$ & 6.5102515 .1704454 .851272 \\
\hline $\mathrm{H}$ & 7.0759896 .4826695 .858805 \\
\hline $\mathrm{N}$ & 10.9601044 .2170125 .143949 \\
\hline $\mathrm{C}$ & 11.2973104 .4847243 .758235 \\
\hline $\mathrm{C}$ & 10.2688143 .9284932 .753519 \\
\hline $\mathrm{O}$ & 10.4034574 .1836731 .564315 \\
\hline $\mathrm{C}$ & 12.6982373 .9460933 .421898 \\
\hline $\mathrm{C}$ & 13.8210944 .7403464 .079047 \\
\hline $\mathrm{C}$ & 15.1961604 .3213153 .533065 \\
\hline $\mathrm{C}$ & 16.2678625 .3360223 .880253 \\
\hline $\mathrm{N}$ & 16.3894045 .4703565 .389753 \\
\hline $\mathrm{H}$ & 11.0214453 .2588815 .475277 \\
\hline $\mathrm{H}$ & 11.2959695 .5784293 .633550 \\
\hline $\mathrm{H}$ & 12.7558002 .8922303 .753774 \\
\hline $\mathrm{H}$ & 12.8215893 .9527902 .327295 \\
\hline $\mathrm{H}$ & 13.6668585 .8176643 .894547 \\
\hline $\mathrm{H}$ & 13.7889254 .5723865 .170767 \\
\hline $\mathrm{H}$ & 15.4756373 .3324383 .938623 \\
\hline $\mathrm{H}$ & 15.1532414 .2420702 .436844 \\
\hline $\mathrm{H}$ & 17.2566905 .0460593 .494665 \\
\hline $\mathrm{H}$ & 15.9994856 .3245913 .486831 \\
\hline $\mathrm{H}$ & 15.5489635 .9263035 .817673 \\
\hline $\mathrm{H}$ & 16.4799724 .5467735 .842301 \\
\hline $\mathrm{H}$ & 17.2089546 .0328515 .663991 \\
\hline $\mathrm{N}$ & 9.3135273 .1176283 .268822 \\
\hline $\mathrm{C}$ & 8.3083702 .4544352 .469643 \\
\hline $\mathrm{C}$ & 6.9538183 .1680152 .562464 \\
\hline $\mathrm{O}$ & 6.8868944 .3913942 .672764 \\
\hline $\mathrm{H}$ & 9.1650373 .0920684 .276025 \\
\hline $\mathrm{H}$ & 8.6511452 .4438911 .423406 \\
\hline $\mathrm{H}$ & 8.2093361 .4125612 .817129 \\
\hline $\mathrm{N}$ & 5.8706192 .3524672 .527452 \\
\hline
\end{tabular}

\begin{tabular}{|c|c|}
\hline $\mathrm{C}$ & 4.5079432 .8290652 .598968 \\
\hline $\mathrm{C}$ & 3.8325772 .1956953 .847328 \\
\hline $\mathrm{O}$ & 3.8572300 .9766983 .993560 \\
\hline $\mathrm{C}$ & 3.7313562 .4616881 .326135 \\
\hline $\mathrm{H}$ & 5.9814491 .3433652 .494871 \\
\hline $\mathrm{H}$ & 4.5453263 .9231592 .697766 \\
\hline $\mathrm{H}$ & 2.6904342 .8100761 .395106 \\
\hline $\mathrm{H}$ & 4.2010882 .9349350 .451391 \\
\hline $\mathrm{H}$ & 3.7160241 .3688731 .195107 \\
\hline $\mathrm{N}$ & 3.1855693 .0353794 .688099 \\
\hline $\mathrm{C}$ & 2.3404012 .5975545 .789894 \\
\hline $\mathrm{C}$ & 0.9853193 .2935955 .545927 \\
\hline $\mathrm{O}$ & 0.9335164 .5302245 .631631 \\
\hline $\mathrm{C}$ & 2.8906042 .9206207 .200298 \\
\hline $\mathrm{C}$ & 4.3569792 .4609907 .390011 \\
\hline $\mathrm{C}$ & 1.9561112 .3433198 .264598 \\
\hline $\mathrm{C}$ & 4.9939992 .8451348 .740056 \\
\hline $\mathrm{H}$ & 3.2165614 .0388724 .534275 \\
\hline $\mathrm{H}$ & 2.2503081 .5048305 .698317 \\
\hline $\mathrm{H}$ & 2.8746024 .0220877 .300533 \\
\hline $\mathrm{H}$ & 4.4039011 .3625217 .277906 \\
\hline $\mathrm{H}$ & 4.9719202 .8879156 .582009 \\
\hline $\mathrm{H}$ & 2.2869502 .6263899 .273657 \\
\hline $\mathrm{H}$ & 0.9231362 .7040498 .148790 \\
\hline $\mathrm{H}$ & 1.9443881 .2402928 .214988 \\
\hline $\mathrm{H}$ & 6.0669152 .5932248 .736630 \\
\hline $\mathrm{H}$ & 4.8899173 .9260788 .940446 \\
\hline $\mathrm{H}$ & 4.5268012 .3160219 .585010 \\
\hline $\mathrm{N}$ & -0.0538742 .5239195 .163522 \\
\hline $\mathrm{C}$ & -1.2954373 .0887544 .620788 \\
\hline $\mathrm{C}$ & -2.4885822 .4368495 .340697 \\
\hline $\mathrm{O}$ & -2.5720281 .2016315 .367473 \\
\hline $\mathrm{C}$ & -1.4351492 .8099913 .096180 \\
\hline $\mathrm{C}$ & -0.1488503 .0956792 .290637 \\
\hline $\mathrm{C}$ & -2.6551103 .5503462 .530535 \\
\hline $\mathrm{C}$ & 0.2157254 .5664012 .100648 \\
\hline $\mathrm{H}$ & -0.0126921 .5113385 .231940 \\
\hline $\mathrm{H}$ & -1.2531364 .1668614 .828840 \\
\hline $\mathrm{H}$ & -1.6204171 .7247253 .016535 \\
\hline $\mathrm{H}$ & 0.6931082 .5877012 .789162 \\
\hline $\mathrm{H}$ & -0.2663552 .6153141 .303263 \\
\hline $\mathrm{H}$ & -2.6789173 .5034811 .431172 \\
\hline $\mathrm{H}$ & -3.5922813 .1142752 .900808 \\
\hline $\mathrm{H}$ & -2.6476224 .6141282 .821196 \\
\hline $\mathrm{H}$ & 1.2136714 .6681861 .640592 \\
\hline $\mathrm{H}$ & -0.5035345 .0809321 .444616 \\
\hline $\mathrm{H}$ & 0.2385135 .1008553 .063487 \\
\hline $\mathrm{N}$ & -3.3880893 .2713435 .906247 \\
\hline $\mathrm{C}$ & -4.6270382 .8142226 .517526 \\
\hline $\mathrm{C}$ & -5.8281763 .3813785 .727359 \\
\hline $\mathrm{O}$ & -5.8973034 .6037115 .565031 \\
\hline $\mathrm{H}$ & -3.2698984 .2762985 .837666 \\
\hline $\mathrm{H}$ & -4.5948921 .7189776 .577133 \\
\hline $\mathrm{H}$ & -4.6921513 .2278777 .538009 \\
\hline $\mathrm{N}$ & -6.7242522 .4900235 .271955 \\
\hline $\mathrm{C}$ & -7.9528032 .8485984 .572134 \\
\hline $\mathrm{C}$ & -9.2457032 .3215675 .322401 \\
\hline $\mathrm{O}$ & -9.0421161 .4430916 .188240 \\
\hline $\mathrm{C}$ & -7.9980612 .2808923 .145168 \\
\hline $\mathrm{C}$ & -6.8613922 .6673922 .166445 \\
\hline $\mathrm{C}$ & -5.7694931 .5854442 .157811 \\
\hline $\mathrm{C}$ & -7.4290662 .8658790 .750705 \\
\hline $\mathrm{O}$ & -10.3136192 .8009134 .892992 \\
\hline $\mathrm{H}$ & -6.5795291 .4991795 .430485 \\
\hline $\mathrm{H}$ & -7.9504103 .9445054 .510745 \\
\hline
\end{tabular}




$\begin{array}{lll}\mathrm{H} & -8.0333381 .1760763 .216289 \\ \mathrm{H} & -8.9757062 .5912012 .747018 \\ \mathrm{H} & -6.4043413 .6201662 .492277 \\ \mathrm{H} & -4.8900641 .8864711 .566040 \\ \mathrm{H} & -5.4251691 .3545983 .177575 \\ \mathrm{H} & -6.1620820 .6511951 .718972 \\ \mathrm{H} & -6.6327233 .0477790 .007769 \\ \mathrm{H} & -7.9812691 .9649280 .428008 \\ \mathrm{H} & -8.1339913 .7122530 .714611 \\ \mathrm{~N} & 4.5310944 .78729418 .031728 \\ \mathrm{C} & 5.6050374 .37170718 .950835 \\ \mathrm{C} & 5.3997705 .04648820 .397072 \\ \mathrm{O} & 4.6734546 .05891520 .388483 \\ \mathrm{C} & 6.9663074 .82010018 .388933 \\ \mathrm{C} & 7.3499604 .16243117 .069600 \\ \mathrm{O} & 7.4055652 .94636616 .929577 \\ \mathrm{O} & 6.0382464 .51761521 .334607 \\ \mathrm{H} & 4.3470165 .78654918 .046573 \\ \mathrm{H} & 5.6056123 .27498418 .986736 \\ \mathrm{H} & 7.7244964 .52954519 .134748 \\ \mathrm{H} & 6.9874115 .92101318 .305909 \\ \mathrm{O} & 14.0318256 .9103756 .811720 \\ \mathrm{H} & 14.0882917 .8309817 .110519 \\ \mathrm{H} & 13.1070916 .7951226 .524810 \\ \mathrm{O} & 5.9193125 .14124324 .395260 \\ \mathrm{H} & 6.0693585 .16537523 .428465 \\ \mathrm{H} & 5.0988154 .63336324 .436513 \\ \mathrm{O} & 12.1846346 .5140100 .251104 \\ \mathrm{H} & 12.6371776 .5466031 .102922 \\ \mathrm{H} & 11.4348635 .9222010 .426974 \\ \mathrm{O} & 14.8542896 .5808711 .321731 \\ \mathrm{H} & 14.7676867 .5329191 .168206 \\ \mathrm{H} & 14.6338956 .2089090 .451689\end{array}$

\section{Quantum refinement with $\mathrm{LSQC} \omega_{\alpha}=\mathbf{0 . 2 6 9}$}

$\mathrm{N} \quad-2.7325004 .03020813 .997801$

C $\quad-1.7022173 .15969613 .384374$

C $\quad-0.4170663 .98377413 .237015$

O $\quad-0.4439575 .21081113 .328637$

C $\quad-2.1724512 .61983711 .986931$

C $\quad-2.2940093 .65500110 .872632$

C $\quad-3.4961014 .34936110 .661546$

C $\quad-1.2161013 .91351110 .010001$

C $\quad-3.6148955 .2885459 .629480$

C $\quad-1.3173854 .8921689 .010694$

C $\quad-2.5248285 .5590508 .805144$

$\mathrm{H} \quad-1.5220082 .30521214 .048010$

$\mathrm{H} \quad-1.4664801 .86740411 .642513$

$\mathrm{H} \quad-3.1478302 .13818312 .121519$

$\mathrm{H} \quad-4.3511474 .14904011 .304262$

$\mathrm{H} \quad-0.2971543 .34121210 .109228$

$\mathrm{H} \quad-4.5529465 .8168839 .483224$

$\mathrm{H} \quad-0.4607665 .1256798 .383997$

$\mathrm{H} \quad-2.6095586 .2915998 .005852$

$\mathrm{H} \quad-3.5970643 .51352614 .143213$

$\mathrm{H} \quad-2.3199734 .53072915 .497219$

$\mathrm{H} \quad-2.8877074 .83142013 .389113$

$\mathrm{N} \quad 0.7277153 .28286912 .997032$

C $\quad 2.0401323 .94527812 .882881$

C $\quad 2.3411144 .79051114 .165614$

O $\quad 2.4461916 .01167214 .141709$

C $\quad 2.1193484 .81195211 .620384$

$\mathrm{H} \quad 0.6878712 .27452012 .952741$
$\mathrm{H}$

$\mathrm{H}$

$\mathrm{H}$

$\mathrm{H}$

$\mathrm{N}$

C

C

$\mathrm{O}$

C

C

$\mathrm{C}$

$\mathrm{O}$

$\mathrm{O}$

$\mathrm{H}$

$\mathrm{H}$

$\mathrm{H}$

$\mathrm{H}$

$\mathrm{H}$

$\mathrm{H}$

N

C

C

O

C

C

$\mathrm{C}$

$\mathrm{H}$

$\mathrm{H}$

$\mathrm{H}$

$\mathrm{H}$

$\mathrm{H}$

$\mathrm{H}$

$\mathrm{H}$

$\mathrm{H}$

$\mathrm{H}$

N

C

C

O

$\mathrm{H}$

$\mathrm{H}$

$\mathrm{H}$

N

C

C

O

C

$\mathrm{O}$

$\mathrm{H}$

$\mathrm{H}$

$\mathrm{H}$

$\mathrm{H}$

$\mathrm{H}$

$\mathrm{N}$

C

C

$\mathrm{O}$

C

C

O

$\mathrm{N}$

H

$\mathrm{H}$

$\mathrm{H}$

H
2.7874083 .14435412 .841810 3.0935275 .30739211 .566264 1.9781744 .19322410 .729474 1.3479995 .58094511 .659118 2.4409474 .05130915 .295615 2.4915514 .58448316 .661843 3.6620723 .94109817 .422560 3.7038542 .70498517 .479756 1.2016394 .26139017 .452837 $-0.0690154 .89709316 .873562$ $-1.3397944 .14130817 .266980$ $-1.3868233 .44060018 .262534$ $-2.4215334 .31466616 .499001$ 2.4698733 .03975915 .248443 2.6272295 .66295316 .551132 1.0846653 .17419117 .483756 1.3365284 .59398418 .485722 $-0.1746115 .93300517 .221608$ $-0.0102354 .95347215 .780298$ 7.6305065 .00651816 .019709 8.1190404 .55155114 .725484 9.5219465 .13872114 .501855 9.7028496 .36254714 .505838 7.1834194 .94709813 .545030 7.7647994 .44550812 .210952 5.7557494 .41448013 .744351 7.5973976 .00588416 .165551 8.1603943 .45869714 .780851 7.1553916 .04499813 .530563 7.0384654 .54552511 .396959 8.6763044 .98221611 .920958 8.0131663 .37668212 .274740 5.1258924 .70653312 .896603 5.7624383 .31824213 .806263 5.2975894 .78995114 .661085 10.5634914 .27551914 .327468 11.9173374 .77436714 .165895 12.1728725 .40619412 .775777 12.3783556 .61184612 .644516 10.4024513 .27846014 .333455 12.1430535 .54910614 .899981 12.5905043 .92235714 .311037 12.1605594 .53352911 .696657 12.4891144 .91311710 .341705 11.5343444 .2568109 .326553 11.3633883 .0375739 .291828 13.9340744 .4670379 .989821 14.2692404 .6958618 .619579 11.9190923 .56071711 .840371 12.4055916 .00231510 .270588 14.6295354 .98858210 .662159 14.0202263 .38893010 .149193 14.3131425 .6548538 .470352 10.8944935 .1160758 .469029 10.0409814 .6712877 .384184 10.5784835 .1636116 .028831 10.6353146 .3734765 .760052 8.5772595 .1440177 .562134 7.7109964 .5809236 .404942 7.7138563 .3751626 .124338 7.0303895 .4940835 .679898 11.0203736 .1123368 .584050 10.0584253 .5808527 .407374 8.2038754 .7802978 .522178 8.5497416 .2387497 .555830 


\begin{tabular}{|c|c|}
\hline $\mathrm{H}$ & 6.4857185 .2035774 .876643 \\
\hline $\mathrm{H}$ & 7.0937666 .4796565 .887021 \\
\hline $\mathrm{N}$ & 10.9632154 .2129745 .142528 \\
\hline $\mathrm{C}$ & 11.2973844 .4766283 .748252 \\
\hline $\mathrm{C}$ & 10.2737633 .9049522 .748061 \\
\hline $\mathrm{O}$ & 10.4011424 .1335901 .554740 \\
\hline $\mathrm{C}$ & 12.6985323 .9334733 .416379 \\
\hline $\mathrm{C}$ & 13.8245844 .7354364 .069601 \\
\hline $\mathrm{C}$ & 15.1912494 .3111483 .487643 \\
\hline $\mathrm{C}$ & 16.2646895 .3348943 .826177 \\
\hline $\mathrm{N}$ & 16.4103755 .4385935 .345472 \\
\hline $\mathrm{H}$ & 11.0187213 .2566405 .472419 \\
\hline $\mathrm{H}$ & 11.3114845 .5615623 .618385 \\
\hline $\mathrm{H}$ & 12.7623972 .8900543 .760067 \\
\hline $\mathrm{H}$ & 12.8252753 .9423972 .328992 \\
\hline $\mathrm{H}$ & 13.6688735 .8064493 .885271 \\
\hline $\mathrm{H}$ & 13.8124164 .5620545 .154623 \\
\hline $\mathrm{H}$ & 15.4841853 .3308913 .887717 \\
\hline $\mathrm{H}$ & 15.1192564 .2351312 .399600 \\
\hline $\mathrm{H}$ & 17.2421975 .0577343 .423294 \\
\hline $\mathrm{H}$ & 15.9843966 .3213393 .457136 \\
\hline $\mathrm{H}$ & 15.5757155 .8776905 .786309 \\
\hline $\mathrm{H}$ & 16.5071164 .5021735 .758285 \\
\hline $\mathrm{H}$ & 17.2290365 .9923175 .617056 \\
\hline $\mathrm{N}$ & 9.3128283 .1007703 .283487 \\
\hline $\mathrm{C}$ & 8.3110902 .4390402 .479364 \\
\hline $\mathrm{C}$ & 6.9578383 .1600842 .558728 \\
\hline $\mathrm{O}$ & 6.8815914 .3877222 .665821 \\
\hline $\mathrm{H}$ & 9.1594493 .0807574 .285562 \\
\hline $\mathrm{H}$ & 8.6652642 .3999251 .444757 \\
\hline $\mathrm{H}$ & 8.2011601 .4087652 .839995 \\
\hline $\mathrm{N}$ & 5.8738632 .3395092 .525562 \\
\hline $\mathrm{C}$ & 4.5137072 .8136102 .602572 \\
\hline $\mathrm{C}$ & 3.8327172 .1857913 .847394 \\
\hline $\mathrm{O}$ & 3.8503840 .9613813 .997924 \\
\hline $\mathrm{C}$ & 3.7217202 .4572131 .334444 \\
\hline $\mathrm{H}$ & 5.9907161 .3360222 .468634 \\
\hline $\mathrm{H}$ & 4.5434233 .9008262 .705253 \\
\hline $\mathrm{H}$ & 2.6886872 .8094941 .417013 \\
\hline $\mathrm{H}$ & 4.1825002 .9380930 .466313 \\
\hline $\mathrm{H}$ & 3.7032351 .3721111 .192056 \\
\hline $\mathrm{N}$ & 3.1835333 .0257234 .688107 \\
\hline $\mathrm{C}$ & 2.3361782 .5937875 .790063 \\
\hline $\mathrm{C}$ & 0.9848343 .2850845 .541081 \\
\hline $\mathrm{O}$ & 0.9327354 .5256625 .631635 \\
\hline $\mathrm{C}$ & 2.8867162 .9131847 .202083 \\
\hline $\mathrm{C}$ & 4.3544912 .4566397 .391565 \\
\hline $\mathrm{C}$ & 1.9539972 .3234328 .264928 \\
\hline $\mathrm{C}$ & 4.9924652 .8412718 .740232 \\
\hline $\mathrm{H}$ & 3.2258704 .0256114 .538549 \\
\hline $\mathrm{H}$ & 2.2374751 .5074595 .710332 \\
\hline $\mathrm{H}$ & 2.8805054 .0088967 .304939 \\
\hline $\mathrm{H}$ & 4.3977961 .3633057 .291374 \\
\hline $\mathrm{H}$ & 4.9663212 .8869996 .590842 \\
\hline $\mathrm{H}$ & 2.2776072 .5853909 .275291 \\
\hline $\mathrm{H}$ & 0.9252712 .6813758 .150472 \\
\hline $\mathrm{H}$ & 1.9483511 .2260278 .193285 \\
\hline $\mathrm{H}$ & 6.0590642 .5887388 .731214 \\
\hline $\mathrm{H}$ & 4.8861553 .9179848 .930999 \\
\hline $\mathrm{H}$ & 4.5197882 .3151929 .576874 \\
\hline $\mathrm{N}$ & -0.0529352 .5132125 .157954 \\
\hline $\mathrm{C}$ & -1.2965383 .0658954 .602756 \\
\hline $\mathrm{C}$ & -2.4905782 .4283655 .333222 \\
\hline $\mathrm{O}$ & -2.5776481 .1874115 .373460 \\
\hline $\mathrm{C}$ & -1.4441062 .7970453 .071511 \\
\hline
\end{tabular}

\begin{tabular}{|c|c|}
\hline $\mathrm{C}$ & -0.1533903 .0680892 .265935 \\
\hline $\mathrm{C}$ & -2.6516023 .5905682 .536036 \\
\hline $\mathrm{C}$ & 0.2325874 .5410232 .102098 \\
\hline $\mathrm{H}$ & -0.0146951 .5080865 .255335 \\
\hline $\mathrm{H}$ & -1.2487164 .1380764 .800631 \\
\hline $\mathrm{H}$ & -1.6424541 .7224812 .966058 \\
\hline $\mathrm{H}$ & 0.6771852 .5423882 .753987 \\
\hline $\mathrm{H}$ & -0.2766362 .6200261 .270559 \\
\hline $\mathrm{H}$ & -2.6806233 .6276111 .444740 \\
\hline $\mathrm{H}$ & -3.5939923 .1631092 .885991 \\
\hline $\mathrm{H}$ & -2.6104904 .6341962 .872743 \\
\hline $\mathrm{H}$ & 1.2278974 .6266411 .648161 \\
\hline $\mathrm{H}$ & -0.4856855 .0631581 .460152 \\
\hline $\mathrm{H}$ & 0.2571135 .0529993 .070681 \\
\hline $\mathrm{N}$ & -3.3889813 .2644895 .895441 \\
\hline $\mathrm{C}$ & -4.6272872 .8085506 .521130 \\
\hline $\mathrm{C}$ & -5.8293193 .3734235 .728718 \\
\hline $\mathrm{O}$ & -5.9114814 .6060955 .595320 \\
\hline $\mathrm{H}$ & -3.2658164 .2660615 .837678 \\
\hline $\mathrm{H}$ & -4.5946161 .7207546 .592112 \\
\hline $\mathrm{H}$ & -4.6782553 .2190557 .535691 \\
\hline $\mathrm{N}$ & -6.7141062 .4814565 .257628 \\
\hline $\mathrm{C}$ & -7.9501692 .8240514 .553467 \\
\hline $\mathrm{C}$ & -9.2487612 .2855145 .298959 \\
\hline $\mathrm{O}$ & $\begin{array}{llll}-9.036181 & 1.432927 & 6.193748\end{array}$ \\
\hline $\mathrm{C}$ & $\begin{array}{ll}-7.967482 & 2.2394803 .133313\end{array}$ \\
\hline $\mathrm{C}$ & 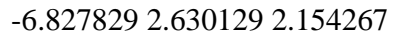 \\
\hline $\mathrm{C}$ & -5.7260681 .5543342 .178453 \\
\hline $\mathrm{C}$ & -7.3962122 .7918560 .734485 \\
\hline $\mathrm{O}$ & -10.3158922 .7454054 .833628 \\
\hline $\mathrm{H}$ & -6.5353071 .4940945 .377937 \\
\hline $\mathrm{H}$ & -7.9654063 .9131384 .490973 \\
\hline $\mathrm{H}$ & -7.9713621 .1400763 .222608 \\
\hline $\mathrm{H}$ & -8.9366372 .5251632 .713514 \\
\hline $\mathrm{H}$ & -6.3855973 .5910252 .455776 \\
\hline $\mathrm{H}$ & -4.8444131 .8450381 .594109 \\
\hline $\mathrm{H}$ & -5.3939881 .3569763 .203767 \\
\hline $\mathrm{H}$ & -6.1061320 .6125411 .759541 \\
\hline $\mathrm{H}$ & $-6.6012372 .965838-0.004463$ \\
\hline $\mathrm{H}$ & -7.9354871 .8827300 .435365 \\
\hline $\mathrm{H}$ & -8.1009583 .6307360 .688475 \\
\hline $\mathrm{N}$ & 4.5246484 .77839618 .025261 \\
\hline $\mathrm{C}$ & 5.6005634 .35682618 .939368 \\
\hline $\mathrm{C}$ & 5.4047505 .02757720 .386478 \\
\hline $\mathrm{O}$ & 4.7016356 .06502720 .373026 \\
\hline $\mathrm{C}$ & 6.9623794 .81510718 .387648 \\
\hline $\mathrm{C}$ & 7.3458164 .15473817 .070452 \\
\hline $\mathrm{O}$ & 7.4186862 .93246716 .933422 \\
\hline $\mathrm{O}$ & 6.0207864 .47091721 .326011 \\
\hline $\mathrm{H}$ & 4.3713925 .77844618 .000319 \\
\hline $\mathrm{H}$ & 5.6006973 .26797318 .975375 \\
\hline $\mathrm{H}$ & 7.7171334 .53776919 .132320 \\
\hline $\mathrm{H}$ & 6.9767385 .91033518 .295159 \\
\hline $\mathrm{O}$ & 14.0536616 .9945756 .866131 \\
\hline $\mathrm{H}$ & 14.1067007 .8600697 .303703 \\
\hline $\mathrm{H}$ & 13.1283356 .9107306 .570712 \\
\hline $\mathrm{O}$ & 5.6293065 .27969324 .881060 \\
\hline $\mathrm{H}$ & 6.3596965 .74410124 .445030 \\
\hline $\mathrm{H}$ & 5.2654524 .76740524 .138963 \\
\hline $\mathrm{O}$ & 11.8569276 .6310940 .565458 \\
\hline $\mathrm{H}$ & 12.7473556 .9527910 .766665 \\
\hline $\mathrm{H}$ & $11.9578826 .139600-0.263804$ \\
\hline $\mathrm{O}$ & 14.8203746 .5553871 .206294 \\
\hline $\mathrm{H}$ & 15.2016187 .4464861 .231386 \\
\hline $\mathrm{H}$ & 14.9306866 .2799380 .281490 \\
\hline
\end{tabular}


Quantum refinement with $\mathrm{xTB} \omega_{\alpha}=1.0$

$\mathrm{N} \quad-2.7751094 .06218514 .015195$

C $\quad-1.7038513 .19375213 .392087$

C $\quad-0.4058343 .99489813 .216370$

O $\quad-0.4371285 .22507813 .323821$

C $\quad-2.1724212 .62356412 .015003$

C $\quad-2.3011113 .66985810 .871448$

C $\quad-3.4968774 .35842210 .649120$

C $\quad-1.2224313 .92207710 .012362$

C $\quad-3.6074765 .2960139 .617996$

C $\quad-1.3333964 .9028429 .000309$

C $\quad-2.5314645 .5625748 .805209$

$\mathrm{H} \quad-1.5062602 .34352914 .050776$

$\mathrm{H} \quad-1.4597221 .88161911 .694320$

$\mathrm{H} \quad-3.1527862 .16572012 .139242$

$\mathrm{H} \quad-4.3573524 .16905111 .277366$

$\mathrm{H} \quad-0.3020443 .36855010 .119996$

$\mathrm{H} \quad-4.5428295 .8123149 .462883$

$\mathrm{H} \quad-0.4983025 .1503678 .367848$

$\mathrm{H} \quad-2.6050596 .2875648 .007108$

$\mathrm{H} \quad-3.6327713 .52131414 .141760$

$\mathrm{H} \quad-2.4588104 .43659115 .003684$

$\mathrm{H} \quad-2.9439584 .86101913 .393683$

$\mathrm{N} \quad 0.7197943 .29037712 .999690$

C $\quad 2.0414083 .94598812 .889766$

C $\quad 2.3453234 .80514614 .158106$

O $\quad 2.4312646 .01945614 .182750$

C $\quad 2.0960414 .82506911 .618670$

$\mathrm{H} \quad 0.7079862 .28584912 .906256$

$\mathrm{H} \quad 2.7928503 .15738912 .831439$

$\mathrm{H} \quad 3.0642215 .31449111 .559447$

H $\quad 1.9488004 .21465810 .732599$

$\mathrm{H} \quad 1.3243155 .58513411 .674790$

$\mathrm{N} \quad 2.4518664 .08420615 .279895$

C $\quad 2.4985454 .57932316 .660193$

C $\quad 3.6536923 .95580317 .441368$

O $\quad 3.7012002 .73005017 .512611$

C $\quad 1.2246024 .24086217 .492668$

C $\quad-0.0614224 .85009616 .981436$

C $\quad-1.3779484 .06150617 .405135$

O $\quad-1.3387903 .37717218 .413050$

O $\quad-2.3275814 .24584816 .613282$

$\mathrm{H} \quad 2.4799663 .07289215 .232304$

$\mathrm{H} \quad 2.6367525 .66125116 .554973$

$\mathrm{H} \quad 1.1074283 .15419117 .505908$

$\mathrm{H} \quad 1.3756054 .58659118 .517268$

$\mathrm{H} \quad-0.1661665 .86943117 .354596$

$\mathrm{H} \quad-0.0326944 .87953115 .888125$

$\mathrm{N} \quad 7.6326134 .99835016 .020928$

C $\quad 8.1271764 .55372014 .708299$

C $\quad 9.5416615 .12996214 .482803$

O $\quad 9.6985966 .37567314 .500992$

C $\quad 7.1884944 .95266013 .540390$

C $\quad 7.7557934 .46623312 .198387$

C $\quad 5.7520854 .42846013 .713902$

$\mathrm{H} \quad 7.6091936 .00054916 .137854$

$\mathrm{H} \quad 8.1698813 .45978614 .744771$

$\mathrm{H} \quad 7.1664246 .04713513 .551846$

$\mathrm{H} \quad 7.0248754 .59894311 .404130$

$\mathrm{H} \quad 8.6595705 .00010211 .919246$

$\mathrm{H} \quad 7.9948483 .40553112 .236154$

$\mathrm{H} \quad 5.1436754 .73861012 .870806$
5.7671913 .33880813 .750914

5.3018474 .78851414 .630899

10.5693774 .30135714 .335579

11.9209974 .79356314 .197395

12.1725665 .40541812 .781347

12.3627656 .62864412 .658417

10.4563833 .29986614 .366733

12.1189325 .58437414 .922099

12.6059463 .95944314 .355703

12.1705834 .55609011 .690123

12.4947414 .92359010 .344911

11.5591864 .2705969 .320613

11.3557543 .0553629 .282445

13.9525814 .46481010 .015804

14.2904284 .6928958 .678009

11.9353473 .58242811 .820034

12.4029346 .01146010 .254175

14.6471104 .99275510 .678327

14.0332703 .38725910 .177901

14.3481965 .6492388 .536122

10.8940925 .1120958 .480782

10.0575634 .6853057 .385752

10.5675195 .1446476 .018221

10.6022566 .3500765 .701343

8.5893485 .1614607 .589487

7.6975604 .6055366 .419784

7.6994073 .4025446 .132662

7.0110945 .5003065 .697928

11.0070506 .1083248 .595840

10.1074523 .5917907 .403700

8.2236484 .8073068 .547049

8.5545266 .2511727 .572428

6.4316905 .2529134 .914204

7.0935806 .4826165 .903854

10.9763164 .2204495 .137807

11.3149734 .4849873 .758842

10.2953653 .9218232 .763903

10.3702694 .2057211 .580989

12.7256663 .9281433 .424011

13.8464884 .7493594 .049158

15.1985384 .2960943 .425759

16.2673625 .3322033 .723394

16.4341095 .4396555 .214313

11.0619573 .2570145 .435742

11.3353395 .5725623 .622332

12.7811992 .8980693 .785809

12.8594093 .9221042 .347955

13.6931065 .8067113 .844644

13.8609374 .5950145 .128577

15.4930343 .3350023 .848335

15.0831134 .1985682 .349929

17.2210015 .0464403 .286437

15.9672926 .2989253 .321211

15.5986865 .8811705 .632002

16.5433034 .5035135 .626750

17.2558386 .0050755 .453464

9.3403743 .1118483 .266598

8.3215772 .4450092 .467853

6.9699393 .1617142 .537505

6.8820144 .3969922 .660177

9.1766153 .0320094 .261209

8.6624232 .4158171 .428808

8.2028241 .4223432 .832357

5.8729652 .3591492 .512165

4.5314482 .8219332 .586790 


\begin{tabular}{|c|c|}
\hline $\mathrm{C}$ & 3.8421222 .2101113 .862521 \\
\hline $\mathrm{O}$ & 3.8476250 .9841464 .020882 \\
\hline $\mathrm{C}$ & 3.7415452 .4606281 .320292 \\
\hline $\mathrm{H}$ & 5.9855841 .3612122 .424864 \\
\hline $\mathrm{H}$ & 4.5505693 .9109622 .709530 \\
\hline $\mathrm{H}$ & 2.7164022 .8083821 .408697 \\
\hline $\mathrm{H}$ & 4.1996862 .9360790 .457121 \\
\hline $\mathrm{H}$ & 3.7402931 .3815231 .189754 \\
\hline $\mathrm{N}$ & 3.1852713 .0245344 .688742 \\
\hline $\mathrm{C}$ & 2.3436142 .6133495 .773770 \\
\hline $\mathrm{C}$ & 0.9923373 .2815035 .527578 \\
\hline $\mathrm{O}$ & 0.9193834 .5374155 .644056 \\
\hline $\mathrm{C}$ & 2.8868952 .9201097 .192107 \\
\hline $\mathrm{C}$ & 4.3608052 .4634737 .404360 \\
\hline $\mathrm{C}$ & 1.9640262 .3440438 .260426 \\
\hline $\mathrm{C}$ & 4.9988042 .8491648 .758232 \\
\hline $\mathrm{H}$ & 3.2375724 .0254854 .557998 \\
\hline $\mathrm{H}$ & 2.2328771 .5244765 .703051 \\
\hline $\mathrm{H}$ & 2.8910574 .0149977 .278904 \\
\hline $\mathrm{H}$ & 4.3960521 .3759937 .328284 \\
\hline $\mathrm{H}$ & 4.9738522 .8889436 .611196 \\
\hline $\mathrm{H}$ & 2.2954102 .6285309 .252948 \\
\hline $\mathrm{H}$ & 0.9452592 .6973058 .124116 \\
\hline $\mathrm{H}$ & 1.9680211 .2542268 .193991 \\
\hline $\mathrm{H}$ & 6.0537532 .5936288 .748055 \\
\hline $\mathrm{H}$ & 4.8931313 .9203768 .922660 \\
\hline $\mathrm{H}$ & 4.5098502 .3180649 .569989 \\
\hline $\mathrm{N}$ & -0.0375552 .5205095 .161906 \\
\hline $\mathrm{C}$ & -1.2861733 .0818014 .607817 \\
\hline $\mathrm{C}$ & -2.4898962 .4598405 .318295 \\
\hline $\mathrm{O}$ & -2.5895251 .2051125 .376069 \\
\hline $\mathrm{C}$ & -1.4237912 .8119203 .080306 \\
\hline $\mathrm{C}$ & -0.1319443 .0824302 .269229 \\
\hline $\mathrm{C}$ & -2.6356123 .5448572 .484795 \\
\hline $\mathrm{C}$ & 0.2383084 .5374632 .053695 \\
\hline $\mathrm{H}$ & -0.0256131 .5162935 .238173 \\
\hline $\mathrm{H}$ & -1.2483884 .1527654 .813041 \\
\hline $\mathrm{H}$ & -1.6019031 .7386713 .001605 \\
\hline $\mathrm{H}$ & 0.7086082 .6106292 .785260 \\
\hline $\mathrm{H}$ & -0.2416252 .6215681 .291250 \\
\hline $\mathrm{H}$ & -2.6440033 .4975231 .406136 \\
\hline $\mathrm{H}$ & -3.5590933 .0957512 .845620 \\
\hline $\mathrm{H}$ & -2.6213554 .5924182 .778298 \\
\hline $\mathrm{H}$ & 1.2294934 .5910571 .607825 \\
\hline $\mathrm{H}$ & -0.4891534 .9985851 .390652 \\
\hline $\mathrm{H}$ & 0.2413195 .0658583 .003518 \\
\hline $\mathrm{N}$ & -3.3832653 .2835615 .912577 \\
\hline $\mathrm{C}$ & -4.6042902 .8100826 .523745 \\
\hline $\mathrm{C}$ & -5.8177633 .3758875 .739857 \\
\hline $\mathrm{O}$ & -5.9620184 .5882575 .601301 \\
\hline $\mathrm{H}$ & -3.2470884 .2828045 .922291 \\
\hline $\mathrm{H}$ & -4.6088841 .7206036 .541281 \\
\hline $\mathrm{H}$ & -4.6622893 .1783077 .554091 \\
\hline $\mathrm{N}$ & -6.7073782 .4770555 .239994 \\
\hline $\mathrm{C}$ & -7.8946662 .8547284 .564025 \\
\hline $\mathrm{C}$ & -9.3058162 .2828115 .306089 \\
\hline $\mathrm{O}$ & -9.0483251 .4606746 .154695 \\
\hline $\mathrm{C}$ & -7.9514672 .2758693 .154466 \\
\hline $\mathrm{C}$ & -6.8177312 .6556942 .158808 \\
\hline $\mathrm{C}$ & -5.7471901 .5543252 .184631 \\
\hline $\mathrm{C}$ & -7.4106712 .8213890 .757402 \\
\hline $\mathrm{O}$ & -10.3042042 .8001024 .871816 \\
\hline $\mathrm{H}$ & -6.4959651 .4873315 .265854 \\
\hline $\mathrm{H}$ & -7.9023793 .9486474 .576161 \\
\hline $\mathrm{H}$ & -7.9552381 .1860313 .239326 \\
\hline
\end{tabular}

$\begin{array}{lll}\mathrm{H} & -8.9027702 .5808142 .723075 \\ \mathrm{H} & -6.3551073 .6057552 .443548 \\ \mathrm{H} & -4.8772301 .8328231 .598275 \\ \mathrm{H} & -5.4090761 .3676113 .202302 \\ \mathrm{H} & -6.1509470 .6306381 .777051 \\ \mathrm{H} & -6.6179672 .9759030 .029643 \\ \mathrm{H} & -7.9635061 .9258020 .481142 \\ \mathrm{H} & -8.0840843 .6731070 .726729 \\ \mathrm{~N} & 4.5288104 .79835218 .033222 \\ \mathrm{C} & 5.6163664 .37566618 .914094 \\ \mathrm{C} & 5.4096345 .08024220 .474762 \\ \mathrm{O} & 4.6652386 .02954520 .456818 \\ \mathrm{C} & 6.9638274 .82710318 .388570 \\ \mathrm{C} & 7.3444524 .17743717 .047323 \\ \mathrm{O} & 7.4140322 .94677116 .943367 \\ \mathrm{O} & 6.0183454 .48324021 .334358 \\ \mathrm{H} & 4.4068115 .79228017 .908191 \\ \mathrm{H} & 5.5929373 .28848918 .979559 \\ \mathrm{H} & 7.7343594 .55173419 .114690 \\ \mathrm{H} & 6.9980205 .91529418 .273015 \\ \mathrm{O} & 13.7599907 .0886537 .023029 \\ \mathrm{H} & 14.1378557 .7535307 .608787 \\ \mathrm{H} & 12.8374587 .3287876 .893679 \\ \mathrm{O} & 5.7703555 .19213124 .080975 \\ \mathrm{H} & 6.4411415 .81969524 .353439 \\ \mathrm{H} & 5.9134564 .98875523 .144135 \\ \mathrm{O} & 11.8951136 .2612830 .385590 \\ \mathrm{H} & 11.2875425 .5908080 .743253 \\ \mathrm{H} & 11.9200506 .142964-0.565979 \\ \mathrm{O} & 14.5376186 .4979601 .013958 \\ \mathrm{H} & 13.5727196 .5372890 .867205 \\ \mathrm{H} & 14.9403846 .4467570 .144850\end{array}$

\section{Quantum refinement with ORCA $\omega_{\alpha}=1.0$}

$\begin{array}{lll}\mathrm{N} & -2.7707124 .05431014 .044637 \\ \mathrm{C} & -1.6996463 .18547213 .409794 \\ \mathrm{C} & -0.4096043 .98871613 .232882 \\ \mathrm{O} & -0.4386295 .22645513 .323579 \\ \mathrm{C} & -2.1760722 .62379812 .018419 \\ \mathrm{C} & -2.3039433 .66733310 .875036 \\ \mathrm{C} & -3.5075474 .36118310 .648452 \\ \mathrm{C} & -1.2132053 .92503710 .011752 \\ \mathrm{C} & -3.6190145 .3057079 .605663 \\ \mathrm{C} & -1.3182454 .9180678 .993402 \\ \mathrm{C} & -2.5304585 .5800828 .791105 \\ \mathrm{H} & -1.5012302 .33156514 .077331 \\ \mathrm{H} & -1.4568131 .88000711 .681444 \\ \mathrm{H} & -3.1637542 .14787012 .147797 \\ \mathrm{H} & -4.3808024 .16608611 .286098 \\ \mathrm{H} & -0.2813883 .35894110 .112971 \\ \mathrm{H} & -4.5688255 .8279869 .446307 \\ \mathrm{H} & -0.4666375 .1780978 .357941 \\ \mathrm{H} & -2.6190766 .3169517 .982890 \\ \mathrm{H} & -3.6280473 .51172714 .183866 \\ \mathrm{H} & -2.4399704 .45337815 .126626 \\ \mathrm{H} & -2.9464414 .85306613 .426145 \\ \mathrm{~N} & 0.7245333 .28400212 .993890 \\ \mathrm{C} & 2.0446703 .94673412 .878634 \\ \mathrm{C} & 2.3488424 .80484414 .160240 \\ \mathrm{O} & 2.4361536 .02870614 .164396 \\ \mathrm{C} & 2.0993604 .81989011 .602483 \\ \mathrm{H} & 0.7044682 .26849712 .936118 \\ \mathrm{H} & 2.8058193 .14920812 .834429\end{array}$




\begin{tabular}{|c|c|}
\hline $\mathrm{H}$ & 944211.529482 \\
\hline $\mathrm{H}$ & 1.9524754 .18905610 .711588 \\
\hline $\mathrm{H}$ & 1.3131135 .58353011 .655082 \\
\hline $\mathrm{N}$ & 2.4544404 .08031915 .281143 \\
\hline $\mathrm{C}$ & 2.4889764 .57280416 .673195 \\
\hline $\mathrm{C}$ & 3.6587863 .96114017 .435663 \\
\hline $\mathrm{O}$ & 3.6996462 .71990617 .499083 \\
\hline $\mathrm{C}$ & 1.2069254 .23845217 .485932 \\
\hline $\mathrm{C}$ & -0.0859454 .83921316 .950264 \\
\hline $\mathrm{C}$ & -1.3695674 .07146217 .341433 \\
\hline $\mathrm{O}$ & -1.3868633 .38494618 .365027 \\
\hline $\mathrm{O}$ & -2.3633424 .23371216 .514365 \\
\hline $\mathrm{H}$ & 2.4966173 .06216015 .220972 \\
\hline $\mathrm{H}$ & 2.6200885 .66230616 .570203 \\
\hline $\mathrm{H}$ & 1.0934433 .14138017 .505725 \\
\hline $\mathrm{H}$ & 1.3478864 .58555118 .524532 \\
\hline $\mathrm{H}$ & -0.1993425 .87815417 .312303 \\
\hline $\mathrm{H}$ & -0.0344964 .87419015 .847664 \\
\hline $\mathrm{N}$ & 7.6334565 .00532016 .018736 \\
\hline $\mathrm{C}$ & 8.1255754 .55571514 .707828 \\
\hline $\mathrm{C}$ & 9.5363905 .12808214 .485554 \\
\hline $\mathrm{O}$ & 9.7021576 .37776714 .500570 \\
\hline $\mathrm{C}$ & 7.1855074 .95628613 .533795 \\
\hline $\mathrm{C}$ & 7.7631844 .46023412 .195995 \\
\hline $\mathrm{C}$ & 5.7437804 .43927713 .713442 \\
\hline $\mathrm{H}$ & 7.6133006 .01272316 .158688 \\
\hline $\mathrm{H}$ & 8.1609973 .45402014 .749607 \\
\hline $\mathrm{H}$ & 7.1678036 .06322613 .536551 \\
\hline $\mathrm{H}$ & 7.0385244 .56765911 .366704 \\
\hline $\mathrm{H}$ & 8.6882964 .98497611 .898354 \\
\hline $\mathrm{H}$ & 8.0031763 .37939912 .242480 \\
\hline $\mathrm{H}$ & 5.1232954 .74780012 .854566 \\
\hline $\mathrm{H}$ & 5.7401173 .33446513 .761401 \\
\hline $\mathrm{H}$ & 5.2759184 .80944714 .636276 \\
\hline $\mathrm{N}$ & 10.5749024 .29218214 .335970 \\
\hline $\mathrm{C}$ & 11.9205144 .79806014 .202257 \\
\hline $\mathrm{C}$ & 12.1734465 .40758812 .785886 \\
\hline $\mathrm{O}$ & 12.3667346 .63306412 .659604 \\
\hline $\mathrm{H}$ & 10.4573313 .28250214 .336484 \\
\hline $\mathrm{H}$ & 12.1420705 .58028614 .939556 \\
\hline $\mathrm{H}$ & 12.6067683 .94453414 .343126 \\
\hline $\mathrm{N}$ & 12.1699574 .54749511 .689315 \\
\hline $\mathrm{C}$ & 12.4975244 .91769310 .345112 \\
\hline $\mathrm{C}$ & 11.5597754 .2719249 .320746 \\
\hline $\mathrm{O}$ & 11.3556873 .0501999 .285794 \\
\hline $\mathrm{C}$ & 13.9505194 .46806710 .018909 \\
\hline $\mathrm{O}$ & 14.2890774 .6816448 .666969 \\
\hline $\mathrm{H}$ & 11.9424253 .56440611 .838302 \\
\hline $\mathrm{H}$ & 12.3984226 .01086010 .249763 \\
\hline $\mathrm{H}$ & 14.6422005 .00709410 .697423 \\
\hline $\mathrm{H}$ & 14.0359383 .38261810 .198795 \\
\hline $\mathrm{H}$ & 14.3344525 .6401998 .521380 \\
\hline $\mathrm{N}$ & 10.8944655 .1197388 .476646 \\
\hline $\mathrm{C}$ & 10.0526234 .6871937 .386597 \\
\hline $\mathrm{C}$ & 10.5711645 .1467296 .021633 \\
\hline $\mathrm{O}$ & 10.6086926 .3580685 .709926 \\
\hline $\mathrm{C}$ & 8.5838215 .1622997 .592956 \\
\hline $\mathrm{C}$ & 7.6950274 .6061736 .421975 \\
\hline $\mathrm{O}$ & 7.6995833 .3949906 .131082 \\
\hline $\mathrm{N}$ & 7.0088585 .5030105 .689552 \\
\hline $\mathrm{H}$ & 11.0226816 .1204938 .609193 \\
\hline $\mathrm{H}$ & 10.0932843 .5907527 .407120 \\
\hline $\mathrm{H}$ & 8.2152054 .8010168 .564057 \\
\hline $\mathrm{H}$ & 8.5502436 .2696767 .577599 \\
\hline $\mathrm{H}$ & 6.4341665 .2431584 .888643 \\
\hline
\end{tabular}

$\mathrm{H} \quad 7.0956096 .4873055 .919811$

$\mathrm{N} \quad 10.9786014 .2126265 .135255$

C $\quad 11.3131874 .4838443 .751760$

C $\quad 10.2923123 .9288792 .759159$

O $\quad 10.3718424 .2146381 .565858$

C $\quad 12.7291333 .9376393 .416582$

C $\quad 13.8459894 .7545394 .045545$

C $\quad 15.2120144 .3183143 .426087$

C $\quad 16.2731795 .3615353 .730735$

$\mathrm{N} \quad 16.4400755 .4697815 .241489$

$\mathrm{H} \quad 11.0440173 .2463825 .456117$

$\mathrm{H} \quad 11.3378585 .5775103 .619475$

$\mathrm{H} \quad 12.7886832 .8883933 .774412$

$\mathrm{H} \quad 12.8655223 .9376992 .325239$

$\mathrm{H} \quad 13.6875025 .8296913 .846757$

$\mathrm{H} \quad 13.8560444 .5943655 .141085$

$\mathrm{H} \quad 15.5158693 .3400893 .847426$

$\mathrm{H} \quad 15.1042324 .2264592 .332467$

$\mathrm{H} \quad 17.2483585 .0872453 .299486$

$\mathrm{H} \quad 15.9637816 .3414613 .333052$

$\mathrm{H} \quad 15.5943945 .9037905 .670472$

$\mathrm{H} \quad 16.5597984 .5274775 .661332$

$\mathrm{H} \quad 17.2635996 .0452275 .497099$

$\mathrm{N} \quad 9.3367143 .1077913 .263953$

C $\quad 8.3182012 .4411442 .468820$

C $\quad 6.9663753 .1618712 .544334$

O $\quad 6.8820004 .4010562 .666983$

$\mathrm{H} \quad 9.1686993 .0468994 .269411$

$\mathrm{H} \quad 8.6585772 .3882421 .422538$

$\mathrm{H} \quad 8.1999071 .4096262 .848907$

$\mathrm{N} \quad 5.8705162 .3514422 .515139$

C $\quad 4.5264182 .8203032 .582650$

C $\quad 3.8431952 .2102893 .860198$

O $\quad 3.8492280 .9795494 .014744$

C $\quad 3.7411022 .4724801 .303992$

$\mathrm{H} \quad 5.9820091 .3400492 .432244$

$\mathrm{H} \quad 4.5486753 .9138062 .703755$

$\mathrm{H} \quad 2.7019592 .8300551 .380278$

$\mathrm{H} \quad 4.2163542 .9624300 .438050$

$\mathrm{H} \quad 3.7334461 .3798301 .161760$

$\mathrm{N} \quad 3.1851883 .0318154 .690739$

C $\quad 2.3442922 .6143525 .784499$

C $\quad 0.9917563 .2793015 .534501$

O $\quad 0.9222704 .5408415 .641338$

C $\quad 2.8878492 .9212767 .200654$

C $\quad 4.3619392 .4647757 .403519$

C $\quad 1.9604792 .3488708 .267906$

C $\quad 5.0014242 .8582718 .761804$

$\mathrm{H} \quad 3.2389624 .0399704 .542829$

$\mathrm{H} \quad 2.2362021 .5203985 .715869$

$\mathrm{H} \quad 2.8921064 .0277947 .290221$

$\mathrm{H} \quad 4.4017491 .3607727 .322961$

$\mathrm{H} \quad 4.9838572 .8908026 .598045$

$\mathrm{H} \quad 2.2796002 .6166259 .287158$

$\mathrm{H} \quad 0.9231252 .7015678 .146185$

$\mathrm{H} \quad 1.9570661 .2414648 .202653$

$\mathrm{H} \quad 6.0749002 .6024278 .759838$

$\mathrm{H} \quad 4.8926103 .9465078 .932992$

$\mathrm{H} \quad 4.5088482 .3296399 .594899$

$\mathrm{N} \quad-0.0403492 .5182625 .163190$

C $\quad-1.2939853 .0785994 .600425$

C $\quad-2.4942752 .4593955 .323117$

O $\quad-2.5833001 .1965645 .369236$

C $\quad-1.4281472 .8059683 .068218$

C $\quad-0.1300193 .0812612 .257412$ 


\begin{tabular}{|c|c|}
\hline $\mathrm{C}$ & -2.6393753 .5642552 .490863 \\
\hline $\mathrm{C}$ & 0.2444654 .5459022 .059265 \\
\hline $\mathrm{H}$ & -0.0143721 .5074075 .267947 \\
\hline $\mathrm{H}$ & -1.2522054 .1569714 .802839 \\
\hline $\mathrm{H}$ & -1.6122981 .7216792 .977825 \\
\hline $\mathrm{H}$ & 0.7178282 .5869972 .769359 \\
\hline $\mathrm{H}$ & -0.2419122 .6191511 .260357 \\
\hline $\mathrm{H}$ & -2.6723833 .5768041 .393600 \\
\hline $\mathrm{H}$ & -3.5824543 .1186682 .847521 \\
\hline $\mathrm{H}$ & -2.6188874 .6248662 .804791 \\
\hline $\mathrm{H}$ & 1.2506944 .6127251 .606699 \\
\hline $\mathrm{H}$ & -0.4915665 .0290991 .394346 \\
\hline $\mathrm{H}$ & 0.2494575 .0742083 .027391 \\
\hline $\mathrm{N}$ & -3.3899793 .2881495 .910775 \\
\hline $\mathrm{C}$ & -4.6158182 .8075916 .529753 \\
\hline $\mathrm{C}$ & -5.8333143 .3745865 .735086 \\
\hline $\mathrm{O}$ & -5.9420734 .6073405 .597364 \\
\hline $\mathrm{H}$ & -3.2679624 .2985025 .907573 \\
\hline $\mathrm{H}$ & -4.6057041 .7108726 .570794 \\
\hline $\mathrm{H}$ & -4.6769113 .1970997 .562593 \\
\hline $\mathrm{N}$ & -6.7331382 .4810145 .274586 \\
\hline $\mathrm{C}$ & -7.9603742 .8478074 .572462 \\
\hline $\mathrm{C}$ & -9.2762552 .3063665 .271939 \\
\hline $\mathrm{O}$ & -9.1067371 .4227416 .141454 \\
\hline $\mathrm{C}$ & -7.9701052 .2886523 .138151 \\
\hline $\mathrm{C}$ & -6.8297232 .6731742 .153089 \\
\hline $\mathrm{C}$ & -5.7441781 .5772142 .178662 \\
\hline $\mathrm{C}$ & -7.4057112 .8498900 .735118 \\
\hline $\mathrm{O}$ & -10.3543332 .7900484 .859267 \\
\hline $\mathrm{H}$ & -6.5207381 .4881905 .337194 \\
\hline $\mathrm{H}$ & -7.9700583 .9476624 .557971 \\
\hline $\mathrm{H}$ & -7.9739241 .1808173 .219292 \\
\hline $\mathrm{H}$ & -8.9379002 .5885602 .704834 \\
\hline $\mathrm{H}$ & -6.3708983 .6358452 .454800 \\
\hline $\mathrm{H}$ & -4.8493201 .8583811 .595784 \\
\hline $\mathrm{H}$ & -5.4085961 .3728683 .210032 \\
\hline $\mathrm{H}$ & -6.1381980 .6347961 .753865 \\
\hline $\mathrm{H}$ & $-6.5997353 .017087-0.004768$ \\
\hline $\mathrm{H}$ & -7.9601061 .9394210 .434964 \\
\hline $\mathrm{H}$ & -8.1014353 .7060780 .691954 \\
\hline $\mathrm{N}$ & 4.5213924 .80331218 .027263 \\
\hline $\mathrm{C}$ & 5.6166794 .37900718 .949606 \\
\hline $\mathrm{C}$ & 5.4196915 .04648020 .426589 \\
\hline $\mathrm{O}$ & 4.6792536 .04659020 .472109 \\
\hline $\mathrm{C}$ & 6.9679734 .82576418 .392973 \\
\hline $\mathrm{C}$ & 7.3493784 .17687917 .068835 \\
\hline $\mathrm{O}$ & 7.4068402 .94113516 .943841 \\
\hline $\mathrm{O}$ & 6.0270894 .48479921 .370311 \\
\hline $\mathrm{H}$ & 4.3782665 .80141417 .934833 \\
\hline $\mathrm{H}$ & 5.6118403 .28481118 .999478 \\
\hline $\mathrm{H}$ & 7.7412984 .55230019 .131172 \\
\hline $\mathrm{H}$ & 7.0006075 .92524118 .281754 \\
\hline $\mathrm{O}$ & 13.7825897 .0577606 .967910 \\
\hline $\mathrm{H}$ & 14.1022537 .8154917 .479989 \\
\hline $\mathrm{H}$ & 12.8522917 .2722996 .782203 \\
\hline $\mathrm{O}$ & 5.6649105 .25063024 .070671 \\
\hline $\mathrm{H}$ & 6.4726685 .72214424 .305663 \\
\hline $\mathrm{H}$ & 5.7674465 .05610723 .104328 \\
\hline $\mathrm{O}$ & 11.8935836 .3079950 .424151 \\
\hline $\mathrm{H}$ & 11.3146055 .5971170 .781471 \\
\hline $\mathrm{H}$ & $12.0013846 .075724-0.508829$ \\
\hline $\mathrm{O}$ & 14.5496916 .4508881 .140632 \\
\hline $\mathrm{H}$ & 13.5796536 .5138320 .973627 \\
\hline $\mathrm{H}$ & 14.9264536 .3207530 .260313 \\
\hline
\end{tabular}

\section{Quantum refinement with $\mathrm{LSQC} \omega_{\alpha}=\mathbf{1 . 0}$}

$\mathrm{N}$

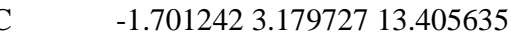

C $\quad-0.4115213 .98648413 .232711$

O $\quad-0.4410325 .22871213 .324690$

C $\quad-2.1760882 .62025412 .014631$

C $\quad-2.3040503 .66789910 .872859$

C $\quad-3.5044294 .36232610 .646555$

C $\quad-1.2175323 .92625210 .013171$

C $\quad-3.6171645 .3073249 .606692$

C $\quad-1.3210554 .9113608 .992588$

C $\quad-2.5280785 .5748258 .791607$

$\mathrm{H} \quad-1.5134052 .33322414 .072541$

$\mathrm{H} \quad-1.4601221 .88348711 .675393$

$\mathrm{H} \quad-3.1558112 .14507112 .143097$

$\mathrm{H} \quad-4.3680564 .16474911 .279689$

$\mathrm{H} \quad-0.2912743 .36867510 .121934$

$\mathrm{H} \quad-4.5586325 .8270679 .454024$

$\mathrm{H} \quad-0.4725645 .1518388 .359619$

$\mathrm{H} \quad-2.6055386 .3048027 .989192$

$\mathrm{H} \quad-3.6226253 .52742814 .191341$

$\mathrm{H} \quad-2.4137964 .44901015 .149284$

$\mathrm{H} \quad-2.9344054 .85790513 .428883$

$\mathrm{N} \quad 0.7244513 .28197412 .997088$

C $\quad 2.0479533 .94545012 .877029$

C $\quad 2.3476124 .80538914 .160973$

O $\quad 2.4368326 .03283114 .162611$

C $\quad 2.0988804 .82168011 .599833$

$\mathrm{H} \quad 0.6949592 .27352612 .931018$

$\mathrm{H} \quad 2.7982903 .15068412 .824769$

$\mathrm{H} \quad 3.0727855 .31718711 .532470$

$\mathrm{H} \quad 1.9515414 .19971110 .712417$

$\mathrm{H} \quad 1.3250915 .58586611 .652511$

$\mathrm{N} \quad 2.4562994 .08012915 .281586$

C $\quad 2.4836374 .57734816 .677017$

C $\quad 3.6566393 .96288117 .433953$

O $\quad 3.6965462 .71694017 .495797$

C $\quad 1.2056254 .22937917 .491298$

C $\quad-0.0811034 .83665516 .953007$

C $\quad-1.3607614 .06887217 .346527$

O $\quad-1.3792433 .38037518 .377212$

O $\quad-2.3716084 .22592816 .532248$

$\mathrm{H} \quad 2.4939023 .06631015 .230694$

$\mathrm{H} \quad 2.6095405 .65895716 .571395$

$\mathrm{H} \quad 1.0984203 .14052917 .515246$

$\mathrm{H} \quad 1.3525914 .56595218 .523915$

$\mathrm{H} \quad-0.1897375 .86975517 .308720$

$\mathrm{H} \quad-0.0341904 .88224215 .858609$

$\mathrm{N} \quad 7.6375145 .01298316 .021253$

C $\quad 8.1240914 .55370414 .707385$

C $\quad 9.5363615 .13202714 .483442$

O $\quad 9.7048786 .38356714 .496895$

C $\quad 7.1854764 .95205213 .533228$

C $\quad 7.7622674 .46248212 .189930$

C $\quad 5.7437044 .43445913 .725655$

$\mathrm{H} \quad 7.6090626 .01565916 .153026$

$\mathrm{H} \quad 8.1601823 .46051714 .758285$

$\mathrm{H} \quad 7.1677906 .05030613 .532028$

$\mathrm{H} \quad 7.0258854 .58650711 .386670$

$\mathrm{H} \quad 8.6772844 .99067311 .899646$

$\mathrm{H} \quad 8.0004893 .39017312 .232460$

$\mathrm{H} \quad 5.1202884 .73257712 .876948$

$\mathrm{H} \quad 5.7449853 .33673613 .775364$ 


\begin{tabular}{|c|c|}
\hline $\mathrm{H}$ & 5.2860754 .79926814 .645604 \\
\hline $\mathrm{N}$ & 10.5730564 .29089914 .329362 \\
\hline $\mathrm{C}$ & 11.9199574 .79669514 .200665 \\
\hline $\mathrm{C}$ & 12.1696595 .41044912 .784894 \\
\hline $\mathrm{O}$ & 12.3692966 .63766912 .659714 \\
\hline $\mathrm{H}$ & 10.4326183 .29003514 .354821 \\
\hline $\mathrm{H}$ & 12.1210995 .58953314 .920276 \\
\hline $\mathrm{H}$ & 12.6022483 .95578014 .363467 \\
\hline $\mathrm{N}$ & 12.1685624 .54872711 .688496 \\
\hline $\mathrm{C}$ & 12.4995804 .92578810 .342314 \\
\hline $\mathrm{C}$ & 11.5607744 .2736679 .320255 \\
\hline $\mathrm{O}$ & 11.3522713 .0487839 .286105 \\
\hline $\mathrm{C}$ & 13.9513354 .47193710 .011277 \\
\hline $\mathrm{O}$ & 14.2869944 .6914468 .651153 \\
\hline $\mathrm{H}$ & 11.9236203 .57465611 .825962 \\
\hline $\mathrm{H}$ & 12.4186606 .01361310 .257285 \\
\hline $\mathrm{H}$ & 14.6428694 .99824010 .683297 \\
\hline $\mathrm{H}$ & 14.0372193 .39381110 .176150 \\
\hline $\mathrm{H}$ & 14.3270065 .6522258 .500851 \\
\hline $\mathrm{N}$ & 10.8963565 .1214898 .474856 \\
\hline $\mathrm{C}$ & 10.0524564 .6813607 .385746 \\
\hline $\mathrm{C}$ & 10.5717385 .1467426 .021751 \\
\hline $\mathrm{O}$ & 10.6107536 .3620445 .712336 \\
\hline $\mathrm{C}$ & 8.5861375 .1607617 .599098 \\
\hline $\mathrm{C}$ & 7.6974104 .6096186 .420469 \\
\hline $\mathrm{O}$ & 7.6992933 .3938326 .129943 \\
\hline $\mathrm{N}$ & 7.0062065 .5031965 .689261 \\
\hline $\mathrm{H}$ & 11.0202256 .1181108 .588238 \\
\hline $\mathrm{H}$ & 10.0826163 .5914897 .407867 \\
\hline $\mathrm{H}$ & 8.2244504 .8014958 .562627 \\
\hline $\mathrm{H}$ & 8.5575496 .2559467 .591480 \\
\hline $\mathrm{H}$ & 6.4535385 .2133194 .892534 \\
\hline $\mathrm{H}$ & 7.0737976 .4907245 .888693 \\
\hline $\mathrm{N}$ & 10.9762884 .2113245 .134861 \\
\hline $\mathrm{C}$ & 11.3144144 .4858703 .750620 \\
\hline $\mathrm{C}$ & 10.2898463 .9242692 .761153 \\
\hline $\mathrm{O}$ & 10.3687264 .2059581 .562432 \\
\hline $\mathrm{C}$ & 12.7300363 .9454243 .399914 \\
\hline $\mathrm{C}$ & 13.8424334 .7719234 .029803 \\
\hline $\mathrm{C}$ & 15.2070154 .3414813 .412006 \\
\hline $\mathrm{C}$ & 16.2679605 .3805683 .715185 \\
\hline $\mathrm{N}$ & 16.4308905 .4859715 .237906 \\
\hline $\mathrm{H}$ & 11.0579713 .2518665 .453719 \\
\hline $\mathrm{H}$ & 11.3218565 .5718393 .625963 \\
\hline $\mathrm{H}$ & 12.7987852 .9038043 .746515 \\
\hline $\mathrm{H}$ & 12.8575473 .9472072 .313358 \\
\hline $\mathrm{H}$ & 13.6758855 .8388463 .838801 \\
\hline $\mathrm{H}$ & 13.8514704 .6090915 .116761 \\
\hline $\mathrm{H}$ & 15.5115743 .3689503 .824834 \\
\hline $\mathrm{H}$ & 15.1062864 .2538302 .326542 \\
\hline $\mathrm{H}$ & 17.2379845 .1094043 .293956 \\
\hline $\mathrm{H}$ & 15.9649426 .3564833 .329089 \\
\hline $\mathrm{H}$ & 15.5921415 .9172555 .666444 \\
\hline $\mathrm{H}$ & 16.5443474 .5536005 .654899 \\
\hline $\mathrm{H}$ & 17.2469906 .0517175 .491151 \\
\hline $\mathrm{N}$ & 9.3361493 .1054493 .269453 \\
\hline $\mathrm{C}$ & 8.3183802 .4403762 .469585 \\
\hline $\mathrm{C}$ & 6.9679233 .1609282 .551000 \\
\hline $\mathrm{O}$ & 6.8848594 .4047772 .673814 \\
\hline $\mathrm{H}$ & 9.1888763 .0601794 .272254 \\
\hline $\mathrm{H}$ & 8.6622362 .4001431 .431413 \\
\hline $\mathrm{H}$ & 8.2133681 .4117492 .833942 \\
\hline $\mathrm{N}$ & 5.8715972 .3522022 .520611 \\
\hline $\mathrm{C}$ & 4.5257012 .8268472 .583705 \\
\hline $\mathrm{C}$ & 3.8455392 .2115013 .861513 \\
\hline
\end{tabular}

\begin{tabular}{|c|c|}
\hline $\mathrm{O}$ & 3.8485960 .9759664 .010672 \\
\hline $\mathrm{C}$ & 3.7345532 .4654491 .312151 \\
\hline $\mathrm{H}$ & 5.9828061 .3457852 .455264 \\
\hline $\mathrm{H}$ & 4.5557803 .9138042 .691323 \\
\hline $\mathrm{H}$ & 2.7019442 .8192631 .392221 \\
\hline $\mathrm{H}$ & 4.1986452 .9408970 .442971 \\
\hline $\mathrm{H}$ & 3.7186061 .3791321 .180402 \\
\hline $\mathrm{N}$ & 3.1844853 .0309394 .690633 \\
\hline $\mathrm{C}$ & 2.3429532 .6080045 .783789 \\
\hline $\mathrm{C}$ & 0.9899293 .2756435 .532445 \\
\hline $\mathrm{O}$ & 0.9220984 .5420115 .644195 \\
\hline $\mathrm{C}$ & 2.8842022 .9198007 .201238 \\
\hline $\mathrm{C}$ & 4.3627582 .4683007 .400781 \\
\hline $\mathrm{C}$ & 1.9591762 .3481958 .274583 \\
\hline $\mathrm{C}$ & 5.0053912 .8483958 .762170 \\
\hline $\mathrm{H}$ & 3.2242264 .0339044 .548757 \\
\hline $\mathrm{H}$ & 2.2451521 .5204405 .708369 \\
\hline $\mathrm{H}$ & 2.8781834 .0169787 .293534 \\
\hline $\mathrm{H}$ & 4.4054231 .3741177 .311007 \\
\hline $\mathrm{H}$ & 4.9739312 .8952136 .598649 \\
\hline $\mathrm{H}$ & 2.2876002 .6274449 .278555 \\
\hline $\mathrm{H}$ & 0.9271032 .6932668 .154473 \\
\hline $\mathrm{H}$ & 1.9590321 .2487878 .217824 \\
\hline $\mathrm{H}$ & 6.0717262 .5964768 .750087 \\
\hline $\mathrm{H}$ & 4.8984893 .9257968 .948858 \\
\hline $\mathrm{H}$ & 4.5255972 .3171959 .590900 \\
\hline $\mathrm{N}$ & -0.0439762 .5155925 .168071 \\
\hline $\mathrm{C}$ & -1.2965683 .0942104 .602165 \\
\hline $\mathrm{C}$ & -2.4965692 .4608535 .318291 \\
\hline $\mathrm{O}$ & -2.5841951 .1928265 .362702 \\
\hline $\mathrm{C}$ & -1.4263792 .8097253 .078014 \\
\hline $\mathrm{C}$ & -0.1302303 .0824312 .271532 \\
\hline $\mathrm{C}$ & -2.6404273 .5467542 .475072 \\
\hline $\mathrm{C}$ & 0.2397774 .5443782 .058120 \\
\hline $\mathrm{H}$ & -0.0301941 .5069515 .248964 \\
\hline $\mathrm{H}$ & -1.2513244 .1647504 .806983 \\
\hline $\mathrm{H}$ & -1.6095771 .7312923 .003427 \\
\hline $\mathrm{H}$ & 0.7105012 .5993062 .789236 \\
\hline $\mathrm{H}$ & -0.2378042 .6075811 .287754 \\
\hline $\mathrm{H}$ & -2.6528703 .4989901 .384927 \\
\hline $\mathrm{H}$ & -3.5764723 .1059152 .831495 \\
\hline $\mathrm{H}$ & -2.6328894 .6060232 .760108 \\
\hline $\mathrm{H}$ & 1.2394144 .6099201 .610954 \\
\hline $\mathrm{H}$ & -0.4855765 .0203451 .388545 \\
\hline $\mathrm{H}$ & 0.2442995 .0871373 .010441 \\
\hline $\mathrm{N}$ & -3.3872173 .2897915 .914213 \\
\hline $\mathrm{C}$ & -4.6165682 .8084046 .530799 \\
\hline $\mathrm{C}$ & -5.8341093 .3704975 .731490 \\
\hline $\mathrm{O}$ & -5.9387924 .6108835 .596889 \\
\hline $\mathrm{H}$ & -3.2741594 .2918175 .877646 \\
\hline $\mathrm{H}$ & -4.5834811 .7194846 .584180 \\
\hline $\mathrm{H}$ & -4.6815433 .2035317 .551695 \\
\hline $\mathrm{N}$ & -6.7314192 .4840105 .265255 \\
\hline $\mathrm{C}$ & -7.9598072 .8528634 .556617 \\
\hline $\mathrm{C}$ & -9.2861702 .2974495 .252243 \\
\hline $\mathrm{O}$ & -9.0937181 .4035166 .113920 \\
\hline $\mathrm{C}$ & -7.9658312 .2789973 .125244 \\
\hline $\mathrm{C}$ & -6.8222122 .6570852 .142747 \\
\hline $\mathrm{C}$ & -5.7345801 .5593232 .171521 \\
\hline $\mathrm{C}$ & -7.3965352 .8266030 .722335 \\
\hline $\mathrm{O}$ & -10.3610112 .7841744 .821961 \\
\hline $\mathrm{H}$ & -6.5378061 .4937375 .338297 \\
\hline $\mathrm{H}$ & -7.9672003 .9431144 .533471 \\
\hline $\mathrm{H}$ & -7.9730011 .1794943 .212426 \\
\hline $\mathrm{H}$ & -8.9288212 .5725492 .696995 \\
\hline
\end{tabular}




$\begin{array}{llll}\mathrm{H} & -6.366239 & 3.613131 & 2.439036 \\ \mathrm{H} & -4.848017 & 1.836867 & 1.588452 \\ \mathrm{H} & -5.404778 & 1.363319 & 3.198205 \\ \mathrm{H} & -6.127762 & 0.622317 & 1.755250 \\ \mathrm{H} & -6.597703 & 2.988425 & -0.015611 \\ \mathrm{H} & -7.947911 & 1.9230750 .428695 \\ \mathrm{H} & -8.0863953 .677249 & 0.680411 \\ \mathrm{~N} & 4.5234664 .80299818 .023868 \\ \mathrm{C} & 5.6169174 .37715818 .953522 \\ \mathrm{C} & 5.4006355 .04320020 .429822 \\ \mathrm{O} & 4.6341476 .03181620 .476740 \\ \mathrm{C} & 6.9688404 .82929118 .392583 \\ \mathrm{C} & 7.3518194 .17938717 .066116 \\ \mathrm{O} & 7.4096562 .94019816 .939514 \\ \mathrm{O} & 6.0243024 .48313221 .370648 \\ \mathrm{H} & 4.3766395 .80016617 .957981\end{array}$

$\mathrm{H} \quad 5.6187443 .29009918 .997136$

$\mathrm{H} \quad 7.7353364 .55778419 .128749$

$\mathrm{H} \quad 6.9923415 .92439018 .289776$

O $\quad 13.7597007 .0541106 .977700$

$\mathrm{H} \quad 13.9278647 .8875097 .447140$

$\mathrm{H} \quad 12.8135277 .0748236 .743593$

O $\quad 5.8599775 .08829824 .126614$

H $\quad 6.4120305 .88355124 .143289$

$\mathrm{H} \quad 5.7738634 .91530923 .151545$

O $\quad 11.8721836 .2900000 .405119$

$\mathrm{H} \quad 11.3323935 .5822980 .828914$

$\mathrm{H} \quad 11.8198906 .100902-0.544137$

O $\quad 14.5304386 .4512591 .125312$

$\mathrm{H} \quad 13.5591816 .4570500 .938050$

H $\quad 14.9297636 .4238920 .242888$ 